NATIONAL LABORATORY

FOR THE DEPARTMENT OF ENERGY

\title{
Development of a SCALE Model for High Flux Isotope Reactor Cycle 400
}

\section{February 2012}

\section{Prepared by}

Dan Ilas

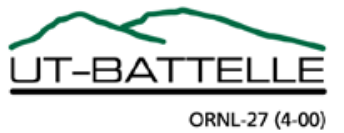




\section{DOCUMENT AVAILABILITY}

Reports produced after January 1, 1996, are generally available free via the U.S. Department of Energy (DOE) Information Bridge.

Web site http://www.osti.gov/bridge

Reports produced before January 1, 1996, may be purchased by members of the public from the following source.

National Technical Information Service

5285 Port Royal Road

Springfield, VA 22161

Telephone 703-605-6000 (1-800-553-6847)

TDD 703-487-4639

Fax 703-605-6900

E-mail info@ntis.gov

Web site http://www.ntis.gov/support/ordernowabout.htm

Reports are available to DOE employees, DOE contractors, Energy Technology Data Exchange (ETDE) representatives, and International Nuclear Information System (INIS) representatives from the following source.

Office of Scientific and Technical Information

P.O. Box 62

Oak Ridge, TN 37831

Telephone 865-576-8401

Fax 865-576-5728

E-mail reports@osti.gov

Web site http://www.osti.gov/contact.html

This report was prepared as an account of work sponsored by an agency of the United States Government. Neither the United States Government nor any agency thereof, nor any of their employees, makes any warranty, express or implied, or assumes any legal liability or responsibility for the accuracy, completeness, or usefulness of any information, apparatus, product, or process disclosed, or represents that its use would not infringe privately owned rights. Reference herein to any specific commercial product, process, or service by trade name, trademark, manufacturer, or otherwise, does not necessarily constitute or imply its endorsement, recommendation, or favoring by the United States Government or any agency thereof. The views and opinions of authors expressed herein do not necessarily state or reflect those of the United States Government or any agency thereof. 


\title{
DEVELOPMENT OF A SCALE MODEL FOR HIGH FLUX ISOTOPE REACTOR CYCLE 400
}

\author{
Dan Ilas
}

Date Published: February 2012

\author{
Prepared by \\ OAK RIDGE NATIONAL LABORATORY \\ Oak Ridge, Tennessee 37831-6283 \\ managed by \\ UT-BATTELLE, LLC \\ for the \\ U.S. DEPARTMENT OF ENERGY \\ under contract DE-AC05-00OR22725
}





\section{CONTENTS}

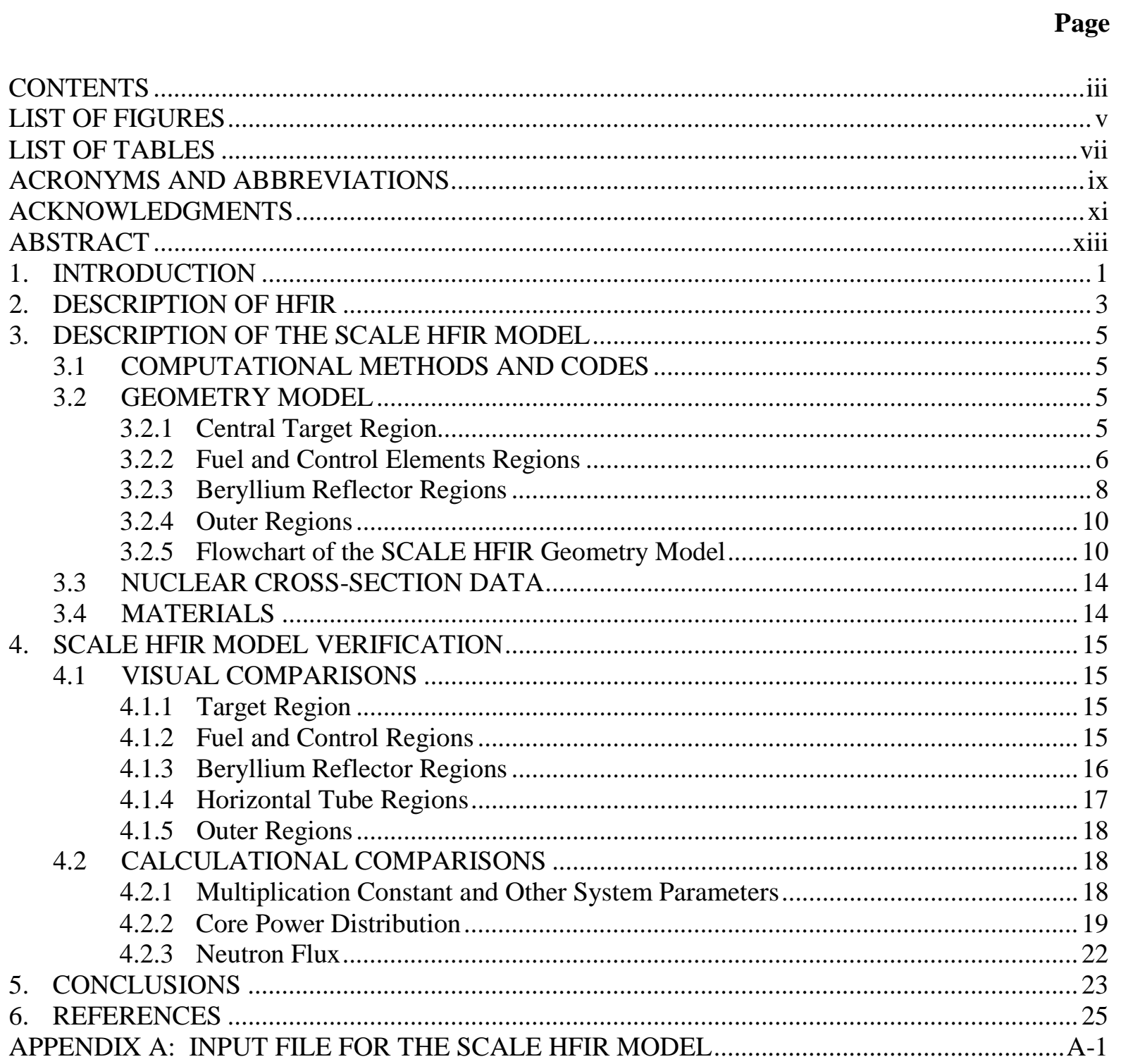




\section{LIST OF FIGURES}

Figure $\quad$ Page

$1 \quad$ HFIR schematic at core midplane (before removal of two engineering facilities)................ 3

2 Numbering scheme for the central target region (radial view) in the SCALE HFIR

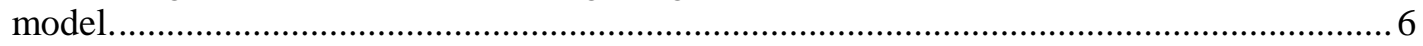

$3 \quad$ Fuel and control regions (axial view) in the SCALE HFIR model................................... 8

$4 \quad$ Beryllium reflector at the horizontal midplane in the Scale HFIR model............................ 9

$5 \quad$ Main outer core components of the SCALE HFIR model............................................... 10

$6 \quad$ Flowchart of the HFIR Scale model (fuel and central target regions). .............................. 12

$7 \quad$ Flowchart of the HFIR Scale model (outside the fuel region)........................................... 13

8 A screenshot of the material block showing several features of the material

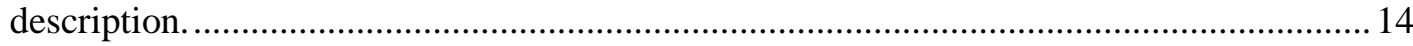

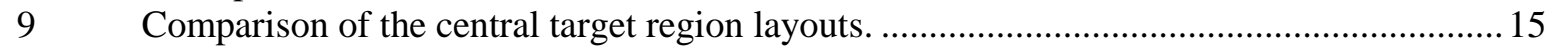

10 Comparison of the fuel and control regions.................................................................. 16

11 Beryllium reflector regions with irradiation facilities, horizontal tubes, and

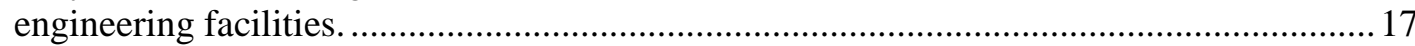

12 Comparison of the HT-4 tip (cold source tube) models................................................... 17

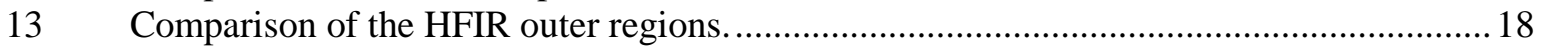

14 Relative $v \Sigma_{\mathrm{f}}$ reaction rate density distributions in IFE and OFE of HFIR. ......................... 19

15 Comparison of relative $v \Sigma_{\mathrm{f}}$ reaction rate density distribution predicted by the SCALE HFIR model and relative fission density distribution predicted by the

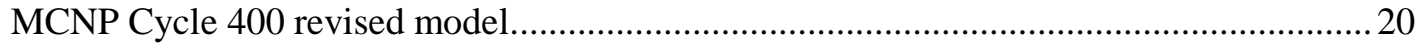




\section{LIST OF TABLES}

Table

Page

$1 \quad$ Radial fuel regions in the SCALE HFIR model ......................................................... 7

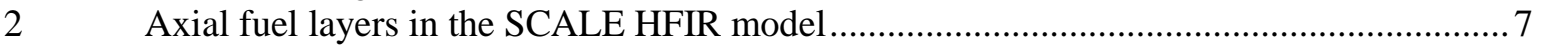

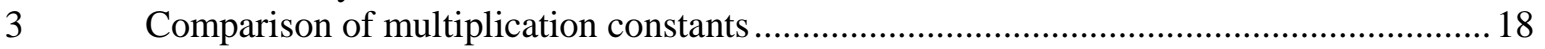

$4 \quad$ Comparison of HFIR system parameters....................................................................... 19

$5 \quad$ Relative $v \Sigma_{\mathrm{f}}$ reaction rate densities predicted by the SCALE HFIR model........................ 21

$6 \quad$ Neutron flux comparison between the SCALE and MCNP models.................................. 22 


\section{ACRONYMS AND ABBREVIATIONS}

$\begin{array}{ll}\text { 2-D } & \text { two dimensional } \\ \text { 3-D } & \text { three dimensional } \\ \text { BOC } & \text { beginning of cycle } \\ \text { CE } & \text { continuous energy } \\ \text { C/E } & \text { calculated to experimental } \\ \text { CR } & \text { control element region } \\ \text { DOE } & \text { U.S. Department of Energy } \\ \text { EOC } & \text { end of cycle } \\ \text { FTT } & \text { flux trap target region } \\ \text { HEU } & \text { high-enriched uranium } \\ \text { HFIR } & \text { High Flux Isotope Reactor } \\ \text { HT } & \text { hydraulic tube } \\ \text { ICE } & \text { inner control element } \\ \text { IFE } & \text { inner fuel element } \\ \text { IRSN } & \text { Institut de Radioprotection et de Sûreté Nucléaire (Institute for Radiological } \\ & \text { Protection and Nuclear Safety) } \\ \text { keff } & \text { effective multiplication constant } \\ \text { LANL } & \text { Los Alamos Nuclear Laboratory } \\ \text { LEU } & \text { low-enriched uranium } \\ \text { OCE } & \text { outer control element } \\ \text { OFE } & \text { outer fuel element } \\ \text { ORNL } & \text { Oak Ridge National Laboratory } \\ \text { pcm } & \text { per cent millirho } \\ \text { PB } & \text { permanent beryllium reflector region } \\ \text { PTP } & \text { peripheral target position } \\ \text { RB } & \text { removable beryllium reflector region } \\ \text { RNSD } & \text { Reactor and Nuclear Systems Division } \\ & \end{array}$




\section{ACKNOWLEDGMENTS}

The author would like to acknowledge the support provided for this project by the Nuclear Science and Engineering Directorate and by the Reactor and Nuclear Systems Division (RNSD). Review of the manuscript by Germina Ilas and Josh Peterson (RNSD), formatting of the document by Hannah Turpin (RNSD), and editing by Amy Harkey (Publishing Services Group) are very much appreciated. 



\begin{abstract}
The development of a comprehensive SCALE computational model for the High Flux Isotope Reactor (HFIR) is documented and discussed in this report. The SCALE model has equivalent features and functionality as the reference MCNP model for Cycle 400 that has been used extensively for HFIR safety analyses and for HFIR experiment design and analyses. Numerical comparisons of the SCALE and MCNP models for the multiplication constant, power density distribution in the fuel, and neutron fluxes at several locations in HFIR indicate excellent agreement between the results predicted with the two models. The SCALE HFIR model is presented in sufficient detail to provide the users of the model with a tool that can be easily customized for various safety analysis or experiment design requirements.
\end{abstract}




\section{INTRODUCTION}

Comprehensive computational neutronic models for the High Flux Isotope Reactor (HFIR) have been developed for the past 10-20 years with numerous developers contributing to this effort. The endeavor culminated with a full HFIR model for the beginning of cycle (BOC) 400, which was developed and documented by Xoubi and Primm in 2004 ${ }^{1}$ and became known as the "Cycle 400 reference model.” The Cycle 400 reference model relied on the older model HFIR-V.2, originally developed by J. C. Gehin, L. A. Smith, and J. A. Bucholz. ${ }^{1}$

The HFIR Cycle 400 reference model is an $\mathrm{MCNP}^{2}$ Monte Carlo model of HFIR that, based on existing documentation and expert opinion, fully describes (most often in terms very close to the physical reality) the central target region, the fueled region, the control elements, and the beryllium reflector, and includes out-of-core components such as irradiation tubes, horizontal tubes, engineering tubes, and the reactor shielding and biological protection, up to an outer radius of $7.20 \mathrm{~m}$ and a total height of $3.00 \mathrm{~m}$. Because of their complexity, the fuel elements required the largest amount of simplification in the model. Homogenization methods were applied in this case to reduce the intricacies of the fuel element's model.

More recently, the Cycle 400 reference model constituted the basis for neutronic and thermalhydraulic computations for the HEU to LEU fuel conversion study for HFIR. ${ }^{3}$ For this latter purpose, the Cycle 400 reference MCNP model was further enhanced by refining the meshing in the fueled region, and then was embedded in a depletion model for use with VESTA ${ }^{4}$ to perform full-core depletion for the duration of the cycle length and analyses at discrete points in time during the fuel cycle. Revisions of the Cycle 400 reference model were made in 2008 and 2010. The 2008 revision corrected a material data card in the beryllium removable reflector. ${ }^{5}$ The 2010 revision added more axial layers (19 instead of 7) in both the inner and the outer fuel elements for depletion purposes and changed the material composition for the control elements to account for the actual irradiation history of the control elements prior to Cycle $400{ }^{6}$ This revised MCNP model will be referred to as the "MCNP Cycle 400 revised model."

Over the past several years, the SCALE computational package ${ }^{7}$, developed and maintained by Oak Ridge National Laboratory (ORNL), has undergone significant development and enhancements in its capabilities. Among these, we can enumerate the following capabilities in the latest release, SCALE 6.1: full, realistic geometry description of complex problems; extension to continuous energy treatment for criticality problems; enhancements in multigroup treatment; full-core depletion capabilities (with multigroup energy treatment); sensitivity and uncertainty analysis capabilities (multigroup); and new or improved nuclear data based primarily on ENDF/B-VII. These capabilities make the SCALE package unique in solving problems that necessitate the coupling of different phenomena in a critical system. With the upcoming enhancements for continuous energy Monte Carlo depletion, shielding, and sensitivity/uncertainty analyses the SCALE package has the potential to become the state of the art in terms of its analysis capabilities for neutronic modeling.

A SCALE model of HFIR with capabilities similar to those of the MCNP Cycle 400 reference model is both possible and desirable. The use of this SCALE model will have a reciprocal advantage for the SCALE developers and the potential users of the model: it allows the current capabilities in SCALE to be benchmarked on a realistic reactor problem of importance to ORNL, and it provides users of the model with a tool that can be easily customized to their needs.

The purpose of the work documented in this report was to develop a SCALE model for HFIR Cycle 400 as an alternative reference that is equivalent to the MCNP model for this same 
configuration. The SCALE model was built, to the extent possible, using the same framework (i.e., no changes) as the MCNP model. Though the similarity of the MCNP and SCALE models was the primary criterion when developing the SCALE model, a secondary criterion focused on developing the SCALE model in a manner that will facilitate future additions, changes, or improvements to the model, such as employing a more modular representation of the configuration.

The MCNP model has been used extensively at HFIR for safety analyses and by HFIR customers for experiment design and analyses. Its performance has been validated against experimental data available from power distributions and destructive isotopic assay measurements. ${ }^{\mathbf{8} 9}$ A SCALE model with equivalent features as the reference MCNP model would facilitate the acceptance of and transition to this new model for HFIR users who are familiar with the MCNP model.

The SCALE model that has been developed for the HFIR is described in this report, and its accuracy is assessed by comparisons with results obtained for the MCNP Cycle 400 revised model. Being based on the MCNP Cycle 400 revised model, the SCALE HFIR model inherits its capability for further use in depletion simulations.

This report is organized as follows. Section 2 contains a brief description of the HFIR. Section 3 describes the SCALE model for HFIR, with a focus on the geometry. Section 4 compares results obtained with the SCALE and the MCNP models. Finally, Section 5 presents a few conclusions. The SCALE input file for the HFIR model is listed as Appendix A. 


\section{DESCRIPTION OF HFIR}

HFIR is a research reactor at ORNL that supports isotope production, material irradiation research, and neutron scattering experiments. It currently operates at $85 \mathrm{MW}(\mathrm{t})$ power. The central flux trap in HFIR provides one of the highest steady-state thermal neutron fluxes among research reactors in the world. ${ }^{\mathbf{1 0}}$

The reactor core consists of a series of concentric regions: the central target region (flux trap), two fuel elements separated by a thin water region, the control elements region, the beryllium reflector, and a water region to the edge of the pressure vessel. The reactor is located in a pool of water. Details of the reactor configuration and operation can be found elsewhere ${ }^{\mathbf{1 1}}$ and also are outlined in the following sections in relation to the SCALE model of the reactor.

There are two fuel elements in HFIR, identified as an inner fuel element (IFE) and an outer fuel element (OFE). They are made up of 171 and 369 involute-shaped thin fuel plates, respectively, and are fuelled with $93.1 \mathrm{wt} \%$ enriched uranium. The fuel plates are separated by water channels.

Because of their particular shape and the fact that the fuel zone in a fuel plate is radially graded, they are most challenging from a modelling point of view. Fig. 1 presents a schematic of HFIR at core midplane, which illustrates the complexity of the reactor geometry. The drawing was made before the removal of two engineering facilities to allow the enlargement of the HB-2 horizontal tube (prior to Cycle 400).

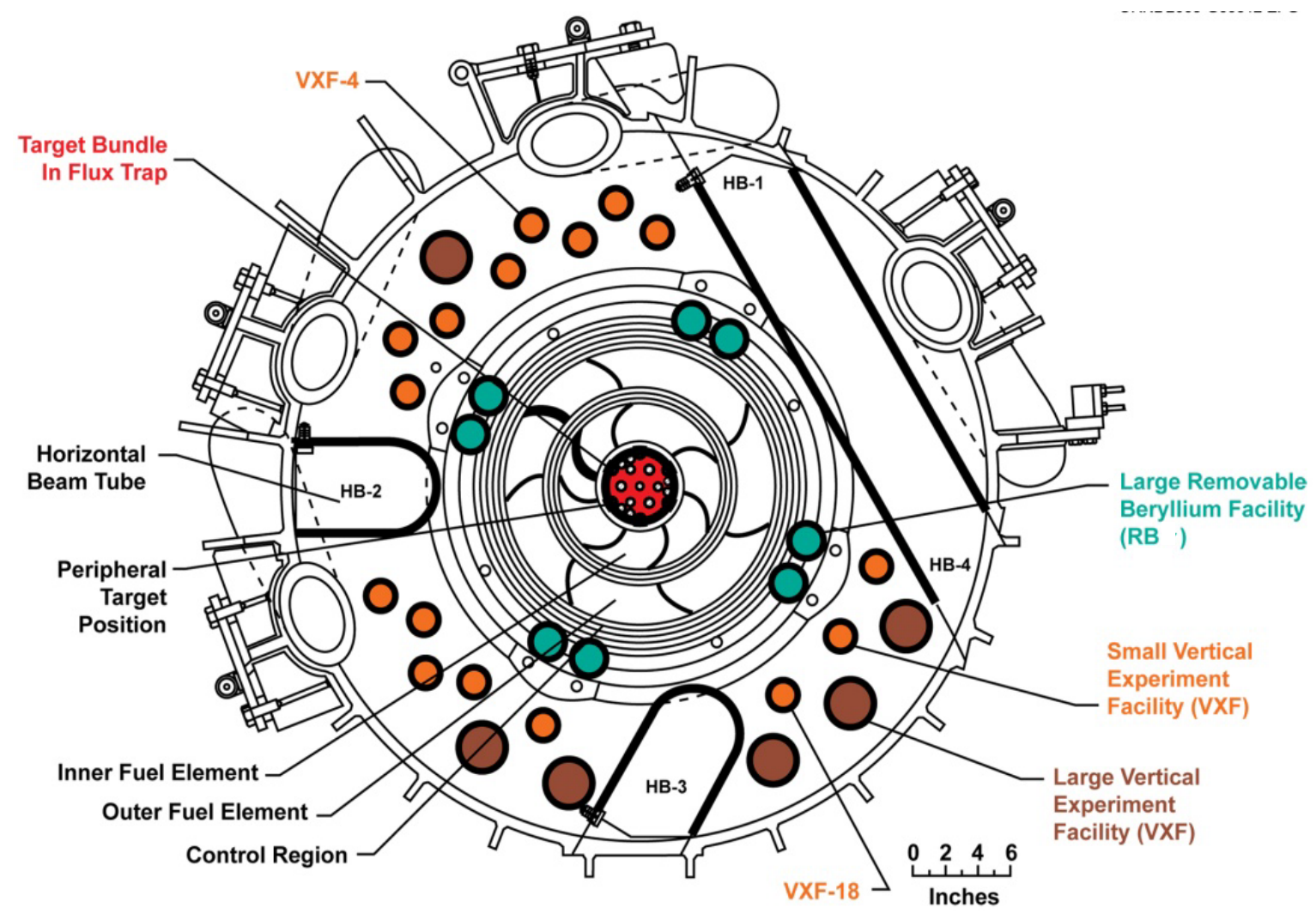

Fig. 1. HFIR schematic at core midplane (before removal of two engineering facilities). 



\section{DESCRIPTION OF THE SCALE HFIR MODEL}

\subsection{COMPUTATIONAL METHODS AND CODES}

The SCALE model for HFIR was developed using the latest release, SCALE 6.1. The SCALE code system uses automated sequences to provide problem-dependent cross-section processing, reactor lattice physics, criticality safety, radiation shielding, and spent fuel characterization analysis capabilities. For this report, the CSAS26/KENO-VI sequence of SCALE with the continuous energy option for neutron transport was used.

The SCALE HFIR model is a large model containing over 10,000 lines of input. Of these, 7,000 are material input lines and $\sim 3,000$ lines contain the geometry model. The input file is heavily commented, containing 1,500 comment lines for easier navigation and identification of the different components of the model to enable user changes or additions to the model, such as may be necessary when a particular target or experiment is inserted into the reactor.

The SCALE model uses the KENO-VI geometry capability, which uses the SCALE generalized geometry package and therefore enables the use of the model as a basis for studies with any other module or sequence in SCALE. The KENO-VI code, a Monte Carlo transport code based on combinational geometry, can accept a wide range of geometry shapes to construct practically any geometric configuration of interest. Because of the particularities of this geometry capability in KENO-VI, there are significant differences between the geometry specifications in the SCALE HFIR model and the MCNP Cycle 400 revised model.

At every step during the development of the SCALE HFIR model, comparisons and checks were performed against the MCNP Cycle 400 revised model. The 5.1.51 version of the MCNP code, developed by Los Alamos National Laboratory, was used for this purpose. MCNP is a generalpurpose Monte Carlo transport code that can simulate the transport of neutrons, photons, and electrons and calculate various quantities of interest for criticality, shielding, and energy deposition studies.

\subsection{GEOMETRY MODEL}

The following sub-sections discuss the geometry of the SCALE HFIR model starting from the center of the HFIR core and moving towards the periphery. A flowchart of the whole model is presented in the last sub-section. The flowchart is intended to facilitate navigation through the geometry of the model and later revisions of the input file.

\subsubsection{Central Target Region}

The central target region (also called the flux trap region) is the central section of the HFIR and includes 37 cylindrical experimental sites arranged in a hexagonal lattice. The arrangement of the target and the labeling scheme is shown in Fig. 2, which illustrates the actual flux trap region in the SCALE HFIR model. Of the 37 experimental sites in the flux trap, 31 are located inside a basket, and 6 are located at the vertices of the hexagonal lattice outside the basket. The experimental sites located outside the basket are identified as peripheral target positions (PTPs), shown as PTP-1 to PTP-6 at locations A-4, D-1, A-1, D-7, G-7, and G-4 in Fig. 2.

The experimental site at location B-3 is occupied by a hydraulic tube (HT) that allows for insertion of experimental specimens during reactor operation. The HT geometry unit, identified as Unit 750 in the SCALE HFIR model, is modeled explicitly to accommodate axially nine specimens, also known as "rabbits". Also shown in Fig. 2 are two stainless steel targets labeled JP-26 and JP-27, 
at locations C-6 and E-2, respectively. The seven dark cylinders at the center of the basket, at locations C-2, D-3, D-4, C-5, E-5, F-5, and E-6, are solid aluminum dummy targets. The other 21 cylindrical units in Fig. 2 are shrouded aluminum dummy targets.

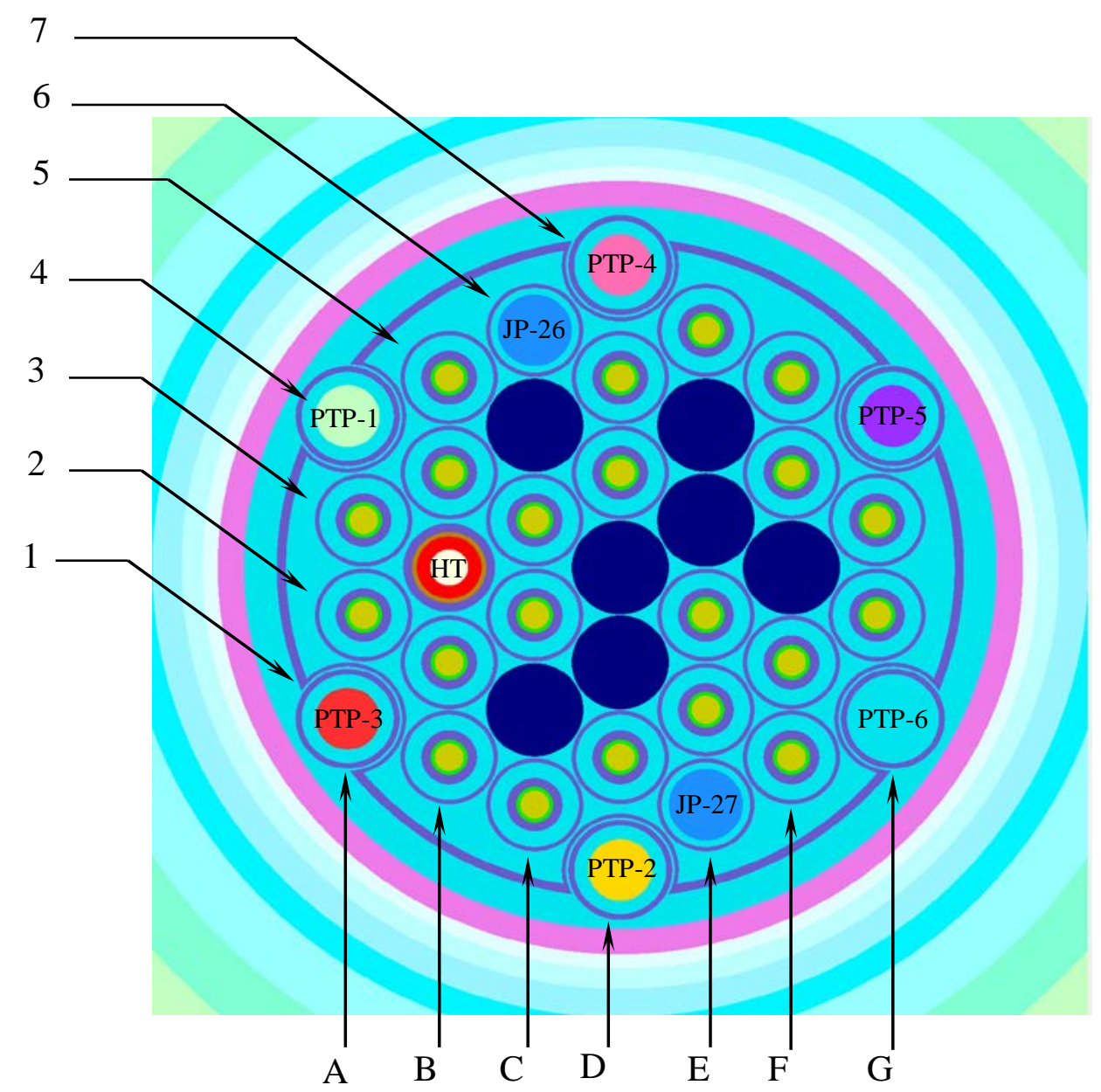

Fig. 2. Numbering scheme for the central target region (radial view) in the SCALE HFIR model.

\subsubsection{Fuel and Control Elements Regions}

As mentioned previously, two fuel elements in HFIR surround the central flux trap region. The IFE consists of 171 fuel plates, each containing ${ }^{1} 15.18 \pm 1 \%$ grams of ${ }^{235} \mathrm{U}$. The OFE consists of 369 fuel plates, each containing $18.44 \pm 1 \%$ grams of ${ }^{235} \mathrm{U}$. For modeling purposes, the fuel plates are homogenized in the MCNP Cycle 400 revised model and the same simplification is used in the SCALE HFIR model. The IFE region is modeled by using $(19$ axial $) \times(8$ radial $)$ homogenized regions, with the ${ }^{235} \mathrm{U}$ concentration varying radially (peaks toward the outer boundary of the IFE) to account for the radial variation of the fuel meat thickness in each fuel plate. The OFE is modeled by using $(19$ axial $) \times\left(9\right.$ radial) homogenized regions, which also have the ${ }^{235} \mathrm{U}$ concentration varying radially (peaks close to the middle of the radius of the OFE). The dimensions for the radial and axial regions in the two fuel elements are shown in Table 1 and Table 2, respectively.

The control element region consists of two concentric annular regions that surround the OFE. They are identified as the inner control element (ICE) and the outer control element (OCE), each 
$0.635 \mathrm{~cm}(1 / 4 \mathrm{in}$.) thick. The ICE is a full cylinder whose main function is to regulate the reactor power; for this reason, it is also called the control or regulating element. The OCE is made up of four quadrants or safety plates. The OCE is also called the safety element. More details on the control elements, including their lifetimes and material compositions, can be found elsewhere. ${ }^{6}$ There are three axial material regions in each of the two control elements; these regions, known by the names "black," "gray," and "white” because of their different neutron-absorption properties, are modeled in the SCALE HFIR model with the same level of detail as in the MCNP Cycle 400 revised model.

Table 1. Radial fuel regions in the SCALE HFIR model

\begin{tabular}{cccccc}
\hline \multicolumn{2}{c}{ IFE } & & \multicolumn{2}{c}{ OFE } \\
\cline { 1 - 2 } \cline { 5 - 5 } Region \# & $\begin{array}{c}\text { Outer radius } \\
(\mathbf{c m})\end{array}$ & & Region \# & $\begin{array}{c}\text { Outer radius } \\
(\mathbf{c m})\end{array}$ \\
\hline $0^{a}$ & 7.14 & & $0^{a}$ & 15.12951 \\
1 & 7.50 & & 1 & 15.50 \\
2 & 8.00 & & 2 & 16.00 \\
3 & 8.50 & & 3 & 16.50 \\
4 & 9.50 & & 4 & 17.50 \\
5 & 10.50 & & 5 & 18.50 \\
6 & 11.50 & & 6 & 19.50 \\
7 & 12.00 & & 7 & 20.00 \\
8 & 12.60 & & 8 & 20.50 \\
& & 9 & 20.978 \\
\hline
\end{tabular}

${ }^{a}$ Region 0 gives the inner radius for IFE and OFE.

Table 2. Axial fuel layers in the SCALE HFIR model

\begin{tabular}{cccc}
\hline Layer \# & $\begin{array}{c}\text { Top boundary } \\
(\mathbf{c m})^{\boldsymbol{a}}\end{array}$ & $\begin{array}{c}\text { Layer } \\
\#\end{array}$ & $\begin{array}{c}\text { Top boundary } \\
(\mathbf{c m})^{\boldsymbol{a}}\end{array}$ \\
\hline 1 & 25.4 & 11 & -1.0 \\
2 & 25.0 & 12 & -4.0 \\
3 & 23.0 & 13 & -7.0 \\
4 & 19.0 & 14 & -10.0 \\
5 & 16.0 & 15 & -13.0 \\
6 & 13.0 & 16 & -16.0 \\
7 & 10.0 & 17 & -19.0 \\
8 & 7.0 & 18 & -23.0 \\
9 & 4.0 & 19 & -25.0 \\
10 & 1.0 & $20^{b}$ & -25.4 \\
\hline${ }^{a}$ Location is with respect to the core midline (at axial location $\left.0.0 \mathrm{~cm}\right)$. \\
${ }^{b}$ Layer 20 gives the lower boundary of the fuel regions. \\
\hline \multicolumn{4}{c}{} \\
\hline
\end{tabular}

An axial cross section of the SCALE HFIR model with details of the fuel region and the control region is shown in Fig. 3. As seen, each of the two fuel elements is bordered radially by sidewalls, and there is a water gap between their adjacent sidewalls. The fueled regions of the fuel elements extend axially for a total height of $50.8 \mathrm{~cm}$ and have unfueled regions above and below them. The geometry unit numbers in the SCALE HFIR model are 2000 and 2300 for the IFE and OFE respectively, and 3000 and 3300 for the ICE and OCE, respectively. 


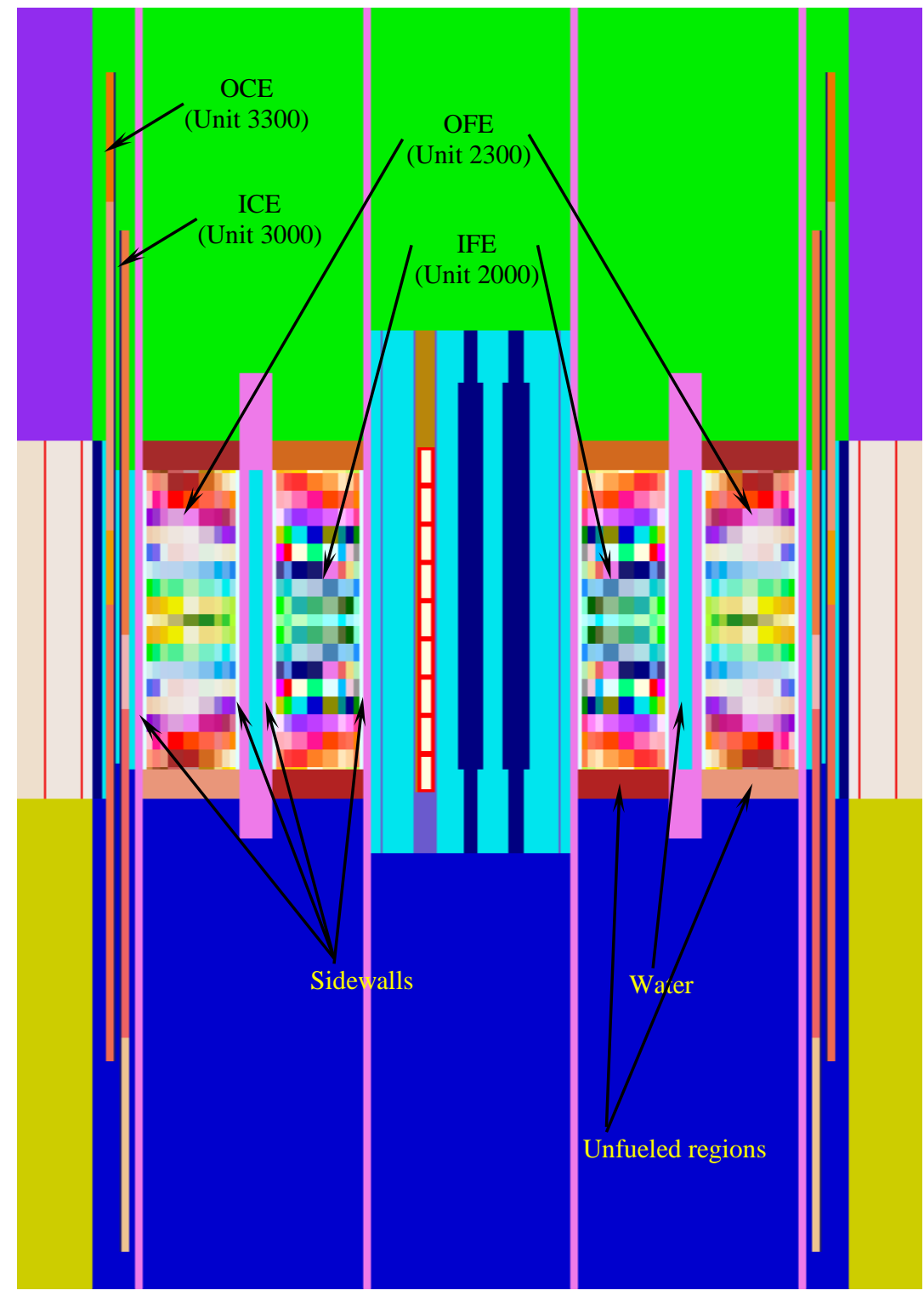

Fig. 3. Fuel and control regions (axial view) in the SCALE HFIR model.

\subsubsection{Beryllium Reflector Regions}

The beryllium reflector that surrounds the HFIR core contains three annular regions, as shown in Fig. 4, which is a cross section of the SCALE HFIR model at the core midplane. The removable reflector, the innermost region in the reflector, is replaced with fresh beryllium every 40 cycles. ${ }^{1}$ It contains four pairs of large experiment facilities (labeled RB-nX, with $n=1,3,5,7$ and $X=A, B$ ) and four small experiment facilities (labeled RB-n, with $n=2,4,6,8$ ). The removable reflector region is surrounded by the semipermanent beryllium reflector region, which is replaced every 80 cycles and contains eight control rod access plug facilities (labeled CR-n, with $n=1$ to 8). The outermost reflector region is the permanent beryllium reflector, which is replaced every 135 cycles and contains 22 vertical experimental facilities (labeled VXF-n, with $n=1$ to 22). Six of the VXF tubes have a 
larger diameter than the other VXF tubes. The permanent reflector also contains the tips of the four horizontal beam tubes and is indented at two places on its outer edge by engineering facility tubes, EF-1 and EF-2.

The beryllium reflector extends vertically for $60.96 \mathrm{~cm}$, symmetrically above and below the horizontal midplane.

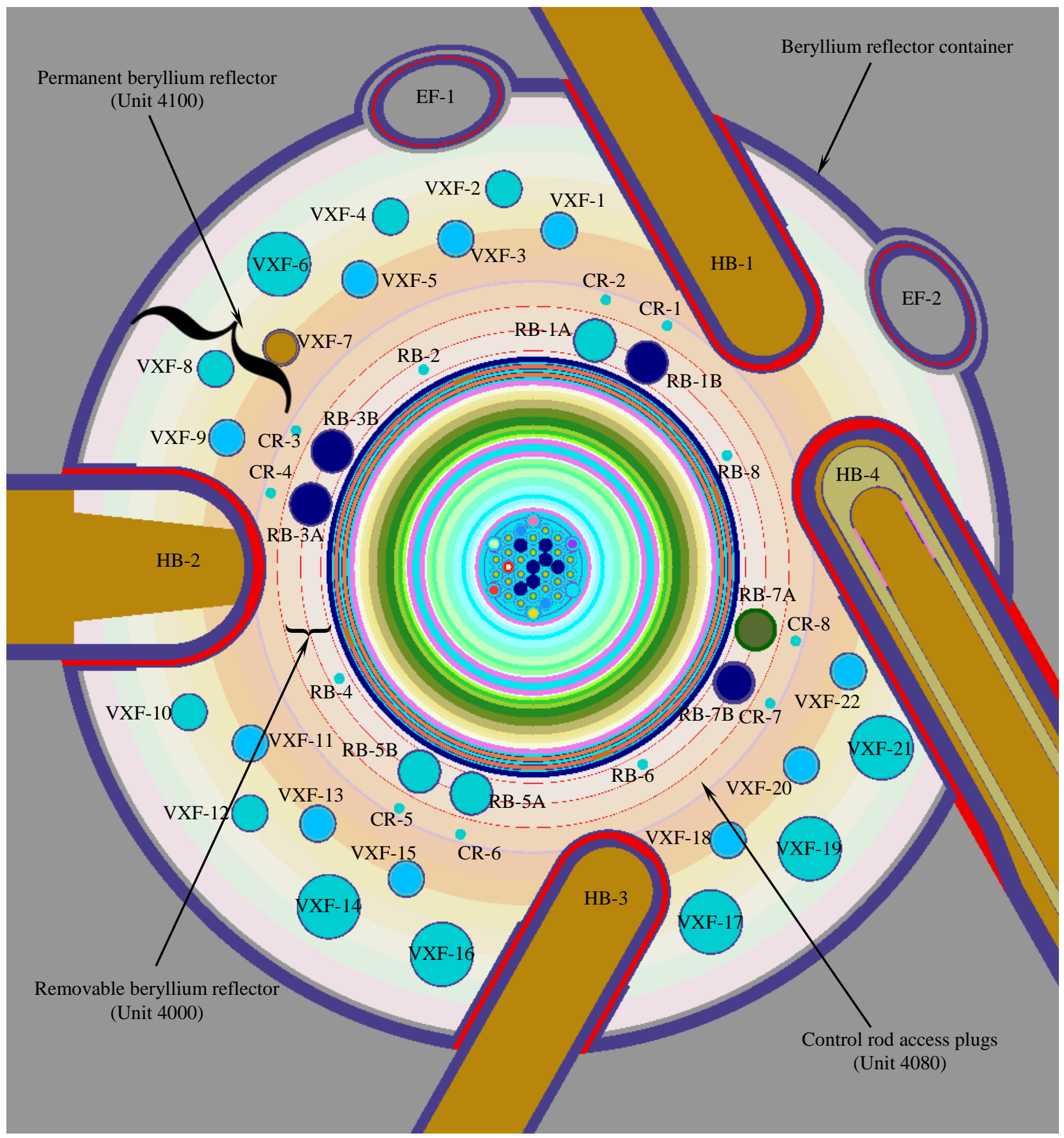

Fig. 4. Beryllium reflector at the horizontal midplane in the Scale HFIR model. 


\subsubsection{Outer Regions}

The main regions outside the beryllium reflector as modeled in the SCALE HFIR model are shown in Fig. 5. The thick biological shielding (Unit 9997 in the geometry model) is made of concrete and is modeled to an outer radius of $720 \mathrm{~cm}$. The water pool (Unit 4170 in the geometry model) is the next major region; it contains the reactor pressure vessel. Inside the pressure vessel and outside the beryllium reflector, there is another region filled with water that provides additional reflection for the reactor core and shielding for the pressure vessel. Cutouts through these regions are filled by the four horizontal beam tubes HB-1 to HB-4.

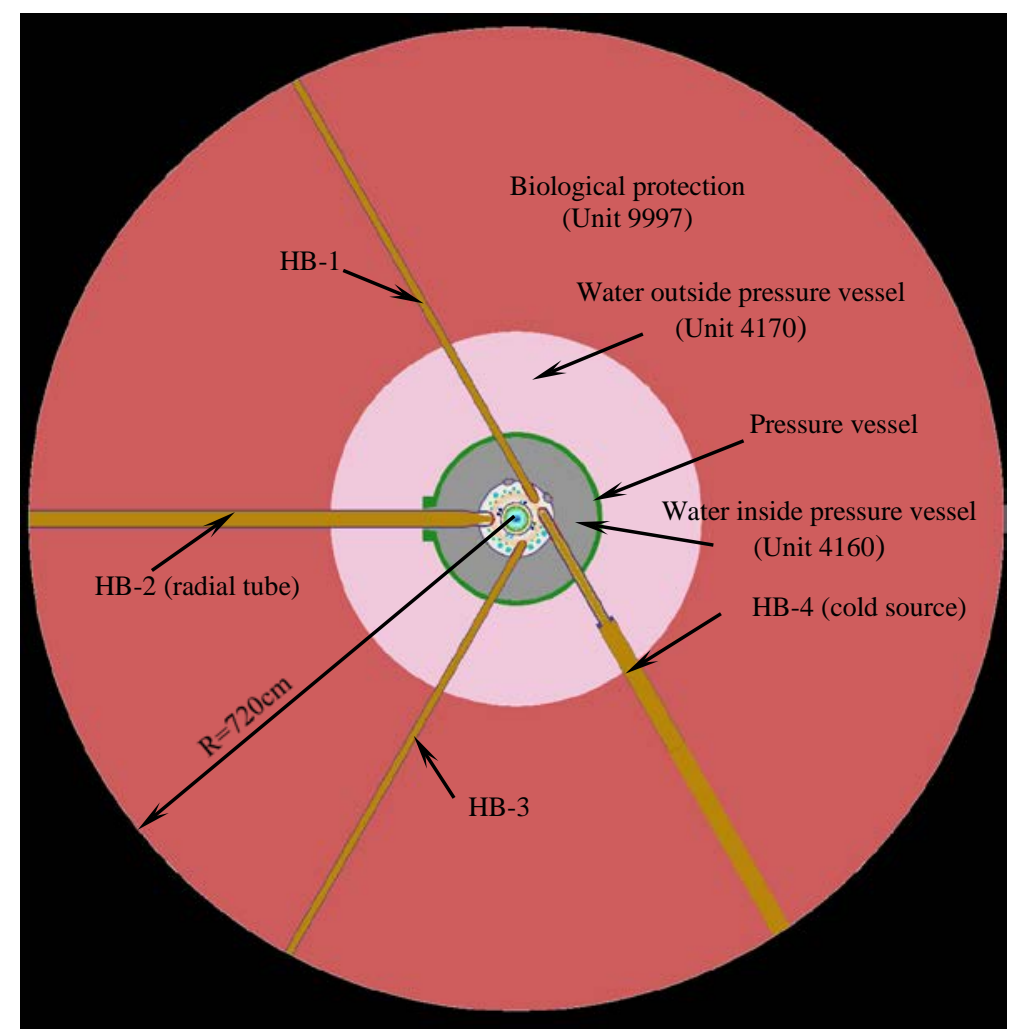

Fig. 5. Main outer core components of the SCALE HFIR model.

\subsubsection{Flowchart of the SCALE HFIR Geometry Model}

The general flowcharts describing the SCALE HFIR geometry model and the corresponding geometry units are shown in Fig. 6 for the HFIR regions that extend radially from the center to the outer edge of the OFE and in Fig. 7 for the HFIR regions that are radially outside the fuel regions. These flowcharts may be used by the expert users of the SCALE HFIR model to model their particular experimental configurations and to facilitate changes to the model.

Similar to the MCNP Cycle 400 revised model, the SCALE HFIR model has a cylindrical overall shape, with a height of $300 \mathrm{~cm}$ (from $-150 \mathrm{~cm}$ to $+150 \mathrm{~cm}$ around the core midplane) and an outer diameter of $1,440 \mathrm{~cm}$ (720 $\mathrm{cm}$ radius).

The general structure of the SCALE HFIR geometry model is based on concentric cylindrical shells. There are 10 such shells corresponding to the major units (italicized terms correspond strictly to ones used in the SCALE/KENO-VI geometry), as described in Fig. 6 and Fig. 7. Each shell has a 
corresponding unit number that uniquely identifies it in the SCALE HFIR model. The outermost unit corresponds to the global unit, and the innermost unit comprises the central target region. Many of the major units include minor units that are embedded in the major ones by using the hole feature of the KENO-VI geometry. In all cases, the minor units are vertical cylinders with their axis parallel to the $Z$ axis of the reactor model.

The fuel materials are made up of radial regions, with each group of radial regions embedded in an axial layer region for both the inner and outer fuel elements, as shown in Fig. 6.

The horizontal tubes, labeled HB-1 to HB-4, span five major geometry units (blue-colored items in Fig. 7), numbered 4080, 4100, 4160, 4170, and 9997. The two engineering facility slant tubes span two major geometry units, numbered 4100 and 4160 (see Fig. 7). 

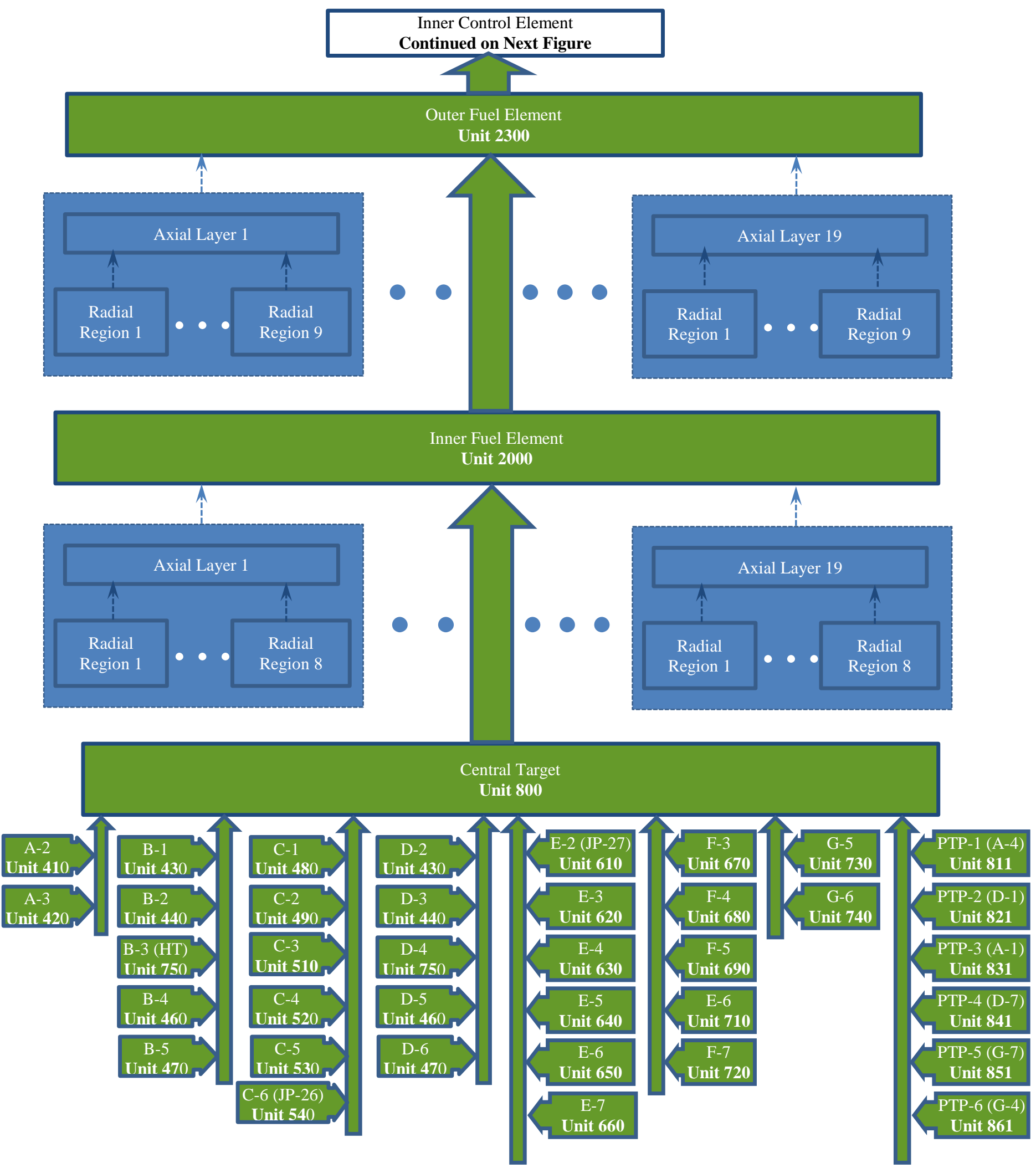

Fig. 6. Flowchart of the HFIR Scale model (fuel and central target regions). 


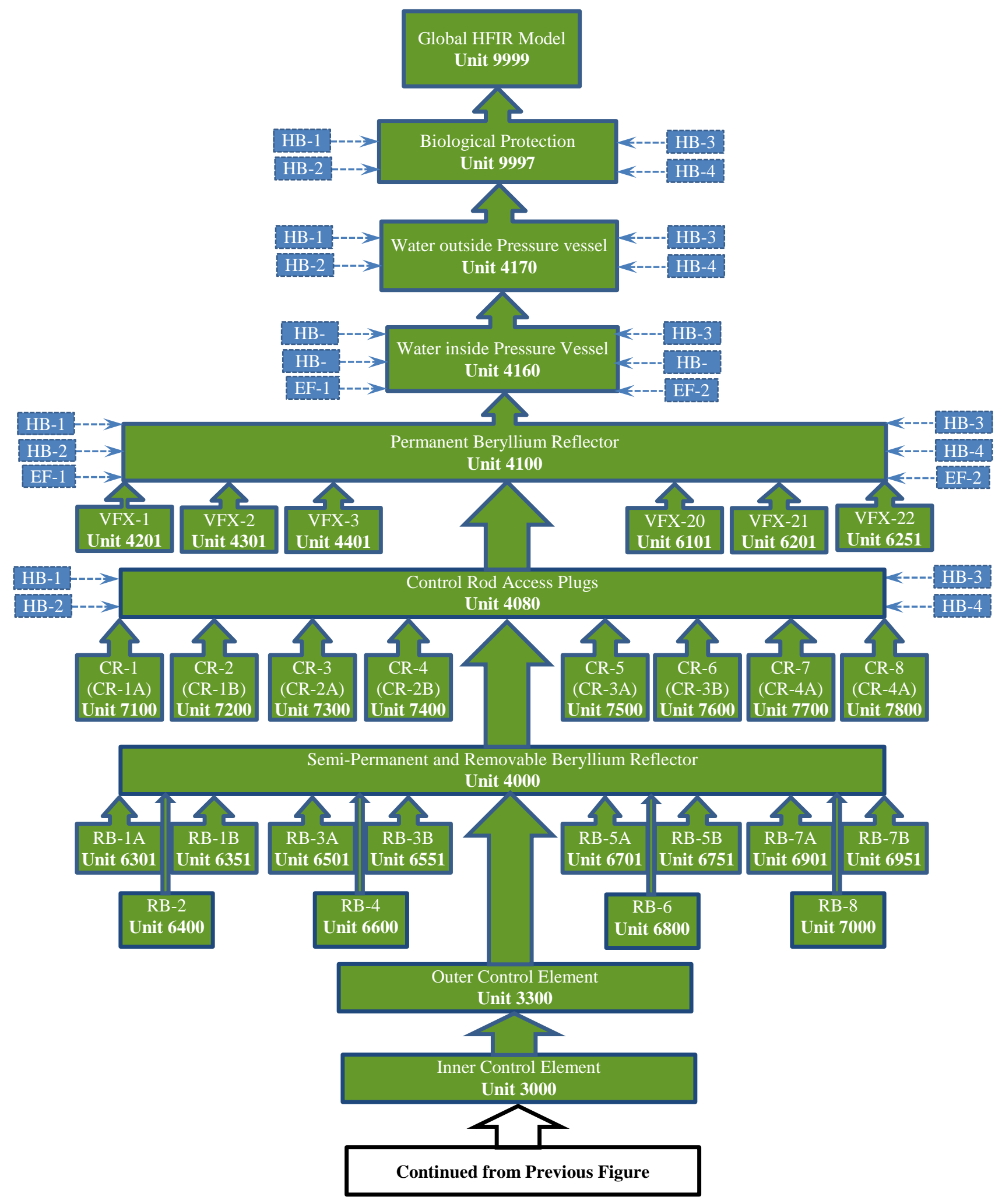

Fig. 7. Flowchart of the HFIR Scale model (outside the fuel region). 


\subsection{NUCLEAR CROSS-SECTION DATA}

Throughout the development of the SCALE HFIR model, the ENDF/B-VII.0 version of the SCALE continuous energy (CE) cross-section library was used; CE ENDF/B-VII.0 data were also used with the MCNP Cycle 400 revised model. The CE library can easily be changed in the SCALE model by simply changing the name of the library. To convert the SCALE HFIR continuous energy model to a multigroup (MG) model, a "celldata" block must be added to the existing input to correctly process the cross sections for the problem. The cross sections in both the SCALE model and the MCNP Cycle 400 revised model are considered at $293.6 \mathrm{~K}$ temperature.

\subsection{MATERIALS}

The SCALE HFIR model contains a total of 264 materials defined by their number densities. Several of the features of the material description in the SCALE HFIR model are illustrated in Fig. 8.

The material numbering scheme in the SCALE model is the same as in the MCNP Cycle 400 revised model with the exception of the SCALE material 100, which was added to account for a different density of the same composition as material 1 (water). Similar MCNP structural materials that had a difference in density of less than $0.1 \%$ have been modeled as the same material. The fuel materials are labeled according to the scheme 2ar or 3ar for the IFE and OFE, respectively, where $a$ is the axial layer number (varying from 0 to 9 , with midplane symmetry considered and with $a=0$ being the central layer and $a=9$ the top layer) and $r$ is the radial region within the axial layer (varying from 1 to 8 [moving from the center to the outside of HFIR] for the IFE and from 1 to 9 for the OFE). The material numbering for the nine axial layers located in the bottom half of the axial fuel region is based on the same scheme as used for the top-half axial layers, with core midline symmetry considered. For example, the same material number is used for the topmost and bottommost axial layers in a fuel element. The total number of materials used in the fuel region is 170, with 80 in the IFE and 90 in the OFE. As a note, the HFIR core is not axially symmetric, but using axially symmetric material distributions is an approximation that is usually considered to hold well.

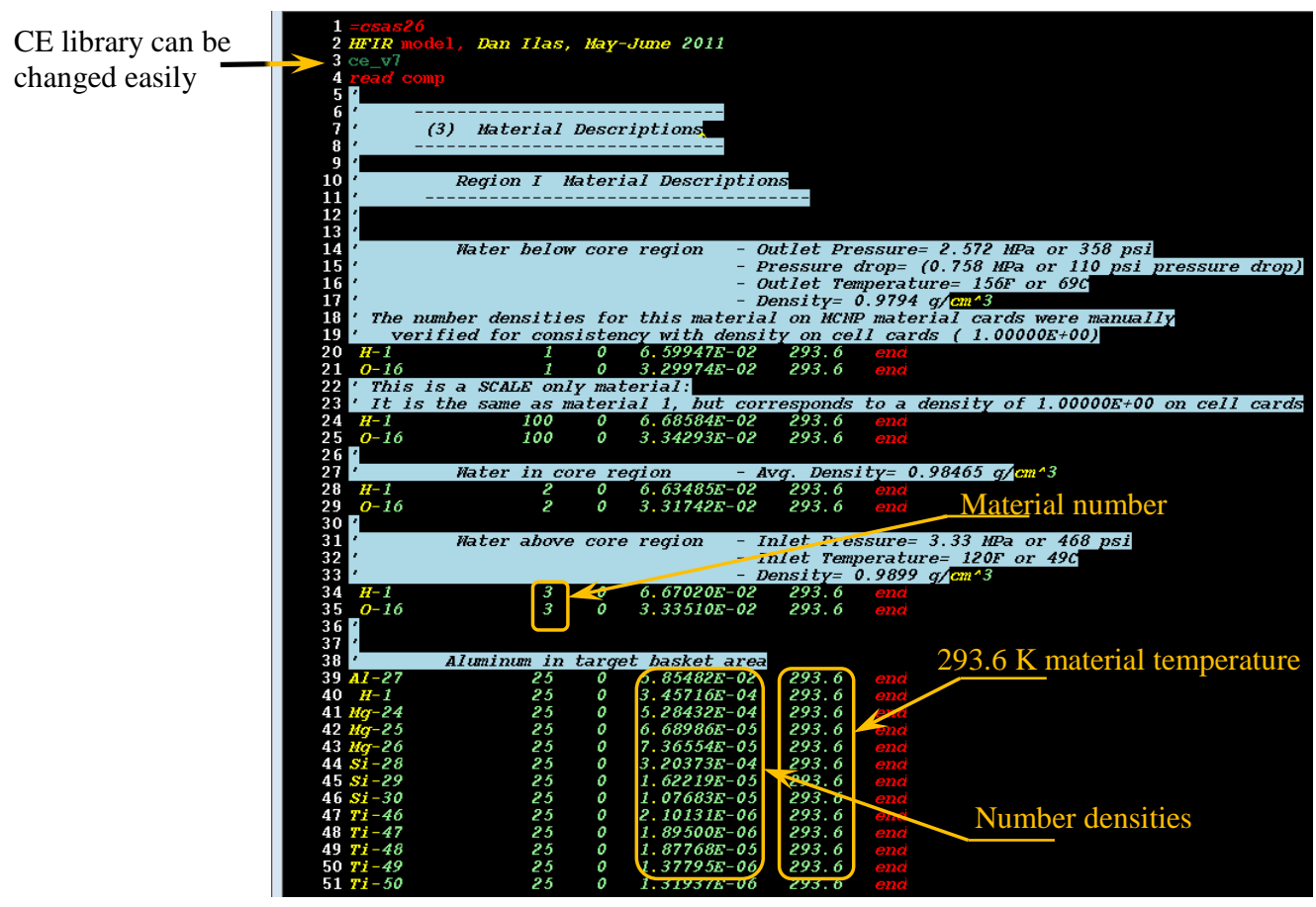

Fig. 8. A screenshot of the material block showing several features of the material description. 


\section{SCALE HFIR MODEL VERIFICATION}

The SCALE HFIR model for Cycle 400 was verified by both visual inspection and numerical comparisons against the MCNP Cycle 400 revised model. The combination of these two approaches gives confidence in the similarity of the two models.

\subsection{VISUAL COMPARISONS}

Visual comparisons were performed for the more complicated geometry regions to confirm the consistency of the geometry modeling in the SCALE and MCNP models. In comparing the plots in the following sub-sections, it should be noted that the MCNP plots include the surface lines while the SCALE plots strictly represent the material regions.

\subsubsection{Target Region}

A comparison between the representation of the central target region in the SCALE HFIR model and the corresponding representation in the MCNP Cycle 400 revised model is shown in Fig. 9 for the horizontal midplane $(\mathrm{z}=0)$. Note that, apart from the different colors used by the plotting capabilities of the two software packages, the SCALE HFIR model reproduces with fidelity the MCNP Cycle 400 revised model representation of the central target region. The actual dimensions were compared by direct inspection of the input files for the two models.

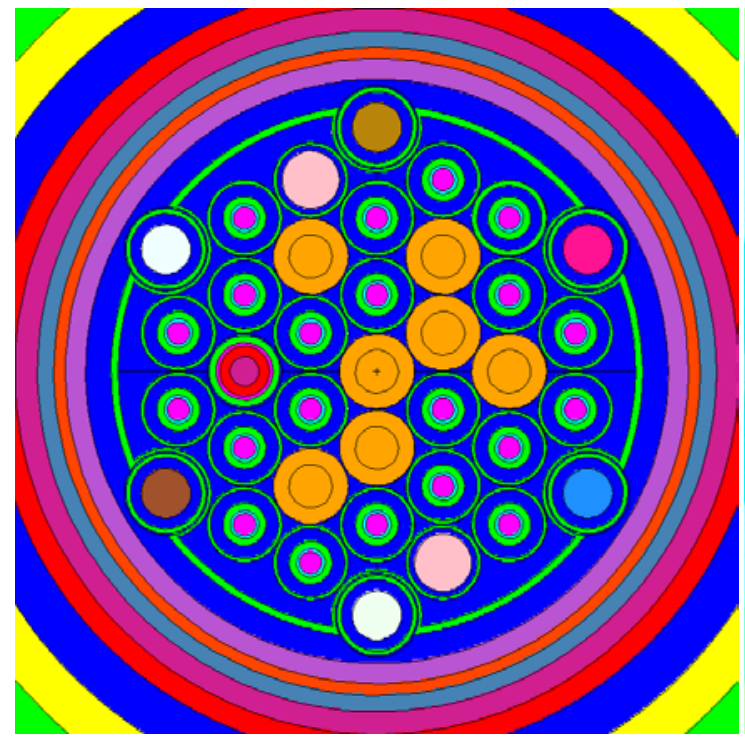

(a) MCNP Cycle 400 revised model

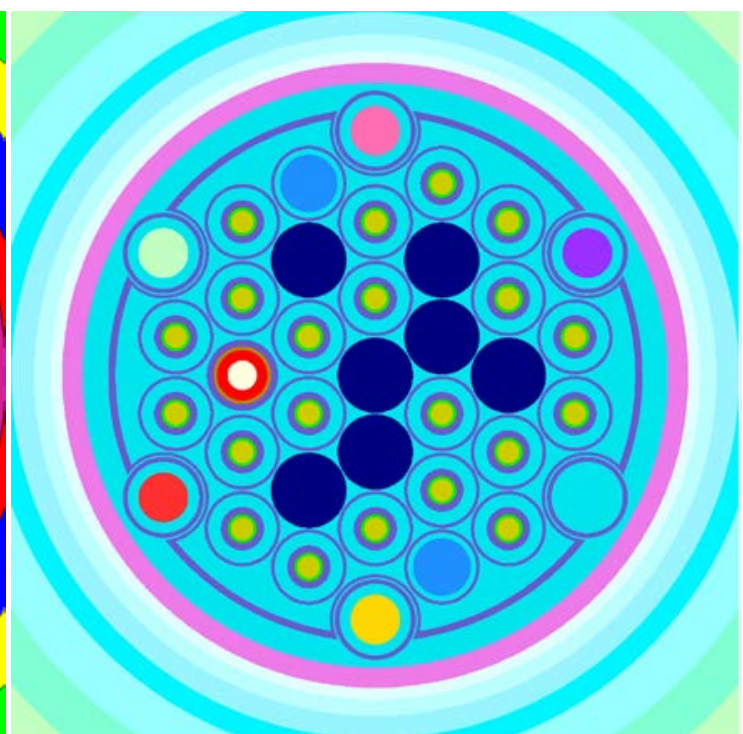

(b) Scale HFIR model

Fig. 9. Comparison of the central target region layouts.

\subsubsection{Fuel and Control Regions}

The fuel region models are compared in Fig. 10 (see Fig. 3 for region identification), where vertical sections of the HFIR are shown for the SCALE HFIR model and the MCNP Cycle 400 revised model in the plane $y=0$ (note that the plots are enlarged in the radial direction to show details). 
Also note the symmetric distribution of the fuel materials with respect to the midplane $\mathrm{z}=0$ and the slight axial asymmetry of the region that separates the two fuel elements.

Also apparent in Fig. 10 is the similar material distribution in the two control regions of the two HFIR models, as well as the axial model of the HT facility and two solid aluminum target facilities in the central flux trap region.

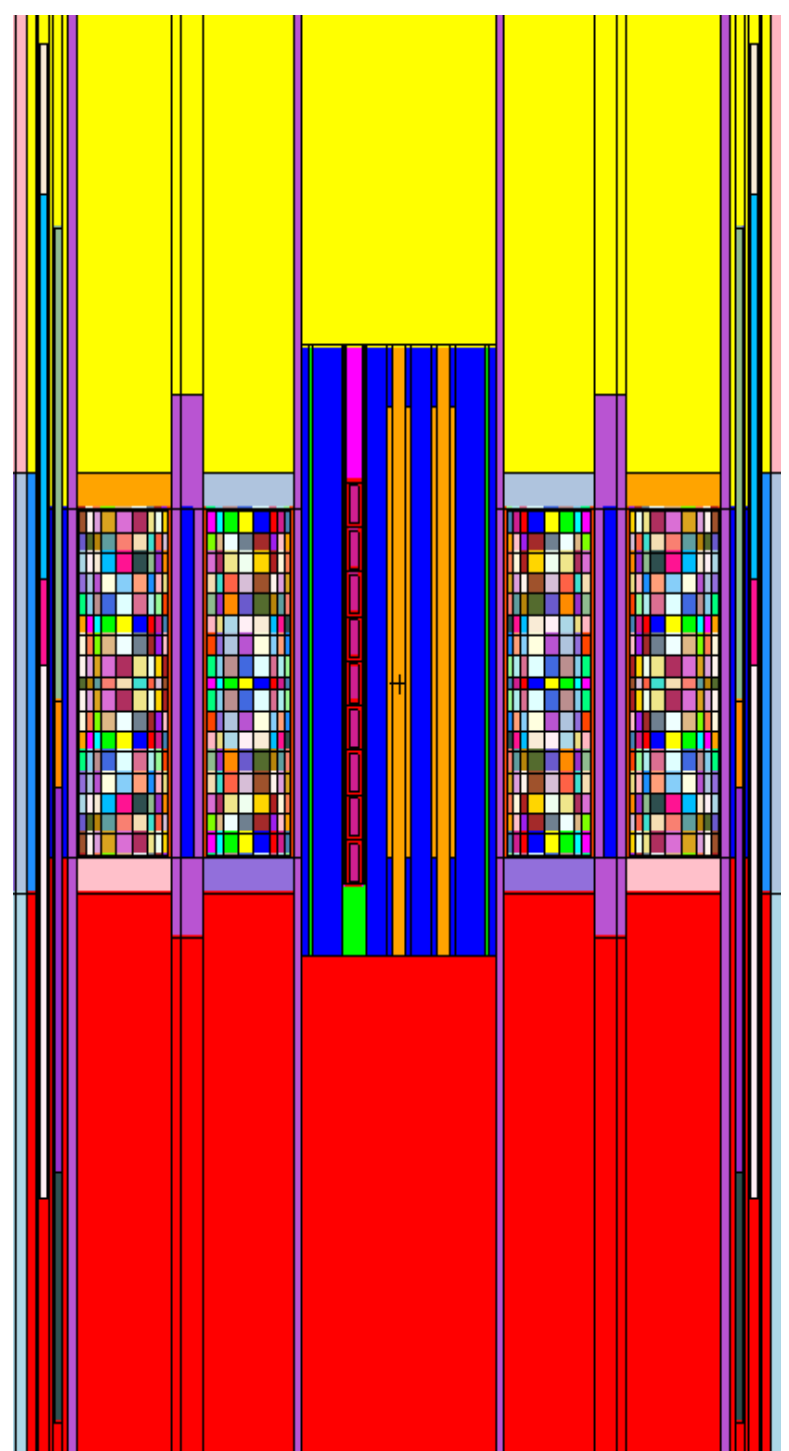

(a) MCNP Cycle 400 revised model

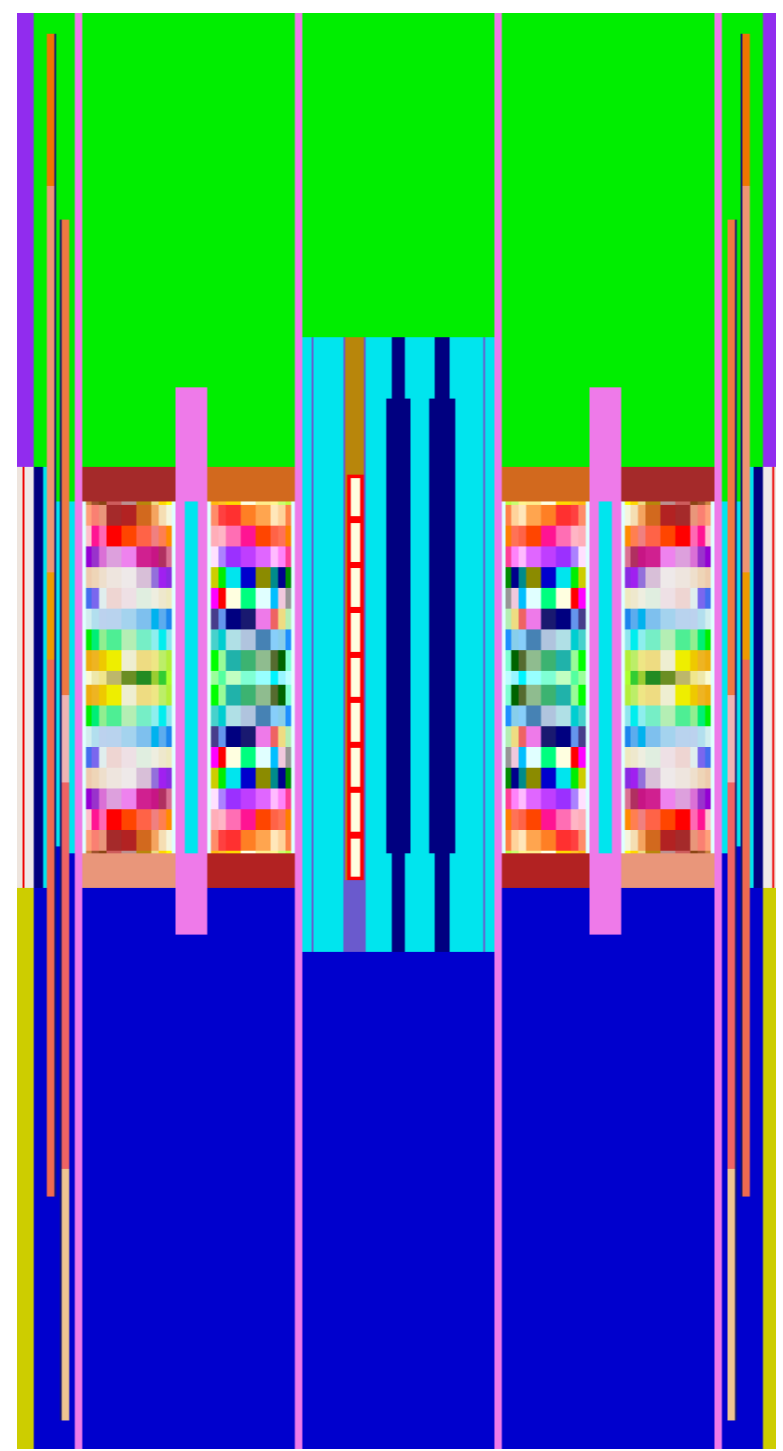

(b) Scale HFIR model

Fig. 10. Comparison of the fuel and control regions.

\subsubsection{Beryllium Reflector Regions}

The similarity between the SCALE HFIR model and the MCNP Cycle 400 revised model, with respect to the distribution of the irradiation facilities that are embedded in the beryllium reflector, is visible in Fig. 11 where a core midplane $(z=0)$ cross section is shown for the two models. The similar positioning of each of the facilities, including the horizontal tubes and the engineering facilities, can be seen. 


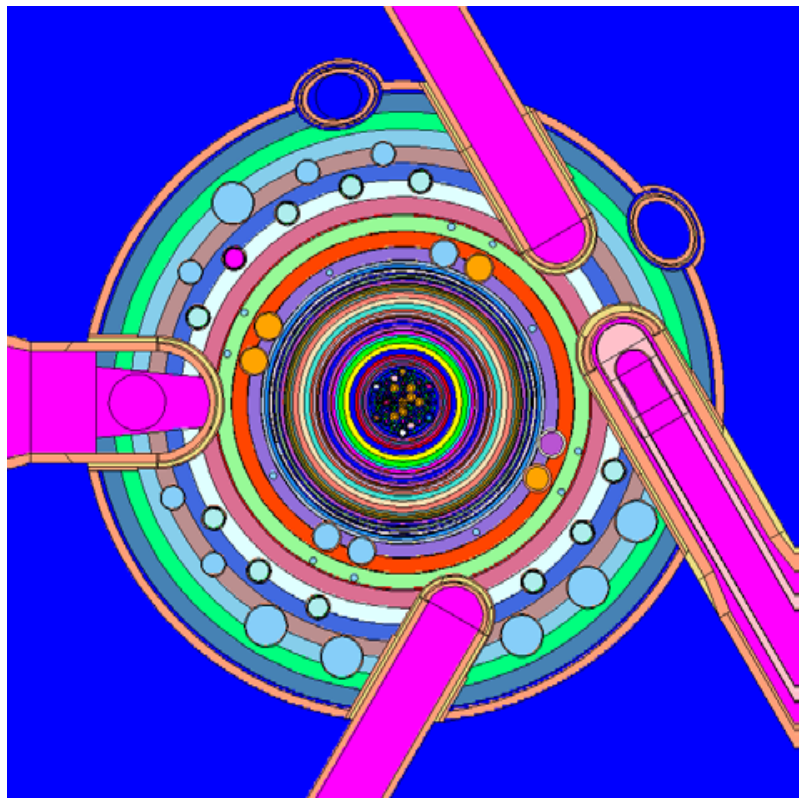

(a) MCNP Cycle 400 revised model

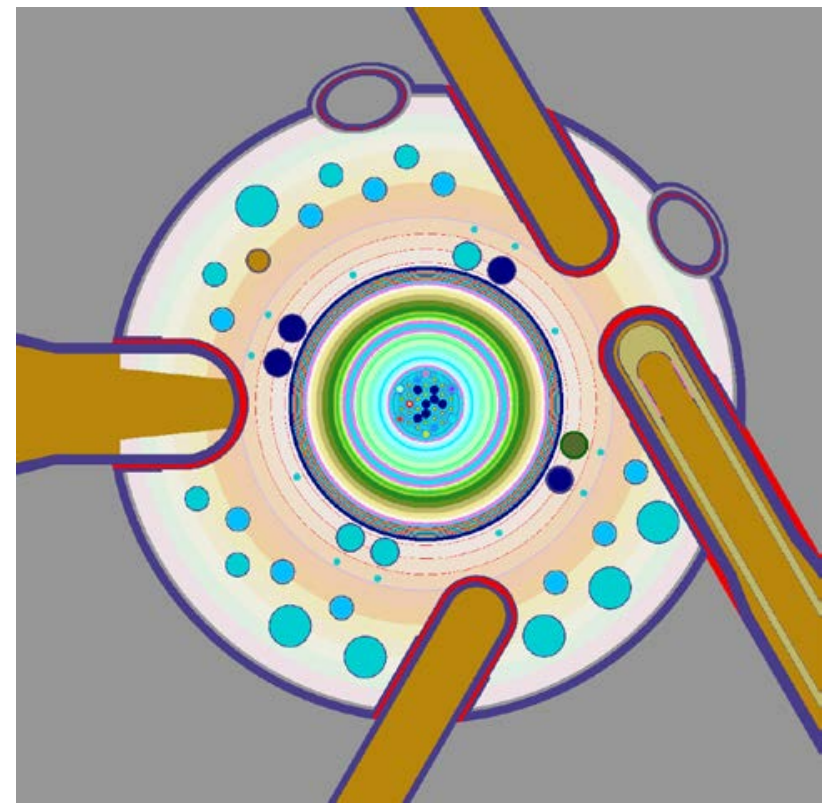

(b) Scale HFIR model

Fig. 11. Beryllium reflector regions with irradiation facilities, horizontal tubes, and engineering facilities.

\subsubsection{Horizontal Tube Regions}

Correct modeling of the horizontal tubes is important to adequately characterize the neutron fluxes along each of the tubes and is especially important for the cold source tube, HB-4. Fig. 12 compares the details of the tip of the HB- 4 tube at $z=0$ in the beryllium reflector, where the similarity of the hydrogen-filled region (light pink for the MCNP model and light yellow for the SCALE model) is apparent.

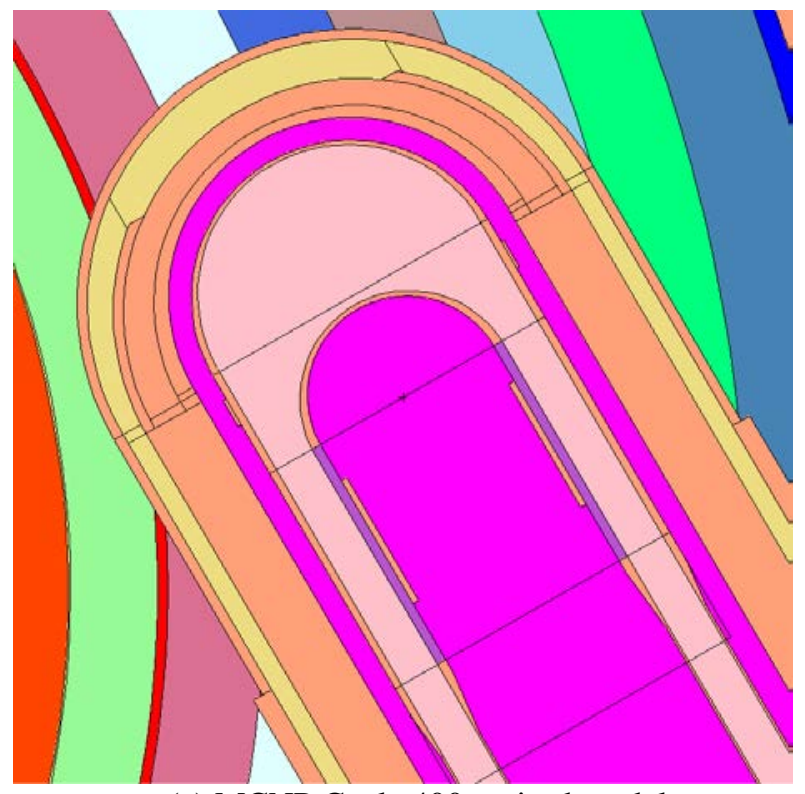

(a) MCNP Cycle 400 revised model

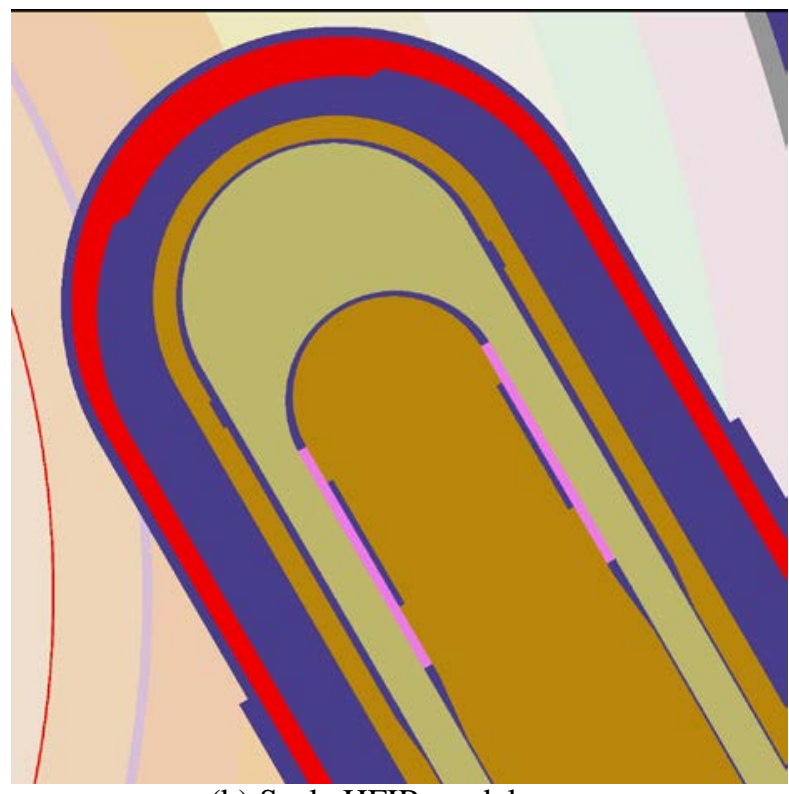

(b) Scale HFIR model

Fig. 12. Comparison of the HT-4 tip (cold source tube) models. 


\subsubsection{Outer Regions}

Layouts of the outer regions of HFIR (outside the pressure vessel), as obtained with the SCALE and MCNP Cycle 400 revised models, are shown in Fig. 13 for a core midplane cross section. The layouts of the four horizontal tubes are identical all the way to the periphery of the model.

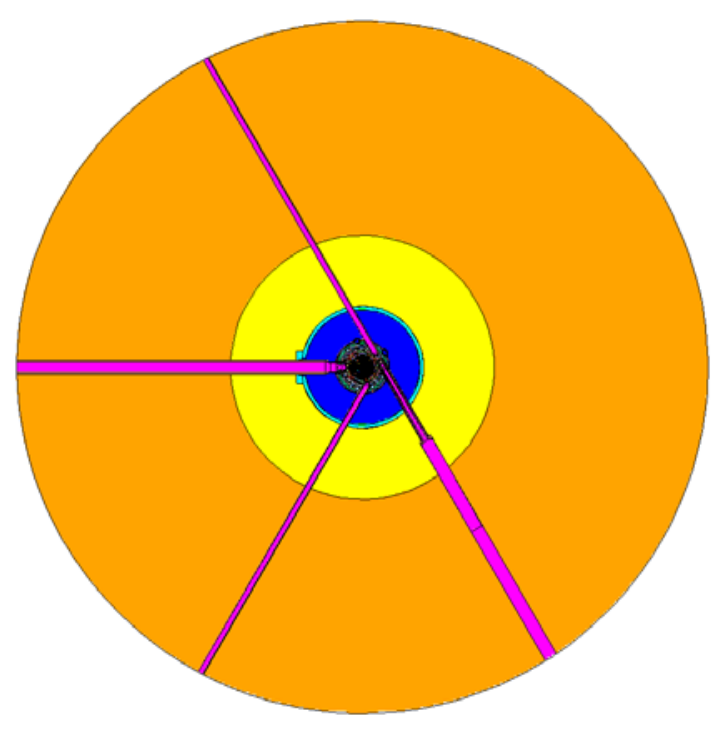

(a) MCNP Cycle 400 revised model

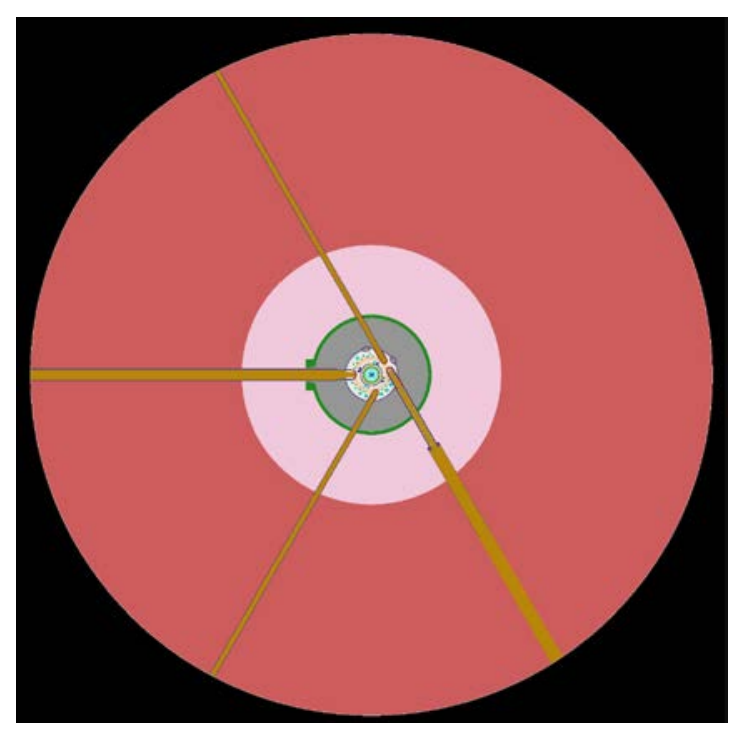

(b) Scale HFIR model

Fig. 13. Comparison of the HFIR outer regions.

\subsection{CALCULATIONAL COMPARISONS}

\subsubsection{Multiplication Constant and Other System Parameters}

The multiplication constants $\left(\mathrm{k}_{\text {eff }}\right)$ predicted by the SCALE HFIR model and the MCNP Cycle 400 revised model and the corresponding standard deviations are shown in Table 3. In each case, 2000 neutron active generations (cycles) were used in the Monte Carlo simulation, with each generation tracking 100,000 particles. A number of 200 generations were initially skipped to achieve a better convergence of the neutron source. As seen, there is excellent agreement between the $k_{\text {eff }}$ values predicted by the two models, with a difference of $124 \mathrm{pcm}$.

Table 3. Comparison of multiplication constants

\begin{tabular}{ccc}
\hline Model & $\mathbf{k}_{\text {eff }}$ & $\boldsymbol{\sigma}$ \\
\hline SCALE HFIR & 0.99630 & 0.00007 \\
MCNP Cycle 400 revised & 0.99754 & 0.00006 \\
\hline
\end{tabular}

Other parameters of interest that globally characterize the HFIR as a critical system are the energy corresponding to the average neutron lethargy causing fission, $\bar{\epsilon}$, and the average number of neutrons produced per fission, $\bar{v}$. Table 4 summarizes the results obtained with the two models. The number of neutrons produced per fission is identical for the two models up to four significant digits. The energy corresponding to the average neutron lethargy causing fission differs slightly (by 2\%) between the SCALE and MCNP models. 
Table 4. Comparison of HFIR system parameters

\begin{tabular}{ccc}
\hline Model & $\overline{\boldsymbol{\epsilon}}(\mathbf{e V})$ & $\overline{\boldsymbol{v}}$ \\
\hline SCALE HFIR & 0.174 & 2.439 \\
MCNP Cycle 400 revised & 0.170 & 2.439 \\
\hline
\end{tabular}

\subsubsection{Core Power Distribution}

It is customary to approximate the relative power distribution in the reactor core with the relative fission reaction rate distribution. In MCNP, the fission reaction rate can be calculated for each cell where fuel is present. This capability is currently lacking in SCALE when used with a continuous energy cross-section library. The $v \Sigma_{\mathrm{f}}$ reaction rate, however, can be extracted directly from the output file for the continuous energy SCALE model for HFIR, for each of the regions that contain fissile material. The relative $v \Sigma_{\mathrm{f}}$ reaction rate density distribution obtained from the SCALE HFIR model output file was normalized to the neutron source in a manner similar to the MCNP results. The relative neutron production rate densities calculated with SCALE are displayed in Fig. 14 both as 3-D surfaces and 2-D contours (at the bottom of the plot), and the corresponding numerical values are given in Table 5. The $v \Sigma_{\mathrm{f}}$ reaction rate peaks at the core midplane in the regions adjacent to the sidewall between the two fuel elements and also near the central flux trap.

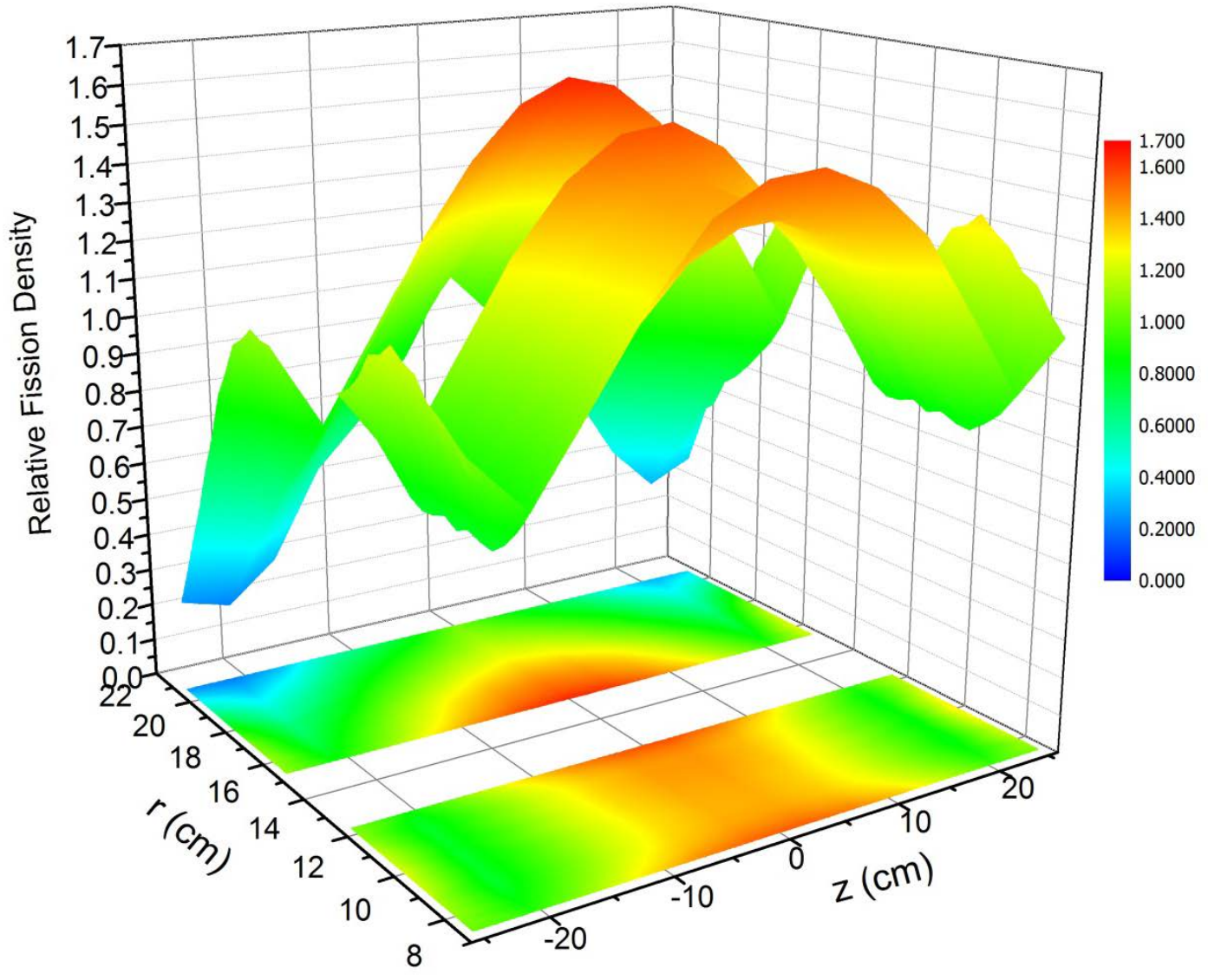

Fig. 14. Relative $v \Sigma_{\mathrm{f}}$ reaction rate density distributions in IFE and OFE of HFIR.

To verify that the relative $v \Sigma_{\mathrm{f}}$ reaction rate density distribution calculated with the SCALE HFIR 
model is consistent with the relative fission density distribution predicted by the MCNP Cycle 400 revised model, a mesh-wise comparison of the two datasets was performed, and is illustrated in Fig. 15. Theoretically, the two distributions are the same when the number of neutrons emitted per fission is independent of energy over the energy range of the incident neutrons where most of the fissions occur. The data illustrated in Fig. 15 was calculated for each mesh as

$$
100 \% \times \frac{S C A L E-M C N P}{M C N P}
$$

where $S C A L E$ is the relative $v \Sigma_{\mathrm{f}}$ reaction rate density calculated with the SCALE model, and MCNP is the relative fission density calculated with the MCNP model. ${ }^{6}$ The data in Fig. 15 is displayed both as 3-D surfaces and 2-D contours (at the bottom of the plot). The difference per mesh between the two models is typically below $0.5 \%$ and increases slightly in the smaller meshes located at the top and bottom of the OFE, most likely due to the poorer statistics in these regions. The arithmetic average of the mesh difference between the two models is $+0.00 \%$ for IFE and $-0.01 \%$ for OFE with maxima of $0.93 \%$ for IFE and $0.99 \%$ for OFE and minima of $-1.44 \%$ for IFE and $-1.19 \%$ for OFE.

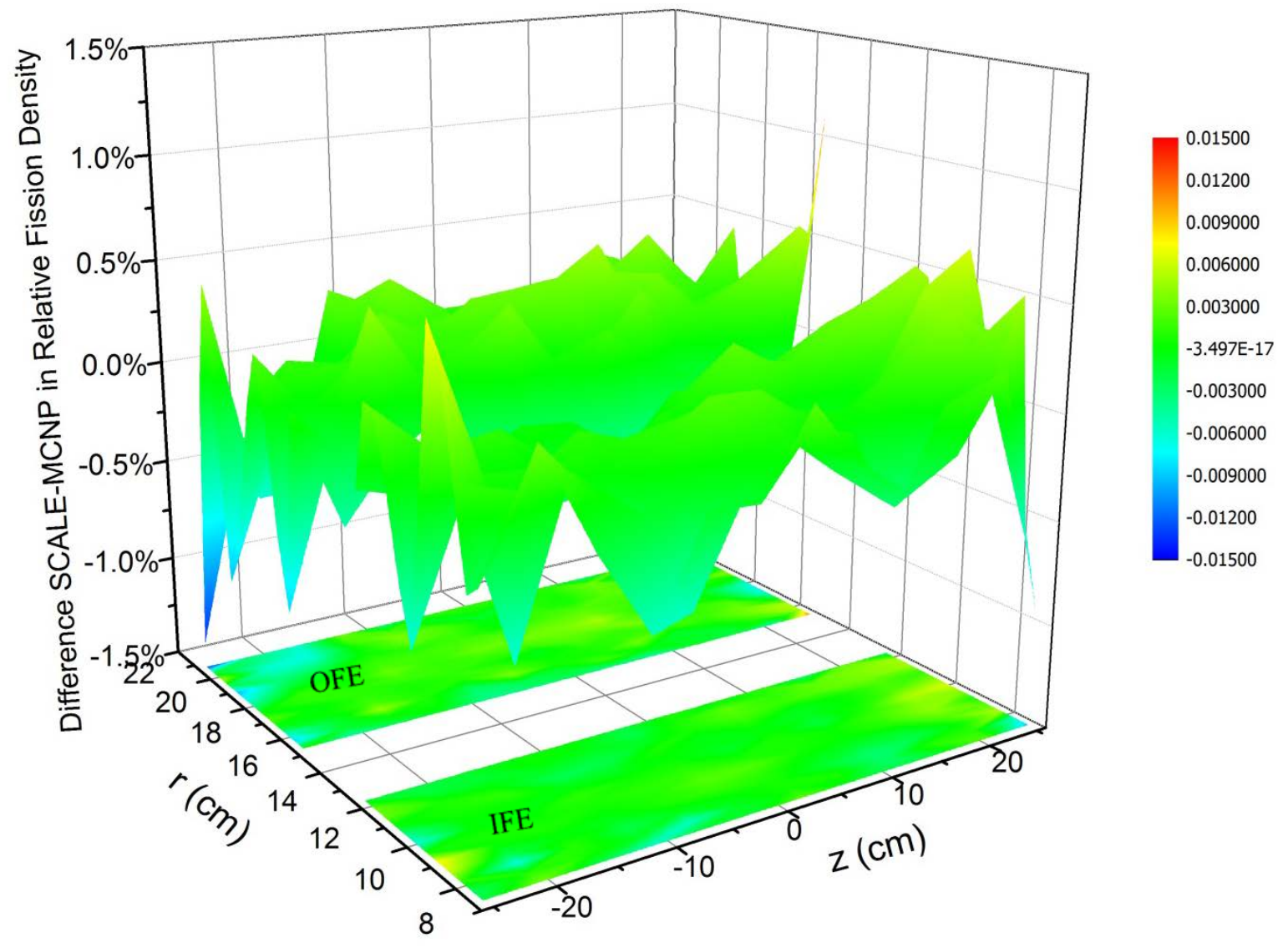

Fig. 15. Comparison of relative $v \Sigma_{f}$ reaction rate density distribution predicted by the SCALE HFIR model and relative fission density distribution predicted by the MCNP Cycle 400 revised model. 
Table 5. Relative $v \Sigma_{\mathrm{f}}$ reaction rate densities predicted by the SCALE HFIR model

\begin{tabular}{|c|c|c|c|c|c|c|c|c|c|c|c|c|c|c|c|c|c|}
\hline \multirow{2}{*}{$\begin{array}{c}\text { Axial }^{a} \\
\text { layer \# }\end{array}$} & \multicolumn{8}{|c|}{ IFE } & \multicolumn{9}{|c|}{ OFE } \\
\hline & $\mathrm{r}=1^{b}$ & $r=2$ & $r=3$ & $r=4$ & $r=5$ & $r=6$ & $r=7$ & $r=8$ & $r=1$ & $r=2$ & $r=3$ & $r=4$ & $r=5$ & $r=6$ & $r=7$ & $r=8$ & $r=9$ \\
\hline 1 & 1.087 & 1.129 & 1.176 & 1.254 & 305 & 266 & 182 & 146 & 1.182 & 1.201 & 1.230 & 1.209 & 1.043 & 0.801 & 0.602 & 0.472 & 0.367 \\
\hline 2 & 0.907 & 0.869 & 0.844 & 0.840 & 843 & 338 & 345 & 0.897 & 33 & 0.875 & 0.829 & 0.759 & 0.646 & 0.523 & 0.424 & 0.357 & 0.294 \\
\hline 3 & 0.952 & 0.896 & 0.857 & 0.834 & 0.832 & 0.845 & 0.863 & 0.925 & 0.953 & 0.896 & 0.850 & 0.776 & 0.662 & 0.542 & 0.444 & 0.379 & 0.313 \\
\hline 4 & 1.088 & 1.033 & 0.992 & 0.971 & 0.974 & 0.986 & 1.007 & 1.070 & 1.103 & 1.047 & 0.995 & 0.922 & 0.793 & 0.657 & 0.545 & 0.471 & 0.397 \\
\hline 5 & 1.237 & 1.175 & 1.128 & 1.105 & 1.113 & 1.127 & 1.145 & 1.219 & 1.262 & 1.197 & 1.147 & 1.067 & 0.934 & 0.796 & 0.686 & 0.614 & 0.541 \\
\hline 6 & 1.363 & 1.301 & 1.245 & 1.226 & 1.232 & 1.248 & 1.270 & 1.353 & 1.400 & 1.335 & 1.275 & 1.195 & 1.054 & 0.920 & 0.815 & 0.744 & 0.676 \\
\hline 7 & 1.460 & 1.387 & 1.336 & 1.315 & 1.323 & 1.343 & 1.372 & 1.451 & 1.515 & 1.443 & 1.383 & 1.298 & 1.152 & 1.015 & 0.907 & 0.836 & 0.762 \\
\hline 8 & 1.513 & 1.435 & 1.393 & 1.376 & 1.392 & 1.410 & 1.443 & 1.531 & 1.595 & 1.519 & 1.461 & 1.372 & 1.228 & 1.093 & 0.990 & 0.922 & 0.848 \\
\hline 9 & 1.546 & 1.473 & 1.424 & 1.404 & 1.424 & 1.447 & 1.477 & 1.571 & 1.638 & 1.564 & 1.507 & 1.420 & 1.284 & 1.163 & 1.081 & 1.029 & 0.973 \\
\hline 10 & 1.552 & 1.482 & 1.425 & 1.408 & 1.430 & 1.450 & 1.488 & 1.578 & 1.642 & 1.572 & 1.513 & 1.429 & 1.295 & 1.187 & 1.121 & 1.073 & 1.033 \\
\hline 11 & 1.543 & 1.467 & 1.416 & 1.397 & 1.413 & 1.435 & 1.467 & 1.557 & 1.620 & 1.545 & 1.484 & 1.400 & 1.261 & 1.135 & 1.040 & 0.978 & 0.916 \\
\hline 12 & 1.502 & 1.429 & 1.370 & 1.352 & 1.366 & 1.386 & 1.413 & 1.503 & 1.557 & 1.482 & 1.423 & 1.336 & 1.185 & 1.038 & 0.920 & 0.840 & 0.748 \\
\hline 13 & 1.427 & 1.358 & 1.307 & 1.280 & 1.291 & 1.304 & 1.331 & 1.413 & 1.462 & 1.395 & 1.335 & 1.248 & 1.098 & 0.950 & 0.832 & 0.753 & 0.670 \\
\hline 14 & 1.309 & 1.252 & 1.205 & 1.177 & 1.189 & 1.199 & 1.225 & 1.293 & 1.343 & 1.274 & 1.219 & 1.135 & 0.994 & 0.851 & 0.742 & 0.664 & 0.588 \\
\hline 15 & 1.184 & 1.119 & 1.080 & 1.057 & 1.064 & 1.073 & 1.094 & 1.157 & 1.195 & 1.134 & 1.083 & 0.999 & 0.860 & 0.717 & 0.605 & 0.526 & 0.447 \\
\hline 16 & 1.040 & 0.989 & 0.948 & 0.925 & 0.929 & 0.937 & 0.952 & 1.007 & 1.041 & 0.981 & 0.929 & 0.849 & 0.715 & 0.568 & 0.447 & 0.364 & 0.282 \\
\hline 17 & 0.912 & 0.857 & 0.815 & 0.793 & 0.789 & 0.793 & 0.812 & 0.862 & 0.889 & 0.831 & 0.783 & 0.710 & 0.589 & 0.460 & 0.355 & 0.284 & 0.217 \\
\hline 18 & 0.865 & 0.827 & 0.798 & 0.788 & 0.787 & 0.781 & 0.786 & 0.830 & 0.858 & 0.799 & 0.753 & 0.682 & 0.566 & 0.438 & 0.334 & 0.264 & 0.200 \\
\hline 19 & 1.014 & 1.052 & 1.109 & 1.180 & 1.223 & 1.176 & 1.094 & 1.054 & 1.076 & 1.085 & 1.111 & 1.070 & 0.898 & 0.665 & 0.473 & 0.360 & 0.251 \\
\hline
\end{tabular}

${ }^{a}$ The axial layer number increases from the top to the bottom of the fuel elements.

${ }^{b} \mathrm{r}=\mathrm{n}$ denotes the radial region number; $\mathrm{n}$ varies from 1 to 8 for the IFE and from 1 to 9 for the OFE, with r=1 corresponding to the smallest radius in the fuel element. 


\subsubsection{Neutron Flux}

The neutron flux level is one of the key parameters for characterizing core performance. A comparison was made of three-group flux data estimated with the SCALE HFIR model and the MCNP Cycle 400 revised model for the HFIR core at 85 MW power for three relevant locations in the HFIR geometry. The flux data comparison is presented in Table 6. The statistical relative standard deviation for the tallied flux is less than $1 \%$ in all cases. The energy group definition for the three-group data is thermal, $<0.625 \mathrm{eV}$; epithermal, $0.625 \mathrm{eV}-100 \mathrm{keV}$; and fast, $100 \mathrm{keV}-20 \mathrm{MeV}$. As the flux results provided by SCALE or MCNP are normalized to the fission source, the values for the flux in $\mathrm{n} / \mathrm{cm}^{2} \mathrm{~s}$ were obtained by multiplying the flux tally values by the total source. The total source $S$ was approximated as

$$
S=\frac{\bar{v} P}{E^{e k_{e f f}}}
$$

where $\bar{v}$ is the average number of neutrons per fission, $P$ is the reactor power in MW, $E$ is the average energy per fission in $\mathrm{MeV}$, and $e$ is a unit conversion factor $\left(1.602 \times 10^{-19} \mathrm{MJ} / \mathrm{MeV}\right)$. An approximate value of $200 \mathrm{MeV}$ was used for $E$, whereas the value for $\mathrm{k}_{\text {eff }}$ and $\bar{v}$ were taken from Table 3 and Table 4 , respectively. The value of the total source is $6.47 \times 10^{18} \mathrm{n} / \mathrm{s}$ for the MCNP Cycle 400 revised model $^{6}$ and $6.49 \times 10^{18} \mathrm{n} / \mathrm{s}$ for the SCALE HFIR model.

The flux data in Table 6 is shown for the following three locations: the central target in the flux trap, the edge of the cold source (hydrogen spherical region at the tip of the cold source), and the VXF-6 location in the beryllium reflector. As observed, the flux values agree well in the SCALE and MCNP models for the central target region and the VXF-6 location in reflector, with differences typically below $2 \%$. The differences between the two models increase slightly to $~ 5 \%$ in the thermal and epithermal components, as well as for the total flux, at the cold source tip.

Table 6. Neutron flux comparison between the SCALE and MCNP models

\begin{tabular}{llcccc}
\hline \multicolumn{1}{c}{ Location } & Model & $\begin{array}{c}\text { Thermal } \\
\text { flux } \\
\left(\mathbf{n} / \mathbf{c m}^{2} \mathbf{s}\right)\end{array}$ & $\begin{array}{c}\text { Epithermal } \\
\text { flux } \\
\left(\mathbf{n} / \mathbf{c m}^{2} \mathbf{s}\right)\end{array}$ & $\begin{array}{c}\text { Fast flux } \\
\left(\mathbf{n} / \mathbf{c m}^{2} \mathbf{s}\right)\end{array}$ & $\begin{array}{c}\text { Total flux } \\
\left(\mathbf{n} / \mathbf{c m}^{2} \mathbf{s}\right)\end{array}$ \\
\hline $\begin{array}{l}\text { Central target } \\
\text { r,Z }=0 \mathrm{~cm}\end{array}$ & SCALE & $2.25 \times 10^{15}$ & $1.33 \times 10^{15}$ & $1.12 \times 10^{15}$ & $4.71 \times 10^{15}$ \\
& MCNP & $2.24 \times 10^{15}$ & $1.30 \times 10^{15}$ & $1.13 \times 10^{15}$ & $4.67 \times 10^{15}$ \\
Reflector & SCALE & $2.72 \times 10^{14}$ & $3.64 \times 10^{13}$ & $9.50 \times 10^{12}$ & $3.18 \times 10^{14}$ \\
VXF-6 & MCNP & $2.67 \times 10^{14}$ & $3.58 \times 10^{13}$ & $9.76 \times 10^{12}$ & $3.13 \times 10^{14}$ \\
& & & & & \\
$\begin{array}{l}\text { Cold source tip } \\
\text { Unit 4100 }\end{array}$ & SCALE & $4.23 \times 10^{14}$ & $9.40 \times 10^{13}$ & $3.81 \times 10^{13}$ & $5.55 \times 10^{14}$ \\
& MCNP & $4.02 \times 10^{14}$ & $9.02 \times 10^{13}$ & $3.80 \times 10^{13}$ & $5.30 \times 10^{14}$ \\
\hline
\end{tabular}




\section{CONCLUSIONS}

The development of a comprehensive SCALE computational model for HFIR is documented and discussed in this report. This SCALE model has equivalent features as the reference MCNP model for Cycle 400, which has been used extensively at HFIR for safety analyses and by HFIR users for experiment design and analyses.

Numerical and visual comparisons are employed to assess the performance of the SCALE HFIR model relative to the MCNP Cycle 400 revised model that is currently used to evaluate relevant reactor performance parameters for the HFIR. Visual comparisons of the two models show excellent geometry matching. Numerical comparisons for the global parameters of the system, power density distribution in the fuel, and neutron fluxes at several locations in the HFIR indicate excellent agreement between the results predicted with the SCALE model and those given by the MCNP model. The difference in $\mathrm{k}_{\mathrm{eff}}$ values calculated with the two models is $124 \mathrm{pcm}$.

The SCALE HFIR model is presented in sufficient detail to provide the users of the model with a tool that can be easily customized for various safety analysis or experiment design requirements. To aid the users of the SCALE HFIR model, a flowchart of the model is included that shows in detail the geometry units describing various sections in the configuration. The input file for the SCALE HFIR model is heavily commented, containing $~ 1,500$ comment lines, for easier navigation and identification of the different components of the model, to enable the user to make changes or to add to the model, such as for the case when a particular target or experiment is inserted in the reactor.

The SCALE HFIR model is based on the SCALE generalized geometry package, and therefore can be used as a basis for studies with other modules or sequences in SCALE, such as for depletion simulations or shielding analyses. Given the diverse capabilities existent or under development in SCALE, there is a potential for more analysis options with SCALE than those provided by MCNP. The SCALE HFIR model provides the modeling basis for future integrated reactor analyses for HFIR and also provides an alternative and a verification tool for the currently used HFIR MCNP model. 


\section{REFERENCES}

1. N. Xoubi and R. T. Primm III, Modelling of the High Flux Isotope Reactor Cycle 400, ORNL/TM2004-251 (2005).

2. X-5 Monte Carlo Team, MCNP-A General Monte Carlo N-Particle Transport Code, Version 5, LA-CP-03-0245, Los Alamos National Laboratory (2003).

3. R. T. Primm III, R. J. Ellis, J. C. Gehin, D. L. Moses, J. L. Binder, and N. Xoubi, Assumptions and Criteria for Performing a Feasibility Study of the Conversion of the High Flux Isotope Reactor Core to Use Low-Enriched Uranium Fuel, ORNL/TM-2005/269, Oak Ridge National Laboratory (2006).

4. W. Haeck, VESTA User's Manual-Version 2.0.0, IRSN Report DSU/SEC/T/2008-331 Indice A, France (2009).

5. R. T. Primm III, D. Chandler, G. Ilas, B. C. Jolly, J. H. Miller, and J. D. Sease, Design Study for a Low-Enriched Uranium Core for the High Flux Isotope Reactor, Annual Report for FY 2008, ORNL/TM-2009/87, Oak Ridge National Laboratory (2009).

6. G. Ilas and R. T. Primm III, Low-Enriched Uranium Fuel Design with Two-Dimensional Grading for the High Flux Isotope Reactor, ORNL/TM-2010/318, Oak Ridge National Laboratory (2011).

7. SCALE: A Comprehensive Modeling and Simulation Suite for Nuclear Safety Analysis and Design, ORNL/TM-2005/39, Version 6.1, June 2011 (Available from Radiation Safety Information Computational Center at Oak Ridge National Laboratory as CCC-785.)

8. D. Chandler, R. T. Primm III, and G. I. Maldonado, Validating MCNP for LEU Fuel Design via Power Distribution Comparisons, ORNL/TM-2008/126, Oak Ridge National Laboratory (2008).

9. D. Chandler, R. T. Primm III, and G. I. Maldonado, Validation of a Monte Carlo Based Depletion Methodology with HFIR Post-Irradiation Examination Data, ORNL/TM-2009/123, Oak Ridge National Laboratory (2009).

10. High Flux Isotope Reactor (HFIR) User Guide: A guide to in-vessel irradiations and experiments. NSUF Revision 1. Oak Ridge National Laboratory (May 2011).

11. R. D. Cheverton and T. M. Sims, HFIR Core Nuclear Design, ORNL-4621, Oak Ridge National Laboratory (1971). 
Appendix A

INPUT FILE FOR THE SCALE HFIR MODEL 

Appendix A. Input File for the SCALE HFIR Model

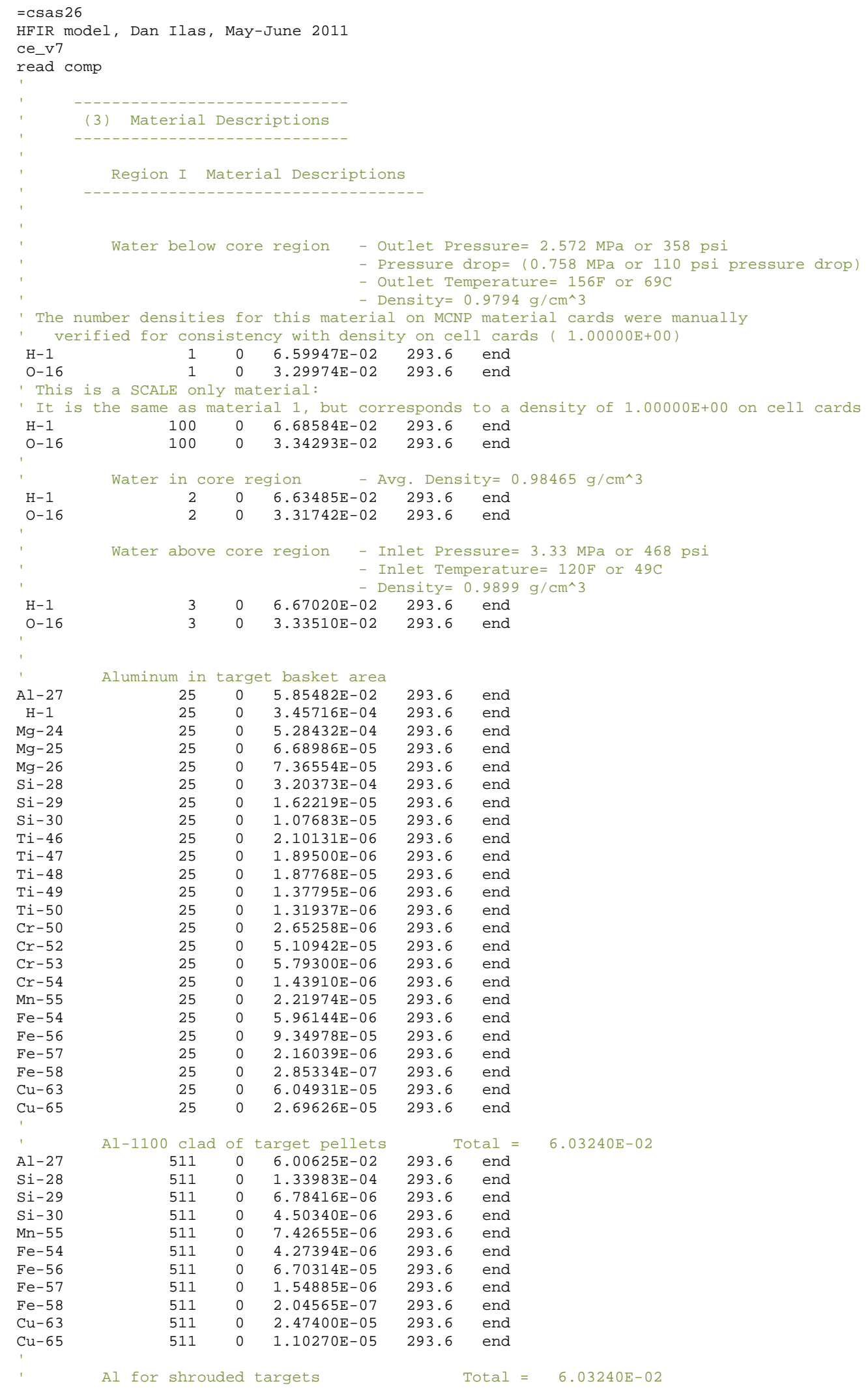




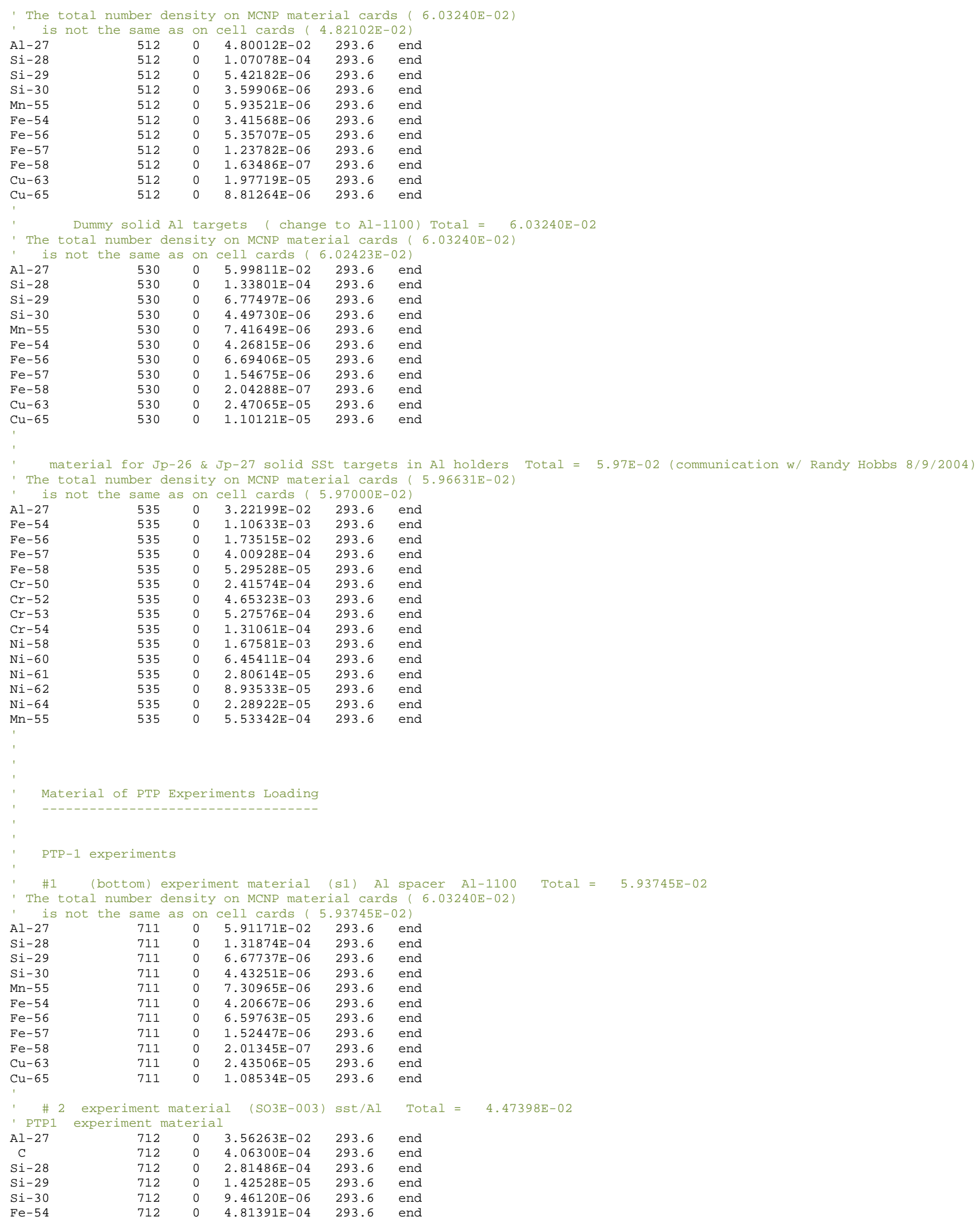




\begin{tabular}{|c|c|c|c|c|c|c|c|}
\hline $\mathrm{Fe}-56$ & 712 & $\odot$ & 7. $55002 \mathrm{E}-03$ & 293.6 & end & & \\
\hline $\mathrm{Fe}-57$ & 712 & $\odot$ & $1.74453 E-04$ & 293.6 & end & & \\
\hline $\mathrm{Fe}-58$ & 712 & $\odot$ & $2.30409 E-\odot 5$ & 293.6 & end & & \\
\hline Mo-92 & 712 & $\odot$ & $2.56880 E-05$ & 293.6 & end & & \\
\hline Mo-94 & 712 & $\odot$ & $1.60118 \mathrm{E}-05$ & 293.6 & end & & \\
\hline Mo-95 & 712 & $\odot$ & $2.75575 \mathrm{E}-05$ & 293.6 & end & & \\
\hline Mo-96 & 712 & $\odot$ & $2.88731 E-05$ & 293.6 & end & & \\
\hline Mo- 97 & 712 & $\odot$ & 1. $65311 E-05$ & 293.6 & end & & \\
\hline Mo-98 & 712 & $\odot$ & 4. 17690E- 05 & 293.6 & end & & \\
\hline Mo -100 & 712 & 0 & $1.66695 \mathrm{E}-05$ & 293.6 & end & & \\
\hline ' & & & & & & & \\
\hline ' \# 3 exp & xperiment & materia & l (NM-634) & Tot & $a l=$ & $3.38204 \mathrm{E}-02$ & \\
\hline W-186 & 713 & 0 & 1. $02360 \mathrm{E}-03$ & 293.6 & end & & \\
\hline $0-16$ & 713 & $\odot$ & 2. $09630 E-\odot 3$ & 293.6 & end & & \\
\hline $\mathrm{Al}-27$ & 713 & $\odot$ & $3.07005 E-\odot 2$ & 293.6 & end & & \\
\hline ' $\quad \# 4 \quad$ exp & xperiment & materia] & (NM-627) & Tot & $a l=$ & $3.36267 E-02$ & \\
\hline W-186 & 714 & $\odot$ & $9.54300 \mathrm{E}-\odot 4$ & 293.6 & end & & \\
\hline $0-16$ & 714 & $\odot$ & 1. $97190 \mathrm{E}-03$ & 293.6 & end & & \\
\hline $\mathrm{Al}-27$ & 714 & $\odot$ & $3.07005 \mathrm{E}-\odot 2$ & 293.6 & end & & \\
\hline ' \# 5 exp & xperiment & materia] & 1 (S2) Mo/V & Total & 2. & $32122 E-02$ & \\
\hline $\mathrm{V}$ & 715 & 0 & $1.72431 E-02$ & 293.6 & end & & \\
\hline Mo- 92 & 715 & $\odot$ & $8.85814 \mathrm{E}-\odot 4$ & 293.6 & end & & \\
\hline Mo-94 & 715 & $\odot$ & $5.52142 E-04$ & 293.6 & end & & \\
\hline Mo-95 & 715 & $\odot$ & $9.50281 E-\odot 4$ & 293.6 & end & & \\
\hline Mo-96 & 715 & $\odot$ & $9.95646 E-\odot 4$ & 293.6 & end & & \\
\hline Mo - 97 & 715 & $\odot$ & $5.70049 E-\odot 4$ & 293.6 & end & & \\
\hline Mo-98 & 715 & $\odot$ & 1. 44०34E- $\odot 3$ & 293.6 & end & & \\
\hline Mo -100 & 715 & $\odot$ & $5.74824 \mathrm{E}-04$ & 293.6 & end & & \\
\hline : \# 6 exp & xperiment & materia] & 1 (NM-659) & $\mathrm{Ra}$ & $\operatorname{tal}=$ & $3.50519 E-02$ & \\
\hline $0-16$ & cross sec & ction & & & & & \\
\hline $0-16$ & 716 & 0 & 1. $\odot \odot \odot \odot 1 E-\odot 7$ & 293.6 & end & & \\
\hline $\mathrm{Al}-27$ & 716 & $\odot$ & $3.50518 \mathrm{E}-02$ & 293.6 & end & & \\
\hline $1 \quad \# 7$ exp & xperiment & materia] & 1 top (T०31) & SST & Tensile & Total = & $6.4716 \mathrm{E}-03$ \\
\hline $\mathrm{Fe}-54$ & 717 & 03 & $3.91600 E-\odot 4$ & 293.6 & end & & \\
\hline $\mathrm{Fe}-56$ & 717 & $\odot$ & $5.92790 E-03$ & 293.6 & end & & \\
\hline $\mathrm{Fe}-57$ & 717 & $\odot$ & $1.34500 E-04$ & 293.6 & end & & \\
\hline $\mathrm{Fe}-58$ & 717 & $\odot$ & $1.76000 \mathrm{E}-\odot 5$ & 293.6 & end & & \\
\hline ' & & & & & & & \\
\hline , & & & & & & & \\
\hline PTP-2 ex & experiment & & & & & & \\
\hline & & & & & & & \\
\hline \#1 (bot & ottom) exp & periment & material & $\mathrm{r} / \mathrm{Al}$ & Total: & $1=5.65355-2$ & \\
\hline Al - 27 & 721 & 0 & $2.85703 E-\odot 2$ & 293.6 & end & & \\
\hline Si-28 & 721 & $\odot$ & $4.25273 E-\odot 4$ & 293.6 & end & & \\
\hline Si-29 & 721 & $\odot$ & $2.15334 \mathrm{E}-05$ & 293.6 & end & & \\
\hline Si-30 & 721 & $\odot$ & $1.42941 E-05$ & 293.6 & end & & \\
\hline $\mathrm{Fe}-54$ & 721 & $\odot$ & $5.17316 \mathrm{E}-05$ & 293.6 & end & & \\
\hline $\mathrm{Fe}-56$ & 721 & $\odot$ & $8.11345 E-04$ & 293.6 & end & & \\
\hline $\mathrm{Fe}-57$ & 721 & $\odot$ & $1.87472 E-05$ & 293.6 & end & & \\
\hline $\mathrm{Fe}-58$ & 721 & $\odot$ & $2.47604 \mathrm{E}-06$ & 293.6 & end & & \\
\hline C-graphite & 721 & $\odot$ & $2.62709 \mathrm{E}-02$ & 293.6 & end & & \\
\hline Mo-92 & 721 & $\odot$ & $5.17768 \mathrm{E}-05$ & 293.6 & end & & \\
\hline Mo-94 & 721 & $\odot$ & $3.22733 E-05$ & 293.6 & end & & \\
\hline Mo-95 & 721 & 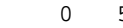 & $5.55449 E-05$ & 293.6 & end & & \\
\hline Mo-96 & 721 & $\odot$ & $5.81965 \mathrm{E}-05$ & 293.6 & end & & \\
\hline Mo-97 & 721 & $\odot$ & $3.33200 \mathrm{E}-05$ & 293.6 & end & & \\
\hline Mo-98 & 721 & $\odot$ & $8.41896 E-05$ & 293.6 & end & & \\
\hline Mo -100 & 721 & $\odot$ & 3. $35991 E-05$ & 293.6 & end & & \\
\hline ' & & & & & & & \\
\hline ' \#2 expe & periment $n$ & material & $\mathrm{SiC} / \mathrm{V}$ & Total= & 4.34300 & $0-2$ & \\
\hline - The total & 1 number & density & on MCNP materi & ial car & ds $(5$. & . 48665E- - 2$)$ & \\
\hline ' is not $t$ & the same & as on $\mathrm{ce}$ & ell cards ( 6 & $.0 \odot \odot \odot \odot \mathrm{E}$ & $=-02)$ & & \\
\hline Al - 27 & 722 & $\odot$ & 1. $90817 \mathrm{E}-02$ & 293.6 & end & & \\
\hline Si-28 & 722 & $\odot$ & $1.15348 \mathrm{E}-02$ & 293.6 & end & & \\
\hline Si-29 & 722 & $\odot$ & $5.84056 \mathrm{E}-\odot 4$ & 293.6 & end & & \\
\hline Si-30 & 722 & $\odot$ & 3. 87703E- 04 & 293.6 & end & & \\
\hline C-graphite & 722 & $\odot$ & $1.25065 \mathrm{E}-02$ & 293.6 & end & & \\
\hline $\mathrm{Fe}-54$ & 722 & $\odot$ & $5.14027 E-\odot 5$ & 293.6 & end & & \\
\hline $\mathrm{Fe}-56$ & 722 & $\odot$ & 8. . $06187 E-\odot 4$ & 293.6 & end & & \\
\hline $\mathrm{Fe}-57$ & 722 & $\odot$ & $1.86280 E-05$ & 293.6 & end & & \\
\hline $\mathrm{Fe}-58$ & 722 & $\odot$ & $2.46030 \mathrm{E}-\odot 6$ & 293.6 & end & & \\
\hline $\mathrm{V}$ & 722 & $\odot$ & $1.48368 \mathrm{E}-02$ & 293.6 & end & & \\
\hline Mo-92 & 722 & $\odot$ & $2.81564 E-05$ & 293.6 & end & & \\
\hline Mo-94 & 722 & 0 & $1.75504 \mathrm{E}-\odot 5$ & 293.6 & end & & \\
\hline
\end{tabular}




\begin{tabular}{|c|c|c|c|c|c|c|}
\hline Mo-95 & 722 & $\odot$ & $3.02055 E-05$ & 293.6 & end & \\
\hline Mo-96 & 722 & 0 & $3.16475 \mathrm{E}-05$ & 293.6 & end & \\
\hline Mo- 97 & 722 & 0 & $1.81196 \mathrm{E}-05$ & 293.6 & end & \\
\hline Mo- 98 & 722 & $\odot$ & $4.57827 E-\odot 5$ & 293.6 & end & \\
\hline Mo- 100 & 722 & $\odot$ & $1.82714 \mathrm{E}-\odot 5$ & 293.6 & end & \\
\hline \multicolumn{7}{|l|}{, } \\
\hline \multicolumn{7}{|l|}{ ' } \\
\hline \multicolumn{7}{|c|}{ ' \#3 experiment material (s1) Al spacer Al-1100 Total $=5.93745 \mathrm{E}-02$} \\
\hline ' The total & mber & density & on MCNP mat & erial ca & $\operatorname{ards}(6.03240 \mathrm{E}-02)$ & \\
\hline ' is not $t$ & same & e as on $\mathrm{c}$ & cell cards ( & 5.93745 & E- - 2 ) & \\
\hline Al-27 & 723 & 30 & $5.91171 \mathrm{E}-02$ & 293.6 & end & \\
\hline Si-28 & 723 & $\odot$ & 1. $31874 \mathrm{E}-\odot 4$ & 293.6 & end & \\
\hline Si-29 & 723 & $\odot$ & $6.67737 \mathrm{E}-06$ & 293.6 & end & \\
\hline Si-30 & 723 & $\odot$ & $4.43251 E-06$ & 293.6 & end & \\
\hline$M n-55$ & 723 & $\odot$ & $7.30965 E-\odot 6$ & 293.6 & end & \\
\hline $\mathrm{Fe}-54$ & 723 & $\odot$ & 4. 20667E-๑6 & 293.6 & end & \\
\hline $\mathrm{Fe}-56$ & 723 & $\odot$ & $6.59763 E-05$ & 293.6 & end & \\
\hline $\mathrm{Fe}-57$ & 723 & $\odot$ & 1. 52447E-๑6 & 293.6 & end & \\
\hline $\mathrm{Fe}-58$ & 723 & $\odot$ & $2.01345 E-07$ & 293.6 & end & \\
\hline $\mathrm{Cu}-63$ & 723 & $\odot$ & $2.43506 \mathrm{E}-\odot 5$ & 293.6 & end & \\
\hline $\mathrm{Cu}-65$ & 723 & $\odot$ & 1. $08534 \mathrm{E}-\odot 5$ & 293.6 & end & \\
\hline ' & & & & & & \\
\hline ' \#4 expe & ment & material & $\mathrm{Gr} / \mathrm{V}$ & Total $=5$ & 5.89491-2 & \\
\hline $\mathrm{Al}-27$ & 724 & 40 & 1. $75354 \mathrm{E}-02$ & 293.6 & end & \\
\hline $\mathrm{Si}-28$ & 724 & $\odot$ & $4.33941 E-\odot 4$ & 293.6 & end & \\
\hline Si-29 & 724 & $\odot$ & $2.19724 \mathrm{E}-\odot 5$ & 293.6 & end & \\
\hline Si-30 & 724 & $\odot$ & 1. $45855 \mathrm{E}-\odot 5$ & 293.6 & end & \\
\hline $\mathrm{Fe}-54$ & 724 & $\odot$ & $5.14741 \mathrm{E}-05$ & 293.6 & end & \\
\hline $\mathrm{Fe}-56$ & 724 & $\odot$ & 8. $07307 \mathrm{E}-\odot 4$ & 293.6 & end & \\
\hline $\mathrm{Fe}-57$ & 724 & $\odot$ & 1. $86539 E-\odot 5$ & 293.6 & end & \\
\hline $\mathrm{Fe}-58$ & 724 & $\odot$ & $2.46372 \mathrm{E}-\odot 6$ & 293.6 & end & \\
\hline C-graphite & 724 & $\odot$ & $2.62254 \mathrm{E}-02$ & 293.6 & end & \\
\hline V & 724 & $\odot$ & 1. $35332 \mathrm{E}-02$ & 293.6 & end & \\
\hline Mo-92 & 724 & $\odot$ & $4.52322 \mathrm{E}-05$ & 293.6 & end & \\
\hline Mo-94 & 724 & $\odot$ & $2.81940 E-\odot 5$ & 293.6 & end & \\
\hline Mo-95 & 724 & $\odot$ & $4.85241 E-\odot 5$ & 293.6 & end & \\
\hline Mo-96 & 724 & $\odot$ & 5. $08405 E-\odot 5$ & 293.6 & end & \\
\hline Mo- 97 & 724 & $\odot$ & 2. $91084 \mathrm{E}-05$ & 293.6 & end & \\
\hline Mo-98 & 724 & $\odot$ & $7.35481 E-05$ & 293.6 & end & \\
\hline Mo - 100 & 724 & $\odot$ & $2.93522 \mathrm{E}-05$ & 293.6 & end & \\
\hline ' & & & & & & \\
\hline ' \#5 expe & ment & material & $\mathrm{Gr} / \mathrm{V}$ & Total $=5$ & $.89252-2$ & \\
\hline $\mathrm{Al}-27$ & 725 & $\odot$ & 1. $74621 \mathrm{E}-02$ & 293.6 & end & \\
\hline Si-28 & 725 & $\odot$ & 4. 32374E- $\odot 4$ & 293.6 & end & \\
\hline Si-29 & 725 & $\odot$ & $2.18930 E-\odot 5$ & 293.6 & end & \\
\hline Si-30 & 725 & $\odot$ & 1. $45328 \mathrm{E}-05$ & 293.6 & end & \\
\hline $\mathrm{Fe}-54$ & 725 & $\odot$ & $5.13221 E-05$ & 293.6 & end & \\
\hline $\mathrm{Fe}-56$ & 725 & $\odot$ & 8. $04923 E-\odot 4$ & 293.6 & end & \\
\hline $\mathrm{Fe}-57$ & 725 & $\odot$ & $1.85988 \mathrm{E}-05$ & 293.6 & end & \\
\hline $\mathrm{Fe}-58$ & 725 & $\odot$ & $2.45644 \mathrm{E}-06$ & 293.6 & end & \\
\hline C-graphite & 725 & $\odot$ & $2.62644 \mathrm{E}-02$ & 293.6 & end & \\
\hline V & 725 & $\odot$ & 1. $34952 \mathrm{E}-02$ & 293.6 & end & \\
\hline Mo- 92 & 725 & $\odot$ & $5.30382 \mathrm{E}-\odot 5$ & 293.6 & end & \\
\hline Mo-94 & 725 & $\odot$ & 3. $30595 \mathrm{E}-\odot 5$ & 293.6 & end & \\
\hline Mo-95 & 725 & $\odot$ & $5.68981 E-05$ & 293.6 & end & \\
\hline Mo-96 & 725 & $\odot$ & $5.96143 E-05$ & 293.6 & end & \\
\hline Mo- 97 & 725 & $\odot$ & $3.41317 \mathrm{E}-05$ & 293.6 & end & \\
\hline Mo-98 & 725 & $\odot$ & $8.62406 E-05$ & 293.6 & end & \\
\hline Mo- 100 & 725 & $\odot$ & $3.44176 \mathrm{E}-05$ & 293.6 & end & \\
\hline ' & & & & & & \\
\hline ' \#6 expe & ment & material & $1 \quad$ (s1) & spacer & Total $=$ & 5. $93745 \mathrm{E}-02$ \\
\hline - The total & mber & density & on MCNP mat & erial ca & $\operatorname{ards}(6.03240 \mathrm{E}-02)$ & \\
\hline ' is not $t$ & same & as on $\mathrm{c}$ & cell cards ( & 5.93745 & $\mathrm{E}-02)$ & \\
\hline Al-27 & 726 & $\odot$ & $5.91171 E-02$ & 293.6 & end & \\
\hline Si-28 & 726 & $\odot$ & 1. $31874 \mathrm{E}-\odot 4$ & 293.6 & end & \\
\hline Si-29 & 726 & $\odot$ & $6.67737 \mathrm{E}-06$ & 293.6 & end & \\
\hline Si-30 & 726 & $\odot$ & $4.43251 E-\odot 6$ & 293.6 & end & \\
\hline$M n-55$ & 726 & $\odot$ & 7. 3०965E- $\odot 6$ & 293.6 & end & \\
\hline $\mathrm{Fe}-54$ & 726 & $\odot$ & 4. $20667 \mathrm{E}-\odot 6$ & 293.6 & end & \\
\hline $\mathrm{Fe}-56$ & 726 & $\odot$ & $6.59763 E-\odot 5$ & 293.6 & end & \\
\hline $\mathrm{Fe}-57$ & 726 & $\odot$ & 1. 52447E-๑6 & 293.6 & end & \\
\hline $\mathrm{Fe}-58$ & 726 & $\odot$ & 2. $01345 E-07$ & 293.6 & end & \\
\hline $\mathrm{Cu}-63$ & 726 & $\odot$ & $2.435 \odot 6 E-\odot 5$ & 293.6 & end & \\
\hline $\mathrm{Cu}-65$ & 726 & $\odot$ & 1. $08534 \mathrm{E}-05$ & 293.6 & end & \\
\hline ' & & & & & & \\
\hline \#7 & $n t$ & material & $\mathrm{Gr} / \mathrm{V}$ & $\mathrm{tal}=6$ & . 03159-2 & \\
\hline
\end{tabular}




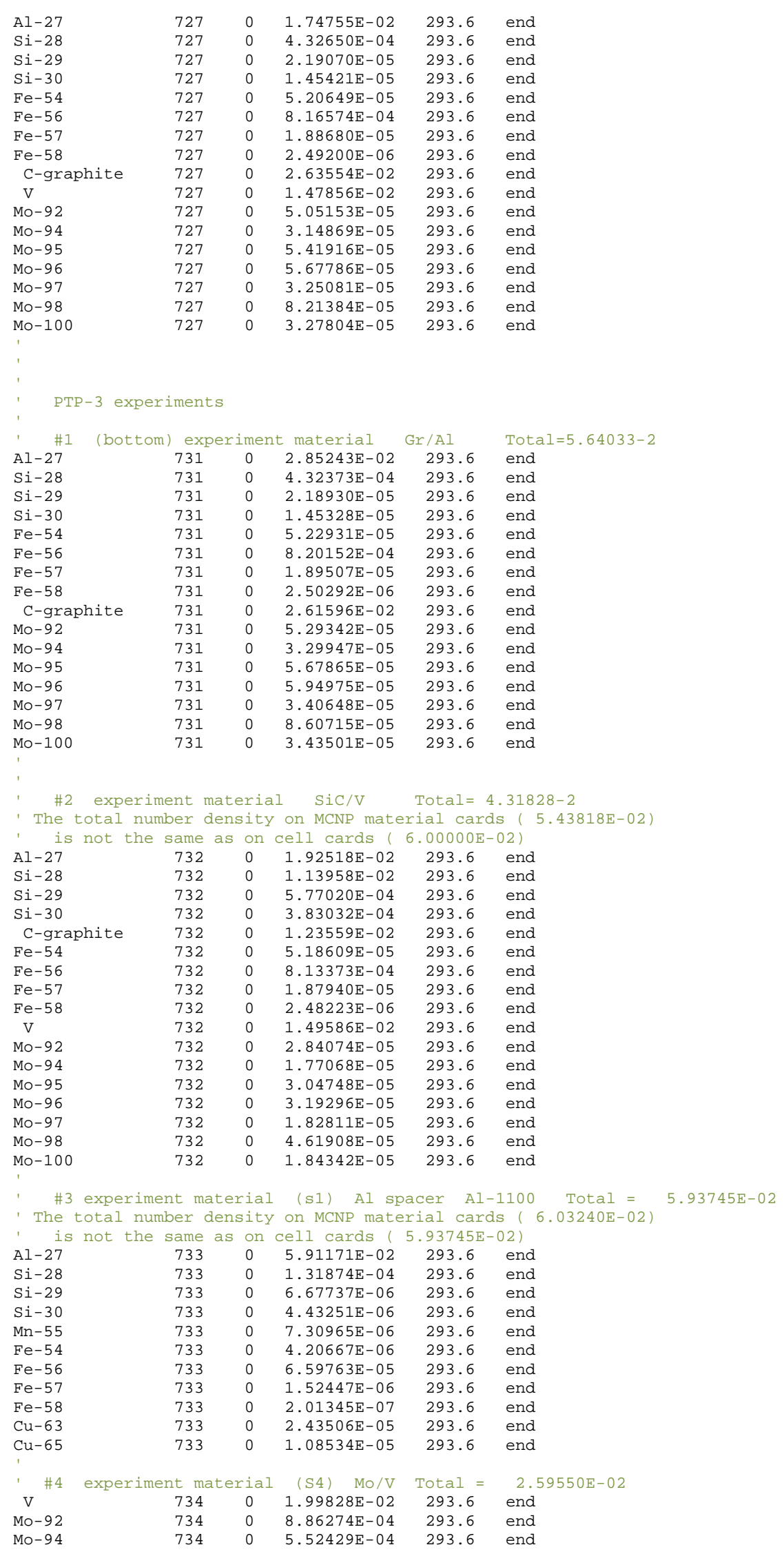




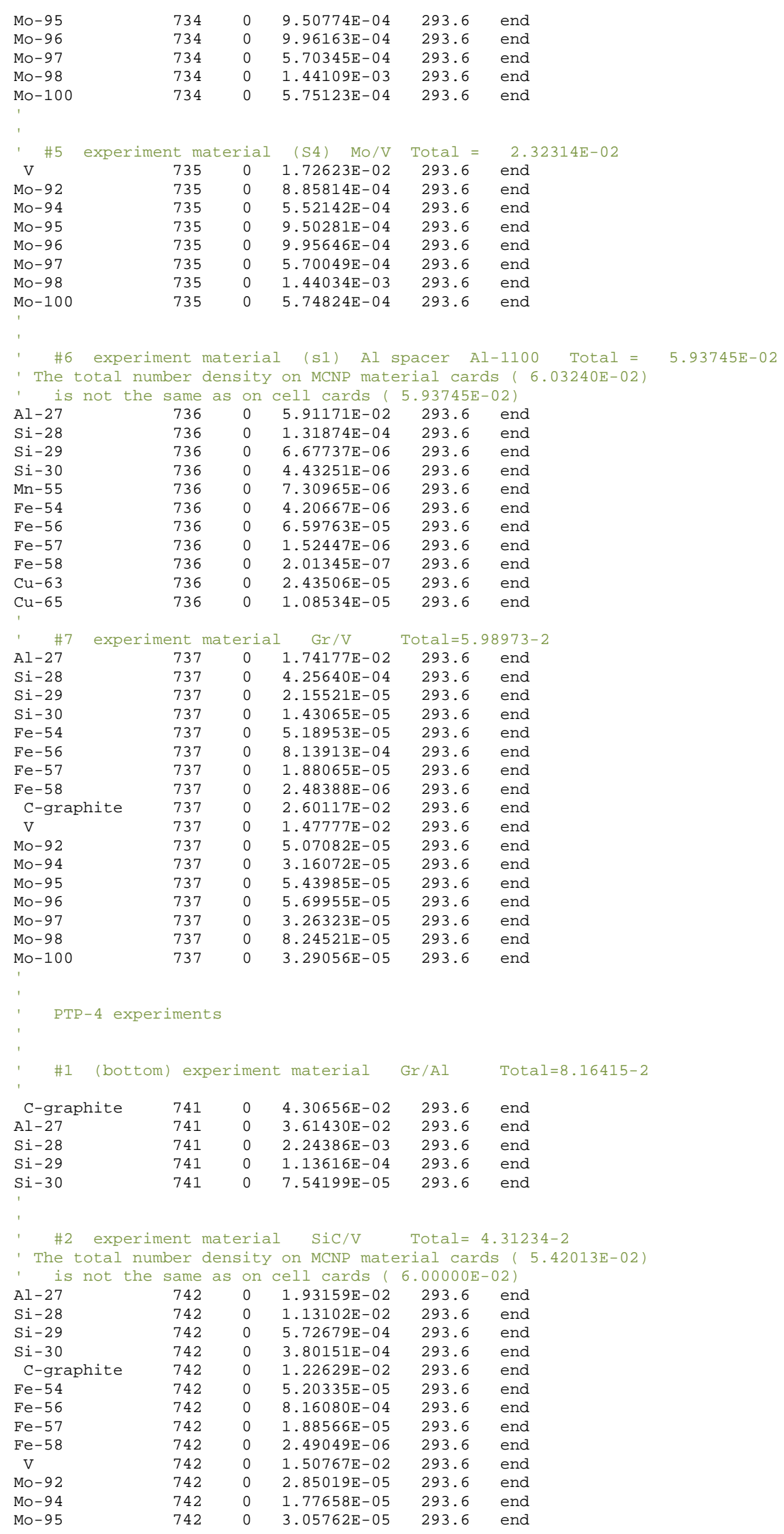




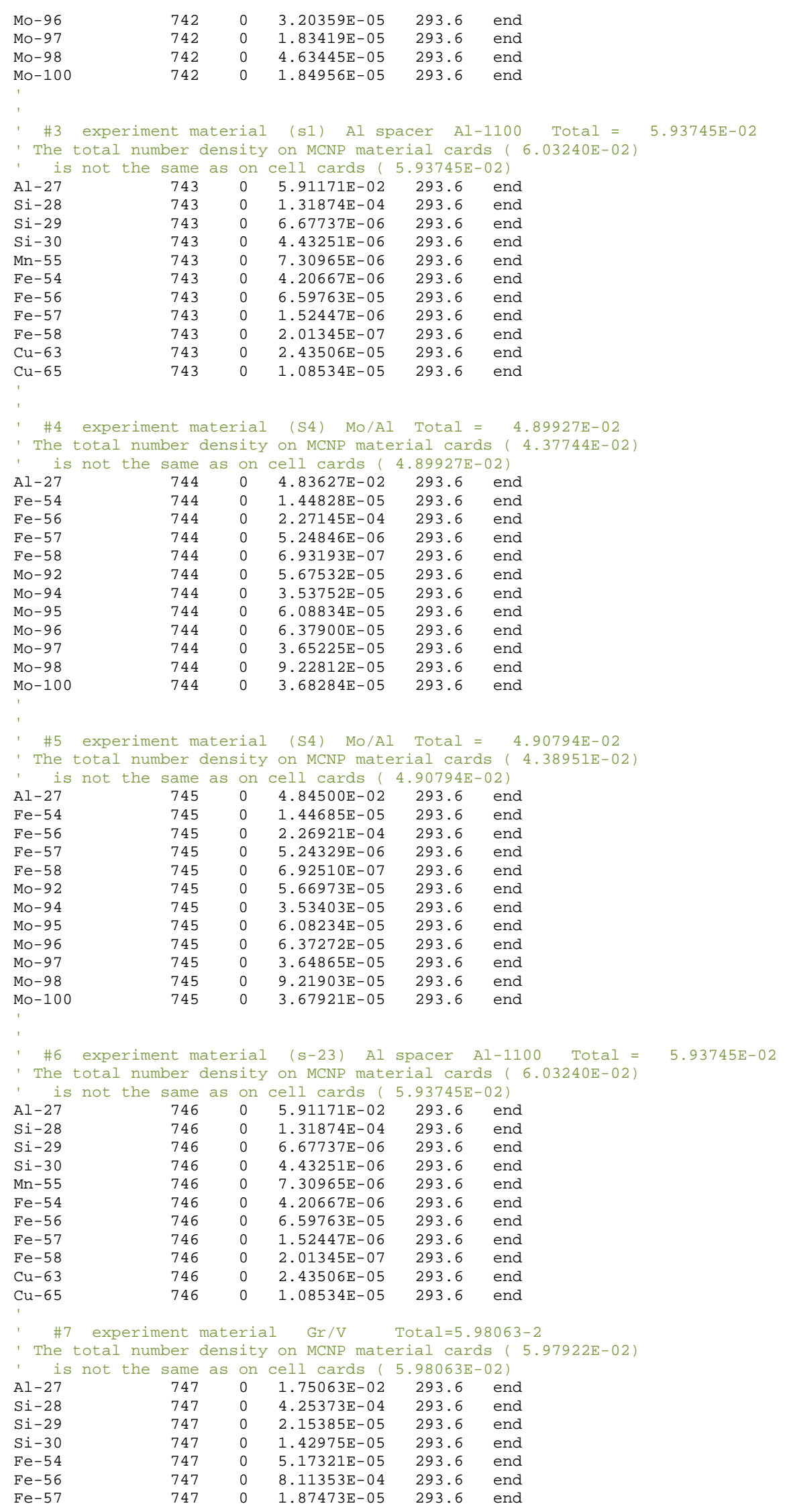




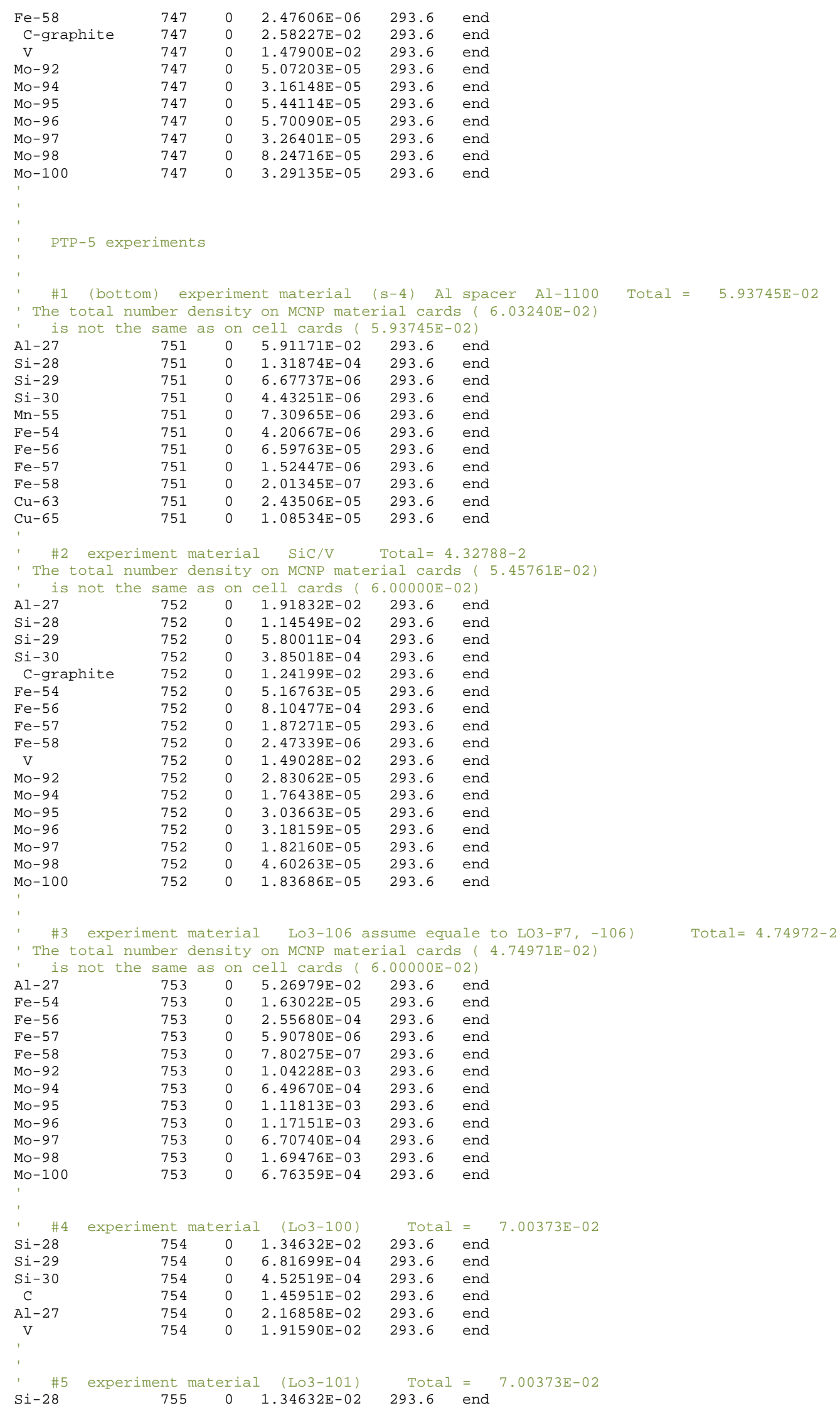




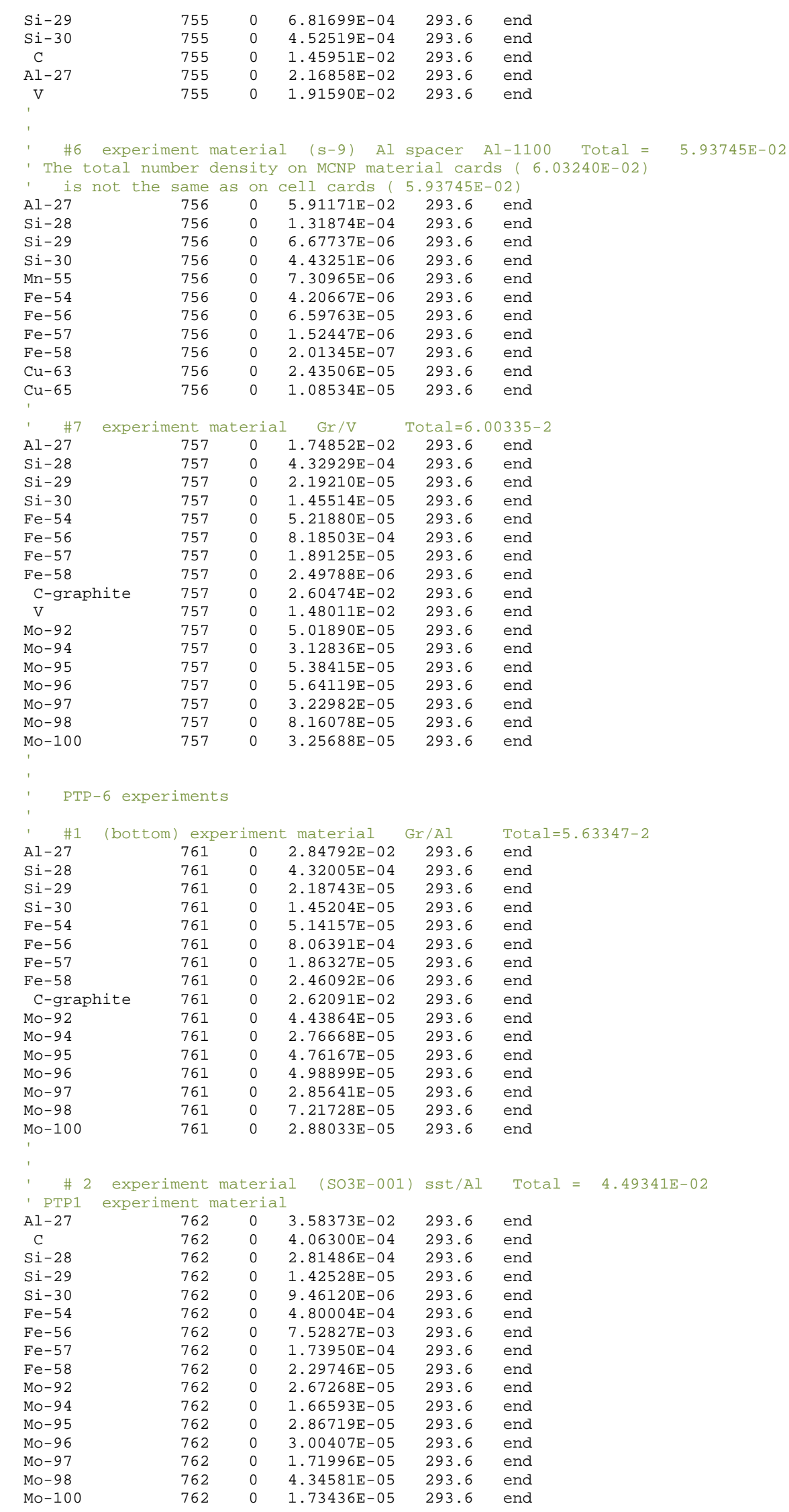




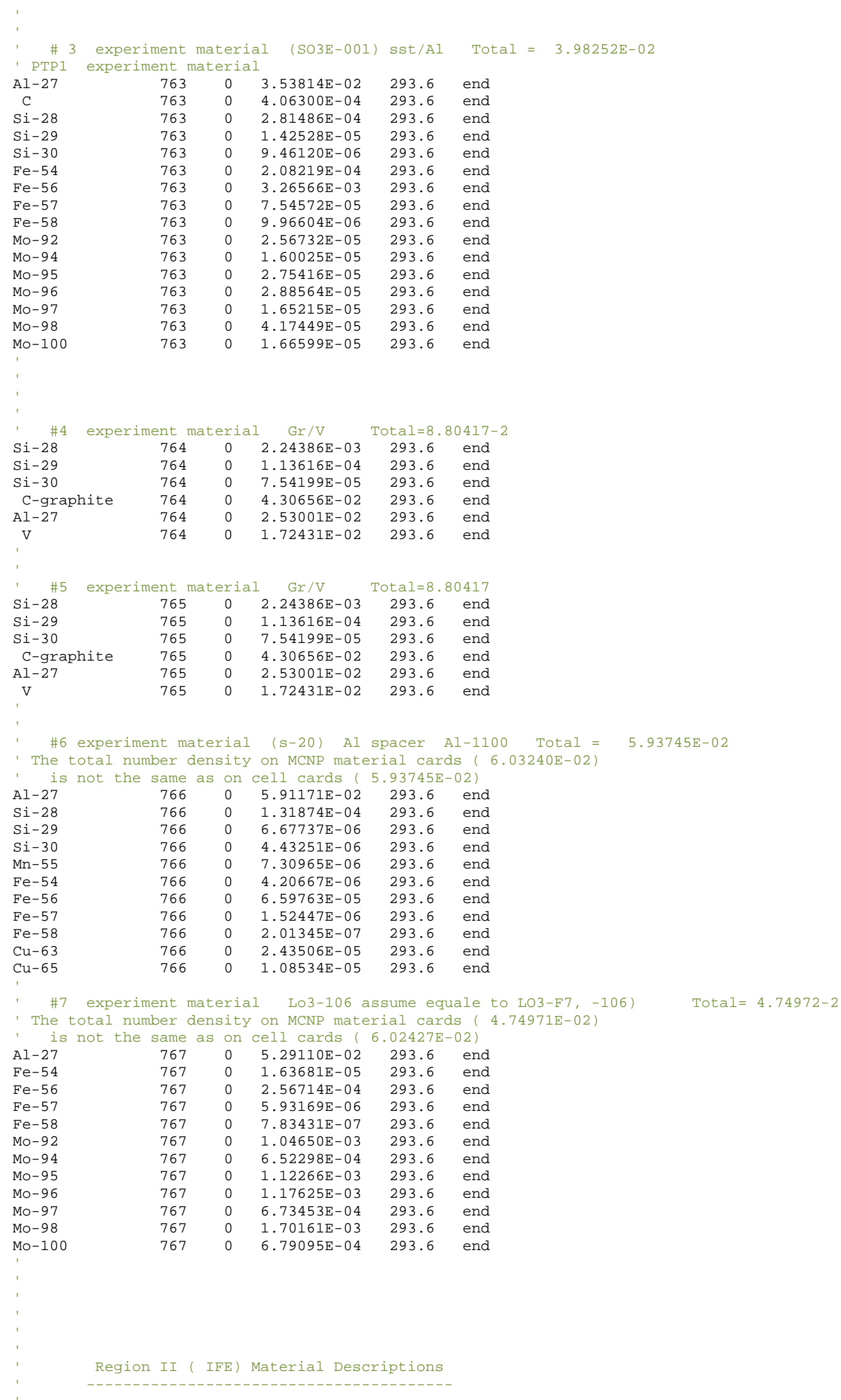


Aluminum Sidewalls

Aluminum fuel element sidewalls

The number densities for this material on MCNP material cards were manually

\begin{tabular}{|c|c|c|c|c|c|}
\hline Al-27 & 20 & 0 & $5.86577 \mathrm{E}-\odot 2$ & 293.6 & end \\
\hline $\mathrm{H}-1$ & 20 & $\odot$ & $3.46363 E-\odot 4$ & 293.6 & end \\
\hline$M g-24$ & 20 & $\odot$ & $5.29421 E-\odot 4$ & 293.6 & \\
\hline$M g-25$ & 20 & 0 & $6.70238 \mathrm{E}-05$ & 293.6 & \\
\hline$M g-26$ & 20 & $\odot$ & $7.37932 \mathrm{E}-05$ & 293.6 & \\
\hline Si-28 & 20 & 0 & $3.20972 \mathrm{E}-04$ & 293.6 & \\
\hline Si-29 & 20 & 0 & $1.62522 \mathrm{E}-05$ & 293.6 & \\
\hline Si-30 & 20 & 0 & 1. $07884 \mathrm{E}-05$ & 293.6 & \\
\hline Ti-46 & 20 & 0 & $2.10524 \mathrm{E}-06$ & 293.6 & \\
\hline Ti-47 & 20 & $\odot$ & 1. 89855E- 06 & 293.6 & \\
\hline Ti-48 & 20 & $\odot$ & $1.88119 \mathrm{E}-05$ & 293.6 & \\
\hline Ti-49 & 20 & 0 & 1. 38053E- $\odot 6$ & 293.6 & \\
\hline Ti-50 & 20 & $\odot$ & 1. 32184E- $\odot 6$ & 293.6 & \\
\hline $\mathrm{Cr}-50$ & 20 & $\odot$ & $2.65754 \mathrm{E}-\odot 6$ & 293.6 & \\
\hline $\mathrm{Cr}-52$ & 20 & $\odot$ & $5.11898 \mathrm{E}-05$ & 293.6 & \\
\hline $\mathrm{Cr}-53$ & 20 & $\odot$ & $5.80384 \mathrm{E}-06$ & 293.6 & \\
\hline $\mathrm{Cr}-54$ & 20 & 0 & 1. 44179E-06 & 293.6 & \\
\hline$M n-55$ & 20 & $\odot$ & $2.22389 \mathrm{E}-05$ & 293.6 & \\
\hline $\mathrm{Fe}-54$ & 20 & $\odot$ & $5.97259 \mathrm{E}-06$ & 293.6 & \\
\hline $\mathrm{Fe}-56$ & 20 & 0 & $9.36727 \mathrm{E}-\odot 5$ & 293.6 & \\
\hline $\mathrm{Fe}-57$ & 20 & $\odot$ & $2.16443 E-06$ & 293.6 & \\
\hline $\mathrm{Fe}-58$ & 20 & $\odot$ & $2.85868 \mathrm{E}-\odot 7$ & 293.6 & \\
\hline $\mathrm{Cu}-63$ & 20 & 0 & $6.06063 E-05$ & 293.6 & \\
\hline Cu-65 & 20 & $\odot$ & $2.70130 \mathrm{E}-\odot 5$ & 293.6 & \\
\hline
\end{tabular}

$\mathrm{Cu}-65$

Upper and lower Unfuelled Core Regions (50\% H20-50\% Al-6061) *

Inner fuel element--lower uncontrolled region

- 3.35240E-02 293.6 end

$0-16 \quad 70 \quad 0 \quad 1.66756 \mathrm{E}-02 \quad 293.6$ end

Al-27 $70 \quad 0 \quad 2.92741 \mathrm{E}-02 \quad 293.6$ end

Si-28 $70 \quad \odot \quad 1.60187 \mathrm{E}-04 \quad 293.6$ end

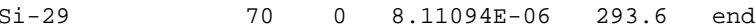

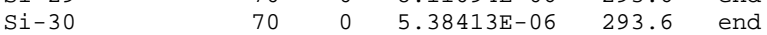

Ti-46 $70 \quad \odot \quad 1.05065 \mathrm{E}-06 \quad 293.6$ end

$\begin{array}{llllll}\text { Ti-47 } & 70 & 0 & 9.47498 \mathrm{E}-07 & 293.6 & \text { end }\end{array}$

$\begin{array}{lllll}\text { Ti-48 } & 70 & \odot & 9.38838 \mathrm{E}-06 & 293.6\end{array}$ end

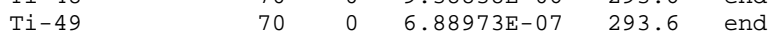

$\begin{array}{lllll}\text { Ti-50 } & 70 & \odot & 6.59682 \mathrm{E}-07 & 293.6\end{array}$ end

$\begin{array}{lllll}\mathrm{Cr}-50 & 70 & 0 & 1.32629 \mathrm{E}-06 & 293.6\end{array}$ end

$\begin{array}{lllll}\mathrm{Cr}-52 & 70 & \odot & 2.55471 \mathrm{E}-05 & 293.6\end{array}$

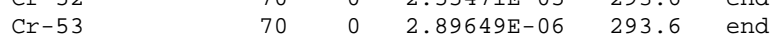

$\begin{array}{llllll}\mathrm{Cr}-54 & 70 & \odot & 7.19549 \mathrm{E}-07 & 293.6 & \text { end }\end{array}$

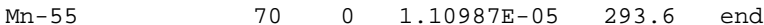

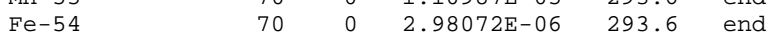

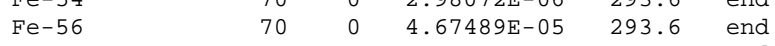

Fe-57 $70 \quad 0 \quad 1.08019 \mathrm{E}-06 \quad 293.6$ end

$\begin{array}{llllll}\mathrm{Fe}-58 & 70 & \odot & 1.42667 \mathrm{E}-07 & 293.6 & \text { end }\end{array}$

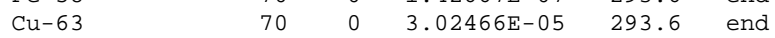

$\begin{array}{lllll}\mathrm{Cu}-65 & 70 & \odot & 1.34813 \mathrm{E}-05 & 293.6\end{array}$ end

$\begin{array}{llllll}\mathrm{Mg}-24 & 70 & \odot & 2.64216 \mathrm{E}-04 & 293.6 & \text { end }\end{array}$

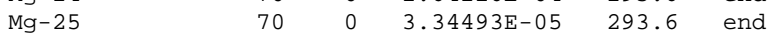

Mg-26 $\quad 70 \quad 0 \quad 3.68277 \mathrm{E}-05 \quad 293.6$ end

$\begin{array}{llllll}\mathrm{H}-1 & 71 & 0 & 3.31702 \mathrm{E}-02 & 293.6 & \text { end }\end{array}$

$\begin{array}{lllll}0-16 & 71 & \odot & 1.64987 \mathrm{E}-02 & 293.6\end{array}$

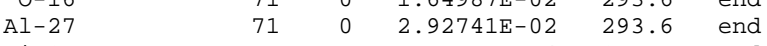

$\begin{array}{llllll}\text { Si-28 } & 71 & \odot & 1.60187 \mathrm{E}-04 & 293.6 & \text { end }\end{array}$

$\begin{array}{lllll}\text { Si-29 } & 71 & 0 & 8.11095 \mathrm{E}-06 & 293.6\end{array}$ end

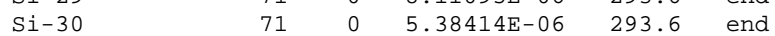

$\mathrm{Ti}-46 \quad 71 \quad \odot \quad 1.05065 \mathrm{E}-06 \quad 293.6$ end

$\begin{array}{llllll}\text { Ti-47 } & 71 & 0 & 9.47499 \mathrm{E}-07 & 293.6 & \text { end }\end{array}$

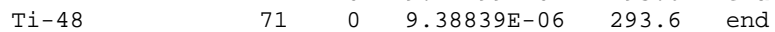

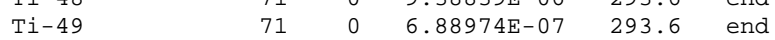

$\begin{array}{lllll}\text { Ti-50 } & 71 & \odot & 6.59683 \mathrm{E}-07 & 293.6\end{array}$ end

$\begin{array}{llllll}\mathrm{Cr}-50 & 71 & 0 & 1.32629 \mathrm{E}-06 & 293.6 & \text { end }\end{array}$

$\begin{array}{lllll}\mathrm{Cr}-52 & 71 & \odot & 2.55471 \mathrm{E}-05 & 293.6\end{array}$ end

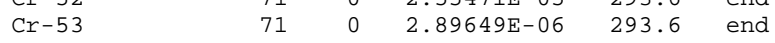

$\begin{array}{lllll}\mathrm{Cr}-54 & 71 & 0 & 7.19550 \mathrm{E}-07 & 293.6\end{array}$ end

$\begin{array}{lllll}\mathrm{Mn}-55 & 71 & 0 & 1.10987 \mathrm{E}-05 & 293.6\end{array}$ end

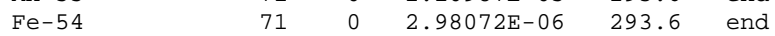

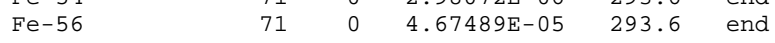

$\begin{array}{llllll}\mathrm{Fe}-57 & 71 & \odot & 1.08019 \mathrm{E}-06 & 293.6 & \text { end }\end{array}$

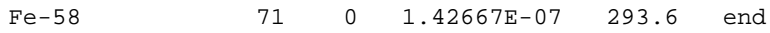

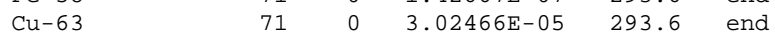

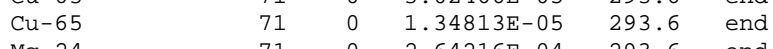

$\begin{array}{lllll}71 & 0 & 2.64216 \mathrm{E}-04 & 293.6 & \text { end }\end{array}$ 


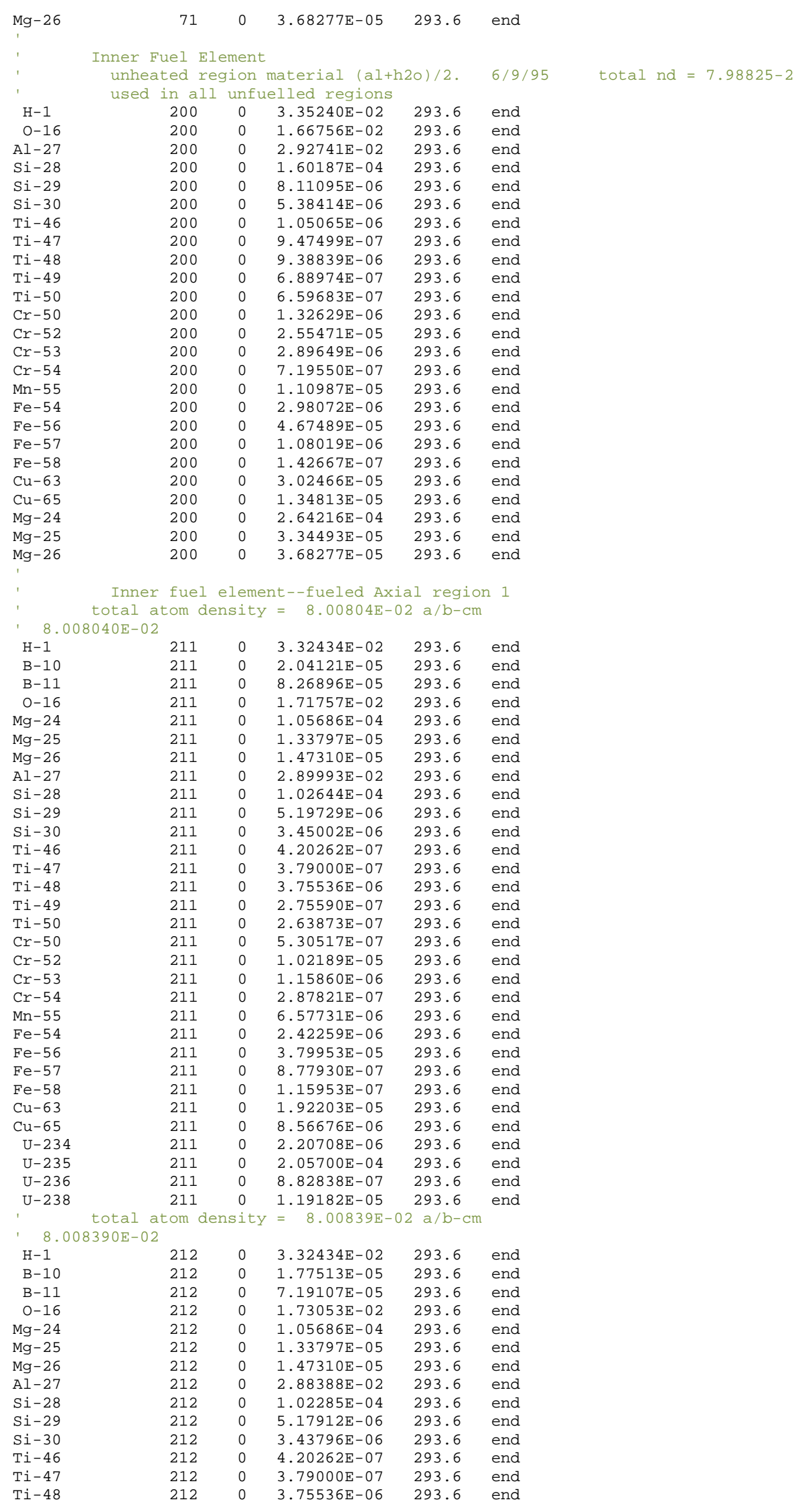




\begin{tabular}{|c|c|c|c|c|c|}
\hline Ti-49 & 212 & $\odot$ & $2.75590 \mathrm{E}-\odot 7$ & 293.6 & end \\
\hline Ti-50 & 212 & $\odot$ & $2.63873 E-07$ & 293.6 & end \\
\hline $\mathrm{Cr}-50$ & 212 & $\odot$ & $5.30517 E-07$ & 293.6 & end \\
\hline $\mathrm{Cr}-52$ & 212 & $\odot$ & 1. $\odot 2189 E-\odot 5$ & 293.6 & end \\
\hline $\mathrm{Cr}-53$ & 212 & $\odot$ & $1.15860 E-06$ & 293.6 & end \\
\hline $\mathrm{Cr}-54$ & 212 & $\odot$ & $2.87821 E-\odot 7$ & 293.6 & end \\
\hline$M n-55$ & 212 & $\odot$ & $6.55746 E-06$ & 293.6 & end \\
\hline $\mathrm{Fe}-54$ & 212 & 0 & $2.41117 \mathrm{E}-06$ & 293.6 & end \\
\hline $\mathrm{Fe}-56$ & 212 & 0 & $3.78162 \mathrm{E}-05$ & 293.6 & end \\
\hline $\mathrm{Fe}-57$ & 212 & 0 & $8.73792 \mathrm{E}-07$ & 293.6 & end \\
\hline $\mathrm{Fe}-58$ & 212 & $\odot$ & $1.15406 \mathrm{E}-07$ & 293.6 & end \\
\hline $\mathrm{Cu}-63$ & 212 & 0 & $1.91542 \mathrm{E}-\odot 5$ & 293.6 & end \\
\hline $\mathrm{Cu}-65$ & 212 & $\odot$ & $8.53729 E-\odot 6$ & 293.6 & end \\
\hline$U-234$ & 212 & $\odot$ & $2.69313 E-\odot 6$ & 293.6 & end \\
\hline$U-235$ & 212 & $\odot$ & $2.510 \odot \odot E-\odot 4$ & 293.6 & end \\
\hline$U-236$ & 212 & $\odot$ & $1.07726 \mathrm{E}-06$ & 293.6 & end \\
\hline$U-238$ & 212 & $\odot$ & $1.45429 E-05$ & 293.6 & end \\
\hline
\end{tabular}

\begin{tabular}{|c|c|c|c|c|c|}
\hline$H-1$ & 213 & $\odot$ & $3.32435 E-02$ & 293.6 & end \\
\hline$B-10$ & 213 & 0 & $1.46911 \mathrm{E}-05$ & 293.6 & end \\
\hline B-11 & 213 & $\odot$ & $5.95139 \mathrm{E}-\odot 5$ & 293.6 & \\
\hline $0-16$ & 213 & $\odot$ & 1. $74543 E-02$ & 293.6 & \\
\hline$M g-24$ & 213 & $\odot$ & 1. $\odot 5686 E-\odot 4$ & 293.6 & \\
\hline$M g-25$ & 213 & $\odot$ & $1.33797 \mathrm{E}-05$ & 293.6 & 10 \\
\hline$M g-26$ & 213 & $\odot$ & 1. $47310 \mathrm{E}-\odot 5$ & 293.6 & \\
\hline $\mathrm{Al}-27$ & 213 & 0 & $2.86541 E-02$ & 293.6 & \\
\hline Si-28 & 213 & $\odot$ & 1. $01874 \mathrm{E}-04$ & 293.6 & \\
\hline Si-29 & 213 & $\odot$ & $5.15831 \mathrm{E}-06$ & 293.6 & \\
\hline Si-30 & 213 & 0 & $3.42415 \mathrm{E}-06$ & 293.6 & \\
\hline Ti-46 & 213 & 0 & $4.20263 E-07$ & 293.6 & \\
\hline Ti-47 & 213 & $\odot$ & $3.79001 E-07$ & 293.6 & \\
\hline Ti-48 & 213 & $\odot$ & $3.75537 \mathrm{E}-06$ & 293.6 & \\
\hline Ti-49 & 213 & $\odot$ & $2.7559 \odot E-\odot 7$ & 293.6 & \\
\hline Ti-50 & 213 & $\odot$ & $2.63873 E-\odot 7$ & 293.6 & \\
\hline $\mathrm{Cr}-50$ & 213 & $\odot$ & $5.30518 \mathrm{E}-\odot 7$ & 293.6 & \\
\hline $\mathrm{Cr}-52$ & 213 & $\odot$ & 1. $\odot 2189 E-\odot 5$ & 293.6 & \\
\hline $\mathrm{Cr}-53$ & 213 & $\odot$ & $1.15860 E-06$ & 293.6 & \\
\hline $\mathrm{Cr}-54$ & 213 & $\odot$ & $2.87821 E-07$ & 293.6 & \\
\hline$M n-55$ & 213 & 0 & $6.53464 \mathrm{E}-06$ & 293.6 & \\
\hline $\mathrm{Fe}-54$ & 213 & $\odot$ & 2. 39803E- $\odot 6$ & 293.6 & \\
\hline $\mathrm{Fe}-56$ & 213 & $\odot$ & $3.76103 E-05$ & 293.6 & \\
\hline $\mathrm{Fe}-57$ & 213 & $\odot$ & $8.69031 \mathrm{E}-07$ & 293.6 & \\
\hline $\mathrm{Fe}-58$ & 213 & $\odot$ & $1.14778 \mathrm{E}-07$ & 293.6 & \\
\hline $\mathrm{Cu}-63$ & 213 & $\odot$ & 1. $90782 \mathrm{E}-\odot 5$ & 293.6 & \\
\hline $\mathrm{Cu}-65$ & 213 & $\odot$ & $8.50342 E-06$ & 293.6 & \\
\hline$U-234$ & 213 & 0 & $3.25215 E-06$ & 293.6 & \\
\hline$U-235$ & 213 & $\odot$ & $3.03100 \mathrm{E}-04$ & 293.6 & \\
\hline$U-236$ & 213 & $\odot$ & 1. $30087 \mathrm{E}-06$ & 293.6 & \\
\hline$U-238$ & 213 & $\odot$ & 1. $75616 \mathrm{E}-\odot 5$ & 293.6 & \\
\hline
\end{tabular}

, total atom density $=8.00937 \mathrm{E}-02 \mathrm{a} / \mathrm{b}-\mathrm{cm}$

$\begin{array}{llllll} & 8.009370 \mathrm{E}-02 & & & & \\ \mathrm{H}-1 & 214 & \odot & 3.32434 \mathrm{E}-02 & 293.6 & \text { end } \\ \mathrm{B}-10 & 214 & \odot & 1.03798 \mathrm{E}-05 & 293.6 & \text { end } \\ \mathrm{B}-11 & 214 & \odot & 4.20486 \mathrm{E}-05 & 293.6 & \text { end } \\ \mathrm{O}-16 & 214 & \odot & 1.76644 \mathrm{E}-02 & 293.6 & \text { end } \\ \mathrm{Mg}-24 & 214 & \odot & 1.05686 \mathrm{E}-04 & 293.6 & \text { end } \\ \mathrm{Mg}-25 & 214 & \odot & 1.33797 \mathrm{E}-05 & 293.6 & \text { end } \\ \mathrm{Mg}-26 & 214 & \odot & 1.47310 \mathrm{E}-05 & 293.6 & \text { end } \\ \mathrm{Al}-27 & 214 & \odot & 2.83940 \mathrm{E}-02 & 293.6 & \text { end } \\ \mathrm{Si}-28 & 214 & \odot & 1.01293 \mathrm{E}-04 & 293.6 & \text { end } \\ \mathrm{Si}-29 & 214 & \odot & 5.12887 \mathrm{E}-06 & 293.6 & \text { end } \\ \mathrm{Si}-3 \odot & 214 & \odot & 3.40461 \mathrm{E}-06 & 293.6 & \text { end } \\ \mathrm{Ti}-46 & 214 & \odot & 4.20262 \mathrm{E}-07 & 293.6 & \text { end } \\ \mathrm{Ti}-47 & 214 & \odot & 3.79000 \mathrm{E}-07 & 293.6 & \text { end } \\ \mathrm{Ti}-48 & 214 & \odot & 3.75536 \mathrm{E}-06 & 293.6 & \text { end } \\ \mathrm{Ti}-49 & 214 & \odot & 2.75590 \mathrm{E}-07 & 293.6 & \text { end } \\ \mathrm{Ti}-50 & 214 & \odot & 2.63873 \mathrm{E}-07 & 293.6 & \text { end } \\ \mathrm{Cr}-5 \odot & 214 & \odot & 5.30517 \mathrm{E}-07 & 293.6 & \text { end } \\ \mathrm{Cr}-52 & 214 & \odot & 1.02189 \mathrm{E}-05 & 293.6 & \text { end } \\ \mathrm{Cr}-53 & 214 & \odot & 1.15860 \mathrm{E}-06 & 293.6 & \text { end } \\ \mathrm{Cr}-54 & 214 & \odot & 2.87821 \mathrm{E}-07 & 293.6 & \text { end } \\ \mathrm{Mn}-55 & 214 & \odot & 6.50246 \mathrm{E}-06 & 293.6 & \text { end } \\ \mathrm{Fe}-54 & 214 & \odot & 2.37952 \mathrm{E}-06 & 293.6 & \text { end } \\ \mathrm{Fe}-56 & 214 & \odot & 3.73198 \mathrm{E}-05 & 293.6 & \text { end } \\ \mathrm{Fe}-57 & 214 & \odot & 8.62321 \mathrm{E}-07 & 293.6 & \text { end } \\ \mathrm{Fe}-58 & 214 & \odot & 1.13891 \mathrm{E}-07 & 293.6 & \text { end } \\ \mathrm{Cu}-63 & 214 & \odot & 1.89710 \mathrm{E}-05 & 293.6 & \text { end } \\ \mathrm{Cu}-65 & 214 & \odot & 8.45562 \mathrm{E}-06 & 293.6 & \text { end } \\ \mathrm{U}-234 & 214 & \odot & 4.03970 \mathrm{E}-06 & 293.6 & \text { end } \\ \mathrm{U}-235 & 214 & \odot & 3.76500 \mathrm{E}-04 & 293.6 & \text { end }\end{array}$




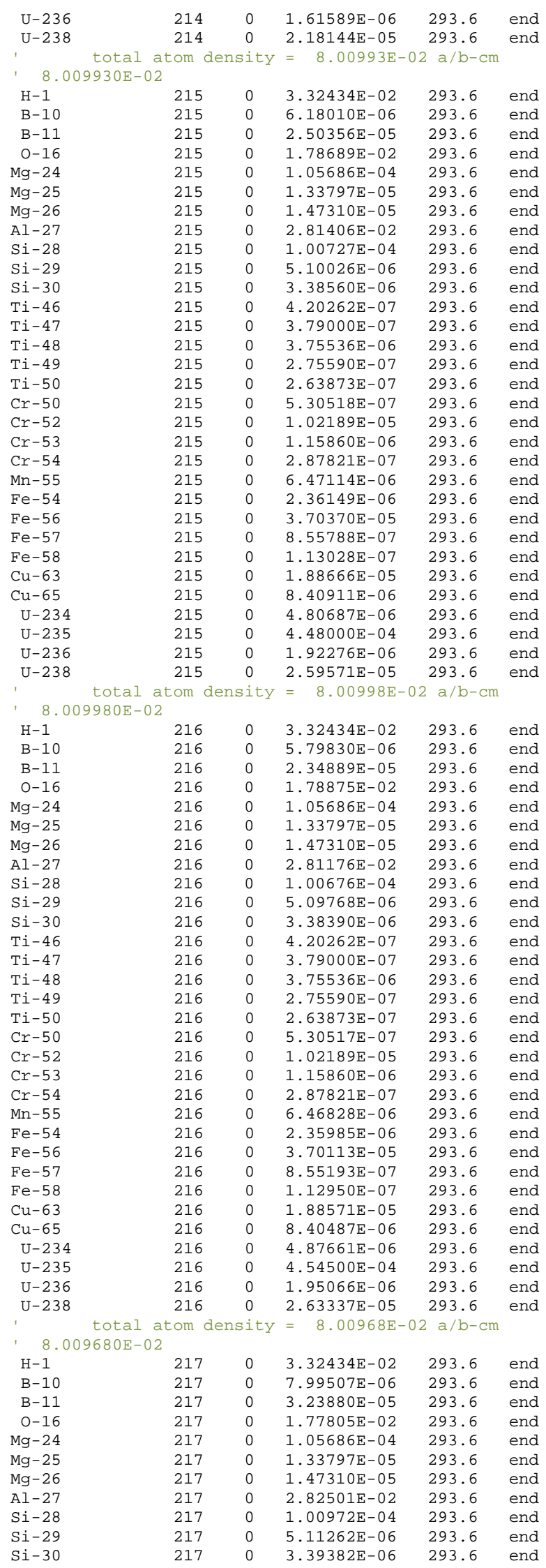




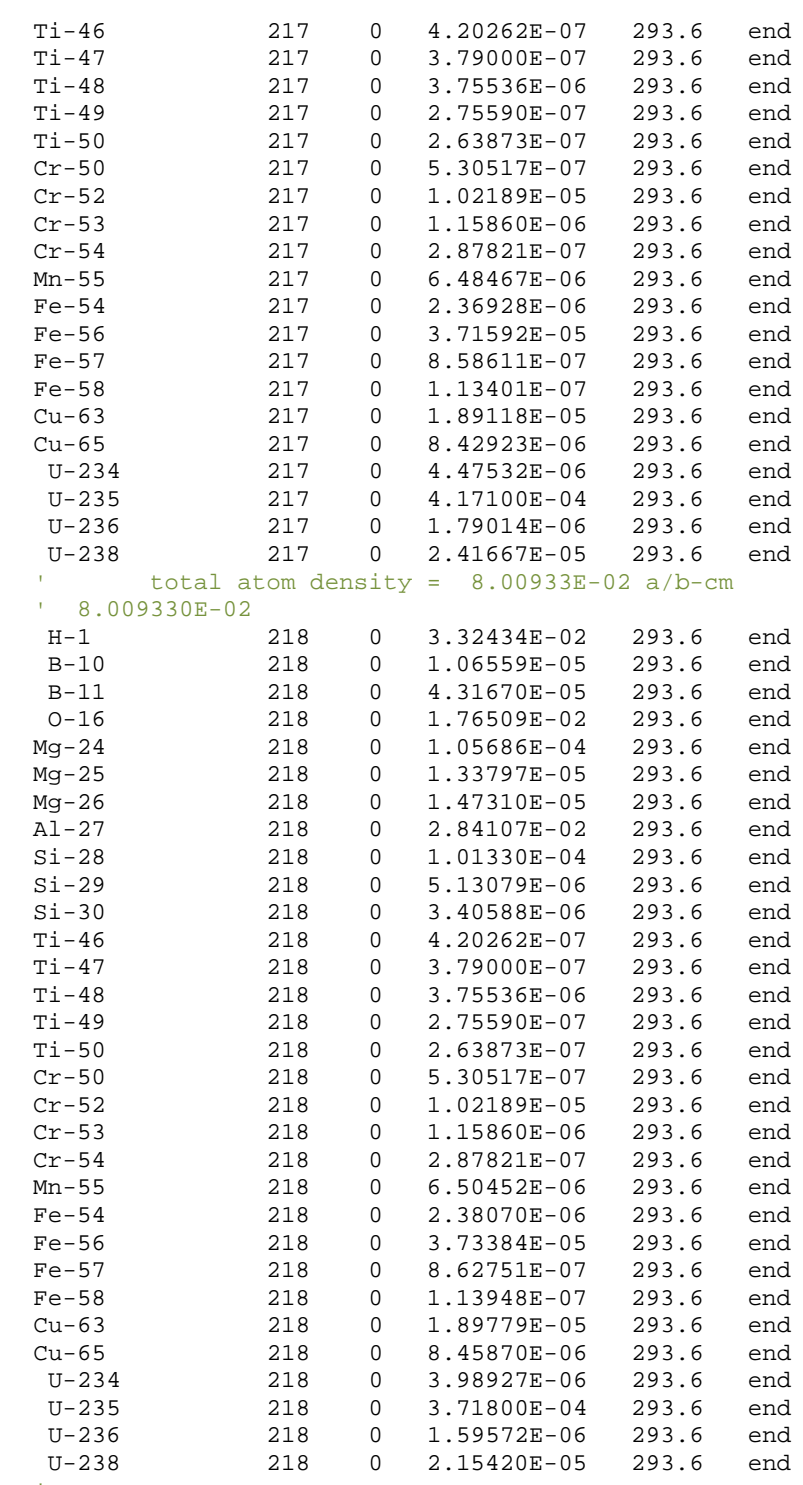

Inner fuel element--fueled Axial region 2 total atom density $=8.00804 \mathrm{E}-02 \mathrm{a} / \mathrm{b}-\mathrm{cm}$ 8. $008040 \mathrm{E}-\odot 2$

\begin{tabular}{|c|c|c|c|c|}
\hline $\mathrm{H}-1$ & 221 & $\odot$ & $3.32434 \mathrm{E}-02$ & 293.6 \\
\hline B -10 & 221 & 0 & $2.04121 E-05$ & 293.6 \\
\hline$B-11$ & 221 & 0 & $8.26896 \mathrm{E}-05$ & 293.6 \\
\hline $0-16$ & 221 & $\odot$ & $1.71757 \mathrm{E}-02$ & 293.6 \\
\hline$M g-24$ & 221 & $\odot$ & $1.05686 E-04$ & 293.6 \\
\hline$M g-25$ & 221 & $\odot$ & 1.33797E-05 & 293.6 \\
\hline $\mathrm{Mg}-26$ & 221 & $\odot$ & $1.47310 \mathrm{E}-05$ & 293.6 \\
\hline Al-27 & 221 & 0 & 2. 89993E-02 & 293.6 \\
\hline Si-28 & 221 & $\odot$ & 1. $02644 E-04$ & 293.6 \\
\hline Si-29 & 221 & $\odot$ & $5.19729 E-06$ & 293.6 \\
\hline $\mathrm{Si}-30$ & 221 & $\odot$ & $3.45002 E-06$ & 293.6 \\
\hline Ti-46 & 221 & $\odot$ & $4.20262 E-07$ & 293.6 \\
\hline Ti-47 & 221 & $\odot$ & 3. $79000 \mathrm{E}-07$ & 293.6 \\
\hline Ti-48 & 221 & $\odot$ & $3.75536 \mathrm{E}-06$ & 293.6 \\
\hline Ti-49 & 221 & $\odot$ & $2.75590 \mathrm{E}-07$ & 293.6 \\
\hline Ti-50 & 221 & $\odot$ & $2.63873 E-07$ & 293.6 \\
\hline $\mathrm{Cr}-50$ & 221 & $\odot$ & $5.30517 \mathrm{E}-07$ & 293.6 \\
\hline $\mathrm{Cr}-52$ & 221 & $\odot$ & 1. $02189 E-05$ & 293.6 \\
\hline $\mathrm{Cr}-53$ & 221 & $\odot$ & $1.15860 E-06$ & 293.6 \\
\hline $\mathrm{Cr}-54$ & 221 & $\odot$ & $2.87821 E-07$ & 293.6 \\
\hline Mn-55 & 221 & $\odot$ & $6.57731 \mathrm{E}-06$ & 293.6 \\
\hline $\mathrm{Fe}-54$ & 221 & $\odot$ & $2.42259 \mathrm{E}-06$ & 293.6 \\
\hline $\mathrm{Fe}-56$ & 221 & $\odot$ & 3. 79953E- 05 & 293.6 \\
\hline $\mathrm{Fe}-57$ & 221 & $\odot$ & $8.77930 \mathrm{E}-07$ & 293.6 \\
\hline
\end{tabular}




\begin{tabular}{|c|c|c|c|c|c|}
\hline $\mathrm{Fe}-58$ & 221 & $\odot$ & $1.15953 E-07$ & 293.6 & end \\
\hline $\mathrm{Cu}-63$ & 221 & $\odot$ & 1. $92203 E-05$ & 293.6 & end \\
\hline $\mathrm{Cu}-65$ & 221 & $\odot$ & $8.56676 \mathrm{E}-06$ & 293.6 & nd \\
\hline$U-234$ & 221 & $\odot$ & $2.20708 E-06$ & 293.6 & \\
\hline$U-235$ & 221 & $\odot$ & $2.05700 E-\odot 4$ & 293.6 & \\
\hline$U-236$ & 221 & $\odot$ & $8.82838 E-07$ & 293.6 & \\
\hline$U-238$ & 221 & $\odot$ & $1.19182 E-05$ & 293.6 & \\
\hline \multicolumn{3}{|c|}{ ' $\quad$ total atom density } & \multicolumn{2}{|c|}{$=8.00839 \mathrm{E}-02 \mathrm{a} / \mathrm{b}-\mathrm{cm}$} & \\
\hline $\mathrm{H}-1$ & 222 & $\odot$ & $3.32434 E-02$ & 293.6 & \\
\hline$B-10$ & 222 & $\odot$ & $1.77513 E-05$ & 293.6 & \\
\hline$B-11$ & 222 & $\odot$ & $7.19107 E-05$ & 293.6 & \\
\hline $0-16$ & 222 & $\odot$ & 1. $73053 E-02$ & 293.6 & \\
\hline$M g-24$ & 222 & 0 & 1. $05686 E-04$ & 293.6 & \\
\hline $\mathrm{Mg}-25$ & 222 & 0 & $1.33797 E-05$ & 293.6 & \\
\hline$M g-26$ & 222 & 0 & $1.47310 E-05$ & 293.6 & \\
\hline Al-27 & 222 & $\odot$ & $2.88388 E-02$ & 293.6 & \\
\hline Si-28 & 222 & $\odot$ & 1. $02285 E-04$ & 293.6 & \\
\hline Si-29 & 222 & $\odot$ & $5.17912 \mathrm{E}-06$ & 293.6 & \\
\hline Si-30 & 222 & 0 & $3.43796 \mathrm{E}-06$ & 293.6 & \\
\hline Ti-46 & 222 & 0 & $4.20262 \mathrm{E}-07$ & 293.6 & \\
\hline Ti-47 & 222 & $\odot$ & 3. $79000 \mathrm{E}-07$ & 293.6 & \\
\hline Ti-48 & 222 & 0 & $3.75536 \mathrm{E}-06$ & 293.6 & \\
\hline Ti-49 & 222 & 0 & $2.75590 E-07$ & 293.6 & \\
\hline Ti-50 & 222 & 0 & $2.63873 E-07$ & 293.6 & \\
\hline $\mathrm{Cr}-50$ & 222 & 0 & $5.30517 E-07$ & 293.6 & \\
\hline $\mathrm{Cr}-52$ & 222 & 0 & 1. $02189 E-05$ & 293.6 & \\
\hline $\mathrm{Cr}-53$ & 222 & $\odot$ & $1.15860 E-06$ & 293.6 & \\
\hline $\mathrm{Cr}-54$ & 222 & $\odot$ & $2.87821 E-07$ & 293.6 & \\
\hline Mn-55 & 222 & $\odot$ & $6.55746 E-06$ & 293.6 & \\
\hline $\mathrm{Fe}-54$ & 222 & $\odot$ & $2.41117 \mathrm{E}-06$ & 293.6 & \\
\hline $\mathrm{Fe}-56$ & 222 & $\odot$ & $3.78162 E-05$ & 293.6 & \\
\hline $\mathrm{Fe}-57$ & 222 & $\odot$ & $8.73792 E-07$ & 293.6 & \\
\hline $\mathrm{Fe}-58$ & 222 & 0 & $1.15406 \mathrm{E}-07$ & 293.6 & \\
\hline $\mathrm{Cu}-63$ & 222 & 0 & 1. $91542 \mathrm{E}-05$ & 293.6 & \\
\hline $\mathrm{Cu}-65$ & 222 & 0 & $8.53729 E-06$ & 293.6 & \\
\hline$U-234$ & 222 & 0 & $2.69313 E-06$ & 293.6 & \\
\hline$U-235$ & 222 & $\odot$ & $2.51000 E-\odot 4$ & 293.6 & \\
\hline$U-236$ & 222 & $\odot$ & 1. $07726 E-\odot 6$ & 293.6 & \\
\hline$U-238$ & 222 & 0 & 1. $45429 E-05$ & 293.6 & \\
\hline \multicolumn{3}{|c|}{ total atom density } & \multirow{2}{*}{\multicolumn{3}{|c|}{$=8.00880 \mathrm{E}-02 \mathrm{a} / \mathrm{b}-\mathrm{cm}$}} \\
\hline \multicolumn{3}{|c|}{ ' $\quad 8 . \odot \odot 88 \odot \odot E-\odot 2$} & & & \\
\hline $\mathrm{H}-1$ & 223 & 0 & $3.32435 E-02$ & 293.6 & \\
\hline$B-10$ & 223 & $\odot$ & 1. 46911E- 05 & 293.6 & \\
\hline$B-11$ & 223 & $\odot$ & $5.95139 E-05$ & 293.6 & \\
\hline $0-16$ & 223 & $\odot$ & 1. $74543 E-02$ & 293.6 & \\
\hline$M g-24$ & 223 & $\odot$ & 1. $05686 E-04$ & 293.6 & \\
\hline$M g-25$ & 223 & $\odot$ & $1.33797 E-05$ & 293.6 & \\
\hline$M g-26$ & 223 & $\odot$ & $1.47310 E-05$ & 293.6 & \\
\hline Al-27 & 223 & $\odot$ & $2.86541 E-\odot 2$ & 293.6 & \\
\hline Si-28 & 223 & $\odot$ & 1. $01874 E-04$ & 293.6 & \\
\hline Si-29 & 223 & 0 & $5.15831 E-06$ & 293.6 & \\
\hline Si-30 & 223 & 0 & $3.42415 E-06$ & 293.6 & \\
\hline $\mathrm{Ti}-46$ & 223 & $\odot$ & 4. 20263E - 07 & 293.6 & \\
\hline Ti-47 & 223 & 0 & $3.79001 \mathrm{E}-07$ & 293.6 & \\
\hline Ti-48 & 223 & 0 & $3.75537 \mathrm{E}-06$ & 293.6 & \\
\hline Ti-49 & 223 & 0 & $2.75590 \mathrm{E}-\odot 7$ & 293.6 & \\
\hline Ti-50 & 223 & $\odot$ & $2.63873 E-07$ & 293.6 & \\
\hline $\mathrm{Cr}-50$ & 223 & $\odot$ & $5.30518 E-07$ & 293.6 & \\
\hline $\mathrm{Cr}-52$ & 223 & $\odot$ & 1. $02189 E-05$ & 293.6 & \\
\hline $\mathrm{Cr}-53$ & 223 & $\odot$ & $1.15860 E-06$ & 293.6 & \\
\hline $\mathrm{Cr}-54$ & 223 & $\odot$ & $2.87821 E-07$ & 293.6 & \\
\hline$M n-55$ & 223 & $\odot$ & $6.53464 \mathrm{E}-06$ & 293.6 & \\
\hline $\mathrm{Fe}-54$ & 223 & $\odot$ & $2.39803 E-06$ & 293.6 & \\
\hline $\mathrm{Fe}-56$ & 223 & 0 & $3.76103 E-05$ & 293.6 & \\
\hline $\mathrm{Fe}-57$ & 223 & 0 & $8.69031 \mathrm{E}-07$ & 293.6 & \\
\hline $\mathrm{Fe}-58$ & 223 & 0 & $1.14778 \mathrm{E}-07$ & 293.6 & \\
\hline $\mathrm{Cu}-63$ & 223 & $\odot$ & 1. $90782 E-05$ & 293.6 & \\
\hline $\mathrm{Cu}-65$ & 223 & $\odot$ & $8.50342 E-06$ & 293.6 & \\
\hline$U-234$ & 223 & $\odot$ & $3.25215 E-06$ & 293.6 & \\
\hline$U-235$ & 223 & $\odot$ & 3. $.3100 E-\odot 4$ & 293.6 & \\
\hline$U-236$ & 223 & $\odot$ & 1. $30087 \mathrm{E}-06$ & 293.6 & \\
\hline$U-238$ & 223 & $\odot$ & $1.75616 E-05$ & 293.6 & \\
\hline & tom & sity & $=8.00937 \mathrm{E}$ & $2 a / b-c l$ & \\
\hline ' 8.00 & & & & & \\
\hline $\mathrm{H}-1$ & 224 & $\odot$ & 3. 32434E- 02 & 293.6 & \\
\hline B - 10 & 224 & 0 & $1.03798 \mathrm{E}-05$ & 293.6 & \\
\hline B-11 & 224 & 0 & $4.20486 E-05$ & 293.6 & \\
\hline $0-16$ & 224 & 0 & $1.76644 E-02$ & 293.6 & \\
\hline$M g-24$ & 224 & 0 & 1. $05686 E-04$ & 293.6 & \\
\hline$M g-25$ & 224 & $\odot$ & $1.33797 E-05$ & 293.6 & \\
\hline
\end{tabular}




\begin{tabular}{|c|c|c|c|c|c|}
\hline$M g-26$ & 224 & $\odot$ & $1.47310 \mathrm{E}-05$ & 293.6 & end \\
\hline Al-27 & 224 & $\odot$ & $2.83940 E-02$ & 293.6 & end \\
\hline Si-28 & 224 & $\odot$ & 1. $01293 E-\odot 4$ & 293.6 & end \\
\hline Si-29 & 224 & $\odot$ & $5.12887 \mathrm{E}-\odot 6$ & 293.6 & end \\
\hline Si-30 & 224 & $\odot$ & $3.4 \odot 461 \mathrm{E}-06$ & 293.6 & end \\
\hline Ti-46 & 224 & 0 & $4.20262 \mathrm{E}-07$ & 293.6 & end \\
\hline Ti-47 & 224 & $\odot$ & 3. $79000 \mathrm{E}-\odot 7$ & 293.6 & and \\
\hline $\mathrm{Ti}-48$ & 224 & $\odot$ & $3.75536 \mathrm{E}-06$ & 293.6 & nd \\
\hline $\mathrm{Ti}-49$ & 224 & $\odot$ & $2.75590 E-\odot 7$ & 293.6 & nd \\
\hline Ti-50 & 224 & $\odot$ & $2.63873 E-07$ & 293.6 & \\
\hline $\mathrm{Cr}-50$ & 224 & $\odot$ & $5.30517 \mathrm{E}-07$ & 293.6 & nd \\
\hline $\mathrm{Cr}-52$ & 224 & 0 & 1. $02189 \mathrm{E}-05$ & 293.6 & end \\
\hline $\mathrm{Cr}-53$ & 224 & $\odot$ & $1.15860 E-06$ & 293.6 & nd \\
\hline $\mathrm{Cr}-54$ & 224 & $\odot$ & $2.87821 \mathrm{E}-07$ & 293.6 & nd \\
\hline Mn-55 & 224 & 0 & $6.50246 \mathrm{E}-06$ & 293.6 & ad \\
\hline $\mathrm{Fe}-54$ & 224 & $\odot$ & $2.37952 \mathrm{E}-06$ & 293.6 & nd \\
\hline $\mathrm{Fe}-56$ & 224 & $\odot$ & $3.73198 \mathrm{E}-05$ & 293.6 & \\
\hline $\mathrm{Fe}-57$ & 224 & $\odot$ & $8.62321 \mathrm{E}-07$ & 293.6 & $\mathrm{hd}$ \\
\hline $\mathrm{Fe}-58$ & 224 & $\odot$ & $1.13891 E-07$ & 293.6 & \\
\hline $\mathrm{Cu}-63$ & 224 & $\odot$ & $1.89710 \mathrm{E}-05$ & 293.6 & id \\
\hline $\mathrm{Cu}-65$ & 224 & $\odot$ & $8.45562 E-\odot 6$ & 293.6 & \\
\hline$U-234$ & 224 & $\odot$ & 4. $03970 E-\odot 6$ & 293.6 & \\
\hline U-235 & 224 & $\odot$ & $3.765 \odot \odot E-\odot 4$ & 293.6 & nd \\
\hline U-236 & 224 & $\odot$ & 1. 61589E- 06 & 293.6 & \\
\hline$U-238$ & 224 & $\odot$ & $2.18144 \mathrm{E}-05$ & 293.6 & \\
\hline \multicolumn{3}{|c|}{$\begin{array}{l}\text { total atom density } \\
\text { 8. } 809930 \mathrm{E}-02\end{array}$} & \multicolumn{3}{|c|}{$=8.00993 \mathrm{E}-02 \mathrm{a} / \mathrm{b}-\mathrm{cm}$} \\
\hline $\mathrm{H}-1$ & 225 & $\odot$ & 3. 32434E- 02 & 293.6 & nd \\
\hline$B-10$ & 225 & 0 & $6.18010 \mathrm{E}-06$ & 293.6 & \\
\hline$B-11$ & 225 & $\odot$ & $2.50356 \mathrm{E}-05$ & 293.6 & \\
\hline $0-16$ & 225 & $\odot$ & $1.78689 \mathrm{E}-02$ & 293.6 & \\
\hline$M g-24$ & 225 & $\odot$ & 1. $05686 \mathrm{E}-\odot 4$ & 293.6 & \\
\hline$M g-25$ & 225 & $\odot$ & $1.33797 \mathrm{E}-05$ & 293.6 & \\
\hline$M g-26$ & 225 & $\odot$ & $1.47310 E-05$ & 293.6 & \\
\hline $\mathrm{Al}-27$ & 225 & $\odot$ & $2.81406 \mathrm{E}-02$ & 293.6 & \\
\hline Si-28 & 225 & $\odot$ & 1. $00727 \mathrm{E}-\odot 4$ & 293.6 & \\
\hline Si-29 & 225 & 0 & $5.10026 \mathrm{E}-06$ & 293.6 & \\
\hline $\mathrm{Si}-30$ & 225 & $\odot$ & $3.38560 \mathrm{E}-06$ & 293.6 & \\
\hline Ti-46 & 225 & $\odot$ & $4.20262 E-07$ & 293.6 & \\
\hline $\mathrm{Ti}-47$ & 225 & $\odot$ & 3. $79000 \mathrm{E}-07$ & 293.6 & \\
\hline $\mathrm{Ti}-48$ & 225 & $\odot$ & $3.75536 \mathrm{E}-\odot 6$ & 293.6 & \\
\hline $\mathrm{Ti}-49$ & 225 & $\odot$ & $2.75590 E-\odot 7$ & 293.6 & \\
\hline Ti-50 & 225 & $\odot$ & $2.63873 E-07$ & 293.6 & \\
\hline $\mathrm{Cr}-50$ & 225 & $\odot$ & $5.30518 E-07$ & 293.6 & \\
\hline $\mathrm{Cr}-52$ & 225 & $\odot$ & 1. $02189 \mathrm{E}-05$ & 293.6 & \\
\hline $\mathrm{Cr}-53$ & 225 & $\odot$ & $1.15860 E-\odot 6$ & 293.6 & \\
\hline $\mathrm{Cr}-54$ & 225 & $\odot$ & $2.87821 E-07$ & 293.6 & \\
\hline Mn-55 & 225 & $\odot$ & $6.47114 \mathrm{E}-06$ & 293.6 & \\
\hline $\mathrm{Fe}-54$ & 225 & $\odot$ & $2.36149 \mathrm{E}-\odot 6$ & 293.6 & \\
\hline $\mathrm{Fe}-56$ & 225 & $\odot$ & 3. $70370 \mathrm{E}-05$ & 293.6 & \\
\hline $\mathrm{Fe}-57$ & 225 & $\odot$ & $8.55788 E-07$ & 293.6 & \\
\hline $\mathrm{Fe}-58$ & 225 & $\odot$ & $1.13028 \mathrm{E}-07$ & 293.6 & \\
\hline $\mathrm{Cu}-63$ & 225 & $\odot$ & $1.88666 \mathrm{E}-05$ & 293.6 & \\
\hline $\mathrm{Cu}-65$ & 225 & $\odot$ & 8. 40911E- 06 & 293.6 & \\
\hline$U-234$ & 225 & $\odot$ & 4. 80687E- 06 & 293.6 & \\
\hline$U-235$ & 225 & $\odot$ & 4. $48000 E-\odot 4$ & 293.6 & \\
\hline$U-236$ & 225 & $\odot$ & 1. $92276 \mathrm{E}-06$ & 293.6 & \\
\hline$U-238$ & 225 & 0 & \multirow{2}{*}{\multicolumn{2}{|c|}{$\begin{array}{l}2.59571 \mathrm{E}-05 \quad 293.6 \\
=\quad 8.00998 \mathrm{E}-02 \mathrm{a} / \mathrm{b}-\mathrm{cm}\end{array}$}} & end \\
\hline \multicolumn{3}{|c|}{ total atom density } & & & \\
\hline $\mathrm{H}-1$ & 226 & $\odot$ & $3.32434 \mathrm{E}-02$ & 293.6 & \\
\hline$B-10$ & 226 & $\odot$ & $5.79830 E-\odot 6$ & 293.6 & \\
\hline$B-11$ & 226 & $\odot$ & $2.34889 E-05$ & 293.6 & \\
\hline $0-16$ & 226 & $\odot$ & $1.78875 \mathrm{E}-02$ & 293.6 & \\
\hline$M g-24$ & 226 & $\odot$ & 1. $05686 E-04$ & 293.6 & \\
\hline$M g-25$ & 226 & $\odot$ & 1. $33797 \mathrm{E}-05$ & 293.6 & \\
\hline$M g-26$ & 226 & $\odot$ & $1.47310 \mathrm{E}-05$ & 293.6 & \\
\hline Al-27 & 226 & $\odot$ & $2.81176 \mathrm{E}-\odot 2$ & 293.6 & \\
\hline Si-28 & 226 & $\odot$ & 1. $00676 E-\odot 4$ & 293.6 & \\
\hline Si-29 & 226 & $\odot$ & $5.09768 E-06$ & 293.6 & \\
\hline Si-30 & 226 & $\odot$ & $3.38390 E-06$ & 293.6 & \\
\hline $\mathrm{Ti}-46$ & 226 & $\odot$ & 4. $20262 E-07$ & 293.6 & \\
\hline $\mathrm{Ti}-47$ & 226 & $\odot$ & $3.79000 E-\odot 7$ & 293.6 & \\
\hline $\mathrm{Ti}-48$ & 226 & $\odot$ & $3.75536 \mathrm{E}-06$ & 293.6 & \\
\hline $\mathrm{Ti}-49$ & 226 & $\odot$ & $2.75590 E-\odot 7$ & 293.6 & \\
\hline $\mathrm{Ti}-50$ & 226 & $\odot$ & $2.63873 E-07$ & 293.6 & \\
\hline $\mathrm{Cr}-50$ & 226 & $\odot$ & $5.30517 \mathrm{E}-07$ & 293.6 & \\
\hline $\mathrm{Cr}-52$ & 226 & $\odot$ & 1. $02189 \mathrm{E}-05$ & 293.6 & \\
\hline $\mathrm{Cr}-53$ & 226 & $\odot$ & $1.15860 E-06$ & 293.6 & \\
\hline $\mathrm{Cr}-54$ & 226 & $\odot$ & $2.87821 E-07$ & 293.6 & \\
\hline Mn-55 & 226 & 0 & $6.46828 \mathrm{E}-06$ & 293.6 & \\
\hline
\end{tabular}




$\begin{array}{llllll}\mathrm{Fe}-54 & 226 & \odot & 2.35985 \mathrm{E}-\odot 6 & 293.6 & \text { end } \\ \mathrm{Fe}-56 & 226 & \odot & 3.70113 \mathrm{E}-05 & 293.6 & \text { end } \\ \mathrm{Fe}-57 & 226 & \odot & 8.55193 \mathrm{E}-\odot 7 & 293.6 & \text { end } \\ \mathrm{Fe}-58 & 226 & \odot & 1.12950 \mathrm{E}-\odot 7 & 293.6 & \text { end } \\ \mathrm{Cu}-63 & 226 & \odot & 1.88571 \mathrm{E}-05 & 293.6 & \text { end } \\ \mathrm{Cu}-65 & 226 & \odot & 8.40487 \mathrm{E}-06 & 293.6 & \text { end } \\ \mathrm{U}-234 & 226 & \odot & 4.87661 \mathrm{E}-\odot 6 & 293.6 & \text { end } \\ \mathrm{U}-235 & 226 & \odot & 4.54500 \mathrm{E}-\odot 4 & 293.6 & \text { end } \\ \mathrm{U}-236 & 226 & \odot & 1.95066 \mathrm{E}-\odot 6 & 293.6 & \text { end } \\ \mathrm{U}-238 & 226 & \odot & 2.63337 \mathrm{E}-\odot 5 & 293.6 & \text { end }\end{array}$

\begin{tabular}{|c|c|c|c|c|c|}
\hline $\mathrm{H}-1$ & 227 & $\odot$ & $3.32434 \mathrm{E}-02$ & 293.6 & end \\
\hline B-10 & 227 & $\odot$ & $7.99507 \mathrm{E}-\odot 6$ & 293.6 & end \\
\hline$B-11$ & 227 & $\odot$ & $3.23880 E-05$ & 293.6 & end \\
\hline $0-16$ & 227 & $\odot$ & $1.77805 \mathrm{E}-02$ & 293.6 & end \\
\hline$M g-24$ & 227 & $\odot$ & 1. $05686 \mathrm{E}-04$ & 293.6 & end \\
\hline$M g-25$ & 227 & $\odot$ & 1. $33797 \mathrm{E}-05$ & 293.6 & end \\
\hline$M g-26$ & 227 & $\odot$ & 1. $47310 \mathrm{E}-05$ & 293.6 & end \\
\hline Al -27 & 227 & $\odot$ & $2.82501 \mathrm{E}-02$ & 293.6 & end \\
\hline Si-28 & 227 & $\odot$ & 1. $\odot \odot 972 E-\odot 4$ & 293.6 & end \\
\hline Si-29 & 227 & $\odot$ & $5.11262 \mathrm{E}-06$ & 293.6 & end \\
\hline Si-30 & 227 & $\odot$ & $3.39382 \mathrm{E}-\odot 6$ & 293.6 & end \\
\hline Ti-46 & 227 & $\odot$ & $4.2 \odot 262 \mathrm{E}-\odot 7$ & 293.6 & end \\
\hline Ti-47 & 227 & $\odot$ & 3. $7900 \odot \mathrm{E}-\odot 7$ & 293.6 & end \\
\hline $\mathrm{Ti}-48$ & 227 & $\odot$ & $3.75536 \mathrm{E}-06$ & 293.6 & end \\
\hline Ti-49 & 227 & $\odot$ & $2.75590 \mathrm{E}-\odot 7$ & 293.6 & end \\
\hline Ti-50 & 227 & $\odot$ & $2.63873 E-07$ & 293.6 & end \\
\hline $\mathrm{Cr}-50$ & 227 & $\odot$ & $5.30517 \mathrm{E}-07$ & 293.6 & end \\
\hline $\mathrm{Cr}-52$ & 227 & $\odot$ & 1. $02189 \mathrm{E}-05$ & 293.6 & end \\
\hline $\mathrm{Cr}-53$ & 227 & $\odot$ & $1.15860 \mathrm{E}-06$ & 293.6 & end \\
\hline $\mathrm{Cr}-54$ & 227 & $\odot$ & $2.87821 \mathrm{E}-07$ & 293.6 & end \\
\hline$M n-55$ & 227 & $\odot$ & $6.48467 \mathrm{E}-\odot 6$ & 293.6 & end \\
\hline $\mathrm{Fe}-54$ & 227 & $\odot$ & $2.36928 \mathrm{E}-\odot 6$ & 293.6 & end \\
\hline $\mathrm{Fe}-56$ & 227 & $\odot$ & $3.71592 \mathrm{E}-05$ & 293.6 & end \\
\hline $\mathrm{Fe}-57$ & 227 & $\odot$ & $8.58611 \mathrm{E}-07$ & 293.6 & end \\
\hline $\mathrm{Fe}-58$ & 227 & $\odot$ & 1. $13401 \mathrm{E}-07$ & 293.6 & end \\
\hline $\mathrm{Cu}-63$ & 227 & $\odot$ & $1.89118 \mathrm{E}-05$ & 293.6 & end \\
\hline $\mathrm{Cu}-65$ & 227 & $\odot$ & $8.42923 E-06$ & 293.6 & end \\
\hline$U-234$ & 227 & $\odot$ & $4.47532 \mathrm{E}-06$ & 293.6 & end \\
\hline$U-235$ & 227 & 0 & $4.1710 \odot \mathrm{E}-\odot 4$ & 293.6 & end \\
\hline $\mathrm{U}-236$ & 227 & $\odot$ & 1. $79014 \mathrm{E}-06$ & 293.6 & end \\
\hline $\mathrm{U}-238$ & 2 & 0 & $2.41667 \mathrm{E}-05$ & 293.6 & end \\
\hline
\end{tabular}

U-238 $227 \quad 0 \quad 2.41667 \mathrm{E}-05 \quad 293.6$ end

total atom density $=8.00933 \mathrm{E}-02 \mathrm{a} / \mathrm{b}-\mathrm{cm}$

\begin{tabular}{|c|c|c|c|c|c|}
\hline $\mathrm{H}-1$ & 228 & $\odot$ & 3. 32434E- - 2 & 293.6 & end \\
\hline$B-10$ & 228 & 0 & 1. $06559 \mathrm{E}-05$ & 293.6 & end \\
\hline$B-11$ & 228 & $\odot$ & $4.31670 E-05$ & 293.6 & end \\
\hline $0-16$ & 228 & $\odot$ & 1. $76509 E-\odot 2$ & 293.6 & end \\
\hline$M g-24$ & 228 & $\odot$ & 1. $\odot 5686 E-\odot 4$ & 293.6 & end \\
\hline$M g-25$ & 228 & $\odot$ & $1.33797 \mathrm{E}-05$ & 293.6 & $\mathrm{ad}$ \\
\hline$M g-26$ & 228 & $\odot$ & $1.47310 \mathrm{E}-05$ & 293.6 & id \\
\hline Al-27 & 228 & $\odot$ & $2.84107 \mathrm{E}-02$ & 293.6 & end \\
\hline Si-28 & 228 & $\odot$ & 1. $01330 E-\odot 4$ & 293.6 & \\
\hline Si-29 & 228 & $\odot$ & $5.13079 E-\odot 6$ & 293.6 & nd \\
\hline Si-30 & 228 & $\odot$ & $3.40588 E-\odot 6$ & 293.6 & ad \\
\hline Ti-46 & 228 & $\odot$ & $4.20262 \mathrm{E}-07$ & 293.6 & $\mathrm{ad}$ \\
\hline Ti-47 & 228 & $\odot$ & $3.7900 \odot E-\odot 7$ & 293.6 & nd \\
\hline Ti-48 & 228 & $\odot$ & $3.75536 \mathrm{E}-06$ & 293.6 & nd \\
\hline Ti-49 & 228 & $\odot$ & $2.75590 E-\odot 7$ & 293.6 & nd \\
\hline Ti-50 & 228 & $\odot$ & $2.63873 E-07$ & 293.6 & nd \\
\hline $\mathrm{Cr}-50$ & 228 & $\odot$ & $5.30517 E-\odot 7$ & 293.6 & \\
\hline $\mathrm{Cr}-52$ & 228 & $\odot$ & 1. $02189 E-05$ & 293.6 & \\
\hline $\mathrm{Cr}-53$ & 228 & $\odot$ & 1. $15860 E-06$ & 293.6 & \\
\hline $\mathrm{Cr}-54$ & 228 & $\odot$ & $2.87821 E-07$ & 293.6 & \\
\hline$M n-55$ & 228 & $\odot$ & $6.50452 E-06$ & 293.6 & \\
\hline $\mathrm{Fe}-54$ & 228 & 0 & $2.38070 \mathrm{E}-\odot 6$ & 293.6 & \\
\hline $\mathrm{Fe}-56$ & 228 & $\odot$ & $3.73384 \mathrm{E}-05$ & 293.6 & \\
\hline $\mathrm{Fe}-57$ & 228 & $\odot$ & $8.62751 E-07$ & 293.6 & \\
\hline $\mathrm{Fe}-58$ & 228 & $\odot$ & $1.13948 E-07$ & 293.6 & $d$ \\
\hline $\mathrm{Cu}-63$ & 228 & $\odot$ & 1. 89779E- 05 & 293.6 & \\
\hline $\mathrm{Cu}-65$ & 228 & $\odot$ & $8.45870 E-06$ & 293.6 & \\
\hline$U-234$ & 228 & $\odot$ & 3. 98927E- 06 & 293.6 & \\
\hline$U-235$ & 228 & $\odot$ & $3.71800 \mathrm{E}-\odot 4$ & 293.6 & \\
\hline$U-236$ & 228 & $\odot$ & $1.59572 \mathrm{E}-06$ & 293.6 & \\
\hline$U-238$ & 228 & $\odot$ & $2.15420 E-05$ & 293.6 & \\
\hline
\end{tabular}

U-238 $228 \quad 2.15420 \mathrm{E}-05 \quad 293.6$ end

Inner fuel element--fueled Axial region 3 total atom density $=8.00804 \mathrm{E}-02 \mathrm{a} / \mathrm{b}-\mathrm{cm}$ $8.008 \odot 40 \mathrm{E}-\odot 2$

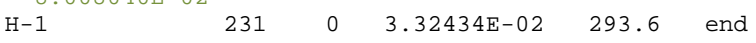




\begin{tabular}{|c|c|c|c|c|c|}
\hline$B-10$ & 231 & $\odot$ & $2.04121 E-05$ & 293.6 & end \\
\hline$B-11$ & 231 & $\odot$ & $8.26896 E-05$ & 293.6 & end \\
\hline $0-16$ & 231 & $\odot$ & $1.71757 \mathrm{E}-02$ & 293.6 & nd \\
\hline$M g-24$ & 231 & $\odot$ & 1. $05686 E-\odot 4$ & 293.6 & \\
\hline$M g-25$ & 231 & $\odot$ & 1. 33797E- 05 & 293.6 & \\
\hline$M g-26$ & 231 & $\odot$ & $1.47310 \mathrm{E}-05$ & 293.6 & \\
\hline Al-27 & 231 & $\odot$ & $2.89993 E-02$ & 293.6 & \\
\hline Si-28 & 231 & $\odot$ & 1. $02644 \mathrm{E}-04$ & 293.6 & \\
\hline Si-29 & 231 & $\odot$ & $5.19729 E-\odot 6$ & 293.6 & \\
\hline Si-30 & 231 & $\odot$ & $3.45002 \mathrm{E}-\odot 6$ & 293.6 & \\
\hline $\mathrm{Ti}-46$ & 231 & $\odot$ & $4.20262 E-07$ & 293.6 & \\
\hline $\mathrm{Ti}-47$ & 231 & $\odot$ & $3.79000 \mathrm{E}-07$ & 293.6 & \\
\hline $\mathrm{Ti}-48$ & 231 & 0 & $3.75536 \mathrm{E}-06$ & 293.6 & \\
\hline $\mathrm{Ti}-49$ & 231 & $\odot$ & $2.75590 \mathrm{E}-07$ & 293.6 & \\
\hline Ti-50 & 231 & 0 & $2.63873 E-07$ & 293.6 & \\
\hline $\mathrm{Cr}-50$ & 231 & $\odot$ & $5.30517 \mathrm{E}-07$ & 293.6 & \\
\hline Cr -52 & 231 & 0 & 1. $02189 E-05$ & 293.6 & \\
\hline $\mathrm{Cr}-53$ & 231 & 0 & $1.15860 \mathrm{E}-06$ & 293.6 & \\
\hline Cr -54 & 231 & $\odot$ & $2.87821 E-07$ & 293.6 & \\
\hline$M n-55$ & 231 & $\odot$ & $6.57731 E-06$ & 293.6 & \\
\hline $\mathrm{Fe}-54$ & 231 & $\odot$ & $2.42259 E-06$ & 293.6 & \\
\hline $\mathrm{Fe}-56$ & 231 & $\odot$ & $3.79953 E-05$ & 293.6 & \\
\hline $\mathrm{Fe}-57$ & 231 & $\odot$ & $8.77930 \mathrm{E}-07$ & 293.6 & \\
\hline $\mathrm{Fe}-58$ & 231 & $\odot$ & $1.15953 E-07$ & 293.6 & \\
\hline $\mathrm{Cu}-63$ & 231 & $\odot$ & 1. $92203 \mathrm{E}-05$ & 293.6 & \\
\hline $\mathrm{Cu}-65$ & 231 & $\odot$ & $8.56676 \mathrm{E}-06$ & 293.6 & \\
\hline $\mathrm{U}-234$ & 231 & 0 & $2.20708 \mathrm{E}-06$ & 293.6 & \\
\hline$U-235$ & 231 & 0 & 2. $05700 \mathrm{E}-\odot 4$ & 293.6 & \\
\hline$U-236$ & 231 & 0 & $8.82838 E-07$ & 293.6 & \\
\hline$U-238$ & 231 & $\odot$ & $1.19182 \mathrm{E}-05$ & 293.6 & \\
\hline \multicolumn{3}{|c|}{$\begin{array}{l}\text { total atom density } \\
\text { 8. } 008390 \mathrm{E}-02\end{array}$} & \multicolumn{2}{|c|}{$=8.00839 \mathrm{E}-02 \mathrm{a} / \mathrm{b}-\mathrm{cm}$} & \\
\hline $\mathrm{H}-1$ & 232 & 0 & $3.32434 \mathrm{E}-02$ & 293.6 & \\
\hline$B-10$ & 232 & $\odot$ & $1.77513 E-05$ & 293.6 & \\
\hline$B-11$ & 232 & $\odot$ & $7.19107 E-05$ & 293.6 & \\
\hline $0-16$ & 232 & 0 & $1.73053 \mathrm{E}-\odot 2$ & 293.6 & \\
\hline$M g-24$ & 232 & 0 & 1. $05686 E-\odot 4$ & 293.6 & \\
\hline Mg-25 & 232 & 0 & 1. 33797E- 05 & 293.6 & \\
\hline$M g-26$ & 232 & 0 & $1.47310 \mathrm{E}-05$ & 293.6 & \\
\hline Al-27 & 232 & 0 & $2.88388 E-02$ & 293.6 & \\
\hline Si-28 & 232 & 0 & $1.02285 E-\odot 4$ & 293.6 & \\
\hline Si-29 & 232 & 0 & $5.17912 \mathrm{E}-\odot 6$ & 293.6 & \\
\hline Si-30 & 232 & $\odot$ & $3.43796 E-06$ & 293.6 & \\
\hline $\mathrm{Ti}-46$ & 232 & $\odot$ & $4.20262 E-\odot 7$ & 293.6 & \\
\hline $\mathrm{Ti}-47$ & 232 & $\odot$ & $3.790 \odot \odot E-\odot 7$ & 293.6 & \\
\hline Ti-48 & 232 & 0 & $3.75536 \mathrm{E}-\odot 6$ & 293.6 & \\
\hline Ti-49 & 232 & $\odot$ & $2.75590 \mathrm{E}-07$ & 293.6 & \\
\hline Ti-50 & 232 & $\odot$ & $2.63873 E-07$ & 293.6 & \\
\hline $\mathrm{Cr}-50$ & 232 & $\odot$ & $5.30517 \mathrm{E}-07$ & 293.6 & \\
\hline Cr -52 & 232 & 0 & 1. $02189 E-05$ & 293.6 & \\
\hline $\mathrm{Cr}-53$ & 232 & $\odot$ & $1.15860 \mathrm{E}-06$ & 293.6 & \\
\hline $\mathrm{Cr}-54$ & 232 & $\odot$ & $2.87821 E-07$ & 293.6 & \\
\hline$M n-55$ & 232 & $\odot$ & $6.55746 \mathrm{E}-06$ & 293.6 & \\
\hline $\mathrm{Fe}-54$ & 232 & $\odot$ & $2.41117 \mathrm{E}-06$ & 293.6 & \\
\hline $\mathrm{Fe}-56$ & 232 & $\odot$ & $3.78162 \mathrm{E}-05$ & 293.6 & \\
\hline $\mathrm{Fe}-57$ & 232 & $\odot$ & $8.73792 E-07$ & 293.6 & \\
\hline $\mathrm{Fe}-58$ & 232 & $\odot$ & $1.15406 \mathrm{E}-07$ & 293.6 & \\
\hline $\mathrm{Cu}-63$ & 232 & $\odot$ & $1.91542 \mathrm{E}-05$ & 293.6 & \\
\hline Cu-65 & 232 & 0 & $8.53729 E-06$ & 293.6 & \\
\hline$U-234$ & 232 & 0 & $2.69313 E-06$ & 293.6 & \\
\hline$U-235$ & 232 & 0 & $2.51000 \mathrm{E}-\odot 4$ & 293.6 & \\
\hline$U-236$ & 232 & 0 & 1. $07726 \mathrm{E}-06$ & 293.6 & \\
\hline$U-238$ & 232 & $\odot$ & $1.45429 E-05$ & 293.6 & \\
\hline \multirow{2}{*}{\multicolumn{5}{|c|}{ ' $\quad$ 8. $008800 \mathrm{E}-02$}} & \\
\hline $\mathrm{H}-1$ & & & & & \\
\hline$B-10$ & 233 & $\begin{array}{l}0 \\
0\end{array}$ & 5 & 2 & \\
\hline $\mathrm{B}-11$ & 233 & $\odot$ & $5.95139 E-\odot 5$ & 293.6 & \\
\hline $0-16$ & 233 & 0 & $1.74543 E-02$ & 293.6 & \\
\hline$M g-24$ & 233 & $\odot$ & $1.05686 E-04$ & 293.6 & \\
\hline$M g-25$ & 233 & $\odot$ & $1.33797 \mathrm{E}-05$ & 293.6 & \\
\hline $\mathrm{Mg}-26$ & 233 & $\odot$ & $1.47310 \mathrm{E}-05$ & 293.6 & \\
\hline $\mathrm{Al}-27$ & 233 & 0 & $2.86541 E-02$ & 293.6 & \\
\hline Si-28 & 233 & 0 & 1. $01874 \mathrm{E}-\odot 4$ & 293.6 & \\
\hline Si-29 & 233 & 0 & $5.15831 E-06$ & 293.6 & \\
\hline Si-30 & 233 & 0 & $3.42415 E-06$ & 293.6 & \\
\hline $\mathrm{Ti}-46$ & 233 & $\odot$ & $4.20263 E-07$ & 293.6 & \\
\hline Ti-47 & 233 & $\odot$ & $3.79001 E-07$ & 293.6 & \\
\hline $\mathrm{Ti}-48$ & 233 & 0 & $3.75537 \mathrm{E}-06$ & 293.6 & \\
\hline Ti-49 & 233 & 0 & $2.75590 \mathrm{E}-07$ & 293.6 & \\
\hline Ti-50 & 233 & 0 & $2.63873 E-07$ & 293.6 & \\
\hline
\end{tabular}




\begin{tabular}{|c|c|c|c|c|c|}
\hline $\mathrm{Cr}-50$ & 233 & $\odot$ & $5.30518 E-07$ & 293.6 & end \\
\hline $\mathrm{Cr}-52$ & 233 & $\odot$ & 1. $02189 E-05$ & 293.6 & end \\
\hline $\mathrm{Cr}-53$ & 233 & $\odot$ & $1.15860 \mathrm{E}-06$ & 293.6 & end \\
\hline $\mathrm{Cr}-54$ & 233 & $\odot$ & $2.87821 E-07$ & 293.6 & end \\
\hline$M n-55$ & 233 & $\odot$ & $6.53464 \mathrm{E}-06$ & 293.6 & end \\
\hline $\mathrm{Fe}-54$ & 233 & $\odot$ & $2.39803 E-\odot 6$ & 293.6 & end \\
\hline $\mathrm{Fe}-56$ & 233 & $\odot$ & $3.76103 E-05$ & 293.6 & end \\
\hline $\mathrm{Fe}-57$ & 233 & $\odot$ & 8. 69031E-๑7 & 293.6 & end \\
\hline $\mathrm{Fe}-58$ & 233 & $\odot$ & $1.14778 \mathrm{E}-07$ & 293.6 & nd \\
\hline $\mathrm{Cu}-63$ & 233 & $\odot$ & 1. $90782 \mathrm{E}-05$ & 293.6 & nd \\
\hline $\mathrm{Cu}-65$ & 233 & $\odot$ & $8.50342 E-06$ & 293.6 & nd \\
\hline$U-234$ & 233 & $\odot$ & $3.25215 E-06$ & 293.6 & nd \\
\hline$U-235$ & 233 & $\odot$ & 3. $03100 E-\odot 4$ & 293.6 & nd \\
\hline$U-236$ & 233 & $\odot$ & 1. $30087 \mathrm{E}-06$ & 293.6 & nd \\
\hline$U-238$ & 233 & $\odot$ & \multirow{2}{*}{\multicolumn{2}{|c|}{$\begin{array}{l}1.75616 \mathrm{E}-05 \quad 293.6 \\
=\quad 8.00937 \mathrm{E}-02 \mathrm{a} / \mathrm{b}-\mathrm{cm}\end{array}$}} & nd \\
\hline \multicolumn{3}{|c|}{ total atom density } & & & ' $\quad 8.009370 \mathrm{E}-02$ \\
\hline $\mathrm{H}-1$ & 234 & $\odot$ & $3.32434 \mathrm{E}-02$ & 293.6 & end \\
\hline B-10 & 234 & $\odot$ & 1. $\odot 3798 E-05$ & 293.6 & end \\
\hline B-11 & 234 & 0 & $4.20486 E-05$ & 293.6 & nd \\
\hline $0-16$ & 234 & $\odot$ & $1.76644 \mathrm{E}-02$ & 293.6 & and \\
\hline $\mathrm{Mg}-24$ & 234 & $\odot$ & 1. $05686 \mathrm{E}-\odot 4$ & 293.6 & end \\
\hline $\mathrm{Mg}-25$ & 234 & $\odot$ & $1.33797 \mathrm{E}-05$ & 293.6 & end \\
\hline$M g-26$ & 234 & $\odot$ & 1. $47310 \mathrm{E}-05$ & 293.6 & and \\
\hline Al-27 & 234 & $\odot$ & $2.83940 \mathrm{E}-\odot 2$ & 293.6 & nd \\
\hline Si-28 & 234 & $\odot$ & 1. $01293 E-\odot 4$ & 293.6 & nd \\
\hline Si-29 & 234 & $\odot$ & $5.12887 E-06$ & 293.6 & nd \\
\hline Si-30 & 234 & $\odot$ & $3.40461 E-06$ & 293.6 & nd \\
\hline $\mathrm{Ti}-46$ & 234 & $\odot$ & 4. $20262 E-07$ & 293.6 & nd \\
\hline Ti-47 & 234 & 0 & $3.79000 \mathrm{E}-\odot 7$ & 293.6 & ad \\
\hline Ti-48 & 234 & 0 & $3.75536 \mathrm{E}-06$ & 293.6 & nd \\
\hline Ti-49 & 234 & $\odot$ & $2.75590 E-\odot 7$ & 293.6 & nd \\
\hline Ti-50 & 234 & $\odot$ & $2.63873 E-07$ & 293.6 & \\
\hline $\mathrm{Cr}-50$ & 234 & $\odot$ & $5.30517 \mathrm{E}-07$ & 293.6 & \\
\hline $\mathrm{Cr}-52$ & 234 & $\odot$ & 1. $\odot 2189 E-05$ & 293.6 & \\
\hline $\mathrm{Cr}-53$ & 234 & $\odot$ & $1.15860 \mathrm{E}-06$ & 293.6 & nd \\
\hline $\mathrm{Cr}-54$ & 234 & $\odot$ & $2.87821 E-07$ & 293.6 & \\
\hline$M n-55$ & 234 & $\odot$ & $6.50246 E-06$ & 293.6 & nd \\
\hline $\mathrm{Fe}-54$ & 234 & $\odot$ & $2.37952 E-\odot 6$ & 293.6 & רd \\
\hline $\mathrm{Fe}-56$ & 234 & $\odot$ & $3.73198 E-05$ & 293.6 & 10 \\
\hline $\mathrm{Fe}-57$ & 234 & 0 & $8.62321 E-07$ & 293.6 & nd \\
\hline $\mathrm{Fe}-58$ & 234 & $\odot$ & $1.13891 \mathrm{E}-07$ & 293.6 & nd \\
\hline $\mathrm{Cu}-63$ & 234 & $\odot$ & 1. $89710 E-05$ & 293.6 & nd \\
\hline $\mathrm{Cu}-65$ & 234 & $\odot$ & $8.45562 E-06$ & 293.6 & nd \\
\hline$U-234$ & 234 & $\odot$ & 4. ๑397०E-๑6 & 293.6 & nd \\
\hline U-235 & 234 & $\odot$ & $3.765 \odot \odot E-\odot 4$ & 293.6 & \\
\hline$U-236$ & 234 & $\odot$ & 1. $61589 E-06$ & 293.6 & १d \\
\hline$U-238$ & 234 & $\odot$ & $2.18144 \mathrm{E}-05$ & 293.6 & \\
\hline \multirow{2}{*}{\multicolumn{3}{|c|}{$\begin{array}{l}\text { total atom density } \\
\text {, } 8.009930 \mathrm{~F}-02\end{array}$}} & \multicolumn{2}{|c|}{$=8.00993 \mathrm{E}-02 \mathrm{a} / \mathrm{b}-\mathrm{cm}$} & \\
\hline ' 8.00 & & & & & \\
\hline $\mathrm{H}-1$ & 235 & $\odot$ & 3. 32434E- 02 & 293.6 & ena \\
\hline$B-10$ & 235 & $\odot$ & $6.18010 E-06$ & 293.6 & \\
\hline$B-11$ & 235 & $\odot$ & $2.50356 \mathrm{E}-05$ & 293.6 & \\
\hline $0-16$ & 235 & $\odot$ & 1. $78689 E-02$ & 293.6 & \\
\hline $\mathrm{Mg}-24$ & 235 & $\odot$ & 1. $05686 E-\odot 4$ & 293.6 & \\
\hline $\mathrm{Mg}-25$ & 235 & $\odot$ & 1. $33797 \mathrm{E}-05$ & 293.6 & \\
\hline$M g-26$ & 235 & $\odot$ & $1.47310 \mathrm{E}-05$ & 293.6 & Ia \\
\hline Al-27 & 235 & $\odot$ & $2.81406 \mathrm{E}-02$ & 293.6 & nd \\
\hline Si-28 & 235 & $\odot$ & 1. $00727 \mathrm{E}-\odot 4$ & 293.6 & d \\
\hline Si-29 & 235 & $\odot$ & $5.10026 \mathrm{E}-06$ & 293.6 & 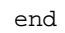 \\
\hline $\mathrm{Si}-30$ & 235 & $\odot$ & $3.38560 \mathrm{E}-06$ & 293.6 & \\
\hline Ti-46 & 235 & $\odot$ & $4.20262 \mathrm{E}-07$ & 293.6 & \\
\hline $\mathrm{Ti}-47$ & 235 & 0 & $3.79000 \mathrm{E}-\odot 7$ & 293.6 & ind \\
\hline $\mathrm{Ti}-48$ & 235 & $\odot$ & $3.75536 E-06$ & 293.6 & \\
\hline Ti-49 & 235 & $\odot$ & $2.75590 \mathrm{E}-07$ & 293.6 & \\
\hline Ti-50 & 235 & $\odot$ & $2.63873 E-07$ & 293.6 & \\
\hline $\mathrm{Cr}-50$ & 235 & $\odot$ & $5.30518 E-07$ & 293.6 & \\
\hline $\mathrm{Cr}-52$ & 235 & $\odot$ & 1. $02189 E-05$ & 293.6 & \\
\hline $\mathrm{Cr}-53$ & 235 & $\odot$ & $1.15860 \mathrm{E}-06$ & 293.6 & \\
\hline $\mathrm{Cr}-54$ & 235 & $\odot$ & $2.87821 E-07$ & 293.6 & \\
\hline$M n-55$ & 235 & $\odot$ & $6.47114 \mathrm{E}-06$ & 293.6 & \\
\hline $\mathrm{Fe}-54$ & 235 & $\odot$ & $2.36149 E-06$ & 293.6 & \\
\hline $\mathrm{Fe}-56$ & 235 & $\odot$ & $3.70370 \mathrm{E}-05$ & 293.6 & \\
\hline $\mathrm{Fe}-57$ & 235 & $\odot$ & $8.55788 E-07$ & 293.6 & \\
\hline $\mathrm{Fe}-58$ & 235 & $\odot$ & $1.13028 E-07$ & 293.6 & \\
\hline $\mathrm{Cu}-63$ & 235 & $\odot$ & $1.88666 \mathrm{E}-05$ & 293.6 & \\
\hline $\mathrm{Cu}-65$ & 235 & $\odot$ & 8. 40911E-०6 & 293.6 & \\
\hline$U-234$ & 235 & $\odot$ & $4.80687 E-06$ & 293.6 & \\
\hline$U-235$ & 235 & $\odot$ & $4.48000 \mathrm{E}-\odot 4$ & 293.6 & \\
\hline$U-236$ & 235 & $\odot$ & 1. $92276 \mathrm{E}-06$ & 293.6 & \\
\hline U-238 & 235 & $\odot$ & $2.59571 E-05$ & 293.6 & nd \\
\hline
\end{tabular}


total atom density $=8.00998 \mathrm{E}-02 \mathrm{a} / \mathrm{b}-\mathrm{cm}$ 8. $009980 \mathrm{E}-02$

\begin{tabular}{|c|c|c|c|c|c|}
\hline$H-1$ & 236 & $\odot$ & 3. 32434E- 02 & 293.6 & end \\
\hline B-10 & 236 & $\odot$ & $5.79830 E-06$ & 293.6 & end \\
\hline B-11 & 236 & $\odot$ & $2.34889 E-05$ & 293.6 & end \\
\hline $0-16$ & 236 & $\odot$ & 1. $78875 \mathrm{E}-02$ & 293.6 & end \\
\hline$M g-24$ & 236 & $\odot$ & $1.05686 \mathrm{E}-\odot 4$ & 293.6 & end \\
\hline$M g-25$ & 236 & $\odot$ & 1. $33797 \mathrm{E}-05$ & 293.6 & end \\
\hline$M g-26$ & 236 & $\odot$ & $1.47310 \mathrm{E}-05$ & 293.6 & end \\
\hline Al-27 & 236 & $\odot$ & $2.81176 \mathrm{E}-02$ & 293.6 & end \\
\hline Si-28 & 236 & $\odot$ & 1. $0 \odot 676 \mathrm{E}-\odot 4$ & 293.6 & end \\
\hline Si-29 & 236 & $\odot$ & $5.09768 \mathrm{E}-06$ & 293.6 & $\mathrm{ad}$ \\
\hline $\mathrm{Si}-30$ & 236 & $\odot$ & 3. 38390E- $\odot 6$ & 293.6 & nd \\
\hline Ti-46 & 236 & $\odot$ & $4.20262 \mathrm{E}-07$ & 293.6 & nd \\
\hline $\mathrm{Ti}-47$ & 236 & $\odot$ & $3.79000 \mathrm{E}-\odot 7$ & 293.6 & nd \\
\hline Ti-48 & 236 & $\odot$ & $3.75536 \mathrm{E}-06$ & 293.6 & nd \\
\hline Ti-49 & 236 & $\odot$ & $2.75590 \mathrm{E}-\odot 7$ & 293.6 & nd \\
\hline Ti-50 & 236 & $\odot$ & $2.63873 E-07$ & 293.6 & nd \\
\hline $\mathrm{Cr}-50$ & 236 & $\odot$ & 5. 30517E- -7 & 293.6 & nd \\
\hline $\mathrm{Cr}-52$ & 236 & $\odot$ & 1. $02189 \mathrm{E}-05$ & 293.6 & nd \\
\hline $\mathrm{Cr}-53$ & 236 & $\odot$ & $1.15860 \mathrm{E}-06$ & 293.6 & nd \\
\hline $\mathrm{Cr}-54$ & 236 & $\odot$ & $2.87821 \mathrm{E}-\odot 7$ & 293.6 & id \\
\hline$M n-55$ & 236 & $\odot$ & $6.46828 E-06$ & 293.6 & id \\
\hline $\mathrm{Fe}-54$ & 236 & $\odot$ & $2.35985 E-06$ & 293.6 & id \\
\hline $\mathrm{Fe}-56$ & 236 & $\odot$ & 3. 70113E- 05 & 293.6 & ad \\
\hline $\mathrm{Fe}-57$ & 236 & $\odot$ & $8.55193 E-07$ & 293.6 & \\
\hline $\mathrm{Fe}-58$ & 236 & $\odot$ & $1.12950 E-07$ & 293.6 & \\
\hline $\mathrm{Cu}-63$ & 236 & $\odot$ & $1.88571 \mathrm{E}-05$ & 293.6 & \\
\hline $\mathrm{Cu}-65$ & 236 & $\odot$ & $8.4 \odot 487 E-\odot 6$ & 293.6 & \\
\hline U-234 & 236 & $\odot$ & $4.87661 \mathrm{E}-06$ & 293.6 & \\
\hline U-235 & 236 & $\odot$ & $4.54500 \mathrm{E}-\odot 4$ & 293.6 & \\
\hline U-236 & 236 & $\odot$ & $1.95066 \mathrm{E}-06$ & 293.6 & \\
\hline U-238 & 236 & $\odot$ & $2.63337 \mathrm{E}-05$ & 293.6 & \\
\hline
\end{tabular}

\begin{tabular}{|c|c|c|c|c|c|}
\hline $\mathrm{H}-1$ & 237 & $\odot$ & $3.32434 \mathrm{E}-\odot 2$ & 293.6 & end \\
\hline B-10 & 237 & $\odot$ & 7. 995०7E-๑6 & 293.6 & end \\
\hline$B-11$ & 237 & $\odot$ & $3.23880 \mathrm{E}-\odot 5$ & 293.6 & end \\
\hline $0-16$ & 237 & $\odot$ & 1. $77805 \mathrm{E}-\odot 2$ & 293.6 & end \\
\hline$M g-24$ & 237 & 0 & 1. $05686 \mathrm{E}-\odot 4$ & 293.6 & end \\
\hline$M g-25$ & 237 & $\odot$ & 1. $33797 \mathrm{E}-\odot 5$ & 293.6 & end \\
\hline$M g-26$ & 237 & $\odot$ & 1. $47310 \mathrm{E}-05$ & 293.6 & end \\
\hline Al-27 & 237 & $\odot$ & $2.82501 E-\odot 2$ & 293.6 & end \\
\hline Si-28 & 237 & $\odot$ & 1. $\odot \odot 972 E-\odot 4$ & 293.6 & end \\
\hline Si-29 & 237 & $\odot$ & $5.11262 E-\odot 6$ & 293.6 & end \\
\hline Si-30 & 237 & $\odot$ & $3.39382 \mathrm{E}-\odot 6$ & 293.6 & end \\
\hline $\mathrm{Ti}-46$ & 237 & $\odot$ & $4.20262 E-07$ & 293.6 & end \\
\hline $\mathrm{Ti}-47$ & 237 & $\odot$ & 3. $7900 \odot E-\odot 7$ & 293.6 & end \\
\hline $\mathrm{Ti}-48$ & 237 & $\odot$ & $3.75536 \mathrm{E}-\odot 6$ & 293.6 & end \\
\hline $\mathrm{Ti}-49$ & 237 & $\odot$ & $2.75590 \mathrm{E}-\odot 7$ & 293.6 & end \\
\hline Ti-50 & 237 & $\odot$ & $2.63873 \mathrm{E}-07$ & 293.6 & end \\
\hline $\mathrm{Cr}-50$ & 237 & $\odot$ & $5.30517 \mathrm{E}-07$ & 293.6 & end \\
\hline $\mathrm{Cr}-52$ & 237 & $\odot$ & 1. $02189 E-05$ & 293.6 & end \\
\hline $\mathrm{Cr}-53$ & 237 & $\odot$ & $1.15860 E-06$ & 293.6 & end \\
\hline $\mathrm{Cr}-54$ & 237 & $\odot$ & $2.87821 \mathrm{E}-07$ & 293.6 & end \\
\hline$M n-55$ & 237 & $\odot$ & $6.48467 \mathrm{E}-\odot 6$ & 293.6 & end \\
\hline $\mathrm{Fe}-54$ & 237 & $\odot$ & $2.36928 \mathrm{E}-\odot 6$ & 293.6 & end \\
\hline $\mathrm{Fe}-56$ & 237 & $\odot$ & $3.71592 \mathrm{E}-05$ & 293.6 & end \\
\hline $\mathrm{Fe}-57$ & 237 & $\odot$ & $8.58611 E-07$ & 293.6 & end \\
\hline $\mathrm{Fe}-58$ & 237 & $\odot$ & $1.13401 \mathrm{E}-07$ & 293.6 & end \\
\hline $\mathrm{Cu}-63$ & 237 & $\odot$ & 1. $89118 \mathrm{E}-05$ & 293.6 & end \\
\hline $\mathrm{Cu}-65$ & 237 & $\odot$ & $8.42923 E-06$ & 293.6 & end \\
\hline$U-234$ & 237 & $\odot$ & $4.47532 \mathrm{E}-06$ & 293.6 & end \\
\hline$U-235$ & 237 & $\odot$ & $4.17100 \mathrm{E}-\odot 4$ & 293.6 & end \\
\hline$U-236$ & 237 & $\odot$ & 1. $79014 \mathrm{E}-06$ & 293.6 & end \\
\hline$U-238$ & 237 & $\odot$ & $2.41667 \mathrm{E}-05$ & 293.6 & 两 \\
\hline
\end{tabular}

$\mathrm{U}-238 \quad 237 \quad 0 \quad 2.41667 \mathrm{E}-05 \quad 293.6$ end

$\begin{array}{llllll}8.009330 \mathrm{E}-02 & & & & \\ \mathrm{H}-1 & 238 & \odot & 3.32434 \mathrm{E}-02 & 293.6 & \text { end } \\ \mathrm{B}-10 & 238 & \odot & 1.06559 \mathrm{E}-05 & 293.6 & \text { end } \\ \mathrm{B}-11 & 238 & \odot & 4.31670 \mathrm{E}-05 & 293.6 & \text { end } \\ 0-16 & 238 & \odot & 1.76509 \mathrm{E}-02 & 293.6 & \text { end } \\ \mathrm{Mg}-24 & 238 & \odot & 1.05686 \mathrm{E}-04 & 293.6 & \text { end } \\ \mathrm{Mg}-25 & 238 & \odot & 1.33797 \mathrm{E}-05 & 293.6 & \text { end } \\ \mathrm{Mg}-26 & 238 & \odot & 1.47310 \mathrm{E}-05 & 293.6 & \text { end } \\ \mathrm{Al}-27 & 238 & \odot & 2.84107 \mathrm{E}-02 & 293.6 & \text { end } \\ \mathrm{Si}-28 & 238 & \odot & 1.01330 \mathrm{E}-04 & 293.6 & \text { end } \\ \mathrm{Si}-29 & 238 & \odot & 5.13079 \mathrm{E}-06 & 293.6 & \text { end } \\ \mathrm{Si}-30 & 238 & \odot & 3.40588 \mathrm{E}-06 & 293.6 & \text { end } \\ \mathrm{Ti}-46 & 238 & \odot & 4.20262 \mathrm{E}-07 & 293.6 & \text { end } \\ \mathrm{Ti}-47 & 238 & \odot & 3.79000 \mathrm{E}-07 & 293.6 & \text { end }\end{array}$




$\begin{array}{llllll}\mathrm{Ti}-48 & 238 & \odot & 3.75536 \mathrm{E}-\odot 6 & 293.6 & \text { end } \\ \mathrm{Ti}-49 & 238 & \odot & 2.75590 \mathrm{E}-\odot 7 & 293.6 & \text { end } \\ \mathrm{Ti}-5 \odot & 238 & \odot & 2.63873 \mathrm{E}-\odot 7 & 293.6 & \text { end } \\ \mathrm{Cr}-5 \odot & 238 & \odot & 5.30517 \mathrm{E}-\odot 7 & 293.6 & \text { end } \\ \mathrm{Cr}-52 & 238 & \odot & 1.02189 \mathrm{E}-\odot 5 & 293.6 & \text { end } \\ \mathrm{Cr}-53 & 238 & \odot & 1.15860 \mathrm{E}-\odot 6 & 293.6 & \text { end } \\ \mathrm{Cr}-54 & 238 & \odot & 2.87821 \mathrm{E}-\odot 7 & 293.6 & \text { end } \\ \mathrm{Mn}-55 & 238 & \odot & 6.50452 \mathrm{E}-\odot 6 & 293.6 & \text { end } \\ \mathrm{Fe}-54 & 238 & \odot & 2.38070 \mathrm{E}-\odot 6 & 293.6 & \text { end } \\ \mathrm{Fe}-56 & 238 & \odot & 3.73384 \mathrm{E}-\odot 5 & 293.6 & \text { end } \\ \mathrm{Fe}-57 & 238 & \odot & 8.62751 \mathrm{E}-\odot 7 & 293.6 & \text { end } \\ \mathrm{Fe}-58 & 238 & \odot & 1.13948 \mathrm{E}-\odot 7 & 293.6 & \text { end } \\ \mathrm{Cu}-63 & 238 & \odot & 1.89779 \mathrm{E}-\odot 5 & 293.6 & \text { end } \\ \mathrm{Cu}-65 & 238 & \odot & 8.45870 \mathrm{E}-\odot 6 & 293.6 & \text { end } \\ \mathrm{U}-234 & 238 & \odot & 3.98927 \mathrm{E}-\odot 6 & 293.6 & \text { end } \\ \mathrm{U}-235 & 238 & \odot & 3.71800 \mathrm{E}-\odot 4 & 293.6 & \text { end } \\ \mathrm{U}-236 & 238 & \odot & 1.59572 \mathrm{E}-\odot 6 & 293.6 & \text { end } \\ \mathrm{U}-238 & 238 & \odot & 2.15420 \mathrm{E}-\odot 5 & 293.6 & \text { end }\end{array}$

Inner fuel element--fueled Axial region 4 total atom density $=8.00804 \mathrm{E}-02 \mathrm{a} / \mathrm{b}-\mathrm{cm}$

8. $\odot \odot 8 \odot 40 \mathrm{E}-\odot 2$

\begin{tabular}{|c|c|c|c|c|c|}
\hline $\mathrm{H}-1$ & 241 & $\odot$ & 3. 32434E- -2 & 293.6 & end \\
\hline$B-10$ & 241 & $\odot$ & $2.04121 E-05$ & 293.6 & end \\
\hline$B-11$ & 241 & $\odot$ & $8.26896 E-05$ & 293.6 & end \\
\hline $0-16$ & 241 & $\odot$ & $1.71757 \mathrm{E}-02$ & 293.6 & dd \\
\hline$M g-24$ & 241 & 0 & 1. $05686 \mathrm{E}-04$ & 293.6 & \\
\hline$M g-25$ & 241 & $\odot$ & $1.33797 \mathrm{E}-05$ & 293.6 & d \\
\hline$M g-26$ & 241 & $\odot$ & $1.47310 \mathrm{E}-05$ & 293.6 & \\
\hline Al-27 & 241 & 0 & $2.89993 E-02$ & 293.6 & nd \\
\hline Si-28 & 241 & 0 & 1. $02644 \mathrm{E}-04$ & 293.6 & nd \\
\hline Si-29 & 241 & 0 & $5.19729 E-\odot 6$ & 293.6 & nd \\
\hline Si-30 & 241 & 0 & $3.45002 \mathrm{E}-\odot 6$ & 293.6 & d \\
\hline Ti-46 & 241 & $\odot$ & $4.20262 E-07$ & 293.6 & \\
\hline Ti-47 & 241 & 0 & $3.79000 \mathrm{E}-07$ & 293.6 & \\
\hline Ti-48 & 241 & 0 & $3.75536 \mathrm{E}-06$ & 293.6 & \\
\hline Ti-49 & 241 & 0 & $2.75590 \mathrm{E}-\odot 7$ & 293.6 & \\
\hline Ti-50 & 241 & $\odot$ & $2.63873 E-07$ & 293.6 & \\
\hline $\mathrm{Cr}-50$ & 241 & 0 & $5.30517 \mathrm{E}-07$ & 293.6 & \\
\hline $\mathrm{Cr}-52$ & 241 & 0 & 1. $02189 E-\odot 5$ & 293.6 & \\
\hline $\mathrm{Cr}-53$ & 241 & $\odot$ & $1.15860 E-06$ & 293.6 & \\
\hline $\mathrm{Cr}-54$ & 241 & 0 & $2.87821 E-07$ & 293.6 & \\
\hline$M n-55$ & 241 & $\odot$ & $6.57731 \mathrm{E}-06$ & 293.6 & \\
\hline $\mathrm{Fe}-54$ & 241 & $\odot$ & $2.42259 E-06$ & 293.6 & end \\
\hline $\mathrm{Fe}-56$ & 241 & $\odot$ & 3. 79953E- 05 & 293.6 & \\
\hline $\mathrm{Fe}-57$ & 241 & 0 & $8.77930 \mathrm{E}-07$ & 293.6 & \\
\hline $\mathrm{Fe}-58$ & 241 & $\odot$ & $1.15953 E-07$ & 293.6 & \\
\hline $\mathrm{Cu}-63$ & 241 & $\odot$ & 1. $92203 E-05$ & 293.6 & \\
\hline $\mathrm{Cu}-65$ & 241 & $\odot$ & $8.56676 \mathrm{E}-06$ & 293.6 & \\
\hline$U-234$ & 241 & 0 & $2.20708 \mathrm{E}-\odot 6$ & 293.6 & \\
\hline$U-235$ & 241 & $\odot$ & 2. $05700 \mathrm{E}-\odot 4$ & 293.6 & \\
\hline$U-236$ & 241 & $\odot$ & $8.82838 E-07$ & 293.6 & \\
\hline$U-238$ & 241 & $\odot$ & 1. $19182 \mathrm{E}-05$ & 293.6 & \\
\hline
\end{tabular}

, total atom density $=8.00839 \mathrm{E}-02 \mathrm{a} / \mathrm{b}-\mathrm{cm}$

' 8. 0०839०E-๑2

$\begin{array}{llllll}\mathrm{H}-1 & 242 & \odot & 3.32434 \mathrm{E}-\odot 2 & 293.6 & \text { end } \\ \mathrm{B}-10 & 242 & \odot & 1.77513 \mathrm{E}-\odot 5 & 293.6 & \text { end } \\ \mathrm{B}-11 & 242 & \odot & 7.19107 \mathrm{E}-\odot 5 & 293.6 & \text { end } \\ \mathrm{O}-16 & 242 & \odot & 1.73053 \mathrm{E}-\odot 2 & 293.6 & \text { end } \\ \mathrm{Mg}-24 & 242 & \odot & 1.05686 \mathrm{E}-\odot 4 & 293.6 & \text { end } \\ \mathrm{Mg}-25 & 242 & \odot & 1.33797 \mathrm{E}-\odot 5 & 293.6 & \text { end } \\ \mathrm{Mg}-26 & 242 & \odot & 1.47310 \mathrm{E}-\odot 5 & 293.6 & \text { end } \\ \mathrm{Al}-27 & 242 & \odot & 2.88388 \mathrm{E}-\odot 2 & 293.6 & \text { end } \\ \mathrm{Si}-28 & 242 & \odot & 1.02285 \mathrm{E}-\odot 4 & 293.6 & \text { end } \\ \mathrm{Si}-29 & 242 & \odot & 5.17912 \mathrm{E}-\odot 6 & 293.6 & \text { end } \\ \mathrm{Si}-3 \odot & 242 & \odot & 3.43796 \mathrm{E}-\odot 6 & 293.6 & \text { end } \\ \mathrm{Ti}-46 & 242 & \odot & 4.20262 \mathrm{E}-\odot 7 & 293.6 & \text { end } \\ \mathrm{Ti}-47 & 242 & \odot & 3.79000 \mathrm{E}-\odot 7 & 293.6 & \text { end } \\ \mathrm{Ti}-48 & 242 & \odot & 3.75536 \mathrm{E}-\odot 6 & 293.6 & \text { end } \\ \mathrm{Ti}-49 & 242 & \odot & 2.75590 \mathrm{E}-\odot 7 & 293.6 & \text { end } \\ \mathrm{Ti}-50 & 242 & \odot & 2.63873 \mathrm{E}-\odot 7 & 293.6 & \text { end } \\ \mathrm{Cr}-50 & 242 & \odot & 5.30517 \mathrm{E}-\odot 7 & 293.6 & \text { end } \\ \mathrm{Cr}-52 & 242 & \odot & 1.02189 \mathrm{E}-\odot 5 & 293.6 & \text { end } \\ \mathrm{Cr}-53 & 242 & \odot & 1.15860 \mathrm{E}-\odot 6 & 293.6 & \text { end } \\ \mathrm{Cr}-54 & 242 & \odot & 2.87821 \mathrm{E}-\odot 7 & 293.6 & \text { end } \\ \mathrm{Mn}-55 & 242 & \odot & 6.55746 \mathrm{E}-\odot 6 & 293.6 & \text { end } \\ \mathrm{Fe}-54 & 242 & \odot & 2.41117 \mathrm{E}-\odot 6 & 293.6 & \text { end } \\ \mathrm{Fe}-56 & 242 & \odot & 3.78162 \mathrm{E}-\odot 5 & 293.6 & \text { end } \\ \mathrm{Fe}-57 & 242 & \odot & 8.73792 \mathrm{E}-\odot 7 & 293.6 & \text { end } \\ \mathrm{Fe}-58 & 242 & \odot & 1.15406 \mathrm{E}-\odot 7 & 293.6 & \text { end } \\ \mathrm{Cu}-63 & 242 & \odot & 1.91542 \mathrm{E}-\odot 5 & 293.6 & \text { end }\end{array}$




\begin{tabular}{|c|c|c|c|c|c|}
\hline $\mathrm{Cu}-65$ & 242 & $\odot$ & $8.53729 E-06$ & 293.6 & end \\
\hline$U-234$ & 242 & $\odot$ & $2.69313 E-06$ & 293.6 & end \\
\hline$U-235$ & 242 & $\odot$ & $2.51000 \mathrm{E}-04$ & 293.6 & רd \\
\hline$U-236$ & 242 & $\odot$ & $1.07726 \mathrm{E}-06$ & 293.6 & 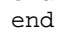 \\
\hline$U-238$ & 242 & $\odot$ & $1.45429 E-05$ & 293.6 & nd \\
\hline \multicolumn{3}{|c|}{$\begin{array}{l}\text { total atom density } \\
8800 \mathrm{E}-02\end{array}$} & \multicolumn{3}{|c|}{$=8.00880 \mathrm{E}-02 \mathrm{a} / \mathrm{b}-\mathrm{cm}$} \\
\hline $\mathrm{H}-1$ & 243 & 0 & $3.32435 \mathrm{E}-02$ & 293.6 & p \\
\hline$B-10$ & 243 & $\odot$ & $1.46911 \mathrm{E}-05$ & 293.6 & \\
\hline B-11 & 243 & 0 & $5.95139 E-05$ & 293.6 & \\
\hline $0-16$ & 243 & $\odot$ & $1.74543 E-02$ & 293.6 & \\
\hline$M g-24$ & 243 & $\odot$ & 1. $05686 E-04$ & 293.6 & \\
\hline $\mathrm{Mg}-25$ & 243 & $\odot$ & $1.33797 \mathrm{E}-05$ & 293.6 & \\
\hline$M g-26$ & 243 & $\odot$ & $1.47310 E-05$ & 293.6 & \\
\hline Al-27 & 243 & 0 & $2.86541 E-02$ & 293.6 & \\
\hline Si-28 & 243 & 0 & 1. $01874 \mathrm{E}-\odot 4$ & 293.6 & \\
\hline Si-29 & 243 & 0 & $5.15831 E-06$ & 293.6 & \\
\hline $\mathrm{Si}-30$ & 243 & 0 & $3.42415 \mathrm{E}-06$ & 293.6 & \\
\hline $\mathrm{Ti}-46$ & 243 & $\odot$ & $4.20263 E-07$ & 293.6 & \\
\hline $\mathrm{Ti}-47$ & 243 & 0 & $3.79001 \mathrm{E}-07$ & 293.6 & \\
\hline $\mathrm{Ti}-48$ & 243 & 0 & $3.75537 \mathrm{E}-06$ & 293.6 & \\
\hline $\mathrm{Ti}-49$ & 243 & $\odot$ & $2.75590 E-07$ & 293.6 & \\
\hline $\mathrm{Ti}-50$ & 243 & $\odot$ & $2.63873 E-07$ & 293.6 & \\
\hline $\mathrm{Cr}-50$ & 243 & 0 & $5.30518 E-07$ & 293.6 & \\
\hline $\mathrm{Cr}-52$ & 243 & $\odot$ & 1. $02189 E-05$ & 293.6 & \\
\hline $\mathrm{Cr}-53$ & 243 & 0 & $1.15860 \mathrm{E}-06$ & 293.6 & \\
\hline $\mathrm{Cr}-54$ & 243 & $\odot$ & $2.87821 E-07$ & 293.6 & \\
\hline$M n-55$ & 243 & $\odot$ & $6.53464 E-06$ & 293.6 & \\
\hline $\mathrm{Fe}-54$ & 243 & 0 & 2. 39803E- 06 & 293.6 & \\
\hline $\mathrm{Fe}-56$ & 243 & 0 & 3. $76103 \mathrm{E}-05$ & 293.6 & \\
\hline $\mathrm{Fe}-57$ & 243 & 0 & 8. 69031E-07 & 293.6 & \\
\hline $\mathrm{Fe}-58$ & 243 & 0 & $1.14778 \mathrm{E}-07$ & 293.6 & \\
\hline $\mathrm{Cu}-63$ & 243 & $\odot$ & 1. $90782 \mathrm{E}-05$ & 293.6 & \\
\hline $\mathrm{Cu}-65$ & 243 & $\odot$ & $8.50342 \mathrm{E}-06$ & 293.6 & \\
\hline U-234 & 243 & 0 & $3.25215 E-06$ & 293.6 & \\
\hline$U-235$ & 243 & $\odot$ & $3 . \odot 310 \odot E-\odot 4$ & 293.6 & \\
\hline $\mathrm{U}-236$ & 243 & 0 & 1. $30087 \mathrm{E}-\odot 6$ & 293.6 & \\
\hline$U-238$ & 243 & 0 & $1.75616 \mathrm{E}-05$ & 293.6 & \\
\hline \multicolumn{3}{|c|}{$\begin{array}{l}\text { total at } \\
1 \quad 8.009370 \mathrm{E}-02\end{array}$} & \multicolumn{2}{|c|}{$=8.00937 \mathrm{E}-02 \mathrm{a} / \mathrm{b}-\mathrm{cm}$} & \\
\hline $\mathrm{H}-1$ & 244 & $\odot$ & 3. 32434E- 02 & 293.6 & \\
\hline$B-10$ & 244 & 0 & $1.03798 \mathrm{E}-05$ & 293.6 & \\
\hline B-11 & 244 & 0 & $4.20486 E-05$ & 293.6 & \\
\hline $0-16$ & 244 & $\odot$ & $1.76644 \mathrm{E}-02$ & 293.6 & \\
\hline$M g-24$ & 244 & $\odot$ & 1. $05686 E-\odot 4$ & 293.6 & \\
\hline$M g-25$ & 244 & 0 & $1.33797 \mathrm{E}-\odot 5$ & 293.6 & \\
\hline$M g-26$ & 244 & $\odot$ & $1.47310 \mathrm{E}-05$ & 293.6 & \\
\hline Al-27 & 244 & $\odot$ & $2.83940 E-02$ & 293.6 & \\
\hline Si-28 & 244 & $\odot$ & 1. $01293 E-04$ & 293.6 & \\
\hline Si-29 & 244 & 0 & $5.12887 \mathrm{E}-\odot 6$ & 293.6 & \\
\hline Si-30 & 244 & 0 & 3. 40461E-06 & 293.6 & \\
\hline Ti-46 & 244 & $\odot$ & 4. $2 \odot 262 E-\odot 7$ & 293.6 & \\
\hline Ti-47 & 244 & $\odot$ & $3.790 \odot \odot E-\odot 7$ & 293.6 & \\
\hline $\mathrm{Ti}-48$ & 244 & $\odot$ & $3.75536 E-06$ & 293.6 & \\
\hline $\mathrm{Ti}-49$ & 244 & $\odot$ & $2.75590 E-07$ & 293.6 & \\
\hline $\mathrm{Ti}-50$ & 244 & $\odot$ & $2.63873 E-07$ & 293.6 & \\
\hline $\mathrm{Cr}-50$ & 244 & $\odot$ & $5.30517 \mathrm{E}-07$ & 293.6 & \\
\hline $\mathrm{Cr}-52$ & 244 & 0 & 1. $02189 E-05$ & 293.6 & \\
\hline $\mathrm{Cr}-53$ & 244 & 0 & $1.15860 E-06$ & 293.6 & \\
\hline $\mathrm{Cr}-54$ & 244 & 0 & $2.87821 E-07$ & 293.6 & \\
\hline$M n-55$ & 244 & 0 & $6.50246 E-06$ & 293.6 & \\
\hline $\mathrm{Fe}-54$ & 244 & $\odot$ & $2.37952 E-06$ & 293.6 & \\
\hline $\mathrm{Fe}-56$ & 244 & $\odot$ & $3.73198 E-05$ & 293.6 & \\
\hline $\mathrm{Fe}-57$ & 244 & $\odot$ & $8.62321 E-07$ & 293.6 & \\
\hline $\mathrm{Fe}-58$ & 244 & $\odot$ & 1. 13891E- - 7 & 293.6 & \\
\hline $\mathrm{Cu}-63$ & 244 & $\odot$ & $1.89710 E-\odot 5$ & 293.6 & \\
\hline $\mathrm{Cu}-65$ & 244 & $\odot$ & $8.45562 \mathrm{E}-06$ & 293.6 & \\
\hline$U-234$ & 244 & $\odot$ & $4.03970 E-\odot 6$ & 293.6 & \\
\hline$U-235$ & 244 & $\odot$ & $3.76500 \mathrm{E}-\odot 4$ & 293.6 & \\
\hline$U-236$ & 244 & $\odot$ & $1.61589 E-06$ & 293.6 & \\
\hline$U-238$ & 244 & $\odot$ & $2.18144 \mathrm{E}-\odot 5$ & 293.6 & \\
\hline 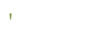 & atom & sity & $=8.00993 \mathrm{E}-$ & $2 a / b-c l$ & \\
\hline \multicolumn{6}{|c|}{ ' $\quad$ 8. $009930 \mathrm{E}-02$} \\
\hline $\mathrm{H}-1$ & 245 & $\odot$ & $3.32434 \mathrm{E}-02$ & 293.6 & \\
\hline B-10 & 245 & 0 & $6.18010 \mathrm{E}-06$ & 293.6 & \\
\hline B-11 & 245 & 0 & $2.5 \odot 356 \mathrm{E}-\odot 5$ & 293.6 & \\
\hline $0-16$ & 245 & $\odot$ & $1.78689 \mathrm{E}-02$ & 293.6 & \\
\hline$M g-24$ & 245 & 0 & $1.05686 E-04$ & 293.6 & \\
\hline$M g-25$ & 245 & $\odot$ & $1.33797 \mathrm{E}-05$ & 293.6 & \\
\hline$M g-26$ & 245 & 0 & $1.47310 \mathrm{E}-05$ & 293.6 & \\
\hline Al-27 & 245 & 0 & $2.81406 \mathrm{E}-02$ & 293.6 & \\
\hline
\end{tabular}




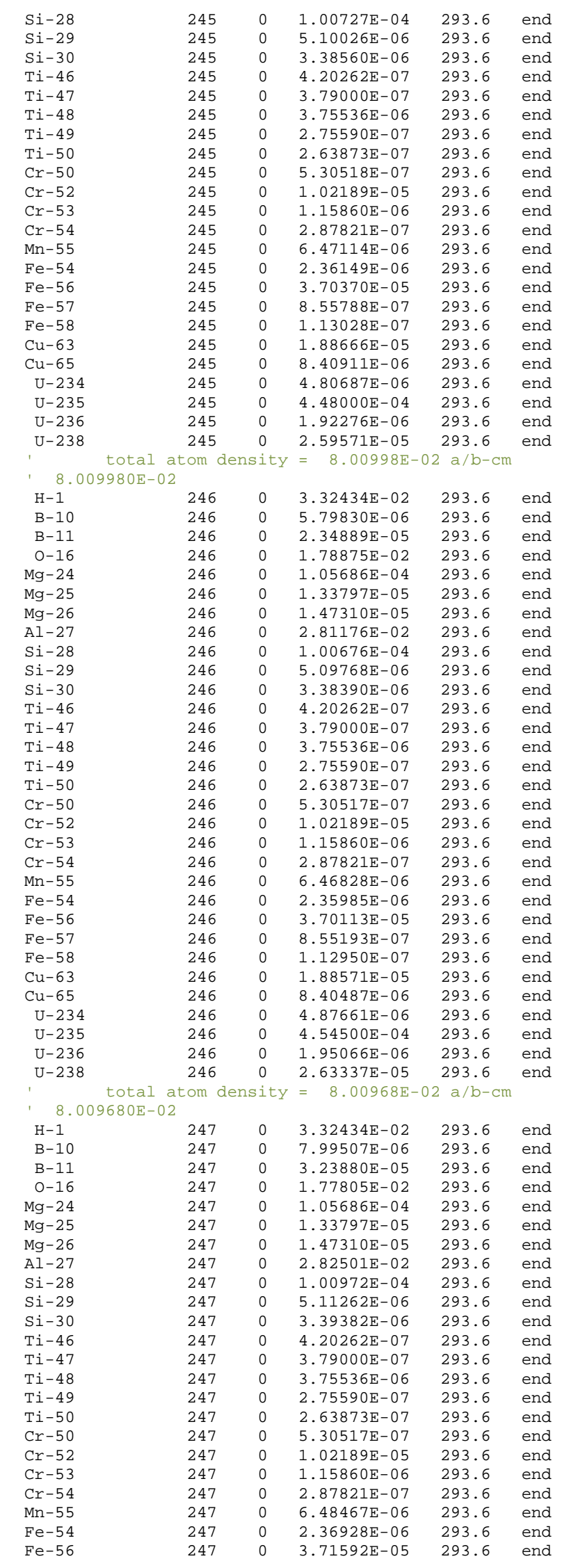




\begin{tabular}{|c|c|c|c|c|c|}
\hline $\mathrm{Fe}-57$ & 247 & $\odot$ & $8.58611 E-07$ & 293.6 & end \\
\hline $\mathrm{Fe}-58$ & 247 & $\odot$ & $1.13401 \mathrm{E}-07$ & 293.6 & \\
\hline $\mathrm{Cu}-63$ & 247 & $\odot$ & 1. $89118 \mathrm{E}-05$ & 293.6 & \\
\hline $\mathrm{Cu}-65$ & 247 & $\odot$ & 8. 42923E- 06 & 293.6 & \\
\hline$U-234$ & 247 & $\odot$ & $4.47532 E-06$ & 293.6 & \\
\hline$U-235$ & 247 & $\odot$ & $4.17100 \mathrm{E}-\odot 4$ & 293.6 & \\
\hline$U-236$ & 247 & $\odot$ & 1. $79014 \mathrm{E}-\odot 6$ & 293.6 & \\
\hline$U-238$ & 247 & $\odot$ & $2.41667 \mathrm{E}-05$ & 293.6 & \\
\hline \multicolumn{3}{|c|}{ total atom density } & \multicolumn{3}{|c|}{$=8.00933 \mathrm{E}-02 \mathrm{a} / \mathrm{b}-\mathrm{cm}$} \\
\hline $\mathrm{H}-1$ & 248 & $\odot$ & $3.32434 \mathrm{E}-02$ & 293.6 & \\
\hline$B-10$ & 248 & $\odot$ & 1. $06559 E-05$ & 293.6 & \\
\hline$B-11$ & 248 & $\odot$ & 4. $31670 \mathrm{E}-05$ & 293.6 & \\
\hline $0-16$ & 248 & $\odot$ & 1. $76509 E-02$ & 293.6 & \\
\hline$M g-24$ & 248 & $\odot$ & 1. $05686 E-04$ & 293.6 & \\
\hline$M g-25$ & 248 & $\odot$ & $1.33797 \mathrm{E}-05$ & 293.6 & \\
\hline$M g-26$ & 248 & $\odot$ & 1. $47310 \mathrm{E}-05$ & 293.6 & \\
\hline Al-27 & 248 & $\odot$ & $2.84107 E-02$ & 293.6 & \\
\hline Si-28 & 248 & $\odot$ & 1. $01330 \mathrm{E}-04$ & 293.6 & \\
\hline Si-29 & 248 & $\odot$ & $5.13079 E-06$ & 293.6 & \\
\hline Si-30 & 248 & $\odot$ & $3.40588 \mathrm{E}-06$ & 293.6 & \\
\hline Ti-46 & 248 & $\odot$ & $4.20262 \mathrm{E}-07$ & 293.6 & \\
\hline Ti-47 & 248 & $\odot$ & $3.79000 \mathrm{E}-\odot 7$ & 293.6 & \\
\hline Ti-48 & 248 & 0 & $3.75536 \mathrm{E}-06$ & 293.6 & \\
\hline Ti-49 & 248 & $\odot$ & $2.75590 \mathrm{E}-\odot 7$ & 293.6 & \\
\hline Ti-50 & 248 & $\odot$ & $2.63873 E-07$ & 293.6 & \\
\hline $\mathrm{Cr}-50$ & 248 & $\odot$ & $5.30517 E-07$ & 293.6 & \\
\hline $\mathrm{Cr}-52$ & 248 & $\odot$ & 1. $\odot 2189 E-\odot 5$ & 293.6 & \\
\hline $\mathrm{Cr}-53$ & 248 & $\odot$ & $1.15860 \mathrm{E}-06$ & 293.6 & \\
\hline $\mathrm{Cr}-54$ & 248 & $\odot$ & $2.87821 E-07$ & 293.6 & \\
\hline Mn - 55 & 248 & $\odot$ & $6.5 \odot 452 \mathrm{E}-06$ & 293.6 & \\
\hline $\mathrm{Fe}-54$ & 248 & $\odot$ & $2.38070 \mathrm{E}-\odot 6$ & 293.6 & \\
\hline $\mathrm{Fe}-56$ & 248 & $\odot$ & $3.73384 E-05$ & 293.6 & \\
\hline $\mathrm{Fe}-57$ & 248 & $\odot$ & $8.62751 E-07$ & 293.6 & \\
\hline $\mathrm{Fe}-58$ & 248 & $\odot$ & $1.13948 \mathrm{E}-07$ & 293.6 & \\
\hline $\mathrm{Cu}-63$ & 248 & $\odot$ & 1. 89779E- 05 & 293.6 & \\
\hline $\mathrm{Cu}-65$ & 248 & $\odot$ & $8.45870 E-06$ & 293.6 & \\
\hline$U-234$ & 248 & $\odot$ & $3.98927 E-06$ & 293.6 & \\
\hline$U-235$ & 248 & $\odot$ & $3.71800 E-\odot 4$ & 293.6 & \\
\hline$U-236$ & 248 & $\odot$ & $1.59572 \mathrm{E}-06$ & 293.6 & \\
\hline$U-238$ & 248 & $\odot$ & $2.15420 E-05$ & 293.6 & \\
\hline
\end{tabular}

Inner fuel element--fueled Axial region 5 total atom density $=8.00804 \mathrm{E}-02 \mathrm{a} / \mathrm{b}-\mathrm{cm}$ $8.008040 \mathrm{E}-\mathrm{-2}$

$\begin{array}{llllll}8.008040 \mathrm{H}-1 & 251 & \odot & 3.32434 \mathrm{E}-\odot 2 & 293.6 & \text { end } \\ \mathrm{B}-10 & 251 & \odot & 2.04121 \mathrm{E}-\odot 5 & 293.6 & \text { end } \\ \mathrm{B}-11 & 251 & \odot & 8.26896 \mathrm{E}-\odot 5 & 293.6 & \text { end } \\ \mathrm{O}-16 & 251 & \odot & 1.71757 \mathrm{E}-\odot 2 & 293.6 & \text { end } \\ \mathrm{Mg}-24 & 251 & \odot & 1.05686 \mathrm{E}-\odot 4 & 293.6 & \text { end } \\ \mathrm{Mg}-25 & 251 & \odot & 1.33797 \mathrm{E}-\odot 5 & 293.6 & \text { end } \\ \mathrm{Mg}-26 & 251 & \odot & 1.47310 \mathrm{E}-\odot 5 & 293.6 & \text { end } \\ \mathrm{Al}-27 & 251 & \odot & 2.89993 \mathrm{E}-\odot 2 & 293.6 & \text { end } \\ \mathrm{Si}-28 & 251 & \odot & 1.02644 \mathrm{E}-\odot 4 & 293.6 & \text { end } \\ \mathrm{Si}-29 & 251 & \odot & 5.19729 \mathrm{E}-\odot 6 & 293.6 & \text { end } \\ \mathrm{Si}-3 \odot & 251 & \odot & 3.45002 \mathrm{E}-\odot 6 & 293.6 & \text { end } \\ \mathrm{Ti}-46 & 251 & \odot & 4.20262 \mathrm{E}-\odot 7 & 293.6 & \text { end } \\ \mathrm{Ti}-47 & 251 & \odot & 3.79000 \mathrm{E}-\odot 7 & 293.6 & \text { end } \\ \mathrm{Ti}-48 & 251 & \odot & 3.75536 \mathrm{E}-\odot 6 & 293.6 & \text { end } \\ \mathrm{Ti}-49 & 251 & \odot & 2.75590 \mathrm{E}-\odot 7 & 293.6 & \text { end } \\ \mathrm{Ti}-5 \odot & 251 & \odot & 2.63873 \mathrm{E}-\odot 7 & 293.6 & \text { end } \\ \mathrm{Cr}-50 & 251 & \odot & 5.30517 \mathrm{E}-\odot 7 & 293.6 & \text { end } \\ \mathrm{Cr}-52 & 251 & \odot & 1.02189 \mathrm{E}-\odot 5 & 293.6 & \text { end } \\ \mathrm{Cr}-53 & 251 & \odot & 1.15860 \mathrm{E}-\odot 6 & 293.6 & \text { end } \\ \mathrm{Cr}-54 & 251 & \odot & 2.87821 \mathrm{E}-\odot 7 & 293.6 & \text { end } \\ \mathrm{Mn}-55 & 251 & \odot & 6.57731 \mathrm{E}-\odot 6 & 293.6 & \text { end } \\ \mathrm{Fe}-54 & 251 & \odot & 2.42259 \mathrm{E}-\odot 6 & 293.6 & \text { end } \\ \mathrm{Fe}-56 & 251 & \odot & 3.79953 \mathrm{E}-\odot 5 & 293.6 & \text { end } \\ \mathrm{Fe}-57 & 251 & \odot & 8.77930 \mathrm{E}-\odot 7 & 293.6 & \text { end } \\ \mathrm{Fe}-58 & 251 & \odot & 1.15953 \mathrm{E}-\odot 7 & 293.6 & \text { end } \\ \mathrm{Cu}-63 & 251 & \odot & 1.92203 \mathrm{E}-\odot 5 & 293.6 & \text { end } \\ \mathrm{Cu}-65 & 251 & \odot & 8.56676 \mathrm{E}-\odot 6 & 293.6 & \text { end } \\ \mathrm{U}-234 & 251 & \odot & 2.20708 \mathrm{E}-\odot 6 & 293.6 & \text { end } \\ \mathrm{U}-235 & 251 & \odot & 2.05700 \mathrm{E}-\odot 4 & 293.6 & \text { end } \\ \mathrm{U}-236 & 251 & \odot & 8.82838 \mathrm{E}-\odot 7 & 293.6 & \text { end } \\ \mathrm{U}-238 & 251 & \odot & 1.19182 \mathrm{E}-\odot 5 & 293.6 & \text { end }\end{array}$

total atom density $=8.00839 \mathrm{E}-02 \mathrm{a} / \mathrm{b}-\mathrm{cm}$

8. $0 \odot 8390 \mathrm{E}-02$

$\begin{array}{llllll}\text { H-1 } & 252 & \odot & 3.32434 \mathrm{E}-02 & 293.6 & \text { end } \\ \text { B-10 } & 252 & \odot & 1.77513 \mathrm{E}-05 & 293.6 & \text { end } \\ \text { B-11 } & 252 & \odot & 7.19107 \mathrm{E}-05 & 293.6 & \text { end }\end{array}$




\begin{tabular}{|c|c|c|c|c|}
\hline $0-16$ & 252 & $\odot$ & 1. $73053 \mathrm{E}-02$ & 293.6 \\
\hline$M g-24$ & 252 & $\odot$ & 1. $05686 E-04$ & 293.6 \\
\hline$M g-25$ & 252 & $\odot$ & 1. $33797 \mathrm{E}-05$ & 293.6 \\
\hline$M g-26$ & 252 & $\odot$ & 1. $47310 \mathrm{E}-05$ & 293.6 \\
\hline Al-27 & 252 & 0 & $2.88388 \mathrm{E}-02$ & 293.6 \\
\hline Si-28 & 252 & $\odot$ & 1. $\odot 2285 E-\odot 4$ & 293.6 \\
\hline Si-29 & 252 & $\odot$ & $5.17912 \mathrm{E}-\odot 6$ & 293.6 \\
\hline $\mathrm{Si}-30$ & 252 & $\odot$ & $3.43796 \mathrm{E}-06$ & 293.6 \\
\hline $\mathrm{Ti}-46$ & 252 & $\odot$ & $4.20262 \mathrm{E}-07$ & 293.6 \\
\hline $\mathrm{Ti}-47$ & 252 & $\odot$ & $3.79000 \mathrm{E}-07$ & 293.6 \\
\hline $\mathrm{Ti}-48$ & 252 & 0 & $3.75536 \mathrm{E}-06$ & 293.6 \\
\hline Ti-49 & 252 & $\odot$ & $2.75590 \mathrm{E}-\odot 7$ & 293.6 \\
\hline Ti-50 & 252 & $\odot$ & $2.63873 E-07$ & 293.6 \\
\hline $\mathrm{Cr}-50$ & 252 & $\odot$ & $5.30517 \mathrm{E}-07$ & 293.6 \\
\hline $\mathrm{Cr}-52$ & 252 & $\odot$ & 1. $02189 E-05$ & 293.6 \\
\hline $\mathrm{Cr}-53$ & 252 & $\odot$ & $1.15860 E-\odot 6$ & 293.6 \\
\hline $\mathrm{Cr}-54$ & 252 & $\odot$ & $2.87821 E-07$ & 293.6 \\
\hline$M n-55$ & 252 & $\odot$ & $6.55746 \mathrm{E}-06$ & 293.6 \\
\hline $\mathrm{Fe}-54$ & 252 & $\odot$ & $2.41117 \mathrm{E}-06$ & 293.6 \\
\hline $\mathrm{Fe}-56$ & 252 & $\odot$ & $3.78162 \mathrm{E}-05$ & 293.6 \\
\hline $\mathrm{Fe}-57$ & 252 & $\odot$ & $8.73792 \mathrm{E}-07$ & 293.6 \\
\hline $\mathrm{Fe}-58$ & 252 & $\odot$ & $1.15406 \mathrm{E}-07$ & 293.6 \\
\hline $\mathrm{Cu}-63$ & 252 & $\odot$ & $1.91542 \mathrm{E}-05$ & 293.6 \\
\hline $\mathrm{Cu}-65$ & 252 & $\odot$ & $8.53729 E-06$ & 293.6 \\
\hline$U-234$ & 252 & $\odot$ & $2.69313 \mathrm{E}-06$ & 293.6 \\
\hline$U-235$ & 252 & $\odot$ & $2.51000 \mathrm{E}-\odot 4$ & 293.6 \\
\hline$U-236$ & 252 & $\odot$ & 1. $07726 \mathrm{E}-06$ & 293.6 \\
\hline$U-238$ & 252 & $\odot$ & 1. $45429 E-05$ & 293.6 \\
\hline
\end{tabular}

total atom density $=8.00880 \mathrm{E}-02 \mathrm{a} / \mathrm{b}-\mathrm{cm}$

\begin{tabular}{|c|c|c|c|c|}
\hline $\mathrm{H}-1$ & 253 & $\odot$ & 3. 32435E- 02 & 293.6 \\
\hline$B-10$ & 253 & $\odot$ & 1. $46911 \mathrm{E}-\odot 5$ & 293.6 \\
\hline$B-11$ & 253 & $\odot$ & $5.95139 \mathrm{E}-\odot 5$ & 293.6 \\
\hline $0-16$ & 253 & $\odot$ & $1.74543 \mathrm{E}-\odot 2$ & 293.6 \\
\hline$M g-24$ & 253 & $\odot$ & 1. $05686 E-\odot 4$ & 293.6 \\
\hline$M g-25$ & 253 & $\odot$ & $1.33797 \mathrm{E}-05$ & 293.6 \\
\hline$M g-26$ & 253 & $\odot$ & 1. $47310 \mathrm{E}-05$ & 293.6 \\
\hline Al-27 & 253 & $\odot$ & $2.86541 E-02$ & 293.6 \\
\hline Si-28 & 253 & 0 & 1. $01874 \mathrm{E}-04$ & 293.6 \\
\hline Si-29 & 253 & 0 & $5.15831 \mathrm{E}-06$ & 293.6 \\
\hline Si-30 & 253 & $\odot$ & $3.42415 \mathrm{E}-\odot 6$ & 293.6 \\
\hline Ti-46 & 253 & $\odot$ & $4.20263 E-07$ & 293.6 \\
\hline $\mathrm{Ti}-47$ & 253 & $\odot$ & 3. $79001 E-07$ & 293.6 \\
\hline $\mathrm{Ti}-48$ & 253 & $\odot$ & $3.75537 \mathrm{E}-06$ & 293.6 \\
\hline Ti-49 & 253 & $\odot$ & $2.7559 \odot E-\odot 7$ & 293.6 \\
\hline Ti-50 & 253 & $\odot$ & $2.63873 E-07$ & 293.6 \\
\hline $\mathrm{Cr}-50$ & 253 & 0 & $5.30518 \mathrm{E}-07$ & 293.6 \\
\hline $\mathrm{Cr}-52$ & 253 & 0 & 1. $02189 E-05$ & 293.6 \\
\hline $\mathrm{Cr}-53$ & 253 & 0 & $1.15860 \mathrm{E}-06$ & 293.6 \\
\hline $\mathrm{Cr}-54$ & 253 & $\odot$ & $2.87821 \mathrm{E}-07$ & 293.6 \\
\hline$M n-55$ & 253 & $\odot$ & $6.53464 \mathrm{E}-06$ & 293.6 \\
\hline $\mathrm{Fe}-54$ & 253 & $\odot$ & $2.39803 E-\odot 6$ & 293.6 \\
\hline $\mathrm{Fe}-56$ & 253 & $\odot$ & 3. $76103 E-05$ & 293.6 \\
\hline $\mathrm{Fe}-57$ & 253 & $\odot$ & $8.69031 E-07$ & 293.6 \\
\hline $\mathrm{Fe}-58$ & 253 & $\odot$ & $1.14778 \mathrm{E}-07$ & 293.6 \\
\hline $\mathrm{Cu}-63$ & 253 & $\odot$ & 1. $90782 \mathrm{E}-05$ & 293.6 \\
\hline $\mathrm{Cu}-65$ & 253 & $\odot$ & $8.5 \odot 342 \mathrm{E}-\odot 6$ & 293.6 \\
\hline$U-234$ & 253 & $\odot$ & $3.25215 E-\odot 6$ & 293.6 \\
\hline$U-235$ & 253 & $\odot$ & 3. $0310 \odot E-\odot 4$ & 293.6 \\
\hline$U-236$ & 253 & $\odot$ & 1. $30087 \mathrm{E}-06$ & 293.6 \\
\hline$U-238$ & 253 & $\odot$ & 1. $75616 \mathrm{E}-05$ & 293.6 \\
\hline
\end{tabular}

, total atom density $=8.00937 \mathrm{E}-02 \mathrm{a} / \mathrm{b}-\mathrm{cm}$

\begin{tabular}{|c|c|c|c|c|c|}
\hline \multicolumn{6}{|c|}{ 8. ๑๑937๑E - ๑2 } \\
\hline $\mathrm{H}-1$ & 254 & $\odot$ & 3. 32434E- 02 & 293.6 & end \\
\hline$B-10$ & 254 & 0 & $1.03798 \mathrm{E}-05$ & 293.6 & end \\
\hline B-11 & 254 & 0 & $4.20486 E-05$ & 293.6 & end \\
\hline $0-16$ & 254 & 0 & 1. $76644 \mathrm{E}-02$ & 293.6 & end \\
\hline$M g-24$ & 254 & 0 & $1.05686 \mathrm{E}-04$ & 293.6 & id \\
\hline$M g-25$ & 254 & 0 & 1.33797E- 05 & 293.6 & id \\
\hline$M g-26$ & 254 & $\odot$ & $1.47310 \mathrm{E}-05$ & 293.6 & \\
\hline $\mathrm{Al}-27$ & 254 & $\odot$ & $2.83940 E-02$ & 293.6 & \\
\hline Si-28 & 254 & 0 & 1. $01293 E-\odot 4$ & 293.6 & \\
\hline Si-29 & 254 & $\odot$ & $5.12887 E-06$ & 293.6 & \\
\hline Si-30 & 254 & 0 & $3.40461 E-06$ & 293.6 & \\
\hline Ti-46 & 254 & $\odot$ & $4.2 \odot 262 \mathrm{E}-\odot 7$ & 293.6 & \\
\hline Ti-47 & 254 & 0 & $3.790 \odot \odot E-\odot 7$ & 293.6 & \\
\hline Ti-48 & 254 & $\odot$ & $3.75536 \mathrm{E}-\odot 6$ & 293.6 & \\
\hline Ti-49 & 254 & $\odot$ & $2.75590 \mathrm{E}-\odot 7$ & 293.6 & \\
\hline Ti-50 & 254 & $\odot$ & $2.63873 E-07$ & 293.6 & \\
\hline $\mathrm{Cr}-50$ & 254 & 0 & $5.30517 \mathrm{E}-07$ & 293.6 & \\
\hline $\mathrm{Cr}-52$ & 254 & 0 & 1. $02189 \mathrm{E}-05$ & 293.6 & \\
\hline
\end{tabular}




$\begin{array}{llllll}\mathrm{Cr}-53 & 254 & \odot & 1.15860 \mathrm{E}-\odot 6 & 293.6 & \text { end } \\ \mathrm{Cr}-54 & 254 & \odot & 2.87821 \mathrm{E}-\odot 7 & 293.6 & \text { end } \\ \mathrm{Mn}-55 & 254 & \odot & 6.50246 \mathrm{E}-\odot 6 & 293.6 & \text { end } \\ \mathrm{Fe}-54 & 254 & \odot & 2.37952 \mathrm{E}-\odot 6 & 293.6 & \text { end } \\ \mathrm{Fe}-56 & 254 & \odot & 3.73198 \mathrm{E}-\odot 5 & 293.6 & \text { end } \\ \mathrm{Fe}-57 & 254 & \odot & 8.62321 \mathrm{E}-\odot 7 & 293.6 & \text { end } \\ \mathrm{Fe}-58 & 254 & \odot & 1.13891 \mathrm{E}-\odot 7 & 293.6 & \text { end } \\ \mathrm{Cu}-63 & 254 & \odot & 1.89710 \mathrm{E}-\odot 5 & 293.6 & \text { end } \\ \mathrm{Cu}-65 & 254 & \odot & 8.45562 \mathrm{E}-\odot 6 & 293.6 & \text { end } \\ \mathrm{U}-234 & 254 & \odot & 4.03970 \mathrm{E}-\odot 6 & 293.6 & \text { end } \\ \mathrm{U}-235 & 254 & \odot & 3.76500 \mathrm{E}-\odot 4 & 293.6 & \text { end } \\ \mathrm{U}-236 & 254 & \odot & 1.61589 \mathrm{E}-\odot 6 & 293.6 & \text { end } \\ \mathrm{U}-238 & 254 & \odot & 2.18144 \mathrm{E}-\odot 5 & 293.6 & \text { end }\end{array}$

, total atom density $=8.00993 \mathrm{E}-02 \mathrm{a} / \mathrm{b}-\mathrm{cm}$

\begin{tabular}{|c|c|c|c|c|c|}
\hline 8. & & & & & \\
\hline $\mathrm{H}-1$ & 255 & $\odot$ & 3. 32434E- 02 & 293.6 & end \\
\hline$B-10$ & 255 & $\odot$ & $6.18010 \mathrm{E}-06$ & 293.6 & end \\
\hline B-11 & 255 & $\odot$ & $2.50356 \mathrm{E}-\odot 5$ & 293.6 & \\
\hline $0-16$ & 255 & $\odot$ & $1.78689 E-02$ & 293.6 & end \\
\hline$M g-24$ & 255 & $\odot$ & 1. $05686 E-\odot 4$ & 293.6 & end \\
\hline$M g-25$ & 255 & $\odot$ & 1. $33797 \mathrm{E}-\odot 5$ & 293.6 & nd \\
\hline$M g-26$ & 255 & $\odot$ & $1.47310 \mathrm{E}-05$ & 293.6 & nd \\
\hline $\mathrm{Al}-27$ & 255 & $\odot$ & $2.81406 \mathrm{E}-\odot 2$ & 293.6 & end \\
\hline Si-28 & 255 & 0 & 1. $00727 \mathrm{E}-\odot 4$ & 293.6 & end \\
\hline Si-29 & 255 & $\odot$ & $5.10026 \mathrm{E}-06$ & 293.6 & end \\
\hline $\mathrm{Si}-30$ & 255 & 0 & $3.38560 \mathrm{E}-06$ & 293.6 & end \\
\hline $\mathrm{Ti}-46$ & 255 & $\odot$ & $4.20262 E-07$ & 293.6 & end \\
\hline $\mathrm{Ti}-47$ & 255 & $\odot$ & $3.79000 \mathrm{E}-\odot 7$ & 293.6 & \\
\hline $\mathrm{Ti}-48$ & 255 & $\odot$ & $3.75536 E-06$ & 293.6 & \\
\hline $\mathrm{Ti}-49$ & 255 & $\odot$ & $2.7559 \odot E-\odot 7$ & 293.6 & \\
\hline $\mathrm{Ti}-50$ & 255 & 0 & $2.63873 E-07$ & 293.6 & end \\
\hline $\mathrm{Cr}-50$ & 255 & 0 & $5.30518 E-07$ & 293.6 & \\
\hline $\mathrm{Cr}-52$ & 255 & 0 & 1. $02189 \mathrm{E}-05$ & 293.6 & \\
\hline $\mathrm{Cr}-53$ & 255 & $\odot$ & $1.15860 E-\odot 6$ & 293.6 & end \\
\hline $\mathrm{Cr}-54$ & 255 & $\odot$ & $2.87821 E-\odot 7$ & 293.6 & \\
\hline$M n-55$ & 255 & 0 & $6.47114 \mathrm{E}-06$ & 293.6 & \\
\hline $\mathrm{Fe}-54$ & 255 & 0 & $2.36149 E-06$ & 293.6 & \\
\hline $\mathrm{Fe}-56$ & 255 & 0 & $3.70370 \mathrm{E}-05$ & 293.6 & \\
\hline $\mathrm{Fe}-57$ & 255 & $\odot$ & $8.55788 E-07$ & 293.6 & \\
\hline $\mathrm{Fe}-58$ & 255 & 0 & $1.13028 \mathrm{E}-07$ & 293.6 & \\
\hline $\mathrm{Cu}-63$ & 255 & $\odot$ & 1. $88666 \mathrm{E}-\odot 5$ & 293.6 & \\
\hline Cu- 65 & 255 & $\odot$ & $8.40911 \mathrm{E}-06$ & 293.6 & end \\
\hline U-234 & 255 & $\odot$ & 4. $80687 E-06$ & 293.6 & end \\
\hline$U-235$ & 255 & $\odot$ & 4. $48000 E-\odot 4$ & 293.6 & end \\
\hline$U-236$ & 255 & 0 & 1. $92276 \mathrm{E}-06$ & 293.6 & end \\
\hline$U-238$ & 255 & 0 & $2.59571 E-05$ & 293.6 & end \\
\hline
\end{tabular}

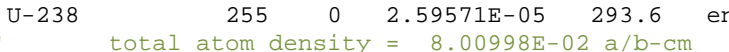

\begin{tabular}{|c|c|c|c|c|}
\hline $\mathrm{H}-1$ & 256 & $\odot$ & 3. 32434E- 02 & 293.6 \\
\hline$B-10$ & 256 & $\odot$ & $5.79830 \mathrm{E}-\odot 6$ & 293.6 \\
\hline B-11 & 256 & $\odot$ & 2. 34889E- 05 & 293.6 \\
\hline $0-16$ & 256 & 0 & 1. $78875 \mathrm{E}-02$ & 293.6 \\
\hline$M g-24$ & 256 & $\odot$ & 1. $05686 E-\odot 4$ & 293.6 \\
\hline $\mathrm{Mg}-25$ & 256 & 0 & $1.33797 \mathrm{E}-05$ & 293.6 \\
\hline $\mathrm{Mg}-26$ & 256 & $\odot$ & 1. $47310 \mathrm{E}-05$ & 293.6 \\
\hline $\mathrm{Al}-27$ & 256 & $\odot$ & $2.81176 \mathrm{E}-\odot 2$ & 293.6 \\
\hline Si-28 & 256 & $\odot$ & 1. $00676 E-\odot 4$ & 293.6 \\
\hline Si-29 & 256 & $\odot$ & $5.09768 \mathrm{E}-\odot 6$ & 293.6 \\
\hline Si-30 & 256 & $\odot$ & $3.38390 E-\odot 6$ & 293.6 \\
\hline Ti-46 & 256 & $\odot$ & $4.20262 E-07$ & 293.6 \\
\hline Ti-47 & 256 & $\odot$ & 3. $7900 \odot E-\odot 7$ & 293.6 \\
\hline Ti-48 & 256 & $\odot$ & $3.75536 \mathrm{E}-06$ & 293.6 \\
\hline Ti-49 & 256 & $\odot$ & $2.7559 \odot E-\odot 7$ & 293.6 \\
\hline Ti-50 & 256 & $\odot$ & $2.63873 E-07$ & 293.6 \\
\hline $\mathrm{Cr}-50$ & 256 & $\odot$ & $5.30517 \mathrm{E}-07$ & 293.6 \\
\hline $\mathrm{Cr}-52$ & 256 & $\odot$ & 1. $02189 E-05$ & 293.6 \\
\hline $\mathrm{Cr}-53$ & 256 & $\odot$ & $1.15860 E-06$ & 293.6 \\
\hline $\mathrm{Cr}-54$ & 256 & $\odot$ & $2.87821 E-07$ & 293.6 \\
\hline$M n-55$ & 256 & $\odot$ & $6.46828 E-06$ & 293.6 \\
\hline $\mathrm{Fe}-54$ & 256 & $\odot$ & $2.35985 E-\odot 6$ & 293.6 \\
\hline $\mathrm{Fe}-56$ & 256 & $\odot$ & 3. 70113E- 05 & 293.6 \\
\hline $\mathrm{Fe}-57$ & 256 & $\odot$ & $8.55193 E-07$ & 293.6 \\
\hline $\mathrm{Fe}-58$ & 256 & 0 & $1.12950 \mathrm{E}-07$ & 293.6 \\
\hline $\mathrm{Cu}-63$ & 256 & $\odot$ & 1. $88571 \mathrm{E}-05$ & 293.6 \\
\hline $\mathrm{Cu}-65$ & 256 & $\odot$ & $8.40487 E-\odot 6$ & 293.6 \\
\hline$U-234$ & 256 & $\odot$ & 4. 87661E-๑6 & 293.6 \\
\hline U-235 & 256 & 0 & $4.54500 E-\odot 4$ & 293.6 \\
\hline$U-236$ & 256 & $\odot$ & $1.95066 \mathrm{E}-06$ & 293.6 \\
\hline U-238 & 256 & $\odot$ & $2.63337 \mathrm{E}-05$ & 293.6 \\
\hline
\end{tabular}

total atom density $=8.00968 \mathrm{E}-02 \mathrm{a} / \mathrm{b}-\mathrm{cm}$

8. $009680 \mathrm{E}-02$ 


\begin{tabular}{|c|c|c|c|c|c|}
\hline $\mathrm{H}-1$ & 257 & $\odot$ & $3.32434 \mathrm{E}-02$ & 293.6 & end \\
\hline$B-10$ & 257 & $\odot$ & $7.99507 E-\odot 6$ & 293.6 & id \\
\hline$B-11$ & 257 & $\odot$ & $3.23880 E-05$ & 293.6 & nd \\
\hline $0-16$ & 257 & 0 & 1. 77805E- 02 & 293.6 & \\
\hline$M g-24$ & 257 & $\odot$ & 1. $05686 E-\odot 4$ & 293.6 & end \\
\hline$M g-25$ & 257 & $\odot$ & $1.33797 E-05$ & 293.6 & \\
\hline$M g-26$ & 257 & $\odot$ & $1.47310 \mathrm{E}-05$ & 293.6 & \\
\hline Al-27 & 257 & $\odot$ & $2.82501 E-02$ & 293.6 & \\
\hline $\mathrm{Si}-28$ & 257 & $\odot$ & 1. $\odot \odot 972 E-\odot 4$ & 293.6 & \\
\hline Si-29 & 257 & $\odot$ & $5.11262 E-06$ & 293.6 & \\
\hline $\mathrm{Si}-30$ & 257 & $\odot$ & $3.39382 E-06$ & 293.6 & \\
\hline $\mathrm{Ti}-46$ & 257 & $\odot$ & $4.20262 E-07$ & 293.6 & \\
\hline $\mathrm{Ti}-47$ & 257 & $\odot$ & $3.79000 \mathrm{E}-07$ & 293.6 & \\
\hline $\mathrm{Ti}-48$ & 257 & $\odot$ & $3.75536 \mathrm{E}-06$ & 293.6 & \\
\hline $\mathrm{Ti}-49$ & 257 & $\odot$ & $2.75590 \mathrm{E}-\odot 7$ & 293.6 & \\
\hline Ti-50 & 257 & $\odot$ & $2.63873 E-07$ & 293.6 & \\
\hline $\mathrm{Cr}-50$ & 257 & $\odot$ & $5.30517 \mathrm{E}-07$ & 293.6 & \\
\hline $\mathrm{Cr}-52$ & 257 & 0 & 1. $02189 \mathrm{E}-05$ & 293.6 & \\
\hline $\mathrm{Cr}-53$ & 257 & 0 & $1.15860 \mathrm{E}-06$ & 293.6 & \\
\hline $\mathrm{Cr}-54$ & 257 & $\odot$ & $2.87821 E-07$ & 293.6 & \\
\hline$M n-55$ & 257 & $\odot$ & $6.48467 E-06$ & 293.6 & \\
\hline $\mathrm{Fe}-54$ & 257 & $\odot$ & $2.36928 E-06$ & 293.6 & \\
\hline $\mathrm{Fe}-56$ & 257 & $\odot$ & $3.71592 \mathrm{E}-05$ & 293.6 & \\
\hline $\mathrm{Fe}-57$ & 257 & $\odot$ & $8.58611 E-07$ & 293.6 & \\
\hline $\mathrm{Fe}-58$ & 257 & $\odot$ & $1.13401 \mathrm{E}-07$ & 293.6 & \\
\hline $\mathrm{Cu}-63$ & 257 & 0 & 1. $89118 \mathrm{E}-05$ & 293.6 & \\
\hline $\mathrm{Cu}-65$ & 257 & 0 & 8. 42923E- 06 & 293.6 & \\
\hline$U-234$ & 257 & $\odot$ & $4.47532 E-06$ & 293.6 & \\
\hline$U-235$ & 257 & 0 & $4.1710 \odot \mathrm{E}-\odot 4$ & 293.6 & \\
\hline$U-236$ & 257 & $\odot$ & 1. 79014E-๑6 & 293.6 & \\
\hline$U-238$ & 257 & 0 & $2.41667 \mathrm{E}-05$ & 293.6 & \\
\hline \multirow{2}{*}{\multicolumn{3}{|c|}{$\begin{array}{l}\text { total atom density } \\
8.009330 \mathrm{E}-02\end{array}$}} & \multicolumn{2}{|c|}{$=8.00933 \mathrm{E}-02 \mathrm{a} / \mathrm{b}-\mathrm{cm}$} & \\
\hline & & & & & \\
\hline $\mathrm{H}-1$ & 258 & $\odot$ & $3.32434 E-02$ & 293.6 & \\
\hline$B-10$ & 258 & 0 & 1. $06559 E-\odot 5$ & 293.6 & \\
\hline$B-11$ & 258 & $\odot$ & $4.31670 E-\odot 5$ & 293.6 & \\
\hline $0-16$ & 258 & $\odot$ & $1.76509 \mathrm{E}-\odot 2$ & 293.6 & \\
\hline$M g-24$ & 258 & $\odot$ & $1.05686 \mathrm{E}-\odot 4$ & 293.6 & \\
\hline$M g-25$ & 258 & $\odot$ & $1.33797 E-05$ & 293.6 & \\
\hline$M g-26$ & 258 & 0 & 1. $47310 \mathrm{E}-05$ & 293.6 & \\
\hline Al-27 & 258 & 0 & $2.84107 \mathrm{E}-02$ & 293.6 & \\
\hline $\mathrm{Si}-28$ & 258 & 0 & 1. $01330 \mathrm{E}-04$ & 293.6 & \\
\hline Si-29 & 258 & 0 & $5.13079 E-06$ & 293.6 & \\
\hline $\mathrm{Si}-30$ & 258 & 0 & $3.40588 E-06$ & 293.6 & \\
\hline Ti-46 & 258 & $\odot$ & $4.20262 E-07$ & 293.6 & \\
\hline $\mathrm{Ti}-47$ & 258 & $\odot$ & $3.790 \odot \odot E-\odot 7$ & 293.6 & \\
\hline $\mathrm{Ti}-48$ & 258 & 0 & $3.75536 \mathrm{E}-06$ & 293.6 & \\
\hline Ti-49 & 258 & 0 & $2.75590 \mathrm{E}-\odot 7$ & 293.6 & \\
\hline Ti-50 & 258 & 0 & $2.63873 E-07$ & 293.6 & \\
\hline $\mathrm{Cr}-50$ & 258 & $\odot$ & $5.30517 E-\odot 7$ & 293.6 & \\
\hline $\mathrm{Cr}-52$ & 258 & $\odot$ & $1.02189 E-05$ & 293.6 & \\
\hline $\mathrm{Cr}-53$ & 258 & $\odot$ & $1.15860 E-06$ & 293.6 & \\
\hline $\mathrm{Cr}-54$ & 258 & 0 & $2.87821 E-07$ & 293.6 & \\
\hline$M n-55$ & 258 & $\odot$ & $6.5 \odot 452 E-\odot 6$ & 293.6 & \\
\hline $\mathrm{Fe}-54$ & 258 & $\odot$ & $2.38070 E-06$ & 293.6 & \\
\hline $\mathrm{Fe}-56$ & 258 & $\odot$ & $3.73384 \mathrm{E}-05$ & 293.6 & \\
\hline $\mathrm{Fe}-57$ & 258 & $\odot$ & $8.62751 E-07$ & 293.6 & \\
\hline $\mathrm{Fe}-58$ & 258 & $\odot$ & $1.13948 E-07$ & 293.6 & \\
\hline $\mathrm{Cu}-63$ & 258 & $\odot$ & $1.89779 E-05$ & 293.6 & \\
\hline $\mathrm{Cu}-65$ & 258 & $\odot$ & $8.45870 \mathrm{E}-06$ & 293.6 & \\
\hline$U-234$ & 258 & $\odot$ & $3.98927 \mathrm{E}-06$ & 293.6 & \\
\hline U-235 & 258 & 0 & $3.71800 \mathrm{E}-\odot 4$ & 293.6 & \\
\hline$U-236$ & 258 & $\odot$ & $1.59572 \mathrm{E}-06$ & 293.6 & \\
\hline$U-238$ & 258 & $\odot$ & $2.15420 \mathrm{E}-05$ & 293.6 & \\
\hline
\end{tabular}

Inner fuel element--fueled Axial region 6 total atom density $=8.00804 \mathrm{E}-02 \mathrm{a} / \mathrm{b}-\mathrm{cm}$ 8. $008040 \mathrm{E}-02$

$\begin{array}{llllll}\mathrm{H}-1 & 261 & \odot & 3.32434 \mathrm{E}-02 & 293.6 & \text { end } \\ \mathrm{B}-10 & 261 & \odot & 2.04121 \mathrm{E}-05 & 293.6 & \text { end } \\ \mathrm{B}-11 & 261 & \odot & 8.26896 \mathrm{E}-05 & 293.6 & \text { end } \\ \mathrm{O}-16 & 261 & \odot & 1.71757 \mathrm{E}-02 & 293.6 & \text { end } \\ \mathrm{Mg}-24 & 261 & \odot & 1.05686 \mathrm{E}-04 & 293.6 & \text { end } \\ \mathrm{Mg}-25 & 261 & \odot & 1.33797 \mathrm{E}-05 & 293.6 & \text { end } \\ \mathrm{Mg}-26 & 261 & \odot & 1.47310 \mathrm{E}-05 & 293.6 & \text { end } \\ \mathrm{Al}-27 & 261 & \odot & 2.89993 \mathrm{E}-02 & 293.6 & \text { end } \\ \mathrm{Si}-28 & 261 & \odot & 1.02644 \mathrm{E}-04 & 293.6 & \text { end } \\ \mathrm{Si}-29 & 261 & \odot & 5.19729 \mathrm{E}-06 & 293.6 & \text { end } \\ \mathrm{Si}-30 & 261 & \odot & 3.45002 \mathrm{E}-06 & 293.6 & \text { end } \\ \mathrm{Ti}-46 & 261 & \odot & 4.20262 \mathrm{E}-07 & 293.6 & \text { end } \\ \mathrm{Ti}-47 & 261 & \odot & 3.79000 \mathrm{E}-07 & 293.6 & \text { end }\end{array}$




\begin{tabular}{|c|c|c|c|c|c|}
\hline Ti-48 & 261 & $\odot$ & $3.75536 \mathrm{E}-06$ & 293.6 & end \\
\hline Ti-49 & 261 & $\odot$ & $2.75590 \mathrm{E}-\odot 7$ & 293.6 & end \\
\hline Ti-50 & 261 & $\odot$ & $2.63873 E-07$ & 293.6 & end \\
\hline $\mathrm{Cr}-50$ & 261 & $\odot$ & $5.30517 \mathrm{E}-07$ & 293.6 & end \\
\hline $\mathrm{Cr}-52$ & 261 & $\odot$ & 1. $02189 E-05$ & 293.6 & end \\
\hline $\mathrm{Cr}-53$ & 261 & $\odot$ & $1.15860 \mathrm{E}-06$ & 293.6 & end \\
\hline $\mathrm{Cr}-54$ & 261 & $\odot$ & $2.87821 E-07$ & 293.6 & end \\
\hline Mn-55 & 261 & $\odot$ & $6.57731 E-06$ & 293.6 & end \\
\hline $\mathrm{Fe}-54$ & 261 & $\odot$ & $2.42259 E-06$ & 293.6 & and \\
\hline $\mathrm{Fe}-56$ & 261 & $\odot$ & $3.79953 E-05$ & 293.6 & nd \\
\hline $\mathrm{Fe}-57$ & 261 & 0 & $8.77930 \mathrm{E}-07$ & 293.6 & nd \\
\hline $\mathrm{Fe}-58$ & 261 & $\odot$ & $1.15953 E-07$ & 293.6 & nd \\
\hline $\mathrm{Cu}-63$ & 261 & $\odot$ & 1. $92203 E-05$ & 293.6 & nd \\
\hline $\mathrm{Cu}-65$ & 261 & $\odot$ & $8.56676 E-06$ & 293.6 & nd \\
\hline U-234 & 261 & $\odot$ & $2.20708 \mathrm{E}-06$ & 293.6 & \\
\hline$U-235$ & 261 & $\odot$ & $2.05700 E-\odot 4$ & 293.6 & nd \\
\hline$U-236$ & 261 & $\odot$ & 8. 82838E- - 7 & 293.6 & nd \\
\hline$U-238$ & 261 & $\odot$ & \multirow{2}{*}{\multicolumn{2}{|c|}{$\begin{array}{l}1.19182 \mathrm{E}-05 \quad 293.6 \\
=\quad 8.00839 \mathrm{E}-02 \mathrm{a} / \mathrm{b}-\mathrm{cm}\end{array}$}} & end \\
\hline \multicolumn{3}{|c|}{ total atom density } & & & \\
\hline $\mathrm{H}-1$ & 262 & $\odot$ & 3. 32434E- 02 & 293.6 & end \\
\hline$B-10$ & 262 & $\odot$ & 1. $77513 E-05$ & 293.6 & \\
\hline B-11 & 262 & 0 & $7.19107 \mathrm{E}-05$ & 293.6 & id \\
\hline $0-16$ & 262 & $\odot$ & $1.73053 E-02$ & 293.6 & nd \\
\hline$M g-24$ & 262 & $\odot$ & 1. $05686 E-04$ & 293.6 & end \\
\hline$M g-25$ & 262 & $\odot$ & $1.33797 \mathrm{E}-05$ & 293.6 & nd \\
\hline$M g-26$ & 262 & $\odot$ & $1.47310 \mathrm{E}-05$ & 293.6 & nd \\
\hline Al-27 & 262 & $\odot$ & $2.88388 \mathrm{E}-\odot 2$ & 293.6 & nd \\
\hline Si-28 & 262 & 0 & $1.02285 E-\odot 4$ & 293.6 & nd \\
\hline Si-29 & 262 & $\odot$ & $5.17912 \mathrm{E}-06$ & 293.6 & nd \\
\hline Si-30 & 262 & $\odot$ & $3.43796 \mathrm{E}-06$ & 293.6 & nd \\
\hline $\mathrm{Ti}-46$ & 262 & $\odot$ & 4. $20262 E-07$ & 293.6 & nd \\
\hline Ti-47 & 262 & $\odot$ & $3.79000 \mathrm{E}-\odot 7$ & 293.6 & nd \\
\hline Ti-48 & 262 & $\odot$ & $3.75536 \mathrm{E}-06$ & 293.6 & nd \\
\hline Ti-49 & 262 & $\odot$ & $2.75590 \mathrm{E}-07$ & 293.6 & nd \\
\hline Ti-50 & 262 & $\odot$ & $2.63873 E-07$ & 293.6 & nd \\
\hline $\mathrm{Cr}-50$ & 262 & $\odot$ & $5.30517 E-07$ & 293.6 & \\
\hline $\mathrm{Cr}-52$ & 262 & $\odot$ & 1. $\odot 2189 E-\odot 5$ & 293.6 & nd \\
\hline $\mathrm{Cr}-53$ & 262 & $\odot$ & $1.15860 \mathrm{E}-\odot 6$ & 293.6 & \\
\hline $\mathrm{Cr}-54$ & 262 & $\odot$ & $2.87821 E-07$ & 293.6 & nd \\
\hline$M n-55$ & 262 & 0 & $6.55746 E-06$ & 293.6 & \\
\hline $\mathrm{Fe}-54$ & 262 & 0 & $2.41117 \mathrm{E}-06$ & 293.6 & \\
\hline $\mathrm{Fe}-56$ & 262 & 0 & $3.78162 \mathrm{E}-05$ & 293.6 & nd \\
\hline $\mathrm{Fe}-57$ & 262 & 0 & $8.73792 \mathrm{E}-07$ & 293.6 & 110 \\
\hline $\mathrm{Fe}-58$ & 262 & 0 & $1.15406 \mathrm{E}-07$ & 293.6 & nd \\
\hline $\mathrm{Cu}-63$ & 262 & 0 & 1. $91542 \mathrm{E}-05$ & 293.6 & nd \\
\hline $\mathrm{Cu}-65$ & 262 & $\odot$ & $8.53729 E-06$ & 293.6 & a \\
\hline U-234 & 262 & $\odot$ & 2. 69313E- - 6 & 293.6 & \\
\hline$U-235$ & 262 & $\odot$ & $2.51000 \mathrm{E}-\odot 4$ & 293.6 & \\
\hline $\mathrm{U}-236$ & 262 & $\odot$ & 1. $07726 E-\odot 6$ & 293.6 & \\
\hline$U-238$ & 262 & 0 & 1. 45429E- 05 & 293.6 & \\
\hline 1 & tom & sity & $=8.00880 \mathrm{E}$. & $2 \mathrm{a} / \mathrm{b}-\mathrm{cm}$ & \\
\hline \multicolumn{6}{|c|}{ ' $\quad 8.0 \odot 88 \odot \odot E-\odot 2$} \\
\hline $\mathrm{H}-1$ & 263 & $\odot$ & $3.32435 E-02$ & 293.6 & $d$ \\
\hline$B-10$ & 263 & 0 & $1.46911 \mathrm{E}-05$ & 293.6 & \\
\hline$B-11$ & 263 & 0 & 5. $95139 E-05$ & 293.6 & nd \\
\hline $0-16$ & 263 & 0 & $1.74543 E-02$ & 293.6 & nd \\
\hline$M g-24$ & 263 & 0 & 1. $05686 E-\odot 4$ & 293.6 & \\
\hline$M g-25$ & 263 & 0 & $1.33797 \mathrm{E}-05$ & 293.6 & 10 \\
\hline$M g-26$ & 263 & 0 & $1.47310 \mathrm{E}-05$ & 293.6 & nd \\
\hline Al-27 & 263 & 0 & $2.86541 E-02$ & 293.6 & d \\
\hline Si-28 & 263 & 0 & 1. $01874 \mathrm{E}-04$ & 293.6 & d \\
\hline Si-29 & 263 & 0 & $5.15831 E-06$ & 293.6 & nd \\
\hline $\mathrm{Si}-30$ & 263 & 0 & $3.42415 \mathrm{E}-06$ & 293.6 & \\
\hline Ti-46 & 263 & 0 & $4.20263 E-07$ & 293.6 & \\
\hline Ti-47 & 263 & 0 & $3.79001 \mathrm{E}-07$ & 293.6 & \\
\hline Ti-48 & 263 & 0 & $3.75537 \mathrm{E}-06$ & 293.6 & \\
\hline Ti-49 & 263 & 0 & $2.75590 E-\odot 7$ & 293.6 & 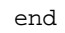 \\
\hline Ti-50 & 263 & 0 & $2.63873 E-07$ & 293.6 & \\
\hline $\mathrm{Cr}-50$ & 263 & 0 & $5.30518 E-07$ & 293.6 & \\
\hline $\mathrm{Cr}-52$ & 263 & 0 & 1. $02189 E-05$ & 293.6 & \\
\hline $\mathrm{Cr}-53$ & 263 & 0 & $1.15860 E-06$ & 293.6 & 10 \\
\hline $\mathrm{Cr}-54$ & 263 & 0 & $2.87821 E-07$ & 293.6 & \\
\hline$M n-55$ & 263 & 0 & $6.53464 E-06$ & 293.6 & \\
\hline $\mathrm{Fe}-54$ & 263 & 0 & $2.39803 E-06$ & 293.6 & \\
\hline $\mathrm{Fe}-56$ & 263 & 0 & $3.76103 E-05$ & 293.6 & \\
\hline $\mathrm{Fe}-57$ & 263 & 0 & 8.69031E- 07 & 293.6 & \\
\hline $\mathrm{Fe}-58$ & 263 & 0 & $1.14778 E-07$ & 293.6 & \\
\hline $\mathrm{Cu}-63$ & 263 & 0 & 1. $90782 \mathrm{E}-05$ & 293.6 & \\
\hline $\mathrm{Cu}-65$ & 263 & 0 & $8.50342 E-06$ & 293.6 & \\
\hline U-234 & 263 & 0 & $3.25215 \mathrm{E}-06$ & 293.6 & \\
\hline
\end{tabular}




\begin{tabular}{|c|c|c|c|c|c|}
\hline$U-235$ & 263 & $\odot$ & $3.03100 \mathrm{E}-\odot 4$ & 293.6 & end \\
\hline$U-236$ & 263 & $\odot$ & 1. $30087 E-06$ & 293.6 & \\
\hline$U-238$ & 263 & $\odot$ & 1. $75616 \mathrm{E}-05$ & 293.6 & \\
\hline \multicolumn{3}{|c|}{$\begin{array}{l}\text { total atom density } \\
\text { 8. } 009370 \mathrm{E}-\mathrm{-} 2\end{array}$} & \multicolumn{2}{|c|}{$=8.00937 \mathrm{E}-02 \mathrm{a} / \mathrm{b}-\mathrm{cm}$} & \\
\hline $\mathrm{H}-1$ & 264 & $\odot$ & $3.32434 \mathrm{E}-02$ & 293.6 & CI \\
\hline$B-10$ & 264 & $\odot$ & $1.03798 E-05$ & 293.6 & \\
\hline$B-11$ & 264 & $\odot$ & $4.20486 E-05$ & 293.6 & \\
\hline $0-16$ & 264 & $\odot$ & $1.76644 \mathrm{E}-02$ & 293.6 & \\
\hline$M g-24$ & 264 & $\odot$ & $1.05686 E-\odot 4$ & 293.6 & \\
\hline $\mathrm{Mg}-25$ & 264 & $\odot$ & 1.33797E- 05 & 293.6 & \\
\hline $\mathrm{Mg}-26$ & 264 & $\odot$ & $1.47310 \mathrm{E}-05$ & 293.6 & \\
\hline Al-27 & 264 & $\odot$ & $2.83940 E-\odot 2$ & 293.6 & \\
\hline Si-28 & 264 & $\odot$ & 1. $01293 E-\odot 4$ & 293.6 & \\
\hline Si-29 & 264 & $\odot$ & $5.12887 E-06$ & 293.6 & \\
\hline Si-30 & 264 & $\odot$ & $3.4 \odot 461 \mathrm{E}-06$ & 293.6 & \\
\hline $\mathrm{Ti}-46$ & 264 & $\odot$ & $4.2 \odot 262 E-07$ & 293.6 & \\
\hline Ti-47 & 264 & $\odot$ & $3.7900 \odot E-\odot 7$ & 293.6 & \\
\hline Ti-48 & 264 & 0 & $3.75536 \mathrm{E}-06$ & 293.6 & \\
\hline Ti-49 & 264 & 0 & $2.75590 \mathrm{E}-07$ & 293.6 & \\
\hline Ti-50 & 264 & 0 & $2.63873 E-07$ & 293.6 & \\
\hline $\mathrm{Cr}-50$ & 264 & $\odot$ & $5.30517 \mathrm{E}-07$ & 293.6 & \\
\hline $\mathrm{Cr}-52$ & 264 & $\odot$ & 1. $02189 E-05$ & 293.6 & \\
\hline $\mathrm{Cr}-53$ & 264 & $\odot$ & $1.15860 E-06$ & 293.6 & \\
\hline $\mathrm{Cr}-54$ & 264 & $\odot$ & $2.87821 E-07$ & 293.6 & \\
\hline$M n-55$ & 264 & $\odot$ & $6.50246 \mathrm{E}-06$ & 293.6 & \\
\hline $\mathrm{Fe}-54$ & 264 & $\odot$ & $2.37952 E-06$ & 293.6 & \\
\hline $\mathrm{Fe}-56$ & 264 & $\odot$ & $3.73198 \mathrm{E}-05$ & 293.6 & \\
\hline $\mathrm{Fe}-57$ & 264 & $\odot$ & $8.62321 E-07$ & 293.6 & \\
\hline $\mathrm{Fe}-58$ & 264 & $\odot$ & $1.13891 E-07$ & 293.6 & \\
\hline $\mathrm{Cu}-63$ & 264 & 0 & 1.89710E-05 & 293.6 & \\
\hline $\mathrm{Cu}-65$ & 264 & $\odot$ & $8.45562 E-06$ & 293.6 & \\
\hline$U-234$ & 264 & $\odot$ & $4.03970 E-\odot 6$ & 293.6 & \\
\hline$U-235$ & 264 & $\odot$ & $3.7650 \odot E-\odot 4$ & 293.6 & \\
\hline$U-236$ & 264 & $\odot$ & $1.61589 \mathrm{E}-06$ & 293.6 & \\
\hline$U-238$ & 264 & $\odot$ & $2.18144 \mathrm{E}-05$ & 293.6 & \\
\hline \multirow{2}{*}{\multicolumn{3}{|c|}{$\begin{array}{l}\text { total atom density } \\
\text { ( } 8.009930 \mathrm{E}-02\end{array}$}} & \multirow{2}{*}{\multicolumn{3}{|c|}{$=8.00993 \mathrm{E}-02 \mathrm{a} / \mathrm{b}-\mathrm{cm}$}} \\
\hline ' $\quad 8 . \odot \odot 9930 E-\odot 2$ & & & & & \\
\hline $\mathrm{H}-1$ & 265 & $\odot$ & $3.32434 \mathrm{E}-02$ & 293.6 & \\
\hline$B-10$ & 265 & $\odot$ & $6.18010 E-06$ & 293.6 & \\
\hline B-11 & 265 & 0 & $2.50356 \mathrm{E}-05$ & 293.6 & \\
\hline $0-16$ & 265 & 0 & 1. 78689E-02 & 293.6 & \\
\hline $\mathrm{Mg}-24$ & 265 & $\odot$ & $1.05686 E-\odot 4$ & 293.6 & \\
\hline $\mathrm{Mg}-25$ & 265 & $\odot$ & 1.33797E- 05 & 293.6 & \\
\hline$M g-26$ & 265 & $\odot$ & $1.47310 \mathrm{E}-05$ & 293.6 & \\
\hline Al-27 & 265 & $\odot$ & $2.81406 \mathrm{E}-02$ & 293.6 & \\
\hline Si-28 & 265 & $\odot$ & $1.00727 E-04$ & 293.6 & \\
\hline Si-29 & 265 & $\odot$ & $5.10026 \mathrm{E}-\odot 6$ & 293.6 & \\
\hline Si-30 & 265 & $\odot$ & $3.38560 E-06$ & 293.6 & \\
\hline $\mathrm{Ti}-46$ & 265 & $\odot$ & $4.20262 E-07$ & 293.6 & \\
\hline Ti-47 & 265 & $\odot$ & $3.79000 \mathrm{E}-07$ & 293.6 & \\
\hline Ti-48 & 265 & $\odot$ & $3.75536 \mathrm{E}-06$ & 293.6 & \\
\hline Ti-49 & 265 & $\odot$ & $2.75590 \mathrm{E}-07$ & 293.6 & \\
\hline Ti-50 & 265 & $\odot$ & $2.63873 E-07$ & 293.6 & \\
\hline $\mathrm{Cr}-50$ & 265 & $\odot$ & $5.30518 \mathrm{E}-07$ & 293.6 & \\
\hline $\mathrm{Cr}-52$ & 265 & $\odot$ & 1. $02189 \mathrm{E}-05$ & 293.6 & \\
\hline $\mathrm{Cr}-53$ & 265 & $\odot$ & $1.15860 E-06$ & 293.6 & \\
\hline $\mathrm{Cr}-54$ & 265 & $\odot$ & $2.87821 E-07$ & 293.6 & \\
\hline$M n-55$ & 265 & $\odot$ & $6.47114 \mathrm{E}-06$ & 293.6 & \\
\hline $\mathrm{Fe}-54$ & 265 & $\odot$ & $2.36149 E-06$ & 293.6 & \\
\hline $\mathrm{Fe}-56$ & 265 & $\odot$ & $3.70370 E-05$ & 293.6 & \\
\hline $\mathrm{Fe}-57$ & 265 & $\odot$ & $8.55788 E-07$ & 293.6 & \\
\hline $\mathrm{Fe}-58$ & 265 & $\odot$ & $1.13028 \mathrm{E}-07$ & 293.6 & \\
\hline Cu-63 & 265 & $\odot$ & $1.88666 \mathrm{E}-05$ & 293.6 & \\
\hline Cu-65 & 265 & $\odot$ & 8. 40911E- 06 & 293.6 & \\
\hline$U-234$ & 265 & $\odot$ & $4.80687 \mathrm{E}-06$ & 293.6 & \\
\hline U-235 & 265 & $\odot$ & $4.48000 \mathrm{E}-\odot 4$ & 293.6 & \\
\hline$U-236$ & 265 & $\odot$ & 1. $92276 \mathrm{E}-\odot 6$ & 293.6 & \\
\hline$U-238$ & 265 & $\odot$ & $2.59571 E-05$ & 293.6 & \\
\hline & tom & sity & $=8.00998 \mathrm{E}$ & $2 a / b-c r$ & \\
\hline ' $\quad 8.00$ & & & & & \\
\hline $\mathrm{H}-1$ & 266 & $\odot$ & $2434 E-\odot 2$ & 293.6 & \\
\hline B-10 & 266 & $\odot$ & $5.7983 \odot E-\odot 6$ & 293.6 & \\
\hline B-11 & 266 & $\odot$ & $2.34889 \mathrm{E}-05$ & 293.6 & \\
\hline $0-16$ & 266 & $\odot$ & 1. 78875E- $\odot 2$ & 293.6 & \\
\hline$M g-24$ & 266 & $\odot$ & $1.05686 \mathrm{E}-\odot 4$ & 293.6 & \\
\hline$M g-25$ & 266 & $\odot$ & $1.33797 \mathrm{E}-05$ & 293.6 & \\
\hline$M g-26$ & 266 & $\odot$ & $1.47310 \mathrm{E}-05$ & 293.6 & \\
\hline Al-27 & 266 & $\odot$ & $2.81176 E-02$ & 293.6 & \\
\hline Si-28 & 266 & $\odot$ & 1. $00676 E-\odot 4$ & 293.6 & \\
\hline Si-29 & 266 & $\odot$ & $5.09768 E-06$ & 293.6 & \\
\hline
\end{tabular}




$\begin{array}{llllll}\mathrm{Si}-3 \odot & 266 & \odot & 3.38390 \mathrm{E}-\odot 6 & 293.6 & \text { end } \\ \mathrm{Ti}-46 & 266 & \odot & 4.20262 \mathrm{E}-\odot 7 & 293.6 & \text { end } \\ \mathrm{Ti}-47 & 266 & \odot & 3.79000 \mathrm{E}-\odot 7 & 293.6 & \text { end } \\ \mathrm{Ti}-48 & 266 & \odot & 3.75536 \mathrm{E}-\odot 6 & 293.6 & \text { end } \\ \mathrm{Ti}-49 & 266 & \odot & 2.75590 \mathrm{E}-\odot 7 & 293.6 & \text { end } \\ \mathrm{Ti}-5 \odot & 266 & \odot & 2.63873 \mathrm{E}-\odot 7 & 293.6 & \text { end } \\ \mathrm{Cr}-5 \odot & 266 & \odot & 5.30517 \mathrm{E}-\odot 7 & 293.6 & \text { end } \\ \mathrm{Cr}-52 & 266 & \odot & 1.02189 \mathrm{E}-05 & 293.6 & \text { end } \\ \mathrm{Cr}-53 & 266 & \odot & 1.15860 \mathrm{E}-\odot 6 & 293.6 & \text { end } \\ \mathrm{Cr}-54 & 266 & \odot & 2.87821 \mathrm{E}-\odot 7 & 293.6 & \text { end } \\ \mathrm{Mn}-55 & 266 & \odot & 6.46828 \mathrm{E}-\odot 6 & 293.6 & \text { end } \\ \mathrm{Fe}-54 & 266 & \odot & 2.35985 \mathrm{E}-\odot 6 & 293.6 & \text { end } \\ \mathrm{Fe}-56 & 266 & \odot & 3.70113 \mathrm{E}-\odot 5 & 293.6 & \text { end } \\ \mathrm{Fe}-57 & 266 & \odot & 8.55193 \mathrm{E}-\odot 7 & 293.6 & \text { end } \\ \mathrm{Fe}-58 & 266 & \odot & 1.12950 \mathrm{E}-\odot 7 & 293.6 & \text { end } \\ \mathrm{Cu}-63 & 266 & \odot & 1.88571 \mathrm{E}-\odot 5 & 293.6 & \text { end } \\ \mathrm{Cu}-65 & 266 & \odot & 8.40487 \mathrm{E}-\odot 6 & 293.6 & \text { end } \\ \mathrm{U}-234 & 266 & \odot & 4.87661 \mathrm{E}-\odot 6 & 293.6 & \text { end } \\ \mathrm{U}-235 & 266 & \odot & 4.54500 \mathrm{E}-\odot 4 & 293.6 & \text { end } \\ \mathrm{U}-236 & 266 & \odot & 1.95066 \mathrm{E}-\odot 6 & 293.6 & \text { end } \\ \mathrm{U}-238 & 266 & \odot & 2.63337 \mathrm{E}-05 & 293.6 & \text { end }\end{array}$

total atom density $=8.00968 \mathrm{E}-02 \mathrm{a} / \mathrm{b}-\mathrm{cm}$

\begin{tabular}{llllll}
\multicolumn{5}{c}{ total atom density } & \multicolumn{3}{c}{$8.00968 \mathrm{E}-02$} & $\mathrm{a} / \mathrm{b}-\mathrm{Cm}$ & \\
$\mathrm{H}-1$ & 267 & $\odot$ & $3.32434 \mathrm{E}-02$ & 293.6 & end \\
$\mathrm{B}-10$ & 267 & $\odot$ & $7.99507 \mathrm{E}-06$ & 293.6 & end \\
$\mathrm{B}-11$ & 267 & $\odot$ & $3.23880 \mathrm{E}-05$ & 293.6 & end \\
$\mathrm{O}-16$ & 267 & $\odot$ & $1.77805 \mathrm{E}-02$ & 293.6 & end \\
$\mathrm{Mg}-24$ & 267 & $\odot$ & $1.05686 \mathrm{E}-04$ & 293.6 & end \\
$\mathrm{Mg}-25$ & 267 & $\odot$ & $1.33797 \mathrm{E}-05$ & 293.6 & end \\
$\mathrm{Mg}-26$ & 267 & $\odot$ & $1.47310 \mathrm{E}-05$ & 293.6 & end \\
$\mathrm{Al}-27$ & 267 & $\odot$ & $2.82501 \mathrm{E}-02$ & 293.6 & end \\
$\mathrm{Si}-28$ & 267 & $\odot$ & $1.00972 \mathrm{E}-04$ & 293.6 & end \\
$\mathrm{Si}-29$ & 267 & $\odot$ & $5.11262 \mathrm{E}-06$ & 293.6 & end \\
$\mathrm{Si}-30$ & 267 & $\odot$ & $3.39382 \mathrm{E}-06$ & 293.6 & end \\
$\mathrm{Ti}-46$ & 267 & $\odot$ & $4.20262 \mathrm{E}-07$ & 293.6 & end \\
$\mathrm{Ti}-47$ & 267 & $\odot$ & $3.79000 \mathrm{E}-07$ & 293.6 & end \\
$\mathrm{Ti}-48$ & 267 & $\odot$ & $3.75536 \mathrm{E}-06$ & 293.6 & end \\
$\mathrm{Ti}-49$ & 267 & $\odot$ & $2.75590 \mathrm{E}-07$ & 293.6 & end \\
$\mathrm{Ti}-50$ & 267 & $\odot$ & $2.63873 \mathrm{E}-07$ & 293.6 & end \\
$\mathrm{Cr}-50$ & 267 & $\odot$ & $5.30517 \mathrm{E}-07$ & 293.6 & end \\
$\mathrm{Cr}-52$ & 267 & $\odot$ & $1.02189 \mathrm{E}-05$ & 293.6 & end \\
$\mathrm{Cr}-53$ & 267 & $\odot$ & $1.15860 \mathrm{E}-06$ & 293.6 & end \\
$\mathrm{Cr}-54$ & 267 & $\odot$ & $2.87821 \mathrm{E}-07$ & 293.6 & end \\
$\mathrm{Mn}-55$ & 267 & $\odot$ & $6.48467 \mathrm{E}-06$ & 293.6 & end \\
$\mathrm{Fe}-54$ & 267 & $\odot$ & $2.36928 \mathrm{E}-06$ & 293.6 & end \\
$\mathrm{Fe}-56$ & 267 & $\odot$ & $3.71592 \mathrm{E}-05$ & 293.6 & end \\
$\mathrm{Fe}-57$ & 267 & $\odot$ & $8.58611 \mathrm{E}-07$ & 293.6 & end \\
$\mathrm{Fe}-58$ & 267 & $\odot$ & $1.13401 \mathrm{E}-07$ & 293.6 & end \\
$\mathrm{Cu}-63$ & 267 & $\odot$ & $1.89118 \mathrm{E}-05$ & 293.6 & end \\
$\mathrm{Cu}-65$ & 267 & $\odot$ & $8.42923 \mathrm{E}-06$ & 293.6 & end \\
$\mathrm{U}-234$ & 267 & $\odot$ & $4.47532 \mathrm{E}-06$ & 293.6 & end \\
$\mathrm{U}-235$ & 267 & $\odot$ & $4.17100 \mathrm{E}-04$ & 293.6 & end \\
$\mathrm{U}-236$ & 267 & $\odot$ & $1.79014 \mathrm{E}-06$ & 293.6 & end \\
$\mathrm{U}-238$ & 267 & $\odot$ & $2.41667 \mathrm{E}-05$ & 293.6 & end
\end{tabular}

total atom density $=4.460$

\begin{tabular}{|c|c|c|c|c|c|}
\hline $\mathrm{H}-1$ & 268 & $\odot$ & 3. 32434E- - 22 & 293.6 & end \\
\hline$B-10$ & 268 & $\odot$ & 1. $06559 E-05$ & 293.6 & end \\
\hline$B-11$ & 268 & $\odot$ & $4.31670 E-05$ & 293.6 & end \\
\hline $0-16$ & 268 & $\odot$ & 1. $76509 E-\odot 2$ & 293.6 & \\
\hline$M g-24$ & 268 & $\odot$ & 1. $05686 E-04$ & 293.6 & \\
\hline$M g-25$ & 268 & $\odot$ & $1.33797 E-05$ & 293.6 & \\
\hline$M g-26$ & 268 & $\odot$ & 1. $47310 E-05$ & 293.6 & id \\
\hline Al- 27 & 268 & $\odot$ & $2.84107 \mathrm{E}-\odot 2$ & 293.6 & nd \\
\hline Si-28 & 268 & $\odot$ & 1. $\odot 1330 E-\odot 4$ & 293.6 & nd \\
\hline Si-29 & 268 & 0 & $5.13079 E-06$ & 293.6 & nd \\
\hline $\mathrm{Si}-30$ & 268 & $\odot$ & $3.40588 E-06$ & 293.6 & nd \\
\hline Ti-46 & 268 & 0 & $4.20262 \mathrm{E}-07$ & 293.6 & \\
\hline Ti-47 & 268 & 0 & $3.79000 \mathrm{E}-\odot 7$ & 293.6 & \\
\hline Ti-48 & 268 & 0 & $3.75536 \mathrm{E}-06$ & 293.6 & \\
\hline Ti-49 & 268 & $\odot$ & $2.75590 \mathrm{E}-\odot 7$ & 293.6 & \\
\hline Ti-50 & 268 & $\odot$ & $2.63873 E-07$ & 293.6 & \\
\hline $\mathrm{Cr}-50$ & 268 & $\odot$ & $5.30517 \mathrm{E}-07$ & 293.6 & \\
\hline $\mathrm{Cr}-52$ & 268 & $\odot$ & 1. $02189 E-\odot 5$ & 293.6 & end \\
\hline $\mathrm{Cr}-53$ & 268 & $\odot$ & $1.15860 \mathrm{E}-\odot 6$ & 293.6 & end \\
\hline $\mathrm{Cr}-54$ & 268 & $\odot$ & $2.87821 E-\odot 7$ & 293.6 & \\
\hline$M n-55$ & 268 & $\odot$ & $6.5 \odot 452 \mathrm{E}-06$ & 293.6 & \\
\hline $\mathrm{Fe}-54$ & 268 & $\odot$ & $2.38070 E-06$ & 293.6 & et \\
\hline $\mathrm{Fe}-56$ & 268 & 0 & $3.73384 \mathrm{E}-05$ & 293.6 & \\
\hline $\mathrm{Fe}-57$ & 268 & 0 & $8.62751 \mathrm{E}-07$ & 293.6 & \\
\hline $\mathrm{Fe}-58$ & 268 & $\odot$ & $1.13948 \mathrm{E}-07$ & 293.6 & \\
\hline
\end{tabular}




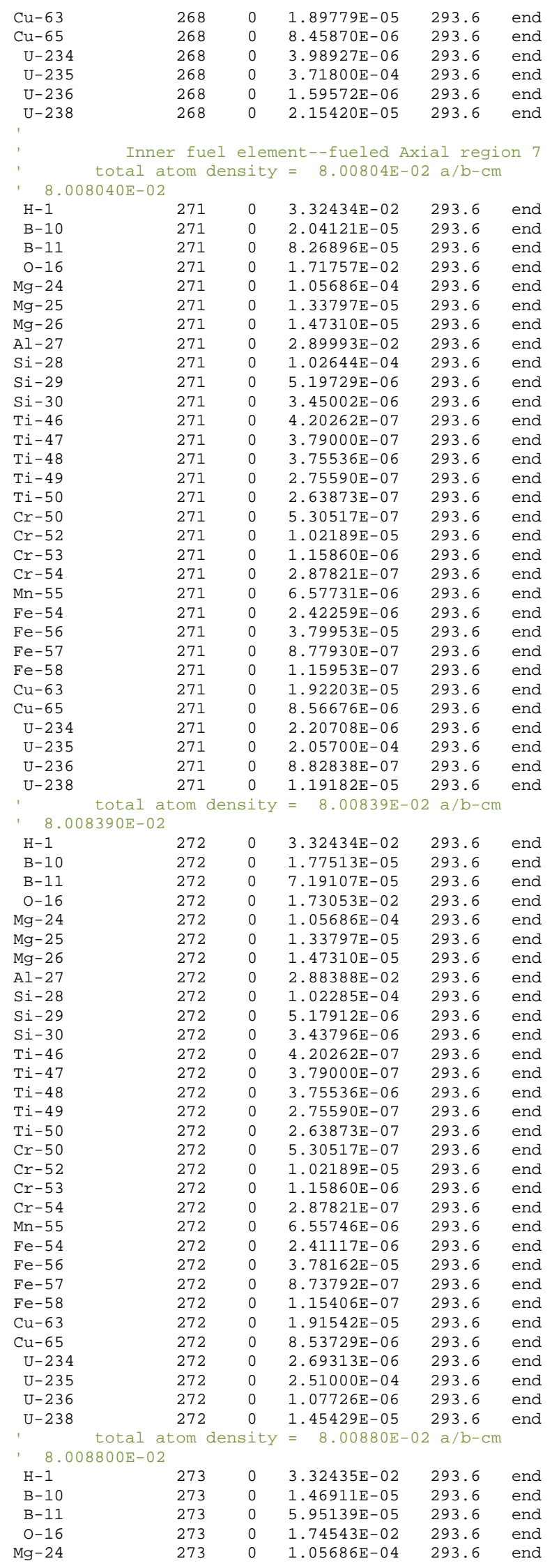




\begin{tabular}{|c|c|c|c|c|c|}
\hline$M g-25$ & 273 & $\odot$ & $1.33797 \mathrm{E}-05$ & 293.6 & end \\
\hline$M g-26$ & 273 & $\odot$ & $1.47310 \mathrm{E}-05$ & 293.6 & end \\
\hline Al-27 & 273 & $\odot$ & $2.86541 E-02$ & 293.6 & end \\
\hline Si-28 & 273 & $\odot$ & 1. $01874 \mathrm{E}-\odot 4$ & 293.6 & end \\
\hline Si-29 & 273 & $\odot$ & $5.15831 \mathrm{E}-06$ & 293.6 & end \\
\hline Si-30 & 273 & 0 & $3.42415 \mathrm{E}-06$ & 293.6 & end \\
\hline Ti-46 & 273 & $\odot$ & $4.20263 E-07$ & 293.6 & and \\
\hline $\mathrm{Ti}-47$ & 273 & $\odot$ & 3. $79001 \mathrm{E}-07$ & 293.6 & nd \\
\hline $\mathrm{Ti}-48$ & 273 & $\odot$ & $3.75537 \mathrm{E}-\odot 6$ & 293.6 & nd \\
\hline $\mathrm{Ti}-49$ & 273 & $\odot$ & $2.75590 E-\odot 7$ & 293.6 & nd \\
\hline Ti-5๑ & 273 & $\odot$ & $2.63873 \mathrm{E}-07$ & 293.6 & nd \\
\hline $\mathrm{Cr}-50$ & 273 & 0 & $5.30518 \mathrm{E}-07$ & 293.6 & end \\
\hline $\mathrm{Cr}-52$ & 273 & $\odot$ & 1. $02189 \mathrm{E}-05$ & 293.6 & nd \\
\hline $\mathrm{Cr}-53$ & 273 & $\odot$ & $1.15860 E-06$ & 293.6 & nd \\
\hline $\mathrm{Cr}-54$ & 273 & 0 & $2.87821 \mathrm{E}-07$ & 293.6 & ad \\
\hline Mn-55 & 273 & $\odot$ & $6.53464 \mathrm{E}-06$ & 293.6 & nd \\
\hline $\mathrm{Fe}-54$ & 273 & $\odot$ & $2.39803 E-06$ & 293.6 & $\mathrm{ad}$ \\
\hline $\mathrm{Fe}-56$ & 273 & $\odot$ & $3.76103 \mathrm{E}-05$ & 293.6 & $\mathrm{hd}$ \\
\hline $\mathrm{Fe}-57$ & 273 & $\odot$ & $8.69031 E-07$ & 293.6 & \\
\hline $\mathrm{Fe}-58$ & 273 & $\odot$ & $1.14778 \mathrm{E}-07$ & 293.6 & nd \\
\hline $\mathrm{Cu}-63$ & 273 & $\odot$ & 1. $90782 \mathrm{E}-05$ & 293.6 & nd \\
\hline Cu-65 & 273 & $\odot$ & $8.5 \odot 342 \mathrm{E}-\odot 6$ & 293.6 & $\mathrm{ad}$ \\
\hline U-234 & 273 & $\odot$ & $3.25215 E-\odot 6$ & 293.6 & \\
\hline U-235 & 273 & $\odot$ & $3.03100 E-\odot 4$ & 293.6 & \\
\hline U-236 & 273 & $\odot$ & 1. $30 \odot 87 \mathrm{E}-\odot 6$ & 293.6 & ad \\
\hline$U-238$ & 273 & $\odot$ & 1. $75616 \mathrm{E}-\odot 5$ & 293.6 & \\
\hline \multicolumn{3}{|c|}{ total atom density } & \multicolumn{3}{|c|}{$=8.00937 \mathrm{E}-02 \mathrm{a} / \mathrm{b}-\mathrm{cm}$} \\
\hline $\mathrm{H}-1$ & 274 & $\odot$ & 3. 32434E- -2 & 293.6 & nd \\
\hline$B-10$ & 274 & $\odot$ & $1.03798 \mathrm{E}-05$ & 293.6 & \\
\hline$B-11$ & 274 & $\odot$ & $4.20486 E-05$ & 293.6 & \\
\hline $0-16$ & 274 & $\odot$ & 1. 76644E- 02 & 293.6 & \\
\hline$M g-24$ & 274 & $\odot$ & 1. $05686 \mathrm{E}-\odot 4$ & 293.6 & \\
\hline$M g-25$ & 274 & $\odot$ & $1.33797 \mathrm{E}-05$ & 293.6 & \\
\hline$M g-26$ & 274 & $\odot$ & $1.47310 \mathrm{E}-05$ & 293.6 & \\
\hline Al-27 & 274 & $\odot$ & $2.83940 E-02$ & 293.6 & \\
\hline Si-28 & 274 & 0 & 1. $01293 E-04$ & 293.6 & \\
\hline Si-29 & 274 & $\odot$ & $5.12887 \mathrm{E}-06$ & 293.6 & \\
\hline Si-30 & 274 & $\odot$ & $3.40461 E-06$ & 293.6 & \\
\hline $\mathrm{Ti}-46$ & 274 & $\odot$ & $4.20262 E-07$ & 293.6 & \\
\hline $\mathrm{Ti}-47$ & 274 & $\odot$ & $3.79000 E-\odot 7$ & 293.6 & \\
\hline $\mathrm{Ti}-48$ & 274 & $\odot$ & $3.75536 \mathrm{E}-06$ & 293.6 & nd \\
\hline $\mathrm{Ti}-49$ & 274 & $\odot$ & $2.75590 E-\odot 7$ & 293.6 & \\
\hline $\mathrm{Ti}-50$ & 274 & $\odot$ & $2.63873 E-07$ & 293.6 & \\
\hline $\mathrm{Cr}-50$ & 274 & $\odot$ & $5.30517 \mathrm{E}-07$ & 293.6 & ad \\
\hline $\mathrm{Cr}-52$ & 274 & $\odot$ & 1. $02189 E-05$ & 293.6 & \\
\hline $\mathrm{Cr}-53$ & 274 & $\odot$ & 1. $15860 E-\odot 6$ & 293.6 & \\
\hline $\mathrm{Cr}-54$ & 274 & $\odot$ & $2.87821 \mathrm{E}-07$ & 293.6 & \\
\hline$M n-55$ & 274 & $\odot$ & $6.50246 \mathrm{E}-06$ & 293.6 & \\
\hline $\mathrm{Fe}-54$ & 274 & $\odot$ & $2.37952 E-06$ & 293.6 & \\
\hline $\mathrm{Fe}-56$ & 274 & $\odot$ & $3.73198 \mathrm{E}-05$ & 293.6 & \\
\hline $\mathrm{Fe}-57$ & 274 & $\odot$ & $8.62321 E-07$ & 293.6 & \\
\hline $\mathrm{Fe}-58$ & 274 & $\odot$ & $1.13891 \mathrm{E}-07$ & 293.6 & \\
\hline $\mathrm{Cu}-63$ & 274 & $\odot$ & 1. $89710 E-05$ & 293.6 & \\
\hline $\mathrm{Cu}-65$ & 274 & $\odot$ & $8.45562 \mathrm{E}-06$ & 293.6 & \\
\hline$U-234$ & 274 & $\odot$ & 4. $03970 E-06$ & 293.6 & \\
\hline$U-235$ & 274 & $\odot$ & 3. 76500E-๑4 & 293.6 & \\
\hline$U-236$ & 274 & $\odot$ & 1. $61589 \mathrm{E}-06$ & 293.6 & \\
\hline$U-238$ & 274 & $\odot$ & $2.18144 \mathrm{E}-05$ & 293.6 & end \\
\hline \multicolumn{3}{|c|}{ total atom density } & $=8.00993 \mathrm{E}$ & $2 a / b-c m$ & \\
\hline $\mathrm{H}-1$ & 275 & 0 & $3.32434 \mathrm{E}-02$ & 293.6 & \\
\hline$B-10$ & 275 & $\odot$ & $6.18010 \mathrm{E}-06$ & 293.6 & \\
\hline$B-11$ & 275 & $\odot$ & $2.50356 \mathrm{E}-\odot 5$ & 293.6 & \\
\hline $0-16$ & 275 & $\odot$ & $1.78689 \mathrm{E}-02$ & 293.6 & \\
\hline$M g-24$ & 275 & $\odot$ & 1. $05686 E-\odot 4$ & 293.6 & \\
\hline$M g-25$ & 275 & $\odot$ & $1.33797 \mathrm{E}-05$ & 293.6 & \\
\hline$M g-26$ & 275 & $\odot$ & $1.47310 \mathrm{E}-05$ & 293.6 & \\
\hline Al-27 & 275 & $\odot$ & $2.81406 \mathrm{E}-\odot 2$ & 293.6 & \\
\hline Si-28 & 275 & $\odot$ & 1. $\odot \odot 727 \mathrm{E}-\odot 4$ & 293.6 & \\
\hline Si-29 & 275 & $\odot$ & $5.10026 \mathrm{E}-06$ & 293.6 & \\
\hline Si-30 & 275 & $\odot$ & $3.38560 E-06$ & 293.6 & \\
\hline $\mathrm{Ti}-46$ & 275 & $\odot$ & 4. $20262 \mathrm{E}-07$ & 293.6 & \\
\hline $\mathrm{Ti}-47$ & 275 & $\odot$ & $3.79000 E-07$ & 293.6 & \\
\hline $\mathrm{Ti}-48$ & 275 & $\odot$ & $3.75536 E-\odot 6$ & 293.6 & \\
\hline $\mathrm{Ti}-49$ & 275 & $\odot$ & $2.75590 \mathrm{E}-07$ & 293.6 & \\
\hline Ti- 50 & 275 & $\odot$ & $2.63873 E-07$ & 293.6 & \\
\hline $\mathrm{Cr}-50$ & 275 & $\odot$ & $5.30518 E-07$ & 293.6 & \\
\hline $\mathrm{Cr}-52$ & 275 & $\odot$ & 1. $02189 E-05$ & 293.6 & \\
\hline $\mathrm{Cr}-53$ & 275 & $\odot$ & 1. $15860 E-06$ & 293.6 & \\
\hline $\mathrm{Cr}-54$ & 275 & 0 & $2.87821 \mathrm{E}-07$ & 293.6 & \\
\hline
\end{tabular}




\begin{tabular}{|c|c|c|c|c|c|}
\hline Mn-55 & 275 & $\odot$ & $6.47114 \mathrm{E}-06$ & 293.6 & end \\
\hline $\mathrm{Fe}-54$ & 275 & $\odot$ & $2.36149 E-06$ & 293.6 & end \\
\hline $\mathrm{Fe}-56$ & 275 & $\odot$ & $3.70370 \mathrm{E}-\odot 5$ & 293.6 & end \\
\hline $\mathrm{Fe}-57$ & 275 & $\odot$ & $8.55788 E-\odot 7$ & 293.6 & end \\
\hline $\mathrm{Fe}-58$ & 275 & $\odot$ & $1.13028 \mathrm{E}-07$ & 293.6 & end \\
\hline $\mathrm{Cu}-63$ & 275 & 0 & $1.88666 \mathrm{E}-05$ & 293.6 & end \\
\hline $\mathrm{Cu}-65$ & 275 & 0 & 8. 40911E- $\odot 6$ & 293.6 & end \\
\hline$U-234$ & 275 & $\odot$ & $4.80687 E-06$ & 293.6 & end \\
\hline$U-235$ & 275 & $\odot$ & 4. 4800०E- - & 293.6 & end \\
\hline$U-236$ & 275 & $\odot$ & 1. $92276 \mathrm{E}-06$ & 293.6 & end \\
\hline$U-238$ & 275 & $\odot$ & $2.59571 \mathrm{E}-05$ & 293.6 & end \\
\hline \multicolumn{3}{|c|}{ total atom density } & \multicolumn{2}{|c|}{$=8.00998 \mathrm{E}-02 \mathrm{a} / \mathrm{b}-\mathrm{cm}$} & \\
\hline $\mathrm{H}-1$ & 276 & $\odot$ & $3.32434 \mathrm{E}-02$ & 293.6 & end \\
\hline$B-10$ & 276 & $\odot$ & $5.79830 \mathrm{E}-\odot 6$ & 293.6 & end \\
\hline$B-11$ & 276 & 0 & $2.34889 E-05$ & 293.6 & end \\
\hline $0-16$ & 276 & 0 & 1. $78875 \mathrm{E}-\mathrm{-} 2$ & 293.6 & end \\
\hline$M g-24$ & 276 & $\odot$ & 1. $05686 \mathrm{E}-\odot 4$ & 293.6 & end \\
\hline Mg - 25 & 276 & 0 & $1.33797 \mathrm{E}-05$ & 293.6 & end \\
\hline Mg-26 & 276 & 0 & 1. $47310 \mathrm{E}-05$ & 293.6 & end \\
\hline $\mathrm{Al}-27$ & 276 & 0 & $2.81176 \mathrm{E}-02$ & 293.6 & end \\
\hline Si-28 & 276 & $\odot$ & 1. $\odot \odot 676 E-\odot 4$ & 293.6 & end \\
\hline Si-29 & 276 & 0 & $5 . \odot 9768 \mathrm{E}-\odot 6$ & 293.6 & end \\
\hline Si-30 & 276 & $\odot$ & 3. 38390E- - & 293.6 & end \\
\hline $\mathrm{Ti}-46$ & 276 & $\odot$ & $4.2 \odot 262 \mathrm{E}-\odot 7$ & 293.6 & end \\
\hline Ti-47 & 276 & 0 & $3.79000 \mathrm{E}-\odot 7$ & 293.6 & end \\
\hline Ti-48 & 276 & 0 & $3.75536 \mathrm{E}-\odot 6$ & 293.6 & end \\
\hline Ti-49 & 276 & 0 & $2.75590 \mathrm{E}-\odot 7$ & 293.6 & end \\
\hline Ti-50 & 276 & 0 & $2.63873 E-07$ & 293.6 & end \\
\hline $\mathrm{Cr}-50$ & 276 & 0 & $5.30517 \mathrm{E}-07$ & 293.6 & end \\
\hline $\mathrm{Cr}-52$ & 276 & 0 & 1. $02189 E-05$ & 293.6 & end \\
\hline $\mathrm{Cr}-53$ & 276 & $\odot$ & $1.15860 \mathrm{E}-\odot 6$ & 293.6 & end \\
\hline $\mathrm{Cr}-54$ & 276 & 0 & $2.87821 E-07$ & 293.6 & end \\
\hline $\mathrm{Mn}-55$ & 276 & $\odot$ & $6.46828 \mathrm{E}-06$ & 293.6 & end \\
\hline $\mathrm{Fe}-54$ & 276 & 0 & $2.35985 E-06$ & 293.6 & end \\
\hline $\mathrm{Fe}-56$ & 276 & $\odot$ & 3. 70113E- 05 & 293.6 & end \\
\hline $\mathrm{Fe}-57$ & 276 & 0 & 8. 55193E- - & 293.6 & end \\
\hline $\mathrm{Fe}-58$ & 276 & $\odot$ & 1. $12950 \mathrm{E}-07$ & 293.6 & end \\
\hline $\mathrm{Cu}-63$ & 276 & 0 & 1. $88571 \mathrm{E}-05$ & 293.6 & end \\
\hline $\mathrm{Cu}-65$ & 276 & 0 & $8.40487 \mathrm{E}-06$ & 293.6 & end \\
\hline$U-234$ & 276 & 0 & $4.87661 \mathrm{E}-06$ & 293.6 & end \\
\hline$U-235$ & 276 & 0 & $4.54500 \mathrm{E}-\odot 4$ & 293.6 & end \\
\hline$U-236$ & 276 & 0 & $1.95066 \mathrm{E}-\odot 6$ & 293.6 & end \\
\hline$U-238$ & 276 & 0 & $2.63337 \mathrm{E}-05$ & 293.6 & end \\
\hline \multicolumn{3}{|c|}{ total atom density } & \multicolumn{2}{|c|}{$=8.00968 \mathrm{E}-02 \mathrm{a} / \mathrm{b}-\mathrm{cm}$} & \\
\hline \\
\hline $\mathrm{H}-1$ & 277 & 0 & 3. 32434E- 02 & 293.6 & end \\
\hline B-10 & 277 & $\odot$ & 7. $99507 E-\odot 6$ & 293.6 & end \\
\hline$B-11$ & 277 & $\odot$ & $3.23880 E-05$ & 293.6 & end \\
\hline $0-16$ & 277 & $\odot$ & 1. 77805E-๑2 & 293.6 & end \\
\hline Mg-24 & 277 & 0 & 1. $05686 \mathrm{E}-04$ & 293.6 & end \\
\hline $\mathrm{Mg}-25$ & 277 & 0 & $1.33797 \mathrm{E}-05$ & 293.6 & end \\
\hline$M g-26$ & 277 & 0 & $1.47310 \mathrm{E}-05$ & 293.6 & end \\
\hline$A l-27$ & 277 & $\odot$ & $2.82501 E-\odot 2$ & 293.6 & end \\
\hline Si-28 & 277 & $\odot$ & 1. $\odot \odot 972 E-\odot 4$ & 293.6 & end \\
\hline Si-29 & 277 & $\odot$ & $5.11262 E-06$ & 293.6 & end \\
\hline $\mathrm{Si}-30$ & 277 & $\odot$ & 3. 39382E- 06 & 293.6 & end \\
\hline $\mathrm{Ti}-46$ & 277 & $\odot$ & 4. $2 \odot 262 \mathrm{E}-\odot 7$ & 293.6 & end \\
\hline $\mathrm{Ti}-47$ & 277 & $\odot$ & $3.79000 \mathrm{E}-\odot 7$ & 293.6 & end \\
\hline Ti-48 & 277 & $\odot$ & $3.75536 \mathrm{E}-\odot 6$ & 293.6 & end \\
\hline Ti-49 & 277 & $\odot$ & $2.75590 \mathrm{E}-\odot 7$ & 293.6 & end \\
\hline Ti-50 & 277 & 0 & $2.63873 \mathrm{E}-07$ & 293.6 & end \\
\hline $\mathrm{Cr}-50$ & 277 & 0 & $5.30517 \mathrm{E}-07$ & 293.6 & end \\
\hline $\mathrm{Cr}-52$ & 277 & $\odot$ & 1. $02189 \mathrm{E}-05$ & 293.6 & end \\
\hline $\mathrm{Cr}-53$ & 277 & $\odot$ & $1.15860 \mathrm{E}-\odot 6$ & 293.6 & end \\
\hline $\mathrm{Cr}-54$ & 277 & $\odot$ & $2.87821 E-\odot 7$ & 293.6 & end \\
\hline$M n-55$ & 277 & $\odot$ & $6.48467 \mathrm{E}-06$ & 293.6 & end \\
\hline $\mathrm{Fe}-54$ & 277 & $\odot$ & $2.36928 \mathrm{E}-06$ & 293.6 & end \\
\hline $\mathrm{Fe}-56$ & 277 & $\odot$ & $3.71592 \mathrm{E}-05$ & 293.6 & end \\
\hline $\mathrm{Fe}-57$ & 277 & 0 & $8.58611 \mathrm{E}-07$ & 293.6 & nd \\
\hline $\mathrm{Fe}-58$ & 277 & 0 & $1.13401 \mathrm{E}-07$ & 293.6 & $d$ \\
\hline $\mathrm{Cu}-63$ & 277 & 0 & 1. $89118 \mathrm{E}-05$ & 293.6 & end \\
\hline $\mathrm{Cu}-65$ & 277 & $\odot$ & $8.42923 E-06$ & 293.6 & end \\
\hline U-234 & 277 & $\odot$ & $4.47532 \mathrm{E}-06$ & 293.6 & end \\
\hline$U-235$ & 277 & $\odot$ & $4.17100 \mathrm{E}-\odot 4$ & 293.6 & end \\
\hline$U-236$ & 277 & $\odot$ & 1. 79014E-๑6 & 293.6 & end \\
\hline$U-238$ & 277 & $\odot$ & $2.41667 \mathrm{E}-05$ & 293.6 & end \\
\hline , $1-200$ & atom & sit & $=8.00933 \mathrm{E}-$ & $2 a / b-c n$ & \\
\hline ' $\quad 8.00$ & & & & & \\
\hline $\mathrm{H}-1$ & 278 & 0 & 3. 32434E- 02 & 293.6 & end \\
\hline$B-10$ & 278 & 0 & 1. $06559 \mathrm{E}-05$ & 293.6 & end \\
\hline
\end{tabular}




\begin{tabular}{|c|c|c|c|c|}
\hline$B-11$ & 278 & $\odot$ & $4.31670 E-05$ & 293.6 \\
\hline $0-16$ & 278 & $\odot$ & 1. $76509 E-02$ & 293.6 \\
\hline$M g-24$ & 278 & $\odot$ & 1. $05686 E-04$ & 293.6 \\
\hline$M g-25$ & 278 & $\odot$ & $1.33797 \mathrm{E}-05$ & 293.6 \\
\hline$M g-26$ & 278 & $\odot$ & 1. $47310 \mathrm{E}-05$ & 293.6 \\
\hline$A l-27$ & 278 & $\odot$ & $2.84107 E-02$ & 293.6 \\
\hline Si-28 & 278 & $\odot$ & 1. $01330 E-04$ & 293.6 \\
\hline Si-29 & 278 & $\odot$ & $5.13079 E-06$ & 293.6 \\
\hline Si-30 & 278 & $\odot$ & $3.40588 \mathrm{E}-06$ & 293.6 \\
\hline $\mathrm{Ti}-46$ & 278 & $\odot$ & 4. $20262 \mathrm{E}-07$ & 293.6 \\
\hline $\mathrm{Ti}-47$ & 278 & $\odot$ & 3. $790 \odot \odot E-\odot 7$ & 293.6 \\
\hline $\mathrm{Ti}-48$ & 278 & $\odot$ & $3.75536 E-06$ & 293.6 \\
\hline $\mathrm{Ti}-49$ & 278 & $\odot$ & $2.75590 E-07$ & 293.6 \\
\hline $\mathrm{Ti}-50$ & 278 & $\odot$ & $2.63873 E-07$ & 293.6 \\
\hline $\mathrm{Cr}-50$ & 278 & $\odot$ & $5.30517 \mathrm{E}-07$ & 293.6 \\
\hline $\mathrm{Cr}-52$ & 278 & $\odot$ & 1. $02189 E-05$ & 293.6 \\
\hline $\mathrm{Cr}-53$ & 278 & $\odot$ & $1.15860 E-06$ & 293.6 \\
\hline $\mathrm{Cr}-54$ & 278 & $\odot$ & $2.87821 E-07$ & 293.6 \\
\hline$M n-55$ & 278 & $\odot$ & $6.50452 \mathrm{E}-06$ & 293.6 \\
\hline $\mathrm{Fe}-54$ & 278 & $\odot$ & $2.38070 E-06$ & 293.6 \\
\hline $\mathrm{Fe}-56$ & 278 & $\odot$ & $3.73384 E-05$ & 293.6 \\
\hline $\mathrm{Fe}-57$ & 278 & $\odot$ & $8.62751 E-07$ & 293.6 \\
\hline $\mathrm{Fe}-58$ & 278 & $\odot$ & $1.13948 E-07$ & 293.6 \\
\hline $\mathrm{Cu}-63$ & 278 & $\odot$ & $1.89779 \mathrm{E}-05$ & 293.6 \\
\hline $\mathrm{Cu}-65$ & 278 & $\odot$ & $8.45870 E-06$ & 293.6 \\
\hline$U-234$ & 278 & $\odot$ & $3.98927 \mathrm{E}-06$ & 293.6 \\
\hline$U-235$ & 278 & $\odot$ & $3.71800 \mathrm{E}-04$ & 293.6 \\
\hline$U-236$ & 278 & $\odot$ & $1.59572 \mathrm{E}-06$ & 293.6 \\
\hline$U-238$ & 278 & $\odot$ & $2.15420 \mathrm{E}-05$ & 293.6 \\
\hline
\end{tabular}

Inner fuel element--fueled Axial region 8 total atom density $=8.00804 \mathrm{E}-02 \mathrm{a} / \mathrm{b}-\mathrm{cm}$

( $8.008040 \mathrm{E}-02$

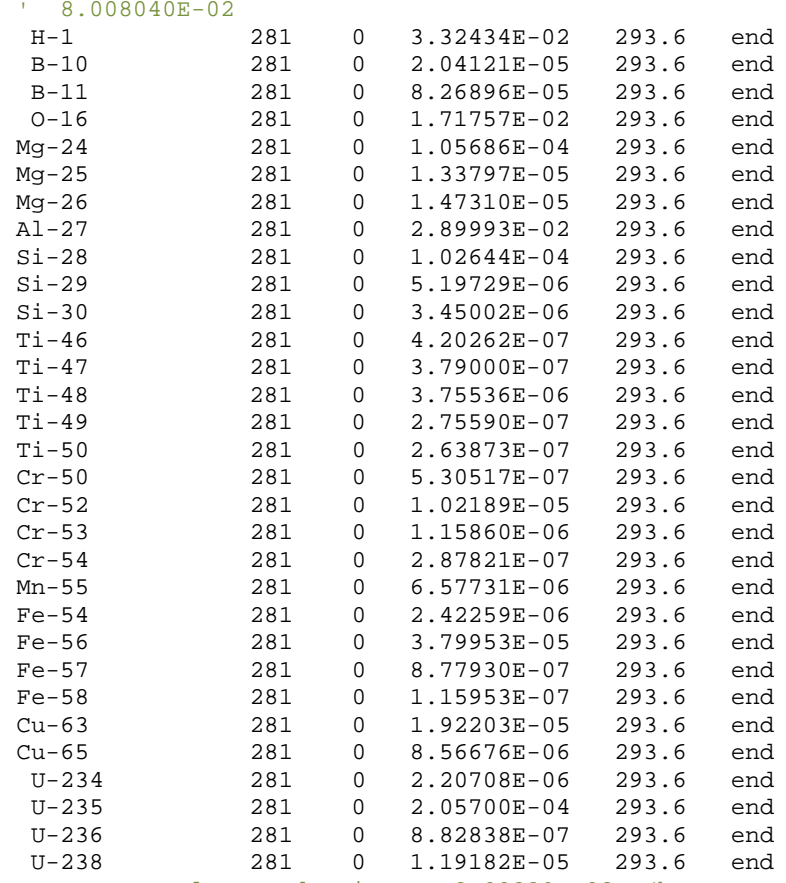

total atom density $=8.00839 \mathrm{E}-02 \mathrm{a} / \mathrm{b}-\mathrm{cm}$

\begin{tabular}{|c|c|c|c|c|c|}
\hline $\mathrm{H}-1$ & 282 & 0 & 3. 32434E- 02 & 293.6 & end \\
\hline$B-10$ & 282 & $\odot$ & $1.77513 \mathrm{E}-05$ & 293.6 & end \\
\hline$B-11$ & 282 & $\odot$ & 7. 19107E- 05 & 293.6 & end \\
\hline $0-16$ & 282 & $\odot$ & $1.73053 \mathrm{E}-02$ & 293.6 & nd \\
\hline$M g-24$ & 282 & 0 & $1.05686 \mathrm{E}-04$ & 293.6 & nd \\
\hline Mg-25 & 282 & 0 & $1.33797 \mathrm{E}-05$ & 293.6 & nd \\
\hline $\mathrm{Mg}-26$ & 282 & 0 & 1. 47310E- 05 & 293.6 & end \\
\hline Al-27 & 282 & 0 & $2.88388 \mathrm{E}-02$ & 293.6 & nd \\
\hline Si-28 & 282 & $\odot$ & 1. $02285 E-04$ & 293.6 & end \\
\hline Si-29 & 282 & $\odot$ & 5.17912E- 06 & 293.6 & end \\
\hline Si-30 & 282 & $\odot$ & 3. 43796E- 06 & 293.6 & end \\
\hline Ti-46 & 282 & $\odot$ & $4.20262 E-07$ & 293.6 & end \\
\hline Ti-47 & 282 & 0 & 3. $7900 \odot \mathrm{E}-\odot 7$ & 293.6 & end \\
\hline Ti-48 & 282 & $\odot$ & $3.75536 \mathrm{E}-06$ & 293.6 & end \\
\hline Ti-49 & 282 & 0 & $2.75590 \mathrm{E}-07$ & 293.6 & end \\
\hline
\end{tabular}




\begin{tabular}{|c|c|c|c|c|c|}
\hline Ti-50 & 282 & $\odot$ & $2.63873 E-07$ & 293.6 & end \\
\hline $\mathrm{Cr}-50$ & 282 & $\odot$ & $5.30517 \mathrm{E}-07$ & 293.6 & end \\
\hline Cr -52 & 282 & 0 & 1. $02189 \mathrm{E}-05$ & 293.6 & end \\
\hline $\mathrm{Cr}-53$ & 282 & $\odot$ & $1.15860 E-06$ & 293.6 & end \\
\hline $\mathrm{Cr}-54$ & 282 & $\odot$ & $2.87821 \mathrm{E}-07$ & 293.6 & end \\
\hline$M n-55$ & 282 & $\odot$ & $6.55746 \mathrm{E}-06$ & 293.6 & end \\
\hline $\mathrm{Fe}-54$ & 282 & $\odot$ & $2.41117 \mathrm{E}-06$ & 293.6 & and \\
\hline $\mathrm{Fe}-56$ & 282 & $\odot$ & $3.78162 \mathrm{E}-05$ & 293.6 & end \\
\hline $\mathrm{Fe}-57$ & 282 & $\odot$ & $8.73792 \mathrm{E}-07$ & 293.6 & nd \\
\hline $\mathrm{Fe}-58$ & 282 & $\odot$ & 1. $15406 \mathrm{E}-\odot 7$ & 293.6 & and \\
\hline $\mathrm{Cu}-63$ & 282 & $\odot$ & $1.91542 \mathrm{E}-05$ & 293.6 & nd \\
\hline $\mathrm{Cu}-65$ & 282 & $\odot$ & $8.53729 E-06$ & 293.6 & end \\
\hline U-234 & 282 & $\odot$ & $2.69313 E-\odot 6$ & 293.6 & ad \\
\hline$U-235$ & 282 & $\odot$ & $2.51000 E-\odot 4$ & 293.6 & ad \\
\hline$U-236$ & 282 & 0 & $1.07726 \mathrm{E}-06$ & 293.6 & nd \\
\hline$U-238$ & 282 & $\odot$ & $1.45429 \mathrm{E}-05$ & 293.6 & nd \\
\hline \multicolumn{3}{|c|}{$\begin{array}{l}\text { total atom density } \\
8800 \mathrm{E}-02\end{array}$} & \multicolumn{3}{|c|}{$=8.00880 \mathrm{E}-02 \mathrm{a} / \mathrm{b}-\mathrm{cm}$} \\
\hline $\mathrm{H}-1$ & 283 & $\odot$ & 3. 32435E- -2 & 293.6 & nd \\
\hline$B-10$ & 283 & $\odot$ & $1.46911 \mathrm{E}-05$ & 293.6 & nd \\
\hline B-11 & 283 & $\odot$ & $5.95139 \mathrm{E}-05$ & 293.6 & end \\
\hline $0-16$ & 283 & $\odot$ & 1. $74543 E-02$ & 293.6 & 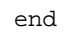 \\
\hline$M g-24$ & 283 & $\odot$ & 1. $05686 E-04$ & 293.6 & d \\
\hline$M g-25$ & 283 & 0 & $1.33797 \mathrm{E}-05$ & 293.6 & \\
\hline$M g-26$ & 283 & $\odot$ & $1.47310 \mathrm{E}-05$ & 293.6 & \\
\hline $\mathrm{Al}-27$ & 283 & $\odot$ & $2.86541 E-02$ & 293.6 & \\
\hline Si-28 & 283 & $\odot$ & 1. $01874 \mathrm{E}-04$ & 293.6 & \\
\hline Si-29 & 283 & $\odot$ & $5.15831 E-06$ & 293.6 & \\
\hline Si-30 & 283 & $\odot$ & $3.42415 E-06$ & 293.6 & Ciru \\
\hline $\mathrm{Ti}-46$ & 283 & $\odot$ & $4.2 \odot 263 E-07$ & 293.6 & nd \\
\hline $\mathrm{Ti}-47$ & 283 & $\odot$ & $3.79001 \mathrm{E}-07$ & 293.6 & mo \\
\hline $\mathrm{Ti}-48$ & 283 & $\odot$ & $3.75537 \mathrm{E}-06$ & 293.6 & nu \\
\hline $\mathrm{Ti}-49$ & 283 & $\odot$ & $2.75590 E-\odot 7$ & 293.6 & \\
\hline $\mathrm{Ti}-50$ & 283 & $\odot$ & $2.63873 E-07$ & 293.6 & \\
\hline $\mathrm{Cr}-50$ & 283 & $\odot$ & $5.30518 \mathrm{E}-07$ & 293.6 & \\
\hline $\mathrm{Cr}-52$ & 283 & 0 & 1. $02189 \mathrm{E}-05$ & 293.6 & \\
\hline $\mathrm{Cr}-53$ & 283 & $\odot$ & $1.15860 E-\odot 6$ & 293.6 & \\
\hline $\mathrm{Cr}-54$ & 283 & $\odot$ & $2.87821 E-07$ & 293.6 & \\
\hline$M n-55$ & 283 & $\odot$ & $6.53464 E-06$ & 293.6 & \\
\hline $\mathrm{Fe}-54$ & 283 & $\odot$ & $2.39803 E-06$ & 293.6 & \\
\hline $\mathrm{Fe}-56$ & 283 & $\odot$ & $3.76103 E-05$ & 293.6 & \\
\hline $\mathrm{Fe}-57$ & 283 & $\odot$ & 8. $69031 \mathrm{E}-07$ & 293.6 & \\
\hline $\mathrm{Fe}-58$ & 283 & $\odot$ & $1.14778 \mathrm{E}-07$ & 293.6 & \\
\hline $\mathrm{Cu}-63$ & 283 & $\odot$ & 1. $90782 \mathrm{E}-05$ & 293.6 & \\
\hline Cu-65 & 283 & 0 & $8.50342 \mathrm{E}-06$ & 293.6 & \\
\hline U-234 & 283 & $\odot$ & $3.25215 E-\odot 6$ & 293.6 & \\
\hline U-235 & 283 & $\odot$ & $3.03100 E-04$ & 293.6 & \\
\hline$U-236$ & 283 & $\odot$ & 1. $30087 \mathrm{E}-\odot 6$ & 293.6 & \\
\hline$U-238$ & 283 & $\odot$ & $1.75616 \mathrm{E}-05$ & 293.6 & end \\
\hline \multicolumn{3}{|c|}{ total atom density } & \multicolumn{2}{|c|}{$=8.00937 \mathrm{E}-02 \mathrm{a} / \mathrm{b}-\mathrm{cm}$} & \\
\hline $\mathrm{H}-1$ & 284 & $\odot$ & 3. $32434 \mathrm{E}-02$ & 293.6 & \\
\hline$B-10$ & 284 & $\odot$ & $1.03798 \mathrm{E}-05$ & 293.6 & \\
\hline$B-11$ & 284 & $\odot$ & $4.20486 \mathrm{E}-05$ & 293.6 & \\
\hline $0-16$ & 284 & $\odot$ & $1.76644 \mathrm{E}-02$ & 293.6 & \\
\hline$M g-24$ & 284 & 0 & 1. $05686 \mathrm{E}-04$ & 293.6 & \\
\hline$M g-25$ & 284 & $\odot$ & 1. $33797 \mathrm{E}-05$ & 293.6 & \\
\hline$M g-26$ & 284 & $\odot$ & 1. $47310 \mathrm{E}-05$ & 293.6 & \\
\hline Al-27 & 284 & $\odot$ & $2.83940 E-02$ & 293.6 & \\
\hline Si-28 & 284 & $\odot$ & 1. $01293 E-\odot 4$ & 293.6 & \\
\hline Si-29 & 284 & $\odot$ & $5.12887 \mathrm{E}-06$ & 293.6 & \\
\hline Si-30 & 284 & $\odot$ & $3.4 \odot 461 \mathrm{E}-06$ & 293.6 & \\
\hline $\mathrm{Ti}-46$ & 284 & $\odot$ & $4.2 \odot 262 \mathrm{E}-07$ & 293.6 & \\
\hline Ti-47 & 284 & $\odot$ & $3.7900 \odot E-\odot 7$ & 293.6 & \\
\hline $\mathrm{Ti}-48$ & 284 & $\odot$ & $3.75536 \mathrm{E}-\odot 6$ & 293.6 & \\
\hline Ti-49 & 284 & $\odot$ & $2.75590 E-\odot 7$ & 293.6 & \\
\hline $\mathrm{Ti}-50$ & 284 & $\odot$ & $2.63873 E-07$ & 293.6 & \\
\hline $\mathrm{Cr}-50$ & 284 & $\odot$ & $5.30517 \mathrm{E}-07$ & 293.6 & \\
\hline Cr -52 & 284 & $\odot$ & 1. $02189 \mathrm{E}-05$ & 293.6 & \\
\hline $\mathrm{Cr}-53$ & 284 & $\odot$ & $1.15860 E-\odot 6$ & 293.6 & \\
\hline $\mathrm{Cr}-54$ & 284 & $\odot$ & $2.87821 E-07$ & 293.6 & \\
\hline$M n-55$ & 284 & $\odot$ & $6.5 \odot 246 E-\odot 6$ & 293.6 & \\
\hline $\mathrm{Fe}-54$ & 284 & $\odot$ & $2.37952 \mathrm{E}-06$ & 293.6 & \\
\hline $\mathrm{Fe}-56$ & 284 & $\odot$ & $3.73198 \mathrm{E}-05$ & 293.6 & \\
\hline $\mathrm{Fe}-57$ & 284 & $\odot$ & $8.62321 \mathrm{E}-07$ & 293.6 & \\
\hline $\mathrm{Fe}-58$ & 284 & $\odot$ & $1.13891 \mathrm{E}-07$ & 293.6 & \\
\hline $\mathrm{Cu}-63$ & 284 & $\odot$ & $1.89710 \mathrm{E}-05$ & 293.6 & \\
\hline $\mathrm{Cu}-65$ & 284 & $\odot$ & $8.45562 \mathrm{E}-06$ & 293.6 & \\
\hline$U-234$ & 284 & $\odot$ & $4.03970 E-06$ & 293.6 & \\
\hline$U-235$ & 284 & $\odot$ & $3.76500 E-\odot 4$ & 293.6 & \\
\hline$U-236$ & 284 & $\odot$ & 1. $61589 E-\odot 6$ & 293.6 & \\
\hline
\end{tabular}




\begin{tabular}{|c|c|c|c|c|c|}
\hline U-238 & 284 & $\odot$ & $2.18144 E-05$ & 293.6 & end \\
\hline ' $\quad 8.0 \odot 993 \odot E-\odot 2$ & \multicolumn{2}{|c|}{$\begin{array}{l}\text { total atom density } \\
9930 \mathrm{E}-02\end{array}$} & \multicolumn{3}{|c|}{$=8.00993 \mathrm{E}-02 \mathrm{a} / \mathrm{b}-\mathrm{cm}$} \\
\hline $\mathrm{H}-1$ & 285 & $\odot$ & $3.32434 \mathrm{E}-02$ & 293.6 & en \\
\hline$B-10$ & 285 & $\odot$ & $6.18010 \mathrm{E}-06$ & 293.6 & end \\
\hline B - 11 & 285 & $\odot$ & $2.50356 \mathrm{E}-05$ & 293.6 & end \\
\hline $0-16$ & 285 & $\Theta$ & $1.78689 \mathrm{E}-02$ & 293.6 & \\
\hline Mg-24 & 285 & 0 & 1. $05686 E-04$ & 293.6 & \\
\hline$M g-25$ & 285 & $\odot$ & $1.33797 \mathrm{E}-05$ & 293.6 & \\
\hline$M g-26$ & 285 & $\odot$ & $1.47310 \mathrm{E}-05$ & 293.6 & \\
\hline $\mathrm{Al}-27$ & 285 & $\odot$ & $2.81406 E-\odot 2$ & 293.6 & \\
\hline $\mathrm{Si}-28$ & 285 & $\odot$ & $1.0 \odot 727 \mathrm{E}-\odot 4$ & 293.6 & \\
\hline Si-29 & 285 & $\odot$ & $5.10026 \mathrm{E}-\odot 6$ & 293.6 & \\
\hline Si-30 & 285 & $\odot$ & $3.3856 \odot E-\odot 6$ & 293.6 & \\
\hline Ti-46 & 285 & $\odot$ & $4.20262 \mathrm{E}-07$ & 293.6 & \\
\hline Ti-47 & 285 & $\odot$ & $3.79000 \mathrm{E}-07$ & 293.6 & \\
\hline Ti-48 & 285 & $\odot$ & $3.75536 \mathrm{E}-06$ & 293.6 & \\
\hline Ti-49 & 285 & $\odot$ & $2.75590 \mathrm{E}-07$ & 293.6 & \\
\hline Ti-50 & 285 & $\odot$ & $2.63873 E-07$ & 293.6 & \\
\hline $\mathrm{Cr}-50$ & 285 & $\odot$ & $5.30518 \mathrm{E}-07$ & 293.6 & \\
\hline $\mathrm{Cr}-52$ & 285 & $\odot$ & 1. $\odot 2189 E-\odot 5$ & 293.6 & \\
\hline $\mathrm{Cr}-53$ & 285 & $\odot$ & $1.1586 \odot E-\odot 6$ & 293.6 & \\
\hline $\mathrm{Cr}-54$ & 285 & $\odot$ & $2.87821 E-07$ & 293.6 & \\
\hline$M n-55$ & 285 & $\odot$ & $6.47114 \mathrm{E}-06$ & 293.6 & \\
\hline $\mathrm{Fe}-54$ & 285 & $\odot$ & $2.36149 E-06$ & 293.6 & \\
\hline $\mathrm{Fe}-56$ & 285 & $\odot$ & $3.70370 \mathrm{E}-05$ & 293.6 & \\
\hline $\mathrm{Fe}-57$ & 285 & $\odot$ & $8.55788 E-07$ & 293.6 & \\
\hline $\mathrm{Fe}-58$ & 285 & $\odot$ & $1.13028 \mathrm{E}-07$ & 293.6 & \\
\hline $\mathrm{Cu}-63$ & 285 & $\odot$ & $1.88666 \mathrm{E}-05$ & 293.6 & \\
\hline $\mathrm{Cu}-65$ & 285 & $\odot$ & 8. 40911E- 06 & 293.6 & \\
\hline$U-234$ & 285 & $\odot$ & 4.80687E- 06 & 293.6 & \\
\hline$U-235$ & 285 & $\odot$ & $4.4800 \odot E-\odot 4$ & 293.6 & \\
\hline$U-236$ & 285 & $\odot$ & $1.92276 \mathrm{E}-\odot 6$ & 293.6 & \\
\hline$U-238$ & 285 & $\odot$ & $2.59571 E-\odot 5$ & 293.6 & \\
\hline ' 8.00 & Lom d & sity & $=8.00998 \mathrm{E}-$ & $2 a / b-c m$ & \\
\hline $\mathrm{H}-1$ & 286 & $\odot$ & 3. 32434E- -2 & 293.6 & \\
\hline$B-10$ & 286 & $\odot$ & $5.79830 \mathrm{E}-06$ & 293.6 & \\
\hline$B-11$ & 286 & $\odot$ & $2.34889 E-\odot 5$ & 293.6 & \\
\hline $0-16$ & 286 & $\odot$ & $1.78875 \mathrm{E}-\odot 2$ & 293.6 & \\
\hline$M g-24$ & 286 & $\odot$ & $1.05686 E-\odot 4$ & 293.6 & \\
\hline $\mathrm{Mg}-25$ & 286 & $\odot$ & $1.33797 \mathrm{E}-05$ & 293.6 & \\
\hline$M g-26$ & 286 & $\odot$ & $1.47310 \mathrm{E}-05$ & 293.6 & \\
\hline$A 1-27$ & 286 & $\odot$ & $2.81176 \mathrm{E}-\odot 2$ & 293.6 & \\
\hline $\mathrm{Si}-28$ & 286 & $\odot$ & $1.00676 \mathrm{E}-\odot 4$ & 293.6 & \\
\hline Si-29 & 286 & $\odot$ & $5.09768 E-\odot 6$ & 293.6 & \\
\hline $\mathrm{Si}-30$ & 286 & $\odot$ & $3.38390 E-06$ & 293.6 & \\
\hline $\mathrm{Ti}-46$ & 286 & $\odot$ & $4.20262 \mathrm{E}-\odot 7$ & 293.6 & \\
\hline $\mathrm{Ti}-47$ & 286 & $\odot$ & $3.79000 \mathrm{E}-\odot 7$ & 293.6 & \\
\hline $\mathrm{Ti}-48$ & 286 & $\odot$ & $3.75536 \mathrm{E}-06$ & 293.6 & \\
\hline Ti-49 & 286 & $\odot$ & $2.75590 \mathrm{E}-07$ & 293.6 & \\
\hline Ti-50 & 286 & $\odot$ & $2.63873 E-07$ & 293.6 & \\
\hline $\mathrm{Cr}-50$ & 286 & $\odot$ & $5.30517 \mathrm{E}-07$ & 293.6 & \\
\hline $\mathrm{Cr}-52$ & 286 & $\odot$ & 1. $\odot 2189 E-\odot 5$ & 293.6 & \\
\hline $\mathrm{Cr}-53$ & 286 & $\odot$ & $1.1586 \odot E-\odot 6$ & 293.6 & \\
\hline $\mathrm{Cr}-54$ & 286 & $\odot$ & $2.87821 E-07$ & 293.6 & \\
\hline$M n-55$ & 286 & $\odot$ & $6.46828 E-06$ & 293.6 & \\
\hline $\mathrm{Fe}-54$ & 286 & $\odot$ & $2.35985 E-06$ & 293.6 & \\
\hline $\mathrm{Fe}-56$ & 286 & $\odot$ & 3. 70113E- 05 & 293.6 & \\
\hline $\mathrm{Fe}-57$ & 286 & $\odot$ & $8.55193 E-07$ & 293.6 & \\
\hline $\mathrm{Fe}-58$ & 286 & 0 & $1.12950 \mathrm{E}-07$ & 293.6 & \\
\hline $\mathrm{Cu}-63$ & 286 & 0 & $1.88571 \mathrm{E}-05$ & 293.6 & \\
\hline $\mathrm{Cu}-65$ & 286 & $\odot$ & $8.40487 \mathrm{E}-06$ & 293.6 & \\
\hline$U-234$ & 286 & $\odot$ & $4.87661 E-\odot 6$ & 293.6 & \\
\hline$U-235$ & 286 & $\odot$ & $4.5450 \odot E-\odot 4$ & 293.6 & \\
\hline $\mathrm{U}-236$ & 286 & $\odot$ & $1.95066 \mathrm{E}-\odot 6$ & 293.6 & \\
\hline$U-238$ & 286 & $\odot$ & $2.63337 \mathrm{E}-05$ & 293.6 & \\
\hline 1 & com d & sity & $=8.00968 \mathrm{E}-$ & $2 a / b-c$ & \\
\hline ' $\quad 8.0 \odot$ & & & & & \\
\hline $\mathrm{H}-1$ & 287 & $\odot$ & $3.32434 \mathrm{E}-02$ & 293.6 & \\
\hline$B-10$ & 287 & $\odot$ & $7.99507 \mathrm{E}-06$ & 293.6 & \\
\hline$B-11$ & 287 & $\odot$ & $3.23880 E-05$ & 293.6 & \\
\hline $0-16$ & 287 & 0 & $1.77805 \mathrm{E}-02$ & 293.6 & \\
\hline $\mathrm{Mg}-24$ & 287 & 0 & 1. $05686 E-04$ & 293.6 & \\
\hline$M g-25$ & 287 & 0 & $1.33797 \mathrm{E}-05$ & 293.6 & \\
\hline $\mathrm{Mg}-26$ & 287 & 0 & $1.47310 \mathrm{E}-05$ & 293.6 & \\
\hline Al-27 & 287 & 0 & $2.82501 E-\odot 2$ & 293.6 & \\
\hline Si-28 & 287 & 0 & 1. $\odot \odot 972 E-\odot 4$ & 293.6 & \\
\hline Si-29 & 287 & 0 & $5.11262 \mathrm{E}-06$ & 293.6 & \\
\hline $\mathrm{Si}-30$ & 287 & 0 & $3.39382 \mathrm{E}-06$ & 293.6 & \\
\hline $\mathrm{Ti}-46$ & 287 & 0 & $4.20262 \mathrm{E}-\odot 7$ & 293.6 & \\
\hline
\end{tabular}




\begin{tabular}{|c|c|c|c|c|c|}
\hline $\mathrm{Ti}-47$ & 287 & $\odot$ & $3.79000 E-\odot 7$ & 293.6 & end \\
\hline $\mathrm{Ti}-48$ & 287 & $\odot$ & $3.75536 \mathrm{E}-06$ & 293.6 & end \\
\hline Ti-49 & 287 & $\odot$ & $2.75590 E-07$ & 293.6 & end \\
\hline Ti-50 & 287 & $\odot$ & $2.63873 E-07$ & 293.6 & end \\
\hline $\mathrm{Cr}-50$ & 287 & $\odot$ & $5.30517 \mathrm{E}-07$ & 293.6 & end \\
\hline Cr -52 & 287 & 0 & 1. $02189 \mathrm{E}-05$ & 293.6 & end \\
\hline $\mathrm{Cr}-53$ & 287 & 0 & $1.15860 \mathrm{E}-06$ & 293.6 & end \\
\hline $\mathrm{Cr}-54$ & 287 & 0 & $2.87821 E-07$ & 293.6 & nd \\
\hline$M n-55$ & 287 & $\odot$ & $6.48467 \mathrm{E}-06$ & 293.6 & nd \\
\hline $\mathrm{Fe}-54$ & 287 & $\odot$ & $2.36928 E-\odot 6$ & 293.6 & \\
\hline $\mathrm{Fe}-56$ & 287 & $\odot$ & $3.71592 \mathrm{E}-\odot 5$ & 293.6 & nd \\
\hline $\mathrm{Fe}-57$ & 287 & 0 & $8.58611 \mathrm{E}-07$ & 293.6 & end \\
\hline $\mathrm{Fe}-58$ & 287 & $\odot$ & $1.13401 \mathrm{E}-07$ & 293.6 & nd \\
\hline Cu-63 & 287 & 0 & $1.89118 \mathrm{E}-05$ & 293.6 & nd \\
\hline Cu-65 & 287 & 0 & $8.42923 E-06$ & 293.6 & ad \\
\hline$U-234$ & 287 & 0 & $4.47532 \mathrm{E}-06$ & 293.6 & nd \\
\hline$U-235$ & 287 & 0 & $4.17100 \mathrm{E}-\odot 4$ & 293.6 & $\mathrm{dd}$ \\
\hline$U-236$ & 287 & 0 & 1. 79014E-06 & 293.6 & $\mathrm{hd}$ \\
\hline$U-238$ & 287 & $\odot$ & $2.41667 \mathrm{E}-05$ & 293.6 & end \\
\hline \multicolumn{3}{|c|}{ total atom density } & \multicolumn{3}{|c|}{$=8.00933 \mathrm{E}-02 \mathrm{a} / \mathrm{b}-\mathrm{cm}$} \\
\hline $\mathrm{H}-1$ & 288 & $\odot$ & 3. 32434E- -2 & 293.6 & end \\
\hline B -10 & 288 & $\odot$ & 1. $06559 \mathrm{E}-\odot 5$ & 293.6 & nd \\
\hline B-11 & 288 & 0 & $4.31670 E-05$ & 293.6 & \\
\hline $0-16$ & 288 & 0 & 1. 76509E- 02 & 293.6 & \\
\hline$M g-24$ & 288 & $\odot$ & 1. $05686 E-\odot 4$ & 293.6 & \\
\hline$M g-25$ & 288 & 0 & $1.33797 \mathrm{E}-05$ & 293.6 & \\
\hline$M g-26$ & 288 & 0 & $1.47310 \mathrm{E}-05$ & 293.6 & \\
\hline Al-27 & 288 & 0 & $2.84107 \mathrm{E}-02$ & 293.6 & \\
\hline Si-28 & 288 & 0 & 1. $01330 E-\odot 4$ & 293.6 & \\
\hline Si-29 & 288 & 0 & $5.13079 \mathrm{E}-06$ & 293.6 & \\
\hline $\mathrm{Si}-30$ & 288 & 0 & $3.40588 \mathrm{E}-06$ & 293.6 & \\
\hline Ti-46 & 288 & 0 & $4.20262 E-07$ & 293.6 & \\
\hline $\mathrm{Ti}-47$ & 288 & 0 & 3. $79000 \mathrm{E}-07$ & 293.6 & \\
\hline $\mathrm{Ti}-48$ & 288 & 0 & $3.75536 \mathrm{E}-06$ & 293.6 & \\
\hline $\mathrm{Ti}-49$ & 288 & $\odot$ & $2.75590 E-\odot 7$ & 293.6 & \\
\hline Ti-50 & 288 & 0 & $2.63873 E-07$ & 293.6 & \\
\hline $\mathrm{Cr}-50$ & 288 & 0 & $5.30517 \mathrm{E}-07$ & 293.6 & \\
\hline $\mathrm{Cr}-52$ & 288 & 0 & 1. $02189 \mathrm{E}-05$ & 293.6 & \\
\hline $\mathrm{Cr}-53$ & 288 & 0 & $1.15860 \mathrm{E}-06$ & 293.6 & end \\
\hline $\mathrm{Cr}-54$ & 288 & $\odot$ & $2.87821 E-07$ & 293.6 & \\
\hline$M n-55$ & 288 & 0 & $6.50452 \mathrm{E}-06$ & 293.6 & end \\
\hline $\mathrm{Fe}-54$ & 288 & $\odot$ & $2.38070 E-\odot 6$ & 293.6 & \\
\hline $\mathrm{Fe}-56$ & 288 & $\odot$ & $3.73384 \mathrm{E}-05$ & 293.6 & nd \\
\hline $\mathrm{Fe}-57$ & 288 & 0 & $8.62751 \mathrm{E}-07$ & 293.6 & ad \\
\hline $\mathrm{Fe}-58$ & 288 & $\odot$ & $1.13948 \mathrm{E}-07$ & 293.6 & \\
\hline $\mathrm{Cu}-63$ & 288 & $\odot$ & $1.89779 \mathrm{E}-05$ & 293.6 & \\
\hline $\mathrm{Cu}-65$ & 288 & 0 & $8.45870 E-06$ & 293.6 & \\
\hline $\mathrm{U}-234$ & 288 & 0 & $3.98927 \mathrm{E}-06$ & 293.6 & \\
\hline$U-235$ & 288 & $\odot$ & $3.71800 E-\odot 4$ & 293.6 & \\
\hline$U-236$ & 288 & $\odot$ & $1.59572 \mathrm{E}-06$ & 293.6 & \\
\hline $\mathrm{U}-238$ & 288 & $\odot$ & $2.15420 \mathrm{E}-05$ & 293.6 & \\
\hline \multicolumn{6}{|c|}{ Inner fuel element--fueled Axial regio } \\
\hline ' & tom & sity & $=8.00804 \mathrm{E}$ & $2 a / b-c m$ & \\
\hline \multicolumn{6}{|c|}{ ' $\quad 8.0 \odot 8 \odot 40 E-\odot 2$} \\
\hline $\mathrm{H}-1$ & 291 & $\odot$ & $3.32434 \mathrm{E}-02$ & 293.6 & end \\
\hline$B-10$ & 291 & $\odot$ & $2.04121 E-05$ & 293.6 & \\
\hline$B-11$ & 291 & $\odot$ & $8.26896 \mathrm{E}-05$ & 293.6 & \\
\hline $0-16$ & 291 & 0 & $1.71757 \mathrm{E}-02$ & 293.6 & \\
\hline $\mathrm{Mg}-24$ & 291 & 0 & 1. $05686 E-\odot 4$ & 293.6 & end \\
\hline$M g-25$ & 291 & $\odot$ & $1.33797 \mathrm{E}-05$ & 293.6 & \\
\hline $\mathrm{Mg}-26$ & 291 & $\odot$ & $1.47310 \mathrm{E}-05$ & 293.6 & \\
\hline Al-27 & 291 & $\odot$ & $2.89993 E-02$ & 293.6 & \\
\hline Si-28 & 291 & 0 & 1. $02644 \mathrm{E}-04$ & 293.6 & \\
\hline Si-29 & 291 & 0 & $5.19729 E-\odot 6$ & 293.6 & \\
\hline Si-30 & 291 & 0 & $3.45 \odot \odot 2 E-\odot 6$ & 293.6 & \\
\hline Ti-46 & 291 & 0 & $4.20262 \mathrm{E}-07$ & 293.6 & \\
\hline Ti-47 & 291 & 0 & $3.79000 \mathrm{E}-\odot 7$ & 293.6 & \\
\hline $\mathrm{Ti}-48$ & 291 & $\odot$ & $3.75536 E-\odot 6$ & 293.6 & \\
\hline Ti-49 & 291 & 0 & $2.75590 E-07$ & 293.6 & \\
\hline Ti-50 & 291 & $\odot$ & $2.63873 E-07$ & 293.6 & \\
\hline $\mathrm{Cr}-50$ & 291 & $\odot$ & 5. 30517E- -7 & 293.6 & \\
\hline $\mathrm{Cr}-52$ & 291 & 0 & 1. $02189 E-05$ & 293.6 & \\
\hline $\mathrm{Cr}-53$ & 291 & $\odot$ & 1. $15860 E-\odot 6$ & 293.6 & and \\
\hline $\mathrm{Cr}-54$ & 291 & 0 & $2.87821 E-07$ & 293.6 & \\
\hline$M n-55$ & 291 & $\odot$ & $6.57731 \mathrm{E}-06$ & 293.6 & \\
\hline $\mathrm{Fe}-54$ & 291 & 0 & $2.42259 E-06$ & 293.6 & \\
\hline $\mathrm{Fe}-56$ & 291 & $\odot$ & $3.79953 E-05$ & 293.6 & \\
\hline $\mathrm{Fe}-57$ & 291 & $\odot$ & $8.77930 E-07$ & 293.6 & \\
\hline $\mathrm{Fe}-58$ & 291 & 0 & $1.15953 \mathrm{E}-07$ & 293.6 & \\
\hline
\end{tabular}




$\begin{array}{cccccr}\text { Cu-63 } & 291 & \odot & 1.92203 \mathrm{E}-\odot 5 & 293.6 & \text { end } \\ \mathrm{Cu}-65 & 291 & \odot & 8.56676 \mathrm{E}-\odot 6 & 293.6 & \text { end } \\ \mathrm{U}-234 & 291 & \odot & 2.20708 \mathrm{E}-\odot 6 & 293.6 & \text { end } \\ \mathrm{U}-235 & 291 & \odot & 2.05700 \mathrm{E}-\odot 4 & 293.6 & \text { end } \\ \mathrm{U}-236 & 291 & \odot & 8.82838 \mathrm{E}-\odot 7 & 293.6 & \text { end } \\ \mathrm{U}-238 & 291 & \odot & 1.19182 \mathrm{E}-\odot 5 & 293.6 & \text { end }\end{array}$

total atom density $=8.00839 \mathrm{E}-02 \mathrm{a} / \mathrm{b}-\mathrm{cm}$

$\begin{array}{llllll}\text { 8.008390E- } 02 & & & & \\ \mathrm{H}-1 & 292 & \odot & 3.32434 \mathrm{E}-02 & 293.6 & \text { end } \\ \mathrm{B}-10 & 292 & \odot & 1.77513 \mathrm{E}-05 & 293.6 & \text { end } \\ \mathrm{B}-11 & 292 & \odot & 7.19107 \mathrm{E}-05 & 293.6 & \text { end } \\ \mathrm{O}-16 & 292 & \odot & 1.73053 \mathrm{E}-02 & 293.6 & \text { end } \\ \mathrm{Mg}-24 & 292 & \odot & 1.05686 \mathrm{E}-04 & 293.6 & \text { end } \\ \mathrm{Mg}-25 & 292 & \odot & 1.33797 \mathrm{E}-05 & 293.6 & \text { end } \\ \mathrm{Mg}-26 & 292 & \odot & 1.47310 \mathrm{E}-05 & 293.6 & \text { end } \\ \mathrm{Al}-27 & 292 & \odot & 2.88388 \mathrm{E}-02 & 293.6 & \text { end } \\ \mathrm{Si}-28 & 292 & \odot & 1.02285 \mathrm{E}-04 & 293.6 & \text { end } \\ \mathrm{Si}-29 & 292 & \odot & 5.17912 \mathrm{E}-06 & 293.6 & \text { end } \\ \mathrm{Si}-30 & 292 & \odot & 3.43796 \mathrm{E}-06 & 293.6 & \text { end } \\ \mathrm{Ti}-46 & 292 & \odot & 4.20262 \mathrm{E}-07 & 293.6 & \text { end } \\ \mathrm{Ti}-47 & 292 & \odot & 3.79000 \mathrm{E}-07 & 293.6 & \text { end } \\ \mathrm{Ti}-48 & 292 & \odot & 3.75536 \mathrm{E}-06 & 293.6 & \text { end } \\ \mathrm{Ti}-49 & 292 & \odot & 2.75590 \mathrm{E}-07 & 293.6 & \text { end } \\ \mathrm{Ti}-50 & 292 & \odot & 2.63873 \mathrm{E}-07 & 293.6 & \text { end } \\ \mathrm{Cr}-5 \odot & 292 & \odot & 5.30517 \mathrm{E}-07 & 293.6 & \text { end } \\ \mathrm{Cr}-52 & 292 & \odot & 1.02189 \mathrm{E}-05 & 293.6 & \text { end } \\ \mathrm{Cr}-53 & 292 & \odot & 1.15860 \mathrm{E}-06 & 293.6 & \text { end } \\ \mathrm{Cr}-54 & 292 & \odot & 2.87821 \mathrm{E}-07 & 293.6 & \text { end } \\ \mathrm{Mn}-55 & 292 & \odot & 6.55746 \mathrm{E}-06 & 293.6 & \text { end } \\ \mathrm{Fe}-54 & 292 & \odot & 2.41117 \mathrm{E}-06 & 293.6 & \text { end } \\ \mathrm{Fe}-56 & 292 & \odot & 3.78162 \mathrm{E}-05 & 293.6 & \text { end } \\ \mathrm{Fe}-57 & 292 & \odot & 8.73792 \mathrm{E}-07 & 293.6 & \text { end } \\ \mathrm{Fe}-58 & 292 & \odot & 1.15406 \mathrm{E}-07 & 293.6 & \text { end } \\ \mathrm{Cu}-63 & 292 & \odot & 1.91542 \mathrm{E}-05 & 293.6 & \text { end } \\ \mathrm{Cu}-65 & 292 & \odot & 8.53729 \mathrm{E}-06 & 293.6 & \text { end } \\ \mathrm{U}-234 & 292 & \odot & 2.69313 \mathrm{E}-06 & 293.6 & \text { end } \\ \mathrm{U}-235 & 292 & \odot & 2.51000 \mathrm{E}-04 & 293.6 & \text { end } \\ \mathrm{U}-236 & 292 & \odot & 1.07726 \mathrm{E}-06 & 293.6 & \text { end } \\ \mathrm{U}-238 & 292 & \odot & 1.45429 \mathrm{E}-05 & 293.6 & \text { end }\end{array}$

U-238 $292 \quad \odot \quad 1.45429 E-05 \quad 293.6$ end

\begin{tabular}{|c|c|c|c|c|c|}
\hline $\mathrm{H}-1$ & 293 & $\odot$ & 3. 32435E- -2 & 293.6 & end \\
\hline B-10 & 293 & $\odot$ & $1.46911 \mathrm{E}-05$ & 293.6 & end \\
\hline$B-11$ & 293 & $\odot$ & $5.95139 E-05$ & 293.6 & end \\
\hline $0-16$ & 293 & $\odot$ & 1. $74543 \mathrm{E}-\odot 2$ & 293.6 & end \\
\hline$M g-24$ & 293 & $\odot$ & 1. $\odot 5686 E-\odot 4$ & 293.6 & end \\
\hline$M g-25$ & 293 & $\odot$ & 1. $33797 \mathrm{E}-\odot 5$ & 293.6 & end \\
\hline$M g-26$ & 293 & $\odot$ & $1.47310 \mathrm{E}-05$ & 293.6 & \\
\hline Al-27 & 293 & $\odot$ & $2.86541 E-\odot 2$ & 293.6 & $\mathrm{dd}$ \\
\hline Si-28 & 293 & $\odot$ & 1. $\odot 1874 E-\odot 4$ & 293.6 & \\
\hline Si-29 & 293 & $\odot$ & $5.15831 E-06$ & 293.6 & d \\
\hline $\mathrm{Si}-30$ & 293 & $\odot$ & $3.42415 \mathrm{E}-06$ & 293.6 & \\
\hline Ti-46 & 293 & $\odot$ & $4.20263 E-07$ & 293.6 & \\
\hline Ti-47 & 293 & $\odot$ & $3.79001 \mathrm{E}-07$ & 293.6 & \\
\hline Ti-48 & 293 & $\odot$ & $3.75537 \mathrm{E}-06$ & 293.6 & \\
\hline Ti-49 & 293 & $\odot$ & $2.75590 E-\odot 7$ & 293.6 & \\
\hline Ti-50 & 293 & $\odot$ & $2.63873 E-07$ & 293.6 & end \\
\hline $\mathrm{Cr}-50$ & 293 & $\odot$ & $5.30518 \mathrm{E}-\odot 7$ & 293.6 & end \\
\hline $\mathrm{Cr}-52$ & 293 & $\odot$ & 1. $\odot 2189 E-\odot 5$ & 293.6 & end \\
\hline $\mathrm{Cr}-53$ & 293 & $\odot$ & 1. $15860 E-\odot 6$ & 293.6 & \\
\hline $\mathrm{Cr}-54$ & 293 & 0 & $2.87821 \mathrm{E}-07$ & 293.6 & \\
\hline Mn-55 & 293 & 0 & $6.53464 \mathrm{E}-06$ & 293.6 & \\
\hline $\mathrm{Fe}-54$ & 293 & 0 & 2. 39803E- - 6 & 293.6 & end \\
\hline $\mathrm{Fe}-56$ & 293 & $\odot$ & $3.76103 \mathrm{E}-05$ & 293.6 & end \\
\hline $\mathrm{Fe}-57$ & 293 & $\odot$ & $8.69031 \mathrm{E}-07$ & 293.6 & end \\
\hline $\mathrm{Fe}-58$ & 293 & $\odot$ & $1.14778 \mathrm{E}-07$ & 293.6 & \\
\hline $\mathrm{Cu}-63$ & 293 & $\odot$ & 1. $90782 \mathrm{E}-05$ & 293.6 & \\
\hline $\mathrm{Cu}-65$ & 293 & $\odot$ & $8.50342 E-06$ & 293.6 & \\
\hline$U-234$ & 293 & $\odot$ & 3. $25215 E-06$ & 293.6 & \\
\hline$U-235$ & 293 & $\odot$ & $3 . \odot 31 \odot \odot E-\odot 4$ & 293.6 & \\
\hline$U-236$ & 293 & $\odot$ & 1. $30087 \mathrm{E}-\odot 6$ & 293.6 & \\
\hline$U-238$ & 293 & $\odot$ & 1. $75616 \mathrm{E}-05$ & 293.6 & \\
\hline
\end{tabular}

total atom density $=8.00937 \mathrm{E}-02 \mathrm{a} / \mathrm{b}-\mathrm{cm}$

$\begin{array}{llllll}8.009370 \mathrm{E}-02 & & & & & \\ \mathrm{H}-1 & 294 & \odot & 3.32434 \mathrm{E}-02 & 293.6 & \text { end } \\ \mathrm{B}-10 & 294 & \odot & 1.03798 \mathrm{E}-05 & 293.6 & \text { end } \\ \mathrm{B}-11 & 294 & \odot & 4.20486 \mathrm{E}-05 & 293.6 & \text { end } \\ 0-16 & 294 & \odot & 1.76644 \mathrm{E}-02 & 293.6 & \text { end } \\ \mathrm{Mg}-24 & 294 & \odot & 1.05686 \mathrm{E}-04 & 293.6 & \text { end } \\ \mathrm{Mg}-25 & 294 & \odot & 1.33797 \mathrm{E}-05 & 293.6 & \text { end } \\ \mathrm{Mg}-26 & 294 & \odot & 1.47310 \mathrm{E}-05 & 293.6 & \text { end }\end{array}$




\begin{tabular}{|c|c|c|c|c|}
\hline Al-27 & 294 & $\odot$ & $2.83940 E-02$ & 293.6 \\
\hline Si-28 & 294 & $\odot$ & 1. $01293 E-04$ & 293.6 \\
\hline Si-29 & 294 & $\odot$ & $5.12887 E-06$ & 293.6 \\
\hline $\mathrm{Si}-30$ & 294 & $\odot$ & $3.40461 E-06$ & 293.6 \\
\hline Ti-46 & 294 & $\odot$ & $4.20262 E-07$ & 293.6 \\
\hline Ti-47 & 294 & $\odot$ & 3. 79000E - 07 & 293.6 \\
\hline Ti-48 & 294 & $\odot$ & $3.75536 E-06$ & 293.6 \\
\hline Ti-49 & 294 & $\odot$ & $2.75590 E-07$ & 293.6 \\
\hline Ti-50 & 294 & $\odot$ & $2.63873 E-07$ & 293.6 \\
\hline $\mathrm{Cr}-50$ & 294 & $\odot$ & $5.30517 E-07$ & 293.6 \\
\hline $\mathrm{Cr}-52$ & 294 & $\odot$ & 1. $02189 E-05$ & 293.6 \\
\hline $\mathrm{Cr}-53$ & 294 & $\odot$ & $1.15860 E-06$ & 293.6 \\
\hline $\mathrm{Cr}-54$ & 294 & $\odot$ & $2.87821 E-07$ & 293.6 \\
\hline$M n-55$ & 294 & $\odot$ & $6.50246 E-06$ & 293.6 \\
\hline $\mathrm{Fe}-54$ & 294 & $\odot$ & $2.37952 E-06$ & 293.6 \\
\hline $\mathrm{Fe}-56$ & 294 & $\odot$ & $3.73198 \mathrm{E}-05$ & 293.6 \\
\hline $\mathrm{Fe}-57$ & 294 & $\odot$ & $8.62321 E-07$ & 293.6 \\
\hline $\mathrm{Fe}-58$ & 294 & $\odot$ & $1.13891 E-07$ & 293.6 \\
\hline $\mathrm{Cu}-63$ & 294 & $\odot$ & $1.89710 \mathrm{E}-05$ & 293.6 \\
\hline Cu-65 & 294 & $\odot$ & $8.45562 \mathrm{E}-06$ & 293.6 \\
\hline$U-234$ & 294 & $\odot$ & 4. $03970 \mathrm{E}-06$ & 293.6 \\
\hline$U-235$ & 294 & $\odot$ & $3.76500 \mathrm{E}-\odot 4$ & 293.6 \\
\hline$U-236$ & 294 & $\odot$ & 1. $61589 E-06$ & 293.6 \\
\hline$U-238$ & 294 & $\odot$ & $2.18144 \mathrm{E}-05$ & 293.6 \\
\hline
\end{tabular}

total atom density $=8.00993 \mathrm{E}-02 \mathrm{a} / \mathrm{b}-\mathrm{cm}$

$\begin{array}{llllll}\text { 8. } 009930 \mathrm{E}-02 & & & & & \\ \mathrm{H}-1 & 295 & \odot & 3.32434 \mathrm{E}-02 & 293.6 & \text { end } \\ \mathrm{B}-10 & 295 & \odot & 6.18010 \mathrm{E}-06 & 293.6 & \text { end } \\ \mathrm{B}-11 & 295 & \odot & 2.50356 \mathrm{E}-05 & 293.6 & \text { end } \\ \mathrm{O}-16 & 295 & \odot & 1.78689 \mathrm{E}-02 & 293.6 & \text { end } \\ \mathrm{Mg}-24 & 295 & \odot & 1.05686 \mathrm{E}-04 & 293.6 & \text { end } \\ \mathrm{Mg}-25 & 295 & \odot & 1.33797 \mathrm{E}-05 & 293.6 & \text { end } \\ \mathrm{Mg}-26 & 295 & \odot & 1.47310 \mathrm{E}-05 & 293.6 & \text { end } \\ \mathrm{Al}-27 & 295 & \odot & 2.81406 \mathrm{E}-02 & 293.6 & \text { end } \\ \mathrm{Si}-28 & 295 & \odot & 1.00727 \mathrm{E}-04 & 293.6 & \text { end } \\ \mathrm{Si}-29 & 295 & \odot & 5.10026 \mathrm{E}-06 & 293.6 & \text { end } \\ \mathrm{Si}-30 & 295 & \odot & 3.38560 \mathrm{E}-06 & 293.6 & \text { end } \\ \mathrm{Ti}-46 & 295 & \odot & 4.20262 \mathrm{E}-07 & 293.6 & \text { end } \\ \mathrm{Ti}-47 & 295 & \odot & 3.79000 \mathrm{E}-07 & 293.6 & \text { end } \\ \mathrm{Ti}-48 & 295 & \odot & 3.75536 \mathrm{E}-06 & 293.6 & \text { end } \\ \mathrm{Ti}-49 & 295 & \odot & 2.75590 \mathrm{E}-07 & 293.6 & \text { end } \\ \mathrm{Ti}-50 & 295 & \odot & 2.63873 \mathrm{E}-07 & 293.6 & \text { end } \\ \mathrm{Cr}-5 \odot & 295 & \odot & 5.30518 \mathrm{E}-07 & 293.6 & \text { end } \\ \mathrm{Cr}-52 & 295 & \odot & 1.02189 \mathrm{E}-05 & 293.6 & \text { end } \\ \mathrm{Cr}-53 & 295 & \odot & 1.15860 \mathrm{E}-06 & 293.6 & \text { end } \\ \mathrm{Cr}-54 & 295 & \odot & 2.87821 \mathrm{E}-07 & 293.6 & \text { end } \\ \mathrm{Mn}-55 & 295 & \odot & 6.47114 \mathrm{E}-06 & 293.6 & \text { end } \\ \mathrm{Fe}-54 & 295 & \odot & 2.36149 \mathrm{E}-06 & 293.6 & \text { end } \\ \mathrm{Fe}-56 & 295 & \odot & 3.70370 \mathrm{E}-05 & 293.6 & \text { end } \\ \mathrm{Fe}-57 & 295 & \odot & 8.55788 \mathrm{E}-07 & 293.6 & \text { end } \\ \mathrm{Fe}-58 & 295 & \odot & 1.13028 \mathrm{E}-07 & 293.6 & \text { end } \\ \mathrm{Cu}-63 & 295 & \odot & 1.88666 \mathrm{E}-05 & 293.6 & \text { end } \\ \mathrm{Cu}-65 & 295 & \odot & 8.40911 \mathrm{E}-06 & 293.6 & \text { end } \\ \mathrm{U}-234 & 295 & \odot & 4.80687 \mathrm{E}-06 & 293.6 & \text { end } \\ \mathrm{U}-235 & 295 & \odot & 4.48000 \mathrm{E}-04 & 293.6 & \text { end } \\ \mathrm{U}-236 & 295 & \odot & 1.92276 \mathrm{E}-06 & 293.6 & \text { end } \\ \mathrm{U}-238 & 295 & \odot & 2.59571 \mathrm{E}-05 & 293.6 & \text { end }\end{array}$

total atom density $=8.00998 \mathrm{E}-02 \mathrm{a} / \mathrm{b}-\mathrm{cm}$

8. $009980 \mathrm{E}-\odot 2$

$\begin{array}{llllll}\text { H-1 } & 296 & \odot & 3.32434 \mathrm{E}-02 & 293.6 & \text { end } \\ \mathrm{B}-10 & 296 & \odot & 5.79830 \mathrm{E}-06 & 293.6 & \text { end } \\ \mathrm{B}-11 & 296 & \odot & 2.34889 \mathrm{E}-05 & 293.6 & \text { end } \\ \mathrm{O}-16 & 296 & \odot & 1.78875 \mathrm{E}-02 & 293.6 & \text { end } \\ \mathrm{Mg}-24 & 296 & \odot & 1.05686 \mathrm{E}-04 & 293.6 & \text { end } \\ \mathrm{Mg}-25 & 296 & \odot & 1.33797 \mathrm{E}-05 & 293.6 & \text { end } \\ \mathrm{Mg}-26 & 296 & \odot & 1.47310 \mathrm{E}-05 & 293.6 & \text { end } \\ \mathrm{Al}-27 & 296 & \odot & 2.81176 \mathrm{E}-\odot 2 & 293.6 & \text { end } \\ \mathrm{Si}-28 & 296 & \odot & 1.00676 \mathrm{E}-04 & 293.6 & \text { end } \\ \mathrm{Si}-29 & 296 & \odot & 5.09768 \mathrm{E}-06 & 293.6 & \text { end } \\ \mathrm{Si}-3 \odot & 296 & \odot & 3.38390 \mathrm{E}-06 & 293.6 & \text { end } \\ \mathrm{Ti}-46 & 296 & \odot & 4.20262 \mathrm{E}-07 & 293.6 & \text { end } \\ \mathrm{Ti}-47 & 296 & \odot & 3.7900 \odot \mathrm{E}-07 & 293.6 & \text { end } \\ \mathrm{Ti}-48 & 296 & \odot & 3.75536 \mathrm{E}-06 & 293.6 & \text { end } \\ \mathrm{Ti}-49 & 296 & \odot & 2.75590 \mathrm{E}-\odot 7 & 293.6 & \text { end } \\ \mathrm{Ti}-50 & 296 & \odot & 2.63873 \mathrm{E}-07 & 293.6 & \text { end } \\ \mathrm{Cr}-50 & 296 & \odot & 5.30517 \mathrm{E}-07 & 293.6 & \text { end } \\ \mathrm{Cr}-52 & 296 & \odot & 1.02189 \mathrm{E}-05 & 293.6 & \text { end } \\ \mathrm{Cr}-53 & 296 & \odot & 1.15860 \mathrm{E}-06 & 293.6 & \text { end } \\ \mathrm{Cr}-54 & 296 & \odot & 2.87821 \mathrm{E}-07 & 293.6 & \text { end } \\ \mathrm{Mn}-55 & 296 & \odot & 6.46828 \mathrm{E}-06 & 293.6 & \text { end } \\ \mathrm{Fe}-54 & 296 & \odot & 2.35985 \mathrm{E}-06 & 293.6 & \text { end }\end{array}$




$\begin{array}{llllll}\mathrm{Fe}-56 & 296 & \odot & 3.70113 \mathrm{E}-05 & 293.6 & \text { end } \\ \mathrm{Fe}-57 & 296 & \odot & 8.55193 \mathrm{E}-\odot 7 & 293.6 & \text { end } \\ \mathrm{Fe}-58 & 296 & \odot & 1.12950 \mathrm{E}-07 & 293.6 & \text { end } \\ \mathrm{Cu}-63 & 296 & \odot & 1.88571 \mathrm{E}-05 & 293.6 & \text { end } \\ \mathrm{Cu}-65 & 296 & \odot & 8.40487 \mathrm{E}-06 & 293.6 & \text { end } \\ \mathrm{U}-234 & 296 & \odot & 4.87661 \mathrm{E}-06 & 293.6 & \text { end } \\ \mathrm{U}-235 & 296 & \odot & 4.5450 \odot \mathrm{E}-\odot 4 & 293.6 & \text { end } \\ \mathrm{U}-236 & 296 & \odot & 1.95066 \mathrm{E}-06 & 293.6 & \text { end } \\ \mathrm{U}-238 & 296 & \odot & 2.63337 \mathrm{E}-\odot 5 & 293.6 & \text { end }\end{array}$

\begin{tabular}{|c|c|c|c|c|c|}
\hline $\mathrm{H}-1$ & 297 & $\odot$ & 3. 32434E- -2 & 293.6 & end \\
\hline B-10 & 297 & 0 & $7.99507 \mathrm{E}-\odot 6$ & 293.6 & end \\
\hline B-11 & 297 & 0 & $3.23880 E-05$ & 293.6 & end \\
\hline $0-16$ & 297 & $\odot$ & $1.77805 \mathrm{E}-\odot 2$ & 293.6 & end \\
\hline$M g-24$ & 297 & $\odot$ & 1. $\odot 5686 E-\odot 4$ & 293.6 & end \\
\hline$M g-25$ & 297 & $\odot$ & $1.33797 \mathrm{E}-05$ & 293.6 & end \\
\hline$M g-26$ & 297 & $\odot$ & $1.47310 \mathrm{E}-05$ & 293.6 & end \\
\hline Al-27 & 297 & $\odot$ & $2.82501 E-02$ & 293.6 & end \\
\hline $\mathrm{Si}-28$ & 297 & $\odot$ & 1. $\odot \odot 972 E-\odot 4$ & 293.6 & nd \\
\hline Si-29 & 297 & 0 & $5.11262 \mathrm{E}-06$ & 293.6 & nd \\
\hline Si-30 & 297 & $\odot$ & $3.39382 \mathrm{E}-06$ & 293.6 & nd \\
\hline Ti-46 & 297 & $\odot$ & $4.2 \odot 262 \mathrm{E}-\odot 7$ & 293.6 & nd \\
\hline $\mathrm{Ti}-47$ & 297 & $\odot$ & $3.79000 \mathrm{E}-\odot 7$ & 293.6 & nd \\
\hline $\mathrm{Ti}-48$ & 297 & $\odot$ & $3.75536 \mathrm{E}-06$ & 293.6 & and \\
\hline Ti-49 & 297 & $\odot$ & $2.75590 \mathrm{E}-\odot 7$ & 293.6 & nd \\
\hline Ti-50 & 297 & $\odot$ & $2.63873 E-07$ & 293.6 & nd \\
\hline $\mathrm{Cr}-50$ & 297 & $\odot$ & $5.30517 \mathrm{E}-07$ & 293.6 & nd \\
\hline $\mathrm{Cr}-52$ & 297 & 0 & 1. $02189 E-05$ & 293.6 & ad \\
\hline $\mathrm{Cr}-53$ & 297 & $\odot$ & $1.15860 \mathrm{E}-06$ & 293.6 & \\
\hline $\mathrm{Cr}-54$ & 297 & 0 & $2.87821 E-07$ & 293.6 & \\
\hline$M n-55$ & 297 & 0 & $6.48467 \mathrm{E}-06$ & 293.6 & end \\
\hline $\mathrm{Fe}-54$ & 297 & 0 & $2.36928 E-06$ & 293.6 & end \\
\hline $\mathrm{Fe}-56$ & 297 & $\odot$ & $3.71592 \mathrm{E}-05$ & 293.6 & end \\
\hline $\mathrm{Fe}-57$ & 297 & $\odot$ & $8.58611 E-\odot 7$ & 293.6 & \\
\hline $\mathrm{Fe}-58$ & 297 & $\odot$ & $1.13401 E-07$ & 293.6 & \\
\hline $\mathrm{Cu}-63$ & 297 & $\odot$ & $1.89118 \mathrm{E}-05$ & 293.6 & \\
\hline $\mathrm{Cu}-65$ & 297 & $\odot$ & 8. 42923E- - 6 & 293.6 & \\
\hline$U-234$ & 297 & $\odot$ & $4.47532 \mathrm{E}-06$ & 293.6 & \\
\hline$U-235$ & 297 & 0 & $4.17100 \mathrm{E}-\odot 4$ & 293.6 & \\
\hline$U-236$ & 297 & 0 & $1.79014 \mathrm{E}-06$ & 293.6 & \\
\hline$U-238$ & 297 & 0 & $2.41667 \mathrm{E}-05$ & 293.6 & 证 \\
\hline
\end{tabular}

$\begin{array}{rrrr}\text { U-238 total atom density }= & 8.00933 \mathrm{E}-02 \mathrm{a} / \mathrm{b}-\mathrm{cm}\end{array}$

1. 8. $009330 \mathrm{E}-02$

$\begin{array}{llllll}\mathrm{H}-1 & 298 & \odot & 3.32434 \mathrm{E}-\odot 2 & 293.6 & \text { end } \\ \mathrm{B}-1 \odot & 298 & \odot & 1.06559 \mathrm{E}-\odot 5 & 293.6 & \text { end } \\ \mathrm{B}-11 & 298 & \odot & 4.31670 \mathrm{E}-\odot 5 & 293.6 & \text { end } \\ \mathrm{O}-16 & 298 & \odot & 1.76509 \mathrm{E}-\odot 2 & 293.6 & \text { end } \\ \mathrm{Mg}-24 & 298 & \odot & 1.05686 \mathrm{E}-\odot 4 & 293.6 & \text { end } \\ \mathrm{Mg}-25 & 298 & \odot & 1.33797 \mathrm{E}-\odot 5 & 293.6 & \text { end } \\ \mathrm{Mg}-26 & 298 & \odot & 1.47310 \mathrm{E}-\odot 5 & 293.6 & \text { end } \\ \mathrm{Al}-27 & 298 & \odot & 2.84107 \mathrm{E}-\odot 2 & 293.6 & \text { end } \\ \mathrm{Si}-28 & 298 & \odot & 1.01330 \mathrm{E}-\odot 4 & 293.6 & \text { end } \\ \mathrm{Si}-29 & 298 & \odot & 5.13079 \mathrm{E}-\odot 6 & 293.6 & \text { end } \\ \mathrm{Si}-3 \odot & 298 & \odot & 3.40588 \mathrm{E}-\odot 6 & 293.6 & \text { end } \\ \mathrm{Ti}-46 & 298 & \odot & 4.20262 \mathrm{E}-\odot 7 & 293.6 & \text { end } \\ \mathrm{Ti}-47 & 298 & \odot & 3.79000 \mathrm{E}-\odot 7 & 293.6 & \text { end } \\ \mathrm{Ti}-48 & 298 & \odot & 3.75536 \mathrm{E}-\odot 6 & 293.6 & \text { end } \\ \mathrm{Ti}-49 & 298 & \odot & 2.75590 \mathrm{E}-\odot 7 & 293.6 & \text { end } \\ \mathrm{Ti}-5 \odot & 298 & \odot & 2.63873 \mathrm{E}-\odot 7 & 293.6 & \text { end } \\ \mathrm{Cr}-5 \odot & 298 & \odot & 5.30517 \mathrm{E}-\odot 7 & 293.6 & \text { end } \\ \mathrm{Cr}-52 & 298 & \odot & 1.02189 \mathrm{E}-\odot 5 & 293.6 & \text { end } \\ \mathrm{Cr}-53 & 298 & \odot & 1.15860 \mathrm{E}-\odot 6 & 293.6 & \text { end } \\ \mathrm{Cr}-54 & 298 & \odot & 2.87821 \mathrm{E}-\odot 7 & 293.6 & \text { end } \\ \mathrm{Mn}-55 & 298 & \odot & 6.50452 \mathrm{E}-\odot 6 & 293.6 & \text { end } \\ \mathrm{Fe}-54 & 298 & \odot & 2.38070 \mathrm{E}-\odot 6 & 293.6 & \text { end } \\ \mathrm{Fe}-56 & 298 & \odot & 3.73384 \mathrm{E}-\odot 5 & 293.6 & \text { end } \\ \mathrm{Fe}-57 & 298 & \odot & 8.62751 \mathrm{E}-\odot 7 & 293.6 & \text { end } \\ \mathrm{Fe}-58 & 298 & \odot & 1.13948 \mathrm{E}-\odot 7 & 293.6 & \text { end } \\ \mathrm{Cu}-63 & 298 & \odot & 1.89779 \mathrm{E}-\odot 5 & 293.6 & \text { end } \\ \mathrm{Cu}-65 & 298 & \odot & 8.45870 \mathrm{E}-\odot 6 & 293.6 & \text { end } \\ \mathrm{U}-234 & 298 & \odot & 3.98927 \mathrm{E}-\odot 6 & 293.6 & \text { end } \\ \mathrm{U}-235 & 298 & \odot & 3.7180 \odot \mathrm{E}-\odot 4 & 293.6 & \text { end } \\ \mathrm{U}-236 & 298 & \odot & 1.59572 \mathrm{E}-\odot 6 & 293.6 & \text { end } \\ \mathrm{U}-238 & & \odot & 2.15420 \mathrm{E}-\odot 5 & 293.6 & \text { end }\end{array}$

Inner fuel element--fueled central Axial region total atom density $=8.00804 \mathrm{E}-02 \mathrm{a} / \mathrm{b}-\mathrm{cm}$ 8. $008040 \mathrm{E}-\odot 2$
$\mathrm{H}-1$
$201 \quad \odot \quad 3.32434 \mathrm{E}-02 \quad 293.6$ end
B-10 $201 \quad 0 \quad 2.04121 \mathrm{E}-05 \quad 293.6$ end 


\begin{tabular}{|c|c|c|c|c|c|}
\hline$B-11$ & 201 & $\odot$ & $8.26896 E-05$ & 293.6 & end \\
\hline $0-16$ & 201 & $\odot$ & $1.71757 \mathrm{E}-02$ & 293.6 & end \\
\hline$M g-24$ & 201 & $\odot$ & 1. $05686 E-04$ & 293.6 & end \\
\hline$M g-25$ & 201 & $\odot$ & $1.33797 E-05$ & 293.6 & end \\
\hline $\mathrm{Mg}-26$ & 201 & $\odot$ & $1.47310 \mathrm{E}-05$ & 293.6 & end \\
\hline Al-27 & 201 & $\odot$ & 2. 89993E- 02 & 293.6 & end \\
\hline Si-28 & 201 & $\odot$ & $1.02644 \mathrm{E}-04$ & 293.6 & end \\
\hline Si-29 & 201 & $\odot$ & $5.19729 E-06$ & 293.6 & end \\
\hline Si-30 & 201 & $\odot$ & $3.450 \odot 2 E-06$ & 293.6 & end \\
\hline Ti-46 & 201 & $\odot$ & $4.20262 E-07$ & 293.6 & end \\
\hline Ti-47 & 201 & $\odot$ & $3.790 \odot \odot E-\odot 7$ & 293.6 & end \\
\hline Ti-48 & 201 & $\odot$ & $3.75536 \mathrm{E}-06$ & 293.6 & end \\
\hline Ti-49 & 201 & $\odot$ & $2.75590 E-07$ & 293.6 & end \\
\hline Ti-50 & 201 & $\odot$ & $2.63873 E-07$ & 293.6 & end \\
\hline $\mathrm{Cr}-50$ & 201 & $\odot$ & $5.30517 \mathrm{E}-07$ & 293.6 & end \\
\hline Cr -52 & 201 & $\odot$ & 1. $02189 E-05$ & 293.6 & end \\
\hline $\mathrm{Cr}-53$ & 201 & $\odot$ & $1.15860 \mathrm{E}-06$ & 293.6 & end \\
\hline $\mathrm{Cr}-54$ & 201 & $\odot$ & $2.87821 E-07$ & 293.6 & end \\
\hline$M n-55$ & 201 & $\odot$ & $6.57731 E-06$ & 293.6 & end \\
\hline $\mathrm{Fe}-54$ & 201 & $\odot$ & $2.42259 E-06$ & 293.6 & end \\
\hline $\mathrm{Fe}-56$ & 201 & $\odot$ & $3.79953 E-05$ & 293.6 & end \\
\hline $\mathrm{Fe}-57$ & 201 & $\odot$ & $8.77930 \mathrm{E}-07$ & 293.6 & end \\
\hline $\mathrm{Fe}-58$ & 201 & $\odot$ & $1.15953 E-07$ & 293.6 & end \\
\hline $\mathrm{Cu}-63$ & 201 & $\odot$ & 1. $92203 E-05$ & 293.6 & end \\
\hline $\mathrm{Cu}-65$ & 201 & $\odot$ & $8.56676 E-06$ & 293.6 & end \\
\hline$U-234$ & 201 & $\odot$ & $2.20708 \mathrm{E}-06$ & 293.6 & end \\
\hline$U-235$ & 201 & $\odot$ & $2.05700 \mathrm{E}-04$ & 293.6 & \\
\hline$U-236$ & 201 & $\odot$ & $8.82838 E-07$ & 293.6 & \\
\hline U-238 & 201 & $\odot$ & 1. 19182E- 05 & 293.6 & end \\
\hline
\end{tabular}

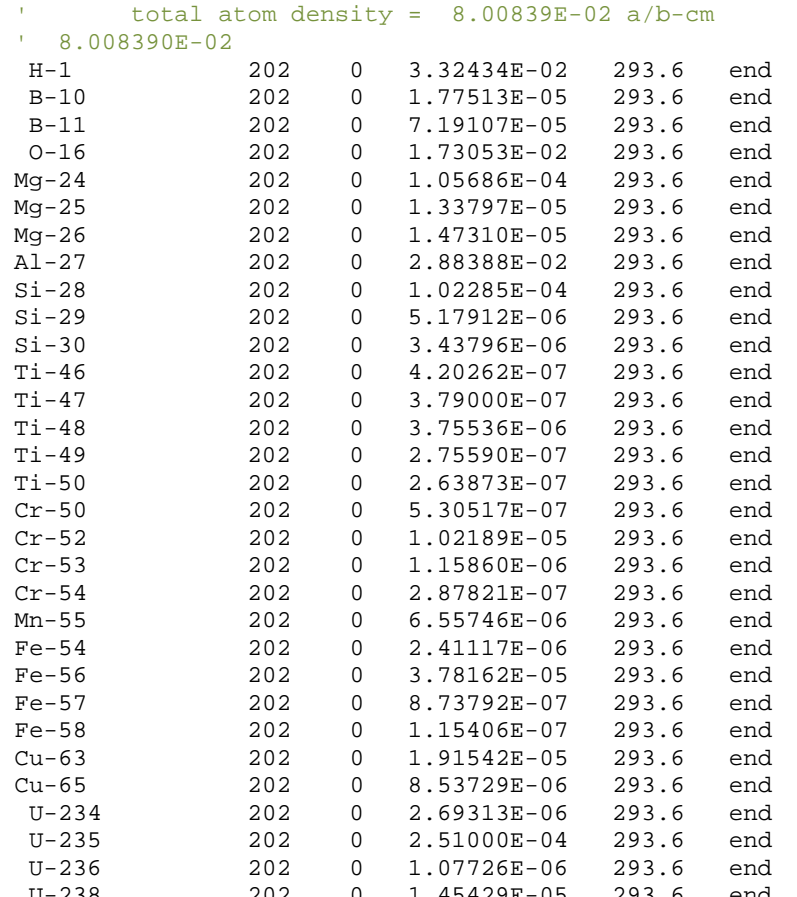

$20201.45429 \mathrm{E}-05 \quad 293.6$ end total atom density $=8.00880 \mathrm{E}-02 \mathrm{a} / \mathrm{b}-\mathrm{cm}$

\begin{tabular}{llllll}
\hline 8. $008800 \mathrm{E}-02$ & & & & & \\
$\mathrm{H}-1$ & 203 & $\odot$ & $3.32435 \mathrm{E}-02$ & 293.6 & end \\
$\mathrm{B}-10$ & 203 & $\odot$ & $1.46911 \mathrm{E}-05$ & 293.6 & end \\
$\mathrm{B}-11$ & 203 & $\odot$ & $5.95139 \mathrm{E}-05$ & 293.6 & end \\
O-16 & 203 & $\odot$ & $1.74543 \mathrm{E}-02$ & 293.6 & end \\
$\mathrm{Mg}-24$ & 203 & $\odot$ & $1.05686 \mathrm{E}-04$ & 293.6 & end \\
$\mathrm{Mg}-25$ & 203 & $\odot$ & $1.33797 \mathrm{E}-05$ & 293.6 & end \\
$\mathrm{Mg}-26$ & 203 & $\odot$ & $1.47310 \mathrm{E}-05$ & 293.6 & end \\
$\mathrm{Al}-27$ & 203 & $\odot$ & $2.86541 \mathrm{E}-02$ & 293.6 & end \\
$\mathrm{Si}-28$ & 203 & $\odot$ & $1.01874 \mathrm{E}-\odot 4$ & 293.6 & end \\
$\mathrm{Si}-29$ & 203 & $\odot$ & $5.15831 \mathrm{E}-06$ & 293.6 & end \\
$\mathrm{Si}-30$ & 203 & $\odot$ & $3.42415 \mathrm{E}-06$ & 293.6 & end \\
$\mathrm{Ti}-46$ & 203 & $\odot$ & $4.20263 \mathrm{E}-07$ & 293.6 & end \\
$\mathrm{Ti}-47$ & 203 & $\odot$ & $3.79001 \mathrm{E}-07$ & 293.6 & end \\
$\mathrm{Ti}-48$ & 203 & $\odot$ & $3.75537 \mathrm{E}-06$ & 293.6 & end \\
$\mathrm{Ti}-49$ & 203 & $\odot$ & $2.75590 \mathrm{E}-07$ & 293.6 & end \\
$\mathrm{Ti}-50$ & 203 & $\odot$ & $2.63873 \mathrm{E}-07$ & 293.6 & end \\
$\mathrm{Cr}-50$ & 203 & $\odot$ & $5.30518 \mathrm{E}-07$ & 293.6 & end
\end{tabular}




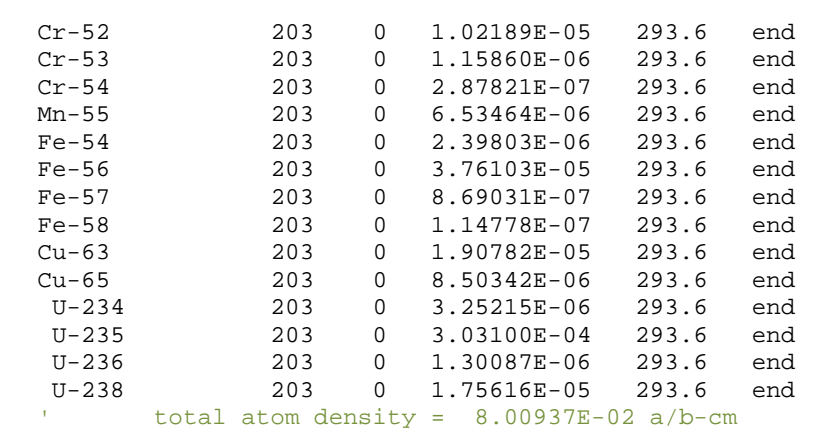

\begin{tabular}{|c|c|c|c|c|c|}
\hline $\mathrm{H}-1$ & 204 & $\odot$ & $3.32434 E-02$ & 293.6 & end \\
\hline B-10 & 204 & $\odot$ & 1. $03798 \mathrm{E}-05$ & 293.6 & end \\
\hline B-11 & 204 & $\odot$ & $4.20486 E-05$ & 293.6 & end \\
\hline $0-16$ & 204 & $\odot$ & $1.76644 E-02$ & 293.6 & end \\
\hline$M g-24$ & 204 & $\odot$ & 1. $05686 E-04$ & 293.6 & end \\
\hline$M g-25$ & 204 & $\odot$ & $1.33797 \mathrm{E}-05$ & 293.6 & end \\
\hline$M g-26$ & 204 & $\odot$ & $1.47310 E-05$ & 293.6 & end \\
\hline Al-27 & 204 & $\odot$ & $2.83940 \mathrm{E}-\odot 2$ & 293.6 & end \\
\hline Si-28 & 204 & $\odot$ & 1. $01293 E-\odot 4$ & 293.6 & end \\
\hline Si-29 & 204 & $\odot$ & $5.12887 E-06$ & 293.6 & end \\
\hline Si-30 & 204 & $\odot$ & $3.40461 E-06$ & 293.6 & end \\
\hline $\mathrm{Ti}-46$ & 204 & $\odot$ & $4.2 \odot 262 E-07$ & 293.6 & end \\
\hline $\mathrm{Ti}-47$ & 204 & $\odot$ & $3.79000 \mathrm{E}-07$ & 293.6 & end \\
\hline Ti-48 & 204 & $\odot$ & $3.75536 \mathrm{E}-06$ & 293.6 & end \\
\hline Ti-49 & 204 & 0 & $2.75590 \mathrm{E}-07$ & 293.6 & end \\
\hline Ti-50 & 204 & $\odot$ & $2.63873 E-07$ & 293.6 & end \\
\hline $\mathrm{Cr}-50$ & 204 & $\odot$ & $5.30517 E-07$ & 293.6 & end \\
\hline $\mathrm{Cr}-52$ & 204 & $\odot$ & 1. $02189 E-05$ & 293.6 & end \\
\hline $\mathrm{Cr}-53$ & 204 & $\odot$ & 1. $15860 E-06$ & 293.6 & end \\
\hline $\mathrm{Cr}-54$ & 204 & $\odot$ & $2.87821 E-07$ & 293.6 & end \\
\hline$M n-55$ & 204 & $\odot$ & $6.50246 E-06$ & 293.6 & end \\
\hline $\mathrm{Fe}-54$ & 204 & $\odot$ & $2.37952 \mathrm{E}-06$ & 293.6 & end \\
\hline $\mathrm{Fe}-56$ & 204 & $\odot$ & $3.73198 \mathrm{E}-05$ & 293.6 & end \\
\hline $\mathrm{Fe}-57$ & 204 & 0 & $8.62321 E-07$ & 293.6 & end \\
\hline $\mathrm{Fe}-58$ & 204 & $\odot$ & $1.13891 E-07$ & 293.6 & end \\
\hline $\mathrm{Cu}-63$ & 204 & $\odot$ & $1.89710 \mathrm{E}-05$ & 293.6 & end \\
\hline Cu-65 & 204 & $\odot$ & $8.45562 E-06$ & 293.6 & end \\
\hline$U-234$ & 204 & $\odot$ & 4. . $3970 E-\odot 6$ & 293.6 & end \\
\hline$U-235$ & 204 & $\odot$ & $3.76500 E-\odot 4$ & 293.6 & end \\
\hline$U-236$ & 204 & $\odot$ & 1. $61589 E-06$ & 293.6 & end \\
\hline$U-238$ & 204 & $\odot$ & $2.18144 \mathrm{E}-05$ & 293.6 & end \\
\hline
\end{tabular}

$204 \quad 2.18144 \mathrm{E}-05 \quad 293.6$ end

1 total atom density $=8.00993 \mathrm{E}-02 \mathrm{a} / \mathrm{b}-\mathrm{cm}$

$\begin{array}{llllll}\text { 8.009930E- } 02 & & & & & \\ \mathrm{H}-1 & 205 & \odot & 3.32434 \mathrm{E}-02 & 293.6 & \text { end } \\ \mathrm{B}-10 & 205 & \odot & 6.18010 \mathrm{E}-06 & 293.6 & \text { end } \\ \mathrm{B}-11 & 205 & \odot & 2.50356 \mathrm{E}-05 & 293.6 & \text { end } \\ \mathrm{O}-16 & 205 & \odot & 1.78689 \mathrm{E}-02 & 293.6 & \text { end } \\ \mathrm{Mg}-24 & 205 & \odot & 1.05686 \mathrm{E}-04 & 293.6 & \text { end } \\ \mathrm{Mg}-25 & 205 & \odot & 1.33797 \mathrm{E}-05 & 293.6 & \text { end } \\ \mathrm{Mg}-26 & 205 & \odot & 1.47310 \mathrm{E}-05 & 293.6 & \text { end } \\ \mathrm{Al}-27 & 205 & \odot & 2.81406 \mathrm{E}-02 & 293.6 & \text { end } \\ \mathrm{Si}-28 & 205 & \odot & 1.00727 \mathrm{E}-04 & 293.6 & \text { end } \\ \mathrm{Si}-29 & 205 & \odot & 5.10026 \mathrm{E}-06 & 293.6 & \text { end } \\ \mathrm{Si}-30 & 205 & \odot & 3.38560 \mathrm{E}-06 & 293.6 & \text { end } \\ \mathrm{Ti}-46 & 205 & \odot & 4.20262 \mathrm{E}-07 & 293.6 & \text { end } \\ \mathrm{Ti}-47 & 205 & \odot & 3.79000 \mathrm{E}-07 & 293.6 & \text { end } \\ \mathrm{Ti}-48 & 205 & \odot & 3.75536 \mathrm{E}-06 & 293.6 & \text { end } \\ \mathrm{Ti}-49 & 205 & \odot & 2.75590 \mathrm{E}-07 & 293.6 & \text { end } \\ \mathrm{Ti}-50 & 205 & \odot & 2.63873 \mathrm{E}-07 & 293.6 & \text { end } \\ \mathrm{Cr}-50 & 205 & \odot & 5.30518 \mathrm{E}-07 & 293.6 & \text { end } \\ \mathrm{Cr}-52 & 205 & \odot & 1.02189 \mathrm{E}-05 & 293.6 & \text { end } \\ \mathrm{Cr}-53 & 205 & \odot & 1.15860 \mathrm{E}-06 & 293.6 & \text { end } \\ \mathrm{Cr}-54 & 205 & \odot & 2.87821 \mathrm{E}-07 & 293.6 & \text { end } \\ \mathrm{Mn}-55 & 205 & \odot & 6.47114 \mathrm{E}-06 & 293.6 & \text { end } \\ \mathrm{Fe}-54 & 205 & \odot & 2.36149 \mathrm{E}-06 & 293.6 & \text { end } \\ \mathrm{Fe}-56 & 205 & \odot & 3.70370 \mathrm{E}-05 & 293.6 & \text { end } \\ \mathrm{Fe}-57 & 205 & \odot & 8.55788 \mathrm{E}-07 & 293.6 & \text { end } \\ \mathrm{Fe}-58 & 205 & \odot & 1.13028 \mathrm{E}-07 & 293.6 & \text { end } \\ \mathrm{Cu}-63 & 205 & \odot & 1.88666 \mathrm{E}-05 & 293.6 & \text { end } \\ \mathrm{Cu}-65 & 205 & \odot & 8.40911 \mathrm{E}-06 & 293.6 & \text { end } \\ \mathrm{U}-234 & 205 & \odot & 4.80687 \mathrm{E}-06 & 293.6 & \text { end } \\ \mathrm{U}-235 & 205 & \odot & 4.48000 \mathrm{E}-04 & 293.6 & \text { end } \\ \mathrm{U}-236 & 205 & \odot & 1.92276 \mathrm{E}-06 & 293.6 & \text { end } \\ \mathrm{U}-238 & 205 & \odot & 2.59571 \mathrm{E}-05 & 293.6 & \text { end }\end{array}$

total atom density $=8.00998 \mathrm{E}-02 \mathrm{a} / \mathrm{b}-\mathrm{cm}$ 


\begin{tabular}{|c|c|c|c|c|c|}
\hline $\mathrm{H}-1$ & 206 & 0 & $3.32434 \mathrm{E}-02$ & 293.6 & end \\
\hline$B-10$ & 206 & 0 & $5.79830 \mathrm{E}-06$ & 293.6 & end \\
\hline$B-11$ & 206 & $\odot$ & $2.34889 E-05$ & 293.6 & \\
\hline $0-16$ & 206 & 0 & 1. $78875 \mathrm{E}-02$ & 293.6 & \\
\hline$M g-24$ & 206 & 0 & 1. $05686 \mathrm{E}-04$ & 293.6 & \\
\hline$M g-25$ & 206 & 0 & 1.33797E-05 & 293.6 & \\
\hline$M g-26$ & 206 & 0 & $1.47310 \mathrm{E}-05$ & 293.6 & \\
\hline Al -27 & 206 & $\odot$ & $2.81176 \mathrm{E}-02$ & 293.6 & \\
\hline Si-28 & 206 & 0 & 1. $00676 \mathrm{E}-04$ & 293.6 & \\
\hline Si-29 & 206 & 0 & $5.09768 \mathrm{E}-06$ & 293.6 & \\
\hline Si-30 & 206 & $\odot$ & 3. $38390 E-06$ & 293.6 & \\
\hline Ti-46 & 206 & 0 & $4.20262 \mathrm{E}-07$ & 293.6 & \\
\hline Ti-47 & 206 & 0 & $3.790 \odot \odot E-07$ & 293.6 & \\
\hline Ti-48 & 206 & 0 & $3.75536 \mathrm{E}-06$ & 293.6 & \\
\hline Ti-49 & 206 & 0 & 2. $75590 \mathrm{E}-07$ & 293.6 & \\
\hline Ti-50 & 206 & 0 & $2.63873 E-07$ & 293.6 & \\
\hline $\mathrm{Cr}-50$ & 206 & 0 & $5.30517 \mathrm{E}-07$ & 293.6 & \\
\hline $\mathrm{Cr}-52$ & 206 & $\odot$ & 1. $02189 E-05$ & 293.6 & \\
\hline $\mathrm{Cr}-53$ & 206 & $\odot$ & $1.15860 \mathrm{E}-06$ & 293.6 & \\
\hline $\mathrm{Cr}-54$ & 206 & $\odot$ & $2.87821 E-07$ & 293.6 & \\
\hline$M n-55$ & 206 & $\odot$ & $6.46828 E-06$ & 293.6 & \\
\hline $\mathrm{Fe}-54$ & 206 & $\odot$ & 2. 35985E- 06 & 293.6 & \\
\hline $\mathrm{Fe}-56$ & 206 & $\odot$ & $3.70113 \mathrm{E}-05$ & 293.6 & \\
\hline $\mathrm{Fe}-57$ & 206 & $\odot$ & 8. 55193E-07 & 293.6 & \\
\hline $\mathrm{Fe}-58$ & 206 & $\odot$ & $1.12950 \mathrm{E}-07$ & 293.6 & \\
\hline $\mathrm{Cu}-63$ & 206 & $\odot$ & $1.88571 \mathrm{E}-05$ & 293.6 & \\
\hline Cu-65 & 206 & $\odot$ & $8.40487 \mathrm{E}-06$ & 293.6 & \\
\hline$U-234$ & 206 & $\odot$ & $4.87661 E-06$ & 293.6 & \\
\hline$U-235$ & 206 & $\odot$ & $4.5450 \odot E-\odot 4$ & 293.6 & \\
\hline$U-236$ & 206 & $\odot$ & $1.95066 \mathrm{E}-06$ & 293.6 & \\
\hline$U-238$ & 206 & $\odot$ & $2.63337 \mathrm{E}-05$ & 293.6 & \\
\hline
\end{tabular}

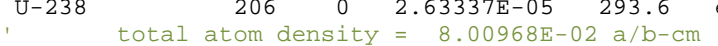

\begin{tabular}{|c|c|c|c|c|c|}
\hline $\mathrm{H}-1$ & 207 & $\odot$ & 3. 32434E- 02 & 293.6 & end \\
\hline$B-10$ & 207 & $\odot$ & $7.99507 E-06$ & 293.6 & end \\
\hline B-11 & 207 & $\odot$ & $3.23880 E-05$ & 293.6 & end \\
\hline $0-16$ & 207 & $\odot$ & 1. $77805 \mathrm{E}-\odot 2$ & 293.6 & end \\
\hline$M g-24$ & 207 & 0 & 1. $05686 E-04$ & 293.6 & end \\
\hline$M g-25$ & 207 & 0 & 1. $33797 \mathrm{E}-05$ & 293.6 & hd \\
\hline$M g-26$ & 207 & 0 & $1.47310 \mathrm{E}-05$ & 293.6 & nd \\
\hline Al-27 & 207 & 0 & $2.82501 \mathrm{E}-02$ & 293.6 & nd \\
\hline $\mathrm{Si}-28$ & 207 & $\odot$ & 1. $\odot \odot 972 E-\odot 4$ & 293.6 & d \\
\hline Si-29 & 207 & $\odot$ & $5.11262 E-06$ & 293.6 & d \\
\hline $\mathrm{Si}-30$ & 207 & $\odot$ & $3.39382 E-06$ & 293.6 & \\
\hline Ti-46 & 207 & $\odot$ & 4. $2 \odot 262 E-\odot 7$ & 293.6 & \\
\hline Ti-47 & 207 & $\odot$ & $3.7900 \odot E-07$ & 293.6 & \\
\hline Ti-48 & 207 & $\odot$ & $3.75536 \mathrm{E}-06$ & 293.6 & \\
\hline Ti-49 & 207 & $\odot$ & $2.7559 \odot \mathrm{E}-\odot 7$ & 293.6 & nd \\
\hline Ti-50 & 207 & $\odot$ & $2.63873 E-07$ & 293.6 & \\
\hline $\mathrm{Cr}-50$ & 207 & $\odot$ & $5.30517 E-07$ & 293.6 & nd \\
\hline $\mathrm{Cr}-52$ & 207 & $\odot$ & 1. $02189 \mathrm{E}-05$ & 293.6 & \\
\hline $\mathrm{Cr}-53$ & 207 & 0 & $1.15860 \mathrm{E}-06$ & 293.6 & \\
\hline $\mathrm{Cr}-54$ & 207 & $\odot$ & $2.87821 \mathrm{E}-07$ & 293.6 & \\
\hline$M n-55$ & 207 & $\odot$ & $6.48467 \mathrm{E}-06$ & 293.6 & \\
\hline $\mathrm{Fe}-54$ & 207 & $\odot$ & $2.36928 E-06$ & 293.6 & \\
\hline $\mathrm{Fe}-56$ & 207 & $\odot$ & $3.71592 E-05$ & 293.6 & \\
\hline $\mathrm{Fe}-57$ & 207 & $\odot$ & $8.58611 E-07$ & 293.6 & \\
\hline $\mathrm{Fe}-58$ & 207 & $\odot$ & $1.13401 E-07$ & 293.6 & \\
\hline $\mathrm{Cu}-63$ & 207 & $\odot$ & 1. $89118 E-05$ & 293.6 & \\
\hline $\mathrm{Cu}-65$ & 207 & $\odot$ & $8.42923 E-06$ & 293.6 & \\
\hline$U-234$ & 207 & $\odot$ & $4.47532 \mathrm{E}-06$ & 293.6 & \\
\hline$U-235$ & 207 & 0 & $4.17100 \mathrm{E}-04$ & 293.6 & \\
\hline$U-236$ & 207 & 0 & 1. $79014 \mathrm{E}-06$ & 293.6 & \\
\hline$U-238$ & 207 & $\odot$ & $2.41667 \mathrm{E}-05$ & 293.6 & \\
\hline
\end{tabular}

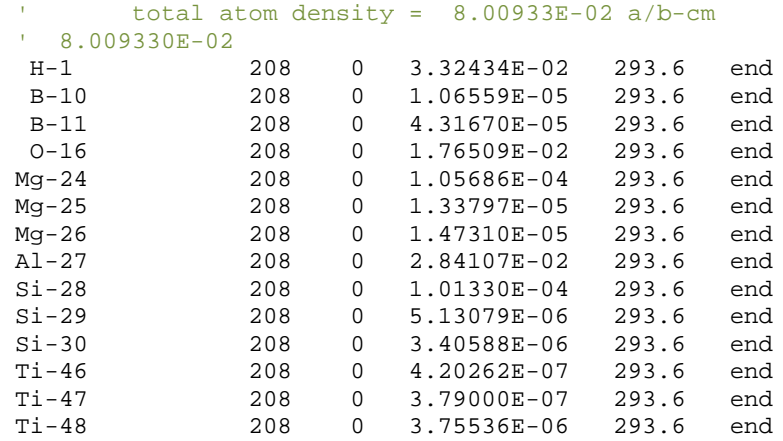




$\begin{array}{llllll}\text { Ti-49 } & 208 & \odot & 2.75590 \mathrm{E}-07 & 293.6 & \text { end } \\ \mathrm{Ti}-5 \odot & 208 & \odot & 2.63873 \mathrm{E}-07 & 293.6 & \text { end } \\ \mathrm{Cr}-5 \odot & 208 & \odot & 5.30517 \mathrm{E}-07 & 293.6 & \text { end } \\ \mathrm{Cr}-52 & 208 & \odot & 1.02189 \mathrm{E}-05 & 293.6 & \text { end } \\ \mathrm{Cr}-53 & 208 & \odot & 1.15860 \mathrm{E}-06 & 293.6 & \text { end } \\ \mathrm{Cr}-54 & 208 & \odot & 2.87821 \mathrm{E}-\odot 7 & 293.6 & \text { end } \\ \mathrm{Mn}-55 & 208 & \odot & 6.50452 \mathrm{E}-06 & 293.6 & \text { end } \\ \mathrm{Fe}-54 & 208 & \odot & 2.38070 \mathrm{E}-06 & 293.6 & \text { end } \\ \mathrm{Fe}-56 & 208 & \odot & 3.73384 \mathrm{E}-05 & 293.6 & \text { end } \\ \mathrm{Fe}-57 & 208 & \odot & 8.62751 \mathrm{E}-07 & 293.6 & \text { end } \\ \mathrm{Fe}-58 & 208 & \odot & 1.13948 \mathrm{E}-07 & 293.6 & \text { end } \\ \mathrm{Cu}-63 & 208 & \odot & 1.89779 \mathrm{E}-05 & 293.6 & \text { end } \\ \mathrm{Cu}-65 & 208 & \odot & 8.45870 \mathrm{E}-06 & 293.6 & \text { end } \\ \mathrm{U}-234 & 208 & \odot & 3.98927 \mathrm{E}-06 & 293.6 & \text { end } \\ \mathrm{U}-235 & 208 & \odot & 3.71800 \mathrm{E}-\odot 4 & 293.6 & \text { end } \\ \mathrm{U}-236 & 208 & \odot & 1.59572 \mathrm{E}-06 & 293.6 & \text { end } \\ \mathrm{U}-238 & 208 & \odot & 2.15420 \mathrm{E}-05 & 293.6 & \text { end } \\ \mathrm{I}-238 & & & & & \end{array}$

Outer Fuel element Material Descriptions

-

Aluminum Sidewalls

$\mathrm{H}-1$ $0-16$

Al -27

Si-28

Si-29

Si-30

Ti-46

$\mathrm{Ti}-47$

$\mathrm{Ti}-48$

Ti-49

Ti- 50

$\mathrm{Cr}-50$

$\mathrm{Cr}-52$

$\mathrm{Cr}-53$

$\mathrm{Cr}-54$

$\mathrm{Mn}-55$

$\mathrm{Fe}-54$

$\mathrm{Fe}-56$

$\mathrm{Fe}-57$

$\mathrm{Fe}-58$

$\mathrm{Cu}-63$

$\mathrm{Cu}-65$

$\mathrm{Mg}-24$

$\mathrm{Mg}-25$

$\mathrm{Mg}-26$

H-1

$0-16$

Al-27

Si-28

Si-29

Si-30

Ti-46

Ti-47

Ti-48

Ti-49

Ti-50

$\mathrm{Cr}-50$

Cr -52

Cr -53

Cr -54

Mn-55

$\mathrm{Fe}-54$

Fe-56

Fe-57

Fe-58

Cu- 63

$\mathrm{Cu}-65$

$\mathrm{Mg}-24$

Mg-25

Mg-26

\begin{tabular}{|c|c|c|c|c|c|}
\hline \multirow{26}{*}{ Outer } & fuel & \multicolumn{3}{|c|}{ element--upper uncontrolled } & \multirow{2}{*}{$\begin{array}{l}\text { region } \\
\text { end }\end{array}$} \\
\hline & 72 & $\odot$ & 3. 35240E- 02 & 293.6 & \\
\hline & 72 & $\odot$ & 1. $66756 \mathrm{E}-02$ & 293.6 & end \\
\hline & 72 & $\odot$ & $2.92741 E-02$ & 293.6 & end \\
\hline & 72 & $\odot$ & 1. $60187 E-\odot 4$ & 293.6 & end \\
\hline & 72 & $\odot$ & $8.11094 \mathrm{E}-06$ & 293.6 & end \\
\hline & 72 & $\odot$ & $5.38413 E-06$ & 293.6 & end \\
\hline & 72 & $\odot$ & 1. $05065 \mathrm{E}-06$ & 293.6 & end \\
\hline & 72 & $\odot$ & $9.47498 \mathrm{E}-07$ & 293.6 & end \\
\hline & 72 & $\odot$ & $9.38838 E-06$ & 293.6 & end \\
\hline & 72 & $\odot$ & $6.88973 E-07$ & 293.6 & end \\
\hline & 72 & $\odot$ & $6.59682 \mathrm{E}-07$ & 293.6 & end \\
\hline & 72 & $\odot$ & $1.32629 E-06$ & 293.6 & end \\
\hline & 72 & $\odot$ & $2.55471 E-05$ & 293.6 & nd \\
\hline & 72 & $\odot$ & $2.89649 E-06$ & 293.6 & end \\
\hline & 72 & $\odot$ & $7.19549 E-07$ & 293.6 & nd \\
\hline & 72 & $\odot$ & $1.10987 \mathrm{E}-05$ & 293.6 & end \\
\hline & 72 & $\odot$ & $2.98072 \mathrm{E}-06$ & 293.6 & end \\
\hline & 72 & $\odot$ & 4.67489E- 05 & 293.6 & end \\
\hline & 72 & 0 & 1. $08019 \mathrm{E}-06$ & 293.6 & end \\
\hline & 72 & $\odot$ & $1.42667 \mathrm{E}-07$ & 293.6 & end \\
\hline & 72 & 0 & $3.02466 \mathrm{E}-05$ & 293.6 & end \\
\hline & 72 & $\odot$ & $1.34813 E-05$ & 293.6 & end \\
\hline & 72 & $\odot$ & $2.64216 \mathrm{E}-04$ & 293.6 & end \\
\hline & 72 & $\odot$ & 3. 34493E- 05 & 293.6 & end \\
\hline & 72 & $\odot$ & $3.68277 \mathrm{E}-05$ & 293.6 & end \\
\hline Outer & fuel & eleme & nt--lower unc & ontrolled & region \\
\hline & 73 & $\odot$ & 3. $31702 \mathrm{E}-02$ & 293.6 & end \\
\hline & 73 & $\odot$ & $1.64987 \mathrm{E}-02$ & 293.6 & end \\
\hline & 73 & $\odot$ & $2.92741 E-02$ & 293.6 & end \\
\hline & 73 & $\odot$ & 1. $60187 E-\odot 4$ & 293.6 & end \\
\hline & 73 & $\odot$ & $8.11095 E-06$ & 293.6 & end \\
\hline & 73 & $\odot$ & $5.38414 \mathrm{E}-06$ & 293.6 & end \\
\hline & 73 & $\odot$ & $1.05065 E-06$ & 293.6 & end \\
\hline & 73 & $\odot$ & $9.47499 E-\odot 7$ & 293.6 & end \\
\hline & 73 & $\odot$ & $9.38839 E-06$ & 293.6 & end \\
\hline & 73 & $\odot$ & $6.88974 \mathrm{E}-\odot 7$ & 293.6 & end \\
\hline & 73 & $\odot$ & $6.59683 E-07$ & 293.6 & end \\
\hline & 73 & $\odot$ & 1. $32629 E-06$ & 293.6 & end \\
\hline & 73 & $\odot$ & $2.55471 E-05$ & 293.6 & end \\
\hline & 73 & $\odot$ & $2.89649 E-06$ & 293.6 & end \\
\hline & 73 & $\odot$ & $7.19550 E-07$ & 293.6 & end \\
\hline & 73 & $\odot$ & 1.10987E-05 & 293.6 & end \\
\hline & 73 & $\odot$ & $2.98072 E-06$ & 293.6 & end \\
\hline & 73 & $\odot$ & $4.67489 E-05$ & 293.6 & end \\
\hline & 73 & $\odot$ & 1. $08019 E-\odot 6$ & 293.6 & end \\
\hline & 73 & $\odot$ & $1.42667 \mathrm{E}-07$ & 293.6 & end \\
\hline & 73 & $\odot$ & $3.02466 \mathrm{E}-\odot 5$ & 293.6 & end \\
\hline & 73 & $\odot$ & 1. $34813 E-\odot 5$ & 293.6 & d \\
\hline & 73 & $\odot$ & $2.64216 E-\odot 4$ & 293.6 & \\
\hline & 73 & $\odot$ & $3.34493 E-05$ & 293.6 & \\
\hline & 73 & 0 & $3.68277 \mathrm{E}-05$ & 293.6 & end \\
\hline
\end{tabular}

Outer Fuel Element fueled region 1 
total atom density $=8.00583 \mathrm{E}-02 \mathrm{a} / \mathrm{b}-\mathrm{cm}$ 8. 005830E- 02

\begin{tabular}{|c|c|c|c|c|c|}
\hline $\mathrm{H}-1$ & 311 & $\odot$ & 3. 32434E- 02 & 293.6 & end \\
\hline $0-16$ & 311 & $\odot$ & 1. $77945 E-\odot 2$ & 293.6 & end \\
\hline $\mathrm{Mg}-24$ & 311 & $\odot$ & 1. $05686 \mathrm{E}-\odot 4$ & 293.6 & end \\
\hline$M g-25$ & 311 & $\odot$ & $1.33797 \mathrm{E}-05$ & 293.6 & nd \\
\hline $\mathrm{Mg}-26$ & 311 & $\odot$ & $1.47310 \mathrm{E}-05$ & 293.6 & $\mathrm{hd}$ \\
\hline Al-27 & 311 & $\odot$ & $2.82328 \mathrm{E}-\odot 2$ & 293.6 & nd \\
\hline Si-28 & 311 & $\odot$ & 1. $0 \odot 933 E-\odot 4$ & 293.6 & nd \\
\hline Si-29 & 311 & $\odot$ & $5.11066 \mathrm{E}-06$ & 293.6 & nd \\
\hline Si-30 & 311 & $\odot$ & 3. 39252E- 06 & 293.6 & nd \\
\hline Ti-46 & 311 & $\odot$ & $4.20262 \mathrm{E}-07$ & 293.6 & nd \\
\hline Ti-47 & 311 & $\odot$ & $3.79000 \mathrm{E}-07$ & 293.6 & nd \\
\hline Ti-48 & 311 & $\odot$ & $3.75536 \mathrm{E}-\odot 6$ & 293.6 & nd \\
\hline Ti-49 & 311 & $\odot$ & $2.75590 E-\odot 7$ & 293.6 & d \\
\hline Ti-50 & 311 & $\odot$ & $2.63873 E-07$ & 293.6 & d \\
\hline $\mathrm{Cr}-50$ & 311 & $\odot$ & $5.30517 E-07$ & 293.6 & id \\
\hline $\mathrm{Cr}-52$ & 311 & $\odot$ & 1. $\odot 2189 E-\odot 5$ & 293.6 & ed \\
\hline $\mathrm{Cr}-53$ & 311 & $\odot$ & 1. $15860 \mathrm{E}-\odot 6$ & 293.6 & d \\
\hline $\mathrm{Cr}-54$ & 311 & $\odot$ & $2.87821 E-07$ & 293.6 & id \\
\hline Mn-55 & 311 & $\odot$ & $6.48252 E-\odot 6$ & 293.6 & \\
\hline $\mathrm{Fe}-54$ & 311 & $\odot$ & $2.368 \odot 4 E-\odot 6$ & 293.6 & \\
\hline $\mathrm{Fe}-56$ & 311 & $\odot$ & $3.71398 \mathrm{E}-05$ & 293.6 & \\
\hline $\mathrm{Fe}-57$ & 311 & $\odot$ & $8.58163 E-07$ & 293.6 & \\
\hline $\mathrm{Fe}-58$ & 311 & $\odot$ & $1.13342 \mathrm{E}-07$ & 293.6 & \\
\hline $\mathrm{Cu}-63$ & 311 & $\odot$ & 1. $89046 \mathrm{E}-05$ & 293.6 & \\
\hline $\mathrm{Cu}-65$ & 311 & $\odot$ & $8.426 \odot 2 E-06$ & 293.6 & \\
\hline$U-234$ & 311 & $\odot$ & $4.52789 E-\odot 6$ & 293.6 & \\
\hline$U-235$ & 311 & $\odot$ & 4. $2200 \odot E-\odot 4$ & 293.6 & \\
\hline$U-236$ & 311 & $\odot$ & 1. $81117 \mathrm{E}-\odot 6$ & 293.6 & \\
\hline$U-238$ & 311 & $\odot$ & $2.44506 \mathrm{E}-05$ & 293.6 & d \\
\hline ' 8.00 & tom c & sity & $=8.00895 \mathrm{E}$ & $2 \mathrm{a} / \mathrm{b}-\mathrm{cm}$ & \\
\hline $\mathrm{H}-1$ & 312 & $\odot$ & 3. 32434E- 02 & 293.6 & end \\
\hline $0-16$ & 312 & $\odot$ & 1. $80329 E-02$ & 293.6 & \\
\hline $\mathrm{Mg}-24$ & 312 & $\odot$ & 1. $05686 E-\odot 4$ & 293.6 & \\
\hline$M g-25$ & 312 & $\odot$ & $1.33797 \mathrm{E}-05$ & 293.6 & \\
\hline $\mathrm{Mg}-26$ & 312 & $\odot$ & 1. $47310 \mathrm{E}-05$ & 293.6 & \\
\hline $\mathrm{Al}-27$ & 312 & $\odot$ & $2.79375 E-\odot 2$ & 293.6 & \\
\hline Si-28 & 312 & $\odot$ & 1. $00274 \mathrm{E}-\odot 4$ & 293.6 & \\
\hline Si-29 & 312 & $\odot$ & $5 . \odot 7732 E-\odot 6$ & 293.6 & id \\
\hline Si-30 & 312 & $\odot$ & $3.37038 \mathrm{E}-\odot 6$ & 293.6 & nd \\
\hline Ti-46 & 312 & $\odot$ & $4.20262 E-07$ & 293.6 & nd \\
\hline $\mathrm{Ti}-47$ & 312 & $\odot$ & $3.79000 \mathrm{E}-\odot 7$ & 293.6 & id \\
\hline Ti-48 & 312 & $\odot$ & $3.75536 \mathrm{E}-06$ & 293.6 & $d$ \\
\hline Ti-49 & 312 & $\odot$ & $2.75590 E-\odot 7$ & 293.6 & d \\
\hline Ti-50 & 312 & $\odot$ & $2.63873 E-07$ & 293.6 & $d$ \\
\hline $\mathrm{Cr}-50$ & 312 & $\odot$ & $5.30517 \mathrm{E}-07$ & 293.6 & dd \\
\hline $\mathrm{Cr}-52$ & 312 & $\odot$ & 1. $\odot 2189 E-\odot 5$ & 293.6 & ed \\
\hline $\mathrm{Cr}-53$ & 312 & $\odot$ & $1.1586 \odot E-\odot 6$ & 293.6 & nd \\
\hline $\mathrm{Cr}-54$ & 312 & $\odot$ & $2.87821 E-07$ & 293.6 & \\
\hline Mn-55 & 312 & $\odot$ & $6.446 \odot 2 E-\odot 6$ & 293.6 & \\
\hline $\mathrm{Fe}-54$ & 312 & $\odot$ & $2.347 \odot 4 \mathrm{E}-\odot 6$ & 293.6 & \\
\hline $\mathrm{Fe}-56$ & 312 & $\odot$ & 3. $68104 \mathrm{E}-\odot 5$ & 293.6 & nd \\
\hline $\mathrm{Fe}-57$ & 312 & 0 & $8.50550 \mathrm{E}-07$ & 293.6 & nd \\
\hline $\mathrm{Fe}-58$ & 312 & 0 & $1.12337 \mathrm{E}-07$ & 293.6 & end \\
\hline $\mathrm{Cu}-63$ & 312 & $\odot$ & $1.87830 \mathrm{E}-05$ & 293.6 & end \\
\hline $\mathrm{Cu}-65$ & 312 & $\odot$ & $8.37182 E-06$ & 293.6 & end \\
\hline$U-234$ & 312 & $\odot$ & $5.42167 E-06$ & 293.6 & end \\
\hline$U-235$ & 312 & $\odot$ & $5.05300 E-\odot 4$ & 293.6 & \\
\hline$U-236$ & 312 & $\odot$ & $2.16868 E-06$ & 293.6 & end \\
\hline$U-238$ & 312 & $\odot$ & 2. $92770 \mathrm{E}-05$ & 293.6 & nd \\
\hline ' 8.01 & tom & sity & $=8.01228 \mathrm{E}$ & $2 \mathrm{a} / \mathrm{b}-\mathrm{cm}$ & \\
\hline $\mathrm{H}-1$ & 313 & $\odot$ & 3. 32434E- 02 & 293.6 & end \\
\hline $0-16$ & 313 & $\odot$ & $1.82870 \mathrm{E}-\odot 2$ & 293.6 & d \\
\hline$M g-24$ & 313 & $\odot$ & 1. $05686 E-\odot 4$ & 293.6 & \\
\hline $\mathrm{Mg}-25$ & 313 & $\odot$ & $1.33797 \mathrm{E}-05$ & 293.6 & \\
\hline$M g-26$ & 313 & $\odot$ & $1.47310 \mathrm{E}-05$ & 293.6 & \\
\hline$A \mathrm{l}-27$ & 313 & $\odot$ & $2.76228 E-02$ & 293.6 & \\
\hline Si-28 & 313 & $\odot$ & $9.95724 \mathrm{E}-\odot 5$ & 293.6 & \\
\hline Si-29 & 313 & $\odot$ & $5.04178 \mathrm{E}-06$ & 293.6 & \\
\hline Si-30 & 313 & $\odot$ & 3. $34679 E-06$ & 293.6 & end \\
\hline Ti-46 & 313 & $\odot$ & $4.20262 E-07$ & 293.6 & end \\
\hline $\mathrm{Ti}-47$ & 313 & $\odot$ & 3. $7900 \odot E-\odot 7$ & 293.6 & end \\
\hline $\mathrm{Ti}-48$ & 313 & $\odot$ & $3.75536 E-06$ & 293.6 & end \\
\hline Ti-49 & 313 & $\odot$ & $2.75590 \mathrm{E}-\odot 7$ & 293.6 & end \\
\hline Ti-50 & 313 & $\odot$ & $2.63873 \mathrm{E}-07$ & 293.6 & \\
\hline $\mathrm{Cr}-50$ & 313 & $\odot$ & $5.30517 \mathrm{E}-07$ & 293.6 & \\
\hline $\mathrm{Cr}-52$ & 313 & $\odot$ & 1. $02189 \mathrm{E}-05$ & 293.6 & \\
\hline $\mathrm{Cr}-53$ & 313 & $\odot$ & $1.15860 \mathrm{E}-\odot 6$ & 293.6 & \\
\hline
\end{tabular}




\begin{tabular}{|c|c|c|c|c|c|}
\hline $\mathrm{Cr}-54$ & 313 & $\odot$ & $2.87821 E-07$ & 293.6 & end \\
\hline Mn-55 & 313 & 0 & $6.40711 \mathrm{E}-06$ & 293.6 & end \\
\hline $\mathrm{Fe}-54$ & 313 & 0 & $2.32464 \mathrm{E}-06$ & 293.6 & end \\
\hline $\mathrm{Fe}-56$ & 313 & $\odot$ & $3.64591 E-05$ & 293.6 & end \\
\hline $\mathrm{Fe}-57$ & 313 & 0 & $8.42433 E-07$ & 293.6 & end \\
\hline $\mathrm{Fe}-58$ & 313 & $\odot$ & 1.11265E- 07 & 293.6 & and \\
\hline $\mathrm{Cu}-63$ & 313 & $\odot$ & 1. $86534 \mathrm{E}-05$ & 293.6 & nd \\
\hline $\mathrm{Cu}-65$ & 313 & $\odot$ & $8.31405 E-06$ & 293.6 & nd \\
\hline$U-234$ & 313 & $\odot$ & $6.37446 E-06$ & 293.6 & nd \\
\hline$U-235$ & 313 & $\odot$ & $5.94100 \mathrm{E}-\odot 4$ & 293.6 & end \\
\hline U-236 & 313 & $\odot$ & $2.54980 E-06$ & 293.6 & nd \\
\hline$U-238$ & 313 & $\odot$ & $3.44221 E-05$ & 293.6 & end \\
\hline \multicolumn{3}{|c|}{ total atom density } & \multicolumn{2}{|c|}{$=8.01530 \mathrm{E}-02 \mathrm{a} / \mathrm{b}-\mathrm{cm}$} & ' $\quad 8.015300 \mathrm{E}-02$ \\
\hline $\mathrm{H}-1$ & 314 & $\odot$ & $3.32434 \mathrm{E}-02$ & 293.6 & end \\
\hline $0-16$ & 314 & $\odot$ & 1. $85176 \mathrm{E}-02$ & 293.6 & \\
\hline$M g-24$ & 314 & $\odot$ & 1. $05686 E-\odot 4$ & 293.6 & \\
\hline$M g-25$ & 314 & $\odot$ & $1.33797 \mathrm{E}-05$ & 293.6 & \\
\hline$M g-26$ & 314 & $\odot$ & $1.47310 \mathrm{E}-05$ & 293.6 & \\
\hline Al-27 & 314 & $\odot$ & $2.73372 \mathrm{E}-02$ & 293.6 & nd \\
\hline Si-28 & 314 & $\odot$ & $9.89350 E-05$ & 293.6 & end \\
\hline Si-29 & 314 & $\odot$ & 5. $00951 E-\odot 6$ & 293.6 & \\
\hline Si-30 & 314 & $\odot$ & 3. 32537E- 06 & 293.6 & \\
\hline $\mathrm{Ti}-46$ & 314 & $\odot$ & 4. $2 \odot 262 \mathrm{E}-07$ & 293.6 & \\
\hline $\mathrm{Ti}-47$ & 314 & $\odot$ & $3.7900 \odot E-\odot 7$ & 293.6 & \\
\hline $\mathrm{Ti}-48$ & 314 & $\odot$ & $3.75536 \mathrm{E}-06$ & 293.6 & \\
\hline Ti-49 & 314 & $\odot$ & $2.75590 E-07$ & 293.6 & \\
\hline Ti-50 & 314 & $\odot$ & $2.63873 E-07$ & 293.6 & \\
\hline $\mathrm{Cr}-50$ & 314 & $\odot$ & $5.30517 \mathrm{E}-07$ & 293.6 & \\
\hline $\mathrm{Cr}-52$ & 314 & $\odot$ & 1. $02189 E-05$ & 293.6 & \\
\hline $\mathrm{Cr}-53$ & 314 & $\odot$ & $1.15860 \mathrm{E}-06$ & 293.6 & \\
\hline $\mathrm{Cr}-54$ & 314 & $\odot$ & $2.87821 \mathrm{E}-07$ & 293.6 & \\
\hline$M n-55$ & 314 & $\odot$ & $6.37178 E-06$ & 293.6 & \\
\hline $\mathrm{Fe}-54$ & 314 & $\odot$ & $2.30432 \mathrm{E}-06$ & 293.6 & \\
\hline $\mathrm{Fe}-56$ & 314 & $\odot$ & $3.61403 E-05$ & 293.6 & \\
\hline $\mathrm{Fe}-57$ & 314 & $\odot$ & $8.35068 \mathrm{E}-07$ & 293.6 & \\
\hline $\mathrm{Fe}-58$ & 314 & $\odot$ & $1.10292 \mathrm{E}-07$ & 293.6 & \\
\hline $\mathrm{Cu}-63$ & 314 & $\odot$ & $1.85357 \mathrm{E}-05$ & 293.6 & \\
\hline $\mathrm{Cu}-65$ & 314 & $\odot$ & 8. 26161E- ๑6 & 293.6 & \\
\hline U-234 & 314 & $\odot$ & $7.23926 \mathrm{E}-06$ & 293.6 & \\
\hline$U-235$ & 314 & 0 & $6.74700 \mathrm{E}-\odot 4$ & 293.6 & \\
\hline$U-236$ & 314 & 0 & $2.89573 E-\odot 6$ & 293.6 & \\
\hline$U-238$ & 314 & 0 & $3.90920 \mathrm{E}-05$ & 293.6 & end \\
\hline \multicolumn{3}{|c|}{ total atom density } & \multicolumn{2}{|c|}{$=8.01437 \mathrm{E}-02 \mathrm{a} / \mathrm{b}-\mathrm{cm}$} & \\
\hline \multicolumn{6}{|c|}{ ' 8.014370E-02 } \\
\hline $\mathrm{H}-1$ & 315 & $\odot$ & $3.32434 \mathrm{E}-02$ & 293.6 & \\
\hline $0-16$ & 315 & $\odot$ & 1. $84469 \mathrm{E}-02$ & 293.6 & \\
\hline$M g-24$ & 315 & $\odot$ & 1. $05686 E-\odot 4$ & 293.6 & \\
\hline$M g-25$ & 315 & $\odot$ & $1.33797 \mathrm{E}-05$ & 293.6 & \\
\hline$M g-26$ & 315 & $\odot$ & 1. $47310 E-05$ & 293.6 & \\
\hline Al-27 & 315 & $\odot$ & $2.74247 \mathrm{E}-02$ & 293.6 & \\
\hline Si-28 & 315 & $\odot$ & $9.91306 \mathrm{E}-05$ & 293.6 & \\
\hline Si-29 & 315 & 0 & $5.01941 E-06$ & 293.6 & \\
\hline Si-30 & 315 & 0 & $3.33194 \mathrm{E}-06$ & 293.6 & \\
\hline $\mathrm{Ti}-46$ & 315 & 0 & $4.20262 \mathrm{E}-07$ & 293.6 & $\mathrm{dd}$ \\
\hline $\mathrm{Ti}-47$ & 315 & 0 & 3. $79000 \mathrm{E}-07$ & 293.6 & \\
\hline $\mathrm{Ti}-48$ & 315 & $\odot$ & $3.75536 \mathrm{E}-\odot 6$ & 293.6 & \\
\hline Ti-49 & 315 & 0 & $2.75590 \mathrm{E}-\odot 7$ & 293.6 & \\
\hline Ti-50 & 315 & $\odot$ & $2.63873 E-07$ & 293.6 & \\
\hline $\mathrm{Cr}-50$ & 315 & $\odot$ & 5. 30517E- -7 & 293.6 & \\
\hline $\mathrm{Cr}-52$ & 315 & $\odot$ & 1. $02189 E-05$ & 293.6 & \\
\hline $\mathrm{Cr}-53$ & 315 & $\odot$ & 1. $15860 E-\odot 6$ & 293.6 & \\
\hline $\mathrm{Cr}-54$ & 315 & $\odot$ & $2.87821 E-07$ & 293.6 & \\
\hline Mn- 55 & 315 & 0 & $6.38261 \mathrm{E}-06$ & 293.6 & \\
\hline $\mathrm{Fe}-54$ & 315 & $\odot$ & $2.31055 E-06$ & 293.6 & \\
\hline $\mathrm{Fe}-56$ & 315 & $\odot$ & $3.62380 E-05$ & 293.6 & \\
\hline $\mathrm{Fe}-57$ & 315 & $\odot$ & $8.37326 E-07$ & 293.6 & \\
\hline $\mathrm{Fe}-58$ & 315 & 0 & $1.10590 \mathrm{E}-07$ & 293.6 & \\
\hline $\mathrm{Cu}-63$ & 315 & 0 & $1.85717 \mathrm{E}-05$ & 293.6 & \\
\hline $\mathrm{Cu}-65$ & 315 & $\odot$ & $8.27767 \mathrm{E}-06$ & 293.6 & \\
\hline$U-234$ & 315 & $\odot$ & $6.97424 \mathrm{E}-\odot 6$ & 293.6 & \\
\hline$U-235$ & 315 & $\odot$ & $6.50000 E-\odot 4$ & 293.6 & \\
\hline$U-236$ & 315 & $\odot$ & $2.78972 \mathrm{E}-\odot 6$ & 293.6 & \\
\hline$U-238$ & 315 & $\odot$ & $3.76609 \mathrm{E}-05$ & 293.6 & \\
\hline & tom & sity & $=8.00985 \mathrm{E}$ & $2 \mathrm{a} / \mathrm{b}-\mathrm{cm}$ & \\
\hline ' $\quad 8.00$ & & & & & \\
\hline $\mathrm{H}-1$ & 316 & 0 & $3.32434 \mathrm{E}-02$ & 293.6 & \\
\hline $0-16$ & 316 & $\odot$ & $1.81013 \mathrm{E}-02$ & 293.6 & \\
\hline$M g-24$ & 316 & $\odot$ & 1. $05686 E-04$ & 293.6 & \\
\hline$M g-25$ & 316 & $\odot$ & 1.33797E- 05 & 293.6 & \\
\hline$M g-26$ & 316 & $\odot$ & $1.47310 \mathrm{E}-05$ & 293.6 & \\
\hline
\end{tabular}




\begin{tabular}{|c|c|c|c|c|c|}
\hline Al-27 & 316 & $\odot$ & $2.78528 E-02$ & 293.6 & end \\
\hline Si-28 & 316 & $\odot$ & 1. $00085 E-\odot 4$ & 293.6 & end \\
\hline Si-29 & 316 & $\odot$ & $5.06774 \mathrm{E}-06$ & 293.6 & end \\
\hline Si-30 & 316 & $\odot$ & $3.36403 E-\odot 6$ & 293.6 & end \\
\hline Ti-46 & 316 & $\odot$ & $4.2 \odot 262 \mathrm{E}-\odot 7$ & 293.6 & end \\
\hline Ti-47 & 316 & 0 & $3.79000 \mathrm{E}-07$ & 293.6 & end \\
\hline Ti-48 & 316 & $\odot$ & $3.75536 \mathrm{E}-\odot 6$ & 293.6 & end \\
\hline Ti-49 & 316 & $\odot$ & $2.75590 E-07$ & 293.6 & nd \\
\hline Ti-50 & 316 & $\odot$ & $2.63873 E-07$ & 293.6 & nd \\
\hline $\mathrm{Cr}-50$ & 316 & $\odot$ & $5.30517 \mathrm{E}-07$ & 293.6 & \\
\hline $\mathrm{Cr}-52$ & 316 & $\odot$ & 1. $02189 \mathrm{E}-\odot 5$ & 293.6 & nd \\
\hline $\mathrm{Cr}-53$ & 316 & $\odot$ & $1.15860 \mathrm{E}-\odot 6$ & 293.6 & end \\
\hline $\mathrm{Cr}-54$ & 316 & $\odot$ & $2.87821 E-07$ & 293.6 & end \\
\hline$M n-55$ & 316 & $\odot$ & $6.43555 \mathrm{E}-06$ & 293.6 & nd \\
\hline $\mathrm{Fe}-54$ & 316 & 0 & $2.34101 \mathrm{E}-06$ & 293.6 & ad \\
\hline $\mathrm{Fe}-56$ & 316 & $\odot$ & $3.67158 \mathrm{E}-05$ & 293.6 & nd \\
\hline $\mathrm{Fe}-57$ & 316 & $\odot$ & $8.48365 E-07$ & 293.6 & nd \\
\hline $\mathrm{Fe}-58$ & 316 & $\odot$ & $1.12048 \mathrm{E}-07$ & 293.6 & $\mathrm{hd}$ \\
\hline $\mathrm{Cu}-63$ & 316 & $\odot$ & $1.87481 E-05$ & 293.6 & \\
\hline $\mathrm{Cu}-65$ & 316 & $\odot$ & $8.35629 E-06$ & 293.6 & \\
\hline U-234 & 316 & $\odot$ & $5.67811 \mathrm{E}-06$ & 293.6 & \\
\hline$U-235$ & 316 & $\odot$ & $5.2920 \odot E-\odot 4$ & 293.6 & \\
\hline$U-236$ & 316 & $\odot$ & $2.27126 \mathrm{E}-\odot 6$ & 293.6 & nd \\
\hline$U-238$ & 316 & $\odot$ & \multirow{2}{*}{\multicolumn{2}{|c|}{ 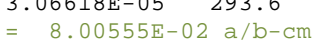 }} & \\
\hline \multirow{2}{*}{\multicolumn{6}{|c|}{ ' $8.005550 \mathrm{E}-02$}} \\
\hline & & & & & \\
\hline$H-1$ & 317 & $\odot$ & $3.32434 \mathrm{E}-02$ & 293.6 & nd \\
\hline $0-16$ & 317 & $\odot$ & $1.77731 \mathrm{E}-02$ & 293.6 & \\
\hline $\mathrm{Mg}-24$ & 317 & $\odot$ & 1. $05686 \mathrm{E}-\odot 4$ & 293.6 & \\
\hline $\mathrm{Mg}-25$ & 317 & $\odot$ & $1.33797 \mathrm{E}-05$ & 293.6 & \\
\hline $\mathrm{Mg}-26$ & 317 & $\odot$ & $1.47310 \mathrm{E}-05$ & 293.6 & \\
\hline Al-27 & 317 & $\odot$ & $2.82593 E-02$ & 293.6 & \\
\hline Si-28 & 317 & $\odot$ & 1. $0 \odot 993 E-\odot 4$ & 293.6 & \\
\hline Si-29 & 317 & $\odot$ & $5.11370 E-06$ & 293.6 & \\
\hline Si-30 & 317 & $\odot$ & 3. 39453E- 06 & 293.6 & \\
\hline $\mathrm{Ti}-46$ & 317 & $\odot$ & 4. $20262 \mathrm{E}-07$ & 293.6 & \\
\hline Ti-47 & 317 & 0 & $3.79000 \mathrm{E}-07$ & 293.6 & \\
\hline Ti-48 & 317 & $\odot$ & $3.75536 \mathrm{E}-06$ & 293.6 & \\
\hline Ti-49 & 317 & $\odot$ & $2.75590 E-07$ & 293.6 & \\
\hline Ti-50 & 317 & $\odot$ & $2.63873 E-07$ & 293.6 & nd \\
\hline $\mathrm{Cr}-50$ & 317 & $\odot$ & 5. 30517E- 07 & 293.6 & \\
\hline $\mathrm{Cr}-52$ & 317 & $\odot$ & 1. $02189 E-05$ & 293.6 & nd \\
\hline $\mathrm{Cr}-53$ & 317 & $\odot$ & $1.1586 \odot E-\odot 6$ & 293.6 & \\
\hline $\mathrm{Cr}-54$ & 317 & $\odot$ & $2.87821 \mathrm{E}-07$ & 293.6 & \\
\hline$M n-55$ & 317 & $\odot$ & $6.48581 \mathrm{E}-06$ & 293.6 & \\
\hline $\mathrm{Fe}-54$ & 317 & $\odot$ & 2. 36993E- $\odot 6$ & 293.6 & \\
\hline $\mathrm{Fe}-56$ & 317 & $\odot$ & $3.71695 \mathrm{E}-05$ & 293.6 & \\
\hline $\mathrm{Fe}-57$ & 317 & $\odot$ & $8.58848 E-07$ & 293.6 & \\
\hline $\mathrm{Fe}-58$ & 317 & $\odot$ & $1.13433 E-07$ & 293.6 & \\
\hline $\mathrm{Cu}-63$ & 317 & $\odot$ & $1.89155 \mathrm{E}-05$ & 293.6 & \\
\hline $\mathrm{Cu}-65$ & 317 & $\odot$ & $8.43090 E-06$ & 293.6 & \\
\hline$U-234$ & 317 & $\odot$ & $4.44742 \mathrm{E}-06$ & 293.6 & \\
\hline$U-235$ & 317 & $\odot$ & $4.14500 E-\odot 4$ & 293.6 & \\
\hline$U-236$ & 317 & $\odot$ & $1.77898 \mathrm{E}-06$ & 293.6 & \\
\hline$U-238$ & 317 & $\odot$ & 2. 40161E- 05 & 293.6 & and \\
\hline \multirow{2}{*}{\multicolumn{6}{|c|}{ ' $8 . \odot \odot 2710 \mathrm{E}-\odot 2$}} \\
\hline & & & & & \\
\hline $\mathrm{H}-1$ & 318 & $\odot$ & 3. 32434E- 02 & 293.6 & \\
\hline $0-16$ & 318 & $\odot$ & $1.75562 \mathrm{E}-02$ & 293.6 & \\
\hline$M g-24$ & 318 & $\odot$ & $1.05686 \mathrm{E}-\odot 4$ & 293.6 & \\
\hline$M g-25$ & 318 & $\odot$ & $1.33797 \mathrm{E}-05$ & 293.6 & \\
\hline$M g-26$ & 318 & $\odot$ & $1.47310 \mathrm{E}-05$ & 293.6 & \\
\hline Al-27 & 318 & $\odot$ & $2.85280 E-02$ & 293.6 & \\
\hline Si-28 & 318 & $\odot$ & 1. $01591 E-\odot 4$ & 293.6 & \\
\hline Si-29 & 318 & $\odot$ & $5.14401 E-06$ & 293.6 & \\
\hline $\mathrm{Si}-30$ & 318 & $\odot$ & $3.41465 E-06$ & 293.6 & \\
\hline $\mathrm{Ti}-46$ & 318 & $\odot$ & 4. $2 \odot 262 \mathrm{E}-07$ & 293.6 & \\
\hline Ti-47 & 318 & $\odot$ & $3.7900 \odot \mathrm{E}-\odot 7$ & 293.6 & \\
\hline Ti-48 & 318 & $\odot$ & $3.75536 \mathrm{E}-06$ & 293.6 & \\
\hline Ti-49 & 318 & $\odot$ & $2.75590 \mathrm{E}-\odot 7$ & 293.6 & \\
\hline Ti-50 & 318 & $\odot$ & $2.63873 E-07$ & 293.6 & \\
\hline $\mathrm{Cr}-50$ & 318 & $\odot$ & $5.30517 \mathrm{E}-07$ & 293.6 & \\
\hline $\mathrm{Cr}-52$ & 318 & $\odot$ & 1. $02189 E-\odot 5$ & 293.6 & \\
\hline $\mathrm{Cr}-53$ & 318 & $\odot$ & 1. $15860 E-06$ & 293.6 & \\
\hline $\mathrm{Cr}-54$ & 318 & $\odot$ & $2.87821 E-\odot 7$ & 293.6 & \\
\hline$M n-55$ & 318 & $\odot$ & $6.51903 E-\odot 6$ & 293.6 & \\
\hline $\mathrm{Fe}-54$ & 318 & $\odot$ & $2.38905 E-06$ & 293.6 & \\
\hline $\mathrm{Fe}-56$ & 318 & $\odot$ & $3.74693 E-05$ & 293.6 & \\
\hline $\mathrm{Fe}-57$ & 318 & $\odot$ & $8.65776 E-07$ & 293.6 & \\
\hline $\mathrm{Fe}-58$ & 318 & $\odot$ & $1.14348 E-07$ & 293.6 & \\
\hline Cu-63 & 318 & 0 & $1.90262 \mathrm{E}-05$ & 293.6 & \\
\hline
\end{tabular}




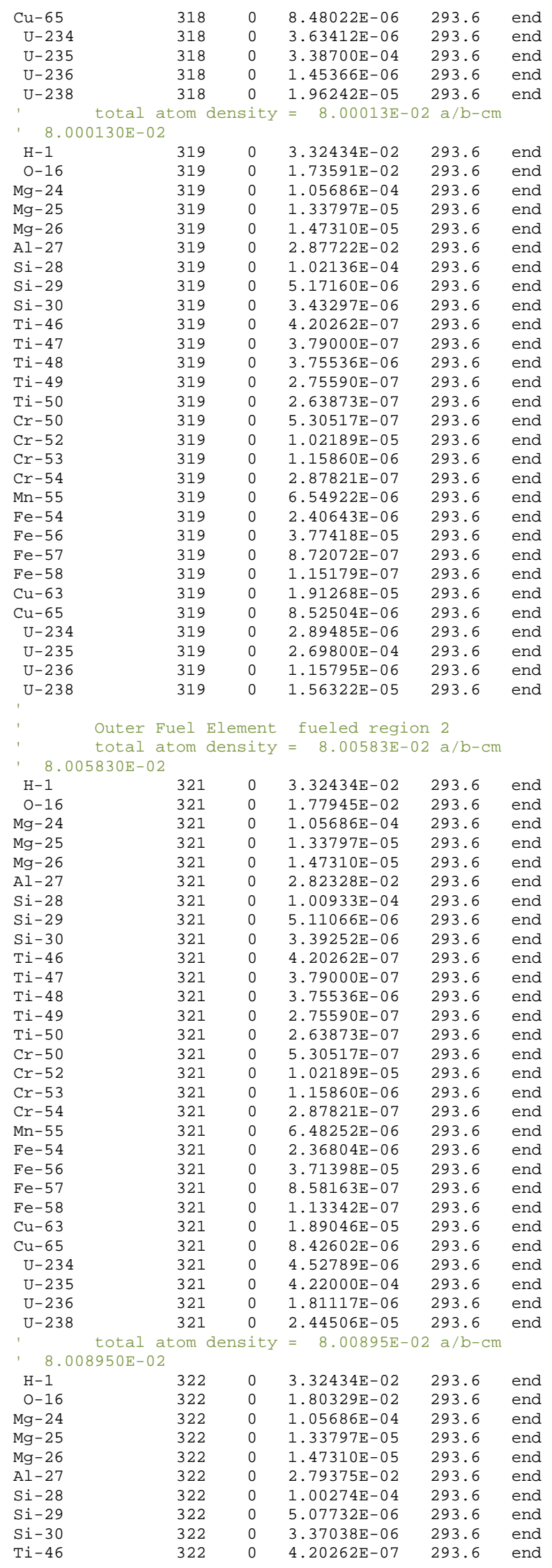




\begin{tabular}{|c|c|c|c|c|c|}
\hline $\mathrm{Ti}-47$ & 322 & $\odot$ & 3. $7900 \odot E-\odot 7$ & 293.6 & end \\
\hline Ti-48 & 322 & $\odot$ & $3.75536 \mathrm{E}-06$ & 293.6 & end \\
\hline $\mathrm{Ti}-49$ & 322 & $\odot$ & $2.75590 \mathrm{E}-07$ & 293.6 & end \\
\hline Ti-50 & 322 & $\odot$ & $2.63873 \mathrm{E}-07$ & 293.6 & end \\
\hline $\mathrm{cr}-50$ & 322 & $\odot$ & $5.30517 \mathrm{E}-07$ & 293.6 & end \\
\hline $\mathrm{Cr}-52$ & 322 & $\odot$ & 1. $02189 \mathrm{E}-05$ & 293.6 & end \\
\hline $\mathrm{Cr}-53$ & 322 & $\odot$ & 1. $15860 E-06$ & 293.6 & nd \\
\hline $\mathrm{Cr}-54$ & 322 & $\odot$ & $2.87821 \mathrm{E}-07$ & 293.6 & nd \\
\hline$M n-55$ & 322 & $\odot$ & $6.446 \odot 2 \mathrm{E}-\odot 6$ & 293.6 & nd \\
\hline $\mathrm{Fe}-54$ & 322 & $\odot$ & $2.347 \odot 4 \mathrm{E}-06$ & 293.6 & nd \\
\hline $\mathrm{Fe}-56$ & 322 & $\odot$ & 3. $68104 \mathrm{E}-05$ & 293.6 & nd \\
\hline $\mathrm{Fe}-57$ & 322 & $\odot$ & $8.50550 E-07$ & 293.6 & nd \\
\hline $\mathrm{Fe}-58$ & 322 & $\odot$ & 1. $12337 \mathrm{E}-07$ & 293.6 & nd \\
\hline $\mathrm{Cu}-63$ & 322 & $\odot$ & $1.87830 \mathrm{E}-05$ & 293.6 & nd \\
\hline $\mathrm{Cu}-65$ & 322 & $\odot$ & $8.37182 \mathrm{E}-06$ & 293.6 & nd \\
\hline $\mathrm{U}-234$ & 322 & $\odot$ & $5.42167 \mathrm{E}-06$ & 293.6 & end \\
\hline U-235 & 322 & $\odot$ & $5.05300 E-\odot 4$ & 293.6 & nd \\
\hline$U-236$ & 322 & $\odot$ & $2.16868 \mathrm{E}-06$ & 293.6 & nd \\
\hline$U-238$ & 322 & $\odot$ & $2.92770 \mathrm{E}-\odot 5$ & 293.6 & end \\
\hline \multicolumn{3}{|c|}{ total atom density } & \multicolumn{3}{|c|}{$=8.01228 \mathrm{E}-02 \mathrm{a} / \mathrm{b}-\mathrm{cm}$} \\
\hline $\mathrm{H}-1$ & 323 & $\odot$ & $3.32434 \mathrm{E}-02$ & 293.6 & \\
\hline $0-16$ & 323 & $\odot$ & 1. $82870 E-02$ & 293.6 & \\
\hline$M g-24$ & 323 & $\odot$ & 1. $05686 \mathrm{E}-04$ & 293.6 & \\
\hline$M g-25$ & 323 & $\odot$ & $1.33797 \mathrm{E}-05$ & 293.6 & ad \\
\hline$M g-26$ & 323 & $\odot$ & 1. $47310 E-05$ & 293.6 & \\
\hline Al-27 & 323 & $\odot$ & $2.76228 \mathrm{E}-02$ & 293.6 & id \\
\hline Si-28 & 323 & 0 & $9.95724 \mathrm{E}-05$ & 293.6 & \\
\hline Si-29 & 323 & $\odot$ & $5.04178 E-06$ & 293.6 & \\
\hline Si-30 & 323 & $\odot$ & $3.34679 E-06$ & 293.6 & \\
\hline $\mathrm{Ti}-46$ & 323 & $\odot$ & $4.20262 \mathrm{E}-07$ & 293.6 & \\
\hline $\mathrm{Ti}-47$ & 323 & $\odot$ & $3.79000 \mathrm{E}-\odot 7$ & 293.6 & \\
\hline $\mathrm{Ti}-48$ & 323 & $\odot$ & $3.75536 \mathrm{E}-06$ & 293.6 & \\
\hline $\mathrm{Ti}-49$ & 323 & $\odot$ & $2.75590 \mathrm{E}-07$ & 293.6 & \\
\hline Ti-50 & 323 & $\odot$ & $2.63873 \mathrm{E}-07$ & 293.6 & \\
\hline $\mathrm{Cr}-50$ & 323 & $\odot$ & $5.30517 \mathrm{E}-07$ & 293.6 & \\
\hline $\mathrm{Cr}-52$ & 323 & $\odot$ & 1. $02189 E-05$ & 293.6 & \\
\hline $\mathrm{Cr}-53$ & 323 & $\odot$ & 1. $15860 E-06$ & 293.6 & \\
\hline $\mathrm{Cr}-54$ & 323 & $\odot$ & $2.87821 \mathrm{E}-07$ & 293.6 & \\
\hline$M n-55$ & 323 & $\odot$ & $6.40711 \mathrm{E}-06$ & 293.6 & \\
\hline $\mathrm{Fe}-54$ & 323 & $\odot$ & $2.32464 \mathrm{E}-06$ & 293.6 & \\
\hline $\mathrm{Fe}-56$ & 323 & $\odot$ & $3.64591 \mathrm{E}-05$ & 293.6 & \\
\hline $\mathrm{Fe}-57$ & 323 & $\odot$ & $8.42433 E-07$ & 293.6 & \\
\hline $\mathrm{Fe}-58$ & 323 & $\odot$ & 1. $11265 \mathrm{E}-07$ & 293.6 & \\
\hline $\mathrm{Cu}-63$ & 323 & $\odot$ & $1.86534 \mathrm{E}-05$ & 293.6 & \\
\hline $\mathrm{Cu}-65$ & 323 & $\odot$ & $8.31405 E-06$ & 293.6 & \\
\hline U-234 & 323 & $\odot$ & $6.37446 E-06$ & 293.6 & \\
\hline U-235 & 323 & $\odot$ & $5.94100 E-04$ & 293.6 & \\
\hline$U-236$ & 323 & $\odot$ & $2.54980 E-06$ & 293.6 & \\
\hline$U-238$ & 323 & $\odot$ & $3.44221 \mathrm{E}-05$ & 293.6 & \\
\hline
\end{tabular}

total atom density $=8.01530 \mathrm{E}-02 \mathrm{a} / \mathrm{b}-\mathrm{cm}$

\begin{tabular}{|c|c|c|c|c|c|}
\hline $\mathrm{H}-1$ & 324 & $\odot$ & 3. $32434 \mathrm{E}-02$ & 293.6 & end \\
\hline $0-16$ & 324 & $\odot$ & 1. $85176 \mathrm{E}-\odot 2$ & 293.6 & end \\
\hline$M g-24$ & 324 & $\odot$ & 1. $05686 E-\odot 4$ & 293.6 & end \\
\hline$M g-25$ & 324 & $\odot$ & $1.33797 \mathrm{E}-\odot 5$ & 293.6 & end \\
\hline$M g-26$ & 324 & $\odot$ & $1.47310 \mathrm{E}-05$ & 293.6 & end \\
\hline Al-27 & 324 & $\odot$ & $2.73372 \mathrm{E}-\odot 2$ & 293.6 & end \\
\hline Si-28 & 324 & $\odot$ & $9.89350 \mathrm{E}-05$ & 293.6 & end \\
\hline Si-29 & 324 & $\odot$ & $5.00951 E-\odot 6$ & 293.6 & ad \\
\hline $\mathrm{Si}-30$ & 324 & $\odot$ & $3.32537 \mathrm{E}-06$ & 293.6 & id \\
\hline Ti-46 & 324 & $\odot$ & $4.20262 \mathrm{E}-07$ & 293.6 & $\mathrm{ad}$ \\
\hline $\mathrm{Ti}-47$ & 324 & $\odot$ & $3.79000 \mathrm{E}-07$ & 293.6 & $\mathrm{ad}$ \\
\hline Ti-48 & 324 & $\odot$ & $3.75536 \mathrm{E}-\odot 6$ & 293.6 & nd \\
\hline $\mathrm{Ti}-49$ & 324 & $\odot$ & $2.75590 E-07$ & 293.6 & nd \\
\hline Ti-50 & 324 & $\odot$ & $2.63873 E-07$ & 293.6 & nd \\
\hline $\mathrm{Cr}-50$ & 324 & $\odot$ & $5.30517 \mathrm{E}-07$ & 293.6 & nd \\
\hline $\mathrm{Cr}-52$ & 324 & $\odot$ & 1. $02189 E-05$ & 293.6 & end \\
\hline $\mathrm{Cr}-53$ & 324 & $\odot$ & $1.15860 E-\odot 6$ & 293.6 & nd \\
\hline $\mathrm{Cr}-54$ & 324 & $\odot$ & $2.87821 E-07$ & 293.6 & nd \\
\hline$M n-55$ & 324 & $\odot$ & $6.37178 E-06$ & 293.6 & \\
\hline $\mathrm{Fe}-54$ & 324 & $\odot$ & $2.30432 \mathrm{E}-06$ & 293.6 & id \\
\hline $\mathrm{Fe}-56$ & 324 & $\odot$ & $3.61403 \mathrm{E}-05$ & 293.6 & $\mathrm{ad}$ \\
\hline $\mathrm{Fe}-57$ & 324 & $\odot$ & $8.35068 \mathrm{E}-07$ & 293.6 & \\
\hline $\mathrm{Fe}-58$ & 324 & $\odot$ & $1.10292 \mathrm{E}-07$ & 293.6 & \\
\hline $\mathrm{Cu}-63$ & 324 & $\odot$ & $1.85357 \mathrm{E}-05$ & 293.6 & \\
\hline $\mathrm{Cu}-65$ & 324 & $\odot$ & $8.26161 E-06$ & 293.6 & \\
\hline$U-234$ & 324 & $\odot$ & $7.23926 \mathrm{E}-06$ & 293.6 & \\
\hline$U-235$ & 324 & $\odot$ & $6.74700 \mathrm{E}-\odot 4$ & 293.6 & \\
\hline$U-236$ & 324 & $\odot$ & $2.89573 E-\odot 6$ & 293.6 & \\
\hline U-238 & 324 & $\odot$ & 3. $90920 \mathrm{E}-\odot 5$ & 293.6 & \\
\hline
\end{tabular}




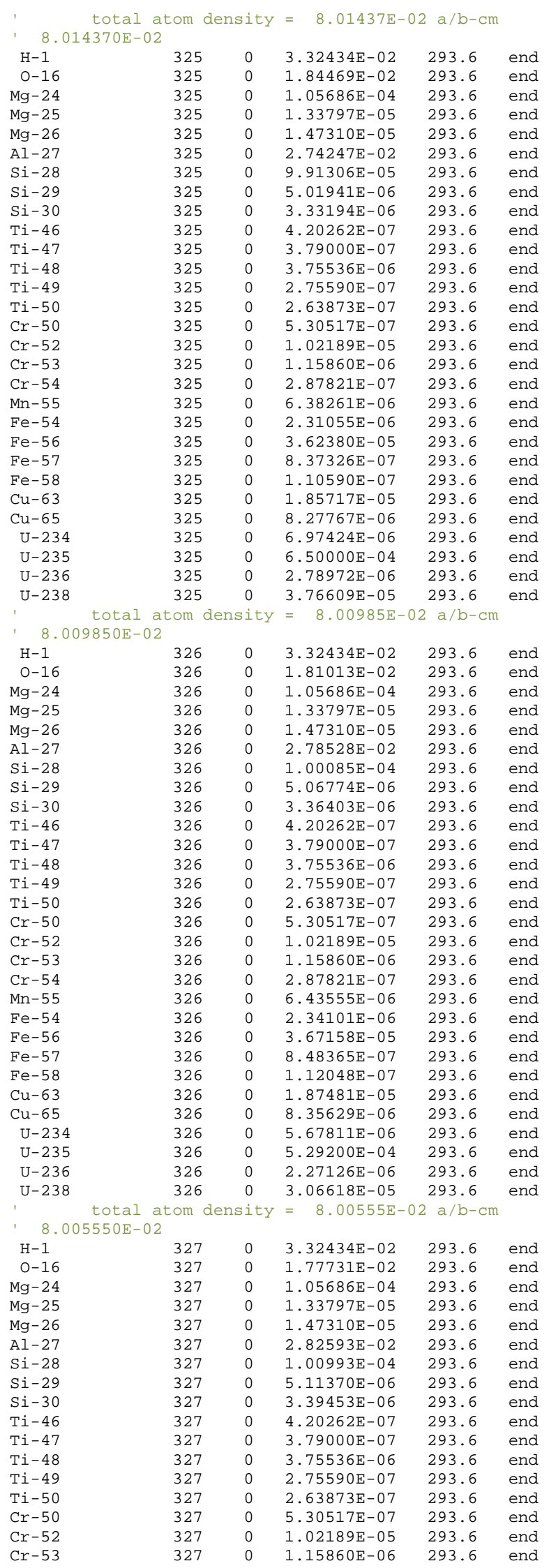




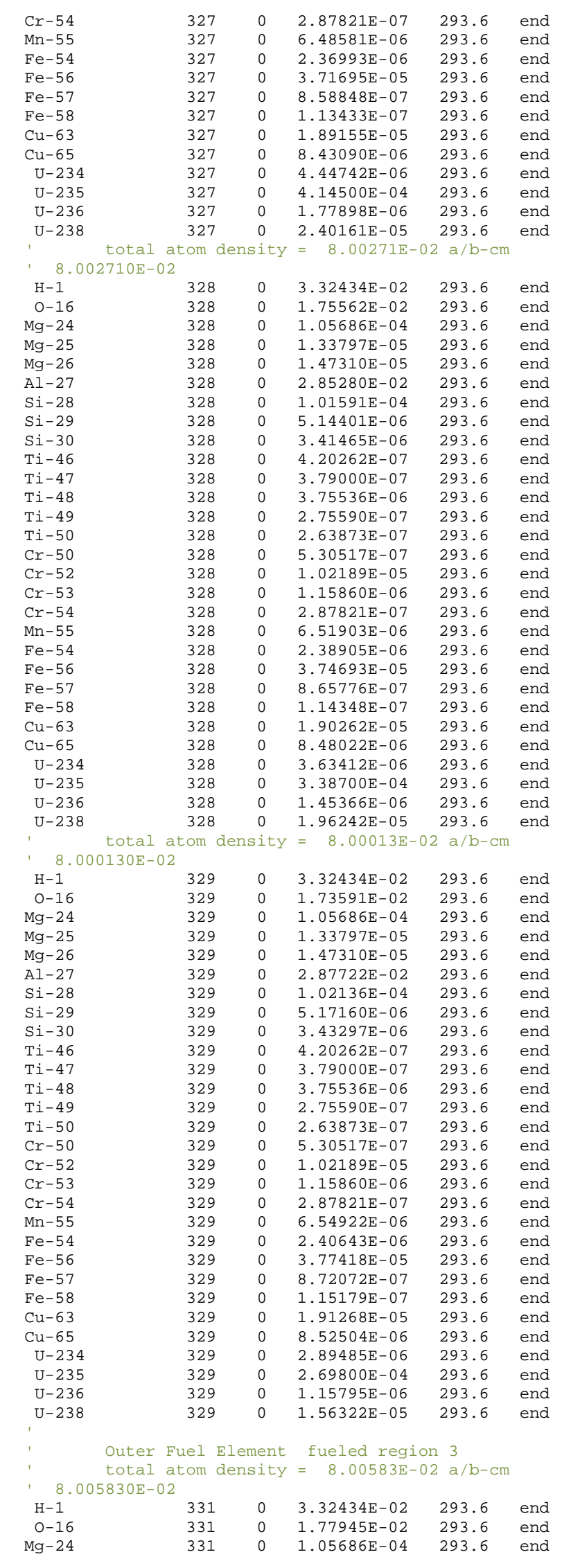




\begin{tabular}{|c|c|c|c|c|c|}
\hline$M g-25$ & 331 & $\odot$ & $1.33797 \mathrm{E}-05$ & 293.6 & end \\
\hline$M g-26$ & 331 & $\odot$ & $1.47310 \mathrm{E}-05$ & 293.6 & end \\
\hline Al-27 & 331 & $\odot$ & $2.82328 E-02$ & 293.6 & end \\
\hline Si-28 & 331 & $\odot$ & 1. $0 \odot 933 E-\odot 4$ & 293.6 & end \\
\hline Si-29 & 331 & $\odot$ & $5.11066 \mathrm{E}-06$ & 293.6 & end \\
\hline Si-30 & 331 & 0 & 3. 39252E- 06 & 293.6 & end \\
\hline Ti-46 & 331 & $\odot$ & $4.20262 \mathrm{E}-\odot 7$ & 293.6 & and \\
\hline $\mathrm{Ti}-47$ & 331 & $\odot$ & 3. $79000 \mathrm{E}-07$ & 293.6 & nd \\
\hline $\mathrm{Ti}-48$ & 331 & $\odot$ & $3.75536 \mathrm{E}-\odot 6$ & 293.6 & nd \\
\hline $\mathrm{Ti}-49$ & 331 & $\odot$ & $2.75590 E-\odot 7$ & 293.6 & \\
\hline Ti-5๑ & 331 & $\odot$ & $2.63873 \mathrm{E}-07$ & 293.6 & nd \\
\hline $\mathrm{Cr}-50$ & 331 & $\odot$ & $5.30517 \mathrm{E}-\odot 7$ & 293.6 & end \\
\hline $\mathrm{Cr}-52$ & 331 & $\odot$ & 1. $02189 \mathrm{E}-05$ & 293.6 & nd \\
\hline $\mathrm{Cr}-53$ & 331 & $\odot$ & $1.15860 E-06$ & 293.6 & nd \\
\hline $\mathrm{Cr}-54$ & 331 & 0 & $2.87821 \mathrm{E}-07$ & 293.6 & ad \\
\hline Mn-55 & 331 & $\odot$ & $6.48252 \mathrm{E}-06$ & 293.6 & nd \\
\hline $\mathrm{Fe}-54$ & 331 & $\odot$ & $2.36804 \mathrm{E}-06$ & 293.6 & $\mathrm{ad}$ \\
\hline $\mathrm{Fe}-56$ & 331 & $\odot$ & $3.71398 \mathrm{E}-05$ & 293.6 & $\mathrm{hd}$ \\
\hline $\mathrm{Fe}-57$ & 331 & $\odot$ & $8.58163 E-07$ & 293.6 & \\
\hline $\mathrm{Fe}-58$ & 331 & $\odot$ & $1.13342 \mathrm{E}-07$ & 293.6 & ad \\
\hline $\mathrm{Cu}-63$ & 331 & $\odot$ & 1. $89046 \mathrm{E}-\odot 5$ & 293.6 & nd \\
\hline $\mathrm{Cu}-65$ & 331 & $\odot$ & $8.42602 E-06$ & 293.6 & \\
\hline U-234 & 331 & $\odot$ & $4.52789 E-\odot 6$ & 293.6 & ad \\
\hline$U-235$ & 331 & $\odot$ & $4.22000 E-\odot 4$ & 293.6 & \\
\hline$U-236$ & 331 & $\odot$ & 1. $81117 \mathrm{E}-06$ & 293.6 & \\
\hline$U-238$ & 331 & $\odot$ & $2.44506 \mathrm{E}-\odot 5$ & 293.6 & \\
\hline \multicolumn{3}{|c|}{ total atom density } & \multicolumn{3}{|c|}{$=8.00895 \mathrm{E}-02 \mathrm{a} / \mathrm{b}-\mathrm{cm}$} \\
\hline $\mathrm{H}-1$ & 332 & $\odot$ & 3. 32434E- -2 & 293.6 & nd \\
\hline $0-16$ & 332 & $\odot$ & 1. $80329 \mathrm{E}-02$ & 293.6 & \\
\hline$M g-24$ & 332 & $\odot$ & 1. $05686 \mathrm{E}-\odot 4$ & 293.6 & \\
\hline$M g-25$ & 332 & $\odot$ & $1.33797 \mathrm{E}-05$ & 293.6 & \\
\hline$M g-26$ & 332 & $\odot$ & $1.47310 \mathrm{E}-05$ & 293.6 & \\
\hline Al-27 & 332 & $\odot$ & $2.79375 \mathrm{E}-02$ & 293.6 & \\
\hline Si-28 & 332 & $\odot$ & 1. $\odot \odot 274 E-\odot 4$ & 293.6 & \\
\hline Si-29 & 332 & $\odot$ & $5.07732 \mathrm{E}-06$ & 293.6 & \\
\hline Si-30 & 332 & $\odot$ & $3.37038 \mathrm{E}-06$ & 293.6 & \\
\hline Ti-46 & 332 & 0 & $4.20262 \mathrm{E}-07$ & 293.6 & \\
\hline $\mathrm{Ti}-47$ & 332 & $\odot$ & 3. $79000 \mathrm{E}-07$ & 293.6 & \\
\hline $\mathrm{Ti}-48$ & 332 & $\odot$ & $3.75536 \mathrm{E}-06$ & 293.6 & \\
\hline Ti-49 & 332 & $\odot$ & $2.75590 E-\odot 7$ & 293.6 & \\
\hline Ti-50 & 332 & $\odot$ & $2.63873 E-07$ & 293.6 & nd \\
\hline $\mathrm{Cr}-50$ & 332 & $\odot$ & 5. 30517E- $\odot 7$ & 293.6 & \\
\hline $\mathrm{Cr}-52$ & 332 & $\odot$ & 1. $02189 E-05$ & 293.6 & \\
\hline $\mathrm{Cr}-53$ & 332 & $\odot$ & $1.15860 \mathrm{E}-06$ & 293.6 & \\
\hline $\mathrm{Cr}-54$ & 332 & $\odot$ & $2.87821 \mathrm{E}-07$ & 293.6 & \\
\hline$M n-55$ & 332 & $\odot$ & $6.44602 \mathrm{E}-06$ & 293.6 & \\
\hline $\mathrm{Fe}-54$ & 332 & $\odot$ & $2.347 \odot 4 \mathrm{E}-\odot 6$ & 293.6 & \\
\hline $\mathrm{Fe}-56$ & 332 & 0 & $3.68104 \mathrm{E}-05$ & 293.6 & \\
\hline $\mathrm{Fe}-57$ & 332 & $\odot$ & $8.50550 E-07$ & 293.6 & \\
\hline $\mathrm{Fe}-58$ & 332 & $\odot$ & $1.12337 \mathrm{E}-07$ & 293.6 & \\
\hline $\mathrm{Cu}-63$ & 332 & $\odot$ & 1. $87830 E-05$ & 293.6 & \\
\hline $\mathrm{Cu}-65$ & 332 & $\odot$ & $8.37182 E-06$ & 293.6 & \\
\hline U-234 & 332 & $\odot$ & $5.42167 \mathrm{E}-06$ & 293.6 & \\
\hline$U-235$ & 332 & $\odot$ & $5.05300 E-04$ & 293.6 & \\
\hline$U-236$ & 332 & $\odot$ & $2.16868 E-06$ & 293.6 & \\
\hline$U-238$ & 332 & $\odot$ & \multicolumn{2}{|c|}{$\begin{array}{l}2.92770 E-05 \quad 293.6 \\
=\quad 801228 E-022 / b-c m\end{array}$} & end \\
\hline \multicolumn{3}{|c|}{$\begin{array}{l}\text { total atom density } \\
\text {. } 8.012280 \mathrm{E}-02\end{array}$} & $=8.01228 \mathrm{E}$ & $2 a / b-c m$ & \\
\hline $\mathrm{H}-1$ & 333 & $\odot$ & 3. 32434E- 02 & 293.6 & \\
\hline $0-16$ & 333 & $\odot$ & $1.82870 E-02$ & 293.6 & \\
\hline$M g-24$ & 333 & $\odot$ & 1. $05686 E-\odot 4$ & 293.6 & \\
\hline$M g-25$ & 333 & $\odot$ & $1.33797 \mathrm{E}-05$ & 293.6 & \\
\hline$M g-26$ & 333 & $\odot$ & 1. $47310 \mathrm{E}-05$ & 293.6 & \\
\hline Al-27 & 333 & $\odot$ & $2.76228 E-02$ & 293.6 & \\
\hline $\mathrm{Si}-28$ & 333 & $\odot$ & $9.95724 \mathrm{E}-\odot 5$ & 293.6 & \\
\hline Si-29 & 333 & $\odot$ & $5.04178 \mathrm{E}-\odot 6$ & 293.6 & \\
\hline Si-30 & 333 & $\odot$ & $3.34679 \mathrm{E}-06$ & 293.6 & \\
\hline Ti-46 & 333 & $\odot$ & 4. $2 \odot 262 \mathrm{E}-\odot 7$ & 293.6 & \\
\hline $\mathrm{Ti}-47$ & 333 & $\odot$ & $3.7900 \odot E-\odot 7$ & 293.6 & \\
\hline $\mathrm{Ti}-48$ & 333 & $\odot$ & $3.75536 \mathrm{E}-06$ & 293.6 & \\
\hline Ti-49 & 333 & $\odot$ & $2.75590 E-07$ & 293.6 & \\
\hline Ti-50 & 333 & $\odot$ & $2.63873 E-07$ & 293.6 & \\
\hline $\mathrm{Cr}-50$ & 333 & $\odot$ & $5.30517 \mathrm{E}-07$ & 293.6 & \\
\hline $\mathrm{Cr}-52$ & 333 & $\odot$ & 1. $02189 E-\odot 5$ & 293.6 & \\
\hline $\mathrm{Cr}-53$ & 333 & $\odot$ & $1.15860 \mathrm{E}-\odot 6$ & 293.6 & \\
\hline $\mathrm{Cr}-54$ & 333 & $\odot$ & $2.87821 \mathrm{E}-07$ & 293.6 & \\
\hline$M n-55$ & 333 & $\odot$ & $6.40711 \mathrm{E}-06$ & 293.6 & \\
\hline $\mathrm{Fe}-54$ & 333 & $\odot$ & $2.32464 \mathrm{E}-06$ & 293.6 & \\
\hline $\mathrm{Fe}-56$ & 333 & $\odot$ & 3. $64591 \mathrm{E}-05$ & 293.6 & \\
\hline $\mathrm{Fe}-57$ & 333 & 0 & $8.42433 E-07$ & 293.6 & \\
\hline
\end{tabular}




\begin{tabular}{|c|c|c|c|c|c|}
\hline $\mathrm{Fe}-58$ & 333 & $\odot$ & $1.11265 E-07$ & 293.6 & end \\
\hline $\mathrm{Cu}-63$ & 333 & 0 & 1. 86534E- 05 & 293.6 & end \\
\hline $\mathrm{Cu}-65$ & 333 & $\odot$ & 8. 31405E- 06 & 293.6 & end \\
\hline$U-234$ & 333 & $\odot$ & $6.37446 E-06$ & 293.6 & end \\
\hline$U-235$ & 333 & $\odot$ & $5.94100 E-04$ & 293.6 & end \\
\hline$U-236$ & 333 & 0 & $2.54980 E-\odot 6$ & 293.6 & nd \\
\hline$U-238$ & 333 & 0 & $3.44221 \mathrm{E}-05$ & 293.6 & end \\
\hline \multicolumn{3}{|c|}{ total atom density } & \multicolumn{3}{|c|}{ ' $\quad 8.015300 \mathrm{E}-02$} \\
\hline $\mathrm{H}-1$ & 334 & $\odot$ & 3. 32434E- 02 & 293.6 & end \\
\hline $0-16$ & 334 & $\odot$ & 1. 85176E- 02 & 293.6 & nd \\
\hline$M g-24$ & 334 & $\odot$ & 1. $05686 \mathrm{E}-\odot 4$ & 293.6 & רd \\
\hline $\mathrm{Mg}-25$ & 334 & $\odot$ & 1. $33797 \mathrm{E}-05$ & 293.6 & רd \\
\hline $\mathrm{Mg}-26$ & 334 & $\odot$ & $1.47310 \mathrm{E}-05$ & 293.6 & רd \\
\hline $\mathrm{Al}-27$ & 334 & $\odot$ & $2.73372 \mathrm{E}-02$ & 293.6 & nd \\
\hline Si-28 & 334 & $\odot$ & $9.89350 E-05$ & 293.6 & nd \\
\hline Si-29 & 334 & $\odot$ & $5.00951 E-\odot 6$ & 293.6 & ad \\
\hline Si-30 & 334 & $\odot$ & $3.32537 \mathrm{E}-06$ & 293.6 & nd \\
\hline Ti-46 & 334 & $\odot$ & $4.20262 \mathrm{E}-\odot 7$ & 293.6 & id \\
\hline $\mathrm{Ti}-47$ & 334 & $\odot$ & $3.79000 \mathrm{E}-07$ & 293.6 & d \\
\hline $\mathrm{Ti}-48$ & 334 & $\odot$ & $3.75536 \mathrm{E}-06$ & 293.6 & \\
\hline Ti-49 & 334 & 0 & $2.75590 \mathrm{E}-07$ & 293.6 & \\
\hline Ti-50 & 334 & 0 & $2.63873 \mathrm{E}-07$ & 293.6 & id \\
\hline $\mathrm{Cr}-50$ & 334 & $\odot$ & $5.30517 \mathrm{E}-07$ & 293.6 & nd \\
\hline $\mathrm{Cr}-52$ & 334 & $\odot$ & 1. $02189 \mathrm{E}-05$ & 293.6 & end \\
\hline $\mathrm{Cr}-53$ & 334 & $\odot$ & $1.15860 \mathrm{E}-06$ & 293.6 & nd \\
\hline $\mathrm{Cr}-54$ & 334 & $\odot$ & $2.87821 E-07$ & 293.6 & nd \\
\hline$M n-55$ & 334 & 0 & $6.37178 \mathrm{E}-\odot 6$ & 293.6 & nd \\
\hline $\mathrm{Fe}-54$ & 334 & $\odot$ & $2.30432 \mathrm{E}-\odot 6$ & 293.6 & nd \\
\hline $\mathrm{Fe}-56$ & 334 & $\odot$ & $3.61403 E-05$ & 293.6 & רd \\
\hline $\mathrm{Fe}-57$ & 334 & $\odot$ & $8.35068 \mathrm{E}-\odot 7$ & 293.6 & १d \\
\hline $\mathrm{Fe}-58$ & 334 & $\odot$ & 1. $10292 \mathrm{E}-07$ & 293.6 & nd \\
\hline $\mathrm{Cu}-63$ & 334 & $\odot$ & $1.85357 \mathrm{E}-05$ & 293.6 & ad \\
\hline $\mathrm{Cu}-65$ & 334 & $\odot$ & $8.26161 \mathrm{E}-06$ & 293.6 & nd \\
\hline$U-234$ & 334 & $\odot$ & $7.23926 \mathrm{E}-06$ & 293.6 & \\
\hline$U-235$ & 334 & $\odot$ & $6.74700 \mathrm{E}-04$ & 293.6 & \\
\hline$U-236$ & 334 & $\odot$ & $2.89573 E-\odot 6$ & 293.6 & \\
\hline$U-238$ & 334 & $\odot$ & 3. $90920 E-\odot 5$ & 293.6 & end \\
\hline \multicolumn{3}{|c|}{ total atom density } & $=8.01437 \mathrm{E}$ & $2 \mathrm{a} / \mathrm{b}-\mathrm{cm}$ & \\
\hline $\mathrm{H}-1$ & 335 & $\odot$ & 3. 32434E- -2 & 293.6 & end \\
\hline $0-16$ & 335 & $\odot$ & 1. $84469 \mathrm{E}-02$ & 293.6 & \\
\hline Mg-24 & 335 & 0 & 1. $05686 \mathrm{E}-\odot 4$ & 293.6 & \\
\hline$M g-25$ & 335 & 0 & $1.33797 \mathrm{E}-05$ & 293.6 & ad \\
\hline $\mathrm{Mg}-26$ & 335 & $\odot$ & $1.47310 \mathrm{E}-05$ & 293.6 & \\
\hline $\mathrm{Al}-27$ & 335 & $\odot$ & $2.74247 \mathrm{E}-02$ & 293.6 & a \\
\hline $\mathrm{Si}-28$ & 335 & $\odot$ & $9.91306 \mathrm{E}-05$ & 293.6 & nd \\
\hline Si-29 & 335 & $\odot$ & 5. $01941 E-06$ & 293.6 & \\
\hline Si-30 & 335 & $\odot$ & $3.33194 \mathrm{E}-\odot 6$ & 293.6 & \\
\hline Ti-46 & 335 & $\odot$ & $4.2 \odot 262 \mathrm{E}-\odot 7$ & 293.6 & \\
\hline Ti-47 & 335 & $\odot$ & $3.7900 \odot E-\odot 7$ & 293.6 & \\
\hline Ti-48 & 335 & $\odot$ & $3.75536 \mathrm{E}-06$ & 293.6 & \\
\hline Ti-49 & 335 & $\odot$ & $2.75590 \mathrm{E}-\odot 7$ & 293.6 & \\
\hline Ti-50 & 335 & $\odot$ & $2.63873 E-07$ & 293.6 & \\
\hline $\mathrm{Cr}-50$ & 335 & 0 & $5.30517 \mathrm{E}-07$ & 293.6 & \\
\hline $\mathrm{Cr}-52$ & 335 & $\odot$ & 1. $02189 \mathrm{E}-05$ & 293.6 & \\
\hline $\mathrm{Cr}-53$ & 335 & $\odot$ & $1.15860 E-06$ & 293.6 & \\
\hline $\mathrm{Cr}-54$ & 335 & $\odot$ & $2.87821 E-07$ & 293.6 & \\
\hline$M n-55$ & 335 & $\odot$ & $6.38261 E-06$ & 293.6 & \\
\hline $\mathrm{Fe}-54$ & 335 & $\odot$ & $2.31055 \mathrm{E}-06$ & 293.6 & nd \\
\hline $\mathrm{Fe}-56$ & 335 & $\odot$ & $3.62380 E-05$ & 293.6 & nd \\
\hline $\mathrm{Fe}-57$ & 335 & $\odot$ & $8.37326 E-07$ & 293.6 & nd \\
\hline $\mathrm{Fe}-58$ & 335 & 0 & 1. 10590E- 07 & 293.6 & \\
\hline Cu-63 & 335 & 0 & $1.85717 \mathrm{E}-05$ & 293.6 & \\
\hline Cu-65 & 335 & 0 & $8.27767 \mathrm{E}-06$ & 293.6 & \\
\hline$U-234$ & 335 & 0 & $6.97424 \mathrm{E}-06$ & 293.6 & \\
\hline U-235 & 335 & 0 & $6.50000 \mathrm{E}-\odot 4$ & 293.6 & \\
\hline$U-236$ & 335 & $\odot$ & $2.78972 \mathrm{E}-06$ & 293.6 & \\
\hline$U-238$ & 335 & 0 & $3.76609 \mathrm{E}-05$ & 293.6 & . \\
\hline ' & tom & sity & $=8.00985 \mathrm{E}$ & $2 a / b-c m$ & \\
\hline \multicolumn{6}{|c|}{ ' $\quad 8.0 \odot 9850 \mathrm{E}-\odot 2$} \\
\hline $\mathrm{H}-1$ & 336 & $\odot$ & 3. 32434E- -2 & 293.6 & \\
\hline $0-16$ & 336 & $\odot$ & 1. $81013 \mathrm{E}-02$ & 293.6 & \\
\hline$M g-24$ & 336 & $\odot$ & 1. $05686 \mathrm{E}-\odot 4$ & 293.6 & \\
\hline$M g-25$ & 336 & $\odot$ & $1.33797 \mathrm{E}-05$ & 293.6 & \\
\hline$M g-26$ & 336 & $\odot$ & $1.47310 \mathrm{E}-05$ & 293.6 & \\
\hline Al-27 & 336 & $\odot$ & $2.78528 \mathrm{E}-02$ & 293.6 & \\
\hline Si-28 & 336 & $\odot$ & 1. $00085 E-\odot 4$ & 293.6 & \\
\hline Si-29 & 336 & $\odot$ & $5.06774 \mathrm{E}-06$ & 293.6 & \\
\hline Si-30 & 336 & $\odot$ & 3. 36403E- 06 & 293.6 & \\
\hline Ti-46 & 336 & 0 & $4.20262 \mathrm{E}-\odot 7$ & 293.6 & \\
\hline
\end{tabular}




\begin{tabular}{|c|c|c|c|c|c|}
\hline $\mathrm{Ti}-47$ & 336 & $\odot$ & 3. $790 \odot \odot E-๑ 7$ & 293.6 & end \\
\hline $\mathrm{Ti}-48$ & 336 & $\odot$ & $3.75536 E-06$ & 293.6 & end \\
\hline Ti-49 & 336 & 0 & $2.75590 \mathrm{E}-\odot 7$ & 293.6 & end \\
\hline Ti-50 & 336 & $\odot$ & $2.63873 E-07$ & 293.6 & end \\
\hline $\mathrm{Cr}-50$ & 336 & 0 & $5.30517 \mathrm{E}-07$ & 293.6 & end \\
\hline $\mathrm{Cr}-52$ & 336 & $\odot$ & 1. $02189 E-05$ & 293.6 & end \\
\hline $\mathrm{Cr}-53$ & 336 & $\odot$ & $1.15860 E-06$ & 293.6 & and \\
\hline $\mathrm{Cr}-54$ & 336 & $\odot$ & $2.87821 E-07$ & 293.6 & end \\
\hline$M n-55$ & 336 & $\odot$ & $6.43555 \mathrm{E}-06$ & 293.6 & nd \\
\hline $\mathrm{Fe}-54$ & 336 & $\odot$ & $2.34101 E-06$ & 293.6 & end \\
\hline $\mathrm{Fe}-56$ & 336 & $\odot$ & $3.67158 \mathrm{E}-05$ & 293.6 & nd \\
\hline $\mathrm{Fe}-57$ & 336 & $\odot$ & $8.48365 \mathrm{E}-07$ & 293.6 & end \\
\hline $\mathrm{Fe}-58$ & 336 & 0 & $1.12048 \mathrm{E}-07$ & 293.6 & nd \\
\hline $\mathrm{Cu}-63$ & 336 & $\odot$ & $1.87481 \mathrm{E}-05$ & 293.6 & רd \\
\hline $\mathrm{Cu}-65$ & 336 & $\odot$ & 8. 35629E - 06 & 293.6 & a \\
\hline U-234 & 336 & $\odot$ & $5.67811 E-06$ & 293.6 & \\
\hline U-235 & 336 & $\odot$ & 5. $29200 E-\odot 4$ & 293.6 & \\
\hline$U-236$ & 336 & $\odot$ & $2.27126 \mathrm{E}-06$ & 293.6 & \\
\hline$U-238$ & 336 & $\odot$ & 3. $06618 E-05$ & 293.6 & \\
\hline \multicolumn{3}{|c|}{$\begin{array}{l}\text { total atom density } \\
: 8.005550 \mathrm{E}-02\end{array}$} & \multicolumn{2}{|c|}{$=8.00555 \mathrm{E}-02 \mathrm{a} / \mathrm{b}-\mathrm{cm}$} & ' $\quad 8.005550 \mathrm{E}-02$ \\
\hline $\mathrm{H}-1$ & 337 & $\odot$ & $3.32434 \mathrm{E}-02$ & 293.6 & id \\
\hline $0-16$ & 337 & $\odot$ & $1.77731 \mathrm{E}-02$ & 293.6 & \\
\hline$M g-24$ & 337 & $\odot$ & 1. $05686 E-\odot 4$ & 293.6 & \\
\hline$M g-25$ & 337 & $\odot$ & $1.33797 \mathrm{E}-05$ & 293.6 & \\
\hline $\mathrm{Mg}-26$ & 337 & $\odot$ & $1.47310 \mathrm{E}-05$ & 293.6 & \\
\hline Al-27 & 337 & $\odot$ & $2.82593 E-02$ & 293.6 & \\
\hline Si-28 & 337 & $\odot$ & 1. $\odot \odot 993 E-\odot 4$ & 293.6 & \\
\hline Si-29 & 337 & $\odot$ & $5.11370 E-06$ & 293.6 & . \\
\hline $\mathrm{Si}-30$ & 337 & $\odot$ & 3. 39453E- 06 & 293.6 & no \\
\hline $\mathrm{Ti}-46$ & 337 & $\odot$ & $4.20262 \mathrm{E}-\odot 7$ & 293.6 & 110 \\
\hline Ti-47 & 337 & $\odot$ & $3.79000 \mathrm{E}-\odot 7$ & 293.6 & 100 \\
\hline $\mathrm{Ti}-48$ & 337 & $\odot$ & $3.75536 \mathrm{E}-06$ & 293.6 & \\
\hline $\mathrm{Ti}-49$ & 337 & $\odot$ & $2.75590 \mathrm{E}-07$ & 293.6 & \\
\hline $\mathrm{Ti}-50$ & 337 & $\odot$ & $2.63873 E-07$ & 293.6 & \\
\hline $\mathrm{Cr}-50$ & 337 & $\odot$ & $5.30517 \mathrm{E}-07$ & 293.6 & \\
\hline $\mathrm{Cr}-52$ & 337 & $\odot$ & 1. $02189 E-05$ & 293.6 & \\
\hline $\mathrm{Cr}-53$ & 337 & $\odot$ & $1.15860 \mathrm{E}-06$ & 293.6 & \\
\hline $\mathrm{Cr}-54$ & 337 & $\odot$ & $2.87821 E-07$ & 293.6 & \\
\hline$M n-55$ & 337 & $\odot$ & $6.48581 E-06$ & 293.6 & \\
\hline $\mathrm{Fe}-54$ & 337 & $\odot$ & $2.36993 E-06$ & 293.6 & \\
\hline $\mathrm{Fe}-56$ & 337 & $\odot$ & $3.71695 E-05$ & 293.6 & \\
\hline $\mathrm{Fe}-57$ & 337 & $\odot$ & $8.58848 \mathrm{E}-07$ & 293.6 & \\
\hline $\mathrm{Fe}-58$ & 337 & $\odot$ & 1. 13433E- 07 & 293.6 & \\
\hline $\mathrm{Cu}-63$ & 337 & $\odot$ & 1. 89155E- 05 & 293.6 & \\
\hline $\mathrm{Cu}-65$ & 337 & $\odot$ & 8. 43090E-०6 & 293.6 & \\
\hline U-234 & 337 & $\odot$ & $4.44742 \mathrm{E}-06$ & 293.6 & \\
\hline$U-235$ & 337 & $\odot$ & $4.14500 E-\odot 4$ & 293.6 & \\
\hline$U-236$ & 337 & $\odot$ & $1.77898 \mathrm{E}-06$ & 293.6 & \\
\hline U-238 & 337 & $\odot$ & $2.40161 \mathrm{E}-05$ & 293.6 & nd \\
\hline 1 & tom & sity & $=8.00271 \mathrm{E}$. & $2 \mathrm{a} / \mathrm{b}-\mathrm{cm}$ & \\
\hline \multicolumn{6}{|c|}{ ' $\quad 8.0 \odot 2710 \mathrm{E}-\odot 2$} \\
\hline $\mathrm{H}-1$ & 338 & $\odot$ & 3. 32434E- 02 & 293.6 & \\
\hline $0-16$ & 338 & $\odot$ & $1.75562 \mathrm{E}-02$ & 293.6 & IU \\
\hline$M g-24$ & 338 & $\odot$ & 1. $05686 E-\odot 4$ & 293.6 & \\
\hline$M g-25$ & 338 & $\odot$ & 1. $33797 \mathrm{E}-05$ & 293.6 & \\
\hline$M g-26$ & 338 & $\odot$ & $1.47310 \mathrm{E}-05$ & 293.6 & \\
\hline Al-27 & 338 & $\odot$ & $2.85280 E-\odot 2$ & 293.6 & \\
\hline Si-28 & 338 & $\odot$ & 1. $01591 E-04$ & 293.6 & \\
\hline Si-29 & 338 & $\odot$ & $5.14401 E-06$ & 293.6 & \\
\hline $\mathrm{Si}-30$ & 338 & $\odot$ & $3.41465 \mathrm{E}-06$ & 293.6 & \\
\hline $\mathrm{Ti}-46$ & 338 & $\odot$ & 4. $20262 E-07$ & 293.6 & \\
\hline $\mathrm{Ti}-47$ & 338 & $\odot$ & $3.7900 \odot E-\odot 7$ & 293.6 & \\
\hline Ti-48 & 338 & $\odot$ & $3.75536 E-06$ & 293.6 & \\
\hline $\mathrm{Ti}-49$ & 338 & $\odot$ & $2.75590 \mathrm{E}-\odot 7$ & 293.6 & \\
\hline Ti-50 & 338 & $\odot$ & $2.63873 E-07$ & 293.6 & \\
\hline $\mathrm{Cr}-50$ & 338 & $\odot$ & $5.30517 \mathrm{E}-07$ & 293.6 & \\
\hline $\mathrm{Cr}-52$ & 338 & $\odot$ & 1. $02189 E-05$ & 293.6 & \\
\hline $\mathrm{Cr}-53$ & 338 & $\odot$ & $1.15860 \mathrm{E}-06$ & 293.6 & \\
\hline $\mathrm{Cr}-54$ & 338 & $\odot$ & $2.87821 E-07$ & 293.6 & \\
\hline$M n-55$ & 338 & $\odot$ & 6. 519๑3E-๑6 & 293.6 & \\
\hline $\mathrm{Fe}-54$ & 338 & $\odot$ & $2.38905 E-06$ & 293.6 & \\
\hline $\mathrm{Fe}-56$ & 338 & $\odot$ & 3. $74693 \mathrm{E}-05$ & 293.6 & \\
\hline $\mathrm{Fe}-57$ & 338 & $\odot$ & $8.65776 E-\odot 7$ & 293.6 & \\
\hline $\mathrm{Fe}-58$ & 338 & $\odot$ & $1.14348 E-07$ & 293.6 & \\
\hline $\mathrm{Cu}-63$ & 338 & $\odot$ & 1. $90262 \mathrm{E}-05$ & 293.6 & \\
\hline $\mathrm{Cu}-65$ & 338 & $\odot$ & $8.48022 \mathrm{E}-\odot 6$ & 293.6 & \\
\hline$U-234$ & 338 & $\odot$ & $3.63412 \mathrm{E}-06$ & 293.6 & \\
\hline$U-235$ & 338 & $\odot$ & $3.38700 \mathrm{E}-\odot 4$ & 293.6 & \\
\hline$U-236$ & 338 & $\odot$ & $1.45366 \mathrm{E}-06$ & 293.6 & \\
\hline U-238 & 338 & $\odot$ & 1. $96242 \mathrm{E}-05$ & 293.6 & \\
\hline
\end{tabular}




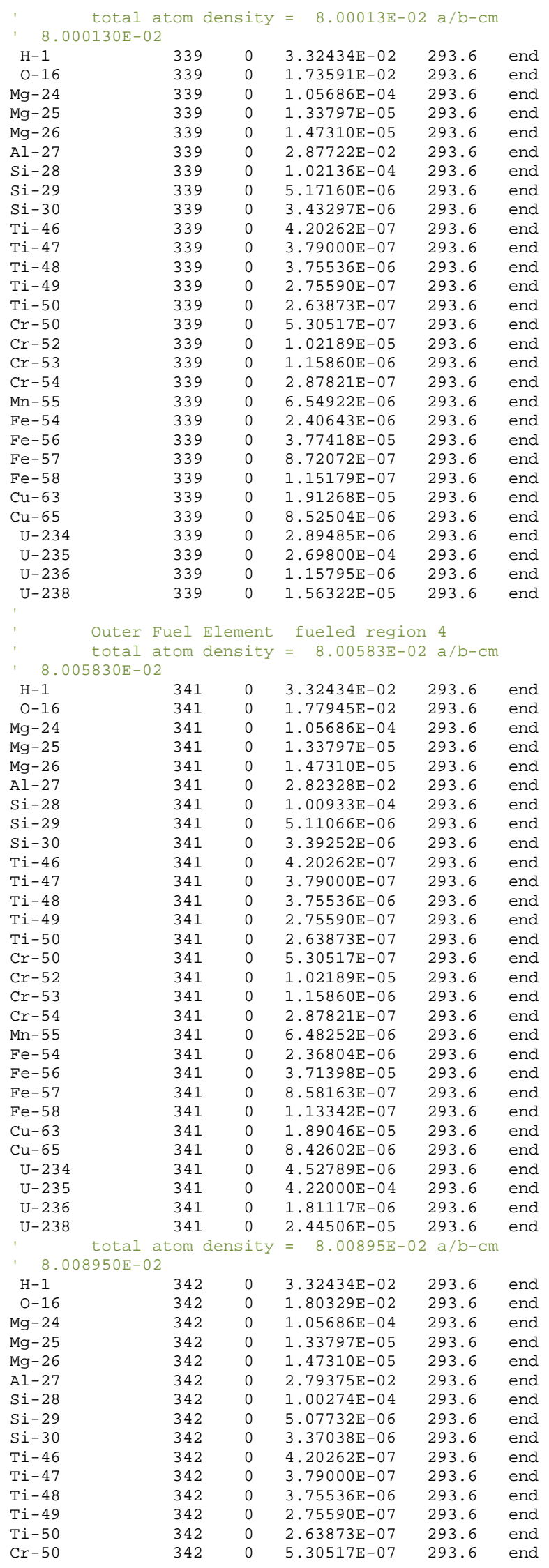




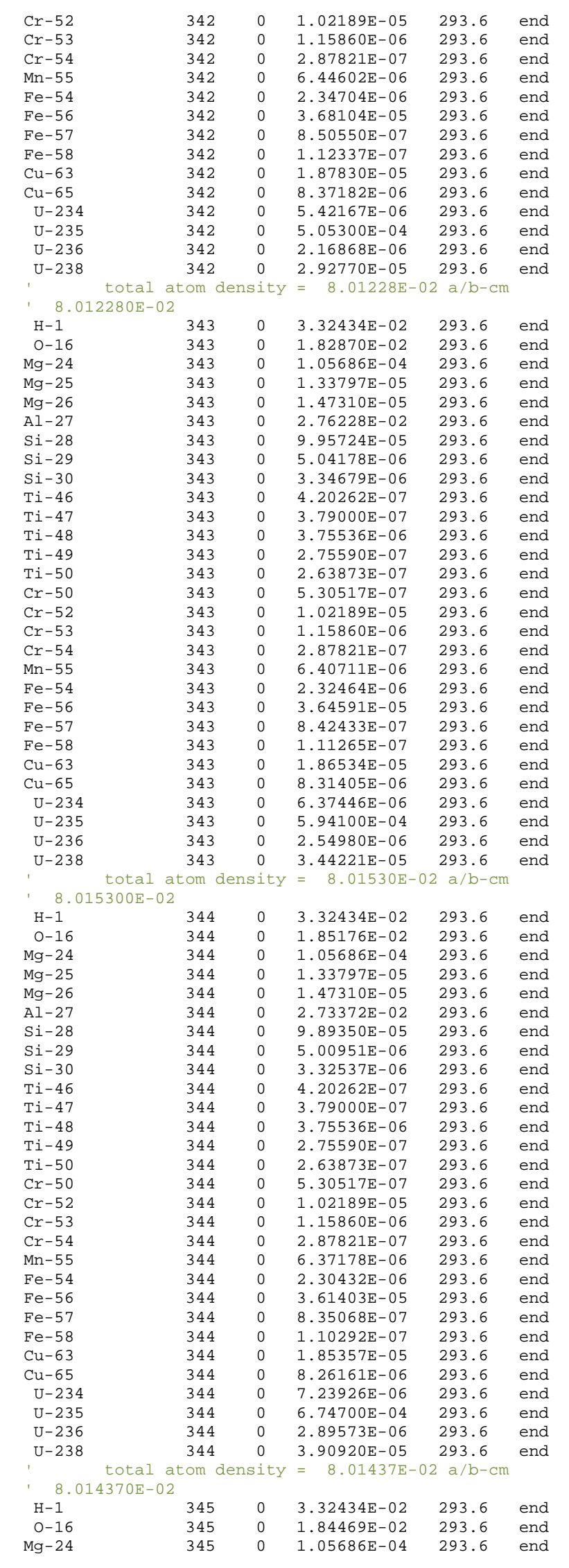




\begin{tabular}{|c|c|c|c|c|c|}
\hline$M g-25$ & 345 & $\odot$ & $1.33797 \mathrm{E}-05$ & 293.6 & end \\
\hline$M g-26$ & 345 & $\odot$ & $1.47310 \mathrm{E}-05$ & 293.6 & end \\
\hline Al-27 & 345 & $\odot$ & $2.74247 \mathrm{E}-02$ & 293.6 & end \\
\hline Si-28 & 345 & $\odot$ & $9.91306 \mathrm{E}-\odot 5$ & 293.6 & end \\
\hline Si-29 & 345 & $\odot$ & $5.01941 E-06$ & 293.6 & end \\
\hline Si-30 & 345 & 0 & 3. 33194E- 06 & 293.6 & end \\
\hline Ti-46 & 345 & $\odot$ & $4.20262 \mathrm{E}-07$ & 293.6 & and \\
\hline $\mathrm{Ti}-47$ & 345 & $\odot$ & 3. $79000 \mathrm{E}-07$ & 293.6 & nd \\
\hline $\mathrm{Ti}-48$ & 345 & $\odot$ & $3.75536 \mathrm{E}-\odot 6$ & 293.6 & \\
\hline $\mathrm{Ti}-49$ & 345 & $\odot$ & $2.75590 E-\odot 7$ & 293.6 & \\
\hline Ti-50 & 345 & $\odot$ & $2.63873 E-07$ & 293.6 & nd \\
\hline $\mathrm{Cr}-50$ & 345 & $\odot$ & $5.30517 \mathrm{E}-07$ & 293.6 & end \\
\hline $\mathrm{Cr}-52$ & 345 & $\odot$ & 1. $02189 \mathrm{E}-05$ & 293.6 & nd \\
\hline $\mathrm{Cr}-53$ & 345 & $\odot$ & $1.15860 E-06$ & 293.6 & nd \\
\hline $\mathrm{Cr}-54$ & 345 & 0 & $2.87821 \mathrm{E}-07$ & 293.6 & ad \\
\hline Mn-55 & 345 & $\odot$ & $6.38261 \mathrm{E}-06$ & 293.6 & nd \\
\hline $\mathrm{Fe}-54$ & 345 & $\odot$ & $2.31055 \mathrm{E}-06$ & 293.6 & $\mathrm{ad}$ \\
\hline $\mathrm{Fe}-56$ & 345 & $\odot$ & $3.62380 E-05$ & 293.6 & $\mathrm{hd}$ \\
\hline $\mathrm{Fe}-57$ & 345 & $\odot$ & $8.37326 E-07$ & 293.6 & \\
\hline $\mathrm{Fe}-58$ & 345 & $\odot$ & 1. $10590 \mathrm{E}-07$ & 293.6 & ad \\
\hline $\mathrm{Cu}-63$ & 345 & $\odot$ & 1. $85717 \mathrm{E}-05$ & 293.6 & nd \\
\hline Cu-65 & 345 & $\odot$ & $8.27767 \mathrm{E}-\odot 6$ & 293.6 & $\mathrm{ad}$ \\
\hline U-234 & 345 & $\odot$ & $6.97424 \mathrm{E}-\odot 6$ & 293.6 & \\
\hline U-235 & 345 & $\odot$ & $6.50 \odot \odot \odot E-\odot 4$ & 293.6 & \\
\hline U-236 & 345 & $\odot$ & $2.78972 \mathrm{E}-\odot 6$ & 293.6 & ad \\
\hline$U-238$ & 345 & $\odot$ & $3.76609 \mathrm{E}-\odot 5$ & 293.6 & \\
\hline \multicolumn{3}{|c|}{ total atom density } & \multicolumn{3}{|c|}{$=8.00985 \mathrm{E}-02 \mathrm{a} / \mathrm{b}-\mathrm{cm}$} \\
\hline $\mathrm{H}-1$ & 346 & $\odot$ & 3. 32434E- -2 & 293.6 & nd \\
\hline $0-16$ & 346 & $\odot$ & $1.81013 \mathrm{E}-02$ & 293.6 & \\
\hline$M g-24$ & 346 & $\odot$ & 1. $05686 \mathrm{E}-\odot 4$ & 293.6 & \\
\hline$M g-25$ & 346 & $\odot$ & $1.33797 \mathrm{E}-05$ & 293.6 & \\
\hline$M g-26$ & 346 & $\odot$ & $1.47310 \mathrm{E}-05$ & 293.6 & \\
\hline Al-27 & 346 & $\odot$ & $2.78528 \mathrm{E}-02$ & 293.6 & \\
\hline Si-28 & 346 & $\odot$ & 1. $00 \odot 85 E-\odot 4$ & 293.6 & \\
\hline Si-29 & 346 & $\odot$ & $5.06774 \mathrm{E}-06$ & 293.6 & \\
\hline Si-30 & 346 & $\odot$ & 3. 36403E- $\odot 6$ & 293.6 & \\
\hline Ti-46 & 346 & 0 & $4.20262 \mathrm{E}-07$ & 293.6 & \\
\hline $\mathrm{Ti}-47$ & 346 & $\odot$ & 3. $79000 \mathrm{E}-07$ & 293.6 & \\
\hline $\mathrm{Ti}-48$ & 346 & $\odot$ & $3.75536 \mathrm{E}-06$ & 293.6 & \\
\hline Ti-49 & 346 & $\odot$ & $2.75590 E-\odot 7$ & 293.6 & \\
\hline Ti-50 & 346 & $\odot$ & $2.63873 E-07$ & 293.6 & nd \\
\hline $\mathrm{Cr}-50$ & 346 & $\odot$ & 5. 30517E- $\odot 7$ & 293.6 & \\
\hline $\mathrm{Cr}-52$ & 346 & $\odot$ & 1. $02189 E-05$ & 293.6 & \\
\hline $\mathrm{Cr}-53$ & 346 & $\odot$ & $1.15860 \mathrm{E}-06$ & 293.6 & \\
\hline $\mathrm{Cr}-54$ & 346 & $\odot$ & $2.87821 \mathrm{E}-07$ & 293.6 & \\
\hline$M n-55$ & 346 & $\odot$ & $6.43555 \mathrm{E}-06$ & 293.6 & \\
\hline $\mathrm{Fe}-54$ & 346 & $\odot$ & $2.34101 \mathrm{E}-\odot 6$ & 293.6 & \\
\hline $\mathrm{Fe}-56$ & 346 & $\odot$ & $3.67158 \mathrm{E}-05$ & 293.6 & \\
\hline $\mathrm{Fe}-57$ & 346 & $\odot$ & $8.48365 E-07$ & 293.6 & \\
\hline $\mathrm{Fe}-58$ & 346 & $\odot$ & $1.12048 \mathrm{E}-07$ & 293.6 & \\
\hline $\mathrm{Cu}-63$ & 346 & $\odot$ & $1.87481 E-05$ & 293.6 & \\
\hline $\mathrm{Cu}-65$ & 346 & $\odot$ & $8.35629 E-06$ & 293.6 & \\
\hline U-234 & 346 & $\odot$ & $5.67811 \mathrm{E}-06$ & 293.6 & \\
\hline$U-235$ & 346 & $\odot$ & $5.29200 E-\odot 4$ & 293.6 & \\
\hline$U-236$ & 346 & $\odot$ & $2.27126 \mathrm{E}-\odot 6$ & 293.6 & \\
\hline$U-238$ & 346 & $\odot$ & \multirow{2}{*}{\multicolumn{2}{|c|}{$\begin{array}{l}3.06618 E-05 \quad 293.6 \\
=\quad 8.00555 E-02 \mathrm{a} / \mathrm{b}-\mathrm{cm}\end{array}$}} & end \\
\hline \multicolumn{3}{|c|}{ ' total atom density } & & & \\
\hline $\mathrm{H}-1$ & 347 & $\odot$ & 3. $32434 \mathrm{E}-02$ & 293.6 & \\
\hline $0-16$ & 347 & $\odot$ & 1. $77731 \mathrm{E}-02$ & 293.6 & \\
\hline$M g-24$ & 347 & $\odot$ & 1. $05686 E-\odot 4$ & 293.6 & \\
\hline$M g-25$ & 347 & $\odot$ & $1.33797 \mathrm{E}-05$ & 293.6 & \\
\hline$M g-26$ & 347 & $\odot$ & 1. $47310 \mathrm{E}-05$ & 293.6 & \\
\hline Al-27 & 347 & $\odot$ & $2.82593 E-02$ & 293.6 & \\
\hline $\mathrm{Si}-28$ & 347 & $\odot$ & 1. $0 \odot 993 E-\odot 4$ & 293.6 & \\
\hline Si-29 & 347 & $\odot$ & $5.11370 E-06$ & 293.6 & \\
\hline Si-30 & 347 & $\odot$ & $3.39453 E-06$ & 293.6 & \\
\hline Ti-46 & 347 & $\odot$ & 4. $2 \odot 262 \mathrm{E}-\odot 7$ & 293.6 & \\
\hline $\mathrm{Ti}-47$ & 347 & $\odot$ & $3.7900 \odot E-\odot 7$ & 293.6 & \\
\hline $\mathrm{Ti}-48$ & 347 & $\odot$ & $3.75536 \mathrm{E}-06$ & 293.6 & \\
\hline Ti-49 & 347 & $\odot$ & $2.75590 E-07$ & 293.6 & \\
\hline Ti-50 & 347 & $\odot$ & $2.63873 E-07$ & 293.6 & \\
\hline $\mathrm{Cr}-50$ & 347 & $\odot$ & $5.30517 \mathrm{E}-07$ & 293.6 & \\
\hline $\mathrm{Cr}-52$ & 347 & $\odot$ & 1. $02189 E-\odot 5$ & 293.6 & \\
\hline $\mathrm{Cr}-53$ & 347 & $\odot$ & $1.15860 \mathrm{E}-\odot 6$ & 293.6 & \\
\hline $\mathrm{Cr}-54$ & 347 & $\odot$ & $2.87821 \mathrm{E}-07$ & 293.6 & \\
\hline$M n-55$ & 347 & $\odot$ & $6.48581 \mathrm{E}-06$ & 293.6 & \\
\hline $\mathrm{Fe}-54$ & 347 & $\odot$ & $2.36993 E-06$ & 293.6 & \\
\hline $\mathrm{Fe}-56$ & 347 & $\odot$ & $3.71695 \mathrm{E}-05$ & 293.6 & \\
\hline $\mathrm{Fe}-57$ & 347 & 0 & $8.58848 \mathrm{E}-07$ & 293.6 & \\
\hline
\end{tabular}




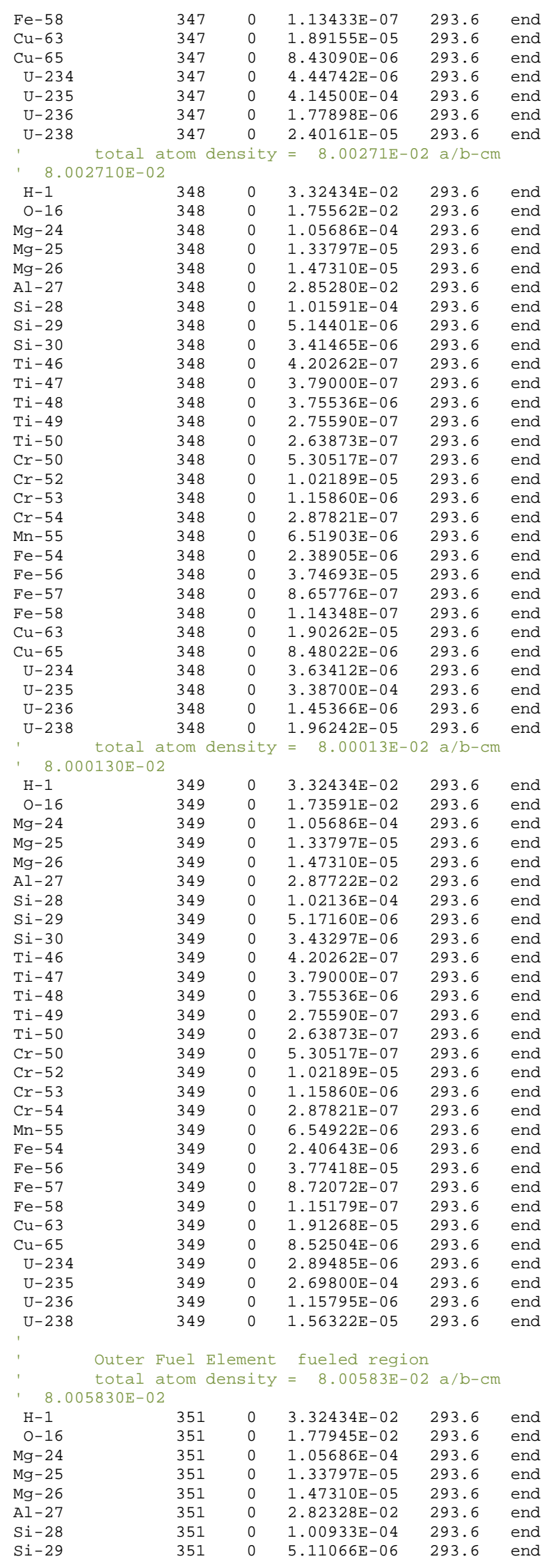




$\begin{array}{llllll}\mathrm{Si}-3 \odot & 351 & \odot & 3.39252 \mathrm{E}-\odot 6 & 293.6 & \text { end } \\ \mathrm{Ti}-46 & 351 & \odot & 4.20262 \mathrm{E}-\odot 7 & 293.6 & \text { end } \\ \mathrm{Ti}-47 & 351 & \odot & 3.79000 \mathrm{E}-\odot 7 & 293.6 & \text { end } \\ \mathrm{Ti}-48 & 351 & \odot & 3.75536 \mathrm{E}-\odot 6 & 293.6 & \text { end } \\ \mathrm{Ti}-49 & 351 & \odot & 2.75590 \mathrm{E}-\odot 7 & 293.6 & \text { end } \\ \mathrm{Ti}-5 \odot & 351 & \odot & 2.63873 \mathrm{E}-\odot 7 & 293.6 & \text { end } \\ \mathrm{Cr}-5 \odot & 351 & \odot & 5.30517 \mathrm{E}-\odot 7 & 293.6 & \text { end } \\ \mathrm{Cr}-52 & 351 & \odot & 1.02189 \mathrm{E}-05 & 293.6 & \text { end } \\ \mathrm{Cr}-53 & 351 & \odot & 1.15860 \mathrm{E}-\odot 6 & 293.6 & \text { end } \\ \mathrm{Cr}-54 & 351 & \odot & 2.87821 \mathrm{E}-\odot 7 & 293.6 & \text { end } \\ \mathrm{Mn}-55 & 351 & \odot & 6.48252 \mathrm{E}-\odot 6 & 293.6 & \text { end } \\ \mathrm{Fe}-54 & 351 & \odot & 2.36804 \mathrm{E}-\odot 6 & 293.6 & \text { end } \\ \mathrm{Fe}-56 & 351 & \odot & 3.71398 \mathrm{E}-\odot 5 & 293.6 & \text { end } \\ \mathrm{Fe}-57 & 351 & \odot & 8.58163 \mathrm{E}-\odot 7 & 293.6 & \text { end } \\ \mathrm{Fe}-58 & 351 & \odot & 1.13342 \mathrm{E}-\odot 7 & 293.6 & \text { end } \\ \mathrm{Cu}-63 & 351 & \odot & 1.89046 \mathrm{E}-\odot 5 & 293.6 & \text { end } \\ \mathrm{Cu}-65 & 351 & \odot & 8.42602 \mathrm{E}-\odot 6 & 293.6 & \text { end } \\ \mathrm{U}-234 & 351 & \odot & 4.52789 \mathrm{E}-\odot 6 & 293.6 & \text { end } \\ \mathrm{U}-235 & 351 & \odot & 4.22000 \mathrm{E}-\odot 4 & 293.6 & \text { end } \\ \mathrm{U}-236 & 351 & \odot & 1.81117 \mathrm{E}-\odot 6 & 293.6 & \text { end } \\ \mathrm{U}-238 & 351 & \odot & 2.44506 \mathrm{E}-05 & 293.6 & \text { end }\end{array}$

total atom density $=8.00895 \mathrm{E}-02 \mathrm{a} / \mathrm{b}-\mathrm{cm}$

\begin{tabular}{|c|c|c|c|c|c|}
\hline \multicolumn{3}{|l|}{ ' } & \multicolumn{3}{|c|}{ 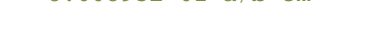 } \\
\hline $\mathrm{H}-1$ & 352 & $\odot$ & $3.32434 \mathrm{E}-02$ & 293.6 & end \\
\hline $0-16$ & 352 & $\odot$ & 1. $80329 E-02$ & 293.6 & end \\
\hline $\mathrm{Mg}-24$ & 352 & $\odot$ & 1. $05686 E-04$ & 293.6 & end \\
\hline $\mathrm{Mg}-25$ & 352 & $\odot$ & $1.33797 \mathrm{E}-05$ & 293.6 & nd \\
\hline $\mathrm{Mg}-26$ & 352 & $\odot$ & $1.47310 \mathrm{E}-05$ & 293.6 & nd \\
\hline Al-27 & 352 & $\odot$ & $2.79375 \mathrm{E}-02$ & 293.6 & nd \\
\hline Si-28 & 352 & $\odot$ & 1. $\odot \odot 274 \mathrm{E}-\odot 4$ & 293.6 & ad \\
\hline Si-29 & 352 & 0 & $5.07732 \mathrm{E}-06$ & 293.6 & \\
\hline Si-30 & 352 & $\odot$ & $3.37038 E-06$ & 293.6 & \\
\hline Ti-46 & 352 & 0 & 4. 20262E- 07 & 293.6 & 110 \\
\hline Ti-47 & 352 & $\odot$ & $3.79000 \mathrm{E}-07$ & 293.6 & nc \\
\hline Ti-48 & 352 & $\odot$ & $3.75536 \mathrm{E}-06$ & 293.6 & \\
\hline Ti-49 & 352 & $\odot$ & $2.75590 \mathrm{E}-07$ & 293.6 & \\
\hline Ti-50 & 352 & $\odot$ & $2.63873 E-07$ & 293.6 & \\
\hline $\mathrm{Cr}-50$ & 352 & $\odot$ & $5.30517 \mathrm{E}-07$ & 293.6 & \\
\hline $\mathrm{Cr}-52$ & 352 & $\odot$ & 1. $02189 E-05$ & 293.6 & \\
\hline $\mathrm{Cr}-53$ & 352 & 0 & $1.15860 E-06$ & 293.6 & \\
\hline $\mathrm{Cr}-54$ & 352 & $\odot$ & $2.87821 E-07$ & 293.6 & \\
\hline$M n-55$ & 352 & 0 & $6.44602 \mathrm{E}-06$ & 293.6 & \\
\hline $\mathrm{Fe}-54$ & 352 & 0 & $2.34704 \mathrm{E}-06$ & 293.6 & \\
\hline $\mathrm{Fe}-56$ & 352 & 0 & $3.68104 \mathrm{E}-05$ & 293.6 & 10 \\
\hline $\mathrm{Fe}-57$ & 352 & $\odot$ & $8.50550 \mathrm{E}-07$ & 293.6 & \\
\hline $\mathrm{Fe}-58$ & 352 & 0 & $1.12337 \mathrm{E}-07$ & 293.6 & \\
\hline $\mathrm{Cu}-63$ & 352 & $\odot$ & 1.87830E-०5 & 293.6 & \\
\hline $\mathrm{Cu}-65$ & 352 & $\odot$ & 8. 37182E- - 66 & 293.6 & \\
\hline U-234 & 352 & $\odot$ & $5.42167 E-06$ & 293.6 & \\
\hline$U-235$ & 352 & $\odot$ & $5.05300 E-\odot 4$ & 293.6 & \\
\hline$U-236$ & 352 & $\odot$ & $2.16868 E-06$ & 293.6 & \\
\hline U- 238 & 352 & $\odot$ & 2. $92770 \mathrm{E}-05$ & 293.6 & \\
\hline 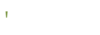 & tom & sity & $=8.01228 \mathrm{E}$ & $2 a / b-c m$ & \\
\hline ' 8.01 & & & & & \\
\hline $\mathrm{H}-1$ & 353 & $\odot$ & $3.32434 \mathrm{E}-02$ & 293.6 & \\
\hline $0-16$ & 353 & $\odot$ & $1.82870 \mathrm{E}-02$ & 293.6 & \\
\hline$M g-24$ & 353 & 0 & $1.05686 \mathrm{E}-04$ & 293.6 & \\
\hline$M g-25$ & 353 & $\odot$ & 1. 33797E- 05 & 293.6 & \\
\hline$M g-26$ & 353 & $\odot$ & $1.47310 \mathrm{E}-05$ & 293.6 & \\
\hline Al-27 & 353 & $\odot$ & $2.76228 E-02$ & 293.6 & \\
\hline Si-28 & 353 & $\odot$ & $9.95724 \mathrm{E}-05$ & 293.6 & \\
\hline Si-29 & 353 & $\odot$ & $5.04178 E-06$ & 293.6 & \\
\hline Si-30 & 353 & $\odot$ & $3.34679 E-06$ & 293.6 & \\
\hline Ti-46 & 353 & $\odot$ & $4.20262 \mathrm{E}-07$ & 293.6 & \\
\hline Ti-47 & 353 & 0 & $3.79000 \mathrm{E}-\odot 7$ & 293.6 & \\
\hline Ti-48 & 353 & $\odot$ & $3.75536 \mathrm{E}-06$ & 293.6 & \\
\hline Ti-49 & 353 & $\odot$ & $2.75590 \mathrm{E}-\odot 7$ & 293.6 & \\
\hline Ti-50 & 353 & $\odot$ & $2.63873 E-07$ & 293.6 & \\
\hline $\mathrm{Cr}-50$ & 353 & $\odot$ & $5.30517 E-07$ & 293.6 & \\
\hline $\mathrm{Cr}-52$ & 353 & $\odot$ & $1.02189 E-05$ & 293.6 & \\
\hline $\mathrm{Cr}-53$ & 353 & $\odot$ & $1.15860 \mathrm{E}-06$ & 293.6 & \\
\hline $\mathrm{Cr}-54$ & 353 & $\odot$ & $2.87821 E-07$ & 293.6 & \\
\hline$M n-55$ & 353 & $\odot$ & $6.40711 \mathrm{E}-06$ & 293.6 & \\
\hline $\mathrm{Fe}-54$ & 353 & $\odot$ & $2.32464 \mathrm{E}-06$ & 293.6 & \\
\hline $\mathrm{Fe}-56$ & 353 & $\odot$ & $3.64591 E-05$ & 293.6 & \\
\hline $\mathrm{Fe}-57$ & 353 & $\odot$ & $8.42433 E-07$ & 293.6 & \\
\hline $\mathrm{Fe}-58$ & 353 & $\odot$ & $1.11265 \mathrm{E}-07$ & 293.6 & \\
\hline $\mathrm{Cu}-63$ & 353 & $\odot$ & 1. 86534E- 05 & 293.6 & \\
\hline $\mathrm{Cu}-65$ & 353 & $\odot$ & 8. 31405E-06 & 293.6 & \\
\hline U-234 & 353 & 0 & $6.37446 E-06$ & 293.6 & \\
\hline U-235 & 353 & 0 & $5.94100 \mathrm{E}-\odot 4$ & 293.6 & \\
\hline
\end{tabular}




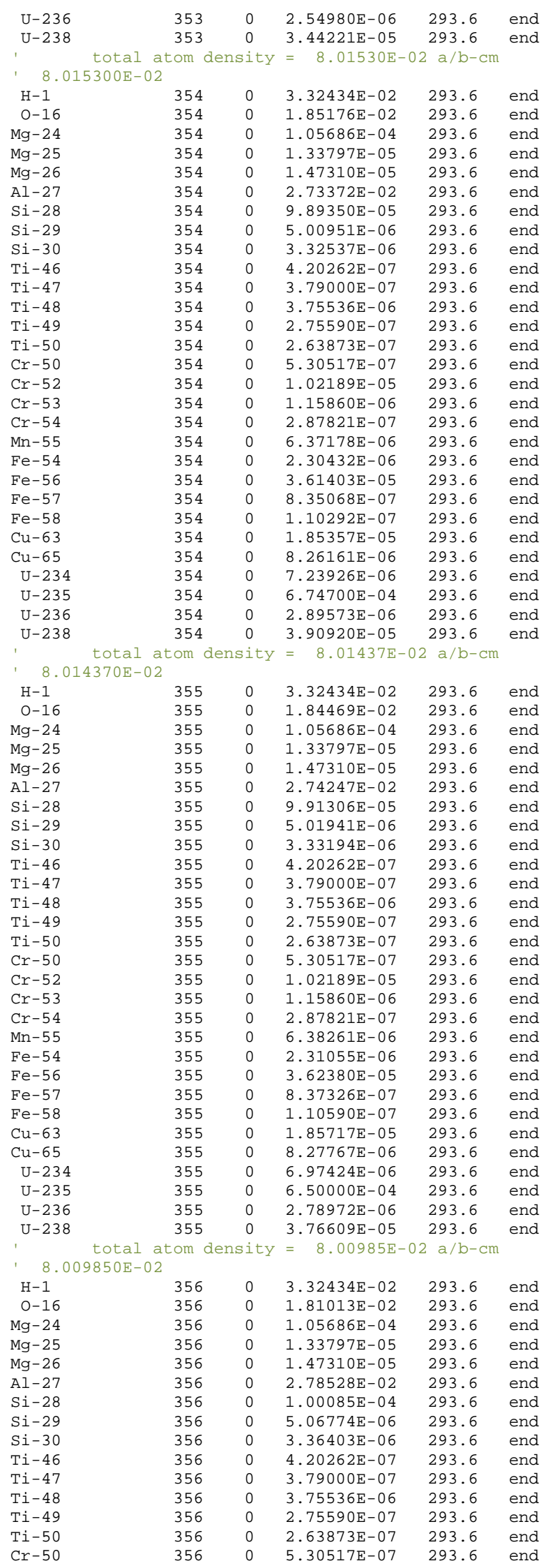




\begin{tabular}{|c|c|c|c|c|c|}
\hline $\mathrm{Cr}-52$ & 356 & $\odot$ & 1. $02189 E-\odot 5$ & 293.6 & end \\
\hline $\mathrm{Cr}-53$ & 356 & $\odot$ & $1.1586 \odot E-\odot 6$ & 293.6 & end \\
\hline $\mathrm{Cr}-54$ & 356 & $\odot$ & $2.87821 E-07$ & 293.6 & end \\
\hline$M n-55$ & 356 & $\odot$ & $6.43555 E-\odot 6$ & 293.6 & end \\
\hline $\mathrm{Fe}-54$ & 356 & $\odot$ & $2.34101 E-06$ & 293.6 & end \\
\hline $\mathrm{Fe}-56$ & 356 & $\odot$ & $3.67158 E-05$ & 293.6 & end \\
\hline $\mathrm{Fe}-57$ & 356 & $\odot$ & $8.48365 E-07$ & 293.6 & nd \\
\hline $\mathrm{Fe}-58$ & 356 & $\odot$ & $1.12048 \mathrm{E}-07$ & 293.6 & end \\
\hline $\mathrm{Cu}-63$ & 356 & $\odot$ & $1.87481 E-05$ & 293.6 & end \\
\hline $\mathrm{Cu}-65$ & 356 & $\odot$ & 8. 35629E- - 6 & 293.6 & and \\
\hline$U-234$ & 356 & $\odot$ & $5.67811 E-\odot 6$ & 293.6 & end \\
\hline$U-235$ & 356 & $\odot$ & $5.29200 \mathrm{E}-\odot 4$ & 293.6 & nd \\
\hline$U-236$ & 356 & $\odot$ & $2.27126 \mathrm{E}-\odot 6$ & 293.6 & d \\
\hline$U-238$ & 356 & $\odot$ & 3. $06618 \mathrm{E}-05$ & 293.6 & end \\
\hline & & & $=8.00555 \mathrm{E}$. & $a / b-c$ & \\
\hline
\end{tabular}

\begin{tabular}{|c|c|c|c|c|c|}
\hline $\mathrm{H}-1$ & 357 & $\odot$ & $3.32434 \mathrm{E}-02$ & 293.6 & end \\
\hline $0-16$ & 357 & $\odot$ & $1.77731 \mathrm{E}-\odot 2$ & 293.6 & end \\
\hline$M g-24$ & 357 & $\odot$ & $1.05686 \mathrm{E}-\odot 4$ & 293.6 & end \\
\hline$M g-25$ & 357 & $\odot$ & $1.33797 \mathrm{E}-05$ & 293.6 & end \\
\hline$M g-26$ & 357 & 0 & $1.47310 \mathrm{E}-05$ & 293.6 & end \\
\hline Al-27 & 357 & $\odot$ & $2.82593 E-02$ & 293.6 & end \\
\hline Si-28 & 357 & $\odot$ & 1. $0 \odot 993 E-\odot 4$ & 293.6 & end \\
\hline Si-29 & 357 & $\odot$ & $5.11370 \mathrm{E}-06$ & 293.6 & end \\
\hline Si-30 & 357 & $\odot$ & 3. 39453E- 06 & 293.6 & end \\
\hline Ti-46 & 357 & $\odot$ & $4.20262 E-07$ & 293.6 & \\
\hline Ti-47 & 357 & $\odot$ & $3.790 \odot \odot E-\odot 7$ & 293.6 & \\
\hline Ti-48 & 357 & $\odot$ & $3.75536 \mathrm{E}-06$ & 293.6 & \\
\hline Ti-49 & 357 & $\odot$ & $2.75590 \mathrm{E}-07$ & 293.6 & \\
\hline Ti-50 & 357 & $\odot$ & $2.63873 E-07$ & 293.6 & \\
\hline $\mathrm{Cr}-50$ & 357 & $\odot$ & $5.30517 \mathrm{E}-07$ & 293.6 & \\
\hline $\mathrm{Cr}-52$ & 357 & $\odot$ & 1. $02189 \mathrm{E}-05$ & 293.6 & a \\
\hline $\mathrm{Cr}-53$ & 357 & $\odot$ & $1.15860 \mathrm{E}-\odot 6$ & 293.6 & id \\
\hline $\mathrm{Cr}-54$ & 357 & $\odot$ & $2.87821 E-\odot 7$ & 293.6 & \\
\hline$M n-55$ & 357 & $\odot$ & $6.48581 E-06$ & 293.6 & \\
\hline $\mathrm{Fe}-54$ & 357 & $\odot$ & $2.36993 E-06$ & 293.6 & \\
\hline $\mathrm{Fe}-56$ & 357 & $\odot$ & $3.71695 \mathrm{E}-05$ & 293.6 & \\
\hline $\mathrm{Fe}-57$ & 357 & 0 & $8.58848 E-07$ & 293.6 & \\
\hline $\mathrm{Fe}-58$ & 357 & $\odot$ & $1.13433 \mathrm{E}-07$ & 293.6 & \\
\hline $\mathrm{Cu}-63$ & 357 & $\odot$ & $1.89155 \mathrm{E}-\odot 5$ & 293.6 & \\
\hline $\mathrm{Cu}-65$ & 357 & $\odot$ & 8. $43090 E-\odot 6$ & 293.6 & \\
\hline$U-234$ & 357 & $\odot$ & $4.44742 \mathrm{E}-06$ & 293.6 & \\
\hline U-235 & 357 & $\odot$ & $4.1450 \odot E-\odot 4$ & 293.6 & \\
\hline$U-236$ & 357 & $\odot$ & $1.77898 \mathrm{E}-\odot 6$ & 293.6 & \\
\hline$U-238$ & 357 & $\Theta$ & 2. 40161E- $\odot 5$ & 293.6 & \\
\hline
\end{tabular}

, total atom density $=8.00271 \mathrm{E}-02 \mathrm{a} / \mathrm{b}-\mathrm{cm}$

' 8. $002710 \mathrm{E}-02$

\begin{tabular}{|c|c|c|c|c|}
\hline $\mathrm{H}-1$ & 358 & 0 & 3. 32434E- 02 & 293.6 \\
\hline $0-16$ & 358 & $\odot$ & $1.75562 \mathrm{E}-02$ & 293.6 \\
\hline$M g-24$ & 358 & $\odot$ & $1.05686 \mathrm{E}-04$ & 293.6 \\
\hline$M g-25$ & 358 & $\odot$ & 1. $33797 \mathrm{E}-05$ & 293.6 \\
\hline $\mathrm{Mg}-26$ & 358 & $\odot$ & $1.47310 \mathrm{E}-05$ & 293.6 \\
\hline Al-27 & 358 & $\odot$ & $2.85280 E-02$ & 293.6 \\
\hline Si-28 & 358 & $\odot$ & 1. $01591 E-04$ & 293.6 \\
\hline Si-29 & 358 & $\odot$ & $5.14401 E-06$ & 293.6 \\
\hline Si-30 & 358 & $\odot$ & $3.41465 E-06$ & 293.6 \\
\hline Ti-46 & 358 & $\odot$ & $4.20262 \mathrm{E}-07$ & 293.6 \\
\hline$i-47$ & 358 & $\odot$ & 3. $79000 \mathrm{E}-07$ & 293.6 \\
\hline$i-48$ & 358 & $\odot$ & $3.75536 \mathrm{E}-06$ & 293.6 \\
\hline Ti-49 & 358 & $\odot$ & $2.75590 E-07$ & 293.6 \\
\hline Ti-50 & 358 & 0 & $2.63873 E-07$ & 293.6 \\
\hline $\mathrm{Cr}-50$ & 358 & $\odot$ & $5.30517 \mathrm{E}-07$ & 293.6 \\
\hline $\mathrm{Cr}-52$ & 358 & $\odot$ & 1. $02189 E-05$ & 293.6 \\
\hline $\mathrm{Cr}-53$ & 358 & $\odot$ & $1.15860 \mathrm{E}-06$ & 293.6 \\
\hline $\mathrm{Cr}-54$ & 358 & $\odot$ & $2.87821 E-07$ & 293.6 \\
\hline$M n-55$ & 358 & $\odot$ & $6.51903 E-06$ & 293.6 \\
\hline $\mathrm{Fe}-54$ & 358 & $\odot$ & $2.38905 E-06$ & 293.6 \\
\hline $\mathrm{Fe}-56$ & 358 & $\odot$ & $3.74693 \mathrm{E}-05$ & 293.6 \\
\hline $\mathrm{Fe}-57$ & 358 & $\odot$ & $8.65776 E-07$ & 293.6 \\
\hline $\mathrm{Fe}-58$ & 358 & $\odot$ & $1.14348 E-07$ & 293.6 \\
\hline $\mathrm{Cu}-63$ & 358 & $\odot$ & 1. $90262 \mathrm{E}-05$ & 293.6 \\
\hline Cu- 65 & 358 & 0 & $8.48022 \mathrm{E}-06$ & 293.6 \\
\hline$U-234$ & 358 & $\odot$ & $3.63412 \mathrm{E}-06$ & 293.6 \\
\hline$U-235$ & 358 & $\odot$ & $3.3870 \odot E-04$ & 293.6 \\
\hline$U-236$ & 358 & $\odot$ & $1.45366 \mathrm{E}-06$ & 293.6 \\
\hline$U-238$ & 358 & $\odot$ & 1. $96242 \mathrm{E}-05$ & 293.6 \\
\hline
\end{tabular}

$\mathrm{U}-238$ total atom density $=8.00013 \mathrm{E}-02 \mathrm{a} / \mathrm{b}-\mathrm{cm}$

8. $000130 \mathrm{E}-\odot 2$

$\begin{array}{cccccr}H-1 & 359 & \odot & 3.32434 \mathrm{E}-02 & 293.6 & \text { end } \\ 0-16 & 359 & \odot & 1.73591 \mathrm{E}-02 & 293.6 & \text { end } \\ \mathrm{Mg}-24 & 359 & \odot & 1.05686 \mathrm{E}-04 & 293.6 & \text { end }\end{array}$




$\begin{array}{llllll}\mathrm{Mg}-25 & 359 & \odot & 1.33797 \mathrm{E}-05 & 293.6 & \text { end } \\ \mathrm{Mg}-26 & 359 & \odot & 1.47310 \mathrm{E}-05 & 293.6 & \text { end } \\ \mathrm{Al}-27 & 359 & \odot & 2.87722 \mathrm{E}-\odot 2 & 293.6 & \text { end } \\ \mathrm{Si}-28 & 359 & \odot & 1.02136 \mathrm{E}-04 & 293.6 & \text { end } \\ \mathrm{Si}-29 & 359 & \odot & 5.17160 \mathrm{E}-06 & 293.6 & \text { end } \\ \mathrm{Si}-3 \odot & 359 & \odot & 3.43297 \mathrm{E}-06 & 293.6 & \text { end } \\ \mathrm{Ti}-46 & 359 & \odot & 4.20262 \mathrm{E}-07 & 293.6 & \text { end } \\ \mathrm{Ti}-47 & 359 & \odot & 3.7900 \odot \mathrm{E}-07 & 293.6 & \text { end } \\ \mathrm{Ti}-48 & 359 & \odot & 3.75536 \mathrm{E}-06 & 293.6 & \text { end } \\ \mathrm{Ti}-49 & 359 & \odot & 2.75590 \mathrm{E}-07 & 293.6 & \text { end } \\ \mathrm{Ti}-5 \odot & 359 & \odot & 2.63873 \mathrm{E}-07 & 293.6 & \text { end } \\ \mathrm{Cr}-5 \odot & 359 & \odot & 5.30517 \mathrm{E}-07 & 293.6 & \text { end } \\ \mathrm{Cr}-52 & 359 & \odot & 1.02189 \mathrm{E}-05 & 293.6 & \text { end } \\ \mathrm{Cr}-53 & 359 & \odot & 1.15860 \mathrm{E}-06 & 293.6 & \text { end } \\ \mathrm{Cr}-54 & 359 & \odot & 2.87821 \mathrm{E}-07 & 293.6 & \text { end } \\ \mathrm{Mn}-55 & 359 & \odot & 6.54922 \mathrm{E}-06 & 293.6 & \text { end } \\ \mathrm{Fe}-54 & 359 & \odot & 2.40643 \mathrm{E}-06 & 293.6 & \text { end } \\ \mathrm{Fe}-56 & 359 & \odot & 3.77418 \mathrm{E}-05 & 293.6 & \text { end } \\ \mathrm{Fe}-57 & 359 & \odot & 8.72072 \mathrm{E}-07 & 293.6 & \text { end } \\ \mathrm{Fe}-58 & 359 & \odot & 1.15179 \mathrm{E}-07 & 293.6 & \text { end } \\ \mathrm{Cu}-63 & 359 & \odot & 1.91268 \mathrm{E}-05 & 293.6 & \text { end } \\ \mathrm{Cu}-65 & 359 & \odot & 8.52504 \mathrm{E}-06 & 293.6 & \text { end } \\ \mathrm{U}-234 & 359 & \odot & 2.89485 \mathrm{E}-06 & 293.6 & \text { end } \\ \mathrm{U}-235 & 359 & \odot & 2.69800 \mathrm{E}-04 & 293.6 & \text { end } \\ \mathrm{U}-236 & 359 & \odot & 1.15795 \mathrm{E}-06 & 293.6 & \text { end } \\ \mathrm{U}-238 & 359 & \odot & 1.56322 \mathrm{E}-05 & 293.6 & \text { end } \\ \mathrm{H}-23 & & & & & \end{array}$

' Outer Fuel Element fueled region 6

total atom density $=8.00583 \mathrm{E}-02 \mathrm{a} / \mathrm{b}-\mathrm{cm}$ 8. $0 \odot 5830 \mathrm{E}-\odot 2$

\begin{tabular}{|c|c|c|c|c|c|}
\hline $\mathrm{H}-1$ & 361 & $\odot$ & 3. 32434E- 02 & 293.6 & end \\
\hline $0-16$ & 361 & $\odot$ & $1.77945 E-\odot 2$ & 293.6 & end \\
\hline Mg-24 & 361 & 0 & 1. $05686 E-04$ & 293.6 & end \\
\hline$M g-25$ & 361 & $\odot$ & $1.33797 \mathrm{E}-05$ & 293.6 & end \\
\hline$M g-26$ & 361 & $\odot$ & $1.47310 \mathrm{E}-05$ & 293.6 & end \\
\hline Al-27 & 361 & $\odot$ & $2.82328 \mathrm{E}-02$ & 293.6 & end \\
\hline Si-28 & 361 & $\odot$ & 1. $\odot \odot 933 E-\odot 4$ & 293.6 & nd \\
\hline Si-29 & 361 & $\odot$ & $5.11066 \mathrm{E}-06$ & 293.6 & nd \\
\hline $\mathrm{Si}-30$ & 361 & 0 & 3. 39252E-๑6 & 293.6 & end \\
\hline Ti-46 & 361 & 0 & 4. 20262E- - 7 & 293.6 & end \\
\hline Ti-47 & 361 & 0 & $3.790 \odot \odot E-\odot 7$ & 293.6 & end \\
\hline $\mathrm{Ti}-48$ & 361 & 0 & $3.75536 \mathrm{E}-06$ & 293.6 & end \\
\hline Ti-49 & 361 & 0 & $2.75590 \mathrm{E}-\odot 7$ & 293.6 & end \\
\hline Ti-50 & 361 & $\odot$ & $2.63873 E-07$ & 293.6 & nd \\
\hline $\mathrm{Cr}-50$ & 361 & $\odot$ & $5.30517 \mathrm{E}-07$ & 293.6 & ad \\
\hline $\mathrm{Cr}-52$ & 361 & $\odot$ & 1. $02189 E-05$ & 293.6 & nd \\
\hline $\mathrm{Cr}-53$ & 361 & $\odot$ & $1.15860 \mathrm{E}-\odot 6$ & 293.6 & nd \\
\hline $\mathrm{Cr}-54$ & 361 & $\odot$ & $2.87821 E-07$ & 293.6 & nd \\
\hline Mn-55 & 361 & $\odot$ & $6.48252 \mathrm{E}-06$ & 293.6 & end \\
\hline $\mathrm{Fe}-54$ & 361 & $\odot$ & $2.36804 \mathrm{E}-06$ & 293.6 & end \\
\hline $\mathrm{Fe}-56$ & 361 & $\odot$ & $3.71398 E-05$ & 293.6 & end \\
\hline $\mathrm{Fe}-57$ & 361 & $\odot$ & $8.58163 E-07$ & 293.6 & nd \\
\hline $\mathrm{Fe}-58$ & 361 & $\odot$ & $1.13342 \mathrm{E}-07$ & 293.6 & id \\
\hline Cu-63 & 361 & $\odot$ & $1.89046 \mathrm{E}-05$ & 293.6 & \\
\hline Cu-65 & 361 & $\odot$ & $8.42602 \mathrm{E}-06$ & 293.6 & \\
\hline$U-234$ & 361 & $\odot$ & $4.52789 E-06$ & 293.6 & d \\
\hline$U-235$ & 361 & $\odot$ & $4.22000 \mathrm{E}-\odot 4$ & 293.6 & nd \\
\hline$U-236$ & 361 & $\odot$ & $1.81117 \mathrm{E}-06$ & 293.6 & nd \\
\hline$U-238$ & 361 & $\odot$ & $2.44506 \mathrm{E}-05$ & 293.6 & nd \\
\hline
\end{tabular}

\begin{tabular}{|c|c|c|c|c|c|}
\hline $\mathrm{H}-1$ & 362 & $\odot$ & $3.32434 \mathrm{E}-02$ & 293.6 & end \\
\hline $0-16$ & 362 & $\odot$ & 1. $80329 \mathrm{E}-\odot 2$ & 293.6 & end \\
\hline$M g-24$ & 362 & $\odot$ & 1. $05686 E-\odot 4$ & 293.6 & end \\
\hline$M g-25$ & 362 & $\odot$ & $1.33797 \mathrm{E}-05$ & 293.6 & end \\
\hline$M g-26$ & 362 & $\odot$ & $1.47310 \mathrm{E}-05$ & 293.6 & ad \\
\hline Al-27 & 362 & $\odot$ & $2.79375 \mathrm{E}-02$ & 293.6 & nd \\
\hline Si-28 & 362 & $\odot$ & 1. $\odot \odot 274 \mathrm{E}-\odot 4$ & 293.6 & nd \\
\hline Si-29 & 362 & $\odot$ & $5.07732 \mathrm{E}-06$ & 293.6 & nd \\
\hline $\mathrm{Si}-30$ & 362 & $\odot$ & $3.37038 \mathrm{E}-06$ & 293.6 & nd \\
\hline Ti-46 & 362 & $\odot$ & $4.20262 E-07$ & 293.6 & \\
\hline Ti-47 & 362 & $\odot$ & $3.79000 \mathrm{E}-07$ & 293.6 & \\
\hline Ti-48 & 362 & $\odot$ & $3.75536 \mathrm{E}-06$ & 293.6 & \\
\hline Ti-49 & 362 & $\odot$ & $2.75590 \mathrm{E}-07$ & 293.6 & \\
\hline Ti-50 & 362 & $\odot$ & $2.63873 E-07$ & 293.6 & \\
\hline $\mathrm{Cr}-50$ & 362 & $\odot$ & 5. 30517E- -7 & 293.6 & \\
\hline $\mathrm{Cr}-52$ & 362 & $\odot$ & 1. $\odot 2189 E-05$ & 293.6 & d \\
\hline $\mathrm{Cr}-53$ & 362 & $\odot$ & $1.15860 E-06$ & 293.6 & d \\
\hline $\mathrm{Cr}-54$ & 362 & $\odot$ & $2.87821 E-07$ & 293.6 & \\
\hline$M n-55$ & 362 & $\odot$ & $6.446 \odot 2 \mathrm{E}-06$ & 293.6 & \\
\hline $\mathrm{Fe}-54$ & 362 & $\odot$ & $2.34704 \mathrm{E}-06$ & 293.6 & \\
\hline
\end{tabular}




$\begin{array}{llllll}\mathrm{Fe}-56 & 362 & \odot & 3.68104 \mathrm{E}-05 & 293.6 & \text { end } \\ \mathrm{Fe}-57 & 362 & \odot & 8.50550 \mathrm{E}-07 & 293.6 & \text { end } \\ \mathrm{Fe}-58 & 362 & \odot & 1.12337 \mathrm{E}-07 & 293.6 & \text { end } \\ \mathrm{Cu}-63 & 362 & \odot & 1.87830 \mathrm{E}-05 & 293.6 & \text { end } \\ \mathrm{Cu}-65 & 362 & \odot & 8.37182 \mathrm{E}-06 & 293.6 & \text { end } \\ \mathrm{U}-234 & 362 & \odot & 5.42167 \mathrm{E}-06 & 293.6 & \text { end } \\ \mathrm{U}-235 & 362 & \odot & 5.05300 \mathrm{E}-04 & 293.6 & \text { end } \\ \mathrm{U}-236 & 362 & \odot & 2.16868 \mathrm{E}-06 & 293.6 & \text { end } \\ \mathrm{U}-238 & 362 & \odot & 2.92770 \mathrm{E}-05 & 293.6 & \text { end }\end{array}$

' ${ }^{-238}$ total atom density $=8.01228 \mathrm{E}-02 \mathrm{a} / \mathrm{b}-\mathrm{cm}$

8. $012280 \mathrm{E}-02$

$\begin{array}{llllll}\mathrm{H}-1 & 363 & \odot & 3.32434 \mathrm{E}-02 & 293.6 & \text { end } \\ \mathrm{O}-16 & 363 & \odot & 1.82870 \mathrm{E}-02 & 293.6 & \text { end } \\ \mathrm{Mg}-24 & 363 & \odot & 1.05686 \mathrm{E}-04 & 293.6 & \text { end } \\ \mathrm{Mg}-25 & 363 & \odot & 1.33797 \mathrm{E}-05 & 293.6 & \text { end } \\ \mathrm{Mg}-26 & 363 & \odot & 1.47310 \mathrm{E}-05 & 293.6 & \text { end } \\ \mathrm{Al}-27 & 363 & \odot & 2.76228 \mathrm{E}-02 & 293.6 & \text { end } \\ \mathrm{Si}-28 & 363 & \odot & 9.95724 \mathrm{E}-05 & 293.6 & \text { end } \\ \mathrm{Si}-29 & 363 & \odot & 5.04178 \mathrm{E}-06 & 293.6 & \text { end } \\ \mathrm{Si}-30 & 363 & \odot & 3.34679 \mathrm{E}-06 & 293.6 & \text { end } \\ \mathrm{Ti}-46 & 363 & \odot & 4.20262 \mathrm{E}-07 & 293.6 & \text { end } \\ \mathrm{Ti}-47 & 363 & \odot & 3.79000 \mathrm{E}-07 & 293.6 & \text { end } \\ \mathrm{Ti}-48 & 363 & \odot & 3.75536 \mathrm{E}-06 & 293.6 & \text { end } \\ \mathrm{Ti}-49 & 363 & \odot & 2.75590 \mathrm{E}-07 & 293.6 & \text { end } \\ \mathrm{Ti}-50 & 363 & \odot & 2.63873 \mathrm{E}-07 & 293.6 & \text { end } \\ \mathrm{Cr}-50 & 363 & \odot & 5.30517 \mathrm{E}-07 & 293.6 & \text { end } \\ \mathrm{Cr}-52 & 363 & \odot & 1.02189 \mathrm{E}-05 & 293.6 & \text { end } \\ \mathrm{Cr}-53 & 363 & \odot & 1.15860 \mathrm{E}-06 & 293.6 & \text { end } \\ \mathrm{Cr}-54 & 363 & \odot & 2.87821 \mathrm{E}-07 & 293.6 & \text { end } \\ \mathrm{Mn}-55 & 363 & \odot & 6.40711 \mathrm{E}-06 & 293.6 & \text { end } \\ \mathrm{Fe}-54 & 363 & \odot & 2.32464 \mathrm{E}-06 & 293.6 & \text { end } \\ \mathrm{Fe}-56 & 363 & \odot & 3.64591 \mathrm{E}-05 & 293.6 & \text { end } \\ \mathrm{Fe}-57 & 363 & \odot & 8.42433 \mathrm{E}-07 & 293.6 & \text { end } \\ \mathrm{Fe}-58 & 363 & \odot & 1.11265 \mathrm{E}-07 & 293.6 & \text { end } \\ \mathrm{Cu}-63 & 363 & \odot & 1.86534 \mathrm{E}-05 & 293.6 & \text { end } \\ \mathrm{Cu}-65 & 363 & \odot & 8.31405 \mathrm{E}-06 & 293.6 & \text { end } \\ \mathrm{U}-234 & 363 & \odot & 6.37446 \mathrm{E}-06 & 293.6 & \text { end } \\ \mathrm{U}-235 & 363 & \odot & 5.94100 \mathrm{E}-04 & 293.6 & \text { end } \\ \mathrm{U}-236 & 363 & \odot & 2.54980 \mathrm{E}-06 & 293.6 & \text { end } \\ \mathrm{U}-238 & 363 & \odot & 3.44221 \mathrm{E}-05 & 293.6 & \text { end } \\ & & & & & \end{array}$

$\begin{array}{lllll}\text { U-238 } & 363 & \odot & 3.44221 \mathrm{E}-05 & 293.6\end{array}$ end

\begin{tabular}{|c|c|c|c|c|c|}
\hline $\mathrm{H}-1$ & 364 & $\odot$ & $3.32434 \mathrm{E}-02$ & 293.6 & end \\
\hline $0-16$ & 364 & $\odot$ & 1. $85176 \mathrm{E}-02$ & 293.6 & end \\
\hline$M g-24$ & 364 & $\odot$ & $1.05686 \mathrm{E}-04$ & 293.6 & end \\
\hline$M g-25$ & 364 & 0 & $1.33797 \mathrm{E}-05$ & 293.6 & nd \\
\hline$M g-26$ & 364 & 0 & 1. $47310 \mathrm{E}-05$ & 293.6 & \\
\hline Al-27 & 364 & 0 & $2.73372 \mathrm{E}-\mathrm{-} 2$ & 293.6 & \\
\hline Si-28 & 364 & 0 & $9.89350 \mathrm{E}-05$ & 293.6 & \\
\hline Si-29 & 364 & 0 & $5.00951 \mathrm{E}-06$ & 293.6 & \\
\hline Si-30 & 364 & 0 & $3.32537 \mathrm{E}-06$ & 293.6 & \\
\hline Ti-46 & 364 & 0 & $4.2 \odot 262 E-07$ & 293.6 & \\
\hline $\mathrm{Ti}-47$ & 364 & 0 & 3. $79000 \mathrm{E}-07$ & 293.6 & \\
\hline Ti-48 & 364 & $\odot$ & $3.75536 \mathrm{E}-06$ & 293.6 & \\
\hline Ti-49 & 364 & $\odot$ & $2.75590 \mathrm{E}-07$ & 293.6 & \\
\hline Ti-50 & 364 & $\odot$ & $2.63873 \mathrm{E}-\odot 7$ & 293.6 & \\
\hline $\mathrm{Cr}-50$ & 364 & $\odot$ & $5.30517 \mathrm{E}-07$ & 293.6 & \\
\hline $\mathrm{Cr}-52$ & 364 & $\odot$ & 1. $02189 \mathrm{E}-05$ & 293.6 & \\
\hline $\mathrm{Cr}-53$ & 364 & $\odot$ & $1.15860 \mathrm{E}-06$ & 293.6 & \\
\hline $\mathrm{Cr}-54$ & 364 & 0 & $2.87821 \mathrm{E}-07$ & 293.6 & \\
\hline$M n-55$ & 364 & 0 & $6.37178 \mathrm{E}-06$ & 293.6 & \\
\hline $\mathrm{Fe}-54$ & 364 & $\odot$ & $2.3 \odot 432 \mathrm{E}-\odot 6$ & 293.6 & \\
\hline $\mathrm{Fe}-56$ & 364 & $\odot$ & $3.61403 \mathrm{E}-05$ & 293.6 & \\
\hline $\mathrm{Fe}-57$ & 364 & $\odot$ & $8.35068 \mathrm{E}-07$ & 293.6 & \\
\hline $\mathrm{Fe}-58$ & 364 & 0 & 1. $10292 \mathrm{E}-07$ & 293.6 & \\
\hline $\mathrm{Cu}-63$ & 364 & 0 & $1.85357 \mathrm{E}-05$ & 293.6 & \\
\hline $\mathrm{Cu}-65$ & 364 & $\odot$ & 8. 26161E-06 & 293.6 & \\
\hline$U-234$ & 364 & 0 & $7.23926 \mathrm{E}-06$ & 293.6 & \\
\hline$U-235$ & 364 & $\odot$ & $6.74700 \mathrm{E}-04$ & 293.6 & \\
\hline$U-236$ & 364 & 0 & $2.89573 E-\odot 6$ & 293.6 & \\
\hline$U-238$ & 364 & $\odot$ & 3. $90920 \mathrm{E}-05$ & 293.6 & \\
\hline \multicolumn{3}{|c|}{$\begin{array}{l}\text { total atom density } \\
4370 \mathrm{E}-\odot 2\end{array}$} & \multicolumn{2}{|c|}{$=8.01437 \mathrm{E}-02 \mathrm{a} / \mathrm{b}-\mathrm{cm}$} & \\
\hline$H-1$ & 365 & $\odot$ & 3. 32434E- 02 & 293.6 & \\
\hline $0-16$ & 365 & 0 & $1.84469 \mathrm{E}-02$ & 293.6 & \\
\hline$M g-24$ & 365 & 0 & 1. $05686 \mathrm{E}-04$ & 293.6 & \\
\hline$M g-25$ & 365 & $\odot$ & $1.33797 \mathrm{E}-05$ & 293.6 & \\
\hline$M g-26$ & 365 & $\odot$ & $1.47310 \mathrm{E}-05$ & 293.6 & \\
\hline$A l-27$ & 365 & 0 & $2.74247 \mathrm{E}-\odot 2$ & 293.6 & \\
\hline Si-28 & 365 & $\odot$ & $9.91306 \mathrm{E}-05$ & 293.6 & \\
\hline Si-29 & 365 & 0 & $5.01941 E-06$ & 293.6 & \\
\hline
\end{tabular}




$\begin{array}{llllll}\mathrm{Si}-3 \odot & 365 & \odot & 3.33194 \mathrm{E}-\odot 6 & 293.6 & \text { end } \\ \mathrm{Ti}-46 & 365 & \odot & 4.20262 \mathrm{E}-\odot 7 & 293.6 & \text { end } \\ \mathrm{Ti}-47 & 365 & \odot & 3.79000 \mathrm{E}-\odot 7 & 293.6 & \text { end } \\ \mathrm{Ti}-48 & 365 & \odot & 3.75536 \mathrm{E}-\odot 6 & 293.6 & \text { end } \\ \mathrm{Ti}-49 & 365 & \odot & 2.75590 \mathrm{E}-\odot 7 & 293.6 & \text { end } \\ \mathrm{Ti}-5 \odot & 365 & \odot & 2.63873 \mathrm{E}-\odot 7 & 293.6 & \text { end } \\ \mathrm{Cr}-5 \odot & 365 & \odot & 5.30517 \mathrm{E}-\odot 7 & 293.6 & \text { end } \\ \mathrm{Cr}-52 & 365 & \odot & 1.02189 \mathrm{E}-05 & 293.6 & \text { end } \\ \mathrm{Cr}-53 & 365 & \odot & 1.15860 \mathrm{E}-\odot 6 & 293.6 & \text { end } \\ \mathrm{Cr}-54 & 365 & \odot & 2.87821 \mathrm{E}-\odot 7 & 293.6 & \text { end } \\ \mathrm{Mn}-55 & 365 & \odot & 6.38261 \mathrm{E}-\odot 6 & 293.6 & \text { end } \\ \mathrm{Fe}-54 & 365 & \odot & 2.31055 \mathrm{E}-\odot 6 & 293.6 & \text { end } \\ \mathrm{Fe}-56 & 365 & \odot & 3.62380 \mathrm{E}-\odot 5 & 293.6 & \text { end } \\ \mathrm{Fe}-57 & 365 & \odot & 8.37326 \mathrm{E}-\odot 7 & 293.6 & \text { end } \\ \mathrm{Fe}-58 & 365 & \odot & 1.10590 \mathrm{E}-\odot 7 & 293.6 & \text { end } \\ \mathrm{Cu}-63 & 365 & \odot & 1.85717 \mathrm{E}-\odot 5 & 293.6 & \text { end } \\ \mathrm{Cu}-65 & 365 & \odot & 8.27767 \mathrm{E}-\odot 6 & 293.6 & \text { end } \\ \mathrm{U}-234 & 365 & \odot & 6.97424 \mathrm{E}-\odot 6 & 293.6 & \text { end } \\ \mathrm{U}-235 & 365 & \odot & 6.50000 \mathrm{E}-\odot 4 & 293.6 & \text { end } \\ \mathrm{U}-236 & 365 & \odot & 2.78972 \mathrm{E}-\odot 6 & 293.6 & \text { end } \\ \mathrm{U}-238 & 365 & \odot & 3.76609 \mathrm{E}-05 & 293.6 & \text { end }\end{array}$

total atom density $=8.00985 \mathrm{E}-02 \mathrm{a} / \mathrm{b}-\mathrm{cm}$

\begin{tabular}{|c|c|c|c|c|c|}
\hline \multicolumn{3}{|l|}{ ' } & \multicolumn{3}{|c|}{ 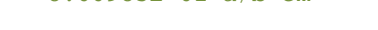 } \\
\hline $\mathrm{H}-1$ & 366 & $\odot$ & $3.32434 \mathrm{E}-02$ & 293.6 & end \\
\hline $0-16$ & 366 & $\odot$ & 1. $81013 \mathrm{E}-02$ & 293.6 & end \\
\hline $\mathrm{Mg}-24$ & 366 & $\odot$ & 1. $05686 E-04$ & 293.6 & end \\
\hline $\mathrm{Mg}-25$ & 366 & $\odot$ & $1.33797 \mathrm{E}-05$ & 293.6 & nd \\
\hline $\mathrm{Mg}-26$ & 366 & $\odot$ & $1.47310 \mathrm{E}-05$ & 293.6 & nd \\
\hline Al-27 & 366 & $\odot$ & $2.78528 E-02$ & 293.6 & nd \\
\hline Si-28 & 366 & $\odot$ & 1. $\odot \odot \odot 85 E-\odot 4$ & 293.6 & ad \\
\hline Si-29 & 366 & 0 & $5.06774 \mathrm{E}-06$ & 293.6 & \\
\hline Si-30 & 366 & $\odot$ & $3.36403 E-06$ & 293.6 & $\mathrm{ad}$ \\
\hline Ti-46 & 366 & 0 & 4. 20262E- 07 & 293.6 & . \\
\hline Ti-47 & 366 & 0 & $3.79000 \mathrm{E}-07$ & 293.6 & nc \\
\hline Ti-48 & 366 & $\odot$ & $3.75536 \mathrm{E}-06$ & 293.6 & \\
\hline Ti-49 & 366 & $\odot$ & $2.7559 \odot E-\odot 7$ & 293.6 & \\
\hline Ti-50 & 366 & $\odot$ & $2.63873 E-07$ & 293.6 & \\
\hline $\mathrm{Cr}-50$ & 366 & $\odot$ & $5.30517 \mathrm{E}-07$ & 293.6 & \\
\hline $\mathrm{Cr}-52$ & 366 & $\odot$ & 1. $02189 E-\odot 5$ & 293.6 & \\
\hline $\mathrm{Cr}-53$ & 366 & $\odot$ & $1.15860 \mathrm{E}-06$ & 293.6 & \\
\hline $\mathrm{Cr}-54$ & 366 & 0 & $2.87821 \mathrm{E}-07$ & 293.6 & \\
\hline$M n-55$ & 366 & 0 & $6.43555 \mathrm{E}-06$ & 293.6 & \\
\hline $\mathrm{Fe}-54$ & 366 & 0 & $2.34101 \mathrm{E}-06$ & 293.6 & \\
\hline $\mathrm{Fe}-56$ & 366 & 0 & $3.67158 \mathrm{E}-05$ & 293.6 & 10 \\
\hline $\mathrm{Fe}-57$ & 366 & $\odot$ & $8.48365 E-07$ & 293.6 & \\
\hline $\mathrm{Fe}-58$ & 366 & 0 & $1.12048 \mathrm{E}-07$ & 293.6 & \\
\hline $\mathrm{Cu}-63$ & 366 & $\odot$ & 1. 87481E- 05 & 293.6 & \\
\hline $\mathrm{Cu}-65$ & 366 & $\odot$ & 8. 35629E- - 66 & 293.6 & \\
\hline U-234 & 366 & $\odot$ & $5.67811 \mathrm{E}-06$ & 293.6 & \\
\hline$U-235$ & 366 & $\odot$ & $5.29200 E-\odot 4$ & 293.6 & \\
\hline$U-236$ & 366 & $\odot$ & $2.27126 \mathrm{E}-06$ & 293.6 & \\
\hline U- 238 & 366 & $\odot$ & 3. $06618 E-05$ & 293.6 & \\
\hline 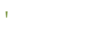 & tom & sity & $=8.00555 \mathrm{E}$ & $2 a / b-c m$ & \\
\hline 8.00 & & & & & \\
\hline $\mathrm{H}-1$ & 367 & $\odot$ & $3.32434 \mathrm{E}-02$ & 293.6 & \\
\hline $0-16$ & 367 & $\odot$ & $1.77731 \mathrm{E}-02$ & 293.6 & \\
\hline$M g-24$ & 367 & 0 & $1.05686 \mathrm{E}-04$ & 293.6 & \\
\hline$M g-25$ & 367 & $\odot$ & 1. 33797E- 05 & 293.6 & \\
\hline$M g-26$ & 367 & $\odot$ & $1.47310 \mathrm{E}-05$ & 293.6 & \\
\hline Al-27 & 367 & $\odot$ & $2.82593 E-02$ & 293.6 & \\
\hline Si-28 & 367 & $\odot$ & 1. $\odot \odot 993 E-\odot 4$ & 293.6 & \\
\hline Si-29 & 367 & $\odot$ & $5.11370 E-06$ & 293.6 & \\
\hline Si-30 & 367 & $\odot$ & $3.39453 E-06$ & 293.6 & \\
\hline Ti-46 & 367 & $\odot$ & $4.20262 \mathrm{E}-07$ & 293.6 & \\
\hline Ti-47 & 367 & 0 & $3.79000 \mathrm{E}-\odot 7$ & 293.6 & \\
\hline Ti-48 & 367 & $\odot$ & $3.75536 \mathrm{E}-06$ & 293.6 & \\
\hline Ti-49 & 367 & $\odot$ & $2.75590 \mathrm{E}-\odot 7$ & 293.6 & \\
\hline Ti-50 & 367 & $\odot$ & $2.63873 E-07$ & 293.6 & \\
\hline $\mathrm{Cr}-50$ & 367 & $\odot$ & $5.30517 E-07$ & 293.6 & \\
\hline $\mathrm{Cr}-52$ & 367 & $\odot$ & $1.02189 E-05$ & 293.6 & \\
\hline $\mathrm{Cr}-53$ & 367 & $\odot$ & $1.15860 \mathrm{E}-06$ & 293.6 & \\
\hline $\mathrm{Cr}-54$ & 367 & $\odot$ & $2.87821 E-07$ & 293.6 & \\
\hline$M n-55$ & 367 & $\odot$ & $6.48581 E-06$ & 293.6 & \\
\hline $\mathrm{Fe}-54$ & 367 & $\odot$ & 2. 36993E- 06 & 293.6 & \\
\hline $\mathrm{Fe}-56$ & 367 & $\odot$ & $3.71695 E-05$ & 293.6 & \\
\hline $\mathrm{Fe}-57$ & 367 & $\odot$ & $8.58848 E-07$ & 293.6 & \\
\hline $\mathrm{Fe}-58$ & 367 & $\odot$ & $1.13433 \mathrm{E}-07$ & 293.6 & \\
\hline $\mathrm{Cu}-63$ & 367 & $\odot$ & 1. 89155E-05 & 293.6 & \\
\hline $\mathrm{Cu}-65$ & 367 & $\odot$ & $8.43090 \mathrm{E}-06$ & 293.6 & \\
\hline U-234 & 367 & 0 & 4. 44742E-๑6 & 293.6 & \\
\hline U-235 & 367 & $\odot$ & $4.14500 \mathrm{E}-\odot 4$ & 293.6 & \\
\hline
\end{tabular}




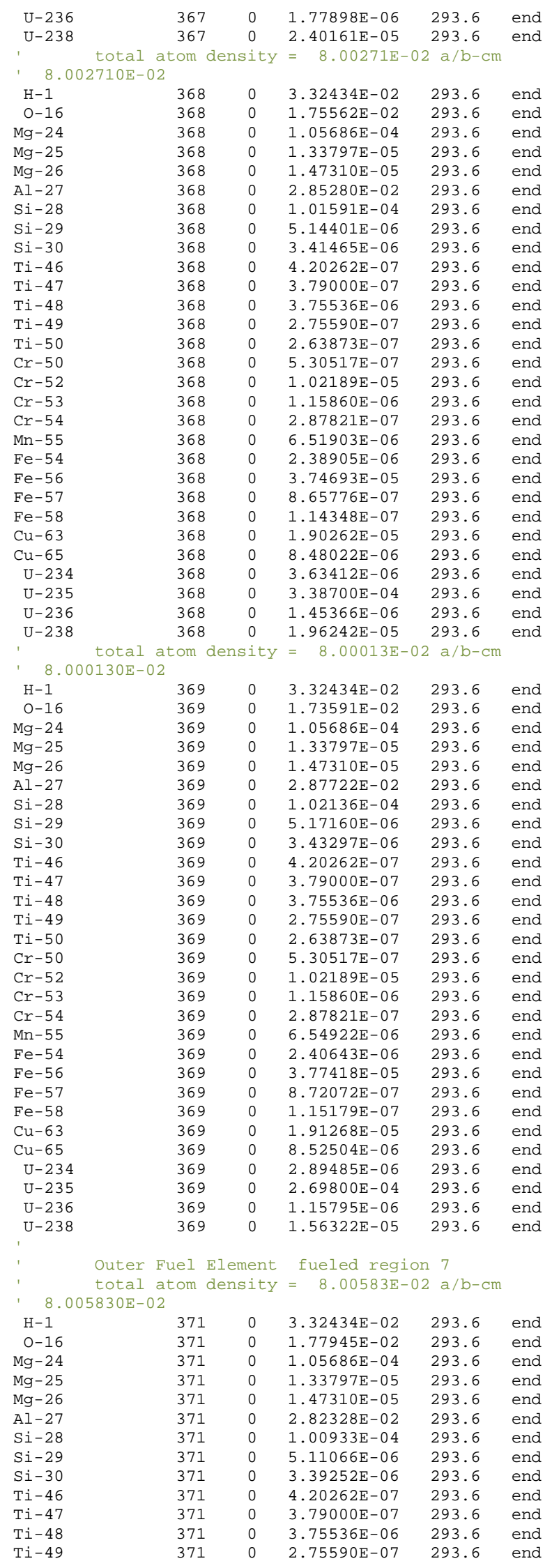




\begin{tabular}{|c|c|c|c|c|c|}
\hline Ti-50 & 371 & $\odot$ & $2.63873 E-\odot 7$ & 293.6 & end \\
\hline $\mathrm{Cr}-50$ & 371 & $\odot$ & $5.30517 \mathrm{E}-07$ & 293.6 & end \\
\hline $\mathrm{Cr}-52$ & 371 & $\odot$ & 1. $02189 E-05$ & 293.6 & end \\
\hline $\mathrm{Cr}-53$ & 371 & $\odot$ & $1.15860 \mathrm{E}-06$ & 293.6 & end \\
\hline $\mathrm{Cr}-54$ & 371 & $\odot$ & $2.87821 E-\odot 7$ & 293.6 & end \\
\hline Mn-55 & 371 & $\odot$ & $6.48252 \mathrm{E}-\odot 6$ & 293.6 & end \\
\hline $\mathrm{Fe}-54$ & 371 & $\odot$ & $2.36804 \mathrm{E}-\odot 6$ & 293.6 & end \\
\hline $\mathrm{Fe}-56$ & 371 & $\odot$ & $3.71398 \mathrm{E}-05$ & 293.6 & end \\
\hline $\mathrm{Fe}-57$ & 371 & $\odot$ & $8.58163 E-07$ & 293.6 & end \\
\hline $\mathrm{Fe}-58$ & 371 & $\odot$ & $1.13342 \mathrm{E}-07$ & 293.6 & end \\
\hline Cu-63 & 371 & $\odot$ & $1.89046 \mathrm{E}-05$ & 293.6 & nd \\
\hline $\mathrm{Cu}-65$ & 371 & $\odot$ & $8.42602 \mathrm{E}-06$ & 293.6 & nd \\
\hline U-234 & 371 & $\odot$ & $4.52789 E-\odot 6$ & 293.6 & nd \\
\hline$U-235$ & 371 & $\odot$ & $4.22000 \mathrm{E}-\odot 4$ & 293.6 & nd \\
\hline$U-236$ & 371 & $\odot$ & $1.81117 \mathrm{E}-06$ & 293.6 & nd \\
\hline$U-238$ & 371 & $\odot$ & $2.44506 \mathrm{E}-05$ & 293.6 & nd \\
\hline \multicolumn{3}{|c|}{$\begin{array}{l}\text { total atom density } \\
\text { ' } 8,008950 \mathrm{E}-02\end{array}$} & \multicolumn{2}{|c|}{$=8.00895 \mathrm{E}-02 \mathrm{a} / \mathrm{b}-\mathrm{cm}$} & \\
\hline $\mathrm{H}-1$ & 372 & $\odot$ & 3. 32434E- 02 & 293.6 & end \\
\hline $0-16$ & 372 & $\odot$ & 1. 80329E- 02 & 293.6 & d \\
\hline$M g-24$ & 372 & $\odot$ & 1. $05686 \mathrm{E}-04$ & 293.6 & id \\
\hline $\mathrm{Mg}-25$ & 372 & $\odot$ & $1.33797 \mathrm{E}-05$ & 293.6 & id \\
\hline$M g-26$ & 372 & $\odot$ & 1. $47310 \mathrm{E}-05$ & 293.6 & end \\
\hline $\mathrm{Al}-27$ & 372 & $\odot$ & $2.79375 \mathrm{E}-02$ & 293.6 & end \\
\hline Si-28 & 372 & $\odot$ & 1. $\odot \odot 274 \mathrm{E}-\odot 4$ & 293.6 & end \\
\hline Si-29 & 372 & $\odot$ & $5.07732 E-06$ & 293.6 & end \\
\hline Si-30 & 372 & $\odot$ & $3.37038 \mathrm{E}-06$ & 293.6 & end \\
\hline Ti-46 & 372 & $\odot$ & $4.2 \odot 262 \mathrm{E}-\odot 7$ & 293.6 & nd \\
\hline Ti-47 & 372 & $\odot$ & $3.79000 \mathrm{E}-\odot 7$ & 293.6 & nd \\
\hline Ti-48 & 372 & $\odot$ & $3.75536 \mathrm{E}-\odot 6$ & 293.6 & and \\
\hline Ti-49 & 372 & $\odot$ & $2.75590 \mathrm{E}-\odot 7$ & 293.6 & nd \\
\hline Ti-50 & 372 & 0 & $2.63873 \mathrm{E}-07$ & 293.6 & nd \\
\hline $\mathrm{Cr}-50$ & 372 & $\odot$ & $5.30517 \mathrm{E}-07$ & 293.6 & nd \\
\hline $\mathrm{Cr}-52$ & 372 & $\odot$ & 1. $02189 E-05$ & 293.6 & nd \\
\hline $\mathrm{Cr}-53$ & 372 & $\odot$ & $1.15860 \mathrm{E}-06$ & 293.6 & nd \\
\hline $\mathrm{Cr}-54$ & 372 & $\odot$ & $2.87821 E-\odot 7$ & 293.6 & nd \\
\hline$M n-55$ & 372 & $\odot$ & $6.44602 \mathrm{E}-\odot 6$ & 293.6 & nd \\
\hline $\mathrm{Fe}-54$ & 372 & $\odot$ & $2.34704 \mathrm{E}-06$ & 293.6 & nd \\
\hline $\mathrm{Fe}-56$ & 372 & $\odot$ & $3.68104 \mathrm{E}-05$ & 293.6 & d \\
\hline $\mathrm{Fe}-57$ & 372 & $\odot$ & $8.50550 E-07$ & 293.6 & nd \\
\hline $\mathrm{Fe}-58$ & 372 & 0 & $1.12337 \mathrm{E}-07$ & 293.6 & nd \\
\hline $\mathrm{Cu}-63$ & 372 & 0 & $1.87830 \mathrm{E}-05$ & 293.6 & nd \\
\hline Cu- 65 & 372 & 0 & $8.37182 \mathrm{E}-06$ & 293.6 & d \\
\hline$U-234$ & 372 & 0 & $5.42167 \mathrm{E}-06$ & 293.6 & nd \\
\hline$U-235$ & 372 & $\odot$ & $5.05300 \mathrm{E}-\odot 4$ & 293.6 & $d$ \\
\hline$U-236$ & 372 & $\odot$ & $2.16868 \mathrm{E}-\odot 6$ & 293.6 & nd \\
\hline$U-238$ & 372 & $\odot$ & $2.92770 \mathrm{E}-05$ & 293.6 & end \\
\hline 1 & tom & sity & $=8.01228 \mathrm{E}-$ & $2 \mathrm{a} / \mathrm{b}-\mathrm{cm}$ & \\
\hline \multicolumn{6}{|c|}{ ' $\quad 8.012280 E-02$} \\
\hline $\mathrm{H}-1$ & 373 & $\odot$ & $3.32434 \mathrm{E}-02$ & 293.6 & nd \\
\hline $0-16$ & 373 & $\odot$ & 1. $82870 \mathrm{E}-02$ & 293.6 & nd \\
\hline$M g-24$ & 373 & $\odot$ & 1. $05686 \mathrm{E}-04$ & 293.6 & nd \\
\hline$M g-25$ & 373 & $\odot$ & 1. 33797E- 05 & 293.6 & nd \\
\hline$M g-26$ & 373 & $\odot$ & $1.47310 \mathrm{E}-05$ & 293.6 & ad \\
\hline $\mathrm{Al}-27$ & 373 & $\odot$ & $2.76228 \mathrm{E}-02$ & 293.6 & nd \\
\hline Si-28 & 373 & $\odot$ & $9.95724 \mathrm{E}-05$ & 293.6 & nd \\
\hline Si-29 & 373 & $\odot$ & $5 . \odot 4178 \mathrm{E}-\odot 6$ & 293.6 & nd \\
\hline Si-30 & 373 & $\odot$ & $3.34679 E-06$ & 293.6 & nd \\
\hline Ti-46 & 373 & $\odot$ & $4.20262 \mathrm{E}-\odot 7$ & 293.6 & nd \\
\hline Ti-47 & 373 & $\odot$ & $3.79000 \mathrm{E}-07$ & 293.6 & ad \\
\hline Ti-48 & 373 & $\odot$ & $3.75536 \mathrm{E}-06$ & 293.6 & nd \\
\hline Ti-49 & 373 & $\odot$ & $2.75590 \mathrm{E}-\odot 7$ & 293.6 & $d$ \\
\hline Ti-50 & 373 & $\odot$ & $2.63873 E-07$ & 293.6 & d \\
\hline $\mathrm{Cr}-50$ & 373 & $\odot$ & $5.30517 \mathrm{E}-07$ & 293.6 & d \\
\hline $\mathrm{Cr}-52$ & 373 & 0 & 1. $02189 \mathrm{E}-05$ & 293.6 & d \\
\hline $\mathrm{Cr}-53$ & 373 & $\odot$ & $1.15860 \mathrm{E}-\odot 6$ & 293.6 & d \\
\hline $\mathrm{Cr}-54$ & 373 & $\odot$ & $2.87821 \mathrm{E}-07$ & 293.6 & d \\
\hline$M n-55$ & 373 & $\odot$ & $6.40711 \mathrm{E}-\odot 6$ & 293.6 & \\
\hline $\mathrm{Fe}-54$ & 373 & $\odot$ & $2.32464 \mathrm{E}-06$ & 293.6 & \\
\hline $\mathrm{Fe}-56$ & 373 & $\odot$ & $3.64591 E-05$ & 293.6 & \\
\hline $\mathrm{Fe}-57$ & 373 & $\odot$ & $8.42433 E-07$ & 293.6 & \\
\hline $\mathrm{Fe}-58$ & 373 & $\odot$ & $1.11265 \mathrm{E}-07$ & 293.6 & nd \\
\hline $\mathrm{Cu}-63$ & 373 & $\odot$ & 1. $86534 \mathrm{E}-05$ & 293.6 & id \\
\hline $\mathrm{Cu}-65$ & 373 & $\odot$ & $8.31405 E-06$ & 293.6 & \\
\hline$U-234$ & 373 & $\odot$ & $6.37446 E-\odot 6$ & 293.6 & nd \\
\hline$U-235$ & 373 & $\odot$ & $5.94100 \mathrm{E}-04$ & 293.6 & end \\
\hline$U-236$ & 373 & $\odot$ & $2.54980 E-\odot 6$ & 293.6 & \\
\hline$U-238$ & 373 & $\odot$ & $3.44221 E-05$ & 293.6 & \\
\hline \multirow{2}{*}{\multicolumn{6}{|c|}{ ' $8.01530 \odot \mathrm{t}-\odot 2$}} \\
\hline & & & & & \\
\hline $\mathrm{H}-1$ & 374 & $\odot$ & $3.32434 \mathrm{E}-02$ & 293.6 & \\
\hline
\end{tabular}




\begin{tabular}{|c|c|c|c|c|c|}
\hline $0-16$ & 374 & $\odot$ & $1.85176 E-02$ & 293.6 & end \\
\hline$M g-24$ & 374 & $\odot$ & 1. $05686 E-\odot 4$ & 293.6 & end \\
\hline$M g-25$ & 374 & $\odot$ & $1.33797 \mathrm{E}-05$ & 293.6 & end \\
\hline$M g-26$ & 374 & $\odot$ & 1. $47310 \mathrm{E}-05$ & 293.6 & end \\
\hline Al-27 & 374 & $\odot$ & $2.73372 \mathrm{E}-\odot 2$ & 293.6 & end \\
\hline Si-28 & 374 & $\odot$ & $9.89350 E-05$ & 293.6 & end \\
\hline Si-29 & 374 & $\odot$ & $5.00951 E-06$ & 293.6 & end \\
\hline Si-30 & 374 & $\odot$ & $3.32537 E-06$ & 293.6 & end \\
\hline Ti-46 & 374 & $\odot$ & $4.20262 E-07$ & 293.6 & end \\
\hline Ti-47 & 374 & $\odot$ & $3.79000 \mathrm{E}-07$ & 293.6 & nd \\
\hline Ti-48 & 374 & $\odot$ & $3.75536 E-06$ & 293.6 & nd \\
\hline Ti-49 & 374 & $\odot$ & $2.75590 E-\odot 7$ & 293.6 & nd \\
\hline Ti-50 & 374 & $\odot$ & $2.63873 E-07$ & 293.6 & nd \\
\hline $\mathrm{Cr}-50$ & 374 & $\odot$ & $5.30517 E-07$ & 293.6 & id \\
\hline $\mathrm{Cr}-52$ & 374 & $\odot$ & 1. $02189 E-05$ & 293.6 & id \\
\hline $\mathrm{Cr}-53$ & 374 & $\odot$ & 1. $15860 E-06$ & 293.6 & id \\
\hline $\mathrm{Cr}-54$ & 374 & $\odot$ & $2.87821 E-07$ & 293.6 & \\
\hline$M n-55$ & 374 & $\odot$ & $6.37178 E-06$ & 293.6 & end \\
\hline $\mathrm{Fe}-54$ & 374 & $\odot$ & $2.30432 \mathrm{E}-\odot 6$ & 293.6 & end \\
\hline $\mathrm{Fe}-56$ & 374 & $\odot$ & $3.61403 E-05$ & 293.6 & \\
\hline $\mathrm{Fe}-57$ & 374 & $\odot$ & $8.35068 \mathrm{E}-07$ & 293.6 & d \\
\hline $\mathrm{Fe}-58$ & 374 & $\odot$ & $1.10292 \mathrm{E}-07$ & 293.6 & \\
\hline $\mathrm{Cu}-63$ & 374 & $\odot$ & $1.85357 \mathrm{E}-05$ & 293.6 & \\
\hline $\mathrm{Cu}-65$ & 374 & $\odot$ & 8. 26161E-๑6 & 293.6 & \\
\hline U-234 & 374 & $\odot$ & $7.23926 E-\odot 6$ & 293.6 & \\
\hline U-235 & 374 & $\odot$ & $6.74700 \mathrm{E}-\odot 4$ & 293.6 & \\
\hline$U-236$ & 374 & $\odot$ & $2.89573 E-06$ & 293.6 & \\
\hline$U-238$ & 374 & $\odot$ & 3. $90920 E-05$ & 293.6 & \\
\hline
\end{tabular}

total atom density $=8.01437 \mathrm{E}-02 \mathrm{a} / \mathrm{b}-\mathrm{cm}$

\begin{tabular}{|c|c|c|c|c|c|}
\hline $\mathrm{H}-1$ & 375 & $\odot$ & $3.32434 \mathrm{E}-02$ & 293.6 & end \\
\hline $0-16$ & 375 & $\odot$ & 1. $84469 E-\odot 2$ & 293.6 & end \\
\hline$M g-24$ & 375 & 0 & 1. $05686 \mathrm{E}-\odot 4$ & 293.6 & nd \\
\hline$M g-25$ & 375 & 0 & 1. 33797E- 05 & 293.6 & \\
\hline$M g-26$ & 375 & 0 & 1. $47310 \mathrm{E}-05$ & 293.6 & \\
\hline Al-27 & 375 & 0 & $2.74247 \mathrm{E}-02$ & 293.6 & \\
\hline Si-28 & 375 & 0 & $9.91306 \mathrm{E}-05$ & 293.6 & \\
\hline Si-29 & 375 & 0 & $5.01941 \mathrm{E}-06$ & 293.6 & \\
\hline Si-30 & 375 & 0 & 3. 33194E- 06 & 293.6 & \\
\hline Ti-46 & 375 & 0 & $4.20262 E-07$ & 293.6 & \\
\hline Ti-47 & 375 & $\odot$ & 3. $79000 \mathrm{E}-\odot 7$ & 293.6 & \\
\hline Ti-48 & 375 & $\odot$ & 3. 75536E- - 66 & 293.6 & \\
\hline Ti-49 & 375 & $\odot$ & $2.75590 \mathrm{E}-\odot 7$ & 293.6 & \\
\hline Ti-50 & 375 & $\odot$ & $2.63873 E-07$ & 293.6 & \\
\hline $\mathrm{Cr}-50$ & 375 & $\odot$ & $5.30517 \mathrm{E}-07$ & 293.6 & \\
\hline $\mathrm{Cr}-52$ & 375 & $\odot$ & 1. $02189 E-05$ & 293.6 & \\
\hline $\mathrm{Cr}-53$ & 375 & $\odot$ & 1. 15860E- 06 & 293.6 & \\
\hline $\mathrm{Cr}-54$ & 375 & 0 & $2.87821 E-\odot 7$ & 293.6 & \\
\hline Mn-55 & 375 & 0 & $6.38261 E-06$ & 293.6 & \\
\hline $\mathrm{Fe}-54$ & 375 & $\odot$ & $2.31055 \mathrm{E}-06$ & 293.6 & \\
\hline $\mathrm{Fe}-56$ & 375 & $\odot$ & $3.62380 E-05$ & 293.6 & \\
\hline $\mathrm{Fe}-57$ & 375 & $\odot$ & $8.37326 \mathrm{E}-07$ & 293.6 & \\
\hline Fe-58 & 375 & $\odot$ & 1. 10590E - 07 & 293.6 & \\
\hline $\mathrm{Cu}-63$ & 375 & $\odot$ & $1.85717 \mathrm{E}-05$ & 293.6 & \\
\hline $\mathrm{Cu}-65$ & 375 & $\odot$ & $8.27767 E-\odot 6$ & 293.6 & \\
\hline$U-234$ & 375 & $\odot$ & $6.97424 \mathrm{E}-06$ & 293.6 & \\
\hline$U-235$ & 375 & $\odot$ & $6.500 \odot \odot E-\odot 4$ & 293.6 & \\
\hline$U-236$ & 375 & 0 & $2.78972 \mathrm{E}-06$ & 293.6 & \\
\hline$U-238$ & 375 & $\odot$ & $3.76609 \mathrm{E}-05$ & 293.6 & \\
\hline \multicolumn{3}{|c|}{$\begin{array}{l}\text { total atom density } \\
850 \mathrm{E}-02\end{array}$} & \multicolumn{3}{|c|}{$=8.00985 \mathrm{E}-02 \mathrm{a} / \mathrm{b}-\mathrm{cm}$} \\
\hline $\mathrm{H}-1$ & 376 & $\odot$ & 3. 32434E- 02 & 293.6 & \\
\hline $0-16$ & 376 & $\odot$ & 1. 81013E- 02 & 293.6 & \\
\hline$M g-24$ & 376 & $\odot$ & 1. $05686 E-\odot 4$ & 293.6 & \\
\hline Mg-25 & 376 & 0 & 1. 33797E- 05 & 293.6 & \\
\hline$M g-26$ & 376 & $\odot$ & $1.47310 \mathrm{E}-05$ & 293.6 & \\
\hline Al-27 & 376 & $\odot$ & $2.78528 \mathrm{E}-02$ & 293.6 & \\
\hline Si-28 & 376 & $\odot$ & 1. $\odot \odot \odot 85 E-\odot 4$ & 293.6 & \\
\hline Si-29 & 376 & 0 & $5.06774 \mathrm{E}-06$ & 293.6 & \\
\hline Si-30 & 376 & 0 & 3. 36403E-०6 & 293.6 & \\
\hline Ti-46 & 376 & $\odot$ & $4.20262 \mathrm{E}-07$ & 293.6 & \\
\hline Ti-47 & 376 & $\odot$ & $3.79000 \mathrm{E}-07$ & 293.6 & \\
\hline Ti-48 & 376 & $\odot$ & $3.75536 \mathrm{E}-06$ & 293.6 & \\
\hline Ti-49 & 376 & 0 & $2.75590 \mathrm{E}-07$ & 293.6 & \\
\hline Ti-50 & 376 & $\odot$ & $2.63873 E-07$ & 293.6 & \\
\hline $\mathrm{Cr}-50$ & 376 & 0 & $5.30517 \mathrm{E}-07$ & 293.6 & \\
\hline $\mathrm{Cr}-52$ & 376 & 0 & 1. $02189 E-05$ & 293.6 & \\
\hline $\mathrm{Cr}-53$ & 376 & 0 & 1. 15860E-०6 & 293.6 & \\
\hline $\mathrm{Cr}-54$ & 376 & 0 & $2.87821 E-\odot 7$ & 293.6 & \\
\hline Mn-55 & 376 & 0 & $6.43555 \mathrm{E}-06$ & 293.6 & \\
\hline $\mathrm{Fe}-54$ & 376 & 0 & $2.34101 \mathrm{E}-06$ & 293.6 & \\
\hline
\end{tabular}




$\begin{array}{llllll}\mathrm{Fe}-56 & 376 & \odot & 3.67158 \mathrm{E}-05 & 293.6 & \text { end } \\ \mathrm{Fe}-57 & 376 & \odot & 8.48365 \mathrm{E}-07 & 293.6 & \text { end } \\ \mathrm{Fe}-58 & 376 & \odot & 1.12048 \mathrm{E}-07 & 293.6 & \text { end } \\ \mathrm{Cu}-63 & 376 & \odot & 1.87481 \mathrm{E}-05 & 293.6 & \text { end } \\ \mathrm{Cu}-65 & 376 & \odot & 8.35629 \mathrm{E}-06 & 293.6 & \text { end } \\ \mathrm{U}-234 & 376 & \odot & 5.67811 \mathrm{E}-06 & 293.6 & \text { end } \\ \mathrm{U}-235 & 376 & \odot & 5.29200 \mathrm{E}-\odot 4 & 293.6 & \text { end } \\ \mathrm{U}-236 & 376 & \odot & 2.27126 \mathrm{E}-\odot 6 & 293.6 & \text { end } \\ \mathrm{U}-238 & 376 & \odot & 3.06618 \mathrm{E}-05 & 293.6 & \text { end }\end{array}$

\begin{tabular}{|c|c|c|c|c|c|}
\hline $\mathrm{H}-1$ & 377 & $\odot$ & 3. 32434E- 02 & 293.6 & end \\
\hline $0-16$ & 377 & $\odot$ & $1.77731 \mathrm{E}-\odot 2$ & 293.6 & end \\
\hline$M g-24$ & 377 & $\odot$ & 1. $05686 E-04$ & 293.6 & end \\
\hline$M g-25$ & 377 & $\odot$ & $1.33797 \mathrm{E}-05$ & 293.6 & end \\
\hline$M g-26$ & 377 & $\odot$ & $1.47310 \mathrm{E}-05$ & 293.6 & end \\
\hline $\mathrm{Al}-27$ & 377 & $\odot$ & $2.82593 E-02$ & 293.6 & id \\
\hline Si-28 & 377 & $\odot$ & 1. $\odot \odot 993 E-\odot 4$ & 293.6 & id \\
\hline Si-29 & 377 & 0 & $5.11370 E-06$ & 293.6 & nd \\
\hline $\mathrm{Si}-30$ & 377 & $\odot$ & 3. 39453E- 06 & 293.6 & nd \\
\hline Ti-46 & 377 & $\odot$ & $4.20262 \mathrm{E}-07$ & 293.6 & nd \\
\hline Ti-47 & 377 & 0 & 3. $79000 \mathrm{E}-\odot 7$ & 293.6 & nd \\
\hline Ti-48 & 377 & $\odot$ & 3. 75536E- - 66 & 293.6 & \\
\hline Ti-49 & 377 & $\odot$ & $2.75590 \mathrm{E}-\odot 7$ & 293.6 & \\
\hline Ti-50 & 377 & $\odot$ & $2.63873 E-07$ & 293.6 & \\
\hline $\mathrm{Cr}-50$ & 377 & $\odot$ & $5.30517 \mathrm{E}-07$ & 293.6 & \\
\hline $\mathrm{Cr}-52$ & 377 & $\odot$ & 1. $02189 E-05$ & 293.6 & \\
\hline $\mathrm{Cr}-53$ & 377 & $\odot$ & 1. 15860E-०6 & 293.6 & \\
\hline $\mathrm{Cr}-54$ & 377 & $\odot$ & $2.87821 E-07$ & 293.6 & \\
\hline Mn-55 & 377 & 0 & $6.48581 E-06$ & 293.6 & \\
\hline $\mathrm{Fe}-54$ & 377 & $\odot$ & $2.36993 E-06$ & 293.6 & \\
\hline $\mathrm{Fe}-56$ & 377 & 0 & $3.71695 \mathrm{E}-05$ & 293.6 & $\mathrm{ad}$ \\
\hline $\mathrm{Fe}-57$ & 377 & $\odot$ & $8.58848 E-07$ & 293.6 & \\
\hline Fe-58 & 377 & $\odot$ & 1. 13433E- 07 & 293.6 & \\
\hline $\mathrm{Cu}-63$ & 377 & $\odot$ & 1. 89155E- 05 & 293.6 & \\
\hline $\mathrm{Cu}-65$ & 377 & $\odot$ & 8. 43०9०E-๑6 & 293.6 & \\
\hline$U-234$ & 377 & 0 & $4.44742 \mathrm{E}-06$ & 293.6 & \\
\hline U-235 & 377 & 0 & $4.14500 \mathrm{E}-\odot 4$ & 293.6 & \\
\hline$U-236$ & 377 & 0 & $1.77898 \mathrm{E}-06$ & 293.6 & \\
\hline$U-238$ & 377 & 0 & $2.40161 \mathrm{E}-\odot 5$ & 293.6 & \\
\hline
\end{tabular}

, total atom density $=8.00271 \mathrm{E}-02 \mathrm{a} / \mathrm{b}-\mathrm{cm}$

\begin{tabular}{|c|c|c|c|c|c|}
\hline $\mathrm{H}-1$ & 378 & $\odot$ & 3. 32434E- 02 & 293.6 & end \\
\hline $0-16$ & 378 & $\odot$ & 1. $75562 \mathrm{E}-02$ & 293.6 & end \\
\hline$M g-24$ & 378 & $\odot$ & $1.05686 E-04$ & 293.6 & end \\
\hline$M g-25$ & 378 & $\odot$ & $1.33797 \mathrm{E}-05$ & 293.6 & nd \\
\hline$M g-26$ & 378 & $\odot$ & 1. $47310 \mathrm{E}-05$ & 293.6 & nd \\
\hline Al-27 & 378 & $\odot$ & $2.85280 E-02$ & 293.6 & \\
\hline Si-28 & 378 & $\odot$ & 1. 01591E- 04 & 293.6 & ad \\
\hline Si-29 & 378 & $\odot$ & $5.14401 \mathrm{E}-06$ & 293.6 & \\
\hline Si-30 & 378 & $\odot$ & $3.41465 E-06$ & 293.6 & \\
\hline Ti-46 & 378 & $\odot$ & $4.20262 \mathrm{E}-07$ & 293.6 & \\
\hline Ti-47 & 378 & $\odot$ & 3. $79000 \mathrm{E}-07$ & 293.6 & \\
\hline $\mathrm{Ti}-48$ & 378 & $\odot$ & $3.75536 \mathrm{E}-06$ & 293.6 & \\
\hline Ti-49 & 378 & $\odot$ & $2.75590 \mathrm{E}-07$ & 293.6 & \\
\hline Ti-50 & 378 & $\odot$ & $2.63873 \mathrm{E}-07$ & 293.6 & \\
\hline $\mathrm{Cr}-50$ & 378 & $\odot$ & 5. 30517E- - 7 & 293.6 & \\
\hline $\mathrm{Cr}-52$ & 378 & $\odot$ & 1. $02189 \mathrm{E}-05$ & 293.6 & \\
\hline $\mathrm{Cr}-53$ & 378 & $\odot$ & $1.15860 \mathrm{E}-06$ & 293.6 & \\
\hline $\mathrm{Cr}-54$ & 378 & $\odot$ & $2.87821 E-07$ & 293.6 & \\
\hline$M n-55$ & 378 & $\odot$ & $6.51903 E-06$ & 293.6 & \\
\hline $\mathrm{Fe}-54$ & 378 & $\odot$ & $2.38905 E-\odot 6$ & 293.6 & \\
\hline $\mathrm{Fe}-56$ & 378 & $\odot$ & $3.74693 \mathrm{E}-05$ & 293.6 & \\
\hline $\mathrm{Fe}-57$ & 378 & $\odot$ & $8.65776 \mathrm{E}-07$ & 293.6 & \\
\hline $\mathrm{Fe}-58$ & 378 & $\odot$ & $1.14348 \mathrm{E}-07$ & 293.6 & \\
\hline $\mathrm{Cu}-63$ & 378 & $\odot$ & 1. $90262 \mathrm{E}-05$ & 293.6 & \\
\hline $\mathrm{Cu}-65$ & 378 & $\odot$ & $8.48022 \mathrm{E}-06$ & 293.6 & \\
\hline$U-234$ & 378 & 0 & 3. $63412 \mathrm{E}-06$ & 293.6 & \\
\hline$U-235$ & 378 & 0 & $3.38700 \mathrm{E}-\odot 4$ & 293.6 & \\
\hline$U-236$ & 378 & $\odot$ & $1.45366 \mathrm{E}-06$ & 293.6 & \\
\hline$U-238$ & 378 & $\odot$ & 1. $96242 \mathrm{E}-05$ & 293.6 & \\
\hline \multicolumn{3}{|c|}{ total atom density } & \multicolumn{3}{|c|}{$=8.00013 \mathrm{E}-02 \mathrm{a} / \mathrm{b}-\mathrm{cm}$} \\
\hline $\mathrm{H}-1$ & 379 & $\odot$ & 3.32 & 29 & \\
\hline $0-16$ & 379 & $\odot$ & $1 E-02$ & 293.6 & \\
\hline$M g-24$ & 379 & $\odot$ & 1. $05686 \mathrm{E}-04$ & 293.6 & \\
\hline$M g-25$ & 379 & $\odot$ & 1.33797E- 05 & 293.6 & \\
\hline$M g-26$ & 379 & $\odot$ & $1.47310 \mathrm{E}-05$ & 293.6 & \\
\hline Al-27 & 379 & $\odot$ & $2.87722 \mathrm{E}-02$ & 293.6 & \\
\hline Si-28 & 379 & $\odot$ & 1. $02136 E-04$ & 293.6 & \\
\hline Si-29 & 379 & $\odot$ & $5.17160 E-06$ & 293.6 & \\
\hline
\end{tabular}




$\begin{array}{llllll}\mathrm{Si}-3 \odot & 379 & \odot & 3.43297 \mathrm{E}-06 & 293.6 & \text { end } \\ \mathrm{Ti}-46 & 379 & \odot & 4.20262 \mathrm{E}-07 & 293.6 & \text { end } \\ \mathrm{Ti}-47 & 379 & \odot & 3.79000 \mathrm{E}-07 & 293.6 & \text { end } \\ \mathrm{Ti}-48 & 379 & \odot & 3.75536 \mathrm{E}-06 & 293.6 & \text { end } \\ \mathrm{Ti}-49 & 379 & \odot & 2.75590 \mathrm{E}-07 & 293.6 & \text { end } \\ \mathrm{Ti}-5 \odot & 379 & \odot & 2.63873 \mathrm{E}-07 & 293.6 & \text { end } \\ \mathrm{Cr}-5 \odot & 379 & \odot & 5.30517 \mathrm{E}-07 & 293.6 & \text { end } \\ \mathrm{Cr}-52 & 379 & \odot & 1.02189 \mathrm{E}-05 & 293.6 & \text { end } \\ \mathrm{Cr}-53 & 379 & \odot & 1.15860 \mathrm{E}-06 & 293.6 & \text { end } \\ \mathrm{Cr}-54 & 379 & \odot & 2.87821 \mathrm{E}-07 & 293.6 & \text { end } \\ \mathrm{Mn}-55 & 379 & \odot & 6.54922 \mathrm{E}-06 & 293.6 & \text { end } \\ \mathrm{Fe}-54 & 379 & \odot & 2.40643 \mathrm{E}-06 & 293.6 & \text { end } \\ \mathrm{Fe}-56 & 379 & \odot & 3.77418 \mathrm{E}-05 & 293.6 & \text { end } \\ \mathrm{Fe}-57 & 379 & \odot & 8.72072 \mathrm{E}-07 & 293.6 & \text { end } \\ \mathrm{Fe}-58 & 379 & \odot & 1.15179 \mathrm{E}-07 & 293.6 & \text { end } \\ \mathrm{Cu}-63 & 379 & \odot & 1.91268 \mathrm{E}-05 & 293.6 & \text { end } \\ \mathrm{Cu}-65 & 379 & \odot & 8.52504 \mathrm{E}-06 & 293.6 & \text { end } \\ \mathrm{U}-234 & 379 & \odot & 2.89485 \mathrm{E}-06 & 293.6 & \text { end } \\ \mathrm{U}-235 & 379 & \odot & 2.6980 \odot \mathrm{E}-\odot 4 & 293.6 & \text { end } \\ \mathrm{U}-236 & 379 & \odot & 1.15795 \mathrm{E}-06 & 293.6 & \text { end } \\ \mathrm{U}-238 & 379 & \odot & 1.56322 \mathrm{E}-05 & 293.6 & \text { end }\end{array}$

' Outer Fuel Element fueled region 8

total atom density $=8.00583 \mathrm{E}-02 \mathrm{a} / \mathrm{b}-\mathrm{cm}$ 8. 005830E- 02

$\begin{array}{llllll}\mathrm{H}-1 & 381 & \odot & 3.32434 \mathrm{E}-02 & 293.6 & \text { end } \\ \mathrm{O}-16 & 381 & \odot & 1.77945 \mathrm{E}-02 & 293.6 & \text { end } \\ \mathrm{Mg}-24 & 381 & \odot & 1.05686 \mathrm{E}-04 & 293.6 & \text { end } \\ \mathrm{Mg}-25 & 381 & \odot & 1.33797 \mathrm{E}-05 & 293.6 & \text { end } \\ \mathrm{Mg}-26 & 381 & \odot & 1.47310 \mathrm{E}-05 & 293.6 & \text { end } \\ \mathrm{Al}-27 & 381 & \odot & 2.82328 \mathrm{E}-02 & 293.6 & \text { end } \\ \mathrm{Si}-28 & 381 & \odot & 1.00933 \mathrm{E}-04 & 293.6 & \text { end } \\ \mathrm{Si}-29 & 381 & \odot & 5.11066 \mathrm{E}-06 & 293.6 & \text { end } \\ \mathrm{Si}-30 & 381 & \odot & 3.39252 \mathrm{E}-06 & 293.6 & \text { end } \\ \mathrm{Ti}-46 & 381 & \odot & 4.20262 \mathrm{E}-07 & 293.6 & \text { end } \\ \mathrm{Ti}-47 & 381 & \odot & 3.79000 \mathrm{E}-07 & 293.6 & \text { end } \\ \mathrm{Ti}-48 & 381 & \odot & 3.75536 \mathrm{E}-06 & 293.6 & \text { end } \\ \mathrm{Ti}-49 & 381 & \odot & 2.75590 \mathrm{E}-07 & 293.6 & \text { end } \\ \mathrm{Ti}-50 & 381 & \odot & 2.63873 \mathrm{E}-07 & 293.6 & \text { end } \\ \mathrm{Cr}-50 & 381 & \odot & 5.30517 \mathrm{E}-07 & 293.6 & \text { end } \\ \mathrm{Cr}-52 & 381 & \odot & 1.02189 \mathrm{E}-05 & 293.6 & \text { end } \\ \mathrm{Cr}-53 & 381 & \odot & 1.15860 \mathrm{E}-06 & 293.6 & \text { end } \\ \mathrm{Cr}-54 & 381 & \odot & 2.87821 \mathrm{E}-07 & 293.6 & \text { end } \\ \mathrm{Mn}-55 & 381 & \odot & 6.48252 \mathrm{E}-06 & 293.6 & \text { end } \\ \mathrm{Fe}-54 & 381 & \odot & 2.36804 \mathrm{E}-06 & 293.6 & \text { end } \\ \mathrm{Fe}-56 & 381 & \odot & 3.71398 \mathrm{E}-05 & 293.6 & \text { end } \\ \mathrm{Fe}-57 & 381 & \odot & 8.58163 \mathrm{E}-07 & 293.6 & \text { end } \\ \mathrm{Fe}-58 & 381 & \odot & 1.13342 \mathrm{E}-07 & 293.6 & \text { end } \\ \mathrm{Cu}-63 & 381 & \odot & 1.89046 \mathrm{E}-05 & 293.6 & \text { end } \\ \mathrm{Cu}-65 & 381 & \odot & 8.42602 \mathrm{E}-06 & 293.6 & \text { end } \\ \mathrm{U}-234 & 381 & \odot & 4.52789 \mathrm{E}-06 & 293.6 & \text { end } \\ \mathrm{U}-235 & 381 & \odot & 4.22000 \mathrm{E}-04 & 293.6 & \text { end } \\ \mathrm{U}-236 & 381 & \odot & 1.81117 \mathrm{E}-06 & 293.6 & \text { end } \\ \mathrm{U}-238 & 381 & \odot & 2.44506 \mathrm{E}-05 & 293.6 & \text { end }\end{array}$

total atom density $=8.00895 \mathrm{E}-02 \mathrm{a} / \mathrm{b}-\mathrm{cm}$

' $8.008950 \mathrm{E}-02$

\begin{tabular}{|c|c|c|c|c|}
\hline $\mathrm{H}-1$ & 382 & $\odot$ & $3.32434 \mathrm{E}-02$ & 293.6 \\
\hline $0-16$ & 382 & 0 & 1. $80329 \mathrm{E}-\odot 2$ & 293.6 \\
\hline $\mathrm{Mg}-24$ & 382 & 0 & 1. $05686 \mathrm{E}-\odot 4$ & 293.6 \\
\hline$M g-25$ & 382 & 0 & $1.33797 \mathrm{E}-05$ & 293.6 \\
\hline $\mathrm{Mg}-26$ & 382 & 0 & $1.47310 \mathrm{E}-05$ & 293.6 \\
\hline Al-27 & 382 & $\odot$ & $2.79375 \mathrm{E}-02$ & 293.6 \\
\hline $\mathrm{Si}-28$ & 382 & 0 & 1. $00274 \mathrm{E}-\odot 4$ & 293.6 \\
\hline Si-29 & 382 & $\odot$ & $5.07732 E-06$ & 293.6 \\
\hline $\mathrm{Si}-30$ & 382 & $\odot$ & $3.37038 E-06$ & 293.6 \\
\hline $\mathrm{Ti}-46$ & 382 & $\odot$ & 4. $20262 \mathrm{E}-07$ & 293.6 \\
\hline $\mathrm{Ti}-47$ & 382 & $\odot$ & $3.79000 \mathrm{E}-07$ & 293.6 \\
\hline $\mathrm{Ti}-48$ & 382 & $\odot$ & $3.75536 \mathrm{E}-06$ & 293.6 \\
\hline Ti-49 & 382 & 0 & $2.75590 \mathrm{E}-07$ & 293.6 \\
\hline Ti-50 & 382 & $\odot$ & $2.63873 E-07$ & 293.6 \\
\hline $\mathrm{Cr}-50$ & 382 & $\odot$ & $5.30517 \mathrm{E}-07$ & 293.6 \\
\hline $\mathrm{Cr}-52$ & 382 & 0 & 1. $02189 \mathrm{E}-05$ & 293.6 \\
\hline $\mathrm{Cr}-53$ & 382 & $\odot$ & $1.15860 E-\odot 6$ & 293.6 \\
\hline $\mathrm{Cr}-54$ & 382 & $\odot$ & $2.87821 E-07$ & 293.6 \\
\hline$M n-55$ & 382 & $\odot$ & $6.446 \odot 2 E-06$ & 293.6 \\
\hline $\mathrm{Fe}-54$ & 382 & $\odot$ & $2.34704 \mathrm{E}-06$ & 293.6 \\
\hline $\mathrm{Fe}-56$ & 382 & $\odot$ & $3.68104 \mathrm{E}-05$ & 293.6 \\
\hline $\mathrm{Fe}-57$ & 382 & $\odot$ & $8.50550 \mathrm{E}-07$ & 293.6 \\
\hline $\mathrm{Fe}-58$ & 382 & $\odot$ & $1.12337 \mathrm{E}-07$ & 293.6 \\
\hline $\mathrm{Cu}-63$ & 382 & 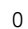 & $1.87830 \mathrm{E}-05$ & 293.6 \\
\hline Cu- 65 & 382 & $\odot$ & $8.37182 \mathrm{E}-06$ & 293.6 \\
\hline
\end{tabular}




\begin{tabular}{|c|c|c|c|c|c|}
\hline$U-234$ & 382 & 0 & $5.42167 \mathrm{E}-06$ & 293.6 & en \\
\hline$U-235$ & 382 & $\odot$ & $5.05300 E-04$ & 293.6 & \\
\hline$U-236$ & 382 & $\odot$ & $2.16868 E-06$ & 293.6 & \\
\hline$U-238$ & 382 & $\odot$ & $2.92770 E-05$ & 293.6 & \\
\hline \multicolumn{3}{|c|}{ total atom density } & \multicolumn{3}{|c|}{$=8.01228 \mathrm{E}-02 \mathrm{a} / \mathrm{b}-\mathrm{cm}$} \\
\hline $\mathrm{H}-1$ & 383 & $\odot$ & $3.32434 E-02$ & 293.6 & en \\
\hline $0-16$ & 383 & 0 & $1.82870 \mathrm{E}-02$ & 293.6 & \\
\hline $\mathrm{Mg}-24$ & 383 & $\odot$ & 1. $05686 E-04$ & 293.6 & \\
\hline $\mathrm{Mg}-25$ & 383 & $\odot$ & $1.33797 E-05$ & 293.6 & \\
\hline$M g-26$ & 383 & $\odot$ & $1.47310 E-05$ & 293.6 & \\
\hline Al-27 & 383 & $\odot$ & $2.76228 E-02$ & 293.6 & \\
\hline Si-28 & 383 & $\odot$ & $9.95724 \mathrm{E}-05$ & 293.6 & \\
\hline Si-29 & 383 & $\odot$ & $5 . \odot 4178 E-\odot 6$ & 293.6 & \\
\hline Si-30 & 383 & 0 & $3.34679 E-06$ & 293.6 & \\
\hline Ti-46 & 383 & 0 & $4.20262 \mathrm{E}-07$ & 293.6 & \\
\hline $\mathrm{Ti}-47$ & 383 & $\odot$ & $3.79000 \mathrm{E}-07$ & 293.6 & \\
\hline $\mathrm{Ti}-48$ & 383 & $\odot$ & $3.75536 E-06$ & 293.6 & \\
\hline Ti-49 & 383 & $\odot$ & $2.75590 E-07$ & 293.6 & \\
\hline Ti-50 & 383 & $\odot$ & $2.63873 E-07$ & 293.6 & \\
\hline $\mathrm{Cr}-50$ & 383 & 0 & $5.30517 E-07$ & 293.6 & \\
\hline $\mathrm{Cr}-52$ & 383 & $\odot$ & 1. $02189 E-05$ & 293.6 & \\
\hline $\mathrm{Cr}-53$ & 383 & $\odot$ & $1.15860 E-06$ & 293.6 & \\
\hline $\mathrm{Cr}-54$ & 383 & $\odot$ & $2.87821 E-07$ & 293.6 & \\
\hline Mn-55 & 383 & $\odot$ & $6.40711 \mathrm{E}-06$ & 293.6 & \\
\hline $\mathrm{Fe}-54$ & 383 & $\odot$ & $2.32464 E-\odot 6$ & 293.6 & \\
\hline $\mathrm{Fe}-56$ & 383 & 0 & $3.64591 \mathrm{E}-05$ & 293.6 & \\
\hline $\mathrm{Fe}-57$ & 383 & 0 & $8.42433 E-07$ & 293.6 & \\
\hline $\mathrm{Fe}-58$ & 383 & 0 & $1.11265 \mathrm{E}-07$ & 293.6 & \\
\hline $\mathrm{Cu}-63$ & 383 & 0 & $1.86534 \mathrm{E}-05$ & 293.6 & \\
\hline Cu- 65 & 383 & 0 & $8.31405 E-06$ & 293.6 & \\
\hline$U-234$ & 383 & $\odot$ & $6.37446 E-06$ & 293.6 & \\
\hline$U-235$ & 383 & $\odot$ & $5.94100 \mathrm{E}-04$ & 293.6 & \\
\hline$U-236$ & 383 & $\odot$ & $2.54980 E-06$ & 293.6 & \\
\hline$U-238$ & 383 & $\odot$ & $3.44221 E-05$ & 293.6 & \\
\hline \multicolumn{3}{|c|}{$\begin{array}{l}\text { total atom density } \\
5300 \mathrm{E}-02\end{array}$} & \multicolumn{3}{|c|}{$=8.01530 \mathrm{E}-02 \mathrm{a} / \mathrm{b}-\mathrm{cm}$} \\
\hline $\mathrm{H}-1$ & 384 & $\odot$ & $3.32434 \mathrm{E}-02$ & 293.6 & \\
\hline $0-16$ & 384 & 0 & $1.85176 \mathrm{E}-02$ & 293.6 & \\
\hline$M g-24$ & 384 & $\odot$ & 1. $05686 E-04$ & 293.6 & \\
\hline$M g-25$ & 384 & 0 & $1.33797 \mathrm{E}-05$ & 293.6 & \\
\hline $\mathrm{Mg}-26$ & 384 & $\odot$ & $1.47310 E-05$ & 293.6 & \\
\hline $\mathrm{Al}-27$ & 384 & $\odot$ & $2.73372 E-\odot 2$ & 293.6 & \\
\hline Si-28 & 384 & $\odot$ & $9.89350 E-05$ & 293.6 & \\
\hline Si-29 & 384 & $\odot$ & 5. $\odot \odot 951 E-\odot 6$ & 293.6 & \\
\hline Si-30 & 384 & $\odot$ & $3.32537 \mathrm{E}-06$ & 293.6 & \\
\hline Ti-46 & 384 & $\odot$ & $4.2 \odot 262 \mathrm{E}-\odot 7$ & 293.6 & \\
\hline Ti-47 & 384 & 0 & $3.79000 \mathrm{E}-07$ & 293.6 & \\
\hline Ti-48 & 384 & 0 & $3.75536 \mathrm{E}-06$ & 293.6 & \\
\hline Ti-49 & 384 & 0 & $2.75590 \mathrm{E}-\odot 7$ & 293.6 & \\
\hline Ti-50 & 384 & 0 & $2.63873 E-07$ & 293.6 & \\
\hline $\mathrm{Cr}-50$ & 384 & $\odot$ & $5.30517 \mathrm{E}-07$ & 293.6 & \\
\hline $\mathrm{Cr}-52$ & 384 & $\odot$ & 1. $02189 E-05$ & 293.6 & \\
\hline $\mathrm{Cr}-53$ & 384 & $\odot$ & $1.15860 E-06$ & 293.6 & \\
\hline $\mathrm{Cr}-54$ & 384 & $\odot$ & $2.87821 E-07$ & 293.6 & \\
\hline$M n-55$ & 384 & $\odot$ & $6.37178 E-06$ & 293.6 & \\
\hline $\mathrm{Fe}-54$ & 384 & $\odot$ & $2.30432 \mathrm{E}-\odot 6$ & 293.6 & \\
\hline $\mathrm{Fe}-56$ & 384 & 0 & $3.61403 E-05$ & 293.6 & \\
\hline $\mathrm{Fe}-57$ & 384 & $\odot$ & $8.35068 E-07$ & 293.6 & \\
\hline $\mathrm{Fe}-58$ & 384 & 0 & $1.10292 \mathrm{E}-07$ & 293.6 & \\
\hline $\mathrm{Cu}-63$ & 384 & 0 & $1.85357 \mathrm{E}-05$ & 293.6 & \\
\hline $\mathrm{Cu}-65$ & 384 & $\odot$ & $8.26161 E-06$ & 293.6 & \\
\hline U-234 & 384 & 0 & $7.23926 E-06$ & 293.6 & \\
\hline U-235 & 384 & $\odot$ & $6.74700 \mathrm{E}-\odot 4$ & 293.6 & \\
\hline$U-236$ & 384 & 0 & $2.89573 E-06$ & 293.6 & \\
\hline$U-238$ & 384 & $\odot$ & 3. $9 \odot 920 E-\odot 5$ & 293.6 & \\
\hline 1 & tom & sity & $=8.01437 \mathrm{E}$ & $2 a / b-c$ & \\
\hline 18.6 & & & & & \\
\hline $\mathrm{H}-1$ & 385 & $\odot$ & 3. 32434E- 02 & 293.6 & \\
\hline $0-16$ & 385 & 0 & 1. $84469 E-02$ & 293.6 & \\
\hline$M g-24$ & 385 & $\odot$ & 1. $05686 E-04$ & 293.6 & \\
\hline$M g-25$ & 385 & 0 & $1.33797 \mathrm{E}-05$ & 293.6 & \\
\hline$M g-26$ & 385 & 0 & $1.47310 \mathrm{E}-05$ & 293.6 & \\
\hline Al-27 & 385 & 0 & $2.74247 \mathrm{E}-02$ & 293.6 & \\
\hline Si-28 & 385 & 0 & $9.91306 \mathrm{E}-05$ & 293.6 & \\
\hline Si-29 & 385 & 0 & $5.01941 E-06$ & 293.6 & \\
\hline $\mathrm{Si}-30$ & 385 & $\odot$ & $3.33194 E-06$ & 293.6 & \\
\hline $\mathrm{Ti}-46$ & 385 & $\odot$ & 4. $20262 E-07$ & 293.6 & \\
\hline Ti-47 & 385 & 0 & $3.790 \odot \odot E-07$ & 293.6 & \\
\hline Ti-48 & 385 & 0 & $3.75536 E-06$ & 293.6 & \\
\hline Ti-49 & 385 & 0 & $2.75590 \mathrm{E}-07$ & 293.6 & \\
\hline
\end{tabular}




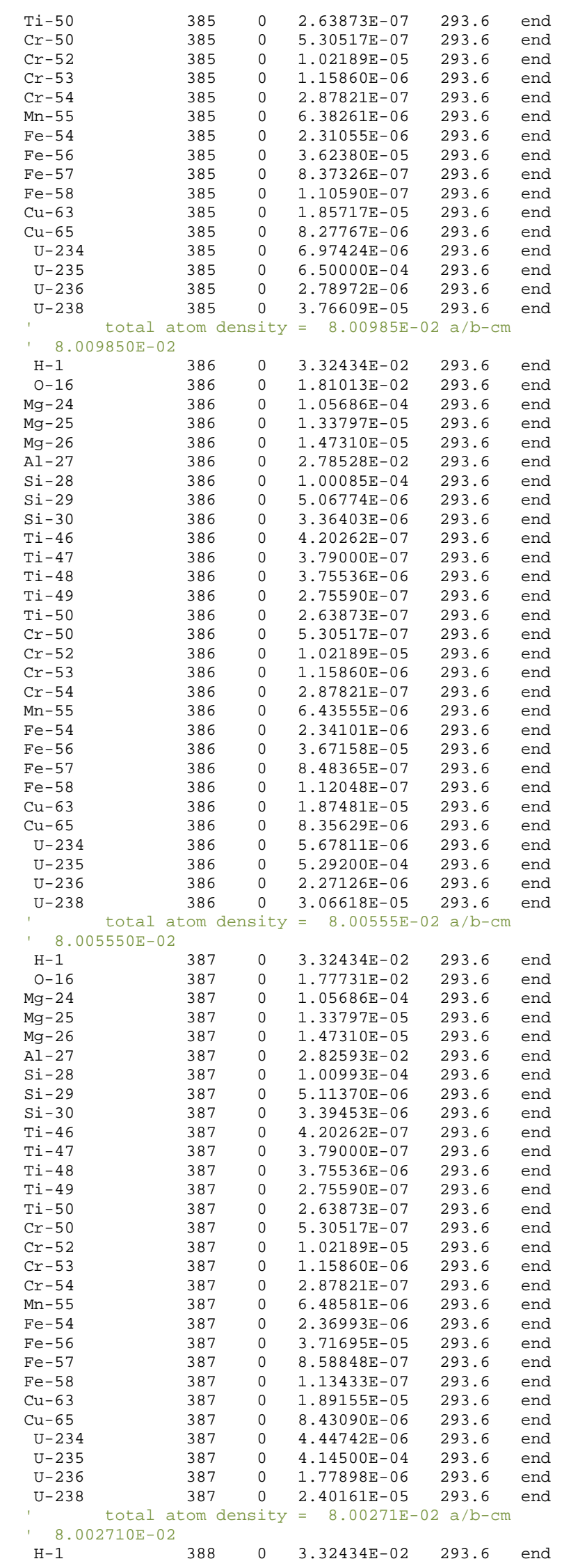




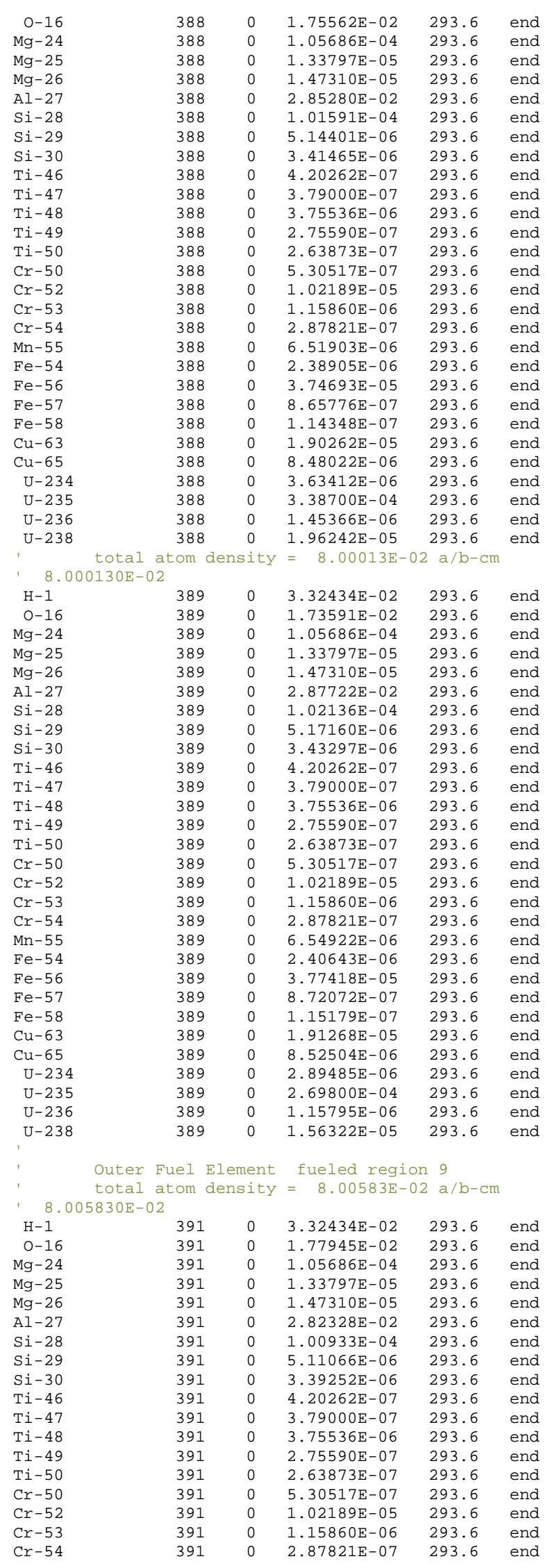




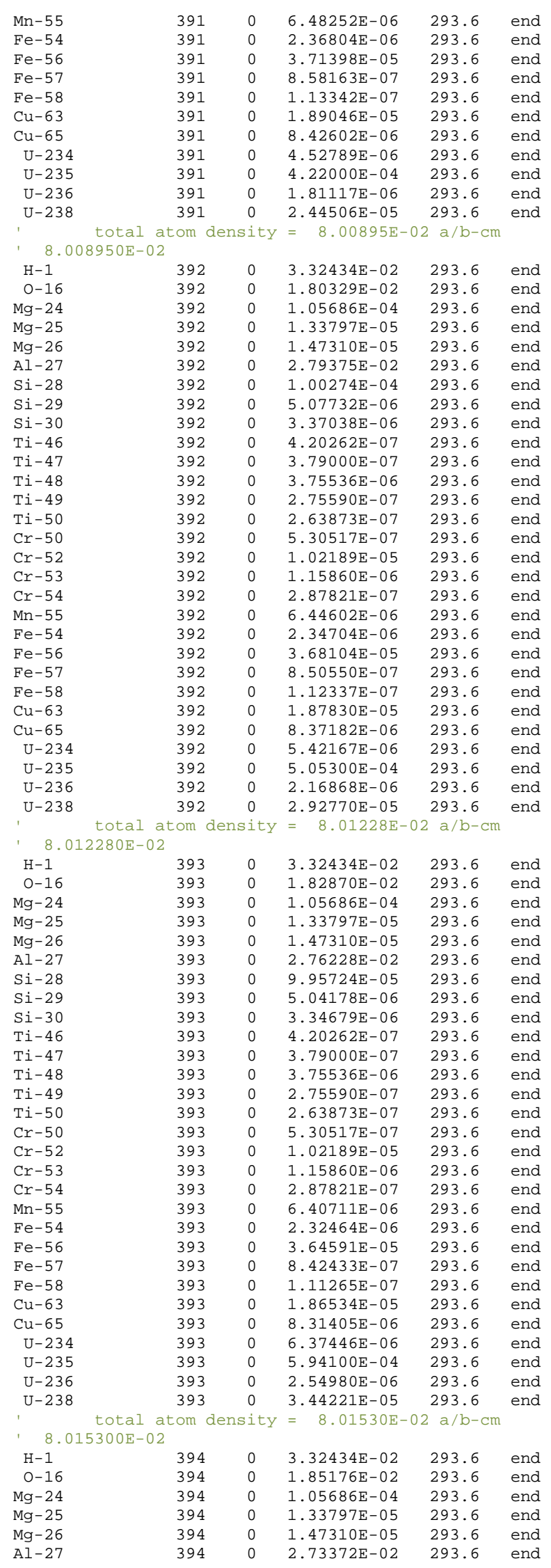




\begin{tabular}{|c|c|c|c|c|c|}
\hline Si-28 & 394 & $\odot$ & $9.89350 E-\odot 5$ & 293.6 & end \\
\hline Si-29 & 394 & $\odot$ & $5.00951 E-06$ & 293.6 & end \\
\hline Si-30 & 394 & $\odot$ & 3. 32537E- 06 & 293.6 & end \\
\hline $\mathrm{Ti}-46$ & 394 & $\odot$ & 4. $20262 \mathrm{E}-07$ & 293.6 & end \\
\hline $\mathrm{Ti}-47$ & 394 & $\odot$ & 3. $79000 \mathrm{E}-07$ & 293.6 & end \\
\hline Ti-48 & 394 & 0 & $3.75536 \mathrm{E}-06$ & 293.6 & end \\
\hline Ti-49 & 394 & 0 & $2.75590 \mathrm{E}-07$ & 293.6 & end \\
\hline Ti-50 & 394 & 0 & $2.63873 E-07$ & 293.6 & nd \\
\hline $\mathrm{Cr}-50$ & 394 & $\odot$ & $5.30517 \mathrm{E}-07$ & 293.6 & nd \\
\hline $\mathrm{Cr}-52$ & 394 & $\odot$ & 1. $02189 E-05$ & 293.6 & nd \\
\hline $\mathrm{Cr}-53$ & 394 & $\odot$ & 1. $15860 E-\odot 6$ & 293.6 & \\
\hline $\mathrm{Cr}-54$ & 394 & $\odot$ & $2.87821 E-07$ & 293.6 & nd \\
\hline$M n-55$ & 394 & $\odot$ & $6.37178 \mathrm{E}-06$ & 293.6 & nd \\
\hline $\mathrm{Fe}-54$ & 394 & $\odot$ & $2.30432 E-06$ & 293.6 & and \\
\hline $\mathrm{Fe}-56$ & 394 & 0 & $3.61403 \mathrm{E}-05$ & 293.6 & nd \\
\hline $\mathrm{Fe}-57$ & 394 & 0 & $8.35068 \mathrm{E}-07$ & 293.6 & nd \\
\hline $\mathrm{Fe}-58$ & 394 & 0 & $1.10292 \mathrm{E}-07$ & 293.6 & ad \\
\hline $\mathrm{Cu}-63$ & 394 & 0 & $1.85357 \mathrm{E}-05$ & 293.6 & nd \\
\hline $\mathrm{Cu}-65$ & 394 & $\odot$ & $8.26161 E-06$ & 293.6 & nd \\
\hline$U-234$ & 394 & $\odot$ & $7.23926 \mathrm{E}-06$ & 293.6 & nd \\
\hline$U-235$ & 394 & $\odot$ & $6.74700 \mathrm{E}-\odot 4$ & 293.6 & \\
\hline$U-236$ & 394 & $\odot$ & $2.89573 E-\odot 6$ & 293.6 & nd \\
\hline$U-238$ & 394 & $\odot$ & 3. $90920 E-\odot 5$ & 293.6 & end \\
\hline & \multicolumn{3}{|c|}{ ' $\quad 8.014370 \mathrm{E}-02$} \\
\hline $\mathrm{H}-1$ & 395 & $\odot$ & $3.32434 \mathrm{E}-02$ & 293.6 & nd \\
\hline $0-16$ & 395 & $\odot$ & $1.84469 E-02$ & 293.6 & nd \\
\hline$M g-24$ & 395 & 0 & $1.05686 \mathrm{E}-04$ & 293.6 & \\
\hline $\mathrm{Mg}-25$ & 395 & $\odot$ & $1.33797 \mathrm{E}-05$ & 293.6 & \\
\hline $\mathrm{Mg}-26$ & 395 & $\odot$ & $1.47310 E-05$ & 293.6 & \\
\hline Al-27 & 395 & $\odot$ & $2.74247 \mathrm{E}-02$ & 293.6 & \\
\hline Si-28 & 395 & $\odot$ & $9.91306 \mathrm{E}-\odot 5$ & 293.6 & \\
\hline Si-29 & 395 & $\odot$ & 5. $01941 \mathrm{E}-\odot 6$ & 293.6 & \\
\hline Si-30 & 395 & 0 & 3. 33194E- 06 & 293.6 & \\
\hline $\mathrm{Ti}-46$ & 395 & 0 & 4. $20262 \mathrm{E}-07$ & 293.6 & \\
\hline $\mathrm{Ti}-47$ & 395 & $\odot$ & $3.79000 E-\odot 7$ & 293.6 & \\
\hline $\mathrm{Ti}-48$ & 395 & $\odot$ & $3.75536 \mathrm{E}-\odot 6$ & 293.6 & \\
\hline $\mathrm{Ti}-49$ & 395 & $\odot$ & $2.75590 E-07$ & 293.6 & \\
\hline Ti-50 & 395 & 0 & $2.63873 E-07$ & 293.6 & \\
\hline $\mathrm{Cr}-50$ & 395 & 0 & $5.30517 \mathrm{E}-07$ & 293.6 & nd \\
\hline $\mathrm{Cr}-52$ & 395 & 0 & 1. $02189 E-05$ & 293.6 & \\
\hline $\mathrm{Cr}-53$ & 395 & 0 & $1.15860 E-06$ & 293.6 & nd \\
\hline $\mathrm{Cr}-54$ & 395 & $\odot$ & $2.87821 E-\odot 7$ & 293.6 & \\
\hline$M n-55$ & 395 & $\odot$ & $6.38261 E-06$ & 293.6 & nd \\
\hline $\mathrm{Fe}-54$ & 395 & $\odot$ & $2.31055 E-06$ & 293.6 & \\
\hline $\mathrm{Fe}-56$ & 395 & $\odot$ & $3.62380 E-05$ & 293.6 & \\
\hline $\mathrm{Fe}-57$ & 395 & $\odot$ & $8.37326 E-07$ & 293.6 & \\
\hline $\mathrm{Fe}-58$ & 395 & $\odot$ & 1. $10590 \mathrm{E}-07$ & 293.6 & \\
\hline $\mathrm{Cu}-63$ & 395 & $\odot$ & $1.85717 \mathrm{E}-05$ & 293.6 & \\
\hline $\mathrm{Cu}-65$ & 395 & $\odot$ & $8.27767 \mathrm{E}-06$ & 293.6 & \\
\hline$U-234$ & 395 & $\odot$ & $6.97424 \mathrm{E}-06$ & 293.6 & \\
\hline U-235 & 395 & $\odot$ & $6.500 \odot \odot E-\odot 4$ & 293.6 & \\
\hline$U-236$ & 395 & $\odot$ & $2.78972 \mathrm{E}-06$ & 293.6 & \\
\hline$U-238$ & 395 & $\odot$ & $3.76609 \mathrm{E}-05$ & 293.6 & nd \\
\hline - -1 & tom & sity & $=8.00985 \mathrm{E}$ & $2 a / b-c m$ & \\
\hline \multicolumn{6}{|c|}{ ' $\quad$ 8. $\odot \odot 985 \odot E-\odot 2$} \\
\hline $\mathrm{H}-1$ & 396 & $\odot$ & 3. 32434E- 02 & 293.6 & \\
\hline $0-16$ & 396 & $\odot$ & 1. $81013 E-02$ & 293.6 & \\
\hline$M g-24$ & 396 & $\odot$ & 1. $05686 \mathrm{E}-04$ & 293.6 & \\
\hline$M g-25$ & 396 & 0 & $1.33797 \mathrm{E}-05$ & 293.6 & \\
\hline$M g-26$ & 396 & $\odot$ & $1.47310 \mathrm{E}-05$ & 293.6 & \\
\hline Al-27 & 396 & $\odot$ & $2.78528 E-02$ & 293.6 & \\
\hline Si-28 & 396 & $\odot$ & 1. $00085 E-\odot 4$ & 293.6 & \\
\hline Si-29 & 396 & $\odot$ & $5.06774 \mathrm{E}-06$ & 293.6 & \\
\hline Si-30 & 396 & $\odot$ & $3.36403 E-06$ & 293.6 & \\
\hline $\mathrm{Ti}-46$ & 396 & $\odot$ & 4. $2 \odot 262 \mathrm{E}-07$ & 293.6 & \\
\hline $\mathrm{Ti}-47$ & 396 & $\odot$ & $3.79000 \mathrm{E}-07$ & 293.6 & \\
\hline Ti-48 & 396 & 0 & $3.75536 \mathrm{E}-06$ & 293.6 & \\
\hline Ti-49 & 396 & 0 & $2.75590 \mathrm{E}-\odot 7$ & 293.6 & \\
\hline Ti-50 & 396 & $\odot$ & $2.63873 E-07$ & 293.6 & \\
\hline $\mathrm{Cr}-50$ & 396 & $\odot$ & $5.30517 \mathrm{E}-07$ & 293.6 & \\
\hline $\mathrm{Cr}-52$ & 396 & $\odot$ & 1. $02189 \mathrm{E}-05$ & 293.6 & \\
\hline $\mathrm{Cr}-53$ & 396 & $\odot$ & 1. $15860 E-\odot 6$ & 293.6 & \\
\hline $\mathrm{Cr}-54$ & 396 & $\odot$ & $2.87821 E-07$ & 293.6 & \\
\hline$M n-55$ & 396 & $\odot$ & $6.43555 E-\odot 6$ & 293.6 & \\
\hline $\mathrm{Fe}-54$ & 396 & $\odot$ & $2.34101 \mathrm{E}-06$ & 293.6 & \\
\hline $\mathrm{Fe}-56$ & 396 & $\odot$ & $3.67158 \mathrm{E}-05$ & 293.6 & \\
\hline $\mathrm{Fe}-57$ & 396 & $\odot$ & $8.48365 E-07$ & 293.6 & \\
\hline $\mathrm{Fe}-58$ & 396 & $\odot$ & $1.12048 \mathrm{E}-07$ & 293.6 & \\
\hline $\mathrm{Cu}-63$ & 396 & $\odot$ & $1.87481 \mathrm{E}-05$ & 293.6 & \\
\hline Cu- 65 & 396 & 0 & $8.35629 E-06$ & 293.6 & \\
\hline
\end{tabular}




\begin{tabular}{|c|c|c|c|c|c|}
\hline$U-234$ & 396 & $\odot$ & $5.67811 E-06$ & 293.6 & end \\
\hline$U-235$ & 396 & $\odot$ & $5.29200 E-\odot 4$ & 293.6 & end \\
\hline$U-236$ & 396 & $\odot$ & $2.27126 \mathrm{E}-06$ & 293.6 & \\
\hline$U-238$ & 396 & 0 & $3.06618 \mathrm{E}-05$ & 293.6 & nd \\
\hline \multicolumn{3}{|c|}{$\begin{array}{l}\text { total atom density } \\
1 \quad 8.005550 \mathrm{E}-02\end{array}$} & \multicolumn{2}{|c|}{$=8.00555 \mathrm{E}-02 \mathrm{a} / \mathrm{b}-\mathrm{cm}$} & \\
\hline $\mathrm{H}-1$ & 397 & $\odot$ & $3.32434 \mathrm{E}-02$ & 293.6 & 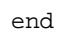 \\
\hline $0-16$ & 397 & 0 & 1. $77731 \mathrm{E}-02$ & 293.6 & \\
\hline $\mathrm{Mg}-24$ & 397 & $\odot$ & 1. $05686 E-04$ & 293.6 & \\
\hline$M g-25$ & 397 & $\odot$ & 1.33797E- 05 & 293.6 & \\
\hline $\mathrm{Mg}-26$ & 397 & $\odot$ & $1.47310 \mathrm{E}-05$ & 293.6 & \\
\hline Al-27 & 397 & $\odot$ & $2.82593 E-02$ & 293.6 & \\
\hline Si-28 & 397 & $\odot$ & 1. $\odot ० 993 E-\odot 4$ & 293.6 & \\
\hline Si-29 & 397 & $\odot$ & $5.11370 E-06$ & 293.6 & \\
\hline Si-30 & 397 & $\odot$ & 3. 39453E- 06 & 293.6 & \\
\hline Ti-46 & 397 & $\odot$ & $4.20262 E-07$ & 293.6 & \\
\hline Ti-47 & 397 & $\odot$ & 3. $79000 \mathrm{E}-07$ & 293.6 & \\
\hline Ti-48 & 397 & $\odot$ & $3.75536 \mathrm{E}-06$ & 293.6 & \\
\hline Ti-49 & 397 & 0 & $2.75590 \mathrm{E}-07$ & 293.6 & \\
\hline Ti-50 & 397 & $\odot$ & $2.63873 E-07$ & 293.6 & \\
\hline $\mathrm{Cr}-50$ & 397 & 0 & $5.30517 \mathrm{E}-07$ & 293.6 & \\
\hline $\mathrm{Cr}-52$ & 397 & $\odot$ & 1. $02189 E-05$ & 293.6 & \\
\hline $\mathrm{Cr}-53$ & 397 & $\odot$ & $1.15860 E-06$ & 293.6 & \\
\hline $\mathrm{Cr}-54$ & 397 & 0 & $2.87821 E-07$ & 293.6 & \\
\hline$M n-55$ & 397 & $\odot$ & $6.48581 E-06$ & 293.6 & \\
\hline $\mathrm{Fe}-54$ & 397 & 0 & $2.36993 E-06$ & 293.6 & \\
\hline $\mathrm{Fe}-56$ & 397 & $\odot$ & $3.71695 \mathrm{E}-05$ & 293.6 & \\
\hline $\mathrm{Fe}-57$ & 397 & $\odot$ & $8.58848 E-07$ & 293.6 & \\
\hline $\mathrm{Fe}-58$ & 397 & $\odot$ & $1.13433 E-07$ & 293.6 & \\
\hline $\mathrm{Cu}-63$ & 397 & 0 & 1. 89155E- 05 & 293.6 & \\
\hline Cu- 65 & 397 & 0 & $8.43090 \mathrm{E}-06$ & 293.6 & \\
\hline$U-234$ & 397 & $\odot$ & $4.44742 E-06$ & 293.6 & \\
\hline$U-235$ & 397 & 0 & $4.14500 E-\odot 4$ & 293.6 & \\
\hline$U-236$ & 397 & $\odot$ & $1.77898 \mathrm{E}-06$ & 293.6 & \\
\hline$U-238$ & 397 & 0 & $2.40161 E-05$ & 293.6 & \\
\hline \multicolumn{3}{|c|}{ total atom density } & \multirow{2}{*}{\multicolumn{3}{|c|}{$=8 . \odot \odot 271 \mathrm{E}-\odot 2 \mathrm{a} / \mathrm{b}-\mathrm{cm}$}} \\
\hline \multicolumn{3}{|c|}{ ' $\quad 8.002710 \mathrm{E}-\odot 2$} & & & \\
\hline $\mathrm{H}-1$ & 398 & $\odot$ & $3.32434 \mathrm{E}-02$ & 293.6 & \\
\hline $0-16$ & 398 & 0 & 1. $75562 \mathrm{E}-02$ & 293.6 & \\
\hline Mg-24 & 398 & 0 & 1. $05686 E-04$ & 293.6 & \\
\hline$M g-25$ & 398 & 0 & 1. 33797E-05 & 293.6 & \\
\hline$M g-26$ & 398 & 0 & 1. $47310 \mathrm{E}-05$ & 293.6 & \\
\hline Al-27 & 398 & $\odot$ & $2.85280 E-02$ & 293.6 & \\
\hline Si-28 & 398 & $\odot$ & 1. $01591 \mathrm{E}-04$ & 293.6 & \\
\hline Si-29 & 398 & $\odot$ & $5.14401 \mathrm{E}-06$ & 293.6 & \\
\hline Si-30 & 398 & $\odot$ & $3.41465 E-06$ & 293.6 & \\
\hline Ti-46 & 398 & 0 & $4.20262 \mathrm{E}-07$ & 293.6 & \\
\hline Ti-47 & 398 & $\odot$ & $3.79000 \mathrm{E}-07$ & 293.6 & \\
\hline Ti-48 & 398 & $\odot$ & $3.75536 \mathrm{E}-06$ & 293.6 & \\
\hline Ti-49 & 398 & $\odot$ & $2.75590 E-07$ & 293.6 & \\
\hline Ti-50 & 398 & 0 & $2.63873 E-07$ & 293.6 & \\
\hline $\mathrm{Cr}-50$ & 398 & 0 & $5.30517 \mathrm{E}-07$ & 293.6 & \\
\hline $\mathrm{Cr}-52$ & 398 & $\odot$ & 1. $02189 E-05$ & 293.6 & \\
\hline $\mathrm{Cr}-53$ & 398 & $\odot$ & $1.15860 E-06$ & 293.6 & \\
\hline $\mathrm{Cr}-54$ & 398 & $\odot$ & $2.87821 E-07$ & 293.6 & \\
\hline$M n-55$ & 398 & 0 & $6.51903 E-06$ & 293.6 & \\
\hline $\mathrm{Fe}-54$ & 398 & $\odot$ & $2.38905 E-06$ & 293.6 & \\
\hline $\mathrm{Fe}-56$ & 398 & 0 & $3.74693 E-05$ & 293.6 & \\
\hline $\mathrm{Fe}-57$ & 398 & $\odot$ & $8.65776 E-07$ & 293.6 & \\
\hline $\mathrm{Fe}-58$ & 398 & $\odot$ & $1.14348 E-07$ & 293.6 & \\
\hline $\mathrm{Cu}-63$ & 398 & $\odot$ & 1. $90262 \mathrm{E}-05$ & 293.6 & \\
\hline Cu-65 & 398 & 0 & $8.48022 \mathrm{E}-06$ & 293.6 & \\
\hline$U-234$ & 398 & 0 & $3.63412 \mathrm{E}-06$ & 293.6 & \\
\hline U-235 & 398 & 0 & $3.38700 \mathrm{E}-04$ & 293.6 & \\
\hline$U-236$ & 398 & 0 & $1.45366 \mathrm{E}-06$ & 293.6 & \\
\hline$U-238$ & 398 & $\odot$ & $1.96242 \mathrm{E}-05$ & 293.6 & \\
\hline & tom & sity & $=8.00013 \mathrm{E}$ & $2 a / b-c m$ & \\
\hline $1 \quad 8.00$ & & & & & \\
\hline $\mathrm{H}-1$ & 399 & $\odot$ & 3. 32434E- 02 & 293.6 & \\
\hline $0-16$ & 399 & $\odot$ & $1.73591 E-02$ & 293.6 & \\
\hline$M g-24$ & 399 & $\odot$ & 1. $05686 E-\odot 4$ & 293.6 & \\
\hline$M g-25$ & 399 & 0 & $1.33797 \mathrm{E}-05$ & 293.6 & \\
\hline$M g-26$ & 399 & 0 & $1.47310 \mathrm{E}-05$ & 293.6 & \\
\hline Al-27 & 399 & 0 & $2.87722 \mathrm{E}-02$ & 293.6 & \\
\hline Si-28 & 399 & 0 & 1. $02136 E-04$ & 293.6 & \\
\hline Si-29 & 399 & $\odot$ & $5.17160 E-06$ & 293.6 & \\
\hline Si-30 & 399 & 0 & $3.43297 E-06$ & 293.6 & \\
\hline Ti-46 & 399 & 0 & $4.20262 E-07$ & 293.6 & \\
\hline $\mathrm{Ti}-47$ & 399 & 0 & 3. 7900०E- -7 & 293.6 & \\
\hline $\mathrm{Ti}-48$ & 399 & 0 & $3.75536 E-06$ & 293.6 & \\
\hline $\mathrm{Ti}-49$ & 399 & 0 & $2.75590 E-07$ & 293.6 & \\
\hline
\end{tabular}




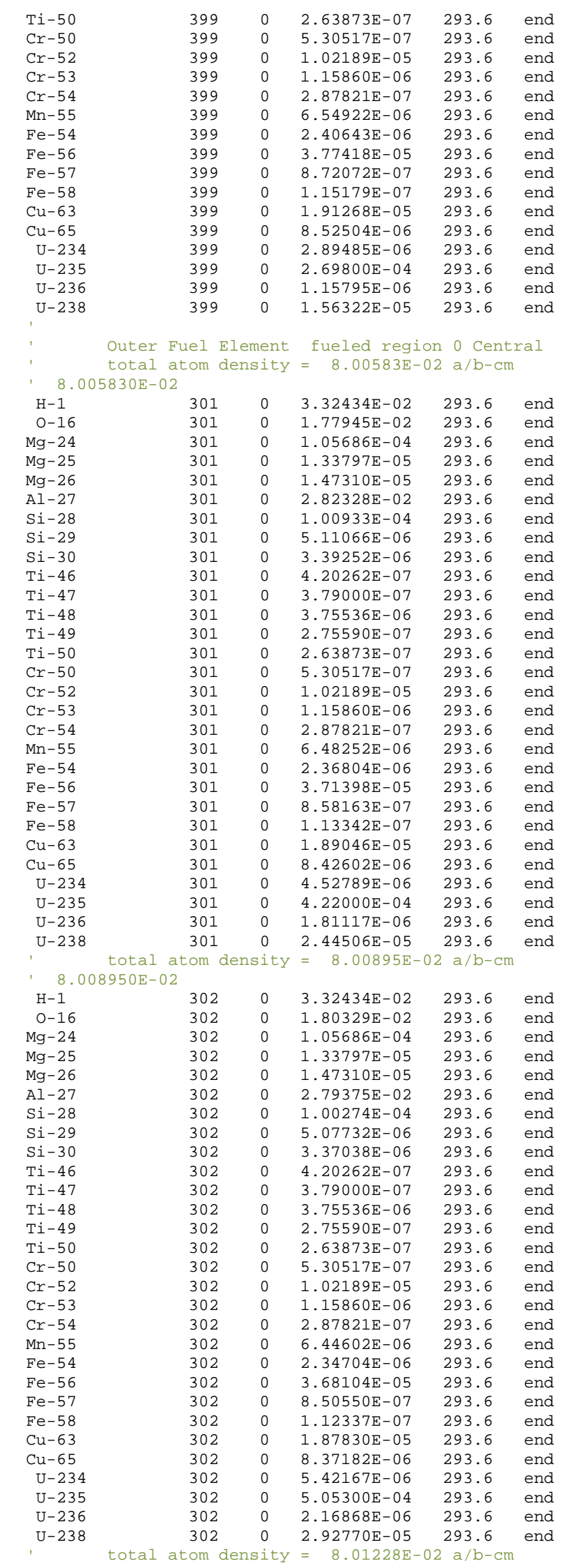




\begin{tabular}{|c|c|c|c|c|c|}
\hline \multicolumn{6}{|c|}{ ' $\quad 8.012280 \mathrm{E}-\odot 2$} \\
\hline $\mathrm{H}-1$ & 303 & $\odot$ & 3. 32434E- 02 & 293.6 & end \\
\hline $0-16$ & 303 & $\odot$ & $1.82870 E-02$ & 293.6 & id \\
\hline$M g-24$ & 303 & $\odot$ & $1.05686 E-04$ & 293.6 & ad \\
\hline$M g-25$ & 303 & $\odot$ & $1.33797 \mathrm{E}-05$ & 293.6 & \\
\hline$M g-26$ & 303 & $\odot$ & $1.47310 \mathrm{E}-05$ & 293.6 & d \\
\hline Al-27 & 303 & $\odot$ & $2.76228 \mathrm{E}-\odot 2$ & 293.6 & \\
\hline Si-28 & 303 & 0 & $9.95724 \mathrm{E}-05$ & 293.6 & \\
\hline Si-29 & 303 & $\odot$ & $5.04178 \mathrm{E}-06$ & 293.6 & \\
\hline Si-30 & 303 & $\odot$ & $3.34679 E-06$ & 293.6 & \\
\hline $\mathrm{Ti}-46$ & 303 & $\odot$ & $4.20262 E-07$ & 293.6 & \\
\hline $\mathrm{Ti}-47$ & 303 & $\odot$ & $3.79000 \mathrm{E}-07$ & 293.6 & \\
\hline $\mathrm{Ti}-48$ & 303 & $\odot$ & $3.75536 \mathrm{E}-06$ & 293.6 & \\
\hline $\mathrm{Ti}-49$ & 303 & $\odot$ & $2.75590 \mathrm{E}-\odot 7$ & 293.6 & \\
\hline Ti-50 & 303 & $\odot$ & $2.63873 \mathrm{E}-07$ & 293.6 & \\
\hline $\mathrm{Cr}-50$ & 303 & $\odot$ & $5.30517 \mathrm{E}-07$ & 293.6 & \\
\hline $\mathrm{Cr}-52$ & 303 & $\odot$ & 1. $02189 \mathrm{E}-05$ & 293.6 & \\
\hline $\mathrm{Cr}-53$ & 303 & $\odot$ & $1.15860 \mathrm{E}-06$ & 293.6 & \\
\hline Cr -54 & 303 & $\odot$ & $2.87821 \mathrm{E}-07$ & 293.6 & \\
\hline$M n-55$ & 303 & $\odot$ & $6.40711 \mathrm{E}-06$ & 293.6 & \\
\hline $\mathrm{Fe}-54$ & 303 & $\odot$ & $2.32464 \mathrm{E}-06$ & 293.6 & \\
\hline $\mathrm{Fe}-56$ & 303 & $\odot$ & $3.64591 \mathrm{E}-05$ & 293.6 & \\
\hline $\mathrm{Fe}-57$ & 303 & $\odot$ & $8.42433 E-07$ & 293.6 & \\
\hline $\mathrm{Fe}-58$ & 303 & $\odot$ & $1.11265 \mathrm{E}-07$ & 293.6 & \\
\hline $\mathrm{Cu}-63$ & 303 & $\odot$ & $1.86534 \mathrm{E}-05$ & 293.6 & \\
\hline $\mathrm{Cu}-65$ & 303 & $\odot$ & $8.31405 \mathrm{E}-06$ & 293.6 & \\
\hline$U-234$ & 303 & $\odot$ & $6.37446 \mathrm{E}-06$ & 293.6 & \\
\hline$U-235$ & 303 & $\odot$ & $5.94100 \mathrm{E}-04$ & 293.6 & \\
\hline$U-236$ & 303 & $\odot$ & $2.54980 \mathrm{E}-06$ & 293.6 & \\
\hline$U-238$ & 303 & 0 & $3.44221 \mathrm{E}-05$ & 293.6 & \\
\hline \multicolumn{3}{|c|}{ total atom density } & \multicolumn{2}{|c|}{$=\quad 8.01530 \mathrm{~F}-02 \mathrm{a} / \mathrm{b}-\mathrm{cm}$} & \\
\hline \multicolumn{6}{|c|}{ ' $8.015300 \mathrm{E}-02$} \\
\hline $\mathrm{H}-1$ & 304 & $\odot$ & $3.32434 \mathrm{E}-02$ & 293.6 & \\
\hline $0-16$ & 304 & $\odot$ & $1.85176 \mathrm{E}-02$ & 293.6 & \\
\hline$M g-24$ & 304 & $\odot$ & 1. $05686 \mathrm{E}-04$ & 293.6 & \\
\hline$M g-25$ & 304 & $\odot$ & $1.33797 \mathrm{E}-05$ & 293.6 & \\
\hline$M g-26$ & 304 & $\odot$ & $1.47310 \mathrm{E}-05$ & 293.6 & \\
\hline Al- -27 & 304 & $\odot$ & $2.73372 \mathrm{E}-02$ & 293.6 & \\
\hline Si-28 & 304 & $\odot$ & $9.89350 \mathrm{E}-05$ & 293.6 & \\
\hline Si-29 & 304 & 0 & $5.00951 \mathrm{E}-06$ & 293.6 & \\
\hline Si-30 & 304 & 0 & $3.32537 \mathrm{E}-06$ & 293.6 & \\
\hline Ti-46 & 304 & $\odot$ & $4.20262 \mathrm{E}-\odot 7$ & 293.6 & \\
\hline Ti-47 & 304 & $\odot$ & $3.790 \odot \odot E-\odot 7$ & 293.6 & \\
\hline Ti-48 & 304 & $\odot$ & $3.75536 \mathrm{E}-\odot 6$ & 293.6 & \\
\hline $\mathrm{Ti}-49$ & 304 & $\odot$ & $2.75590 \mathrm{E}-\odot 7$ & 293.6 & \\
\hline Ti-50 & 304 & $\odot$ & $2.63873 \mathrm{E}-07$ & 293.6 & \\
\hline $\mathrm{Cr}-50$ & 304 & $\odot$ & $5.30517 \mathrm{E}-07$ & 293.6 & \\
\hline $\mathrm{Cr}-52$ & 304 & $\odot$ & 1. $02189 \mathrm{E}-05$ & 293.6 & \\
\hline $\mathrm{Cr}-53$ & 304 & $\odot$ & $1.15860 \mathrm{E}-06$ & 293.6 & \\
\hline $\mathrm{Cr}-54$ & 304 & $\odot$ & $2.87821 \mathrm{E}-07$ & 293.6 & \\
\hline$M n-55$ & 304 & $\odot$ & $6.37178 \mathrm{E}-06$ & 293.6 & \\
\hline $\mathrm{Fe}-54$ & 304 & $\odot$ & $2.30432 \mathrm{E}-\odot 6$ & 293.6 & \\
\hline $\mathrm{Fe}-56$ & 304 & $\odot$ & $3.61403 E-05$ & 293.6 & \\
\hline $\mathrm{Fe}-57$ & 304 & $\odot$ & $8.35068 \mathrm{E}-07$ & 293.6 & \\
\hline $\mathrm{Fe}-58$ & 304 & $\odot$ & $1.10292 \mathrm{E}-07$ & 293.6 & \\
\hline $\mathrm{Cu}-63$ & 304 & $\odot$ & $1.85357 \mathrm{E}-05$ & 293.6 & \\
\hline $\mathrm{Cu}-65$ & 304 & $\odot$ & $8.26161 \mathrm{E}-06$ & 293.6 & \\
\hline$U-234$ & 304 & $\odot$ & $7.23926 \mathrm{E}-06$ & 293.6 & \\
\hline$U-235$ & 304 & $\odot$ & $6.74700 \mathrm{E}-04$ & 293.6 & \\
\hline$U-236$ & 304 & $\odot$ & $2.89573 \mathrm{E}-06$ & 293.6 & \\
\hline$U-238$ & 304 & $\odot$ & $3.90920 \mathrm{E}-05$ & 293.6 & \\
\hline 1 & tom & sity & $=\quad 8.01437 \mathrm{E}$ & $2 a / b-c m$ & \\
\hline \multicolumn{6}{|c|}{ ' $8.014370 \mathrm{E}-\odot 2$} \\
\hline $\mathrm{H}-1$ & 305 & 0 & $3.32434 \mathrm{E}-02$ & 293.6 & \\
\hline $0-16$ & 305 & 0 & $1.84469 \mathrm{E}-02$ & 293.6 & \\
\hline$M g-24$ & 305 & $\odot$ & 1. $05686 E-\odot 4$ & 293.6 & \\
\hline$M g-25$ & 305 & $\odot$ & $1.33797 \mathrm{E}-05$ & 293.6 & \\
\hline$M g-26$ & 305 & $\odot$ & 1. $47310 \mathrm{E}-05$ & 293.6 & \\
\hline Al- 27 & 305 & $\odot$ & $2.74247 \mathrm{E}-02$ & 293.6 & \\
\hline $\mathrm{Si}-28$ & 305 & $\odot$ & $9.91306 \mathrm{E}-05$ & 293.6 & \\
\hline Si-29 & 305 & $\odot$ & 5. $01941 \mathrm{E}-06$ & 293.6 & \\
\hline $\mathrm{Si}-30$ & 305 & $\odot$ & $3.33194 \mathrm{E}-06$ & 293.6 & \\
\hline Ti-46 & 305 & $\odot$ & $4.20262 \mathrm{E}-\odot 7$ & 293.6 & \\
\hline Ti-47 & 305 & $\odot$ & $3.7900 \odot \mathrm{E}-\odot 7$ & 293.6 & \\
\hline Ti-48 & 305 & $\odot$ & $3.75536 \mathrm{E}-\odot 6$ & 293.6 & \\
\hline Ti-49 & 305 & $\odot$ & $2.75590 \mathrm{E}-\odot 7$ & 293.6 & \\
\hline $\mathrm{Ti}-50$ & 305 & $\odot$ & $2.63873 \mathrm{E}-07$ & 293.6 & \\
\hline $\mathrm{Cr}-50$ & 305 & $\odot$ & $5.30517 \mathrm{E}-07$ & 293.6 & \\
\hline $\mathrm{Cr}-52$ & 305 & $\odot$ & 1. $02189 \mathrm{E}-05$ & 293.6 & \\
\hline $\mathrm{Cr}-53$ & 305 & $\odot$ & $1.15860 \mathrm{E}-06$ & 293.6 & \\
\hline $\mathrm{Cr}-54$ & 305 & 0 & $2.87821 \mathrm{E}-07$ & 293.6 & \\
\hline
\end{tabular}




\begin{tabular}{|c|c|c|c|c|c|}
\hline Mn-55 & 305 & $\odot$ & $6.38261 E-06$ & 293.6 & end \\
\hline $\mathrm{Fe}-54$ & 305 & $\odot$ & $2.31055 E-06$ & 293.6 & \\
\hline $\mathrm{Fe}-56$ & 305 & $\odot$ & $3.62380 E-05$ & 293.6 & . \\
\hline $\mathrm{Fe}-57$ & 305 & $\odot$ & $8.37326 E-07$ & 293.6 & \\
\hline $\mathrm{Fe}-58$ & 305 & $\odot$ & 1. 10590E - 07 & 293.6 & \\
\hline $\mathrm{Cu}-63$ & 305 & $\odot$ & $1.85717 \mathrm{E}-05$ & 293.6 & \\
\hline $\mathrm{Cu}-65$ & 305 & $\odot$ & $8.27767 \mathrm{E}-06$ & 293.6 & \\
\hline$U-234$ & 305 & $\odot$ & $6.97424 \mathrm{E}-06$ & 293.6 & \\
\hline$U-235$ & 305 & $\odot$ & $6.50000 E-\odot 4$ & 293.6 & \\
\hline$U-236$ & 305 & $\odot$ & $2.78972 \mathrm{E}-06$ & 293.6 & \\
\hline$U-238$ & 305 & $\odot$ & $3.76609 E-05$ & 293.6 & \\
\hline \multicolumn{3}{|c|}{ total atom density } & \multicolumn{2}{|c|}{$=8.00985 \mathrm{E}-\odot 2 \mathrm{a} / \mathrm{b}-\mathrm{cm}$} & \\
\hline $\mathrm{H}-1$ & 306 & 0 & $3.32434 \mathrm{E}-02$ & 293.6 & \\
\hline $0-16$ & 306 & $\odot$ & 1. $81013 E-02$ & 293.6 & \\
\hline$M g-24$ & 306 & $\odot$ & $1.05686 E-\odot 4$ & 293.6 & \\
\hline$M g-25$ & 306 & $\odot$ & $1.33797 \mathrm{E}-05$ & 293.6 & \\
\hline$M g-26$ & 306 & $\odot$ & $1.47310 \mathrm{E}-05$ & 293.6 & \\
\hline Al-27 & 306 & 0 & $2.78528 \mathrm{E}-02$ & 293.6 & \\
\hline Si-28 & 306 & 0 & 1. $00085 E-04$ & 293.6 & \\
\hline Si-29 & 306 & 0 & $5.06774 \mathrm{E}-06$ & 293.6 & \\
\hline $\mathrm{Si}-30$ & 306 & $\odot$ & $3.36403 E-06$ & 293.6 & \\
\hline Ti-46 & 306 & $\odot$ & $4.20262 \mathrm{E}-\odot 7$ & 293.6 & \\
\hline $\mathrm{Ti}-47$ & 306 & $\odot$ & $3.79000 \mathrm{E}-\odot 7$ & 293.6 & \\
\hline $\mathrm{Ti}-48$ & 306 & $\odot$ & $3.75536 \mathrm{E}-06$ & 293.6 & \\
\hline Ti-49 & 306 & $\odot$ & $2.75590 E-\odot 7$ & 293.6 & \\
\hline Ti-50 & 306 & $\odot$ & $2.63873 E-07$ & 293.6 & \\
\hline $\mathrm{Cr}-50$ & 306 & $\odot$ & $5.3 \odot 517 \mathrm{E}-\odot 7$ & 293.6 & \\
\hline $\mathrm{Cr}-52$ & 306 & $\odot$ & 1. $02189 E-05$ & 293.6 & \\
\hline $\mathrm{Cr}-53$ & 306 & $\odot$ & $1.15860 \mathrm{E}-06$ & 293.6 & \\
\hline $\mathrm{Cr}-54$ & 306 & 0 & $2.87821 E-07$ & 293.6 & \\
\hline$M n-55$ & 306 & $\odot$ & $6.43555 \mathrm{E}-06$ & 293.6 & \\
\hline $\mathrm{Fe}-54$ & 306 & 0 & $2.34101 \mathrm{E}-06$ & 293.6 & \\
\hline $\mathrm{Fe}-56$ & 306 & $\odot$ & $3.67158 \mathrm{E}-05$ & 293.6 & \\
\hline $\mathrm{Fe}-57$ & 306 & $\odot$ & $8.48365 E-07$ & 293.6 & \\
\hline $\mathrm{Fe}-58$ & 306 & $\odot$ & $1.12048 \mathrm{E}-07$ & 293.6 & \\
\hline $\mathrm{Cu}-63$ & 306 & $\odot$ & $1.87481 E-05$ & 293.6 & \\
\hline $\mathrm{Cu}-65$ & 306 & $\odot$ & $8.35629 E-\odot 6$ & 293.6 & \\
\hline U-234 & 306 & 0 & $5.67811 E-06$ & 293.6 & \\
\hline$U-235$ & 306 & 0 & $5.29200 E-\odot 4$ & 293.6 & \\
\hline$U-236$ & 306 & 0 & $2.27126 \mathrm{E}-06$ & 293.6 & \\
\hline$U-238$ & 306 & $\odot$ & $3.06618 E-05$ & 293.6 & \\
\hline \multicolumn{3}{|c|}{$\begin{array}{l}\text { total atom density } \\
18.005550 \mathrm{E}-02\end{array}$} & \multicolumn{2}{|c|}{$=8.00555 \mathrm{E}-02 \mathrm{a} / \mathrm{b}-\mathrm{cm}$} & \\
\hline $\mathrm{H}-1$ & 307 & 0 & $3.32434 \mathrm{E}-02$ & 293.6 & \\
\hline $0-16$ & 307 & $\odot$ & $1.77731 \mathrm{E}-02$ & 293.6 & \\
\hline$M g-24$ & 307 & $\odot$ & 1. $05686 \mathrm{E}-04$ & 293.6 & \\
\hline $\mathrm{Mg}-25$ & 307 & $\odot$ & 1.33797E- 05 & 293.6 & \\
\hline$M g-26$ & 307 & $\odot$ & $1.47310 \mathrm{E}-05$ & 293.6 & \\
\hline $\mathrm{Al}-27$ & 307 & $\odot$ & $2.82593 E-02$ & 293.6 & \\
\hline Si-28 & 307 & $\odot$ & 1. $00993 E-\odot 4$ & 293.6 & \\
\hline Si-29 & 307 & $\odot$ & $5.11370 E-06$ & 293.6 & \\
\hline Si-30 & 307 & $\odot$ & $3.39453 E-06$ & 293.6 & \\
\hline $\mathrm{Ti}-46$ & 307 & $\odot$ & $4.20262 \mathrm{E}-07$ & 293.6 & \\
\hline Ti-47 & 307 & $\odot$ & $3.7900 \odot E-\odot 7$ & 293.6 & \\
\hline $\mathrm{Ti}-48$ & 307 & $\odot$ & $3.75536 \mathrm{E}-06$ & 293.6 & \\
\hline $\mathrm{Ti}-49$ & 307 & $\odot$ & $2.75590 E-\odot 7$ & 293.6 & \\
\hline Ti-50 & 307 & $\odot$ & $2.63873 E-07$ & 293.6 & \\
\hline $\mathrm{Cr}-50$ & 307 & $\odot$ & $5.30517 E-07$ & 293.6 & \\
\hline $\mathrm{Cr}-52$ & 307 & $\odot$ & 1. $02189 E-05$ & 293.6 & \\
\hline $\mathrm{Cr}-53$ & 307 & $\odot$ & $1.15860 E-06$ & 293.6 & \\
\hline $\mathrm{Cr}-54$ & 307 & 0 & $2.87821 \mathrm{E}-07$ & 293.6 & \\
\hline Mn-55 & 307 & 0 & $6.48581 E-06$ & 293.6 & \\
\hline $\mathrm{Fe}-54$ & 307 & 0 & $2.36993 \mathrm{E}-06$ & 293.6 & \\
\hline $\mathrm{Fe}-56$ & 307 & 0 & $3.71695 \mathrm{E}-05$ & 293.6 & \\
\hline $\mathrm{Fe}-57$ & 307 & $\odot$ & $8.58848 E-07$ & 293.6 & \\
\hline $\mathrm{Fe}-58$ & 307 & $\odot$ & $1.13433 E-07$ & 293.6 & \\
\hline $\mathrm{Cu}-63$ & 307 & $\odot$ & 1. $89155 \mathrm{E}-05$ & 293.6 & \\
\hline $\mathrm{Cu}-65$ & 307 & $\odot$ & $8.43090 E-06$ & 293.6 & \\
\hline U-234 & 307 & $\odot$ & $4.44742 E-06$ & 293.6 & \\
\hline U-235 & 7 & $\odot$ & $E-\odot 4$ & 293.6 & \\
\hline$U-236$ & 307 & $\odot$ & $1.77898 \mathrm{E}-\odot 6$ & 293.6 & \\
\hline$U-238$ & 307 & 0 & $2.40161 \mathrm{E}-05$ & 293.6 & \\
\hline & atom & sity & $=8.00271 \mathrm{E}$ & $2 a / b-c$ & \\
\hline \multicolumn{6}{|c|}{ ' $\quad 8 . \odot \odot 271 \odot \mathrm{E}-\odot 2$} \\
\hline $\mathrm{H}-1$ & 308 & $\odot$ & 3. 32434E- 02 & 293.6 & \\
\hline $0-16$ & 308 & $\odot$ & $1.75562 \mathrm{E}-\mathrm{O} 2$ & 293.6 & \\
\hline$M g-24$ & 308 & $\odot$ & $1.05686 E-\odot 4$ & 293.6 & \\
\hline$M g-25$ & 308 & $\odot$ & $1.33797 \mathrm{E}-05$ & 293.6 & \\
\hline$M g-26$ & 308 & $\odot$ & $1.47310 \mathrm{E}-05$ & 293.6 & \\
\hline Al-27 & 308 & $\odot$ & $2.85280 E-02$ & 293.6 & \\
\hline
\end{tabular}




\begin{tabular}{|c|c|c|c|c|c|}
\hline Si-28 & 308 & $\odot$ & 1. $01591 E-\odot 4$ & 293.6 & end \\
\hline Si-29 & 308 & $\odot$ & $5.14401 \mathrm{E}-\odot 6$ & 293.6 & end \\
\hline Si-30 & 308 & $\odot$ & $3.41465 E-\odot 6$ & 293.6 & end \\
\hline Ti-46 & 308 & 0 & $4.20262 \mathrm{E}-\odot 7$ & 293.6 & end \\
\hline Ti-47 & 308 & 0 & $3.790 \odot \odot E-\odot 7$ & 293.6 & end \\
\hline $\mathrm{Ti}-48$ & 308 & $\odot$ & $3.75536 \mathrm{E}-\odot 6$ & 293.6 & end \\
\hline $\mathrm{Ti}-49$ & 308 & $\odot$ & $2.75590 E-07$ & 293.6 & end \\
\hline Ti-50 & 308 & $\odot$ & $2.63873 E-07$ & 293.6 & end \\
\hline $\mathrm{Cr}-50$ & 308 & 0 & $5.30517 \mathrm{E}-07$ & 293.6 & end \\
\hline $\mathrm{Cr}-52$ & 308 & 0 & 1. $02189 E-05$ & 293.6 & end \\
\hline $\mathrm{Cr}-53$ & 308 & $\odot$ & 1. $15860 E-\odot 6$ & 293.6 & end \\
\hline $\mathrm{Cr}-54$ & 308 & $\odot$ & $2.87821 E-07$ & 293.6 & end \\
\hline$M n-55$ & 308 & $\odot$ & $6.51903 E-\odot 6$ & 293.6 & end \\
\hline $\mathrm{Fe}-54$ & 308 & $\odot$ & $2.38905 E-\odot 6$ & 293.6 & nd \\
\hline $\mathrm{Fe}-56$ & 308 & 0 & $3.74693 \mathrm{E}-05$ & 293.6 & nd \\
\hline $\mathrm{Fe}-57$ & 308 & $\odot$ & $8.65776 E-07$ & 293.6 & end \\
\hline $\mathrm{Fe}-58$ & 308 & 0 & $1.14348 \mathrm{E}-07$ & 293.6 & end \\
\hline $\mathrm{Cu}-63$ & 308 & 0 & $1.90262 \mathrm{E}-05$ & 293.6 & end \\
\hline $\mathrm{Cu}-65$ & 308 & 0 & $8.48022 \mathrm{E}-06$ & 293.6 & end \\
\hline$U-234$ & 308 & $\odot$ & $3.63412 \mathrm{E}-\odot 6$ & 293.6 & end \\
\hline U-235 & 308 & $\odot$ & $3.38700 \mathrm{E}-\odot 4$ & 293.6 & end \\
\hline$U-236$ & 308 & $\odot$ & 1. 45366E- $\odot 6$ & 293.6 & end \\
\hline$U-238$ & 308 & $\odot$ & 1. $96242 \mathrm{E}-05$ & 293.6 & end \\
\hline 1 & tom & sity & $=8.00013 \mathrm{E}$ & $2 a / b-c m$ & \\
\hline $\mathrm{H}-1$ & 309 & 0 & $3.32434 \mathrm{E}-02$ & 293.6 & end \\
\hline $0-16$ & 309 & 0 & $1.73591 \mathrm{E}-02$ & 293.6 & nd \\
\hline$M g-24$ & 309 & $\odot$ & 1. $05686 E-\odot 4$ & 293.6 & nd \\
\hline$M g-25$ & 309 & 0 & $1.33797 \mathrm{E}-05$ & 293.6 & nd \\
\hline$M g-26$ & 309 & 0 & $1.47310 \mathrm{E}-05$ & 293.6 & nd \\
\hline Al-27 & 309 & 0 & $2.87722 \mathrm{E}-02$ & 293.6 & nd \\
\hline Si-28 & 309 & $\odot$ & 1. $02136 E-\odot 4$ & 293.6 & nd \\
\hline Si-29 & 309 & $\odot$ & $5.17160 E-06$ & 293.6 & nd \\
\hline Si-30 & 309 & $\odot$ & $3.43297 \mathrm{E}-06$ & 293.6 & end \\
\hline $\mathrm{Ti}-46$ & 309 & 0 & 4. $20262 \mathrm{E}-07$ & 293.6 & nd \\
\hline $\mathrm{Ti}-47$ & 309 & 0 & $3.79000 E-\odot 7$ & 293.6 & id \\
\hline $\mathrm{Ti}-48$ & 309 & $\odot$ & $3.75536 \mathrm{E}-\odot 6$ & 293.6 & and \\
\hline $\mathrm{Ti}-49$ & 309 & $\odot$ & $2.75590 E-07$ & 293.6 & nd \\
\hline Ti-50 & 309 & 0 & $2.63873 \mathrm{E}-07$ & 293.6 & d \\
\hline $\mathrm{Cr}-50$ & 309 & 0 & $5.30517 \mathrm{E}-07$ & 293.6 & end \\
\hline $\mathrm{Cr}-52$ & 309 & 0 & 1. $02189 E-05$ & 293.6 & end \\
\hline $\mathrm{Cr}-53$ & 309 & 0 & $1.15860 E-06$ & 293.6 & end \\
\hline $\mathrm{Cr}-54$ & 309 & $\odot$ & $2.87821 E-\odot 7$ & 293.6 & en \\
\hline$M n-55$ & 309 & $\odot$ & $6.54922 \mathrm{E}-06$ & 293.6 & \\
\hline $\mathrm{Fe}-54$ & 309 & $\odot$ & $2.40643 E-06$ & 293.6 & \\
\hline $\mathrm{Fe}-56$ & 309 & $\odot$ & $3.77418 \mathrm{E}-05$ & 293.6 & nd \\
\hline $\mathrm{Fe}-57$ & 309 & $\odot$ & $8.72072 \mathrm{E}-07$ & 293.6 & \\
\hline $\mathrm{Fe}-58$ & 309 & $\odot$ & 1. 15179E- $\odot 7$ & 293.6 & \\
\hline $\mathrm{Cu}-63$ & 309 & 0 & $1.91268 \mathrm{E}-05$ & 293.6 & \\
\hline $\mathrm{Cu}-65$ & 309 & 0 & $8.52504 \mathrm{E}-06$ & 293.6 & end \\
\hline$U-234$ & 309 & $\odot$ & $2.89485 E-06$ & 293.6 & \\
\hline$U-235$ & 309 & $\odot$ & $2.69800 E-\odot 4$ & 293.6 & \\
\hline$U-236$ & 309 & $\odot$ & $1.15795 \mathrm{E}-06$ & 293.6 & \\
\hline$U-238$ & 309 & $\odot$ & $1.56322 \mathrm{E}-05$ & 293.6 & nd \\
\hline
\end{tabular}

Region IV Control Element Material Descriptions

\begin{tabular}{lccccc}
\multicolumn{7}{c}{ Aluminum clad of } & control elements & \\
$\mathrm{Al}-27$ & 21 & $\odot$ & $5.85482 \mathrm{E}-\odot 2$ & 293.6 & end \\
$\mathrm{H}-1$ & 21 & $\odot$ & $3.45716 \mathrm{E}-\odot 4$ & 293.6 & end \\
$\mathrm{Mg}-24$ & 21 & $\odot$ & $5.28432 \mathrm{E}-\odot 4$ & 293.6 & end \\
$\mathrm{Mg}-25$ & 21 & $\odot$ & $6.68986 \mathrm{E}-05$ & 293.6 & end \\
$\mathrm{Mg}-26$ & 21 & $\odot$ & $7.36554 \mathrm{E}-\odot 5$ & 293.6 & end \\
$\mathrm{Si}-28$ & 21 & $\odot$ & $3.20373 \mathrm{E}-\odot 4$ & 293.6 & end \\
$\mathrm{Si}-29$ & 21 & $\odot$ & $1.62219 \mathrm{E}-\odot 5$ & 293.6 & end \\
$\mathrm{Si}-3 \odot$ & 21 & $\odot$ & $1.07683 \mathrm{E}-\odot 5$ & 293.6 & end \\
$\mathrm{Ti}-46$ & 21 & $\odot$ & $2.10131 \mathrm{E}-\odot 6$ & 293.6 & end \\
$\mathrm{Ti}-47$ & 21 & $\odot$ & $1.89500 \mathrm{E}-\odot 6$ & 293.6 & end \\
$\mathrm{Ti}-48$ & 21 & $\odot$ & $1.87768 \mathrm{E}-\odot 5$ & 293.6 & end \\
$\mathrm{Ti}-49$ & 21 & $\odot$ & $1.37795 \mathrm{E}-\odot 6$ & 293.6 & end \\
$\mathrm{Ti}-5 \odot$ & 21 & $\odot$ & $1.31937 \mathrm{E}-\odot 6$ & 293.6 & end \\
$\mathrm{Cr}-5 \odot$ & 21 & $\odot$ & $2.65258 \mathrm{E}-\odot 6$ & 293.6 & end \\
$\mathrm{Cr}-52$ & 21 & $\odot$ & $5.10942 \mathrm{E}-\odot 5$ & 293.6 & end \\
$\mathrm{Cr}-53$ & 21 & $\odot$ & $5.7930 \odot \mathrm{E}-\odot 6$ & 293.6 & end \\
$\mathrm{Cr}-54$ & 21 & $\odot$ & $1.43910 \mathrm{E}-\odot 6$ & 293.6 & end
\end{tabular}




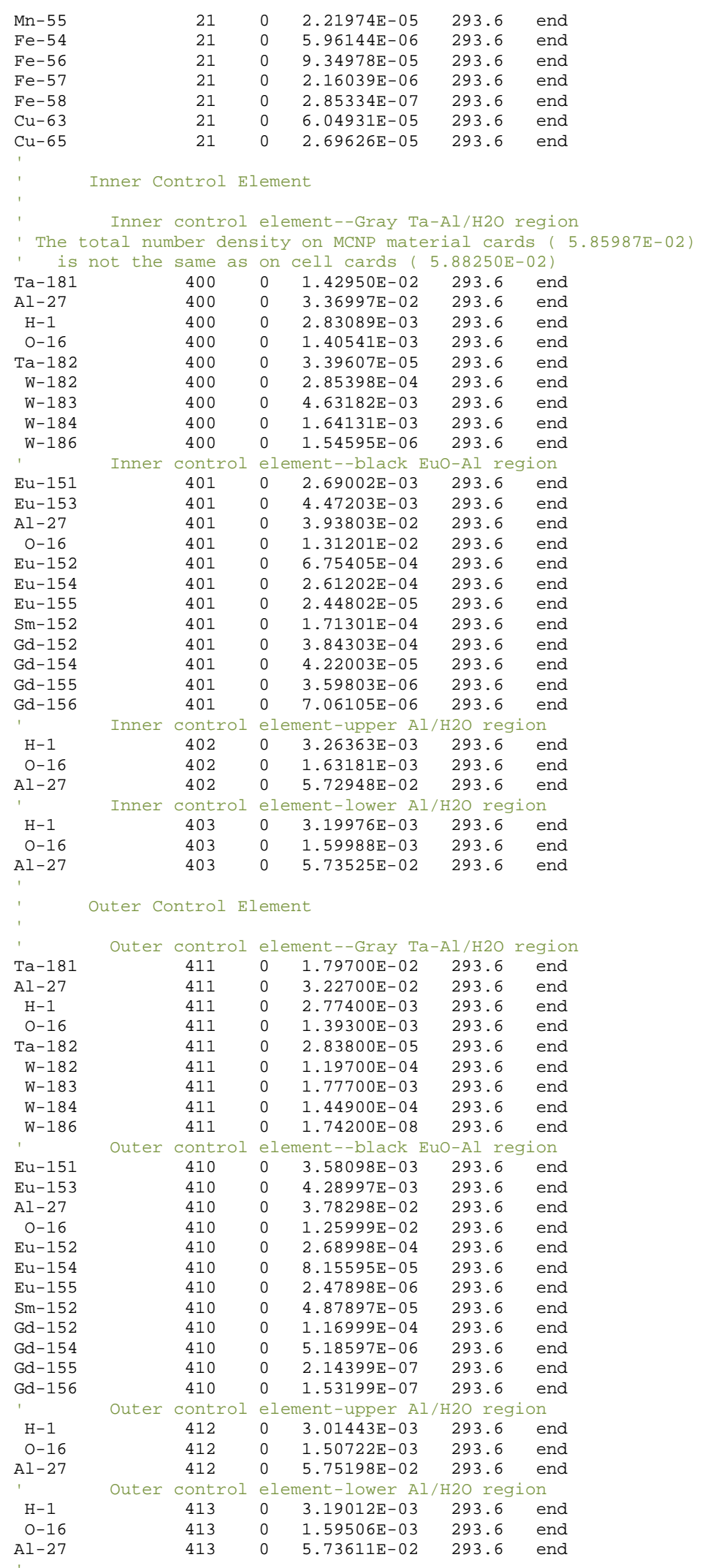




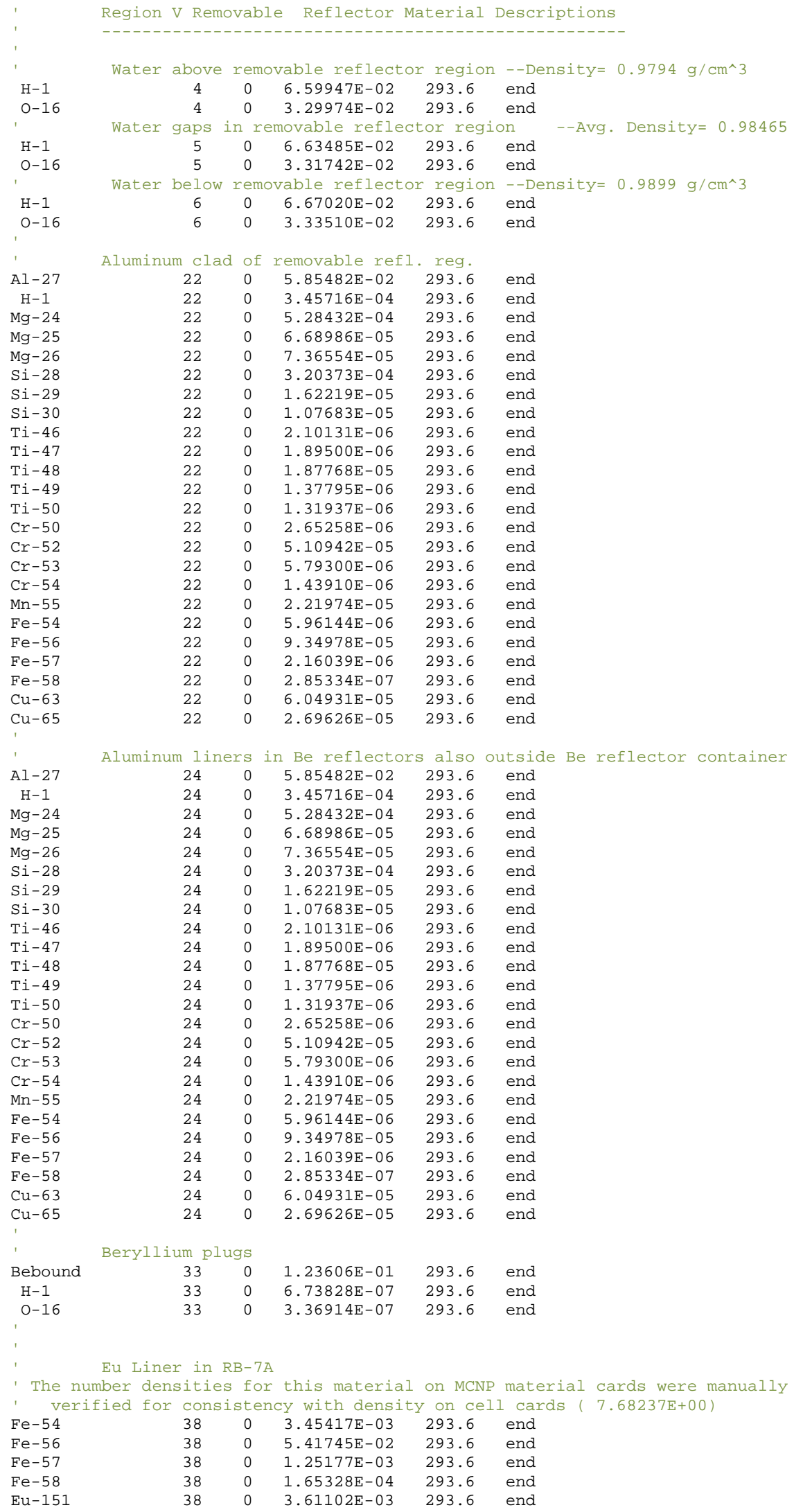

$\begin{array}{llllll}\mathrm{Fe}-54 & 38 & \odot & 3.45417 \mathrm{E}-03 & 293.6 & \text { end } \\ \mathrm{Fe}-56 & 38 & \odot & 5.41745 \mathrm{E}-02 & 293.6 & \text { end } \\ \mathrm{Fe}-57 & 38 & \odot & 1.25177 \mathrm{E}-\odot 3 & 293.6 & \text { end } \\ \mathrm{Fe}-58 & 38 & \odot & 1.65328 \mathrm{E}-\odot 4 & 293.6 & \text { end }\end{array}$

$\begin{array}{llll}\text { Eu-151 } 38 \quad \odot \quad 3.61102 \mathrm{E}-03 & 293.6 & \text { end }\end{array}$ 


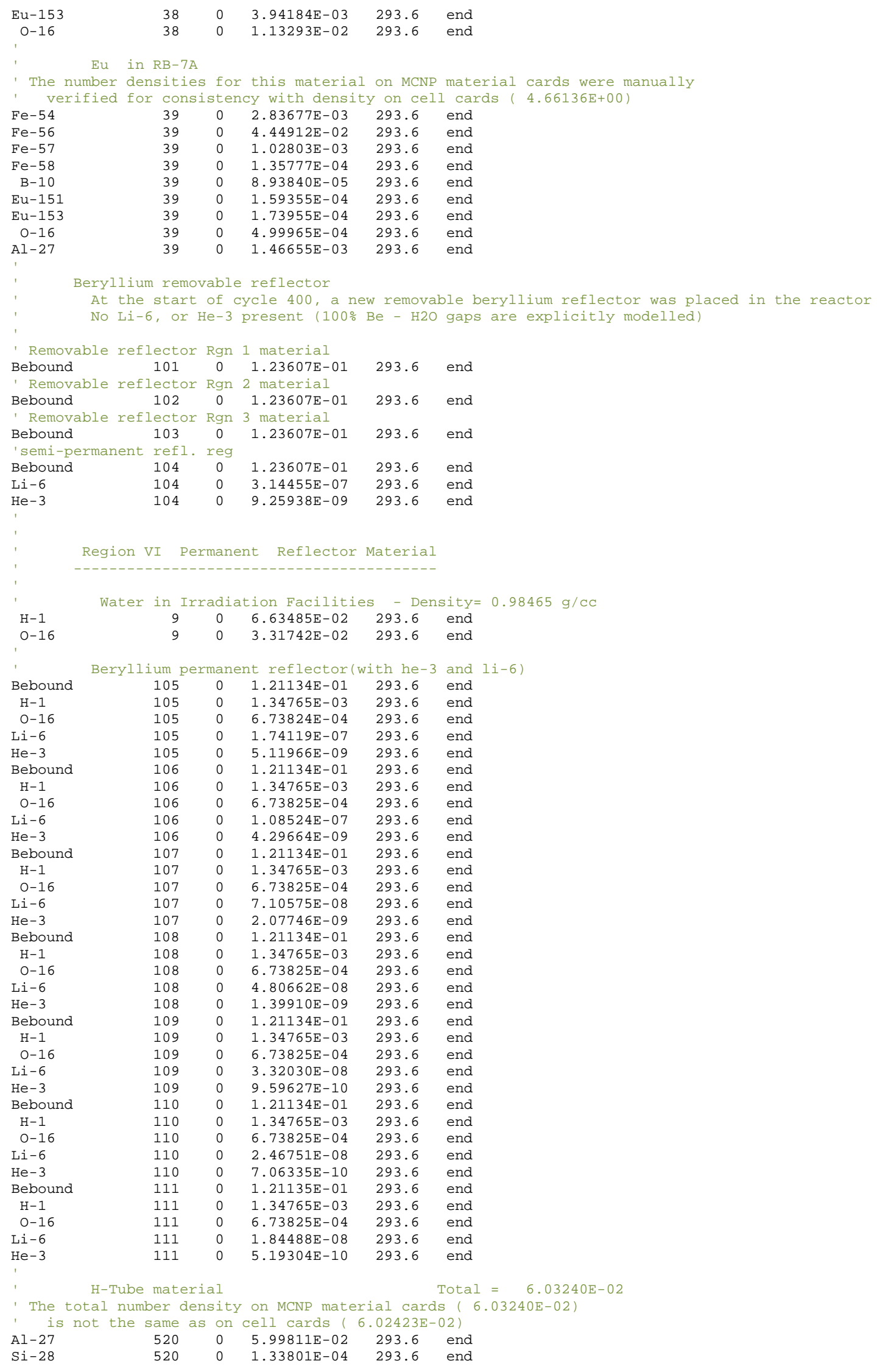




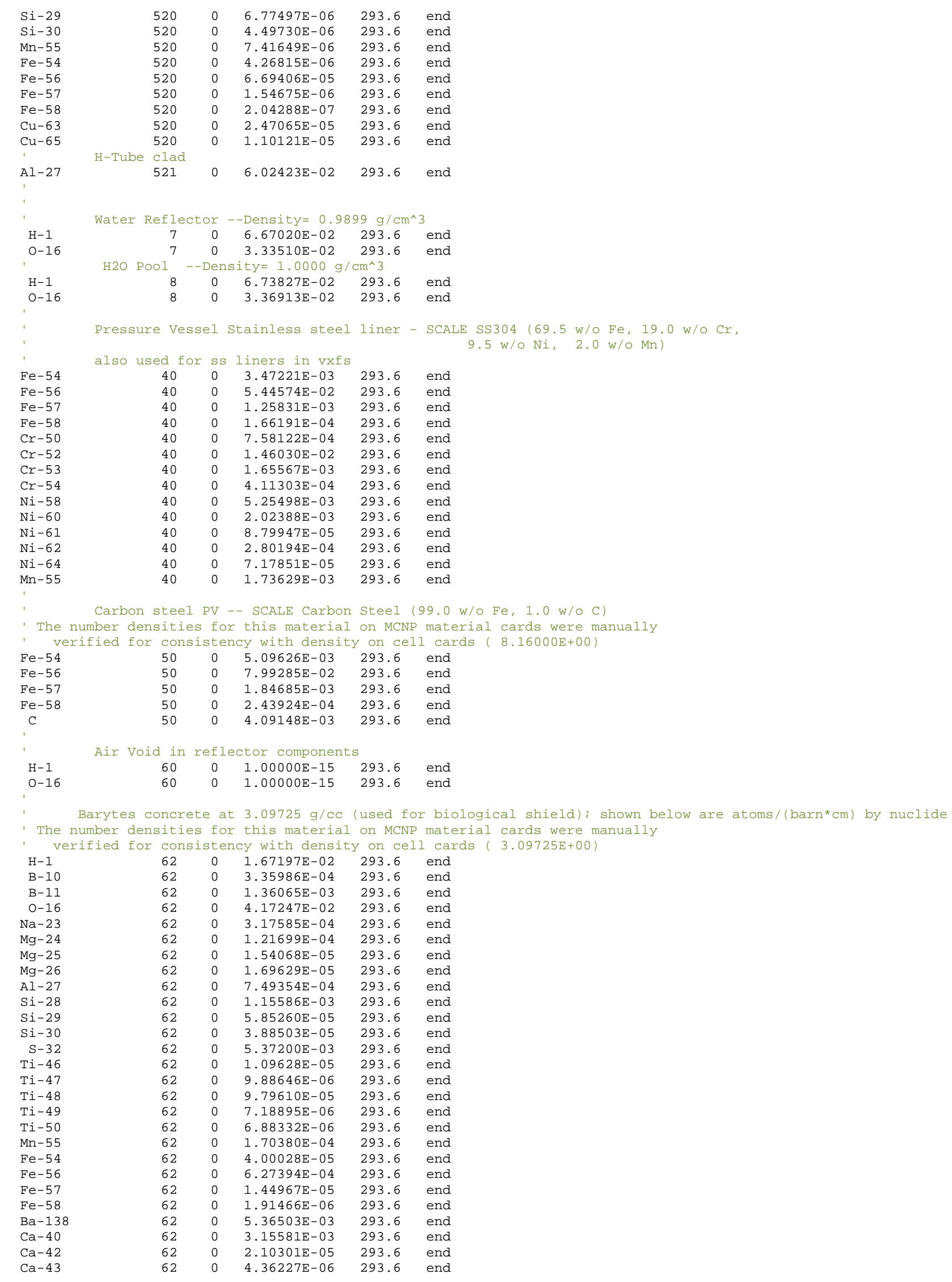




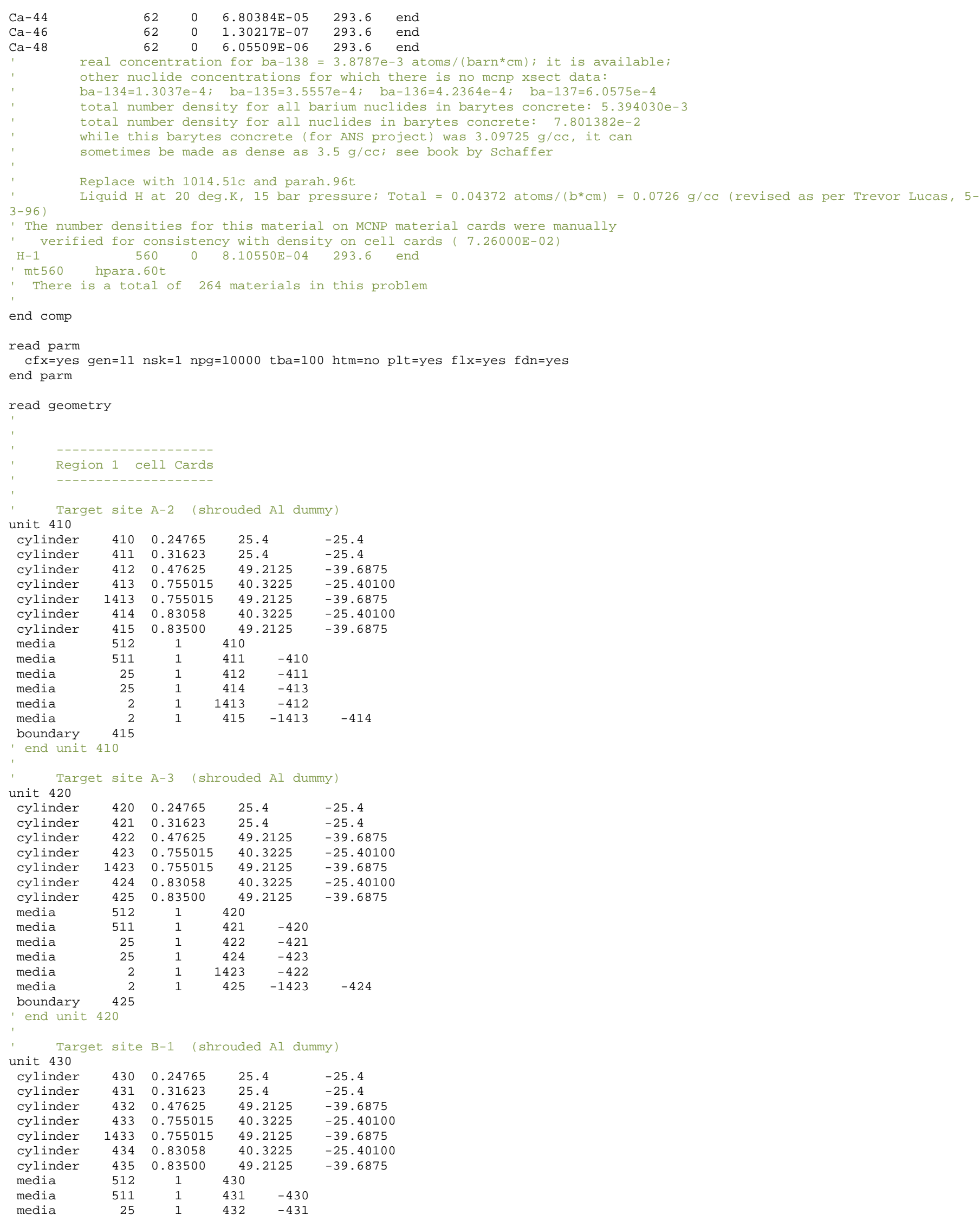




\begin{tabular}{|c|c|c|c|c|c|}
\hline media & 25 & 1 & 434 & -433 & \\
\hline media & 2 & 1 & 1433 & -432 & \\
\hline media & 2 & 1 & 435 & -1433 & -434 \\
\hline boundary & 435 & & & & \\
\hline ' end unit & 430 & & & & \\
\hline Targe & te site & B-2 (sh & rouded & Al dum & my) \\
\hline unit 440 & & & & & \\
\hline cylinder & 440 & $\odot .24765$ & 25. & & -25.4 \\
\hline cylinder & 441 & 0.31623 & 25. & & -25.4 \\
\hline cylinder & 442 & 0.47625 & 49. & 2125 & -39.6875 \\
\hline cylinder & 443 & $\odot .755015$ & 40. & 3225 & -25.40100 \\
\hline cylinder & 1443 & $\odot .755015$ & 49. & 2125 & -39.6875 \\
\hline cylinder & 444 & 0.83058 & 40. & 3225 & -25.40100 \\
\hline cylinder & 445 & 0.83500 & 49. & 2125 & -39.6875 \\
\hline media & 512 & 1 & 440 & & \\
\hline media & 511 & 1 & 441 & -440 & \\
\hline media & 25 & 1 & 442 & -441 & \\
\hline media & 25 & 1 & 444 & -443 & \\
\hline media & 2 & 1 & 1443 & -442 & \\
\hline media & 2 & 1 & 445 & -1443 & -444 \\
\hline boundary & 445 & & & & \\
\hline ' end unit & $44 \odot$ & & & & \\
\hline ' & & & & & \\
\hline Targe & t site & $\mathrm{B}-3 \quad$ (HT & tube) & & \\
\hline unit 750 & & & & & \\
\hline cylinder & 750 & 0.32385 & 28. & 80800 & 23.251795 \\
\hline cylinder & 751 & $\odot .32385$ & 22. & $30 \odot 565$ & 16.744315 \\
\hline cylinder & 752 & 0.32385 & 15. & 793085 & 10.236835 \\
\hline cylinder & 753 & 0.32385 & & 285605 & 3.729355 \\
\hline cylinder & 754 & 0.32385 & & 778125 & -2.778125 \\
\hline cylinder & 755 & 0.32385 & -3. & 729355 & -9.285605 \\
\hline cylinder & 756 & 0.32385 & -10 & 236835 & -15.793085 \\
\hline cylinder & 757 & 0.32385 & -16. & 744315 & -22.300565 \\
\hline cylinder & 758 & 0.32385 & -23. & 251795 & -28.80800 \\
\hline cylinder & 760 & $\odot .55499$ & 29. & 28366 & 22.77618 \\
\hline cylinder & 761 & 0.55499 & 29. & 28366 & 16.26870 \\
\hline cylinder & 762 & 0.55499 & 29. & 28366 & 9.76122 \\
\hline cylinder & 763 & 0.55499 & 29. & 28366 & 3.25374 \\
\hline cylinder & 764 & $\odot .55499$ & 29. & 28366 & -3.25374 \\
\hline cylinder & 765 & 0.55499 & 29. & 28366 & -9.76122 \\
\hline cylinder & 766 & 0.55499 & 29. & 28366 & -16.26870 \\
\hline cylinder & 767 & $\odot .55499$ & 29. & 28366 & -22.77618 \\
\hline , cylinder & 768 & $\odot .55499$ & 29. & 28366 & -29.28366 \\
\hline cylinder & 452 & 0.63500 & 29. & 28366 & -29.28366 \\
\hline cylinder & 453 & $\odot .787400$ & 29. & 28366 & -29.28366 \\
\hline , cylinder & 454 & 0.83500 & 29. & 28366 & -29.28366 \\
\hline cylinder & 455 & $\odot .78740 \odot$ & 29. & 28366 & -39.6875 \\
\hline , cylinder & 456 & 0.83500 & 29. & 28366 & -39.6875 \\
\hline cylinder & 446 & $\odot .55499$ & 49. & 2125 & -29.28366 \\
\hline cylinder & 447 & 0.63500 & 49. & 2125 & -29.28366 \\
\hline cylinder & 448 & 0.787400 & 49. & 2125 & -39.6875 \\
\hline cylinder & 449 & 0.83500 & 49. & 2125 & -39.6875 \\
\hline ' & & & & & \\
\hline media & 520 & 1 & 750 & & \\
\hline media & 520 & 1 & 751 & & \\
\hline media & 520 & 1 & 752 & & \\
\hline media & 520 & 1 & 753 & & \\
\hline media & 520 & 1 & 754 & & \\
\hline media & 520 & 1 & 755 & & \\
\hline media & 520 & 1 & 756 & & \\
\hline media & 520 & 1 & 757 & & \\
\hline media & 520 & 1 & 758 & & \\
\hline media & 521 & 1 & 760 & -750 & \\
\hline media & 521 & 1 & 761 & -751 & -760 \\
\hline media & 521 & 1 & 762 & -752 & -761 \\
\hline media & 521 & 1 & 763 & -753 & -762 \\
\hline media & 521 & 1 & 764 & -754 & -763 \\
\hline media & 521 & 1 & 765 & -755 & -764 \\
\hline media & 521 & 1 & 766 & -756 & -765 \\
\hline media & 521 & 1 & 767 & -757 & -766 \\
\hline media & 521 & 1 & 768 & -758 & -767 \\
\hline media & 60 & 1 & 452 & -768 & \\
\hline media & 25 & 1 & 453 & -452 & \\
\hline media & 2 & 1 & 454 & -453 & \\
\hline media & 25 & 1 & 455 & -453 & \\
\hline media & 2 & 1 & 456 & -455 & -454 \\
\hline
\end{tabular}




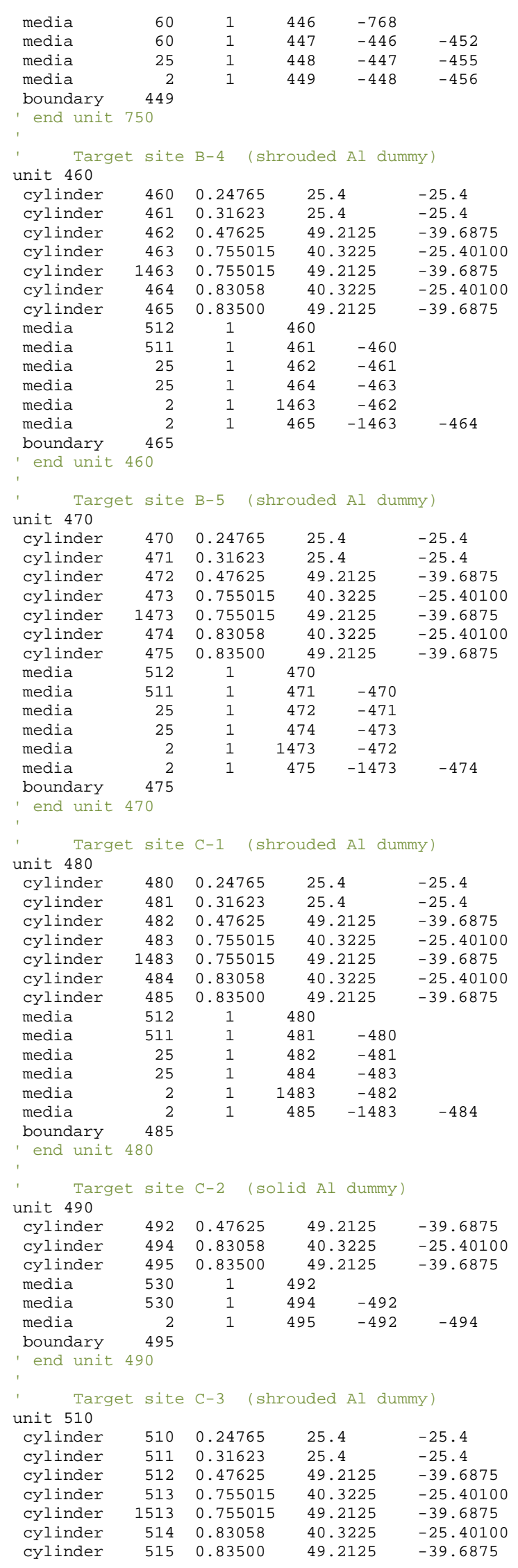




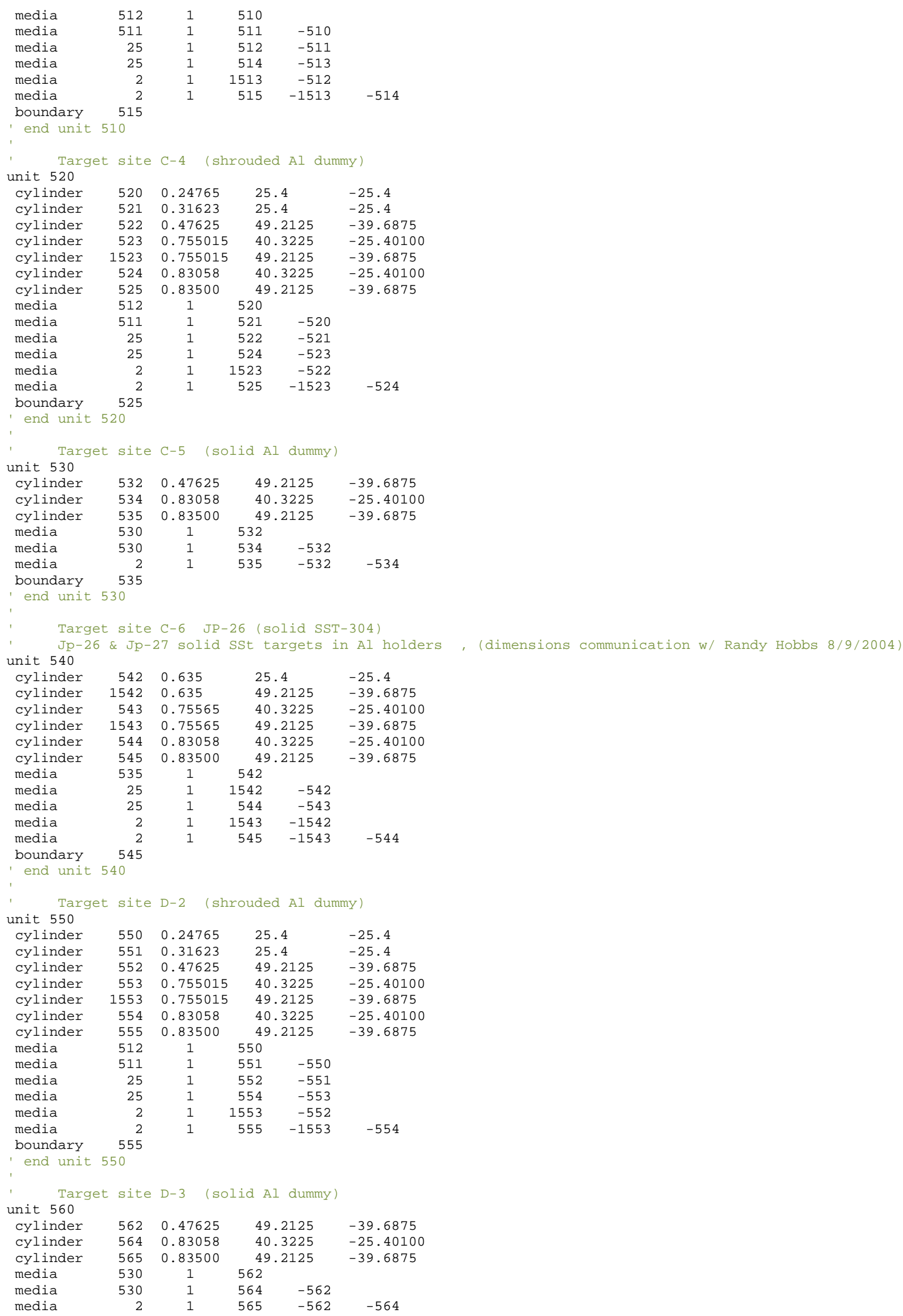




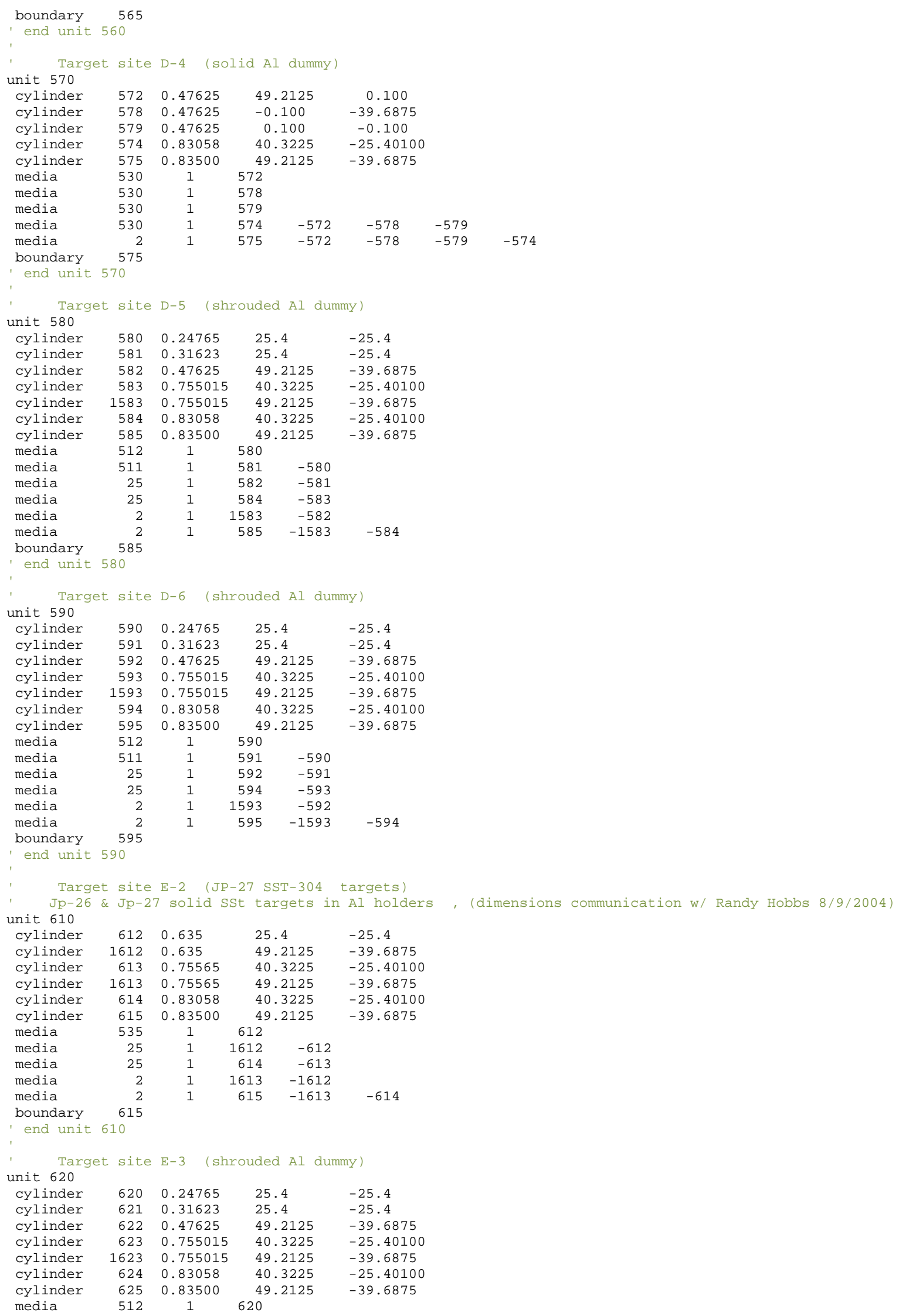




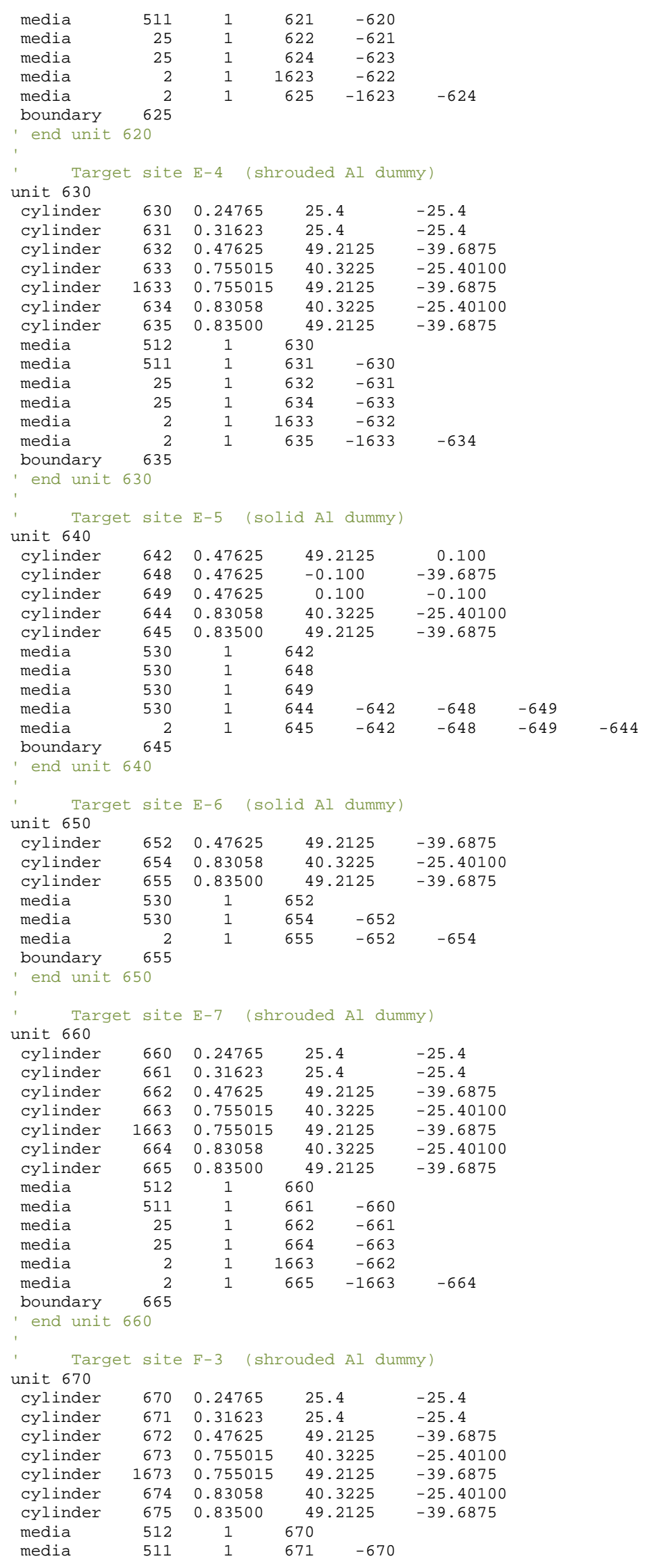




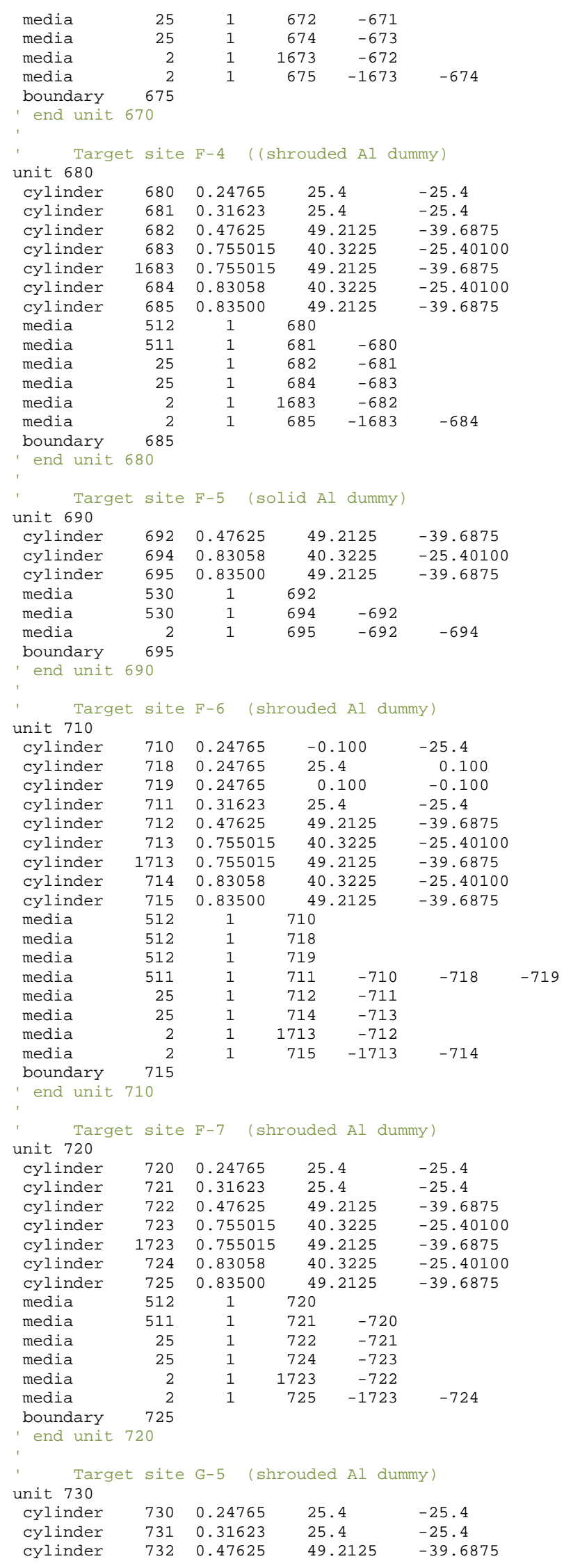




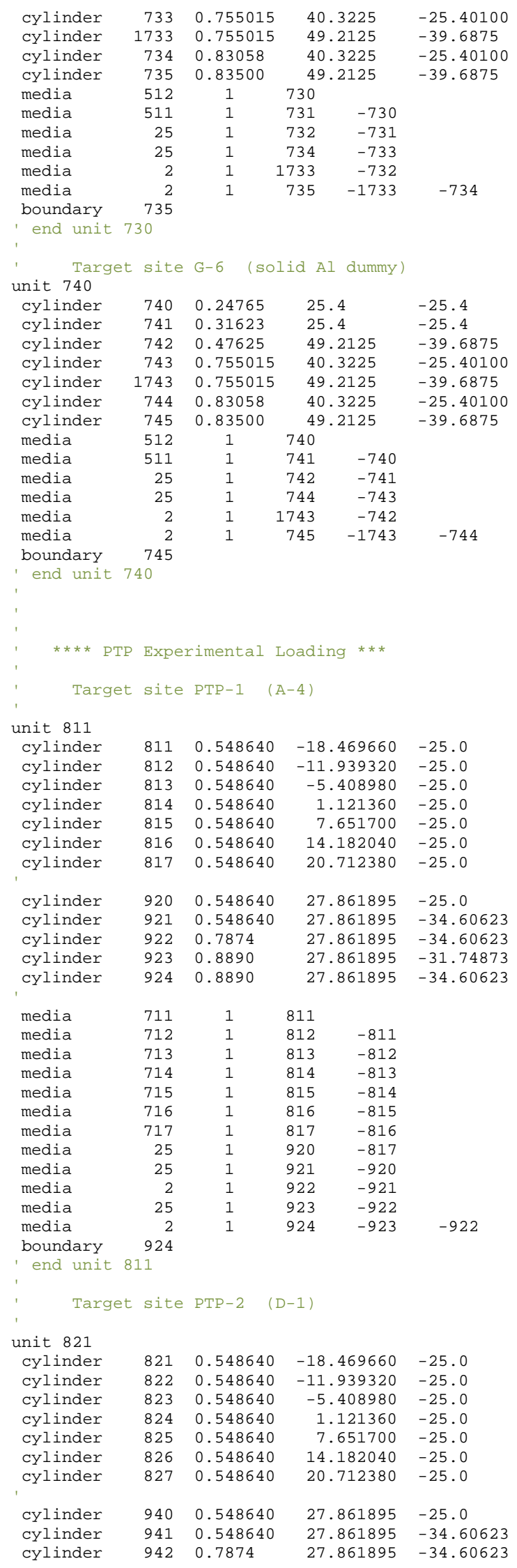




\begin{tabular}{|c|c|c|c|c|c|}
\hline cylinder & 943 & $\odot .8890$ & \multicolumn{2}{|c|}{27.861895} & -31.74873 \\
\hline cylinder & 944 & $\odot .8890$ & \multicolumn{2}{|c|}{27.861895} & -34.60623 \\
\hline media & 721 & 1 & \multicolumn{2}{|l|}{821} & \\
\hline media & 722 & 1 & 822 & -821 & \\
\hline media & 723 & 1 & 823 & -822 & \\
\hline media & 724 & 1 & 824 & -823 & \\
\hline media & 725 & 1 & 825 & -824 & \\
\hline media & 726 & 1 & 826 & -825 & \\
\hline media & 727 & 1 & 827 & -826 & \\
\hline media & 25 & 1 & 940 & -827 & \\
\hline media & 25 & 1 & 941 & -940 & \\
\hline media & 2 & 1 & 942 & -941 & \\
\hline media & 25 & 1 & 943 & -942 & \\
\hline media & 2 & 1 & 944 & -943 & -942 \\
\hline boundary & 944 & & & & \\
\hline \multicolumn{2}{|c|}{ ' end unit 821} & & & & \\
\hline \multicolumn{2}{|c|}{ Target site } & \multicolumn{3}{|c|}{ PTP-3 (A-1) } & \\
\hline \multicolumn{6}{|l|}{ unit 831} \\
\hline cylinder & 831 & 0.548640 & \multirow{2}{*}{\multicolumn{2}{|c|}{$\begin{array}{l}-18.469660 \\
-11.939320\end{array}$}} & -25.0 \\
\hline cylinder & 832 & 0.548640 & & & -25.0 \\
\hline cylinder & 833 & 0.548640 & \multicolumn{2}{|c|}{-5.408980} & -25.0 \\
\hline cylinder & 834 & 0.548640 & \multicolumn{2}{|c|}{1.121360} & -25.0 \\
\hline cylinder & 835 & 0.548640 & \multicolumn{2}{|c|}{7.651700} & -25.0 \\
\hline cylinder & 836 & $\odot .548640$ & & 82040 & -25.0 \\
\hline cylinder & 837 & $\odot .548640$ & & 12380 & $-25 . \odot$ \\
\hline cylinder & 930 & 0.548640 & & 61895 & -25.0 \\
\hline cylinder & 931 & 0.548640 & & 61895 & -34.60623 \\
\hline cylinder & 932 & 0.7874 & & 61895 & -34.60623 \\
\hline cylinder & 933 & $\odot .8890$ & & 61895 & -31.74873 \\
\hline cylinder & 934 & $\odot .8890$ & & 61895 & -34.60623 \\
\hline media & 731 & 1 & 831 & & \\
\hline media & 732 & 1 & 832 & -831 & \\
\hline media & 733 & 1 & 833 & -832 & \\
\hline media & 734 & 1 & 834 & -833 & \\
\hline media & 735 & 1 & 835 & -834 & \\
\hline media & 736 & 1 & 836 & -835 & \\
\hline media & 737 & 1 & 837 & -836 & \\
\hline media & 25 & 1 & 930 & -837 & \\
\hline media & 25 & 1 & 931 & -930 & \\
\hline media & 2 & 1 & 932 & -931 & \\
\hline media & 25 & 1 & 933 & -932 & \\
\hline media & 2 & 1 & 934 & -933 & -932 \\
\hline $\begin{array}{l}\text { boundary } \\
\text { end unit }\end{array}$ & $\begin{array}{l}934 \\
31\end{array}$ & & & & \\
\hline Targe & site & PTP-4 (D- & 7) & & \\
\hline unit 841 & & & & & \\
\hline cylinder & 841 & 0.548640 & -18 & 69660 & -25.0 \\
\hline cylinder & 842 & 0.548640 & -11 & 39320 & -25.0 \\
\hline cylinder & 843 & 0.548640 & & 08980 & -25.0 \\
\hline cylinder & 844 & 0.548640 & & 21360 & -25.0 \\
\hline cylinder & 845 & 0.548640 & & 51700 & -25.0 \\
\hline cylinder & 846 & 0.548640 & & 82040 & -25.0 \\
\hline cylinder & 847 & $\odot .548640$ & & 12380 & -25.0 \\
\hline cylinder & 910 & $\odot .548640$ & & 61895 & -25.0 \\
\hline cylinder & 911 & 0.548640 & & 61895 & -34.60623 \\
\hline cylinder & 912 & $\odot .7874$ & & 61895 & -34.60623 \\
\hline cylinder & 913 & $\odot .8890$ & & 61895 & -31.74873 \\
\hline cylinder & 914 & $\odot .8890$ & & 61895 & -34.60623 \\
\hline media & 741 & 1 & 841 & & \\
\hline media & 742 & 1 & 842 & -841 & \\
\hline media & 743 & 1 & 843 & -842 & \\
\hline media & 744 & 1 & 844 & -843 & \\
\hline media & 745 & 1 & 845 & -844 & \\
\hline media & 746 & 1 & 846 & -845 & \\
\hline media & 747 & 1 & 847 & -846 & \\
\hline media & 25 & 1 & 910 & -847 & \\
\hline media & 25 & 1 & 911 & -910 & \\
\hline media & 2 & 1 & 912 & -911 & \\
\hline media & 25 & 1 & 913 & -912 & \\
\hline media & 2 & 1 & 914 & -913 & -912 \\
\hline bounda & 914 & & & & \\
\hline
\end{tabular}

end unit 841 
Target site PTP-5 (G-7)

unit 851

cylinder

cylinder

$851 \quad 0.548640$

8520.548640

cylinder

$853 \odot .548640$

cylinder

$854 \quad 0.548640$

$855 \quad 0.54864 \odot$

8560.548640

cylinder

$857 \quad 0.548640$

$-18.469660-25.0$

$-11.939320-25.0$

$-5.408980-25.0$

$1.121360-25.0$

$7.651700-25.0$

$14.182040-25.0$

$20.712380-25.0$

$\begin{array}{lllll}\text { cylinder } & 900 & 0.548640 & 27.861895 & -25.0\end{array}$

$\begin{array}{lllll}\text { cylinder } & 901 & 0.548640 & 27.861895 & -34.60623\end{array}$

$\begin{array}{lllll}\text { cylinder } & 902 & 0.7874 & 27.861895 & -34.60623\end{array}$

$\begin{array}{lllll}\text { cylinder } & 903 & 0.8890 & 27.861895 & -31.74873\end{array}$

$\begin{array}{lllll}\text { cylinder } & 904 & 0.8890 & 27.861895 & -34.60623\end{array}$

$\begin{array}{llll}\text { media } & 751 & 1 & 851\end{array}$

$\begin{array}{lllll}\text { media } & 752 & 1 & 852 & -851\end{array}$

$\begin{array}{lllll}\text { media } & 753 & 1 & 853 & -852\end{array}$

$\begin{array}{lllll}\text { media } & 754 & 1 & 854 & -853\end{array}$

$\begin{array}{lllll}\text { media } & 755 & 1 & 855 & -854\end{array}$

$\begin{array}{lllll}\text { media } & 756 & 1 & 856 & -855\end{array}$

$\begin{array}{lllll}\text { media } & 757 & 1 & 857 & -856\end{array}$

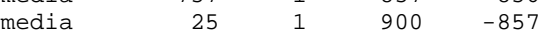

$\begin{array}{lllll}\text { media } & 25 & 1 & 901 & -900\end{array}$

$\begin{array}{lllll}\text { media } & 2 & 1 & 902 & -901\end{array}$

media

$25-1$

$904-903 \quad-902$

end unit 851

Target site PTP-6 (G-4 Al dummy )

unit 861

$\begin{array}{lllll}\text { cylinder } & 861 & 0.548640 & -18.469660 & -25.0\end{array}$

$\begin{array}{lllll}\text { cylinder } & 862 & 0.548640 & -11.939320 & -25.0\end{array}$

$\begin{array}{lllll}\text { cylinder } & 863 & 0.548640 & -5.408980 & -25.0\end{array}$

$\begin{array}{lllll}\text { cylinder } & 864 & 0.548640 & 1.121360 & -25.0\end{array}$

$\begin{array}{lllll}\text { cylinder } & 865 & 0.548640 & 7.651700 & -25.0\end{array}$

$\begin{array}{lllll}\text { cylinder } & 866 & 0.548640 & 14.182040 & -25.0\end{array}$

$\begin{array}{lllll}\text { cylinder } & 867 & 0.548640 & 20.712380 & -25.0\end{array}$

$\begin{array}{lllll}\text { cylinder } \quad 950 & 0.548640 & 27.861895 & -25.0\end{array}$

$\begin{array}{lllll}\text { cylinder } & 951 & 0.548640 & 27.861895 & -34.60623\end{array}$

$\begin{array}{lllll}\text { cylinder } & 952 & 0.7874 & 27.861895 & -34.60623\end{array}$

$\begin{array}{lllll}\text { cylinder } & 953 & 0.8890 & 27.861895 & -31.74873\end{array}$

$\begin{array}{lllll}\text { cylinder } & 954 & 0.8890 & 27.861895 & -34.60623\end{array}$

$\begin{array}{lrrrrr}\text { media } & 761 & 1 & 861 & & \\ \text { media } & 762 & 1 & 862 & -861 & \\ \text { media } & 763 & 1 & 863 & -862 & \\ \text { media } & 764 & 1 & 864 & -863 & \\ \text { media } & 765 & 1 & 865 & -864 & \\ \text { media } & 766 & 1 & 866 & -865 & \\ \text { media } & 767 & 1 & 867 & -866 & \\ \text { media } & 25 & 1 & 950 & -867 & \\ \text { media } & 25 & 1 & 951 & -950 & \\ \text { media } & 2 & 1 & 952 & -951 & \\ \text { media } & 25 & 1 & 953 & -952 & -952 \\ \text { media } & 2 & 1 & 954 & -953 & -952 \\ \text { boundary } & 954 & & & & \end{array}$

end unit 861

Target basket and water above and below target area

unit 800

$\begin{array}{lllll}\text { cylinder } & 800 & 5.71500 & 49.2125 & -39.6875\end{array}$

hole 410 origin $x=-4.388400 \quad y=-0.844550$

hole 420 origin $x=-4.388400 \quad y=\odot .844550$

hole 430 origin $x=-2.925600 \quad y=-3.378200$

hole 440 origin $x=-2.925600 \quad y=-1.689100$

hole 750 origin $x=-2.925600 \quad y=0.000000$

hole 460 origin $x=-2.925600 \quad y=1.689100$

hole 470 origin $x=-2.925600 \quad y=3.378200$

hole 480 origin $x=-1.462800 \quad y=-4.222750$

hole 490 origin $x=-1.462800 \quad y=-2.533650$

hole 510 origin $x=-1.46280 \odot \quad y=-0.844550$

hole 520 origin $x=-1.462800 \quad y=0.844550$

hole 530 origin $x=-1.462800 \quad y=2.533650$ 


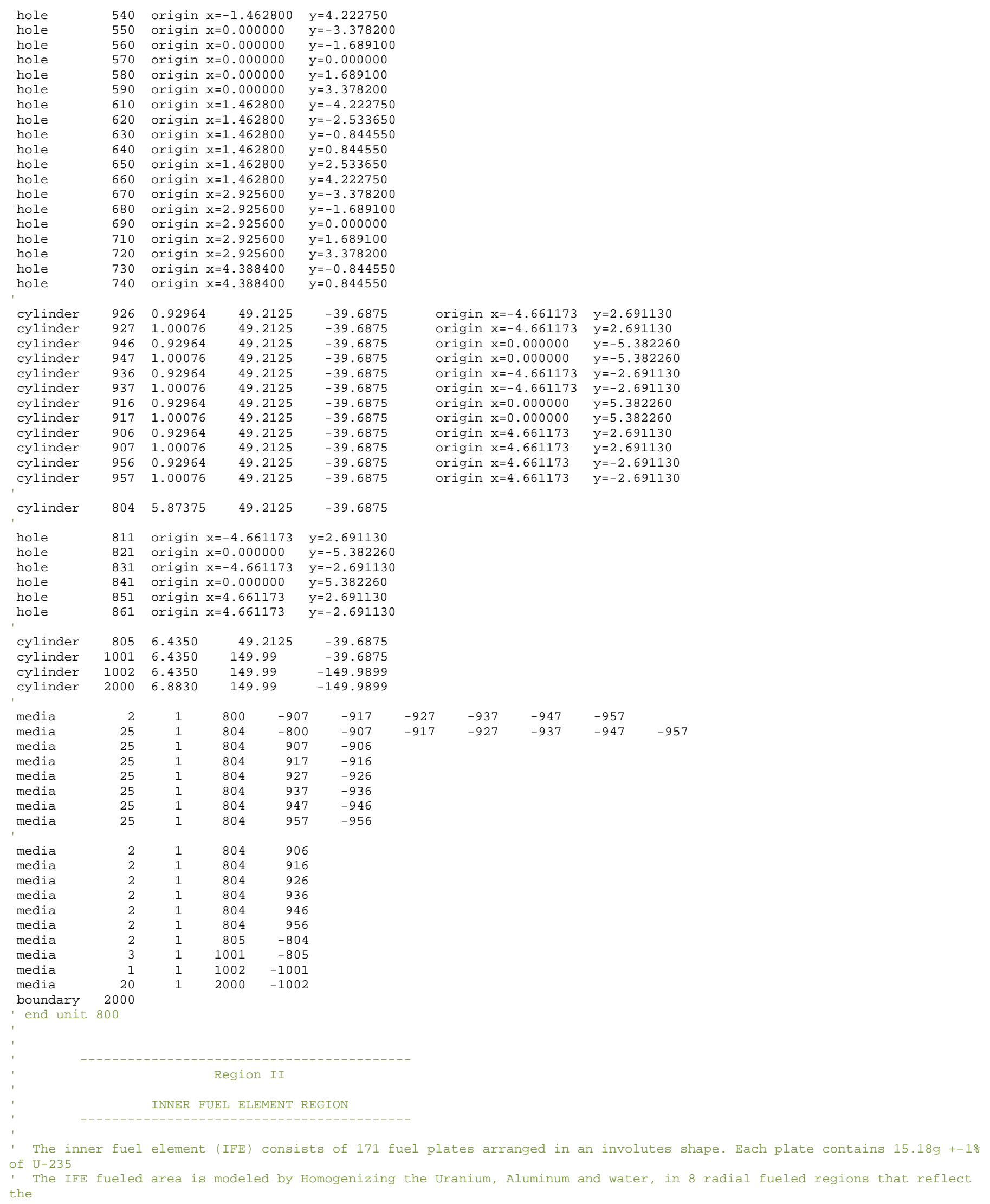




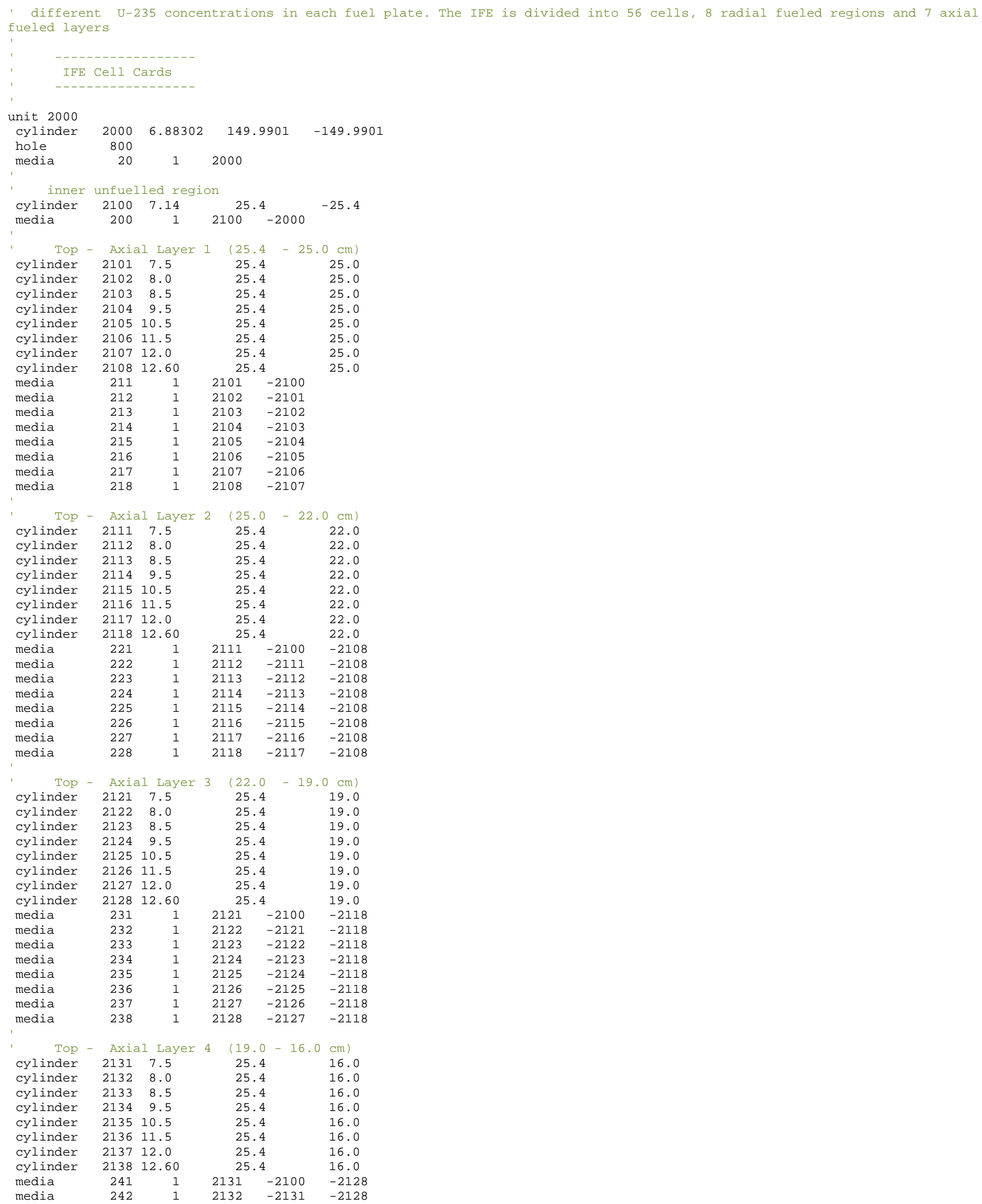




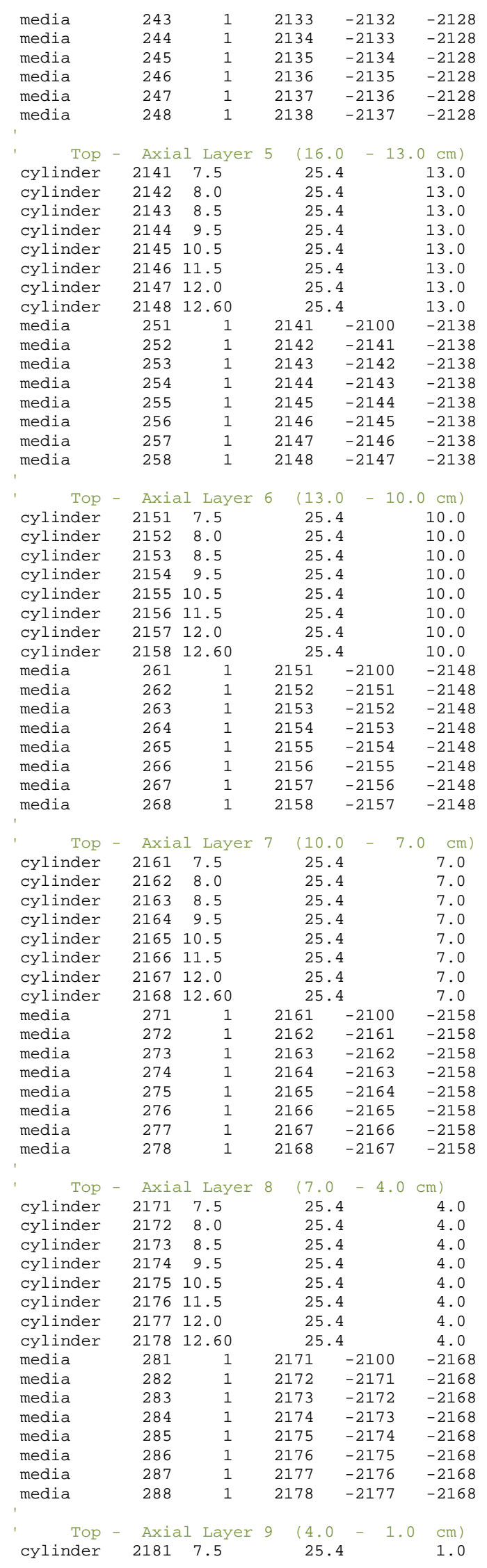




\begin{tabular}{|c|c|c|c|c|c|}
\hline cylinder & 2182 & 8.0 & \multicolumn{2}{|c|}{25.4} & 1.0 \\
\hline cylinder & 2183 & 8.5 & \multicolumn{2}{|c|}{25.4} & 1.0 \\
\hline cylinder & 2184 & 9.5 & \multicolumn{2}{|c|}{25.4} & 1.0 \\
\hline cylinder & 2185 & 10.5 & \multicolumn{2}{|c|}{25.4} & 1.0 \\
\hline cylinder & 2186 & 11.5 & \multicolumn{2}{|c|}{25.4} & 1.0 \\
\hline cylinder & 2187 & 12.0 & \multicolumn{2}{|c|}{25.4} & 1.0 \\
\hline cylinder & 2188 & 12.60 & \multicolumn{2}{|c|}{25.4} & 1.0 \\
\hline media & 291 & 1 & 2181 & -2100 & -2178 \\
\hline media & 292 & 1 & 2182 & -2181 & -2178 \\
\hline media & 293 & 1 & 2183 & -2182 & -2178 \\
\hline media & 294 & 1 & 2184 & -2183 & -2178 \\
\hline media & 295 & 1 & 2185 & -2184 & -2178 \\
\hline media & 296 & 1 & 2186 & -2185 & -2178 \\
\hline media & 297 & 1 & 2187 & -2186 & -2178 \\
\hline media & 298 & 1 & 2188 & -2187 & -2178 \\
\hline Top & Axia & al Layer & 10 & .0 & $-1.0 \mathrm{~cm})$ \\
\hline cylinder & 2191 & 7.5 & & & -1.0 \\
\hline cylinder & 2192 & 8.0 & & & -1.0 \\
\hline cylinder & 2193 & 8.5 & & & -1.0 \\
\hline cylinder & 2194 & 9.5 & & & -1.0 \\
\hline cylinder & 2195 & 10.5 & & & -1.0 \\
\hline cylinder & 2196 & 11.5 & & & -1.0 \\
\hline cylinder & 2197 & 12.0 & & & -1.0 \\
\hline cylinder & 2198 & 12.60 & & & -1.0 \\
\hline media & 201 & 1 & 2191 & -2100 & -2188 \\
\hline media & 202 & 1 & 2192 & -2191 & -2188 \\
\hline media & 203 & 1 & 2193 & -2192 & -2188 \\
\hline media & 204 & 1 & 2194 & -2193 & -2188 \\
\hline media & 205 & 1 & 2195 & -2194 & -2188 \\
\hline media & 206 & 1 & 2196 & -2195 & -2188 \\
\hline media & 207 & 1 & 2197 & -2196 & -2188 \\
\hline media & 208 & 1 & 2198 & -2197 & -2188 \\
\hline Top & Axia & al Layer & 11 & .0 & $-4 . \odot \mathrm{cm})$ \\
\hline cylinder & 2201 & 7.5 & & & -4.0 \\
\hline cylinder & 2202 & 8.0 & & & -4.0 \\
\hline cylinder & 2203 & 8.5 & & & -4.0 \\
\hline cylinder & 2204 & 9.5 & & & -4.0 \\
\hline cylinder & 2205 & 10.5 & & & -4.0 \\
\hline cylinder & 2206 & 11.5 & & & -4.0 \\
\hline cylinder & 2207 & 12.0 & & & -4.0 \\
\hline cylinder & 2208 & 12.60 & & & -4.0 \\
\hline media & 291 & 1 & 2201 & -2100 & -2198 \\
\hline media & 292 & 1 & 2202 & -2201 & -2198 \\
\hline media & 293 & 1 & 2203 & -2202 & -2198 \\
\hline media & 294 & 1 & 2204 & -2203 & -2198 \\
\hline media & 295 & 1 & 2205 & -2204 & -2198 \\
\hline media & 296 & 1 & 2206 & -2205 & -2198 \\
\hline media & 297 & 1 & 2207 & -2206 & -2198 \\
\hline media & 298 & 1 & 2208 & -2207 & -2198 \\
\hline Top & Axia & al Layer & 12 & .0 & $7.0 \mathrm{~cm})$ \\
\hline cylinder & 2211 & 7.5 & & & -7.0 \\
\hline cylinder & 2212 & 8.0 & & & -7.0 \\
\hline cylinder & 2213 & 8.5 & & & -7.0 \\
\hline cylinder & 2214 & 9.5 & & & -7.0 \\
\hline cylinder & 2215 & 10.5 & & & -7.0 \\
\hline cylinder & 2216 & 11.5 & & & -7.0 \\
\hline cylinder & 2217 & 12.0 & & & -7.0 \\
\hline cylinder & 2218 & 12.60 & & & -7.0 \\
\hline media & 281 & 1 & 2211 & -2100 & -2208 \\
\hline media & 282 & 1 & 2212 & -2211 & -2208 \\
\hline media & 283 & 1 & 2213 & -2212 & -2208 \\
\hline media & 284 & 1 & 2214 & -2213 & -2208 \\
\hline media & 285 & 1 & 2215 & -2214 & -2208 \\
\hline media & 286 & 1 & 2216 & -2215 & -2208 \\
\hline media & 287 & 1 & 2217 & -2216 & -2208 \\
\hline media & 288 & 1 & 2218 & -2217 & -2208 \\
\hline Top & Axia & al Layer & 13 & .0 & $-10.0 \mathrm{~cm})$ \\
\hline cylinder & 2221 & 7.5 & & & -10.0 \\
\hline cylinder & 2222 & 8.0 & & & -10.0 \\
\hline cylinder & 2223 & 8.5 & & & -10.0 \\
\hline cylinder & 2224 & 9.5 & & & -10.0 \\
\hline cylinder & 2225 & 10.5 & & & -10.0 \\
\hline cylinder & 2226 & 11.5 & & & -10.0 \\
\hline cylinder & 2227 & 12.0 & & & -10.0 \\
\hline cylinder & 2228 & 12.60 & & & -10.0 \\
\hline media & 271 & 1 & 2221 & -2100 & -2218 \\
\hline media & 272 & 1 & 2222 & -2221 & -2218 \\
\hline
\end{tabular}




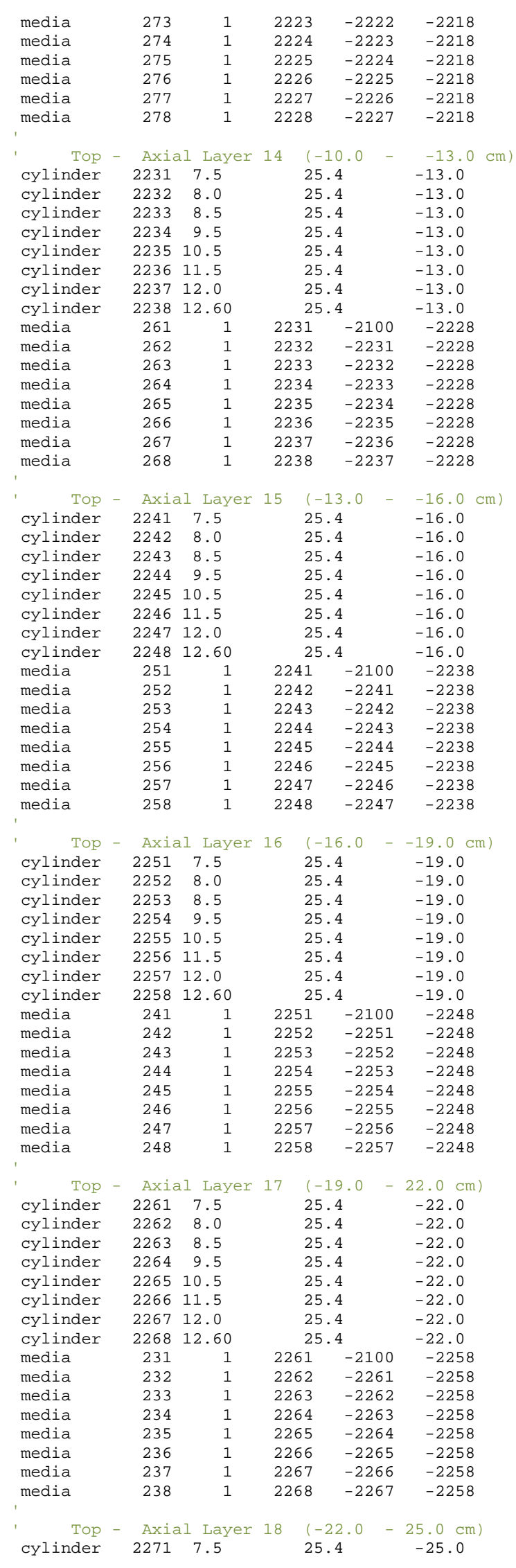




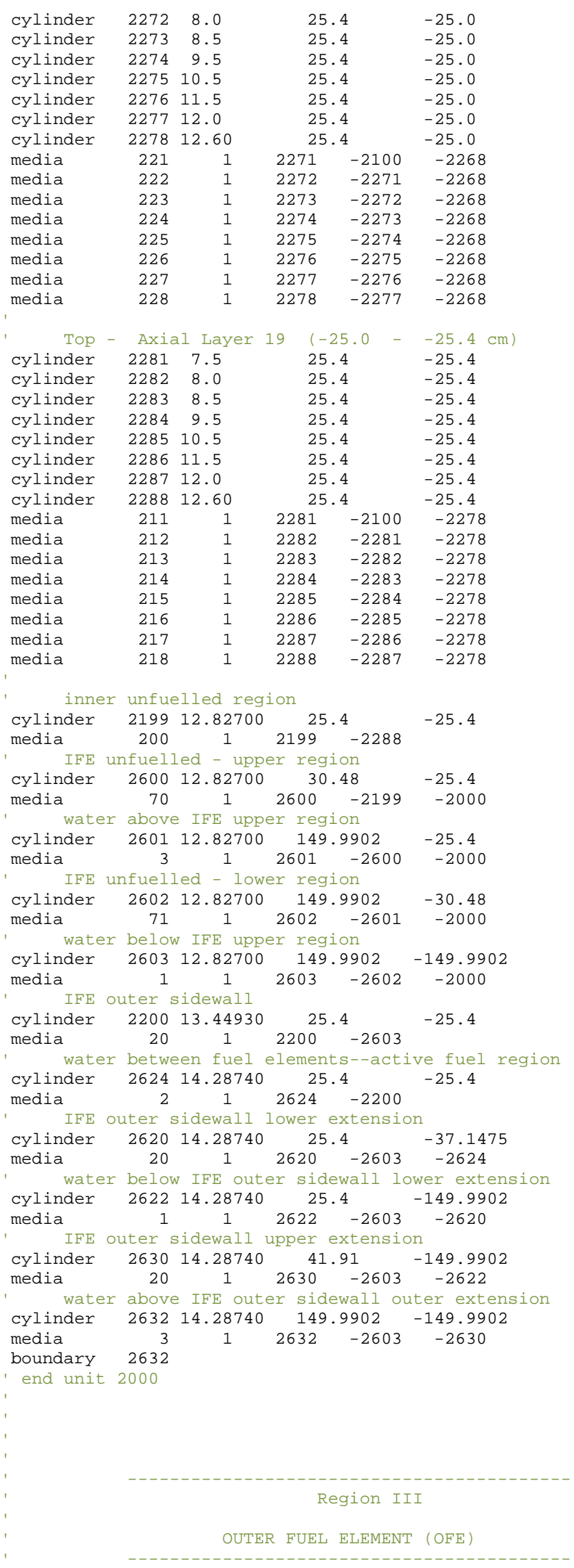

The outer fuel element is region-3 


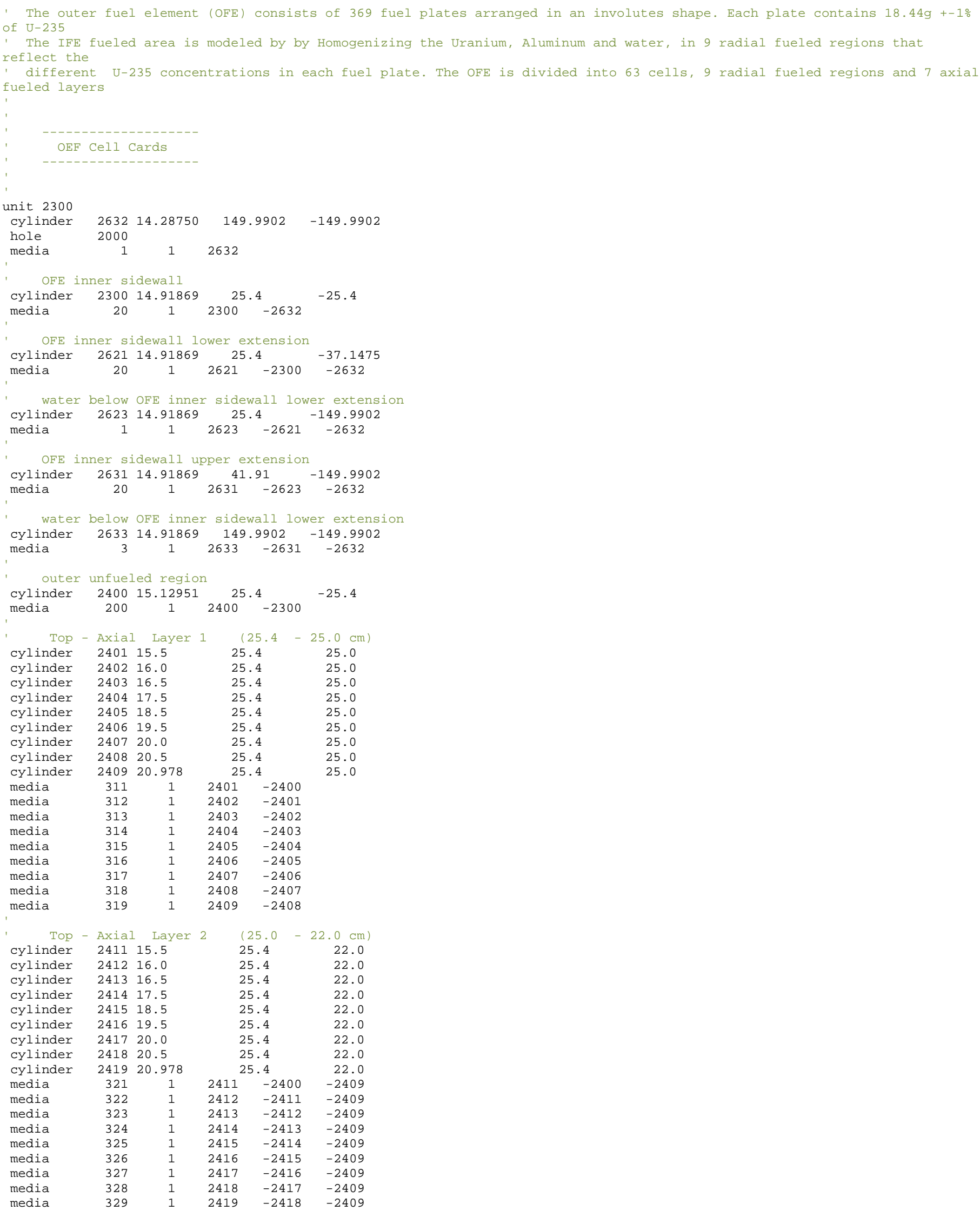




\begin{tabular}{|c|c|c|c|c|c|}
\hline Top & - Axial & Layer & \multirow{2}{*}{\multicolumn{2}{|c|}{$\begin{array}{l}(22.0 \\
25.4\end{array}$}} & $-19.0 \mathrm{~cm})$ \\
\hline cylinder & 2421 & 15.5 & \multirow{2}{*}{\multicolumn{2}{|c|}{$\begin{array}{l}25.4 \\
25.4\end{array}$}} & 19.0 \\
\hline cylinder & 2422 & 16.0 & & & 19.0 \\
\hline cylinder & 2423 & 16.5 & \multicolumn{2}{|c|}{25.4} & 19.0 \\
\hline cylinder & 2424 & 17.5 & \multicolumn{2}{|c|}{25.4} & 19.0 \\
\hline cylinder & 2425 & 18.5 & \multicolumn{2}{|c|}{25.4} & 19.0 \\
\hline cylinder & 2426 & 19.5 & \multicolumn{2}{|c|}{25.4} & 19.0 \\
\hline cylinder & 2427 & 20.0 & \multicolumn{2}{|c|}{25.4} & 19.0 \\
\hline cylinder & 2428 & 20.5 & \multicolumn{2}{|c|}{25.4} & 19.0 \\
\hline cylinder & 2429 & 20.978 & & & 19.0 \\
\hline media & 331 & 1 & 2421 & -2400 & -2419 \\
\hline media & 332 & 1 & 2422 & -2421 & -2419 \\
\hline media & 333 & 1 & 2423 & -2422 & -2419 \\
\hline media & 334 & 1 & 2424 & -2423 & -2419 \\
\hline media & 335 & 1 & 2425 & -2424 & -2419 \\
\hline media & 336 & 1 & 2426 & -2425 & -2419 \\
\hline media & 337 & 1 & 2427 & -2426 & -2419 \\
\hline media & 338 & 1 & 2428 & -2427 & -2419 \\
\hline media & 339 & 1 & 2429 & -2428 & -2419 \\
\hline Top & - Axial & Layer & 4 & 9.0 & $-16 . \odot \mathrm{cm})$ \\
\hline cylinder & 2431 & 15.5 & & & 16.0 \\
\hline cylinder & 2432 & 16.0 & & & 16.0 \\
\hline cylinder & 2433 & 16.5 & & & 16.0 \\
\hline cylinder & 2434 & 17.5 & & & 16.0 \\
\hline cylinder & 2435 & 18.5 & & & 16.0 \\
\hline cylinder & 2436 & 19.5 & & & 16.0 \\
\hline cylinder & 2437 & 20.0 & & & 16.0 \\
\hline cylinder & 2438 & 20.5 & & & 16.0 \\
\hline cylinder & 2439 & 20.978 & & & 16.0 \\
\hline media & 341 & 1 & 2431 & -2400 & -2429 \\
\hline media & 342 & 1 & 2432 & -2431 & -2429 \\
\hline media & 343 & 1 & 2433 & -2432 & -2429 \\
\hline media & 344 & 1 & 2434 & -2433 & -2429 \\
\hline media & 345 & 1 & 2435 & -2434 & -2429 \\
\hline media & 346 & 1 & 2436 & -2435 & -2429 \\
\hline media & 347 & 1 & 2437 & -2436 & -2429 \\
\hline media & 348 & 1 & 2438 & -2437 & -2429 \\
\hline media & 349 & 1 & 2439 & -2438 & -2429 \\
\hline Top & - Axial & Layer & 5 & 6.0 & $-13 . \odot \mathrm{cm})$ \\
\hline cylinder & 2441 & 15.5 & & & 13.0 \\
\hline cylinder & 2442 & 16.0 & & & 13.0 \\
\hline cylinder & 2443 & 16.5 & & & 13.0 \\
\hline cylinder & 2444 & 17.5 & & & 13.0 \\
\hline cylinder & 2445 & 18.5 & & & 13.0 \\
\hline cylinder & 2446 & 19.5 & & & 13.0 \\
\hline cylinder & 2447 & 20.0 & & & 13.0 \\
\hline cylinder & 2448 & 20.5 & & & 13.0 \\
\hline cylinder & 2449 & 20.978 & & .4 & 13.0 \\
\hline media & 351 & 1 & 2441 & -2400 & -2439 \\
\hline media & 352 & 1 & 2442 & -2441 & -2439 \\
\hline media & 353 & 1 & 2443 & -2442 & -2439 \\
\hline media & 354 & 1 & 2444 & -2443 & -2439 \\
\hline media & 355 & 1 & 2445 & -2444 & -2439 \\
\hline media & 356 & 1 & 2446 & -2445 & -2439 \\
\hline media & 357 & 1 & 2447 & -2446 & -2439 \\
\hline media & 358 & 1 & 2448 & -2447 & -2439 \\
\hline media & 359 & 1 & 2449 & -2448 & -2439 \\
\hline Top & - Axial & L Layer & 6 & 3.0 & $-10 . \odot \mathrm{cm})$ \\
\hline cylinder & 2451 & 15.5 & & & 10.0 \\
\hline cylinder & 2452 & 16.0 & & & 10.0 \\
\hline cylinder & 2453 & 16.5 & & & 10.0 \\
\hline cylinder & 2454 & 17.5 & & & 10.0 \\
\hline cylinder & 2455 & 18.5 & & & 10.0 \\
\hline cylinder & 2456 & 19.5 & & & 10.0 \\
\hline cylinder & 2457 & 20.0 & & & 10.0 \\
\hline cylinder & 2458 & 20.5 & & & 10.0 \\
\hline cylinder & 2459 & 20.978 & & .4 & 10.0 \\
\hline media & 361 & 1 & 2451 & -2400 & -2449 \\
\hline media & 362 & 1 & 2452 & -2451 & -2449 \\
\hline media & 363 & 1 & 2453 & -2452 & -2449 \\
\hline media & 364 & 1 & 2454 & -2453 & -2449 \\
\hline media & 365 & 1 & 2455 & -2454 & -2449 \\
\hline media & 366 & 1 & 2456 & -2455 & -2449 \\
\hline media & 367 & 1 & 2457 & -2456 & -2449 \\
\hline media & 368 & 1 & 2458 & -2457 & -2449 \\
\hline media & 369 & 1 & 2459 & -2458 & -2449 \\
\hline
\end{tabular}




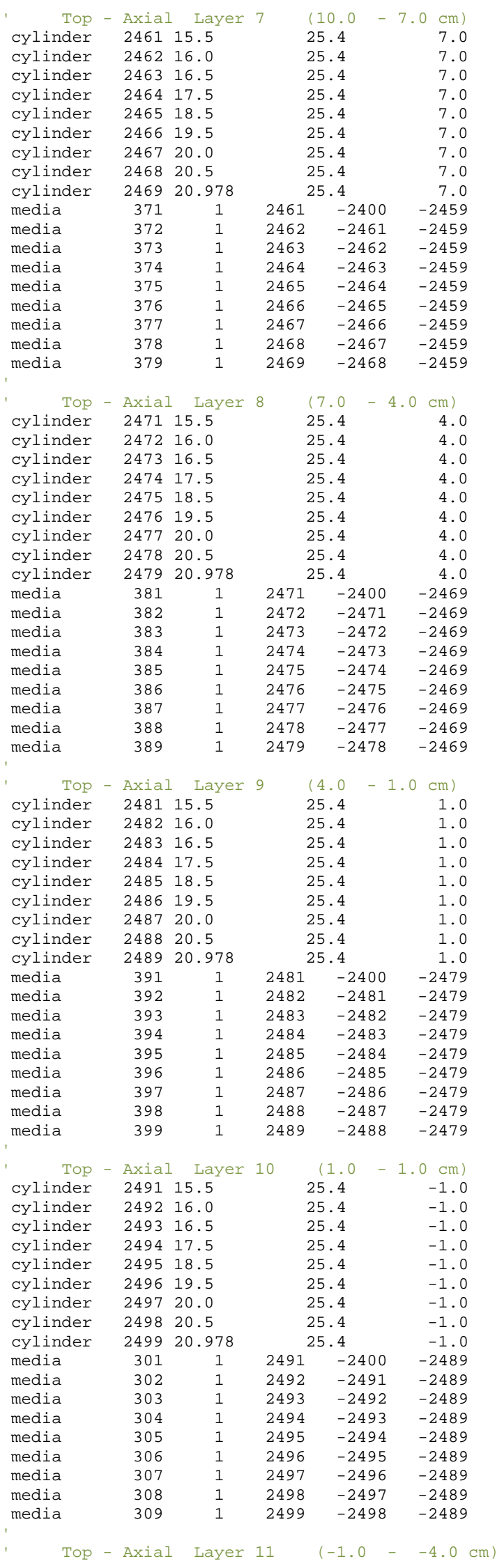




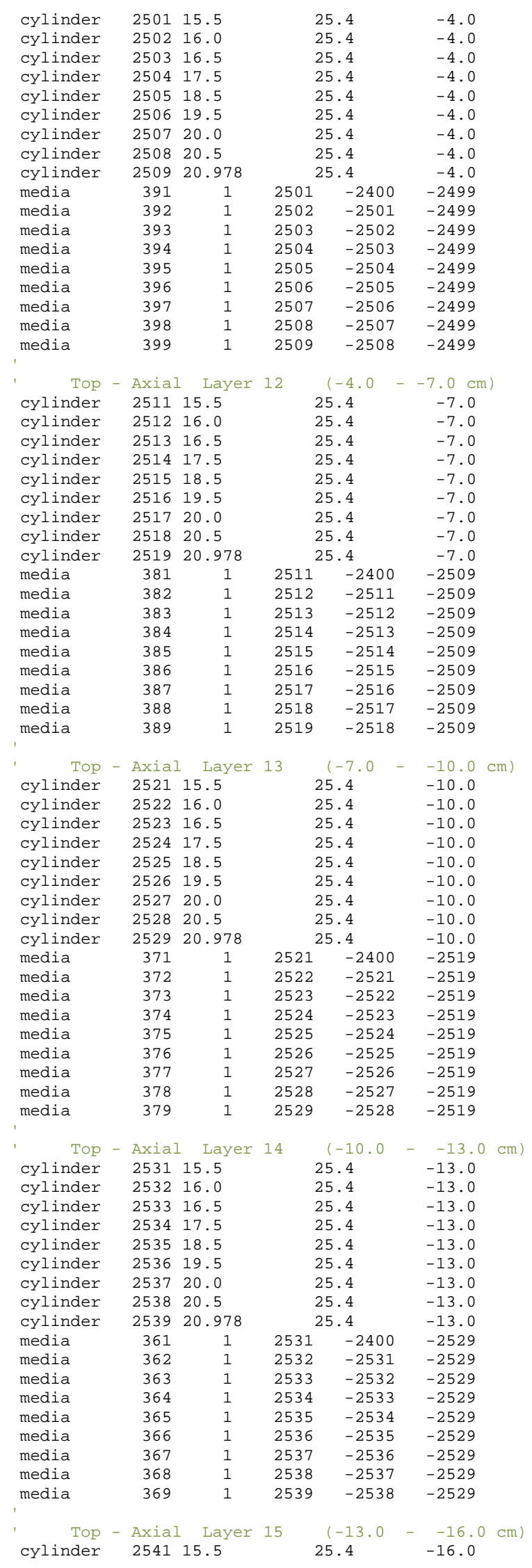




\begin{tabular}{|c|c|c|c|c|c|}
\hline cylinder & 2542 & 16.0 & \multicolumn{2}{|c|}{25.4} & -16.0 \\
\hline cylinder & 2543 & & \multicolumn{2}{|r|}{25.4} & -16.0 \\
\hline cylinder & 2544 & 17.5 & \multicolumn{2}{|r|}{25.4} & -16.0 \\
\hline cylinder & 2545 & 18.5 & & 25.4 & -16.0 \\
\hline cylinder & 2546 & 19.5 & & 25.4 & -16.0 \\
\hline cylinder & 2547 & 20.0 & & 25.4 & -16.0 \\
\hline cylinder & 2548 & 20.5 & & 25.4 & -16.0 \\
\hline cylinder & 2549 & 20.978 & & 25.4 & -16.0 \\
\hline media & 351 & 1 & 2541 & -2400 & -2539 \\
\hline media & 352 & 1 & 2542 & -2541 & -2539 \\
\hline media & 353 & 1 & 2543 & -2542 & -2539 \\
\hline media & 354 & 1 & 2544 & -2543 & -2539 \\
\hline media & 355 & 1 & 2545 & -2544 & -2539 \\
\hline media & 356 & 1 & 2546 & -2545 & -2539 \\
\hline media & 357 & 1 & 2547 & -2546 & -2539 \\
\hline media & 358 & 1 & 2548 & -2547 & -2539 \\
\hline media & 359 & 1 & 2549 & -2548 & -2539 \\
\hline Top & - Axial & Layer & 16 & $(-16.0$ & - $19.0 \mathrm{~cm})$ \\
\hline cylinder & 2551 & 15.5 & & 25.4 & -19.0 \\
\hline cylinder & 2552 & 16.0 & & 25.4 & -19.0 \\
\hline cylinder & 2553 & 16.5 & & 25.4 & -19.0 \\
\hline cylinder & 2554 & 17.5 & & 25.4 & -19.0 \\
\hline cylinder & 2555 & 18.5 & & 25.4 & -19.0 \\
\hline cylinder & 2556 & 19.5 & & 25.4 & -19.0 \\
\hline cylinder & 2557 & 20.0 & & 25.4 & -19.0 \\
\hline cylinder & 2558 & 20.5 & & 25.4 & -19.0 \\
\hline cylinder & 2559 & 20.978 & & 25.4 & -19.0 \\
\hline media & 341 & 1 & 2551 & -2400 & -2549 \\
\hline media & 342 & 1 & 2552 & -2551 & -2549 \\
\hline media & 343 & 1 & 2553 & -2552 & -2549 \\
\hline media & 344 & 1 & 2554 & -2553 & -2549 \\
\hline media & 345 & 1 & 2555 & -2554 & -2549 \\
\hline media & 346 & 1 & 2556 & -2555 & -2549 \\
\hline media & 347 & 1 & 2557 & -2556 & -2549 \\
\hline media & 348 & 1 & 2558 & -2557 & -2549 \\
\hline media & 349 & 1 & 2559 & -2558 & -2549 \\
\hline Top & - Axial & Layer & 17 & $(-19.0$ & $-22 . \odot \mathrm{cm})$ \\
\hline cylinder & 2561 & 15.5 & & 25.4 & -22.0 \\
\hline cylinder & 2562 & 16.0 & & 25.4 & -22.0 \\
\hline cylinder & 2563 & 16.5 & & 25.4 & -22.0 \\
\hline cylinder & 2564 & 17.5 & & 25.4 & -22.0 \\
\hline cylinder & 2565 & 18.5 & & 25.4 & -22.0 \\
\hline cylinder & 2566 & 19.5 & & 25.4 & -22.0 \\
\hline cylinder & 2567 & 20.0 & & 25.4 & -22.0 \\
\hline cylinder & 2568 & 20.5 & & 25.4 & -22.0 \\
\hline cylinder & 2569 & 20.978 & & 25.4 & -22.0 \\
\hline media & 331 & 1 & 2561 & -2400 & -2559 \\
\hline media & 332 & 1 & 2562 & -2561 & -2559 \\
\hline media & 333 & 1 & 2563 & -2562 & -2559 \\
\hline media & 334 & 1 & 2564 & -2563 & -2559 \\
\hline media & 335 & 1 & 2565 & -2564 & -2559 \\
\hline media & 336 & 1 & 2566 & -2565 & -2559 \\
\hline media & 337 & 1 & 2567 & -2566 & -2559 \\
\hline media & 338 & 1 & 2568 & -2567 & -2559 \\
\hline media & 339 & 1 & 2569 & -2568 & -2559 \\
\hline Top & - Axial & 1 Layer & 18 & $(-22.0$ & $-25.0 \mathrm{~cm})$ \\
\hline cylinder & 2571 & 15.5 & & 25.4 & -25.0 \\
\hline cylinder & 2572 & 16.0 & & 25.4 & -25.0 \\
\hline cylinder & 2573 & 16.5 & & 25.4 & -25.0 \\
\hline cylinder & 2574 & 17.5 & & 25.4 & -25.0 \\
\hline cylinder & 2575 & 18.5 & & 25.4 & -25.0 \\
\hline cylinder & 2576 & 19.5 & & 25.4 & -25.0 \\
\hline cylinder & 2577 & 20.0 & & 25.4 & -25.0 \\
\hline cylinder & 2578 & 20.5 & & 25.4 & -25.0 \\
\hline cylinder & 2579 & 20.978 & & 25.4 & -25.0 \\
\hline media & 321 & 1 & 2571 & -2400 & -2569 \\
\hline media & 322 & 1 & 2572 & -2571 & -2569 \\
\hline media & 323 & 1 & 2573 & -2572 & -2569 \\
\hline media & 324 & 1 & 2574 & -2573 & -2569 \\
\hline media & 325 & 1 & 2575 & -2574 & -2569 \\
\hline media & 326 & 1 & 2576 & -2575 & -2569 \\
\hline media & 327 & 1 & 2577 & -2576 & -2569 \\
\hline media & 328 & 1 & 2578 & -2577 & -2569 \\
\hline media & 329 & 1 & 2579 & -2578 & -2569 \\
\hline & - Axial & 1 Layer & 19 & $(-25,0$ & $-\quad-25.4 \mathrm{~cm})$ \\
\hline cylinder & 2581 & 15.5 & & 25.4 & -25.4 \\
\hline cylinder & 2582 & 16.0 & & 25.4 & -25.4 \\
\hline
\end{tabular}




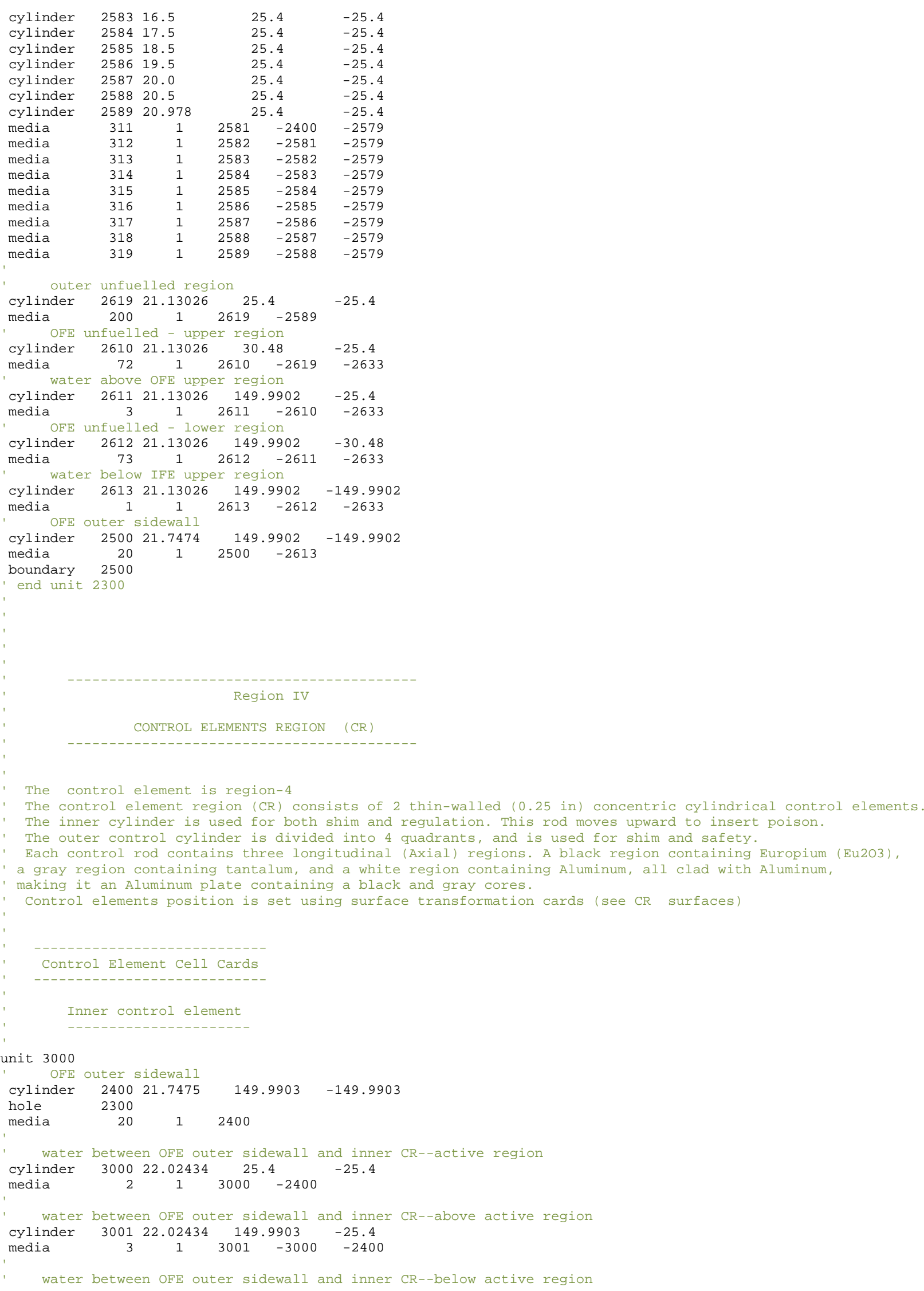




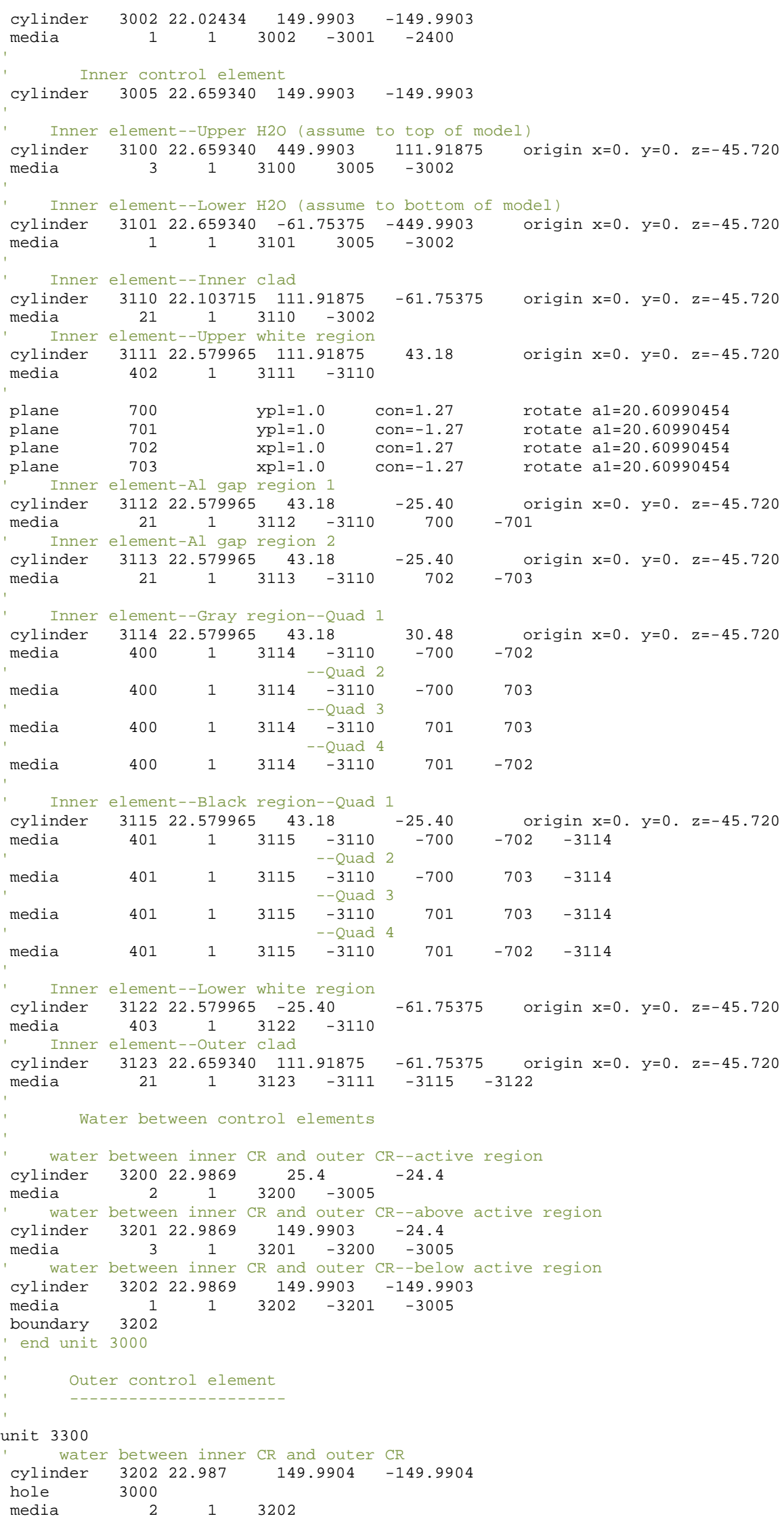




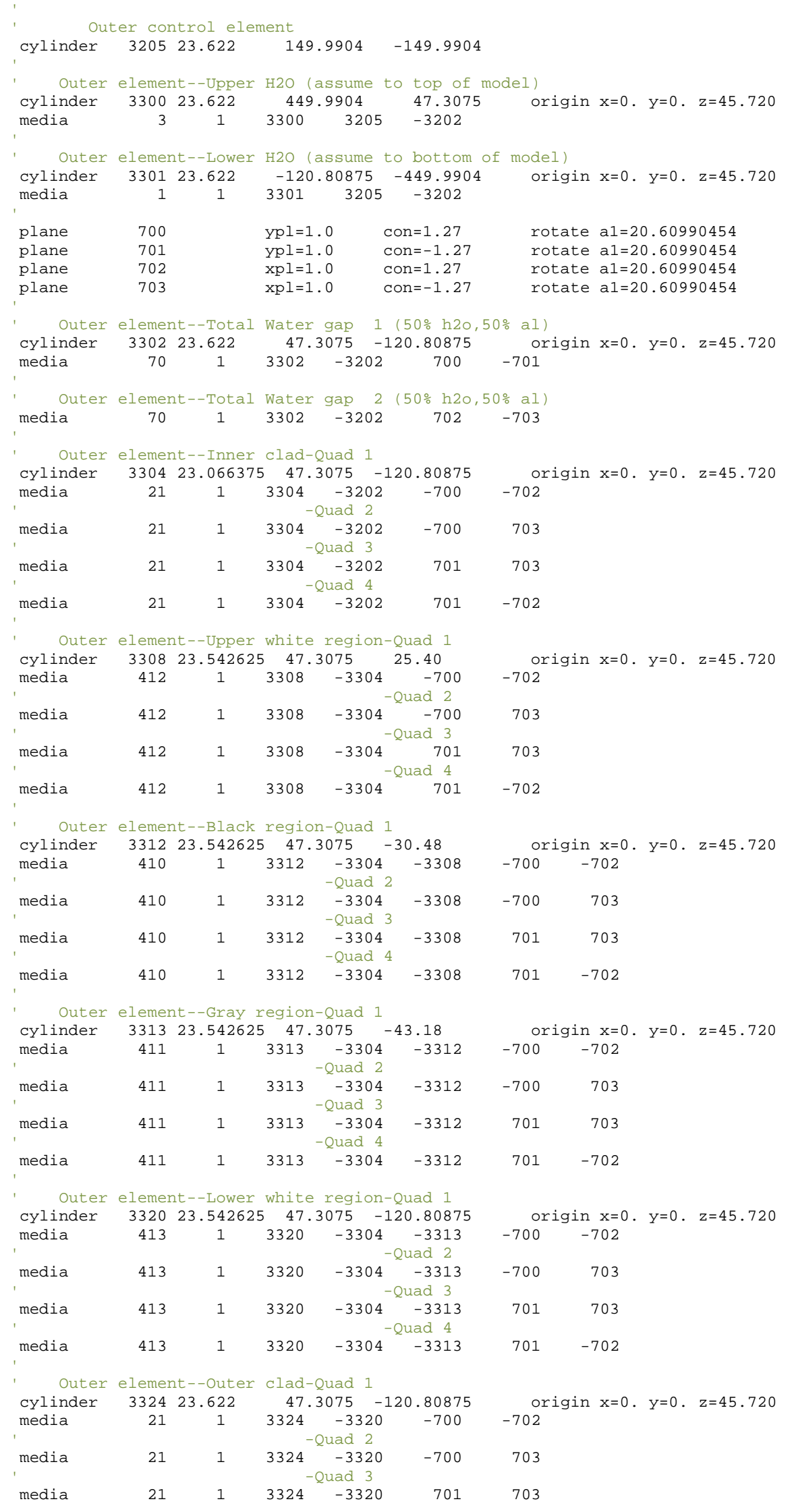




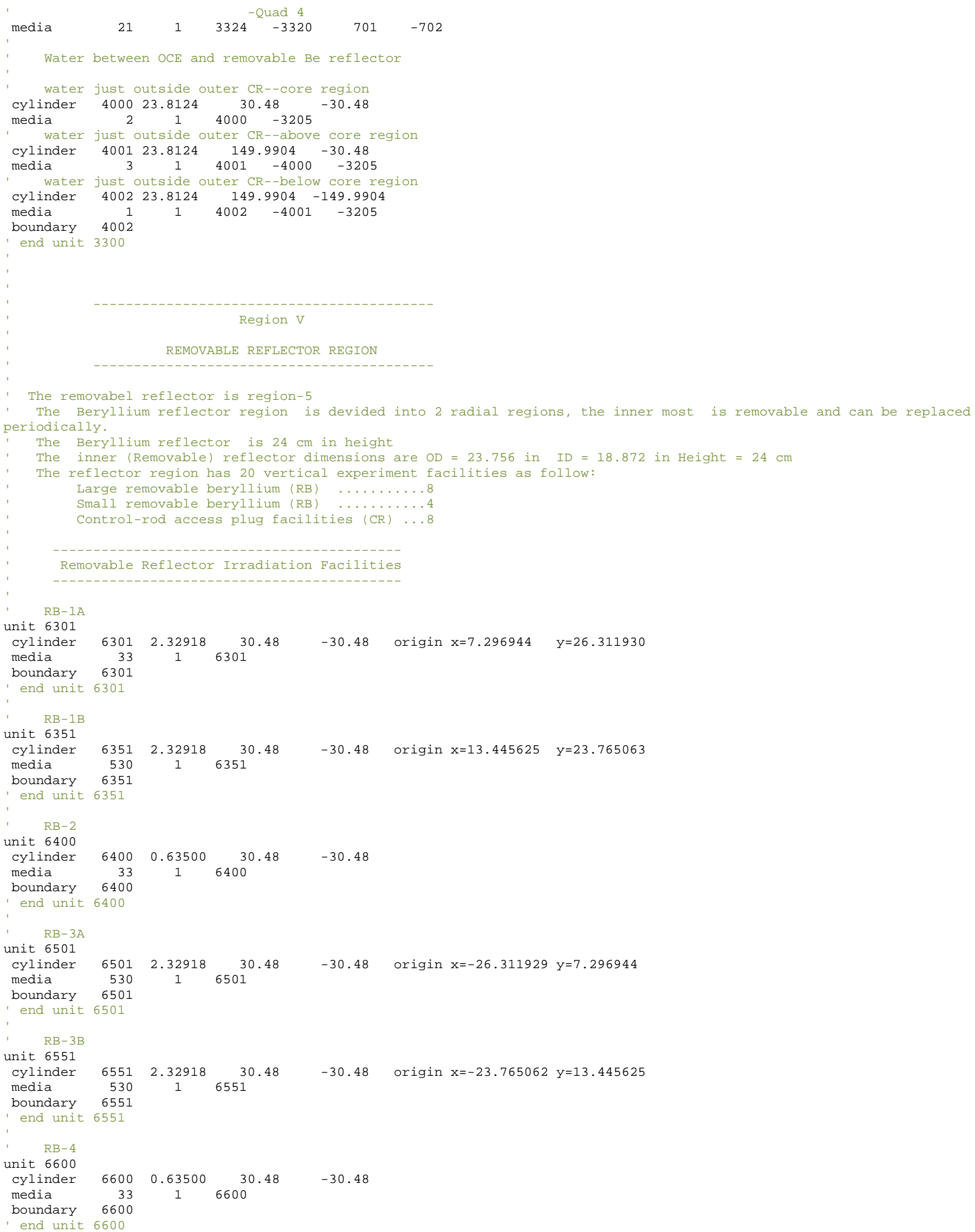




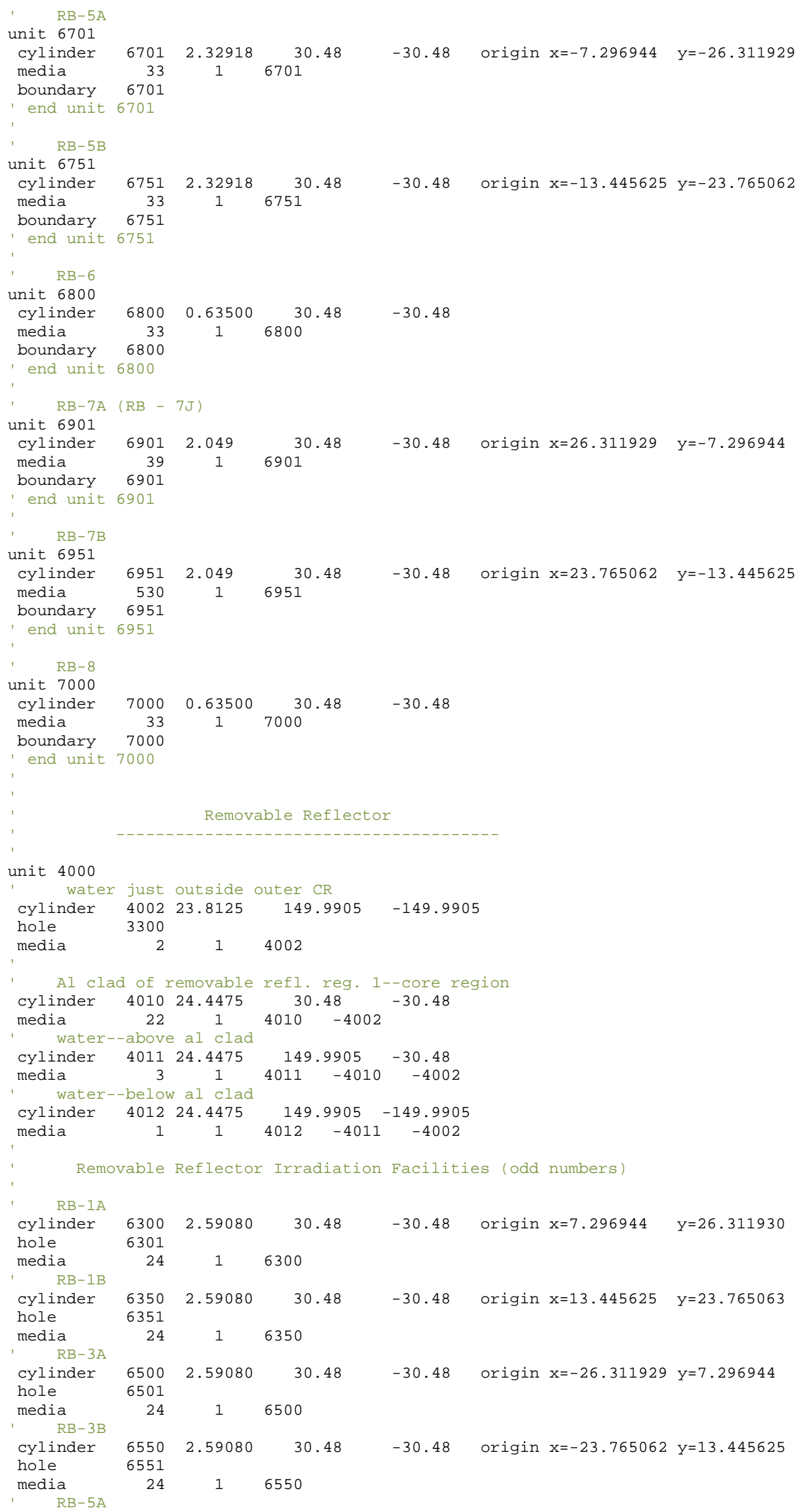

$$
R B-5 A
$$




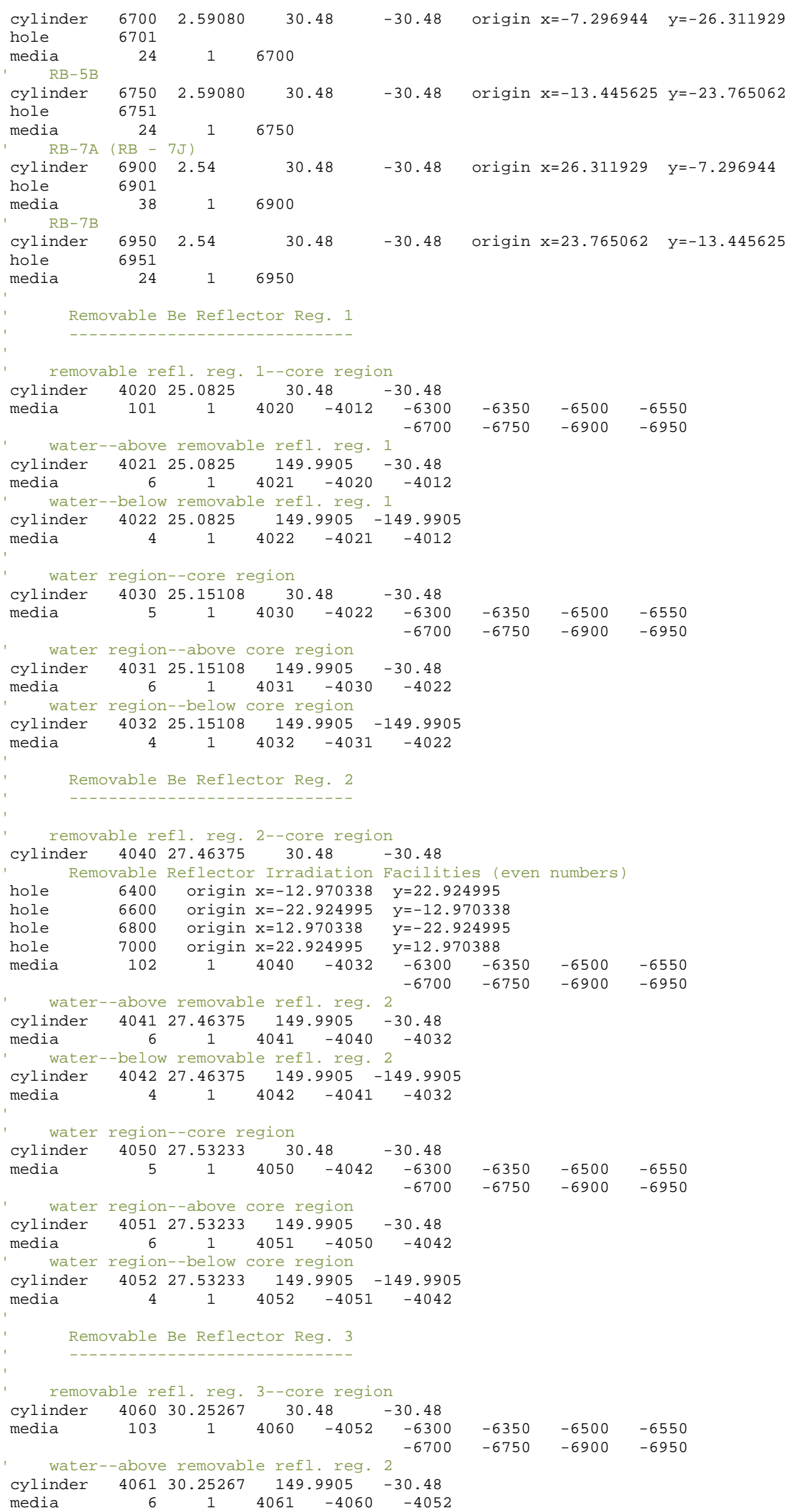




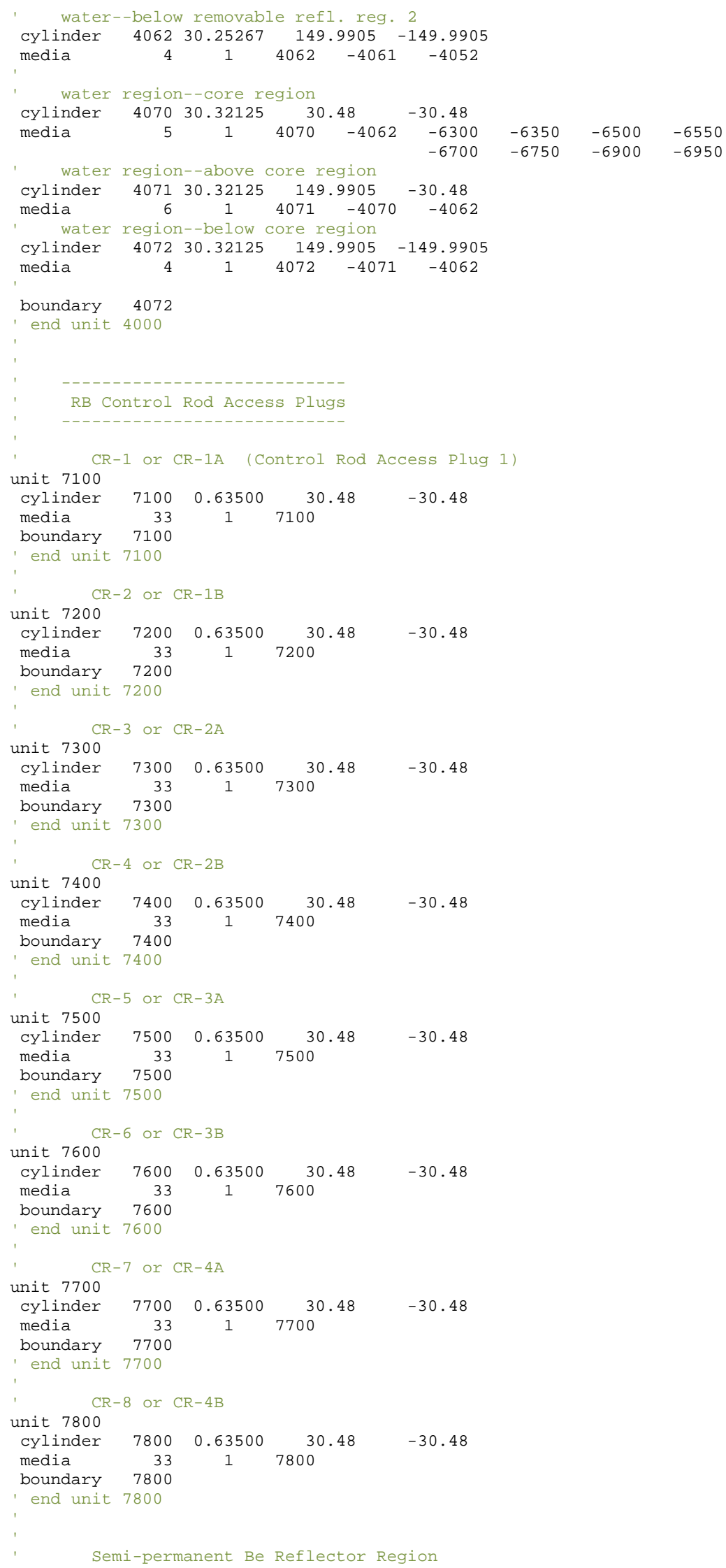




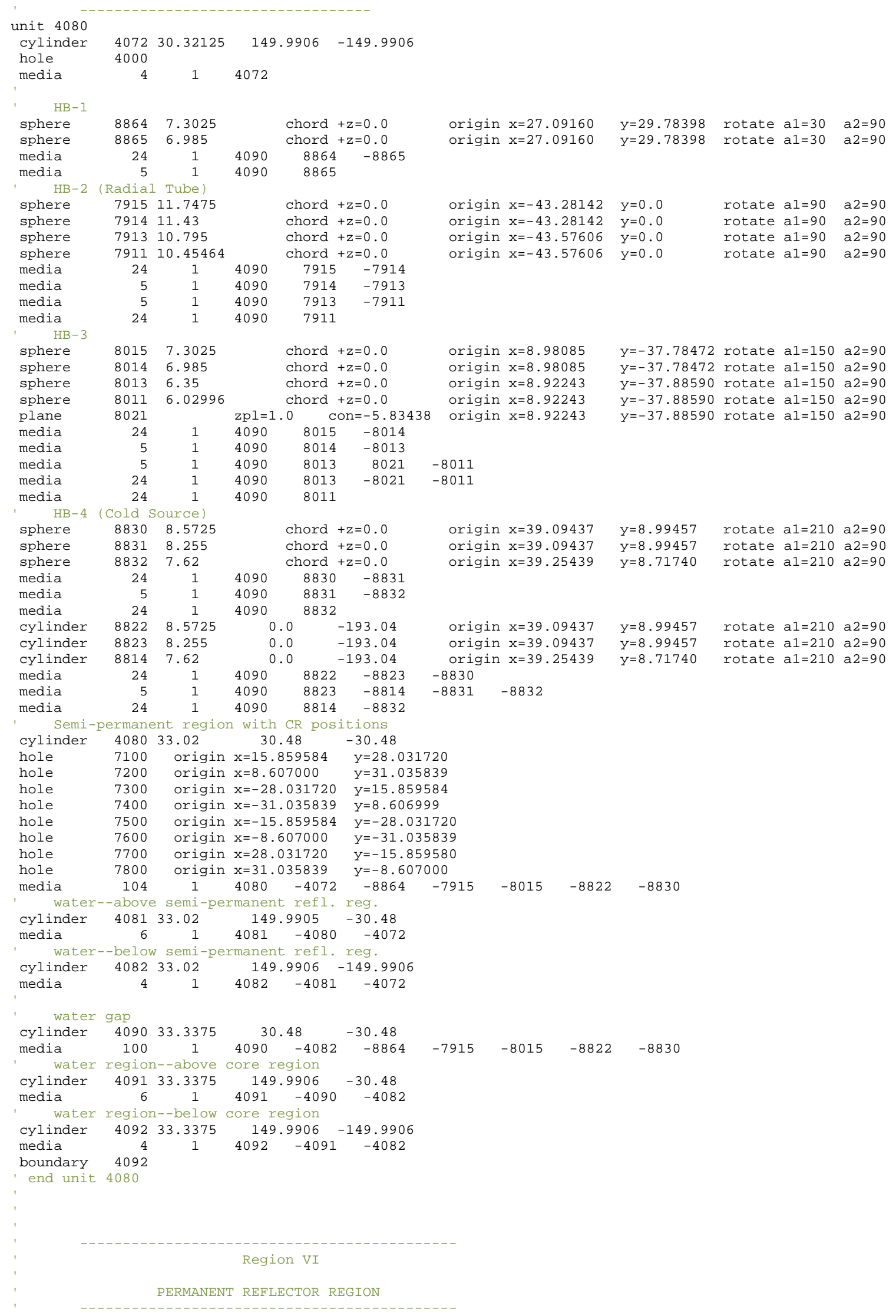




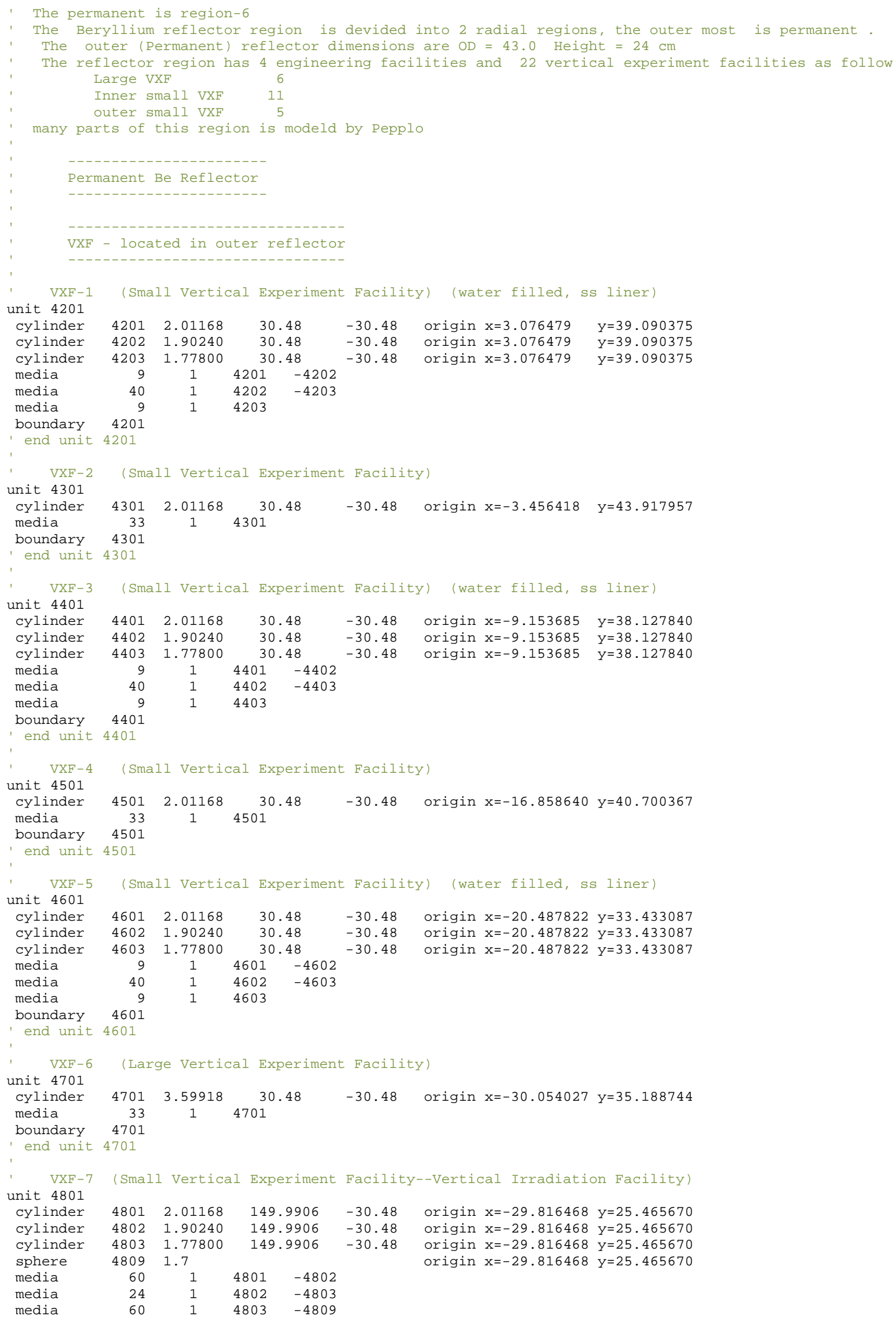




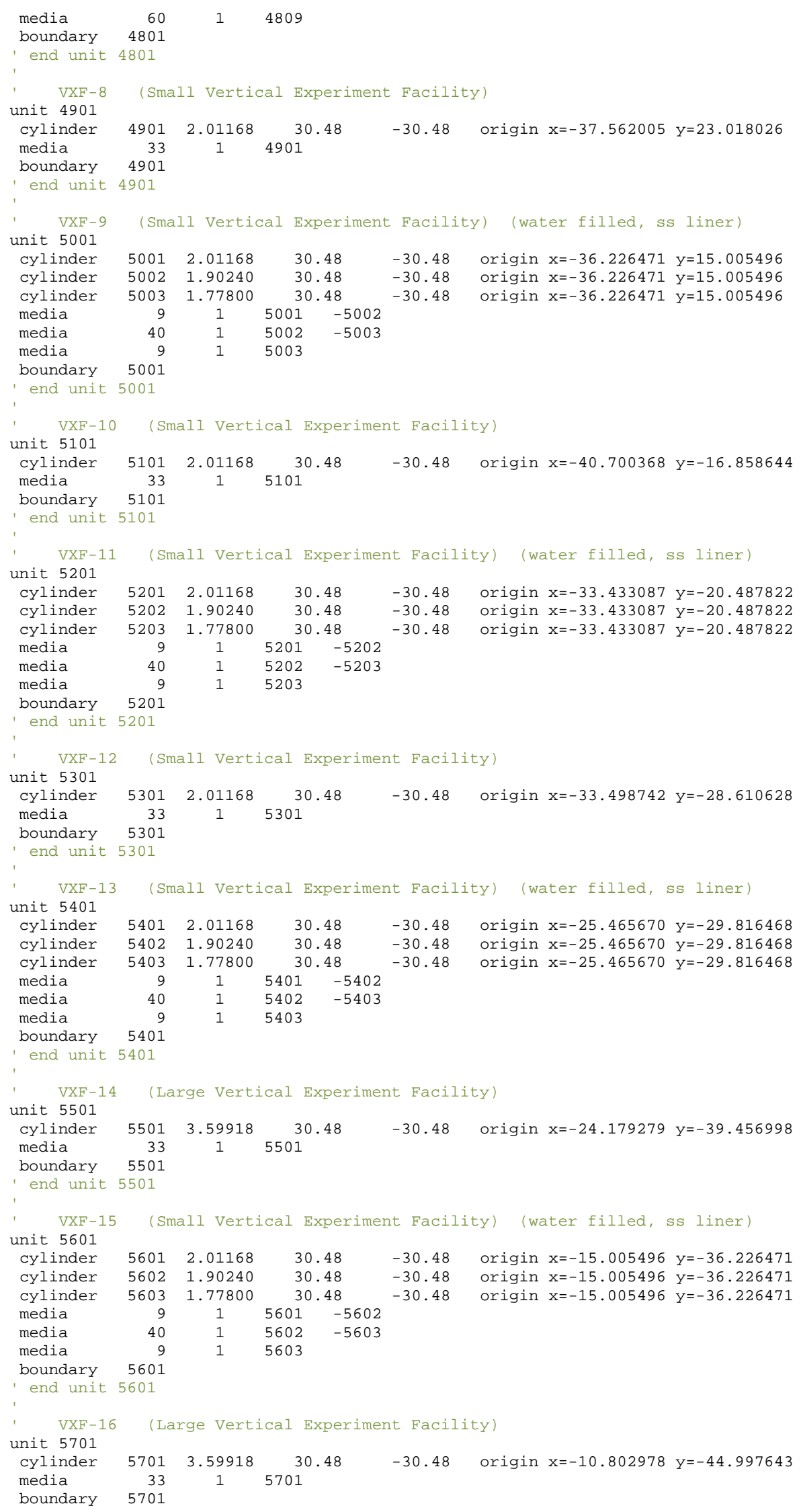




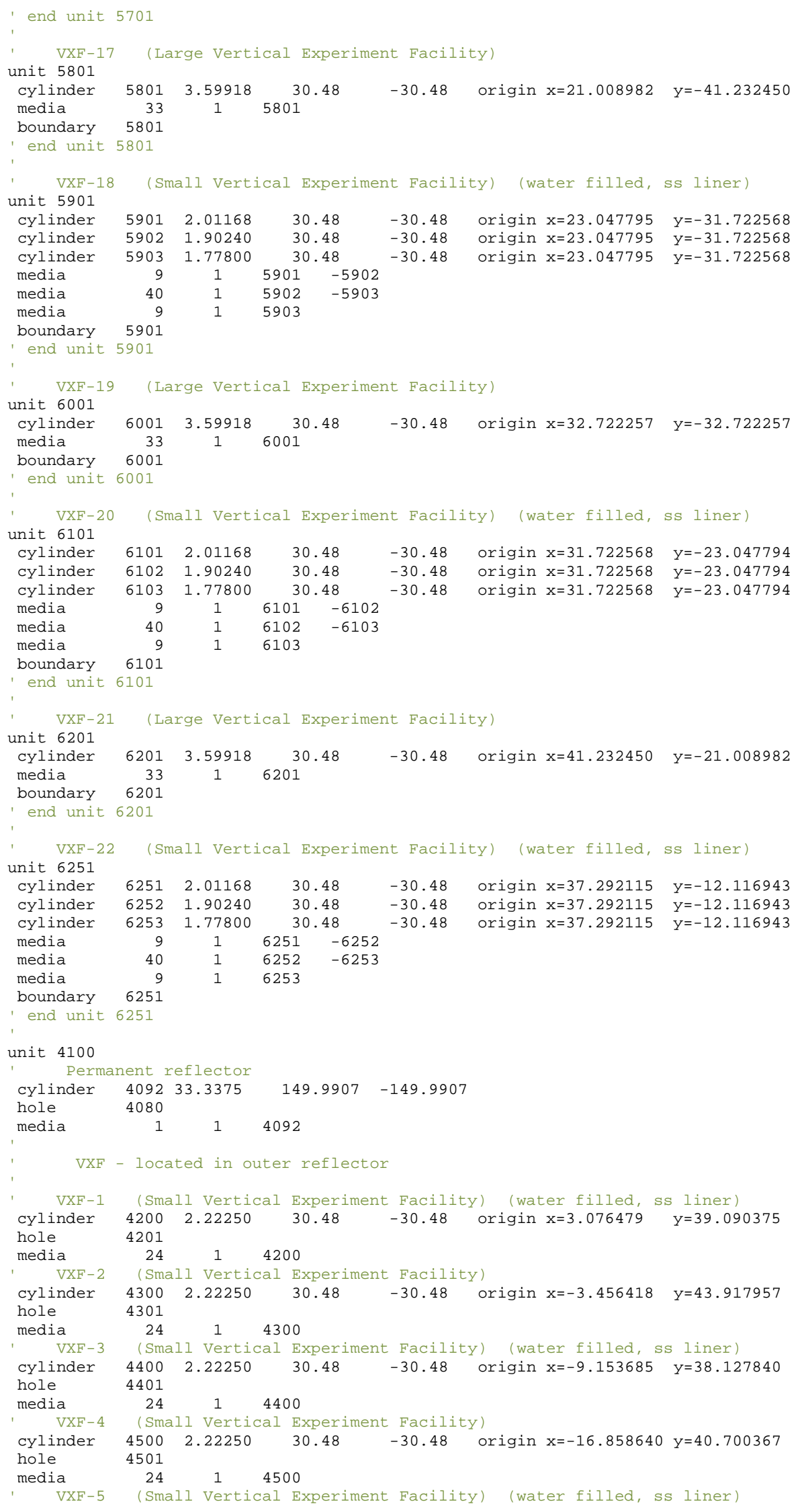




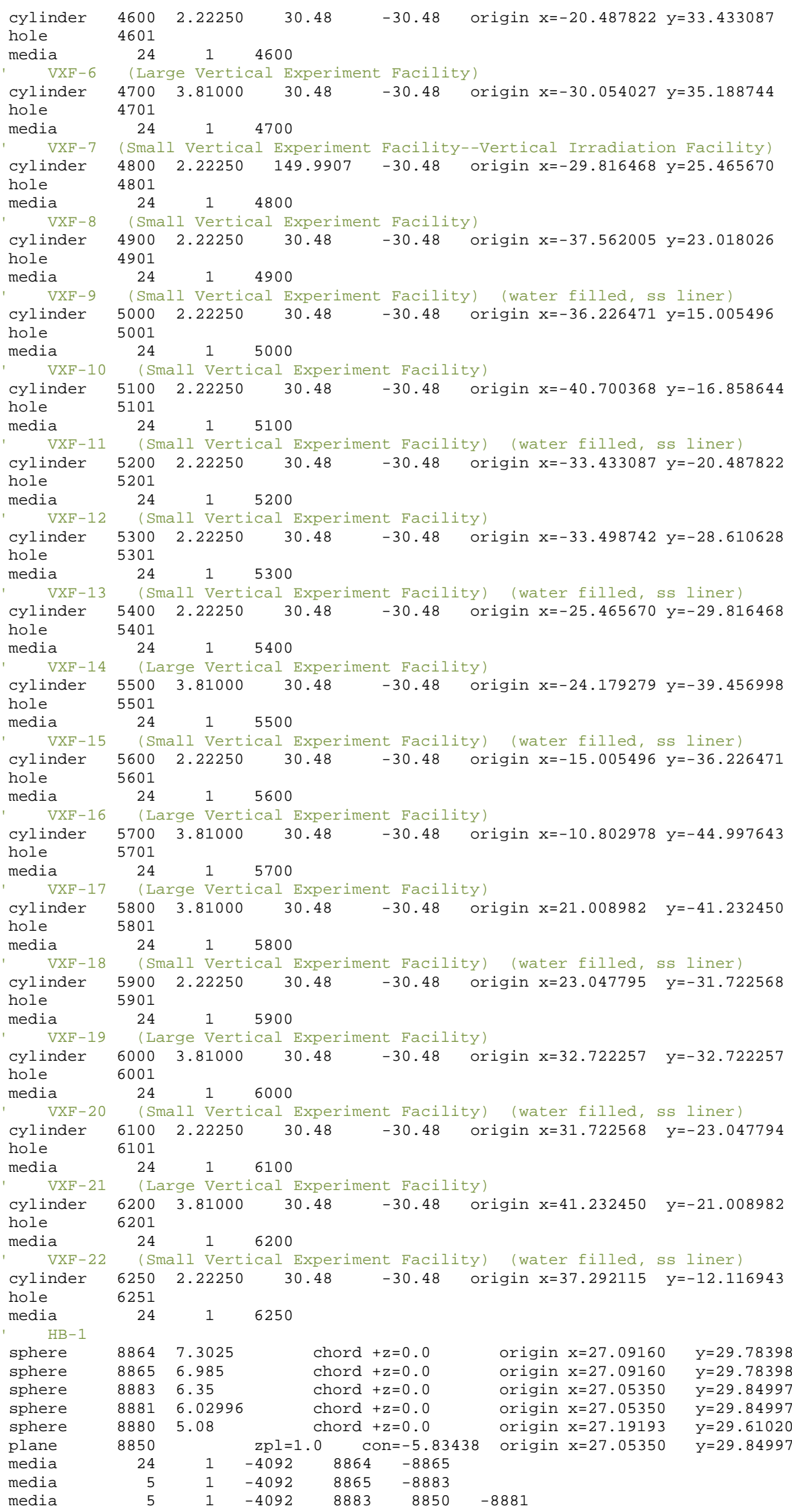

chord $+z=0.0$ chord $+z=0.0$ chord $+z=\odot$. 0 chord $+z=0.0$ 


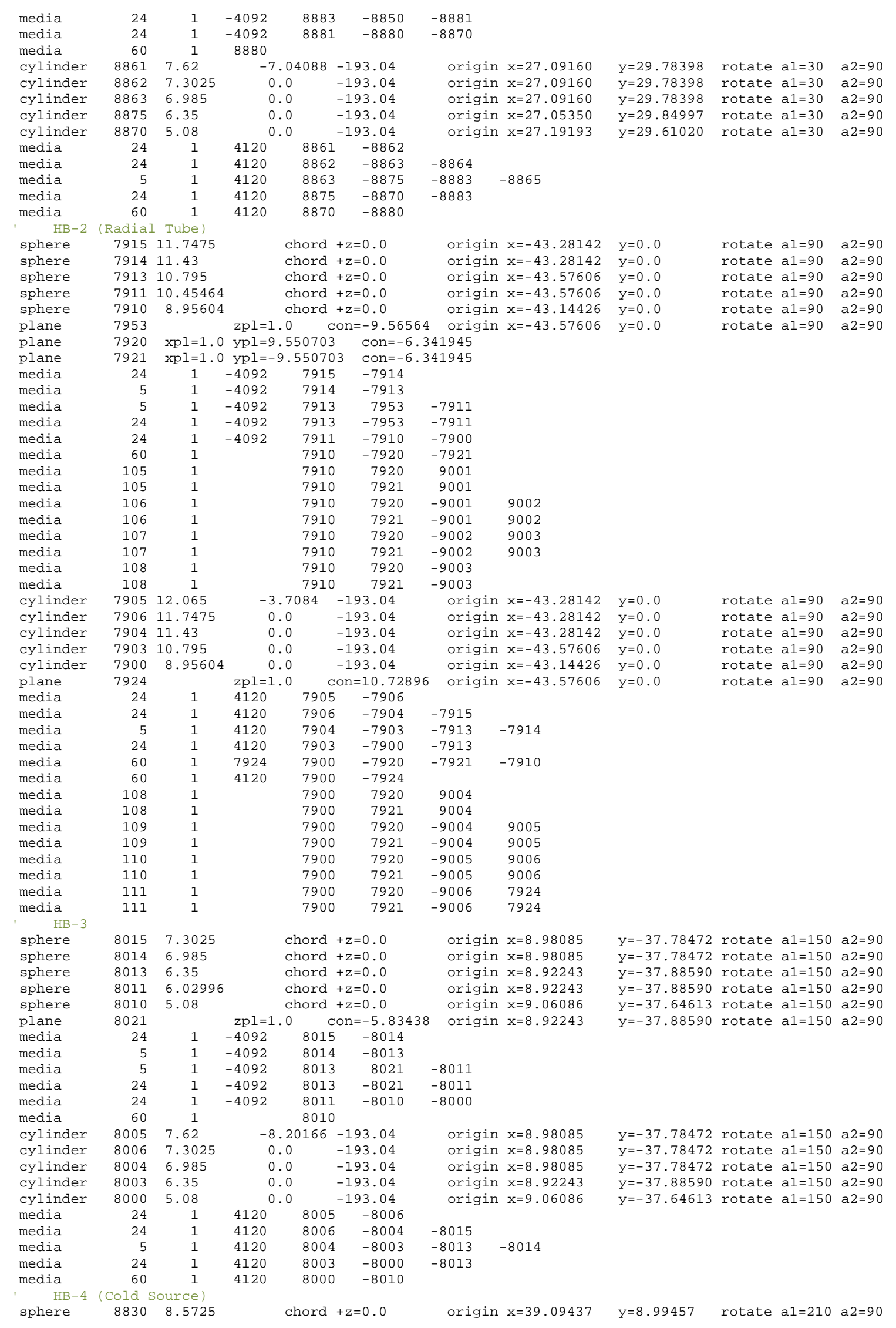




\begin{tabular}{|c|c|c|c|c|c|c|c|c|c|c|c|c|c|}
\hline sphere & 8831 & \multicolumn{2}{|l|}{8.255} & \multicolumn{3}{|c|}{ chord $+z=\odot . \odot$} & \multicolumn{3}{|c|}{ origin $x=39.09437$} & $y=8.99457$ & rotate & $\mathrm{a} 1=210$ & $a 2=9 \odot$ \\
\hline sphere & 8832 & 7.62 & & chord $+z=$ & $=\odot . \odot$ & & origin & $x=39$. & 25439 & $y=8.71740$ & rotate & $a 1=210$ & $a 2=9 \odot$ \\
\hline sphere & 8833 & 7.30250 & & chord $+z=$ & $=\odot . \odot$ & & origin & $x=39$. & 25439 & $y=8.71740$ & rotate & $a 1=210$ & a2 $=90$ \\
\hline sphere & 8835 & 5.63245 & & chord $+z=$ & $=\odot . \odot$ & & origin & $x=38$. & 99277 & $y=9.17054$ & rotate & $a 1=210$ & a2 $=90$ \\
\hline sphere & 8602 & 4.92125 & & chord $+z=$ & $=\odot . \odot$ & & origin & $x=38$. & 99277 & $y=9.17054$ & rotate & $a 1=210$ & a2 $=90$ \\
\hline sphere & 8601 & 4.67360 & & chord $+z=$ & $=0.0$ & & origin & $x=38$. & 93054 & $y=9.27833$ & rotate & $a 1=210$ & $a 2=9 \odot$ \\
\hline plane & 8841 & & $\mathrm{zpl}=1$ & $\operatorname{con}=$ & $=-5.9$ & 309 & origin & $x=39$. & 25439 & $y=8.71740$ & rotate & $a 1=210$ & a2 $=90$ \\
\hline media & 24 & 1 & -4092 & 8830 & -883 & & & & & & & & \\
\hline media & 5 & 1 & -4092 & 8831 & -883 & & & & & & & & \\
\hline media & 5 & 1 & & 8832 & 884 & & -8833 & & & & & & \\
\hline media & 24 & 1 & -4092 & 8832 & -884 & & -8833 & & & & & & \\
\hline media & 24 & 1 & -4092 & 8833 & -883 & & -8817 & & & & & & \\
\hline media & 60 & 1 & & 8835 & -860 & & & & & & & & \\
\hline media & 24 & 1 & & 8602 & -860 & & -8603 & & & & & & \\
\hline media & 560 & 1 & & 8601 & & & & & & & & & \\
\hline media & 560 & 1 & & 8602 & -860 & & 8603 & & & & & & \\
\hline cylinder & 8800 & 8.89 & -9 & 2075 & 93.04 & & origin & $x=39$. & 99437 & $y=8.99457$ & rotate & $\mathrm{a} 1=210$ & a2 $=90$ \\
\hline cylinder & 8822 & 8.5725 & & $-1 s$ & 93.04 & & origin & $x=39$. & 99437 & $y=8.99457$ & rotate & $\mathrm{a} 1=210$ & a2 $=90$ \\
\hline cylinder & 8823 & 8.255 & & -10 & 93.04 & & origin & $x=39$. & 9437 & $y=8.99457$ & rotate & $\mathrm{a} 1=210$ & $\mathrm{a} 2=90$ \\
\hline cylinder & 8814 & 7.62 & & -15 & 93.04 & & origin & $x=39$. & 25439 & $y=8.71740$ & rotate & $a 1=210$ & a2 $=90$ \\
\hline cylinder & 8817 & 5.63245 & & -15 & 93.04 & & origin & $x=38$. & 99277 & $y=9.17054$ & rotate & $a 1=210$ & a2 $=90$ \\
\hline cylinder & 8604 & 4.92125 & & -15 & 93.04 & & origin & $x=38$ & 99277 & $y=9.17054$ & rotate & $a 1=210$ & a2 $=90$ \\
\hline cylinder & 8603 & 4.67360 & & -19 & 93.04 & & origin & $x=38$. & 93054 & $y=9.27833$ & rotate & $a 1=210$ & a2 $=90$ \\
\hline cylinder & 86254 & 5.02031 & & $\odot$ & -0.93 & 98 & origin & $x=39$. & 41314 & $y=8.44244$ & rotate & $a 1=210$ & a2 $=90$ \\
\hline media & 24 & 1 & & 4120 & 880 & & -8822 & & & & & & \\
\hline media & 24 & 1 & -4092 & 4120 & 882 & & -8823 & - 8830 & & & & & \\
\hline media & 5 & 1 & -4092 & 4120 & 882 & & -8814 & -8831 & -8832 & & & & \\
\hline media & 24 & 1 & -4092 & 4120 & 881 & & -8817 & -8832 & & & & & \\
\hline media & 60 & 1 & & 8553 & 881 & & -8604 & -8835 & -86254 & & & & \\
\hline media & 24 & 1 & 8553 & -8616 & & & -8618 & 8604 & -8603 & $-86 \odot 2$ & & & \\
\hline media & 60 & 1 & 8553 & 8616 & & & & 8604 & -8603 & & & & \\
\hline media & 60 & 1 & 8553 & & & & 8618 & 8604 & -8603 & & & & \\
\hline media & 24 & 1 & & & 8625 & & -8604 & & & & & & \\
\hline media & 560 & 1 & 8626 & & & & & & 8603 & $-86 \odot 2$ & 8606 & & \\
\hline media & $56 \odot$ & 1 & 8553 & -8626 & -861 & & & -8617 & & 8603 & & -8612 & \\
\hline media & 24 & 1 & 8553 & -8626 & 861 & & -8616 & & & 8603 & & -8612 & \\
\hline media & 24 & 1 & 8553 & -8626 & & & & 8617 & -8618 & 8603 & & -8612 & \\
\hline media & $6 \odot$ & 1 & 8553 & -8626 & & & 8616 & & & 8603 & & -8612 & \\
\hline media & 60 & 1 & 8553 & -8626 & & & & & 8618 & 8603 & & -8612 & \\
\hline media & 60 & 1 & -8553 & 8629 & 881 & & -8604 & & & & & & \\
\hline media & 60 & 1 & -8553 & 8629 & & & 8604 & 86221 & -86241 & & & & \\
\hline media & 60 & 1 & -8629 & 4120 & 881 & & -8622 & -8624 & & & & & \\
\hline$\quad---\mathrm{CC}$ & ld sou & rce & & & & & & & & & & & \\
\hline ellipsoid & 8605 & 3.103880 & & 8514245 & 3.10 & 3880 & origin & $x=40$. & 76188 & $y=6.10636$ & rotate & $a 1=210$ & $a 2=9 \odot$ \\
\hline ellipsoid & 8606 & 3.403600 & & 1528462 & 3.40 & 3600 & origin & $x=40$. & 33554 & $y=5.97877$ & rotate & $a 1=210$ & $a 2=9 \odot$ \\
\hline sphere & 8607 & 3.97764 & & & & & origin & $x=40$. & 76188 & $y=6.10636$ & rotate & $a 1=210$ & a2 $=90$ \\
\hline sphere & 8608 & 4.27736 & & & & & origin & $x=40$ & 33554 & $y=5.97877$ & rotate & $a 1=210$ & $a 2=9 \odot$ \\
\hline plane & 8626 & & $\mathrm{zpl}=1$ & & & & origin & $x=40$. & 76188 & $y=6.10636$ & rotate & $a 1=210$ & a2 $=9 \odot$ \\
\hline media & 60 & 1 & & 8626 & $86 \odot$ & & 8607 & & & & & & \\
\hline media & 24 & 1 & & 8626 & 860 & & 8608 & -8605 & 8607 & & & & \\
\hline media & 24 & 1 & & 8626 & 860 & & 8608 & & -8607 & & & & \\
\hline media & 560 & 1 & & 8626 & 860 & & -8608 & 8603 & & & & & \\
\hline ecylinder & 8611 & 3.103880 & & 8514245 & 1. & $-99 . \odot$ & $\odot$ origin & $x=40$. & 76188 & $y=6.10636$ & rotate & $\mathrm{a} 1=210$ & $a 2=90$ \\
\hline ecylinder & 8612 & 3.403600 & & 1528462 & 1. & -99.0 & $\odot$ origin & $x=40$. & 33554 & $y=5.97877$ & rotate & $a 1=210$ & a2 $=90$ \\
\hline cylinder & 8609 & 3.97764 & & & 1. & -99.0 & $\odot$ origin & $x=40$. & 76188 & $y=6.10636$ & rotate & $a 1=210$ & a2 $=90$ \\
\hline cylinder & 8610 & 4.27736 & & & 1. & -99.0 & $\odot$ origin & $x=40$. & 33554 & $y=5.97877$ & rotate & $a 1=210$ & a2 $=90$ \\
\hline cylinder & 8627 & 2.2225 & & & $99 . \odot$ & -99.0 & $\odot$ origin & $x=42$. & 51829 & $y=3.06416$ & rotate & $a 1=120$ & a2 $=90$ \\
\hline cylinder & 86271 & 7.12216 & & & $99 . \odot$ & -99.0 & $\odot$ origin & $x=29$. & 56403 & $y=17.06880$ & rotate & $a 1=210$ & $a 2=9 \odot$ \\
\hline cylinder & 86272 & 7.12216 & & & $99 . \odot$ & -99.0 & $\odot$ origin & $x=36$. & 36705 & $y=21.28520$ & rotate & $a 1=210$ & a2 $=90$ \\
\hline plane & 8553 & & $\mathrm{zpl}=1$ & & & & origin & $x=44$. & 64554 & $y=-0.62034$ & rotate & $a 1=210$ & a2 $=9 \odot$ \\
\hline plane & 8615 & $x p l=0.5$ & $z p l=0$ & 8660254 & $\operatorname{con}=$ & -11.9 & 97937 or $i$ & igin $x$ & $=33.21554$ & $4 y=19.177$ & rotate & $a 1=300$ & \\
\hline plane & 8616 & $\mathrm{xpl}=0.5$ & $z p l=0$ & 8660254 & $\operatorname{con}=$ & -12.2 & 27909 ori & igin $x$ & $=33.21554$ & $4 y=19.177$ & rotate & $a 1=300$ & \\
\hline plane & 8617 & $\mathrm{xpl}=0.5$ & $\mathrm{zpl}=-$ & .8660254 & con $=$ & -11.9 & 97937 ori & igin $x$ & $=33.21554$ & $4 y=19.177$ & rotate & $a 1=300$ & \\
\hline plane & 8618 & $\mathrm{xpl}=0.5$ & $\mathrm{zpl}=-$ & .8660254 & con $=$ & -12.2 & 27909 ori & igin $x$ & $=33.21554$ & $4 y=19.177$ & rotate & $a 1=300$ & \\
\hline plane & 8629 & & $\mathrm{zpl}=1$ & & & & origin & $x=45$. & 91554 & $y=-2.82005$ & rotate & $\mathrm{a} 1=210$ & $a 2=90$ \\
\hline cylinder & 8621 & 0.635 & & & $99 . \odot$ & -99.0 & $\odot$ origin & $x=36$. & 71307 & $y=21.19630$ & rotate & $a 1=210$ & a2 $=90$ \\
\hline cylinder & 8622 & $\odot .71501$ & & & $99 . \odot$ & -99.0 & $\odot$ origin & $x=36$. & 71307 & $y=21.19630$ & rotate & $a 1=210$ & a2 $=90$ \\
\hline cone & 86221 & 1.0807 & 3.5 & 0.7 & 70456 & -0.1 & 1 origin & $x=49$. & 41307 & $y=-0.80075$ & rotate & $a 1=210$ & a2 $=9 \odot$ \\
\hline cylinder & 8623 & 0.635 & & & $99 . \odot$ & $-99 . \odot$ & $\odot$ origin & $x=29$. & 71801 & $y=17.15770$ & rotate & $a 1=210$ & $a 2=9 \odot$ \\
\hline cylinder & 8624 & $\odot .71501$ & & & 99.0 & -99.0 & $\odot$ origin & $x=29$. & 71801 & $y=17.15770$ & rotate & $a 1=210$ & a2 $=90$ \\
\hline cone & 86241 & 1.0807 & 3.5 & 0.7 & 70456 & -0.1 & 1 origin & $x=42$. & 41801 & $y=-4.83935$ & rotate & $\mathrm{a} 1=210$ & $\mathrm{a} 2=90$ \\
\hline media & 60 & 1 & 8553 & -8626 & 861 & & 8609 & -8627 & & & & & \\
\hline media & 60 & 1 & 8553 & -8626 & 861 & & 8609 & 8627 & 86271 & 86272 & & & \\
\hline media & 20 & 1 & 8553 & -8626 & & & -8616 & & -8618 & 8612 & 8610 & -8611 & 8609 \\
\hline media & 60 & 1 & 8553 & -8626 & & & 8616 & & & 8612 & 8610 & -8611 & 8609 \\
\hline media & 60 & 1 & 8553 & -8626 & & & & & 8618 & 8612 & 8610 & -8611 & 8609 \\
\hline media & 20 & 1 & 8553 & -8626 & & & -8616 & & -8618 & 8612 & 8610 & & -8609 \\
\hline media & 60 & 1 & 8553 & -8626 & & & 8616 & & & 8612 & 8610 & & -8609 \\
\hline media & 60 & 1 & 8553 & -8626 & & & & & 8618 & 8612 & 8610 & & -8609 \\
\hline
\end{tabular}




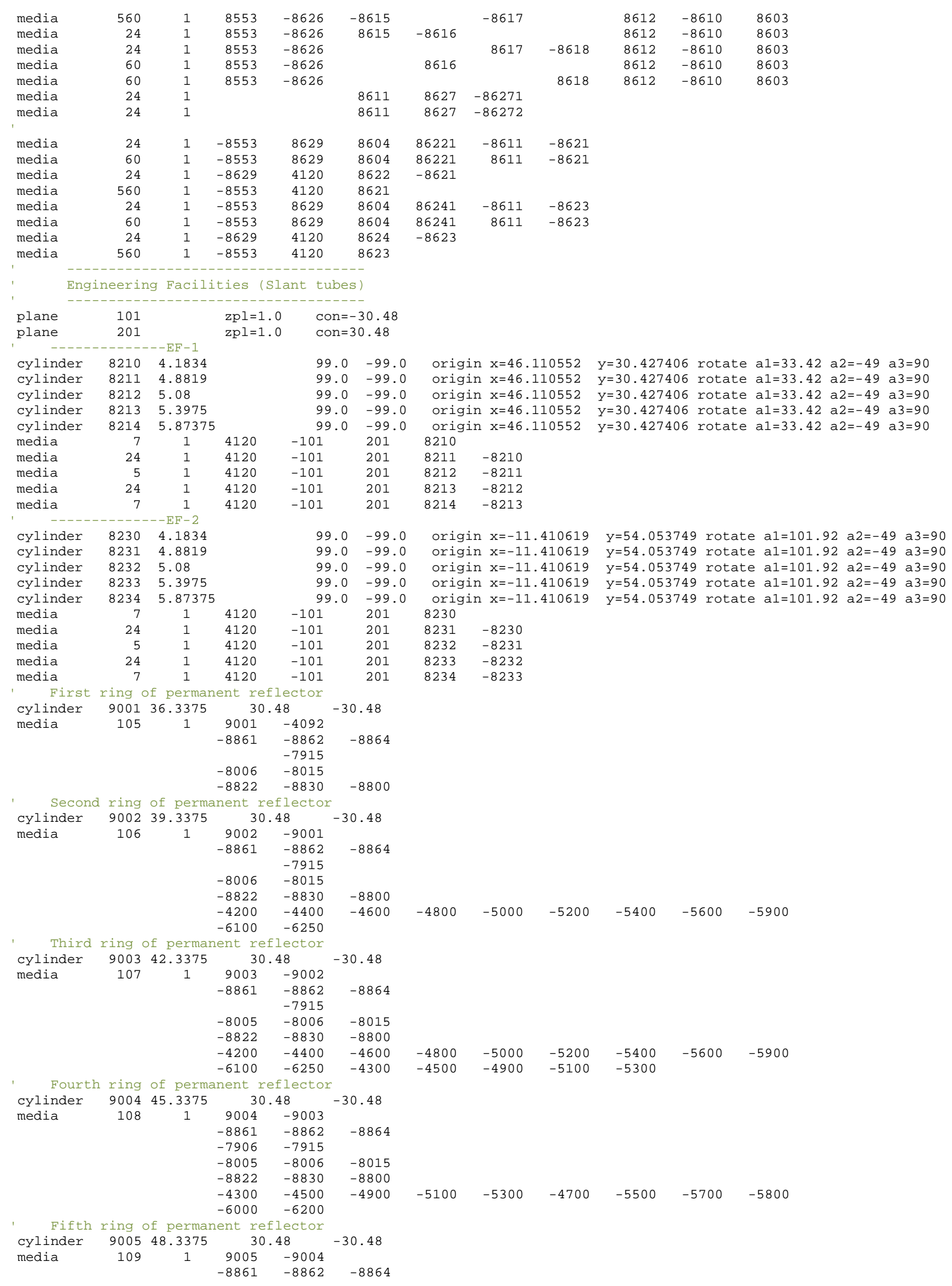




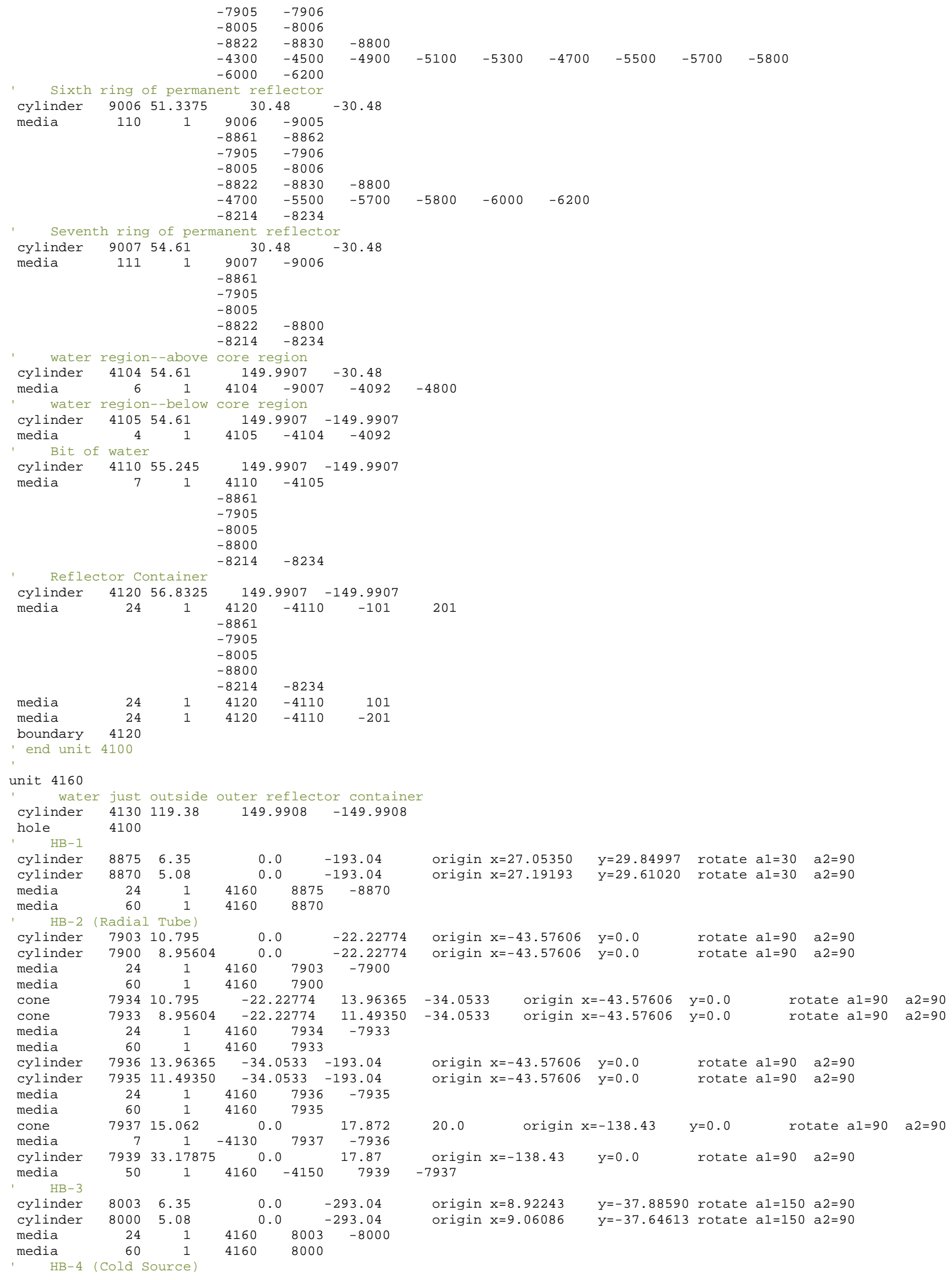




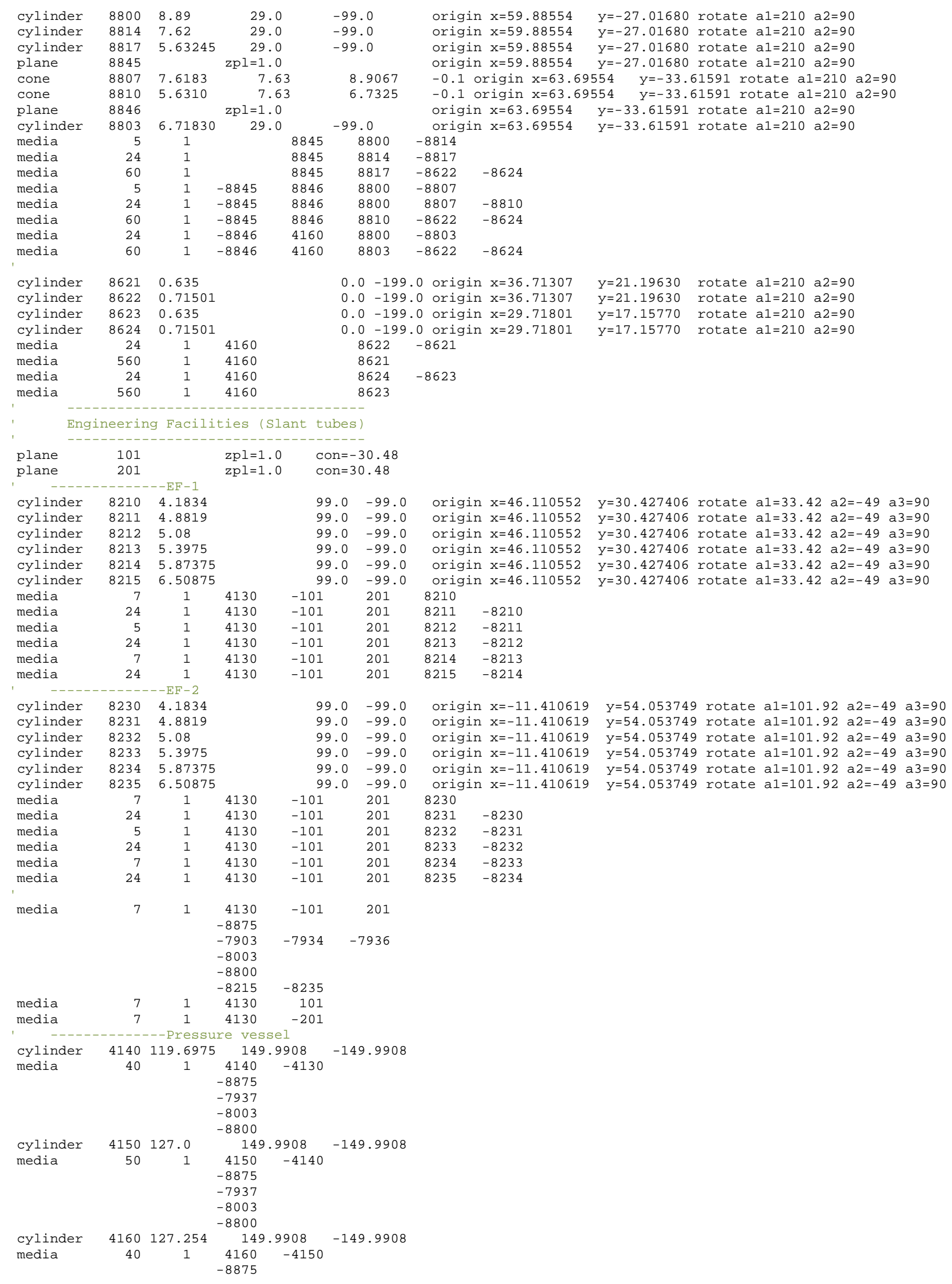




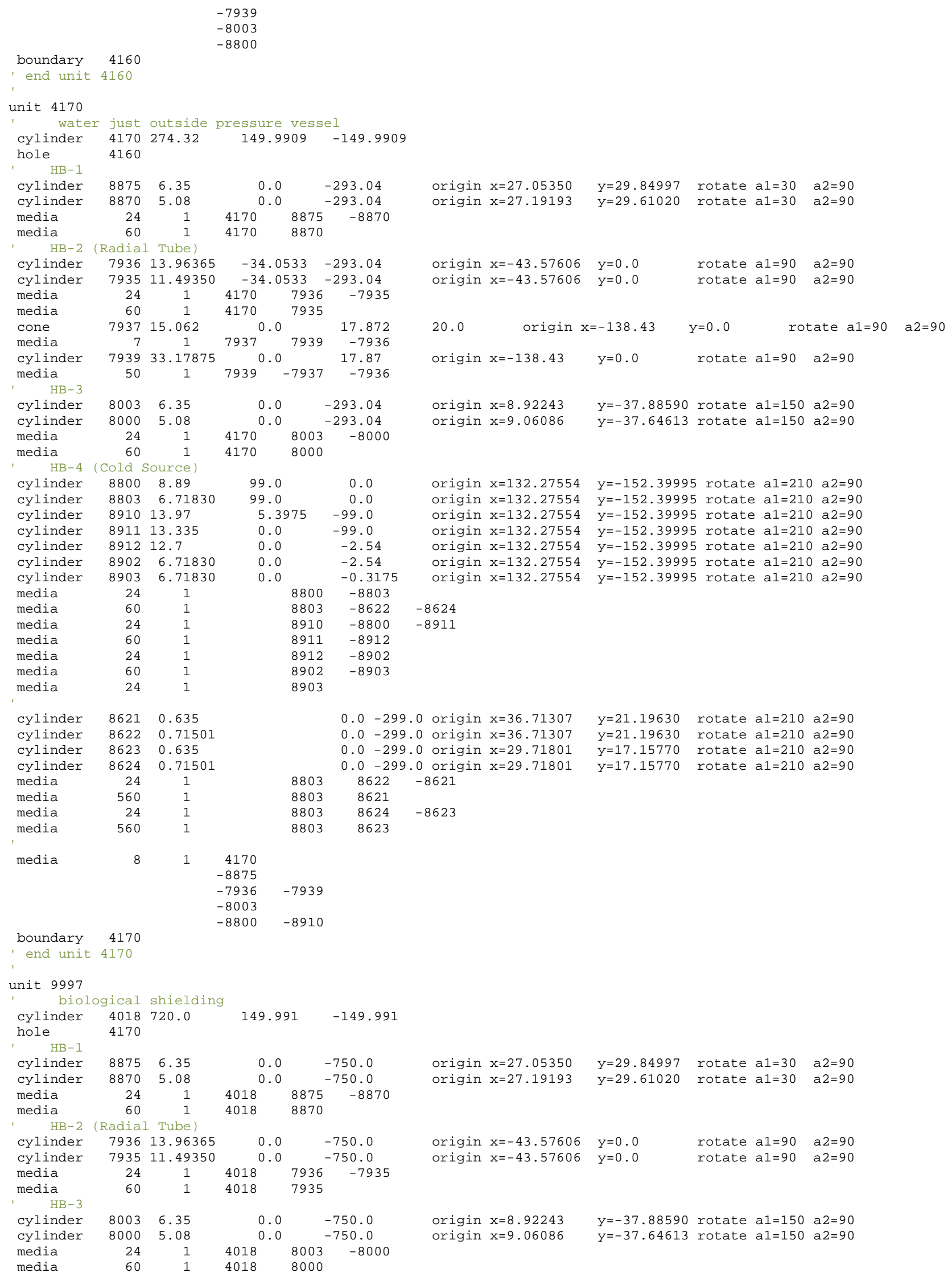




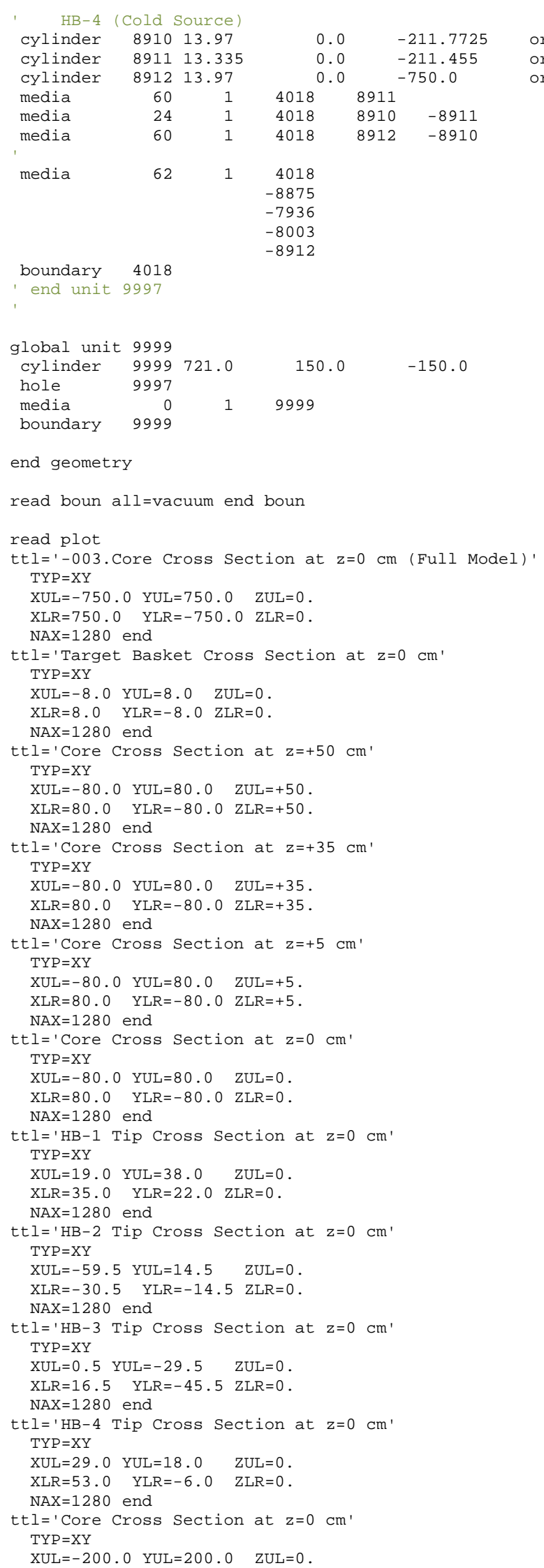


$X L R=20 \odot . \odot \quad Y L R=-20 \odot . \odot Z L R=\odot$.

$\mathrm{NAX}=1280$ end

tt $l=$ 'Core Cross Section at $z=-5 \mathrm{~cm}$ '

TYP $=X Y$

$X U L=-8 \odot . \odot \quad Y U L=8 \odot . \odot \quad Z U L=-5$.

$X L R=80, \odot \quad Y L R=-80, \odot \quad Z L R=-5$.

$\mathrm{NAX}=1280$ end

$\mathrm{ttl}=$ 'Core Cross Section at $z=-35 \mathrm{~cm}$ '

$\mathrm{TYP}=\mathrm{XY}$

$X U L=-80, \odot \quad Y U L=80, \odot \quad Z U L=-35$.

$X L R=80 . \odot \quad Y L R=-80 . \odot \quad Z L R=-35$.

$N A X=1280$ end

ttl $=$ 'Core Cross Section at $z=-50 \mathrm{~cm}$ '

$\mathrm{TYP}=\mathrm{XY}$

$X U L=-80 . \odot \quad Y U L=80 . \odot \quad Z U L=-50$.

$X L R=8 \odot . \odot \quad Y L R=-8 \odot . \odot \quad Z L R=-5 \odot$.

$N A X=1280$ end

ttl='Vertical View of the HFIR Core, $y=\odot \mathrm{cm}$ '

TYP $=X Z$

$X U L=-8 \odot . \odot \quad Y U L=\odot . \quad Z U L=151$

$X L R=8 \odot . \odot \quad Y L R=\odot ， Z L R=-151$

$U A X=1 . \odot W D N=-1 . \odot$ NAX $=1280$ end

ttl='Vertical View of the HFIR Core, $y=7.296944 \mathrm{~cm}$ ' $\mathrm{TYP}=\mathrm{XZ}$

$X U L=-80 . \odot \quad Y U L=7.296944 \quad Z U L=151$

$X L R=80 . \odot \quad Y L R=7.296944 \quad Z L R=-151$

$U A X=1 . \odot$ WDN $=-1 . \odot$ NAX $=1280$ end

$\mathrm{ttl}=$ 'Vertical View of the HFIR Core, $y=13.445625 \mathrm{~cm}$ ' TYP $=X Z$

$X U L=-80 . \odot \quad Y U L=13.445625 \quad Z U L=151$.

$X L R=80 . \odot \quad Y L R=13.445625 \quad Z L R=-151$

$U A X=1 . \odot W D N=-1 . \odot N A X=1280$ end

ttl='Vertical View of the HFIR Core, $y=23.765063 \mathrm{~cm}$ ' TYP $=X Z$

$X U L=-80.0 \quad Y U L=23.765063 \quad Z U L=151$

$X L R=80 . \odot \quad Y L R=23.765063 \quad Z L R=-151$

$U A X=1 . \odot W D N=-1 . \odot$ NAX $=1280$ end

ttl='Vertical View of the HFIR Core, $y=26.311930 \mathrm{~cm}$ '

TYP $=X Z$

$X U L=-80 . \odot \quad Y U L=26.311930 \quad Z U L=151$

$X L R=80 . \odot \quad Y L R=26.311930 \quad Z L R=-151$.

$U A X=1 . \odot W D N=-1 . \odot \quad N A X=1280$ end

end plot

end data

end 



\section{INTERNAL DISTRIBUTION}

1. B. J. Ade (adebj@ornl.gov)

2. J. L. Binder (binderjl@ornl.gov)

3. S. M. Bowman (bowmansm@ornl.gov)

4. B. L. Broadhead (broadheadbl@ornl.gov)

5. C. Bryan (bryancd@ornl.gov)

6. D. Chandler (chandlerd@ornl.gov)

7. D.H. Cook (cookdh@ornl.gov)

8. R.A. Crone (cronera@ornl.gov)

9. M.E. Dunn (dunnme@ornl.gov)

10. R.J. Ellis (ellisrj@ornl.gov)

11. J.D. Freels (freelsjd@ornl.gov)

12. I.C. Gauld (gauldi@ornl.gov)

13. J.C. Gehin (gehinjc@ornl.gov)

14. R.E. Grove (grovere@ornl.gov)

15. G.J. Hirtz (hirtzgj@ornl.gov)

16. R.W. Hobbs (hobbsrw@ornl.gov)

17. A.S. Icenhour (icenhouras@ornl.gov)

18. D. Ilas (ilasd@ornl.gov)
19. G. Ilas (ilasg@ornl.gov)

20. M.A. Jessee (jesseema@ornl.gov)

21. G.I. Maldonado (maldonadogi@ornl.gov)

22. G.T. Mays (maysgt@ornl.gov)

23. J.L. McDuffee (mcduffeej@ornl.gov)

24. C.V. Parks (parkscv@ornl.gov)

25. J.L. Peterson (petersonjl@ornl.gov)

26. T. Powers (powerstp@ornl.gov)

27. B.T. Rearden (reardenb@ornl.gov)

28. I. Remec (remeci@ornl.gov)

29. J.M. Risner (risnerjm@ornl.gov)

30. K.A. Smith (smithka@ornl.gov)

31. T.E. Valentine (valentinete@ornl.gov)

32. J.C. Wagner (wagnerjc@ornl.gov)

33. M.L. Williams (williamsml@ornl.gov)

34. G.L. Yoder (yodergljr@ornl.gov)

35. S.J. Zinkle (zinklesj@ornl.gov)

36. ORNL Laboratory Records (hamrindr@ornl.gov)

None 